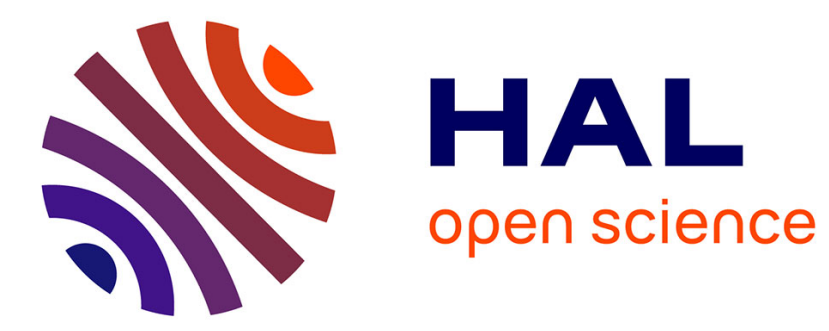

\title{
Adsorption and self-assembly of bio-organic molecules at model surfaces: A route towards increased complexity
}

Dominique Costa, Claire-Marie Pradier, Frederik Tielens, Letizia Savio

\section{To cite this version:}

Dominique Costa, Claire-Marie Pradier, Frederik Tielens, Letizia Savio. Adsorption and self-assembly of bio-organic molecules at model surfaces: A route towards increased complexity. Surface Science Reports, 2015, 70 (4), pp.449-553. 10.1016/j.surfrep.2015.10.002 . hal-01275043

\section{HAL Id: hal-01275043 \\ https://hal.science/hal-01275043}

Submitted on 5 Sep 2016

HAL is a multi-disciplinary open access archive for the deposit and dissemination of scientific research documents, whether they are published or not. The documents may come from teaching and research institutions in France or abroad, or from public or private research centers.
L'archive ouverte pluridisciplinaire HAL, est destinée au dépôt et à la diffusion de documents scientifiques de niveau recherche, publiés ou non, émanant des établissements d'enseignement et de recherche français ou étrangers, des laboratoires publics ou privés. 


\title{
Adsorption and Self-Assembly of Bio-Organic Molecules at Model Surfaces: A Route towards Increased Complexity
}

\author{
Dominique Costa $^{1}$, Claire Marie Pradier $^{2}$, Frederik Tielens ${ }^{3}$ and Letizia Savio ${ }^{4 *}$
}

\author{
${ }^{1}$ Institut de Recherches de Chimie de Paris UMR 8247 ENSCP Chimie Paristech, \\ 11 rue P. Et M. Curie, 75005 Paris, France. \\ ${ }^{2}$ Sorbonne Université, UPMC Univ Paris 06, UMR CNRS 7197, Laboratoire de Réactivité de \\ Surface, 4 Place Jussieu, 75231-Paris cedex 05, France. \\ ${ }^{3}$ Sorbonne Université, UPMC Univ Paris 06, UMR 7574, Laboratoire Chimie de la Matière \\ Condensée, Collège de France, 11 place Marcelin Berthelot, 75231 Paris Cedex 05, France \\ ${ }^{4}$ Istituto dei Materiali per l'Elettronica e il Magnetismo, Consiglio Nazionale delle Ricerche, \\ U.O.S. Genova, Via Dodecaneso 33, 16146 Genova, Italy.
}

*corresponding author: letizia.savio@imem.cnr.it

\begin{abstract}
Understanding the bio-physical-chemical interactions at nanostructured biointerfaces and the assembling mechanisms of so-called hybrid nano-composites is nowadays a keyissue for nanoscience in view of the many possible applications foreseen.

The contribution of surface science in this field is noteworthy since, using a bottom-up approach, it allows the investigation of the fundamental processes at the basis of complex interfacial phenomena and thus it helps to unravelthe elementary mechanisms governing them.

Nowadays it is well demonstrated that a wide variety of different molecular assemblies can form upon adsorption of small biomolecules at surfaces. The geometry of such self-organized structures can often be tuned by a careful control of the experimental conditions during the deposition process. Indeed an impressive number of studies exist (both experimental and - to a lesser extend theoretical), which demonstrate the ability of molecular self-assembly to create different structural motifs in a more or less predictable manner, by tuning the molecular building blocks as well as the metallic substrate.

In this frame, amino acidsand small peptides at surfaces are key, basic, systems to be studied. The amino acidsstructure is simple enough to serve as a model for the chemisorption of biofunctional molecules, but their adsorption at surfaces has applications in surface functionalization, in enantiospecific catalysis, biosensing, nanoparticles shape control or in emerging fields such as "green" corrosion inhibition.

In this paper we review the most recent advancements in this field. We will start from amino acids adsorption at metal surfaces and we will evolve then in the direction of more complex systems, in thelight of the latest improvements of surface science techniques and of computational methods. On one side, we will focus on amino acids adsorption at oxide surfaces, on the other on peptide adsorption both at metal and oxide substrates. Particular attention will be drawn to the added value provided by the combination of several experimental surface science techniques and to the precious contribution of advanced complementary computational methods to resolve the details of systems of increased complexity. Finally, some hints into experiments performed in the presence of water and then characterized in UHV and in the related theoretical work will be presented. This is a further step towards abetter approximation of real biological systems. However, since the methods employed are often not typical of surface science, this topic is not developed in details.
\end{abstract}




\section{Table of Contents}

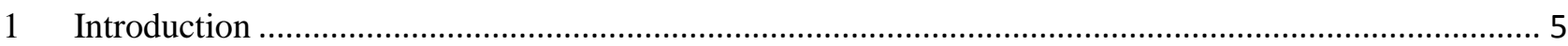

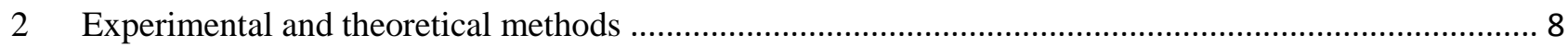

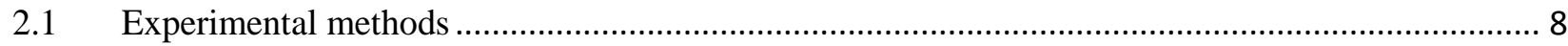

2.1.1 Amino acid species and deposition techniques ............................................................... 8

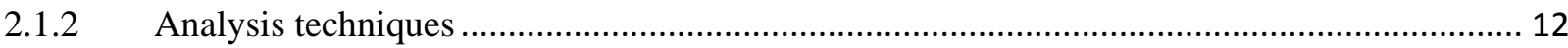

2.1.2.1 Desorption techniques:Temperature Programmed Desorption (TPD) and Thermal

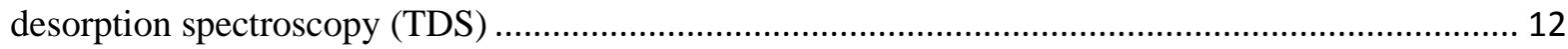

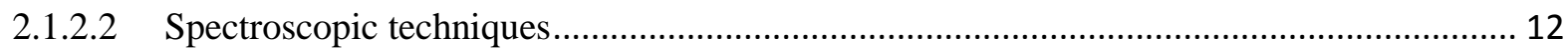

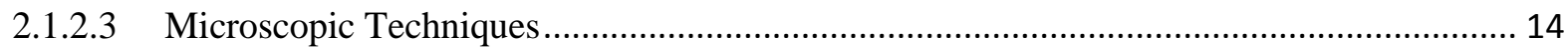

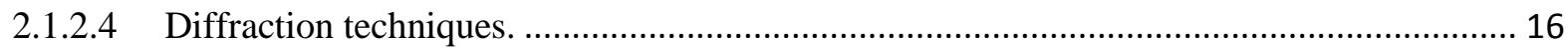

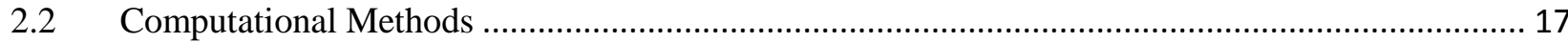

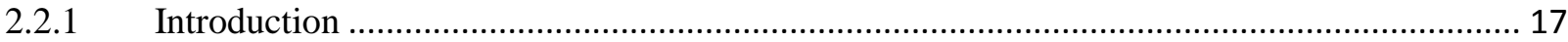

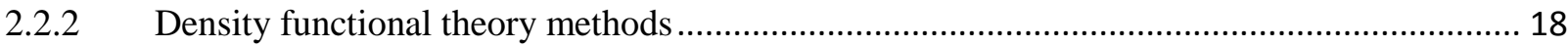

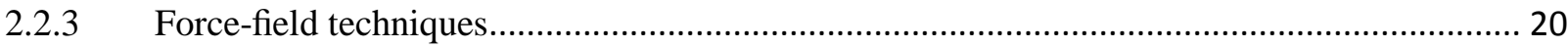

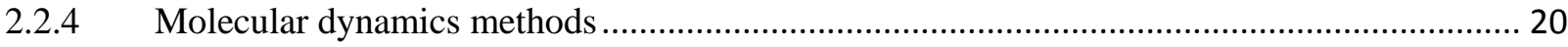

2.2.5 Joined experimental and theoretical studies .................................................................... 21

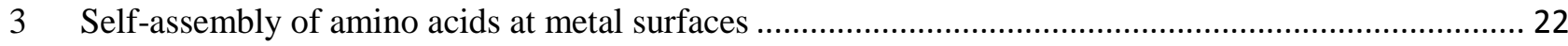

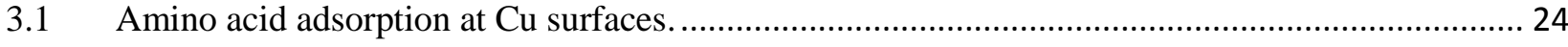

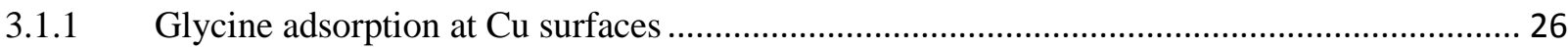

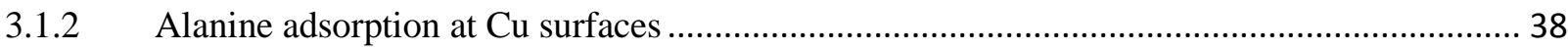

3.1.3 Amino acids with a linear side chain and one additional functional group. ........................... 46

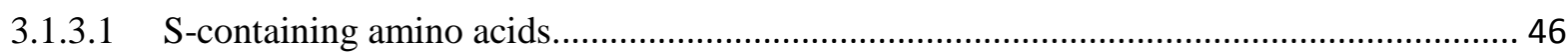

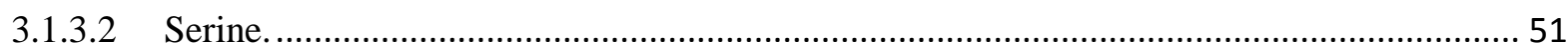

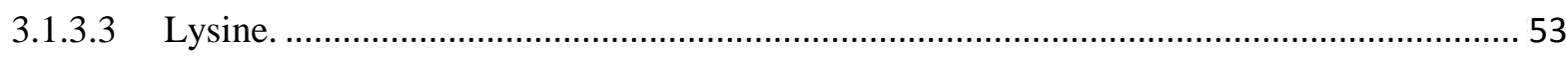

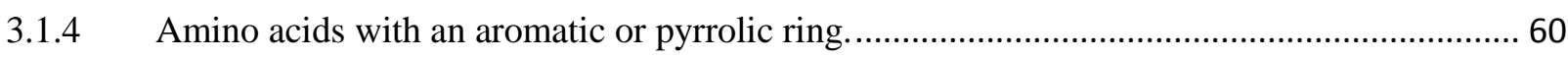

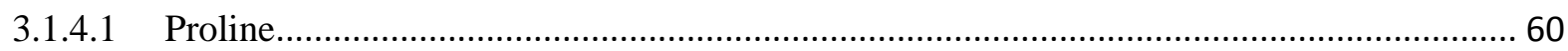

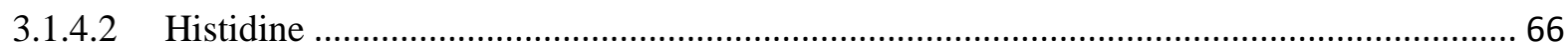

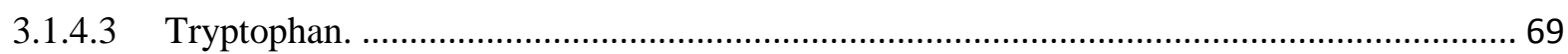

3.1.5 Amino acid adsorption on stepped and chiral $\mathrm{Cu}$ surfaces.................................................. 71

3.1.5.1 Step faceting and bunching of LMI surfaces upon amino acid adsorption.......................... 72

3.1.5.2 Amino acid adsorption at intrinsically chiral metal surfaces............................................ 74

3.2 Amino acid interaction with poorly reactive surfaces …....................................................... 80

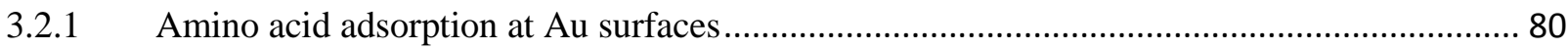

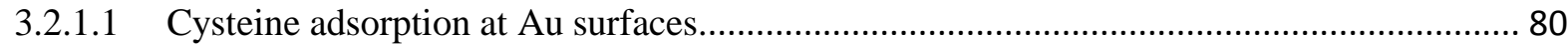




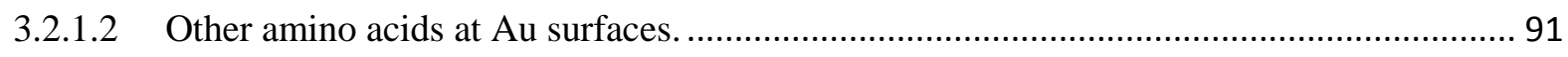

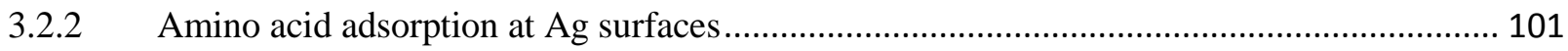

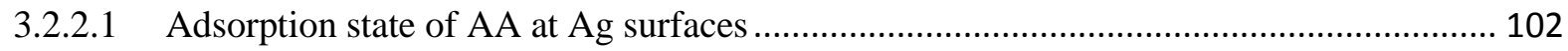

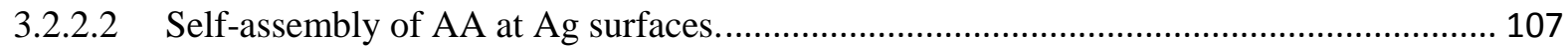

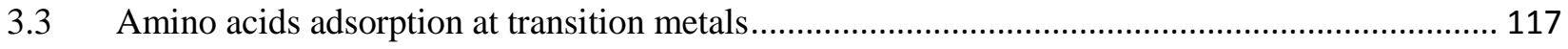

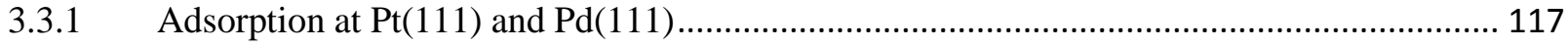

3.3.2 Amino acids as chiral modifiers: the case of Glutamic acid at Ni and Ni/Au surfaces......... 124

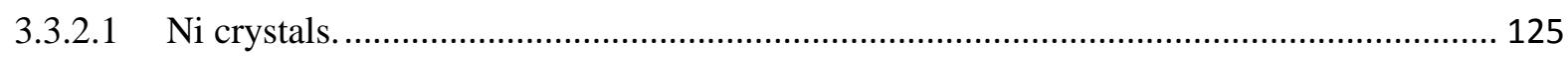

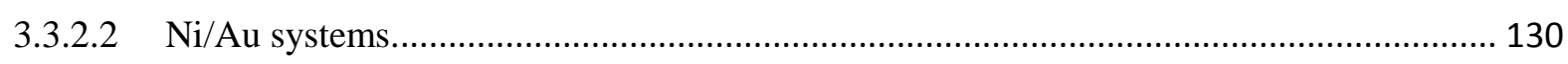

3.3.3 Adsorption of other amino acids at Ni surfaces.............................................................. 132

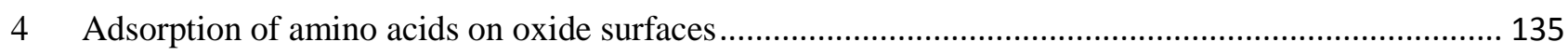

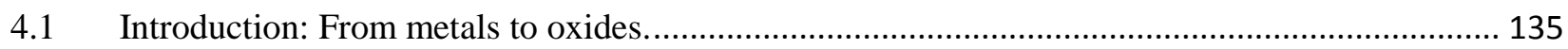

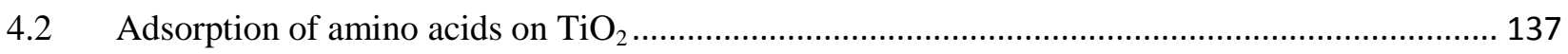

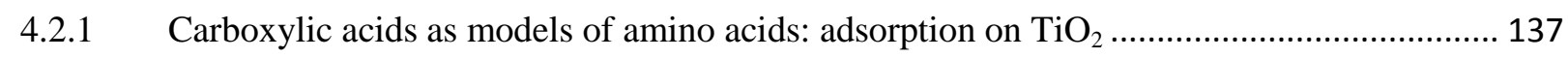

4.2.2 Amines as models of amino acids adsorbed on $\mathrm{TiO}_{2}$...................................................... 139

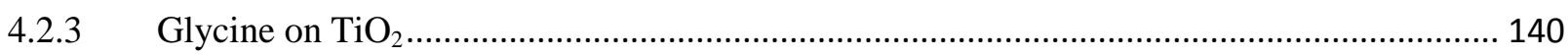

4.2.4 Glycine and water on $\mathrm{TiO}_{2}$ from the gas phase …....................................................... 143

4.2.5 Other amino acids adsorbed on $\mathrm{TiO}_{2}$ single crystals from the gas phase ............................. 145

4.3 Adsorption of amino acids on other oxides ............................................................................... 150

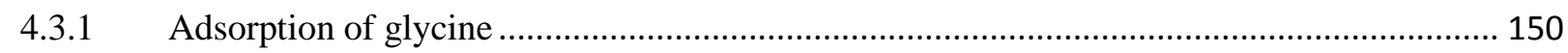

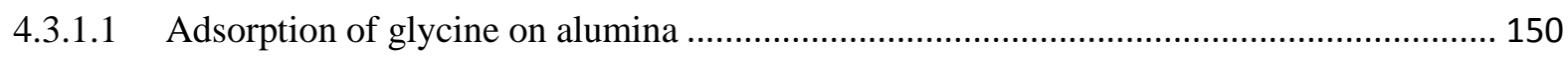

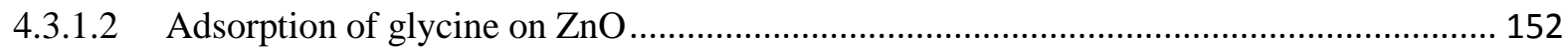

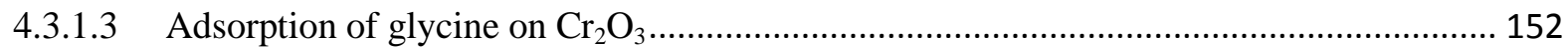

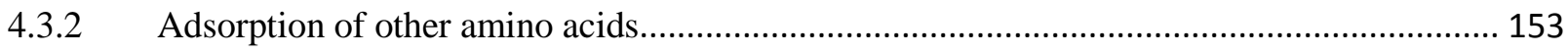

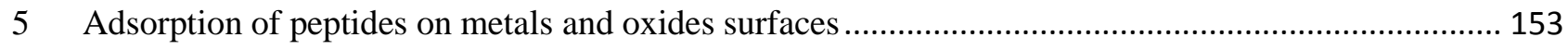

$5.1 \quad$ Why studying peptides at metals and oxides surfaces? ......................................................... 153

5.2 Systems of very low complexity (short peptides on single crystal surfaces, adsorbed under UHV

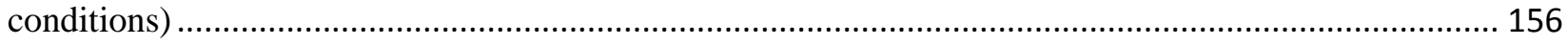

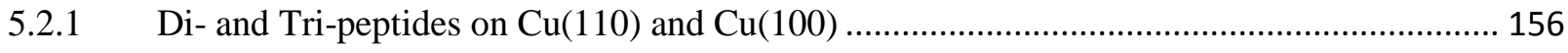

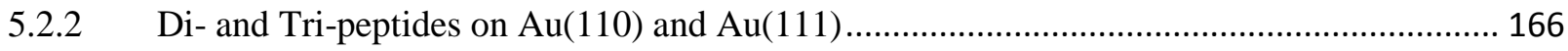

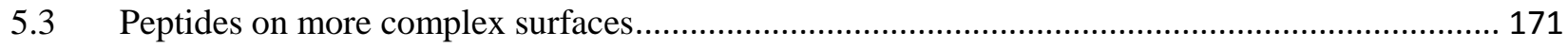

5.3.1 Adsorption of peptides on oxides from the gas phase ........................................................ 171

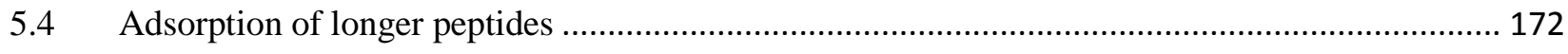

5.4.1 Adsorption of longer peptides on metal surfaces .............................................................. 172

5.4.2 Adsorption of longer peptides on $\mathrm{TiO}_{2}$ and other oxides ................................................ 175

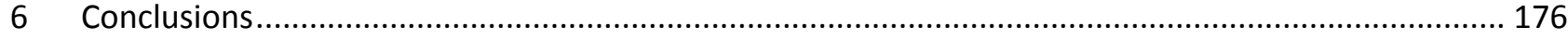


7 Acknowledgements

Erreur ! Signet non défini.

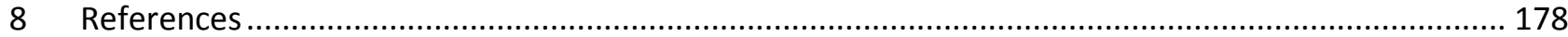

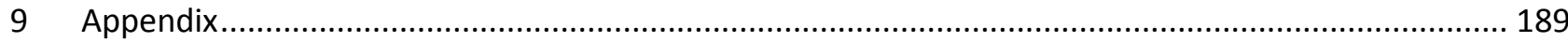


Unravelling the mechanism of interaction of aminoacids (AA) and of peptides with surfaces is of high relevance both for expanding the limits of knowledge in the field of surface science and for opening new perspectives towards improving the behaviour (either reactivity or stability) of materials in a biological environment.Various possible applications exploiting the hybrid organicinorganic interaction are foreseen in nanoelectronics $[1 ; 2]$, in the preparation of hybrid organicinorganic nano-objects [3], in the design of biosensors and in the production of bio-materials of medical and pharmacological use [4]. On the other hand, adsorption of peptides and proteins is a preliminary step for the further growth of bio-films on solid surfaces [5] and must be prevented when addressing fouling, hygiene and bio-corrosion issues.

As tangible example of application, we mention the deposition and immobilization of fullerene molecules on a template formed by a self-assembled glycine layer on $\mathrm{Cu}(111)$ [6]. Fullerenes and their derivatives on metal electrodes are prospective building blocks for functional nanostructures but, at a low coverage, they are usually too mobile to be stable at terraces of a metal surface. Therefore, it is usually difficult to obtain the isolated molecular condition that is essential for achieving molecular devices.

Amino acids are the basic constituents of proteins. Short peptides are the simplest organized structure formed by AAs and still do not present the additional complication of folding, typical of proteins or of long peptides. Their structure is therefore simple enough to serve as a model for the chemisorption of biofunctional molecules but "complex" enough to provide valuable information on the fundamental mechanisms governing the hybrid organic-inorganic interface. Adsorption of these small biomolecules may be ruled by various factors, such as Van der Waals, ionic or electrostatic interactions, but also by geometrical factors as well as intermolecular forces and surface hydrophobicity. Finally, they are all chiral molecules except glycine. Since it is emerging that the surface reactivity can be modified by exploiting the intrisic chirality of AA and peptides, their action as chiral modifier is a crucial issue for chiral separation or enantioselective heterogeneous catalysis.

This review aims at providing a quasi-exhaustive description of the state of the art on the interaction of AA and small peptides with metal and oxide planar surfaces, limiting the analysis to systems investigated experimentally by those means typical of surface science and to calculations performed without solvent. The discussion will evidence how the results obtained are sometimes complementary, sometimes controversial, but how they all contribute to give a comprehensive view of the present knowledge and progresses in the field of biointerfaces at a molecular level. We will face the problem moving from the simplest systems (AA on metals) and proceeding towards an increasing degree of complexity (AA on oxides, peptides on metals, peptides on oxides). In this path, we will try to emphasize the possible bridges between the different systems described and approaches used and also to fill a bit of the gap which is always present between surface science studies and ambient conditions.

Being a surface science analysis, the attention will focus on the biomolecule-surface interaction at the molecular level (nature of the bonds formed and orientation of the molecule at the surface)and on the self-organisation (or surface-induced organisation) of the adsorbed AA and peptides. More macroscopic data (amount of biomolecule adsorbed, surface charges...), often measured at the 
solid-liquid interface, are mentioned when necessary to complement the picture at the molecular level. In the same spirit, on the theoretical side, most of the works cited consist in periodic Density Functional Theory (DFT) calculations, that allow describing bond making and molecular selfassembly and structuration on model, crystalline surfaces.

We believe it is a good moment for a review on this topic since recent advances in surface characterization techniques have led to spectacular improvements in the description of adsorbed systems. Low Energy Electron Diffraction (LEED) and Scanning Tunnelling Microscopy (STM) are the techniques most exploited to describe the geometrical arrangement of adsorbed biomolecules at conductive substrates, while Photoelectron Spectroscopy (PES), High Resolution Electron Loss Spectroscopy (HREELS) and Reflection-Absorption Infrared Spectroscopy (RAIRS) are successfully used to determine the chemical state of molecules on a surface, even sometimes allowing their quantification. Old techniques as thermal programmed desorption (TPD) proved also precious to retrieve information on the characteristic adsorption energies and coverages. In situ analyses were performed in some cases, thus giving access to the mechanism and kinetics of the formation of amino acid and peptide layers on a solid material.

Experimental achievements have been recently sided and reinforced by modelling and calculation, which can be used to shed light on the structure and properties of bio-molecules at surface and on the chirality transfer induced by the adsorption of a chiral species. These theoretical studies typically address the following questions: (i) What is the nature of the intermolecular interactions at play in molecular layers at surfaces? (ii) What is the structure of the layer? (iii) If the adsorbate is chiral, what are the mechanisms by which the chirality at the molecular level is expressed in the two-dimensional system? (iv) Does chiral recognition occurs upon exposure to a racemic mixture?

Combined with experimental data or alone, the theoretical approach can bring spectacular breakthroughs in the description of adsorbed molecules. In many examples, and more particularly on metal surfaces, it has provided invaluable information to interpret experimental data and better understand the structural and environmental factors affecting amino acid/peptide adsorption and ordering on a surface. In the following chapters the reader will find several examples of the exceptional dialog between STM structural data and first principles periodic calculations. Complementarity between spectroscopies (XPS, HREELS, PM IRRAS) and calculations will also be highlighted. Not surprisingly, most of the theoretical results mentioned are obtained with DFT (and the more recent DFT-D approach which includes the dispersion forces in the Hamiltonian). Indeed, such method has proven to be efficient in the description of breaking/making bonds and electronic properties analysis.

The DFT framework is also the appropriate tool to predict the thermodynamic phase diagram of adsorption/desorption, as will be illustrated by some works. The main reason for that is the small size of the biomolecules described here, that allow to neglect (at least partly) entropic effects. In some rare works entropic and even kinetics effects were considered, opening the way to multi-scale approaches.

The content of this review is presented in such a way that the reader will have a detailed description of both the basic and the up-to-date available results concerning simple biomolecule interactions with metal and oxide surfaces. Illustrated by classical but also by very recent examples, we hope it will constitute a useful reference tool for surface chemists, biochemists and advanced biotechnology engineers. Firstly, experimental and theoretical methods are described. Then the paper is divided 
into three main parts, dealing with (i) the adsorption and self- assembly of amino acids at metal surfaces and (ii) at oxide surfaces and with (iii) the adsorption of peptides on metal and oxide substrates.

In the first part, the interaction of AA with several metal surfaces is elucidated. The reasons and perspectives linked to the choice of each substrate will be explained in the corresponding sections. We mention here the dominant role of copper, for which several crystallographic faces and almost all the AA species have been studied. This richness of results allows a comparative analysis of the role of the surface geometry and of the amino acid side chain in the adsorption and self-assembly process. Besides $\mathrm{Cu}$, two different categories of metals are made object of investigation, namely noble metals ( $\mathrm{Au}$ and $\mathrm{Ag}$ ) and reactive transition metals (mainly $\mathrm{Pt}, \mathrm{Pd}$ and $\mathrm{Ni}$ ). They have completely different electronic properties and thus exhibit a significantly different behaviour with respect to AA adsorption. The analysis of these systems reveals two determining factors: the ability or inability of the metal to promote carboxylic acid dehydrogenation and the affinity to sulphur atoms, which governs the interaction of cysteine and methionine (the two S-containing amino acids).

In the subsequent section the results on AAadsoption at oxide surfaces are reported. The majority of the studies were performed on $\mathrm{TiO}_{2}$, anhydrous or sometimes in the presence of water molecules, providing some valuable hints into the possible behaviour of a more « real » biomaterial in contact with a biological solution. Another very studied system, $\mathrm{SiO}_{2}$, was not considered here, as adsorption of biomolecules on silica was recently thoroughly revised [7]. For each metal/oxide, a short introduction recalls the possible application domains. As for AA on metals, the nature and energetics of bond formation were scrutinized, as well as the resulting molecular conformation at the surface. With respect to the AA/metal section, the reader will notice the change in the dominant experimental techniques employed for the characterisation and, perhaps, a larger fraction of purely theoretical works. This is a consequence of the increased complexity of the system: the poorly conductive $\left(\mathrm{TiO}_{2}\right)$ or insulating nature of the substrate does not allow the use of electronic techniques and requires the development of suitable approaches. Attention was paid to joined theory and experimental works, which are however seldom, or, more often, to the role of calculations to clarify the characterizations at the molecular level. As in the case of metals, it will be illustrated that carboxylate groups are the most frequent moieties bonding to the oxide surfaces, whereas in this case SH functionalities (in cysteine and methionine) play no key role. To the best of our knowledge, no strictly speaking "self-assembly" of AA takes place at oxide surfaces, as will be discussed later in the text.

The last section deals with peptides. Peptides constituted by two or three AA can now be investigated with the same surface science approach used for single AA. For peptides beyond that size, only examples where information at a molecular level could be obtained are reported. The chemical state of adsorbed peptides, the preservation of their molecular structure, as well as their possible folding or unfolding when in contact with a solid material are emerging as key interrogations for their biocompatible or antibiofilm properties; this is one of the issues addressed in this review. In this part, due to the larger dimensions of the peptides as compared to AA, papers using classical force field methods are more numerous than in the section on AA on metals. However only few works were performed without explicit solvent.

In the end, in the conclusive section we summarise the general view of the reactivity of amino acids and peptides with metal and oxide surfaces, based on all systems detailed in the report. 
Having limited the content of the present work to those studies in which the information could be obtained at the molecular level and with the tools of surface science makes this review necessary incomplete. The characterisation of the hybrid organic-inorganic interface formed by small protein constituents goes much beyond the scope we have delimited:

1) To maintain an acceptable length, we are not going to discuss about AA adsorption on semiconducting surfaces, for which quite a lot of literature is already present [8-13]. Indeed the study of the interface between biomolecules and semiconducting surfaces is mainly motivated by the possible applications in molecular electronics and bio-compatible electronic devices, which would make the content of the present review diverge from its central focus. We have also neglected the emerging field of adsorption at low-dimensional carbon nanostructures as graphene and carbon-nanotubes. The topic is still quite controversial - e.g. the biocompatibility of graphene layers is still under discussion - and a much less systematic analysis than for metals and oxides is available at present [14-19]. Adsorption on non-planar surfaces (porous oxides) and nanoparticles (often done in solvent) was also excluded.

2) We have omitted the case of long peptides. In general, these more complex systems are investigated with methods typical of biophysics instead of surface science. Furthermore, it still difficult to obtain information at the molecular level for these systems[20;21].

3) As mentioned in the text, a lot of studies were performed in liquid solution and as a function of $\mathrm{pH}$. In this case the analysis techniques and the calculation methods are necessarily different, and once again, the information obtained is not always at the molecular level. Nonetheless, this is an essential, intermediate step in the passage from the ideal systems to the real ones and thus for the exploitation of the basic knowledge in many fields in which this kind of interfaces is employed. We refer the reader to other specific reviews on the topic [22-32].

In spite of these severe limitations, we believe that the present work demonstrates the ability of the surface science approach to address rather complex systems of the biological world and is helpful to the reader to understand and anticipate the behaviour of some relevant systems.

\section{Experimental and theoretical methods}

In this chapter, we offer a quick overview of the experimental and theoretical methods which are most widely used in the study of amino acids and peptides adsorption at surfaces. We are not going to focus on experimental and computational details, for which more specific books and reviews are recommended, but we aim at clarifying which kind of information can be obtained by each technique.

\subsection{Experimental methods}

\subsubsection{Amino acid species and deposition techniques}


The most general formula to represent an $\alpha$-amino acid is reported in Figure 1a: the molecule is characterised by an $\alpha-\mathrm{C}$ atom in position 2 , to which both the amino $\left(\mathrm{NH}_{2}\right)$ and carboxylic $(\mathrm{COOH})$ groups are bound. The rest of the molecule is generally represented as a radical (-R), the structure and composition of which change for each of the $20 \alpha$-amino acid species present in nature (schematically represented in Figure 2 in their zwitterionic form). The different AA species show quite different characteristics from the point of view of their polarity (Timberlake classification scheme [33]) and of their structure (e.g the Koolman classification scheme [34]). Indeed, they may have either a non-polar or a polar (either neutral or charged) termination. They can be aliphatic (e.g. alanine), or present additional functional groups, either a carboxylic (e.g. glutamic acid), an amino (e.g. lysine) or a sulphur group (e.g. cysteine). Furthermore, they can have a linear structure (e.g. glutamic acid) or their radical termination may contain one heterocycle (e.g. proline) and/or aromatic (e.g. tyrosine) ring.

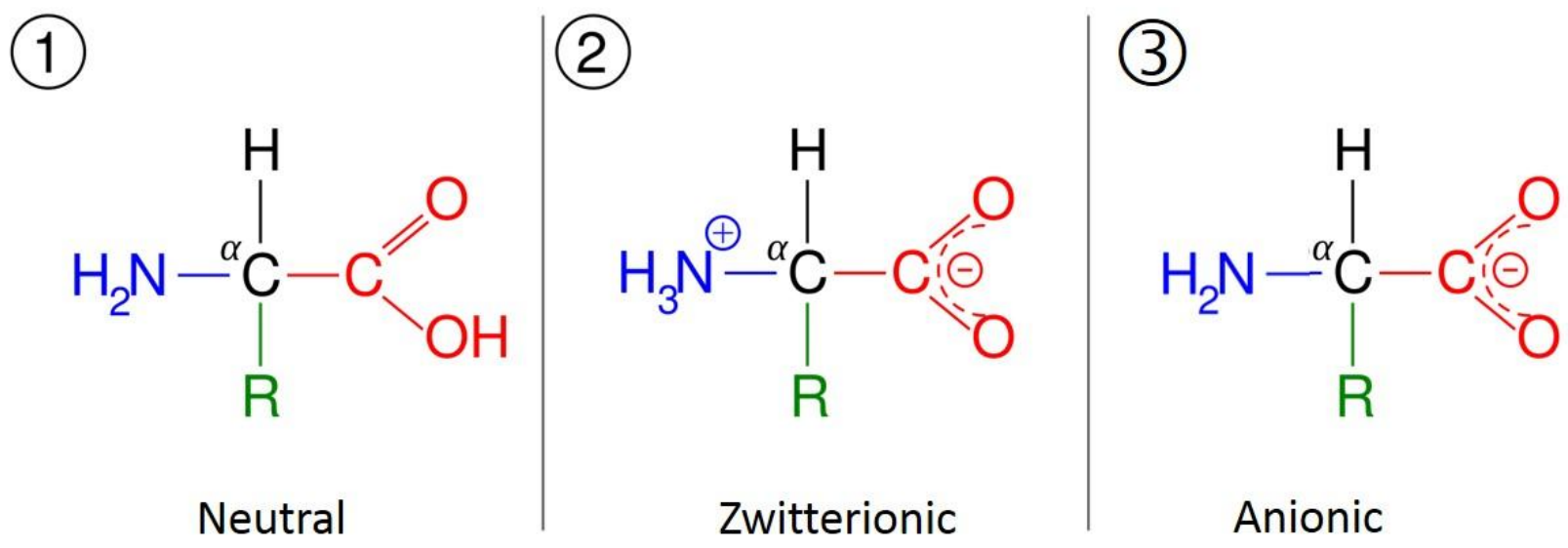

Figure 1.General scheme of an $\alpha$-amino acid in the (1) neutral, (2) zwitterionic and (3) anionic form. The amino group is represented in blue, the carboxylic/carboxylate group is in red, the $\alpha$ carbon is in black and the radical side chain $\mathrm{R}$ is in green. 


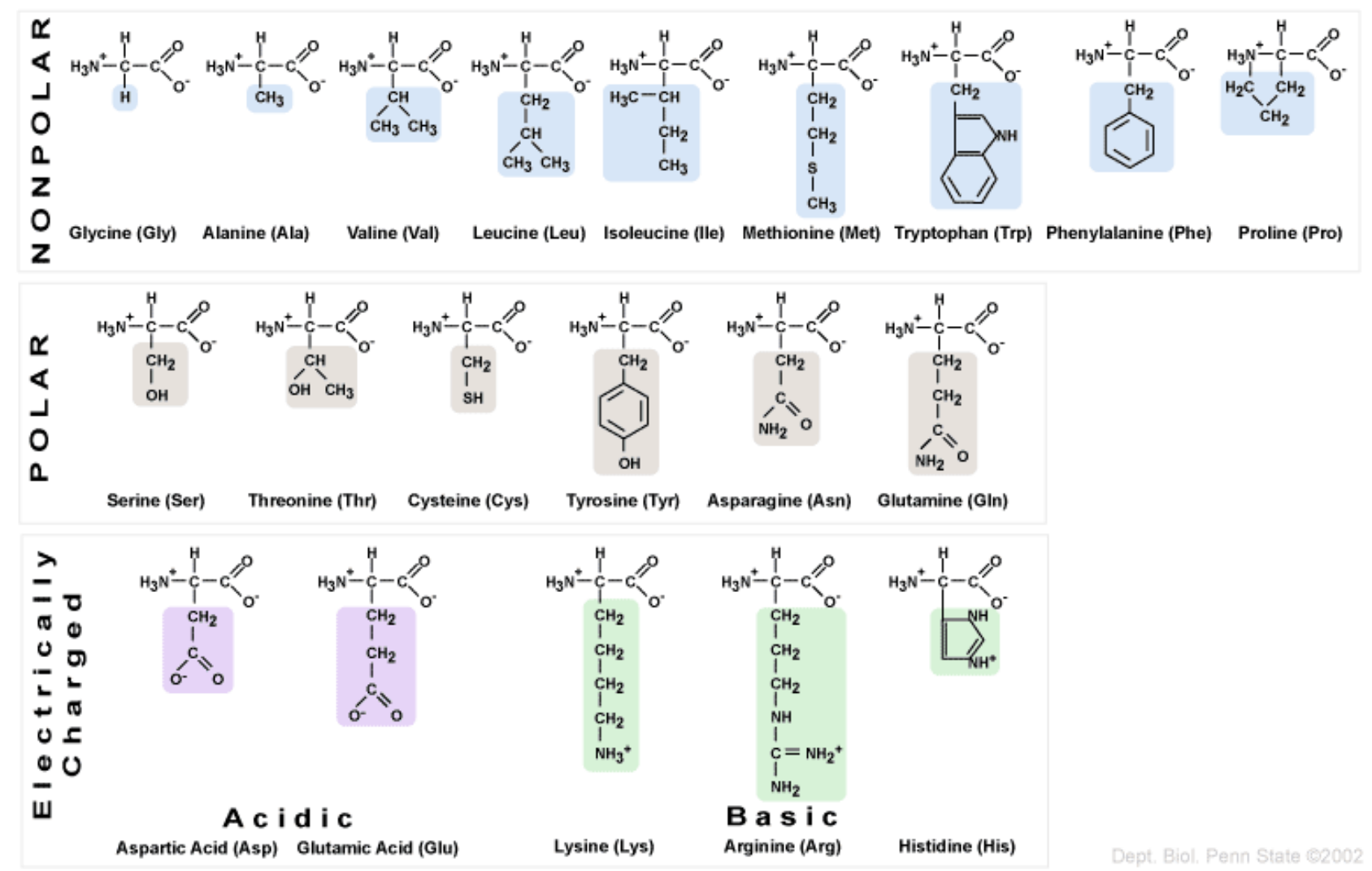

Figure 2. Scheme of the $\alpha$-amino acid species present in nature, represented in their zwitterionic form and grouped according to their polarity and electrical charge. Taken with permission from "http://www.personal.psu.edu/staff/m/b/mbt102/bisci4online/chemistry/chemistry8.htm"

Besides glycine, which is the simplest and smallest molecule of the family, all other AA are chiral. Since the (L)-enantiomer is the one chosen by evolution to be compatible with life, and hence it is the only one present in living bodies, it is a natural consequence that the large majority of studies have been performed on (L)-type molecules. In the following, we will assume that this is always the enantiomer employed in investigations, unless otherwise explicitly stated.

Amino acids are present in nature in three possible chemical forms (see Figure 1), depending on the environmental conditions of the molecule: 1) the neutral form, typical of the isolated molecule (usually in the gas phase); 2) the zwitterionic form, typical for solid AA crystals and, in general, for molecules at poorly reactive surfaces. In this case a proton is transferred from the carboxylic group to the amino group of the same molecule, which remains globally neutral. 3) The anionic (and sometimes the radical state), in which deprotonation has occurred. This is found mainly for AA strongly interacting with a substrate, leading to the breaking/formation of chemical bonds. The distinction between the zwitterionic and the non-zwitterionic form is straightforward since the $-\mathrm{NH}_{2}$ and $-\mathrm{NH}_{3}{ }^{+}$groups have different photoemission and vibrational signatures. The most common indicator used in literature is the N1s binding energy, which is expected at $\mathrm{E}_{\mathrm{b}}(\mathrm{N} 1 \mathrm{~s}) \sim 400 \mathrm{eV}$ for the neutral amino group and up-shifts by $\sim 1 \mathrm{eV}$ or more if the group is charged.

Other chemical forms can be encountered when protonation/deprotonation of side groups occurs. 
In line towards more complex and also more "realistic" biomolecules, one finds the peptides, which are polymers of amino acids linked to each other by peptidic bonds (CO-NH). As an example, a dipeptide results from the condensation of two amino acids, i.e. the reaction between the $\mathrm{COOH}$ end of one AA and the $\mathrm{NH}_{2}$ functionality of the second one, with the elimination of one water molecule. Peptides can be a chain of similar (homopeptides) or various (heteropeptides) amino acids. Their length can range from dipeptides to a chain of several tenths of aminoacids; proteins are the "summum" of a peptide chain where the sequence of aminoacids, their location and their threedimensional arrangement control the biological activity of the molecule.

Very short peptides ( 2 to 4 AA) on metals can be investigated with the usually called surface science tools, even under ultra high vacuum conditions, since these low molecular weight molecules can be sublimated in the analysis chamber. Ab initio calculations have also brought lots of information on these small systems. The interaction of longer peptides with metal surfaces has often been investigated by combining several chemical analysis techniques, e.g. chromatography. Here, we will limit our report on the long peptide systems to a few cases in which, despite their size, information concerning their interactions with a metal could be drawn at the molecular level.

If the sample is prepared in vacuum, the most common technique for producing the organic layer is the sublimation from a crucible. The latter is usually resistively heated and can be made of a vitreous material (glass, quartz, Pyrex ${ }^{\circledR}$ ), of a ceramic material (e.g. boron nitride) or of a suitable metal non-interacting with the chemical species to be sublimated. Amino acids or small peptides, which are present in nature in the solid form, can be sublimated by heating the crucible to a temperature $\left(\mathrm{T}_{\text {sub }}\right)$ which depends on the amino acid species. The choice of the optimal $\mathrm{T}_{\text {sub }}$ is a key issue in each experiment; in fact it must be high enough to guarantee a reasonable sublimation rate, but lower than the critical value leading to the melting of the AA powders and to the consequent possible damage of the molecules. Since AA are quite hygroscopic, in the optimal configuration the crucible is separated from the preparation chamber by a valve and it is differentially pumped, in order to allow for a careful outgassing of the powders.

We mention that, in the past, molecular beams of aminoacids were produced by laser ablation and employed for gas phase experiments aiming at conformational studies of the isolated molecules [35; 36]. More recently, a source for production of supersonic molecular beams (SMB) of AA has been developed with the scope of applying it to the study of the molecule-surface interactions. While the source is operative, a comparative analysis of the overlayer obtained by traditional effusive deposition and by deposition of hyperthermal molecules is not available. This latter SMB source is not based on laser ablation but it is more similar to the one used for pentacene [37]: the AA vapour is created by resistive heating and the molecules are carried in a stream of inert gas through a nozzle, which produces the supersonic expansion.

Amino acids and peptides may also be deposited, and thus let interact, in solution. While the analysis of the bio-interface in wet or atmospheric conditions is out of the scope of the present review, in the following we will report a few examples of samples prepared in this way and then dried and investigated under UHV. 


\subsubsection{Analysis techniques}

\subsubsection{Desorption techniques:Temperature Programmed Desorption (TPD) and Thermal desorption spectroscopy (TDS)}

The technique, indicated either as TPD or TDS, monitors the desorption products as a function of sample temperature when the surface is heated at a constant, known rate. The multiple desorption peaks correspond to adsorbates in different chemical states (physisorbed, chemisorbed, dissociated, in single- or in multi-layer etc...) and hence characterised by different adsorption energies. Due to the relatively simple setup required by this technique, it is among the most common from the early days of surface science and has been employed quite extensively also for the characterisation of AA and peptides adsorption at surfaces [38-43].

\subsubsection{Spectroscopic techniques}

Spectroscopic techniques are widely employed in the investigation of organic layers at surfaces in order to elucidate the final state (and sometimes the total amount and the orientation) of stable adsorbed species and to deduce information on the possible adsorption sites.

Photoemission and vibrational spectroscopy are often complementary. In fact, on the one hand, the core level shifts of atoms in molecules at different adsorption sites (e.g. at a terrace or at undercoordinated sites) and/or in different chemical states (e.g. neutral or anionic) may be very similar; on the other hand, the unambiguous assignment of molecule/surface vibrations is often not easy. When taken together, results from the two techniques can be unambiguously interpreted.

$\mathrm{X}$-ray photoemission spectroscopy allows to investigate the core levels of the atoms present at the surface, giving information on the chemical composition of the adlayer and of the first surface layers. Furthermore, the analysis of core-level shift provides precious information on the chemical environment, while the quantitative analysis of the peak intensities gives a reliable indication on the adsorbate coverage. With respect to the AA/surface systems, XPS is the most common technique employed to determine the chemical nature of the organic layer and it has been applied to the large majority of the experimentally investigated systems (see Table 3). In particular, the zwitterionic or non-zwitterionic form of the adsorbate is immediately revealed by the binding energy of the N1s level $\left(\mathrm{E}_{\mathrm{b}} \sim 400 \mathrm{eV}\right.$ for $\mathrm{NH}_{2} ; \mathrm{E}_{\mathrm{b}} \geq 401 \mathrm{eV}$ for $\left.\mathrm{NH}_{3}{ }^{+}\right)$. Additional qualitative and quantitative information is gained from a detailed fitting and analysis of the XPS spectra. For example, if two different states of the same molecule co-exist on the surface (neutral and anionic, or anionic and zwitterionic), it is possible to estimate their relative amounts.

Photoemission experiments can be performed either with conventional laboratory sources or with Synchrotron light. In the latter case, the high photon flux allows time-resolved and/or high energyresolution measurements. In addition, the availability of a tunable X-ray source offers the possibility to perform also edge spectroscopies, in particular Near Edge X-ray Absorption Fine Structure (NEXAFS). Providing significant information on the molecular bonds orientation, this 
technique is very helpful for the characterisation of the structural conformation of the adsorbed molecules [44-46].

The basic principle of vibrational spectroscopy is that each molecule absorbs energy when there is a resonance with its vibrational modes, and this occurs at characteristic frequencies. When the molecule is adsorbed, such frequencies are modified with respect to the gas-phase ones due to the interaction with the substrate. They remain however characteristic of the chemical group and of the adsorption state. The analysis of the phonon spectrum allows therefore the determination of the adsorbed chemical state of the stable products. Since the characteristic frequencies are often structure-dependent and influenced by the geometry of the adsorption site, vibrational spectroscopy proved to be an extremely useful tool for this kind of information. The assignment of modes by vibrational spectroscopy is based on selection rules, on isotope labelling and on a detailed analysis of the cross section and, in the case of HREELS, of the scattering mechanism (dipolar vs impact) involved.

For vibrational spectroscopy on metal surfaces, either infrared reflection absorption spectroscopy (IRAS or RAIRS - see Figure 3a) [47-51] or high resolution electron energy loss (HREELS - see Figure 3b) [52-54] detection can be used. The former technique relies on the amplification of the electric field at the surface, in the direction normal to it; like basic IR spectroscopy, IRAS underwent a rapid technical evolution over the last years, so that detection of adsorbates at a few percent of monolayer coverage is now feasible thanks to Fourier Transform-based acquisition (FTIRAS).

Beyond IRAS or RAIRS, Polarization Modulation (PM)-IRRAS has been developed with the objective of characterizing adsorbed layers in air or in the presence of a gas phase, for example during the sublimation of amino acids or peptides. In such a setup, a $\mathrm{ZnSe}$ grid polarizer and a $\mathrm{ZnSe}$ photoelastic modulator, placed prior to the sample, are used to modulate the incident beam between $\mathrm{p}$ and s polarization at a high frequency (modulation frequency $=50 \mathrm{kHz}$ ). This obviously enables in situ characterization of a surface during the formation of the adlayer (see several examples later in this report $[55 ; 56])$.

IRAS or PM-IRRAS are operated at grazing angle on a reflective surface. Under this condition, the signal strictly originates from dipole moments, which have a component normal to the surface; a direct consequence is that interesting information about the orientation of the bonds, and thus about the geometry of adsorbed molecules, is provided by the relative intensities of the vibration modes of the molecule[57].

Another powerful IR-based technique is Attenuated Total Reflection Infrared Spectroscopy (ATRIR), in which the adsorbed layer is probed by the evanescent wave after reflection of the IR beam on a special crystal ( $\mathrm{Si}, \mathrm{Ge} . .$.$) .$

HREELS has a higher sensitivity then IRAS (in dipole and in resonant scattering) and allows the investigation of phonon dispersion curves when used in the impact scattering mode. It needs however a UHV environment. In a HREEL spectrometer a beam of monochromatic, low-energy electrons is accelerated to the chosen primary energy $\left(E_{e}\right)$ and focussed on the sample by a series of electrostatic lenses. The reflected beam is collected by a similar lens system, analysed in energy through a single- or a double-pass analyser and detected by a channeltron. When electrons exchange 
energy with the surface, e.g. by exciting electronic or vibrational modes of characteristic energy $E_{\text {loss }}$, they are reflected with an energy $E_{\text {out }}=E_{e}-E_{\text {loss }}$ and loss peaks appear in the spectrum.

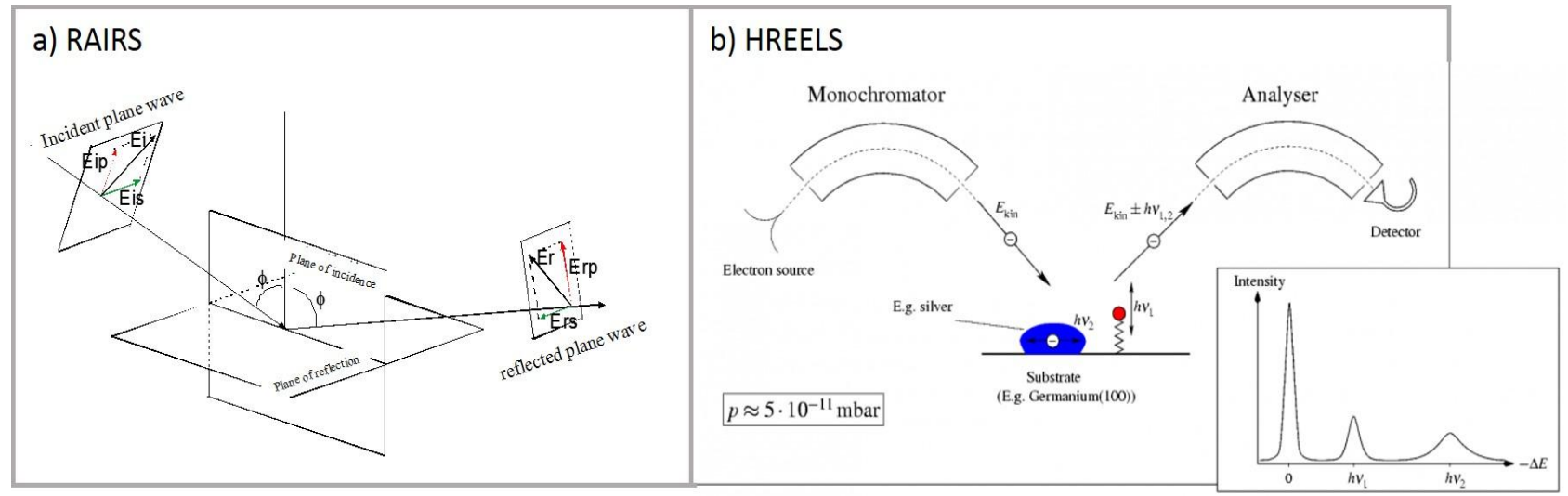

Figure 3. Schematic principle of operation for the main vibrational spectroscopy techniques: a) RAIRS. The incident beam is split into two components, $E_{i p}$ (in the plane of incidence) and $E_{i s}$ (normal to the plane of incidence); the latter undergoes a phase change of $180^{\circ}$ upon reflection, while the phase change of $\mathrm{E}_{\mathrm{ip}}$ depends on the angle of incidence $\phi$. At the surface, the resulting electric field is maximum for $\phi$ close to $80-86^{\circ}$. B) HREELS. The electron beam is monochromatised and accelerate onto the sample, where some electrons exchange energy with it by exciting surface and/or adsorbate phonons and/or collective electronic modes (e.g. surface plasmons). The scattered electrons are then analysed in energy and collected by an electron multiplier. The inset shows a typical HREEL spectrum. Taken with permission from http://www.kip.uni-heidelberg.de/ag_pucci/instrumentation/hreels.

We mention that, both for amino acids and peptides, vibrational (especially IR) spectroscopy has been widely used to characterise the adsorption of the organic layer (see tables Table 1, Erreur ! Source du renvoi introuvable., Table 12), in particular to determine the final state of stable adsorbed species and to deduce information on the possible adsorption sites. However, due to the complexity of the systems, derived from the molecular dimensions and from the consequently large number of possible vibrational modes, complementary techniques are often necessary for a convincing characterisation of the adsorbed system.

\subsubsection{Microscopic Techniques}

High resolution scanning probe microscopy (SPM) has known a huge development in the last few years and has proved to be an extremely powerful tool for a direct characterisation of surface topography and structure [58]. The main advantage of SPM techniques lies in their ability to give a direct, spatially resolved image of the surface structure, the comprehension of which - at a first glance - is much more immediate than the analysis of spectroscopic or diffraction data. Among the available SPM techniques, Scanning Tunnelling Miscroscopy (STM) is by far the most widely employed in the study of amino acids and small peptides at metal surfaces (see tables AA systems and Peptides systems), since it guarantees the highest lateral resolution and the best sensitivity to surface corrugation.

A scheme of a STM is reported in Figure 4. Measuring the tunnelling current between a conductive sample and a metallic tip scanning at a few $\AA$ distance from it allows to record a high resolution 
image of the surface electronic density and corrugation. The tunnelling current, I, is determined by the superposition of the wave functions, $\psi$, of the two electrodes. In the simplified hypothesis of a 1D barrier of widths, one gets the approximate formula:

$$
I \propto \sum_{\mu v}\left|\psi_{\mu}^{0}\right|^{2}\left|\psi_{v}^{0}\right|^{2} e^{-2 k s}
$$

The high sensitivity to surface corrugation is therefore guaranteed by the exponential decay of the tunnelling current as a function of the barrier width (i.e. of tip-surface distance). On the contrary the lateral resolution depends both on the surface corrugation and on the shape of the tip. The latter should end ideally with a single atom, but its shape is often more complicated and may affect the quality of the recorded image. The limit of atomic resolution is easily reached in last generation STMs, which are characterised by a better vibrational damping and thermal stability.

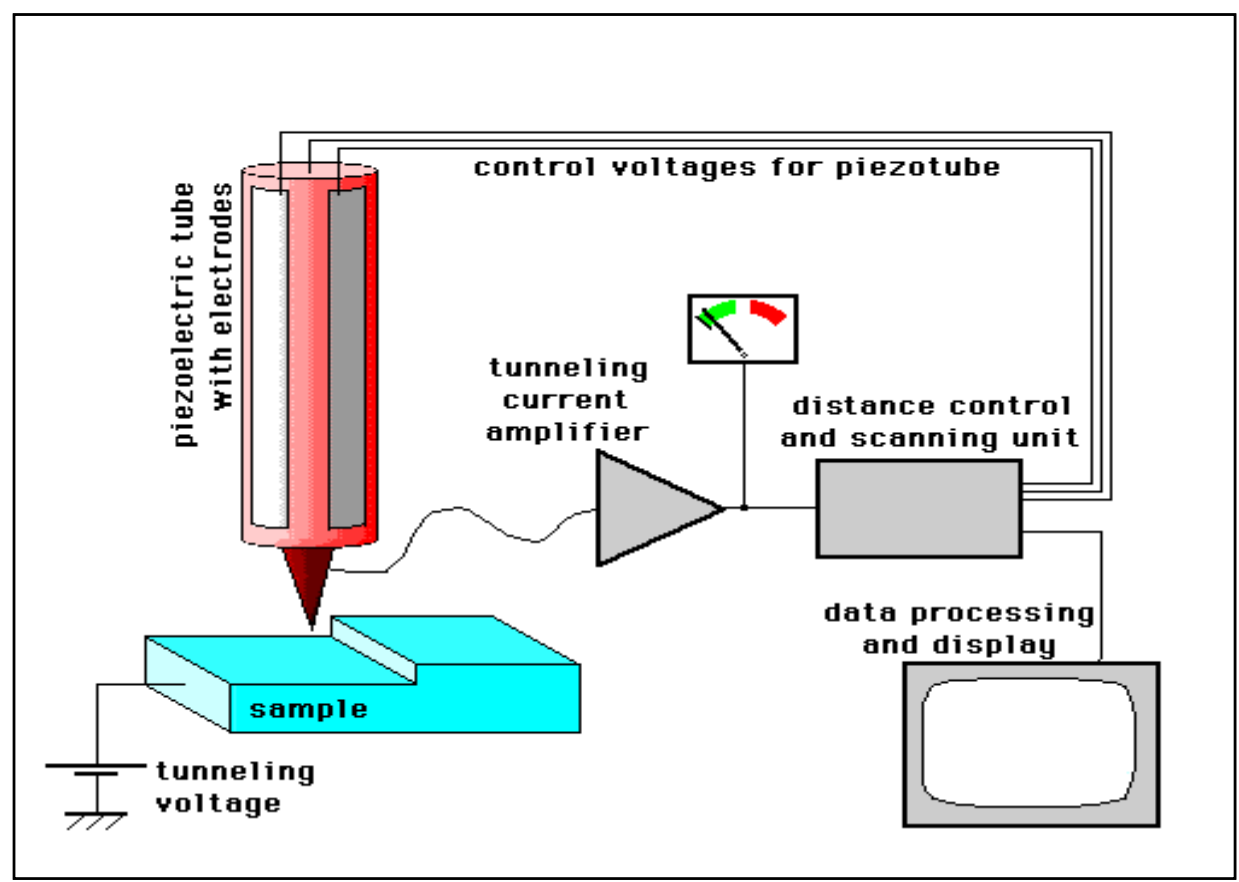

Figure 4. Scheme of the basic elements of an STM: the tip mounted on piezoelectric elements, the current amplifier, the feedback circuit and the data storage unit are shown.

The structure of the self-assembled layers of organic molecules can thus be described at a molecular level, and such analysis is often the basis for empirical models [54] or for more accurate calculations [45; 59-63]. Of course, due to the fragile nature of the molecules, some precautions must be taken to avoid damage of the organic layer by the strong electric field induced by the tip. Not all self-assembled structures are indeed stable upon scanning, so that the use of low bias voltage may be necessary in some conditions. Similarly, in the case of AA, it is extremely difficult to image a molecular multilayer since the topmost amino acids are weakly bound and thus strongly interacting with the tip. 
STM studies represent a significant amount of the literature on AA at metal surfaces, while they are less frequent on the more complex peptide/metal systems. Since the obvious limit of the STM technique is the need of a conductive/semiconductive sample, very little work has been done on oxide surfaces: to the best of our knowledge, only one paper reporting on Gly adsorption at $\mathrm{TiO}_{2}$ is present in literature [64].

The other, very popular SPM technique, atomic force microscopy (AFM), is also very common for the characterisation of organic/biological molecules at surfaces. Being based on Van der Waals interactions, it should perturb less the investigated systems and thus it should be, in principle, even more suitable for the analysis of AA and peptides adsorption at surfaces. However, the resolution of AFM is not as good as that of STM. Moreover, AFM is preferentially applied when images in air/liquid/controlled atmosphere are required and the results obtained are more often related to the bio-physics context, or combined with electrochemical characterisations, than to the surface science approach on which we are focussing the present review.

\subsubsection{Diffraction techniques.}

Various diffraction techniques are employed in solid state physics for structural characterisation. Either electrons, X-rays or He atoms can be used as probes, since their characteristic wavelengths are comparable with typical crystal lattice spacings, so that the latter ones behave as diffraction gratings.

Hard X-rays are used mainly in the determination of the bulk structure, due to their large penetration depth; they are therefore unsuitable for soft adsorbate layers as those we are dealing with. X-rays at grazing incidence are, on the contrary, useful for the investigation of surface structure, but synchrotron light is needed. Among these techniques we mention X-ray photoelectron diffraction (XPD), which is seldom used for the characterisation of AA at metal surfaces [65-67]. However, attention must be taken in order to rule out possible damages of the organic layer by the intense photon beam.

He atom scattering (HAS) is extremely surface sensitive since He atoms do not penetrate into the crystal. Well collimated, low energy (few meV) beams are produced by supersonic molecular beam apparatuses. The diffraction pattern of elastically scattered He atoms was widely employed in the past for the structural characterisation of surfaces, also in presence of adsorbate layers and/or superstructures. Although powerful, this technique is rarely used in the investigation of AA and peptides at surfaces [63] for the combination of two factors: i) The complex interpretation of the results. In fact HAS, as all diffraction techniques, gives information on the reciprocal lattice of the surface. ii) The competition of SPM techniques. In fact, the interest of the surface science community on the AA and peptides interaction with surfaces developed when the use of the alternative scanning probe microscopies - of more immediate interpretation - was already quite widespread. 
Low Energy Electron Diffraction (LEED) is much more common. At $10-200 \mathrm{eV}$ the penetration depth of electrons is limited to few atomic layers, so that a good surface sensitivity is guaranteed. Inspection of the diffraction pattern of elastically scattered electrons at crystal surfaces is the first check to understand the surface symmetry and to evaluate the presence of superstructures both on LMI and on HMI planes. In the study of amino acids adsorption at metal surfaces, LEED is often employed for a qualitative description of the overlayer geometry [42; 67-69]. These are delicate measurements, since the interaction with the electron beam may damage the layer of molecules and create disorder/desorption. For this reason the primary electron energy is set at the lowest possible value compatible with the available optics. Maximum values of a few tenths of $\mathrm{eV}$ are reported in literature.

\subsection{Computational Methods}

\subsubsection{Introduction}

20 years after the development of STM in the beginning of the ' 80 s, and a decade after turning quantum chemical methods into practical tools to study surfaces (periodic DFT developed at the end of the same decade), theoretical studies on molecular self-assemblies at surfaces began. A seminal theoretical study on this topic was dedicated to the adsorption of tartaric acid on $\mathrm{Cu}(110)[70]$. The interest in tartaric acid finds its origin in its chiral properties. The adsorption of so called chiral modifier on metal surfaces revealed that each isomer forms different chemisorption domains, being mirror images from each other on the surface. From this study by Barbosa and Sautet, some insights about the formation and stability of the different bitartrate domains for the R,R and the S,S isomers were revealed. The most stable adsorption configuration on $\mathrm{Cu}(110)$ was predicted and a difference in stability between the isomers in the same domains was calculated. An energy difference of 10 $\mathrm{kJ} / \mathrm{mol}$ was found to be enough to produce the distinct chiral assemblies observed after adsorption of each optical isomer on the $\mathrm{Cu}$ surface. In conclusion, it was found that the molecular stability of the assembly is promoted by two factors: i) The capability to organize its inter- and intra- molecular H-bonds, and hence to reach the chemisorption structure with moderate cost in deformation energy. ii)The packing energy cost for the molecules in a specific domain. The stress generated after adsorption on the $\mathrm{Cu}$ atoms was found to be a potential explanation for the formation of the empty channels in the adsorption pattern.

Later on, organic molecules and biomolecules with a sulphur head group started to attract considerable interest because of their wide use in nanotechnology-related fields, like surface patterning and functionalization[71; 72] and molecular electronics[73]. Besides other interesting applications, the strong affinity of sulphur to metals can be exploited to link other species to a supporting metallic surface or to form well-ordered self-assembled monolayers (SAMs)[74]. Among biomolecules, the S-containing cysteine and cystine amino acids are of special interest for often being on the border of large proteins, so that they provide a link to anchor these proteins to inorganic supports. For instance, a stable functional monolayer of pseudomonas aeruginosa azurin can be formed on $\mathrm{Au}(111)$ [75] and also on a functionalized $\mathrm{SiO}_{2}$ substrate by attaching the protein to the surface via its Cys3- Cys26 disulfide group.[76] 
Since the first, pioneering studies, several first principles theoretical calculations on amino acid interaction with metallic and oxide surfaces have been performed. In the following parts of this work we will give an overview of the molecular modelling approaches focused on the use of ab initio/DFT methods or force field methods applied on amino acids and, to a less extent, on small peptides and proteins in general. An interesting overview of the computational techniques in biomineralisation studies was published in 2008 by Harding et al.[77] A more recent description can be found in reference[7].

The first step in molecular modelling,after building the molecular model,consists in selecting the most appropriate method to describe molecules and molecular systems interacting with a surface at the optimal price/quality rate. When the electronic properties of a material have to be studied, quantum-mechanical methods must be used. Among them, the DFT methods are very popular as they are less computationally intensive than other ab-initio methods with similar accuracy. Besides for electronic properties, DFT is particularly appropriate for describing chemisorption phenomena (bond breaking and making).The DFT calculations yield detailed information on all aspects of chemical bonding: the bonding site(s), the electron density redistribution between the adsorbate and the surface, the atomic orbitals involved (provided a localized basis set is used), as well as theoretical vibrational spectra. The DFT approach can also be used to simulate STM images. Since a few years, dispersion forces, which play a critical role in physisorbed systems, have been included in DFT approaches.

Although computer hardware and calculation algorithms keep improving, quantum chemical methods are intractable for systems exceeding thousands of atoms, and can still be advantageously substituted by force-field (FF) methods which are several orders of magnitude faster.This allows to describe larger systems and larger time scales (typically picoseconds for DFT and nanoseconds for classical methods). Besides the gain in calculation time, FF allows to include non-covalent intermolecular interactions, which play a critical role in physisorbed systems, and are too expensive to be reproduced accurately with quantum-mechanical methods.

\subsubsection{Density functional theory methods}

Without going into too much detail, the central concept of DFT is that the electronic energy of a system can be determined from the electron density $\rho(r)$ rather than from the wave function, through the following relation:

$$
E[\rho(\mathrm{r})]=\rho(\mathrm{r}) \mathrm{U}_{\mathrm{ext}}(\mathrm{r})+F_{\mathrm{HK}}[\rho]
$$

$v_{\text {ext }}(\rho)$ is the external potential generated by the nuclear configuration and $F_{\mathrm{HK}}[\rho]$ is a universal functional, which is not known exactly and is not associated to any external potential. To define a practical functional, Kohn and Sham described the electronic density in terms of mono-electronic molecular orbitals $\varphi_{i}(r)$ :

$$
\rho(r)=\sum_{i=1}^{o c c}\left|\varphi_{i}(r)\right|^{2}
$$


The total electronic energy of the system is expressed as the energy of a system of non-interacting electrons plus a new energy term $E_{\mathrm{xc}}$, called exchange-correlation energy. Approximations are required in order to compute the exchange-correlation energy. The most common one is the socalled local density approximation (LDA). In this case the exchange correlation term $E_{\mathrm{xc}}$ is calculated from the exchange-correlation energy for a homogeneous gas of electrons $\epsilon_{x c}[\rho(r)]$ having a density, $\rho$, equal to the local density $\rho(\mathrm{r})$ :

$$
E_{x c}[\rho(r)]=\int \rho(r) \epsilon_{x c}[\rho(r)] d r
$$

The LDA approximation yields reliable molecular geometries and vibrational frequencies, but overestimates the bond energies. To overcome this problem, the exchange-correlation term can be decomposed in separate exchange and correlation contributions. This allows combining the advantages of Hartree-Fock methods (which account for the exact exchange) and DFT methods (which include electron correlation). This combined approach relies on hybrid functionals: the exchange is calculated in part with Hartree-Fock and in part with DFT and the correlation is calculated with DFT.

Geometry optimisation algorithms can also be coupled to DFT calculations in order to obtain the most stable structure for the system under study. Because DFT describes the electron density, it can intrinsically account for changes due for instance to strong, chemical interactions between a molecule and a metal surface.

From the modelling point of view, a molecular layer on a surface is a quasi-infinite two-dimensional system. To treat it computationally, two approaches are possible:

(i) One can consider that the adsorbed layer is a perfectly regular 2D system for which a structural unit cell can be defined. Periodic boundary conditions (PBC) can then be applied, both at the DFT and force-field levels; then the calculations deal only with one elementary cell surrounded by equivalent cells, which strongly reduces the complexity of the problem and bypasses finite size effects. In order to define the unit cell, this approach requires that the adsorbate and the surface are commensurate, which is always the case for chemisorbed systems, but may not be the case for physisorbed ones. Most of the studies presented here use this periodic approach. So in the following, when no precisions to DFT method are specified, we refer to PBC studies. We notify only when cluster calculations are performed.

(ii) A fragment can be 'carved off' the system and the calculations can be carried out on that fragment. In this approach, the atoms and molecules in the periphery of the fragment are not representative of the actual situation because they miss neighbouring sites; this is called the 'edge effect'. For the results to be relevant, the fragment should therefore be large enough so that its central part can be considered as unaffected by the edges. Because no unit cell is defined, this approach allows to overcome the commensurability problem mentioned above and to take into account specific situations, e.g. the presence of defects.

However, standard density functionals areunable to cope with long-range dispersion interactions, which are essential to properly describe the adsorption of biomolecules on surfaces and the selfassembling of organic molecules. Since a few years, the DFTD approach has been developed, especially the so-called DFT D2 and D3 approaches developed by S. Grimme.[78-86] More details can be found in the original papers and a summary in ref.[7]. The beauty of the DFT-D approach is that it leaves unaltered the original accuracy of the DFT functional for short range interactions 
(bond breaking/making), while it dramatically improves the long-range tail of the interaction dominated by London forces. Recently, the vdW-DF has been implemented in periodic DFT codes[87].Dion et al. proposed a non-local correlation functional that approximately accounts for dispersion interactions[88].

With bond breaking and making, the DFT approaches allow to evaluate quantitative data as adsorption energies $\left(\mathrm{E}_{\mathrm{ads}}\right.$, which refers to the difference between reactants and products, $\mathrm{E}_{\mathrm{ads}}=$

$E_{\text {products }}-E_{\text {reactants }}$ ), which are reported all along this review. Since the adsorption energies are typically calculated as the difference between electronic energies of the products (adsorbed molecules) and the reactants (surface and molecule in vacuum), a negative value indicates an exothermicprocess. For sake of clarity and for internal coherence of the paper, we reported negative values of $\mathrm{E}_{\mathrm{ads}}$ also in those cases in which the absolute value is indicated in the reference paper.

\subsubsection{Force-field techniques}

Asthey are generally less computationally demanding, force-field methods can be applied to very large systems (i.e., several thousand atoms) while DFT techniques are usually restricted to a few hundred atoms. With force fields, the time evolution of the system at a given temperature can be investigated theoretically.

While in quantum mechanics, according to the Born-Oppenheimer approximation, it is usual to study the electronic structure in the field of frozen nuclei, the opposite approach is used in forcefield-based methods. The electrons are not explicitly treated and are assumed to surround the nuclei appropriately, generating a potential field in which the nuclei are located. This force-field is a set of equations whose mathematical form is derived from classical mechanics. Atoms are approximated as soft spheres bonded to each other with springs. Once a force-field is parameterized to correctly reproduce experimental or quantum-chemical results, the potential energy surface can be explored to find the lowest energy situations. However this point is all but trivial for complex, reactive surfaces.

A force-field is a mathematical translation of the old basic idea that bonds and angles between atoms in a molecule tend to have "natural" values. The interatomic interactions are mimicked by a set of empirical formulas, and the accuracy of the results depends on the way the parameters and functional forms of the energy expression have been chosen. Practically, a force-field is tuned to best reproduce experimental or calculated data of a series of simple molecules, and its applicability domain is then assumed to extend to other similar systems according to the principle of transferability of the parameters. However one main drawback of many force fields is that no bonds are broken and formed, which is the main event in chemistry. Very few reactive force fields exist; the most popular is ReaxFF [89], which has been used to study biomolecules adsorption on surfaces [90-92].

Adsorption energies can be evaluated with FF in the same way as with DFT.

\subsubsection{Molecular dynamics methods}


Molecular dynamics methods (MD) calculate the time-dependent behaviour of a molecular system. The starting point is Newton's equation of motion. The force is expressed here as the gradient of the potential energy E. Integrating these equations provides the positions, velocities, and accelerations of the atoms as they vary with time; this collection of data constitutes the trajectory, which is a source of information at the microscopic level, from which macroscopic observables (internal energy, pressure...) can be extracted. The initial positions usually correspond to an energy minimum that has been obtained by Molecular Mechanics (MM), while the initial velocities are often chosen from a Maxwell-Boltzmann distribution reflecting the temperature at which the simulation is performed. The velocities vary during the simulation, as kinetic energy is changed into potential energy and vice versa, and temperature also changes. Hence, different temperature control methods periodically adjust the velocities to maintain the temperature, while reproducing the correct statistical ensemble (NVE, i.e., constant number of particles, volume and energy is called the microcanonical ensemble; NVT, the canonical ensemble; NPT, the isobaric-isothermal ensemble). The integration of Newton's laws of motion is solved numerically on the basis of the finitedifference method. The positions, velocities and accelerations are determined at a time $t+\Delta t$ from their values at time t. Ideally, the time step should be large, in order to generate as much trajectory time as possible for a given computational effort, but it must also be shorter than the typical time for the fastest atomic movements. Typically, a time step of $10^{-15} \mathrm{~s}$ is chosen, i.e., ten times shorter than a $\mathrm{C}-\mathrm{H}$ vibration. With such a small time step, MD simulations can be very time consuming and computationally expensive, depending on the time scale of the dynamic process investigated. Among the fastest processes are the local motions $\left(0.01\right.$ to $5 \AA, 10^{-15}$ to $\left.10^{-1} \mathrm{~s}\right)$ such as atomic fluctuations, the motion of short molecular segments and loop motions in proteins. At longer time scale, there are the rigid body motions ( 1 to $10 \AA, 10^{-9}$ to $1 \mathrm{~s}$ ), i.e., the motion of molecular parts that are locally organized, such as the helices and domains in proteins. Finally, the large-scale motions (45 $\AA, 10^{-7}$ to $10^{4} \mathrm{~s}$ ), for instance protein folding or unfolding processes, the dissociation/association of molecular complexes, or the helix-coil transitions, are the slowest and are still out of reach of atomistic MD simulations. In these cases coarse grain methods are more appropriate but they will not be discussed here (See e.g.[93]).

\subsubsection{Joined experimental and theoretical studies}

Nowadays, the development of periodic density functional theory (DFT), accompanied by more efficient computing resources, allow reliable modelling of complex interfaces. As a consequence, many works present joined experimental and theoretical studies. The comparison between experience and theory is based, in the case of adsorption on metal single crystals, mainly on structural data obtained with STM or LEED. In the case of oxide surfaces, comparison between calculated and experimental spectroscopic data (e.g. IR with calculated vibration frequencies) is much more common.

For what concerns the self-assembly of molecules on a surface, it is worth to mention that it is generally intractable for DFT to calculate, without any structural information originating from experimental data, the nature of the chemical form and organization of molecules adsorbed under a particular condition, due to the too large number of possible geometrical and chemical configurations. Thus, good experimental data on these aspects are absolutely necessary to provide a 
robust starting point for the calculations. Once this is achieved, the calculation can then go on to provide further insights on the bonding, intermolecular interactions, bond lengths, detailed geometry, etc. that would have been inaccessible from experimentation. For metal surfaces, to bridge the gap and propose/refine structural models that reproduce the STM results, atomistic modelling (i.e. theoretical methods that explicitly consider all atoms) is needed. This implies a strong feedback between measurement and modelling: the experimental adsorption patterns can be used as input for the calculations. The geometry of the system is then optimized towards a stable (i.e. low energy) situation and the theoretical results are then confronted with the original data for consistency checks. Combined with the STM data, the results of the calculations then yield a full structural description of the adsorbed molecular layers. Examples of this joint 'STM-modelling' approach will be given in the following sections.

Besides the structural information, atomistic modelling is most useful to get a deep insight on the energetics of the systems under study. First, it is possible to calculate the binding energy of a given molecule to the surface; this implies, for instance, the energetics of metal-molecule bonding in the case of chemisorption, or weak $\mathrm{CH}-\pi$ or $\pi-\pi$ interactions for systems physisorbed. Second, the internal cohesion of the adsorbed layer can be evaluated, by computing the molecule-molecule interaction energy; this includes van der Waals interactions, as well as H-bonding or $\pi-\pi$ interactions. Third, one can compare different molecular organizations, and identify the most probable one on the basis of the energy criterion. Fourth, since energy barriers can also be calculated, the occurrence of transitions can be assessed at the molecular scale (e.g., between different conformations) or at the supramolecular scale (e.g., between different supramolecular arrangements).

\section{Self-assembly of amino acids at metal surfaces}

The study of the interaction of bio-molecules with metal surfaces, at the molecular level, represents a large fraction of the analysis of hybrid organic-inorganic interface. For amino acids and small peptides, this is evident at a first glance by comparing the - necessarily incomplete - Table 1 with Erreur ! Source du renvoi introuvable. andTable 12, reported in the following chapters. Although the investigation of these simplified systems may seem quite academic since pure and perfectly clean metals are rarely found in real life, we recall that many compounds in living bodies (e.g. many enzymes and proteins) contain a metal atom [94]. Furthermore hybrid compounds and/or functionalised metal nanoparticles appear more and more promising in pharmacological applications, as drug delivery and imaging techniques with contrast medium.

It is true, however, that the richness of information present on AA/metal systems in the surface science literature is mainly due to their reduced complexity. On the one hand, the substrate is conductive and can therefore be easily analysed with all the most common surface science techniques. On the other hand, the bio-molecule is sufficiently small to allow a detailed understanding of both the internal conformation of the molecule and the intermolecular and molecule-substrate interactions. In this respect, as it is already evident from Table 1, the joint efforts of experimental techniques and ab-initio theoretical methods revealed to be an extremely precious tool for the unravelling of the detailed adsorption and self-assembly mechanisms. We also note the 
preponderant role of imaging techniques, especially STM, with respect to the case of adsorption at semiconducting or insulating surfaces.

In the following we divide the section in three separate sub-sections, devoted to amino acids adsorption at $\mathrm{Cu}$ surfaces (section3.1), at noble metal surfaces (section3.2) and at transition metal surfaces (section 3.3), respectively. The reasons leading to the choice of each substrate are detailed in the corresponding sub-sections.

\begin{tabular}{|c|c|c|c|c|c|c|c|c|c|c|}
\hline & $\stackrel{\varrho}{\stackrel{\Xi}{0}}$ & 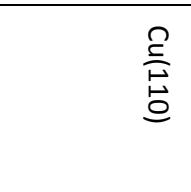 & $\stackrel{\varrho}{\stackrel{\Xi}{巳}}$ & 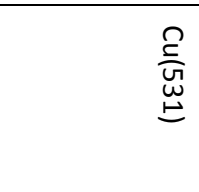 & 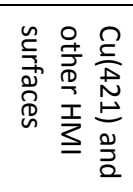 & 站 & 点 & 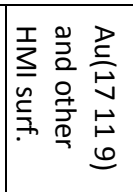 & 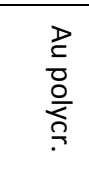 & $\stackrel{?}{\stackrel{R}{E}}$ \\
\hline Gly & $\begin{array}{l}\text { STM, } \\
\text { RAIRS, } \\
\text { XPD [66; } \\
95 ; \\
\text { 96][95; } \\
\text { 97-99] } \\
\text { DFT[100 } \\
\text { ] }\end{array}$ & $\begin{array}{l}\text { STM, RAIRS, } \\
\text { XPS, XPD, } \\
\text { XES, NEXAFS, } \\
\text { LEED, DFT } \\
\text { [42; 67; 101- } \\
\text { 103][104][10 } \\
5 ; \\
\text { 106][100][10 } \\
7], \text { XPD [108] }\end{array}$ & $\begin{array}{l}\text { XPS, XPD, } \\
\text { RAIRS, DFT } \\
\text { [96; 109] } \\
\text { STM[6; 95; } \\
\text { 97; 99;110], } \\
\text { XPD [66; } \\
111]\end{array}$ & $\begin{array}{l}\text { LEED, NEXAFS, } \\
\text { XPS, TPD } \\
{[112]}\end{array}$ & & $\begin{array}{l}\text { STM } \\
{[113]}\end{array}$ & & & & $\begin{array}{l}\text { XPS } \\
{[114]}\end{array}$ \\
\hline Cys & & $\begin{array}{l}\text { FT-RAIRS, } \\
\text { XPS [48] }\end{array}$ & & $\begin{array}{l}\text { XPS, Nexafs } \\
{[115]}\end{array}$ & & $\begin{array}{l}\text { STM, } \\
\text { XPS, UPS, } \\
\text { NEXAFS, } \\
\text { HREELS, } \\
\text { DFT } \\
{[116-} \\
123] \\
{[124][12} \\
\text { 5]. [126] }\end{array}$ & $\begin{array}{l}\text { XPS, } \\
\text { HREELS } \\
{[52],} \\
\text { DFT, } \\
\text { STM } \\
{[127-} \\
129] ; \\
{[130 ;} \\
131][13 \\
2][133]\end{array}$ & \begin{tabular}{|l|}
$X P D$ \\
{$[65]$} \\
DFT \\
{$[134][6$} \\
$5 ; 135]$
\end{tabular} & & \\
\hline Ala & $\begin{array}{l}\text { RAIRS, } \\
\text { LEED, } \\
\text { STM } \\
{[136][13} \\
6- \\
138] \cdot[13 \\
9]\end{array}$ & $\begin{array}{l}\text { RAIRS, LEED, } \\
\text { STM, XPS, } \\
\text { NEXAFS, DFT } \\
\text { [69; 140; } \\
\text { 141][50; 69; } \\
105][142]\end{array}$ & & $\begin{array}{l}\text { LEED, XPS, } \\
\text { NEXAFS[112; } \\
\text { 143] } \\
\text { DFT[144] }\end{array}$ & $\begin{array}{l}\text { LEED, } \\
\text { NEXAFS, } \\
\text { XPS } \\
\text { [145] } \\
\text { DFT[146] }\end{array}$ & & & & & \\
\hline His & & $\begin{array}{l}\text { AES, LEED, } \\
\text { RAIRS, XPS, } \\
\text { NEXAFS [46; } \\
147 ; 148]\end{array}$ & & & & & MD 380 & & $\begin{array}{l}\text { XPS } \\
\text { [149] }\end{array}$ & \\
\hline Lys & $\begin{array}{l}\text { STM } \\
{[150]}\end{array}$ & $\begin{array}{l}\text { XPS, NEXAFS, } \\
\text { RAIRS[151; } \\
152] \\
\text { DFT[61] }\end{array}$ & & & & & $\begin{array}{l}\text { STM } \\
{[153]}\end{array}$ & & & \\
\hline Glu & & & & & & & & & & \\
\hline Met & & XPS[154] & $\begin{array}{l}\text { STM, HAS, } \\
\text { XPS [62] }\end{array}$ & $\begin{array}{l}\text { XPS, } \\
\text { NEXAFS[115] }\end{array}$ & & & $\begin{array}{l}\text { XPS, } \\
\text { RAIRS, } \\
\text { LEED, } \\
\text { STM, } \\
\text { DFT } \\
{[68 ;} \\
155]\end{array}$ & & & \\
\hline
\end{tabular}




\begin{tabular}{|l|l|l|l|l|l|l|l|l|l|}
\hline Tyr & & & & & & & & \\
\hline Pro & & $\begin{array}{l}\text { RAIRS, LEED, } \\
\text { STM, } \\
\text { DFT[156- } \\
\text { 158] }\end{array}$ & & & & & $\begin{array}{l}\text { STM, } \\
\text { XPS, } \\
\text { HREELS } \\
{[159]}\end{array}$ & \\
& & & & & & & & \\
\hline Ser & STM[16 & $\begin{array}{l}\text { XPS, NEXAFS, } \\
\text { LEED, STM } \\
\text { [43] }\end{array}$ & & $\begin{array}{l}\text { LEED, XPS, } \\
\text { NEXAFS, DFT } \\
\text { [144] }\end{array}$ & & & & & \\
\hline Trp & STM & & & & & & & & \\
\end{tabular}

\begin{tabular}{|c|c|c|c|c|c|c|c|c|c|}
\hline & $\frac{\sqrt{0}}{\stackrel{0}{8}}$ & 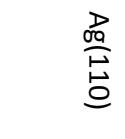 & $\begin{array}{l}\stackrel{D}{o g} \\
\stackrel{P}{E} \\
巳\end{array}$ & $\begin{array}{l}\text { D } \\
\text { oa } \\
\text { ס } \\
\frac{0}{\alpha}\end{array}$ & $\frac{\substack{\text { da } \\
\complement}}{\complement}$ & 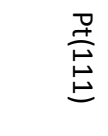 & 异 & 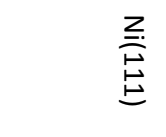 & 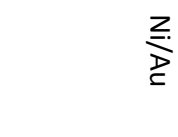 \\
\hline Gly & & & & & $\begin{array}{l}\text { STM } \\
{[162]}\end{array}$ & $\begin{array}{l}\text { TDS, } \\
\text { XPS } \\
{[38]} \\
\end{array}$ & $\begin{array}{l}\text { TPD, XPS } \\
\text { [40], } \\
\text { DFT[100] }\end{array}$ & & \\
\hline Cys & & & $\begin{array}{l}\text { STM, XPS, } \\
\text { NEXAFS [44] } \\
\text { DFT [163-166] }\end{array}$ & & & & & & \\
\hline Ala & & & & & & & TPD, XPS [39] & & \\
\hline His & & & & & & & & & \\
\hline Lys & & & & & & & & & \\
\hline Glu & $\begin{array}{l}\text { STM, XPS, } \\
\text { HREELS, DFT } \\
{[54 ; 59]}\end{array}$ & $\begin{array}{l}\text { RAIRS, } \\
\text { STM } \\
\text { DFT [51; } \\
\text { 167] }\end{array}$ & $\begin{array}{l}\text { STM, XPS, } \\
\text { HREELS, DFT } \\
{[53]}\end{array}$ & & & & & $\begin{array}{l}\text { RAIRS, STM } \\
{[168-170]}\end{array}$ & $\begin{array}{l}\text { RAIRS, TPD, } \\
\text { MEIS, STM } \\
{[171]}\end{array}$ \\
\hline Met & & & $\begin{array}{l}\text { STM, XPS, } \\
\text { HAS, MM [62; } \\
63]\end{array}$ & & & & & & \\
\hline Tyr & & & $\begin{array}{l}\text { STM, XPS, } \\
\text { NEXAFS [45] }\end{array}$ & & & & & & \\
\hline Pro & & & & & & & TPD, XPS [41] & & \\
\hline Ser & & & & & & & & & \\
\hline Many & & & & $\begin{array}{l}\text { SERS } \\
{[172]}\end{array}$ & & & & & \\
\hline
\end{tabular}

Table 1. Summary of the amino acid metal systems described in the present report with the list of employed techniques and the reference to the main related papers.

\subsection{Amino acid adsorption at $\mathrm{Cu}$ surfaces.}

So far, the large majority of the investigations on the adsorption of amino acids at metal surfaces under controlled, UHV conditions has been performedon $\mathrm{Cu}$ samples. Data are present in literature for almost all amino acids species and, in several cases, on more than one $\mathrm{Cu}$ surface.

The reason for the choice of copper as privileged substrate for fundamental studies is both historical and functional. In fact, $\mathrm{Cu}$ surfaces were already very well characterised when, in the $90 \mathrm{~s}$, the first 
surface science investigations of the hybrid metal-organic interface begun. This offered the advantage of starting a new field of research using a well-known substrate.

The comparison among results on the many $\mathrm{AA} / \mathrm{Cu}$ systems allows to draw a global picture in which one can address both the common features and the differences related to the surface geometry and/or the molecular structure. This is not possible for other AA/metal systems, which have been explored in a much less systematic way. The first trivial question is: "Does the molecule adsorb? And in which form?" The general trend for amino acids on $\mathrm{Cu}$ is that at room temperature they adsorb in themonolayer regime, while multilayers are observed at $300 \mathrm{~K}$ only in a few cases and after prolonged exposure [48; 157]. Multilayers easily form, on the contrary, at lower T. Both XPS and RAIRS data agree that the molecules directly interacting with the surface are in general in the anionic form, while zwitterionic adsorption is characteristic of the multilayer.

Secondly, it is possible to identify common adsorption configurations of the different amino acids. As it will be detailed in the following, the simplest amino acids bind to the $\mathrm{Cu}$ surface in a $\mu 2$ or $\mu 3$ configuration, depending on coverage and annealing temperature (see, e.g., Figure 5 and Figure 11). The former geometry corresponds to the situation in which two binding points are present, namely the $\mathrm{N}$ atom and one $\mathrm{O}$ atom of the carboxylate group, while the second oxygen atom protrudes into the vacuum. The three binding points configuration $(\mu 3)$ is more stable, since the $\mathrm{N}$ atom and both carboxylate $\mathrm{O}$ atoms are bound to the surface; the oxygen atoms sit on adjacent $\mathrm{Cu}$ atoms in the $<1$ $10>$ directions, in nearly on top position, while the $\mathrm{N}$ atom is nearly on top one $\mathrm{Cu}$ atom of the nearest $\mathrm{Cu}$ row (see Figure 5). In case of the simplest amino acids, like Gly and Ala, the radical group protrudes out from the surface plane; if the radical chain is more complex and contains additional functionalities, on the contrary, it may be involved in hydrogen bonds or in an extra bond with the metal substrate. Therefore it may orient close to the surface and establish a fourth binding point with it. This is the $\mu 4$ conformation observed, e.g., for $\mathrm{Lys} / \mathrm{Cu}(110)$ [152] and $\mathrm{Ser} / \mathrm{Cu}(531)$ [144].

The similarity in the adsorption conformation leads for these molecules to very similar long range adsorption patterns, as demonstrated by LEED and STM. Three distinctly different phases may form on $\mathrm{Cu}$ surfaces with increasing molecular coverage: the 2D gas, the intermediate, and the solid phase. The gas phase is driven by repulsive interaction among the molecules, which are "standing" or "surfing" on the substrate and can diffuse frequently. In the intermediate phase, the "standing" molecules align into short rows, which are stabilised by intermolecular H-bonds. In the solid phase the amino acid units lay flat on the surface arranging in extended, ordered superstructures. For Gly and Ala on $\mathrm{Cu}(110)$ the most stable assembly is the $(3 \times 2)$ superstructure $[104 ; 105 ; 173]$ (see Figure 8 and Figure 15d), which is also observed for a more complex molecule such as Lys [174]. The most stable adsorption patterns observed for the other amino acids at saturation coverage are, generally, less dense and can be explained by the necessity to accommodate larger molecules. In all cases hydrogen bonding is essential for the stabilization of the layer. In addition, some amino acids may cause magnificent step faceting and bunching of the surface or even exhibit some enantioselectivity.

The overall picture tells that the adsorption mechanism is the same - or very similar - for all amino acids and it is mainly ruled by the amino acid functionalities although a contribution may come from additional reactive terminations. 
Adsorption of amino acids at $\mathrm{Cu}$ surfaces was reviewed by S.M. Barlow and R. Raval in 2003 [175], in a report which was mainly focussed on the chiral organisation of molecules at metal surfaces but which also offered an accurate summary of the state of the art of the AA/Cu systems. To avoid overlapping with already existing information, we provide in this review a quick overview of the state of the art until 2003 and focus on the progresses achieved from that moment on. We start with the simplest non-chiral (glycine, section 3.1.1) and chiral (alanine, section 3.1.2) molecules, for which the largest information is available. In the following sections, we describe the adsorption behaviour of more complex amino acids, which are grouped according to some common characteristics of the molecule or of the surface. Therefore, in section 3.1.3, we review the interaction of $\mathrm{Cu}$ surfaces with amino acids having a lateral side chain with an additional functional group. In this frame it is particularly interesting to see how the presence of the - potentially reactive- molecular tail affects the $\mu 3$ adsorption configuration. Section 3.1.4 is devoted to amino acids characterised by a ring-shaped radical tail, i.e. having an aromatic and/or a pyrrolic ring besides the amino-acid functionalities. This particular feature may induce a molecular rigidity which, especially in the case of proline, significantly influences the adsorption pattern. Finally, in section 3.1.5 we discuss the adsorption at HMI surfaces, with particular attention to adsorbateinduced step-faceting and to enantioselectivity effects induced by the use of chiral surfaces.

\subsubsection{Glycine adsorption at $\mathrm{Cu}$ surfaces}

Glycine chemisorbs on $\mathrm{Cu}(110)$ in the $300-420 \mathrm{~K}$ temperature range. RAIRS measurements (see Table 2 for a description of the observed vibrational frequencies and their assignments) [42] demonstrated that at $\mathrm{T}_{\mathrm{ev}}=300 \mathrm{~K}$ dissociative adsorption occurs, with the production of a glycinate (anionic) species and of atomic $\mathrm{H}$, which eventually recombines and desorbs as $\mathrm{H}_{2}$. The orientation of the adsorbed species changes as the coverage increases (see Figure 5). The bonding occurs initially via equivalent oxygen atoms of the carboxylate group (I), oriented broadly perpendicular to the surface in a similar way to the one found for simple deprotonated carboxylic acids such as formate or acetate [67]. At saturation coverage the molecular orientation changes and a $\mu 2$ conformation (II), associated to a faint $(3 \times 2)$ g LEED pattern, is assumed. Finally, upon annealing the saturated layer to $420 \mathrm{~K}$, no significant desorption occurs, the $(3 \times 2) \mathrm{g}$ LEED pattern sharpens and the molecules accommodate in a $\mu 3$ adsorption geometry (III). An analogous structure is proposed also upon deposition of Gly on $\mathrm{Cu}(110)$ at $400 \mathrm{~K}$ [67].

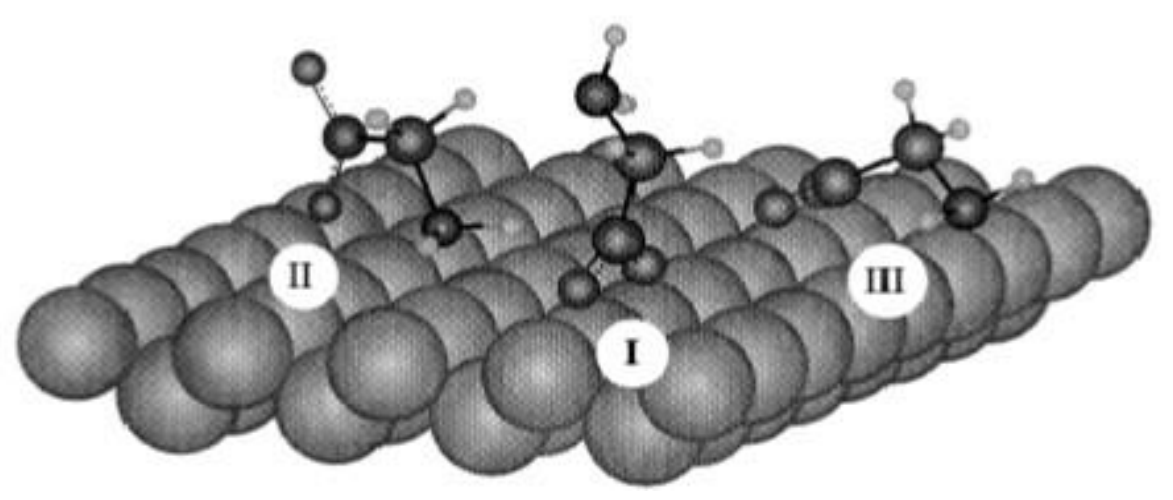


Figure 5. Proposed conformational models for the glycinate species adsorbed on $\mathrm{Cu}(110)$ as deduced from RAIRS. (I) Low Gly coverage at T = $300 \mathrm{~K}$; (II) Saturation coverage at $\mathrm{T}=300 \mathrm{~K}$; (III) annealing to $\mathrm{T}=420 \mathrm{~K}$. Taken with permission from ref. [42].

Below room temperature, multilayers of zwitterionic glycine form. Desorption occurs in two stages, since the second layer is stable up to $340 \mathrm{~K}$ while the subsequent ones give a maximum at $\mathrm{T}=320$ $\mathrm{K}$. At $85 \mathrm{~K}$ some of the glycine moleculesare present on the surface in their acid form, as evidenced by the presence of a carbonyl $(\mathrm{CNO})$ stretch.

The different adsorption structures proposed for deprotonated glycine on $\mathrm{Cu}(110)$ (Figure 5) were modelled using cluster models within the DFT framework [103]. The most stable structure was found to be the flat one (structure III in Figure 5), in which the adsorbate binds to the surface through both its functional groups ( $\mu 3$ configuration) and is $0.73 \mathrm{eV}$ more stable than the bridge conformation (structure I inFigure 5). The formation of the $\mu 3$ species only at $\mathrm{T} \geq 400 \mathrm{~K}$ is related to the removal of surface or subsurface hydrogen at this temperature, which allows the adsorbate to lie down on the surface. The precise location of the $\mathrm{O}$ and $\mathrm{N}$ atoms with respect to the $\mathrm{Cu}(110)$ substrate was determined with high accuracy by photoelectron diffraction $(\mathrm{PhD})$ and the resulting configuration is summarised in Figure 6A [66; 176]. A subsequent structural LEED study [173] substantially confirms the model.

The Gly/Cu(110) overlayer was analysed from the electronic point of view by combining DFT with XES and NEXAFS $[67 ; 102]$.By performing angular resolved measurements, it was possible to separate the $2 p$ density of states into three different spatial directions for all the four atoms of interest in the molecule. The orbitals of importance for the interaction of glycine are strongly localized on the functional groups and essentially similar to the small molecule models (formate, acetate, ammonia). This experimental approach is expected to be applicable to more complex systems, e.g., protein and peptide adsorption on metal and oxide surfaces. 


\begin{tabular}{|c|c|c|c|c|c|}
\hline a) & \multicolumn{5}{|c|}{ Glycine } \\
\hline \multirow[t]{2}{*}{ Vibrational mode } & \multicolumn{3}{|l|}{$\mathrm{Cu}(110)(\mathrm{a})$} & \multirow{2}{*}{$\begin{array}{l}\mathrm{Cu}(100)(\mathrm{b}) \\
\Theta_{\text {sat }} \text { at } 300 \mathrm{~K}+ \\
\text { annealing to } 420 \\
\mathrm{~K}\end{array}$} & \multirow{2}{*}{$\begin{array}{l}\mathrm{Cu}(111)(\mathrm{b}) \\
\Theta_{\text {sat }} \text { at } 300 \mathrm{~K}+ \\
\text { annealing to } 420 \\
\mathrm{~K}\end{array}$} \\
\hline & Low $\Theta, 300 \mathrm{~K}$ & $\Theta_{\text {sat }}, 300 \mathrm{~K}$ & $\begin{array}{l}\Theta_{\text {sat }} \text { at } 300 \mathrm{~K}+ \\
\text { annealing to } 420 \\
\mathrm{~K}\end{array}$ & & \\
\hline$v_{\text {asym }}\left(\mathrm{CH}_{2}\right)$ & 2906 & 2910 & 2920 & 2915 & 2915 \\
\hline$v_{\text {sym }}\left(\mathrm{CH}_{2}\right)$ & 2860 & 2860 & 2858 & 2850 & 2850 \\
\hline$v_{\text {asym }}\left(\mathrm{COO}^{-}\right)$ & --- & 1630 & 1630 & 1630 & 1643 \\
\hline$\delta_{\text {sciss }}\left(\mathrm{NH}_{2}\right)$ & --- & 1578 & 1578 & $(1580)$ & (1580) \\
\hline$\delta_{\text {sciss }}\left(\mathrm{CH}_{2}\right)$ & --- & 1441 & 1441 & $(1440)$ & (1425) \\
\hline$v_{\text {sym }}\left(\mathrm{COO}^{-}\right)$ & 1417 & 1422 & 1414 & 1412 & 1410 \\
\hline$\omega\left(\mathrm{CH}_{2}\right)$ & 1332 & 1320 & 1323 & 1335 & 1333 \\
\hline$\omega\left(\mathrm{NH}_{2}\right), v(\mathrm{CN})$ & $1088 / 1024$ & 1105 & 1102 & $1105 / 1025$ & $1110 / 1033$ \\
\hline$v(C C)$ & 969 & 985 & 985 & 963 & 970 \\
\hline $\mathrm{r}\left(\mathrm{CH}_{2}\right)$ & 945 & 902 & --- & 924 & 915 \\
\hline b) & \multicolumn{4}{|c|}{ Alanine } & \\
\hline \multirow[t]{2}{*}{ Vibrational mode } & \multicolumn{3}{|l|}{$\mathrm{Cu}(110)(\mathrm{c})$} & $\mathrm{Cu}(100)(\mathrm{d})$ & \\
\hline & Phase I & Phase II and III & Phase IV & $\Theta_{\text {sat }}$ & \\
\hline$v_{\text {asym }}\left(\mathrm{CH}_{3}\right)$ & & 2985 & 2978 & 2970 & \\
\hline$v_{\text {sym }}\left(\mathrm{CH}_{3}\right)$ & (2935) & 2931 & 2931 & 2929 & \\
\hline $2 \times \delta_{a}\left(C_{3}\right)$ & 2876 & 2876 & 2876 & & \\
\hline$v_{\text {asym }}\left(\mathrm{COO}^{-}\right)$ & (1613) & 1626 & & & \\
\hline$\delta_{\text {sciss }}\left(\mathrm{NH}_{2}\right)$ & & 1576 & & & \\
\hline$\delta_{\text {asym }}\left(\mathrm{CH}_{3}\right)$ & $(1466)$ & 1462 & 1456 & 1459 & \\
\hline$v_{\mathrm{sym}}\left(\mathrm{COO}^{-}\right)+\delta_{\mathrm{s}}\left(\mathrm{CH}_{3}\right)$ & 1411 & 1415 & 1415 & 1409 & \\
\hline$\delta_{\text {sym }}\left(\mathrm{CH}_{3}\right)+v_{\text {sym }}\left(\mathrm{COO}^{-}\right)$ & (1373) & 1382 & (1373) & 1367 & \\
\hline $\begin{array}{l}\delta(\mathrm{CH})+v_{\text {asym }}\left(\mathrm{COO}^{-}\right) \\
\omega\left(\mathrm{CH}_{2}\right)\end{array}$ & 1302 & 1289 & 1300 & 1299 & \\
\hline$\omega\left(\mathrm{NH}_{2}\right)+v_{\text {sym }}\left(\mathrm{OCO}^{-}\right)$ & 1141 & 1167 & 1145 & 1147 & \\
\hline$v_{\text {asym }}(\mathrm{CCN})$ & 1083 & 1086 & 1074 & & \\
\hline$v(\mathrm{CN})+v_{\text {sym }}\left(\mathrm{OCO}^{-}\right)+$ & 1036 & 1036 & 1048 & & \\
\hline$v(\mathrm{CN})+\delta_{\text {sym }}(\mathrm{OCO})$ & 919 & 919 & 919 & & \\
\hline$\delta_{\text {sym }}(\mathrm{OCO})+v(\mathrm{CN})$ & & 847 & & & \\
\hline$\delta_{\text {sym }}(O C O)+v(C C)$ & (771) & 771 & (771) & & \\
\hline
\end{tabular}


Table 2.a) Gly/Cu. Adsorption bands measured upon Gly adsorption at LMI Cu surfaces, expressed in $\mathrm{cm}^{-1}$. Values in brackets correspond to poorly resolved features in the RAIR spectra. Data taken from: (a) ref. [42], (b) ref. [96]. b) Same as a) for the $\mathrm{Ala} / \mathrm{Cu}(110)$ and $\mathrm{Ala} / \mathrm{Cu}(100)$ systems. Data taken from: (c) refs. [50; 105], (d) ref. [136].

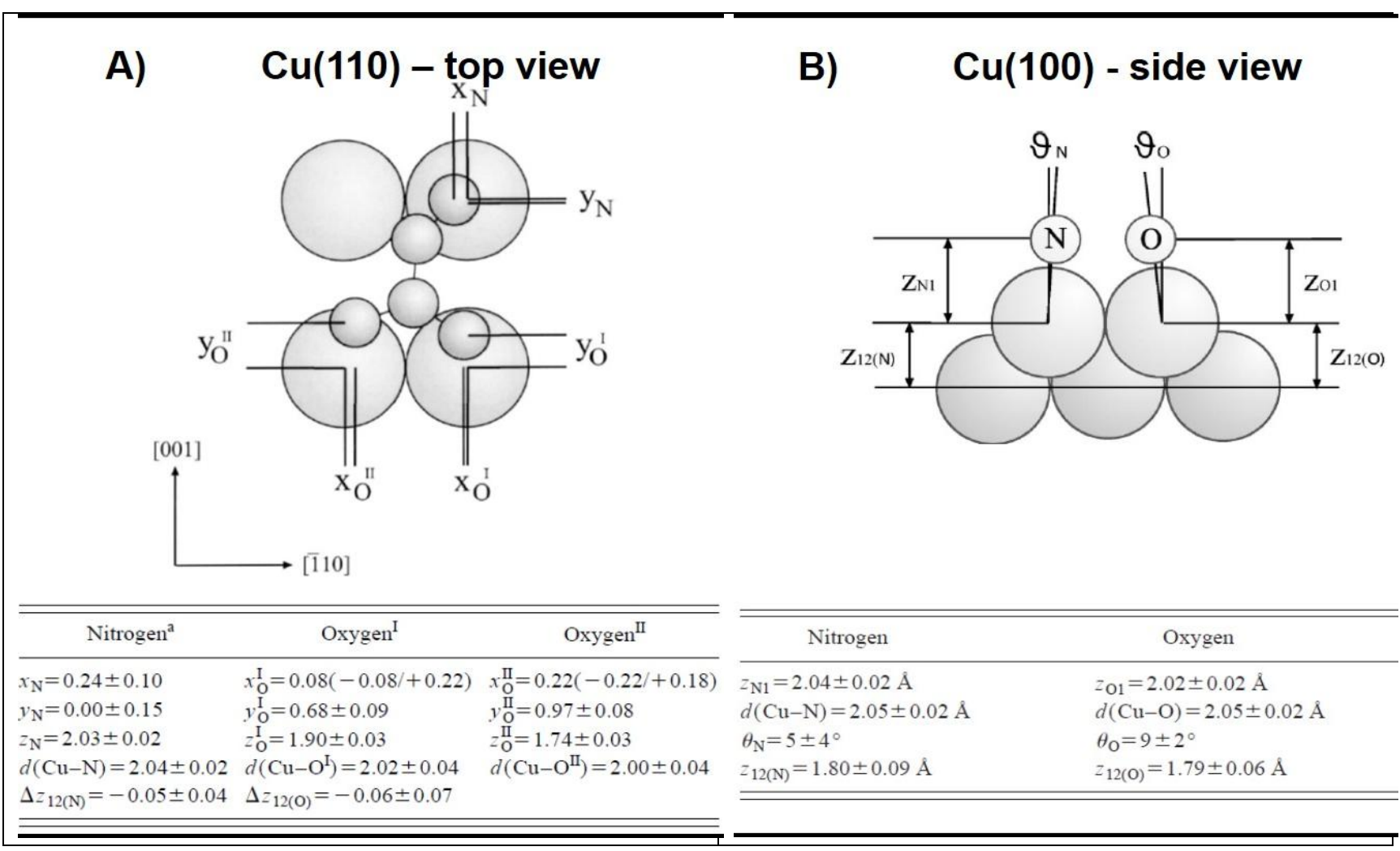

Figure 6. A) Top view of a glycinate molecule adsorbed on $\mathrm{Cu}(110)$ in the most stable, $\mu 3$ configuration. The values for the structural parameters are given in the table at the bottom (in $\AA$, $x$ and $y$ - directions are along one of the $\langle-110\rangle$ and $\langle 001\rangle$ directions, respectively). The adsorption geometry is derived by combining theoretical results for the relative positions of the atoms with the $\mathrm{PhD}$ results for the quantitative offsets of the oxygen and nitrogen atoms from atop sites. While $\mathrm{N}$ is located in a nearly atop site, the two non-equivalent $\mathrm{O}$ atoms are bound to adjacent atoms of the next $\mathrm{Cu}$ row, significantly displaced in the $<001>$ direction. B) Schematic side view defining key structural parameters of the nitrogen and oxygen binding sites of glycinate on $\mathrm{Cu}(100)$. The optimum structural parameters values are reported in the table at the bottom of each panel. The $\mathrm{Cu}-$ $\mathrm{N}$ and $\mathrm{Cu}-\mathrm{O}$ bond lengths deduced from XPD analysis are very similar for $\mathrm{Gly} / \mathrm{Cu}(110)$ and $\mathrm{Gly} / \mathrm{Cu}(100)$. This contrasts with the shorter $\mathrm{Cu}-\mathrm{O}$ bond lengths found for carboxylates on $\mathrm{Cu}(110)$; the difference may arise from the 'lying-down' geometry of the amino acids, associated with the extra substrate bonding through the $\mathrm{N}$ atom. Taken with permission from ref. [66]

Since a well-defined LEED pattern is present for the flat configuration, an empirical model of molecular ordering is proposed in which interadsorbate bonding is dominated by $\mathrm{CH} \cdots \mathrm{O}$ and $\mathrm{N}-$ $\mathrm{H} \cdots \mathrm{O}$ hydrogen bonds similar to those found in bulk glycine [42]. The centred $(3 \times 2)$ structure with two molecules per unit cell results from a compromise between optimal adsorbate site, intermolecular hydrogen bonding and maximum adsorbate density. The threefold and twofold 
periodicities along the $\langle-110\rangle$ and $<001>$ directions, respectively, lead to an almost square unit cell; this structure is quite common for small $\alpha$-amino acids on $\mathrm{Cu}(110)$, since the molecules are bound to the surface through the AA functional groups and let the radical tail protrude far from the surface. The corresponding STM analysis of the $(3 \times 2)$ g-Gly/Cu(110) overlayer showed both homochiral and heterochiral domains at the surface [101]. This may sound odd, since glycine is non-chiral. Indeed it is often indicated as a pro-chiral molecule. This means that the stable gas-phase conformation of glycine does not have a mirror plane, so it might formally be considered chiral in that its mirror image is not superimposable on itself; however, the energy barrier for interconversion of the two enantiomers is low, and racemization easily occurs at room temperature. This barrier is raised dramatically upon strong chemisorption on a metal surface, such as $\mathrm{Cu}(110)$, and this effect causes the chemisorbed species to become efficiently chiral. This is a general phenomenon, which applies to any molecule which loses all mirror planes on chemisorption. In principle, an achiral substrate is not enantioselective, so the D- and L-enantiomers should be present in equal amount on the surface and form a racemic layer. Contrary to this prediction, Figure 7A clearly shows the presence of two different domains on the $(3 \times 2) \mathrm{g}-\mathrm{Gly} / \mathrm{Cu}(110)$ surface, which are incommensurable with respect to each other. Detailed analysis of the images (Figure 7B) allows to identify a homochiral, pseudo centred structure (Figure 7, top of panel B and panel C) and a heterochiral structure with a glide plane (Figure 7, bottom of panel B and panel D). In both cases, each unit cell contains two molecules. This work also confirmed the existence of only one adsorption site for the amino group, identified with the only STM feature commensurate between different domains. The other features are associated to the carboxylate groups, which bind therefore at two different sites, leading to four non-equivalent $\mathrm{O}$ atoms within each unit cell. A possible involvement of copper atom displacements in the final structures is not ruled out but it is not discussed due to lack of data. We mention that the chiral recognition process described for $\mathrm{Gly} / \mathrm{Cu}(110)$ is quite common when dealing with chiral molecules but, since it is not typical of amino acids, an accurate description goes beyond the scope of the present review. We,thus,recommendto the reader specific literature on the topic $[175 ; 177 ; 178]$.

Finally, we mention that the results of ref. [101] are not in perfect agreement with subsequent works aimed at determining the long range order of the $(3 \times 2)-\mathrm{Gly} / \mathrm{Cu}(110) \mu 3$ structure on the basis of diffraction methods $[66 ; 173]$. The authors of these latter studiessuggest that homochiral domains may be present but in minorityon the surface compared to heterochiral ones. In one of the cited papers[66], they explicitly raise doubts on the interpretation of STM images. We are not going to comment on this point, leaving the interested reader free to judge and appreciate the debate. 


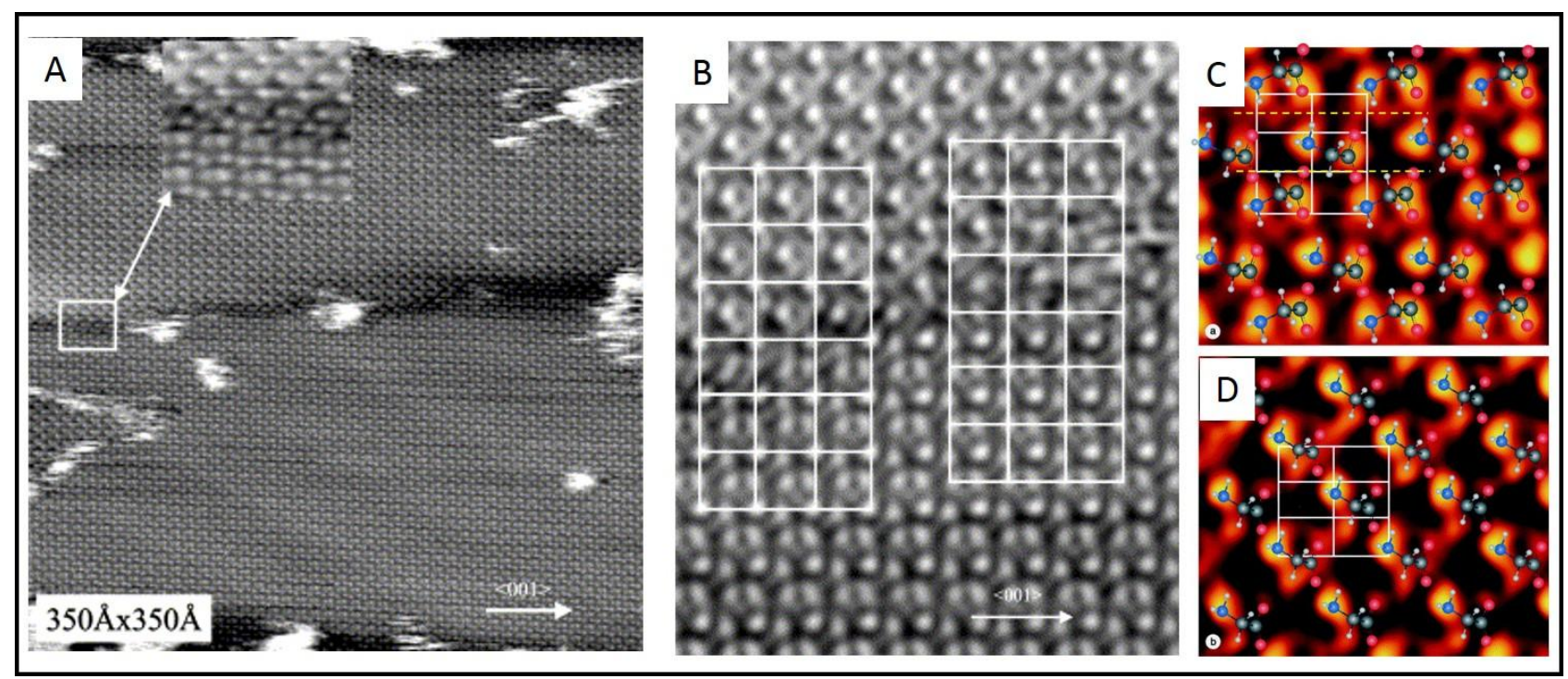

Figure 7. A) STM image $\left(350 \times 350 \AA^{2}, V=-0.06 \mathrm{~V}, \mathrm{I}=4.28 \mathrm{nA}\right)$ of a saturated monolayer of glycinate on $\mathrm{Cu}(110)$. Two domains with different structure, although with the same $(3 \times 2)$ periodicity, are present on the terrace. Their boundaries are sometimes decorated with disordered molecules, appearing as the whitish cloudy features. A magnification of the region at the domain boundary, which shows the relationship between the two different types of domain, is shown in the inset. B) High pass filtered STM image $\left(65 \times 80 \AA^{2}, V=-0.06 \mathrm{~V}, \mathrm{I}=4.28 \mathrm{nA}\right)$ of the same glycinate layer, which emphasizes the different internal structure of the two regions. The upper domain in the image has a pseudo-centered structure with two dominant features per unit cell (marked by the grid). The lower domain has four features per unit cell and a more evident glide plane symmetry along the <001> direction. Both unit cells contain two molecules, in accord with previous studies [42; 67; 175; 176]. C) and D) Models of the heterochiral and homochiral unit cells superimposed tohigh resolution STM images. Taken with permission from Ref. [101].

Plane wave density functional theory (DFT) has also been applied to determine the structure and relative energy of heterochiral and homochiral $(3 \times 2)$ glycine adlayers on $\mathrm{Cu}(110)$ [104].

The calculated heterochiral structure is in quantitative agreement with X-ray photoelectron diffraction experiments, but the calculated homochiral structure was found to be inconsistent with experimental data (two different $\mathrm{Cu}-\mathrm{O}$ distances versus four in experiment). The calculations also indicate that the heterochiral $(3 \times 2)$ adlayer is more stable than the homochiral $(3 \times 2)$ one: the predicted adsorption energy is $-2.82 \mathrm{eV}$ for the former and $-2.76 \mathrm{eV}$ for the latter. This results indicated that large regions of the homochiral $(3 \times 2)$ glycine adlayer are unlikely to exist on $\mathrm{Cu}(110)$, in agreement with the conclusions of refs. [66; 173] . 

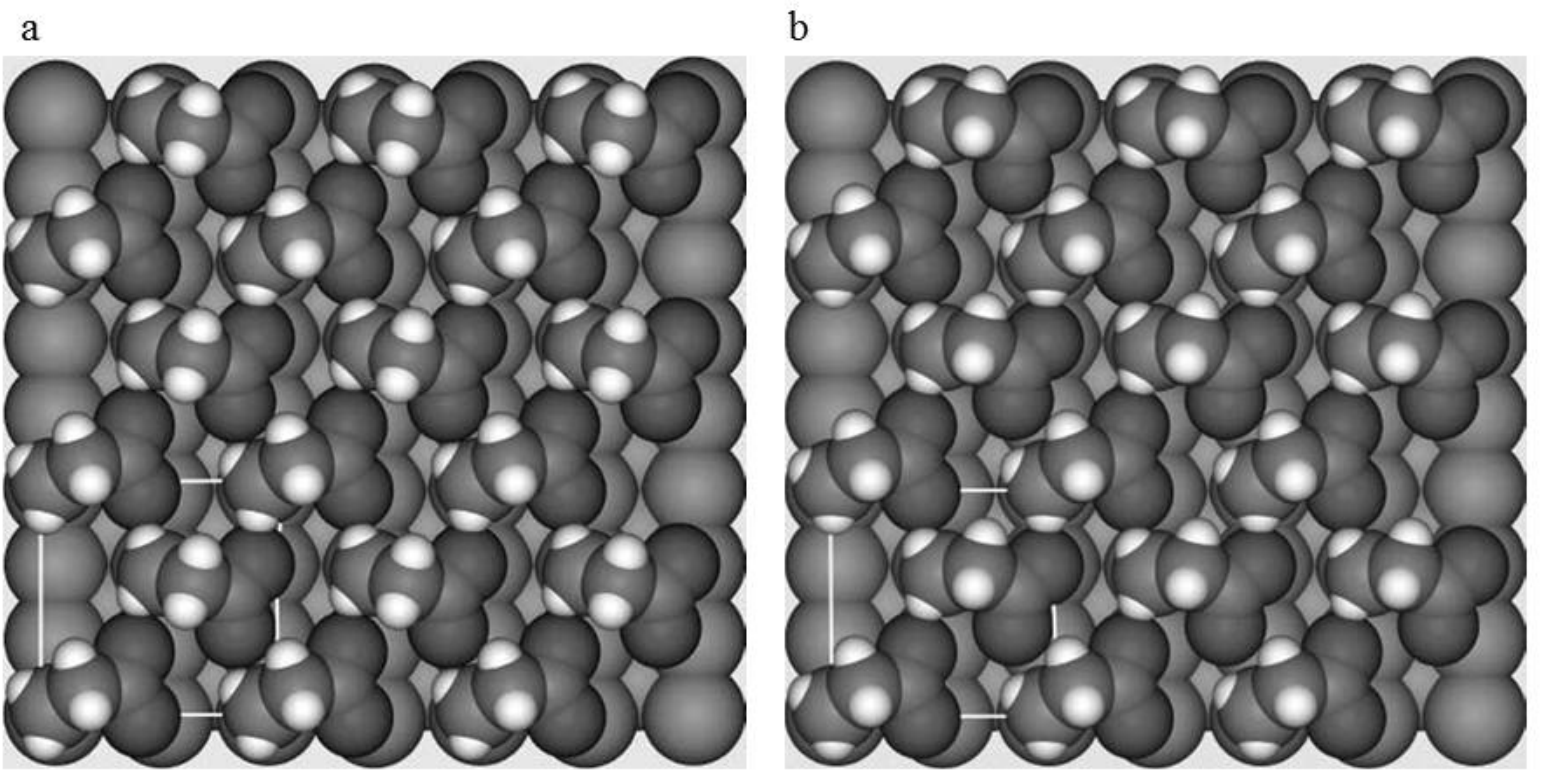

Figure 8 . The optimal geometry of the heterochiral (a) and homochiral (b) (3×2) glycinate adlayer on $\mathrm{Cu}(110)$ as calculated from GGA-DFT. The white box indicates the surface unit cell. Taken with permission from ref. [104].

a)

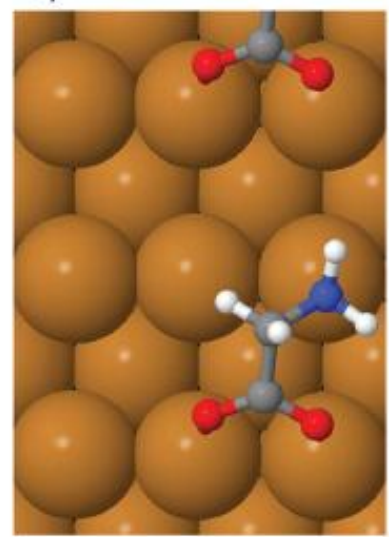

e)

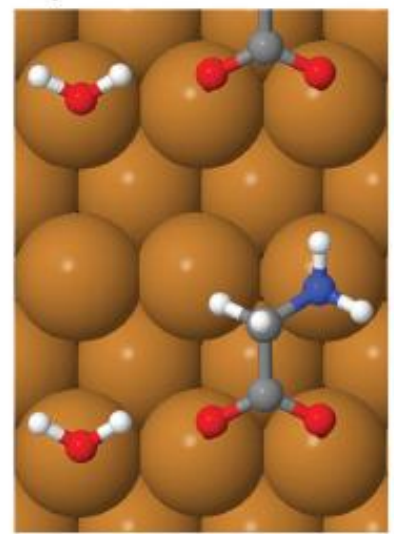

b)

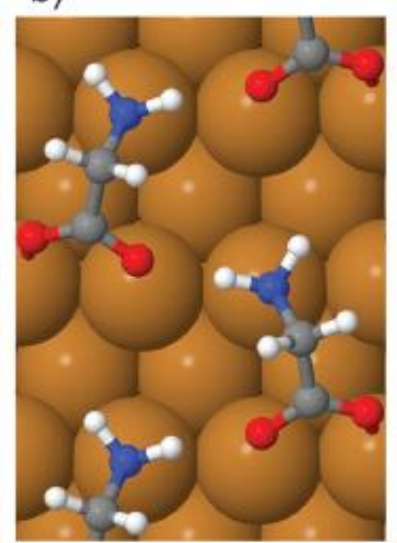

f)

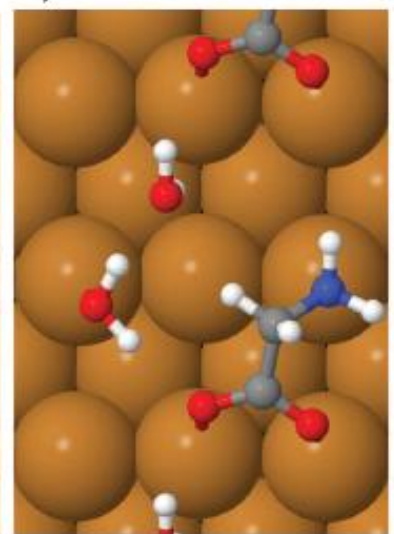

c)

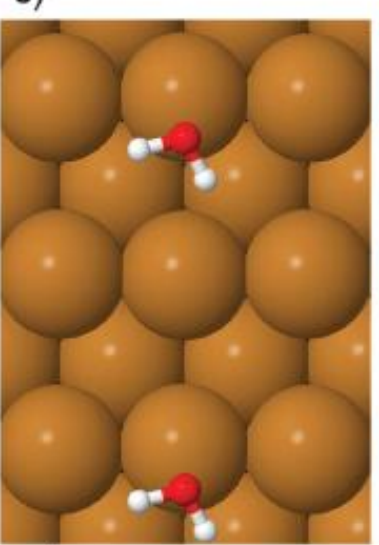

g)

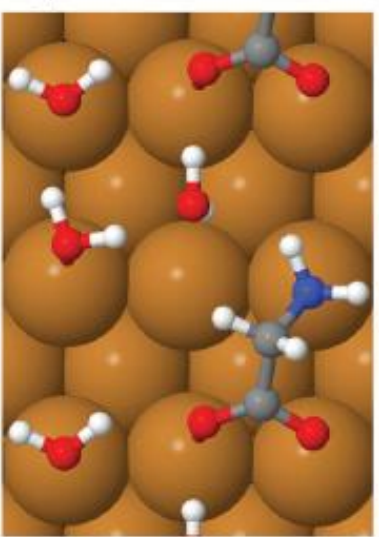

d)

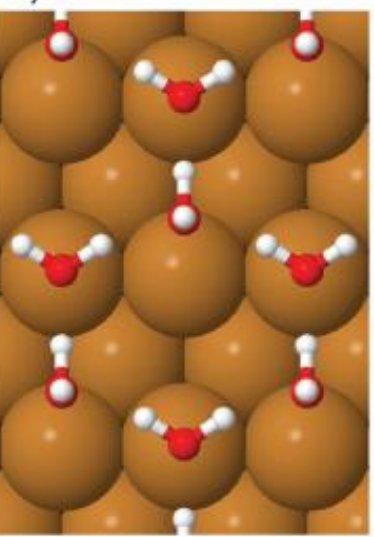

h)

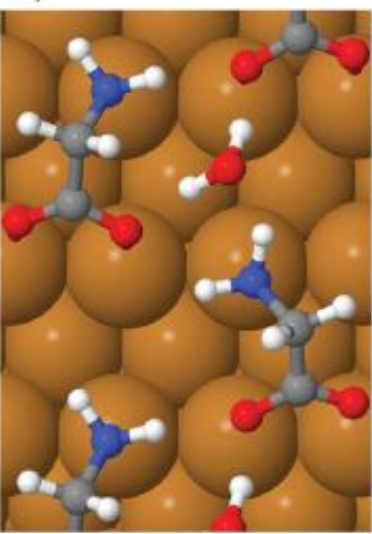

Figure 9. Preferred glycine and water structures on $\mathrm{Cu}(110)$ obtained by DFT. (a) Single glycine monomer (Gly); (b) glycine monolayer (2Gly); (c) water monomer $\left(\mathrm{H}_{2} \mathrm{O}\right)$; (d) water monolayer $\left(6 \mathrm{H}_{2} \mathrm{O}\right)$; (e) mono-hydrated glycine $\left(\mathrm{Gly}-\mathrm{H}_{2} \mathrm{O}\right)$; (f) bi-hydrated glycine $\left(\mathrm{Gly}+2 \mathrm{H}_{2} \mathrm{O}\right)$; (g) tri hydrated glycine $\left(\mathrm{Gly}+3 \mathrm{H}_{2} \mathrm{O}\right)$; (h) glycine monolayer mono-hydrated $\left(2 \mathrm{Gly}+\mathrm{H}_{2} \mathrm{O}\right)$. Taken with permission from ref. [106] 
Interestingly, recently the $\mathrm{Gly} / \mathrm{Cu}(110)$ system was studied at the DFT-D level considering coadsorption with water (see Figure 9)[106]. Sacchi et al. [106] calculated a phase diagram using a thermodynamics approach, showing that under UHV conditions and for a wide range of temperatures, a pure glycine monolayer is more stable than either mixed Gly-water phases or pure water (ice) monolayers. However for high water pressures half-dissociated water layers can appear on the surface at low and intermediate temperatures. To build this phase diagram various $\mathrm{Gly} / \mathrm{H}_{2} \mathrm{O}$ surface structures where calculated as a function of the water concentration, which are shown in Figure 9.
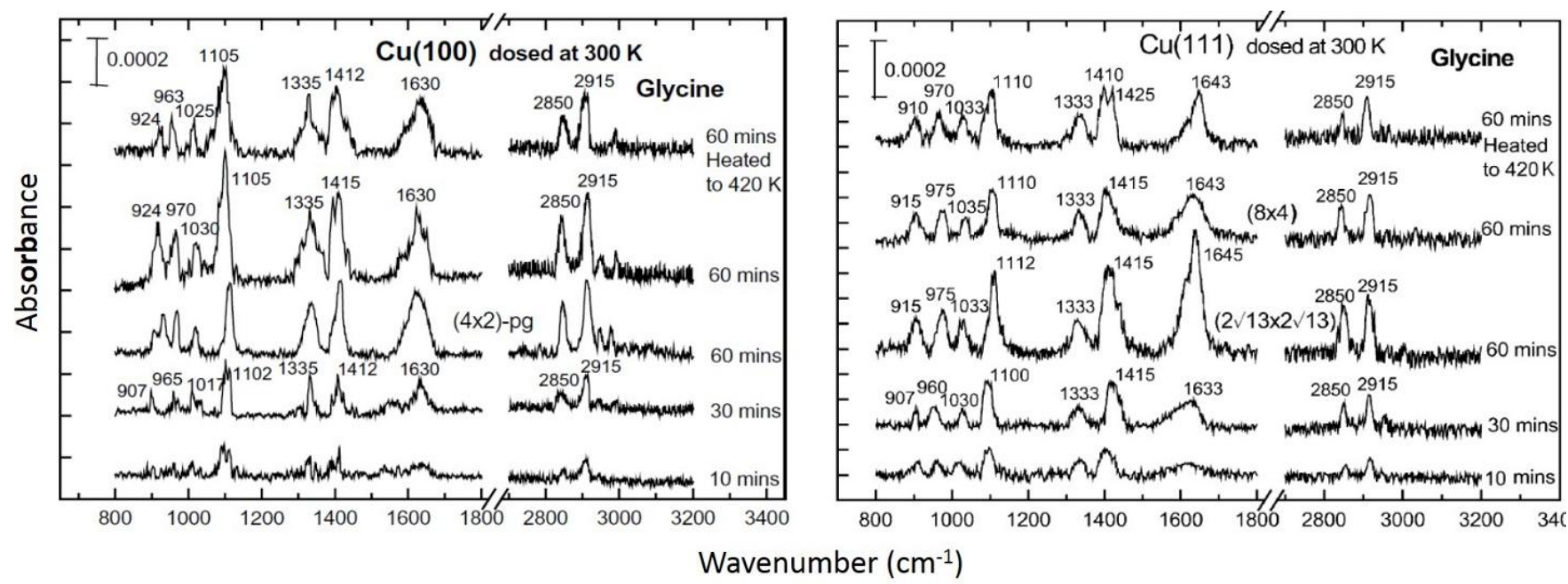

Figure 10. RAIR spectra obtained from $\mathrm{Cu}(100)$ and $\mathrm{Cu}(111)$ following different Gly exposures and - in the topmost spectra- annealingto $420 \mathrm{~K}$. Taken with permission from ref. [96].

The interaction of glycine with $\mathrm{Cu}(100)$ and $\mathrm{Cu}(111)$ initially attracted less attention and was developed in detail comparable to the one of the $\mathrm{Gly} / \mathrm{Cu}(110)$ system only in the last years. An early qualitative LEED analysis [179] identified high-coverage and long-range order $(4 \times 2)$ and $(8 \times 8)$ adsorption phases on $\mathrm{Cu}(100)$ and $\mathrm{Cu}(111)$, respectively. More recently, STM [6; 95; 97; 99; 110], photoelectron diffraction [66; 111] and infrared spectroscopy [96] analyses have been performed.

The main adsorption lines observed in RAIRS spectra of Gly-covered low Miller index Cu surfaces are shown in Figure 10 and summarised in Table 2[96]. The strong similarity of the vibrational frequencies measured for $\mathrm{Cu}(100)$ and $\mathrm{Cu}(111)$ with those seen on $\mathrm{Cu}(110)$ clearly indicates that the adsorbed species is alwaysglycinate.

According to photoelectron diffraction data [66], glycinate organizes on $\mathrm{Cu}(100)$ in extended layers with $(4 \times 2)$ periodicity and a glide plane in the $<0-11>$ direction. As on $\mathrm{Cu}(110)$, both the $\mathrm{N}$ and the $\mathrm{O}$ atoms are bound to the surface, causing a flat orientation of the molecular backbone. The position of the $\mathrm{N}$ and $\mathrm{O}$ atoms with respect to the $\mathrm{Cu}$ atoms underneath was determined with great accuracy as reported in Figure $6 \mathrm{~B}$. Both the $\mu 2$ and $\mu 3$ adsorption configurations are possible. By taking into account the additional structural information available from STM and LEED and the adsorption geometry previously determined for $\mathrm{Gly} / \mathrm{Cu}(110)$, the authors conclude that the $\mu 3$ configuration proposed in Figure 11 is the most likely. In such a geometry, the formation of hydrogen-bond chains of molecules in heterochiral domains highly stabilizes the structure[98]. The possible 
coexistence of these domains with a minority of molecules arranged in a homochiral $\mathrm{c}(2 \times 4)$ phase is however not ruled out.

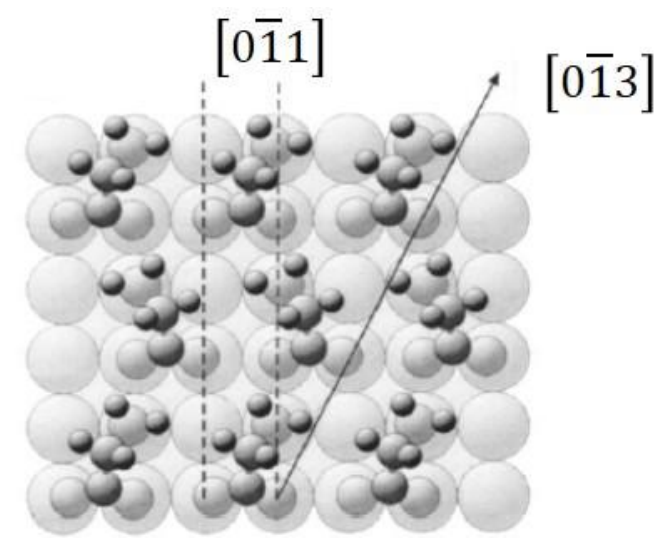

Figure 11. Schematic plan view of the most likely geometry for an overlayer of glycinate on $\mathrm{Cu}(100)$ : heterochiral $(2 \times 4)$-glide plane geometry. The detailed atomic positions in the model is schematic; the internal conformation is based on the results of calculations performed for glycinate on $\mathrm{Cu}(110)$ [103]. Taken with permission from ref. [66].

For glycinate on $\mathrm{Cu}(100)$ the coexistence of two different geometries, namely $\mathrm{c}(2 \times 4)$ and $\mathrm{p}(4 \times 2)$, was indeed observed by STM [95; 97-99] (see Figure 12AB). The two structures correspond to identical local coverage values, namelyone Gly unit every four surface unit cells. The $\mathrm{p}(4 \times 2)$ phase is dominant at large terraces, while the $\mathrm{c}(4 \times 2)$ one is observed mainly in proximity of the steps [98] since it favours the formation of <103> steps[99].

In the $\mathrm{c}(2 \times 4)$ and $\mathrm{p}(2 \times 4)$ superstructures, the difference in H-bond orientation does not seem to influence the specific surface free energy, as confirmed by aDFT study [180]. On the other hand, the empirical models proposed in Figure 12, and based on comparative analysis of the STM and LEED data, suggest that:

- The $(4 \times 2)$ superstructure contains 2 molecules per unit cell, each one being the mirror image of the other. $\mathrm{NH} \cdots \mathrm{O}$ hydrogen bonds develop here in the $\langle 110\rangle$ direction.

- In the $\mathrm{c}(4 \times 2)$ structure, $\mathrm{NH} \cdots \mathrm{O}$ hydrogen bonds form between neighbouring molecules along the $\langle 310\rangle$ directions, responsible for stabilization of the layer. This explains why the formation of $c(2 \times 4)$ islands is strictly related to the presence of $<103>$ steps or $(3117)$ facets, as it will be discussed inSection 3.1.5.

The difference in the electronic structure between the two molecular arrangements (almost isoenergetic) was characterised by STS [99]. The presence of an electronic state for the $p(2 \times 4)$ structure at $5 \mathrm{~K}$ (appearing as a sharp edge in the STS spectrum) is the origin of the mechanism that stabilizes this phase as the ground state of the system, since it is the result of the interaction between the molecular layer and the $\mathrm{Cu}(100)$ substrate.

In conclusion, adsorption of Gly on $\mathrm{Cu}(100)$ not only leads to the formation of self-assembled structures of particular geometry but also causes a significant modification of the surface morphology (see Figure 12C). The same effect, as well as the formation of superstructures determined mainly by the same kind of bonds, were observed for other AA species, in particular for alanine/Cu(001)[139] and lysine/Cu(001)[181] (see section3.1.5). 


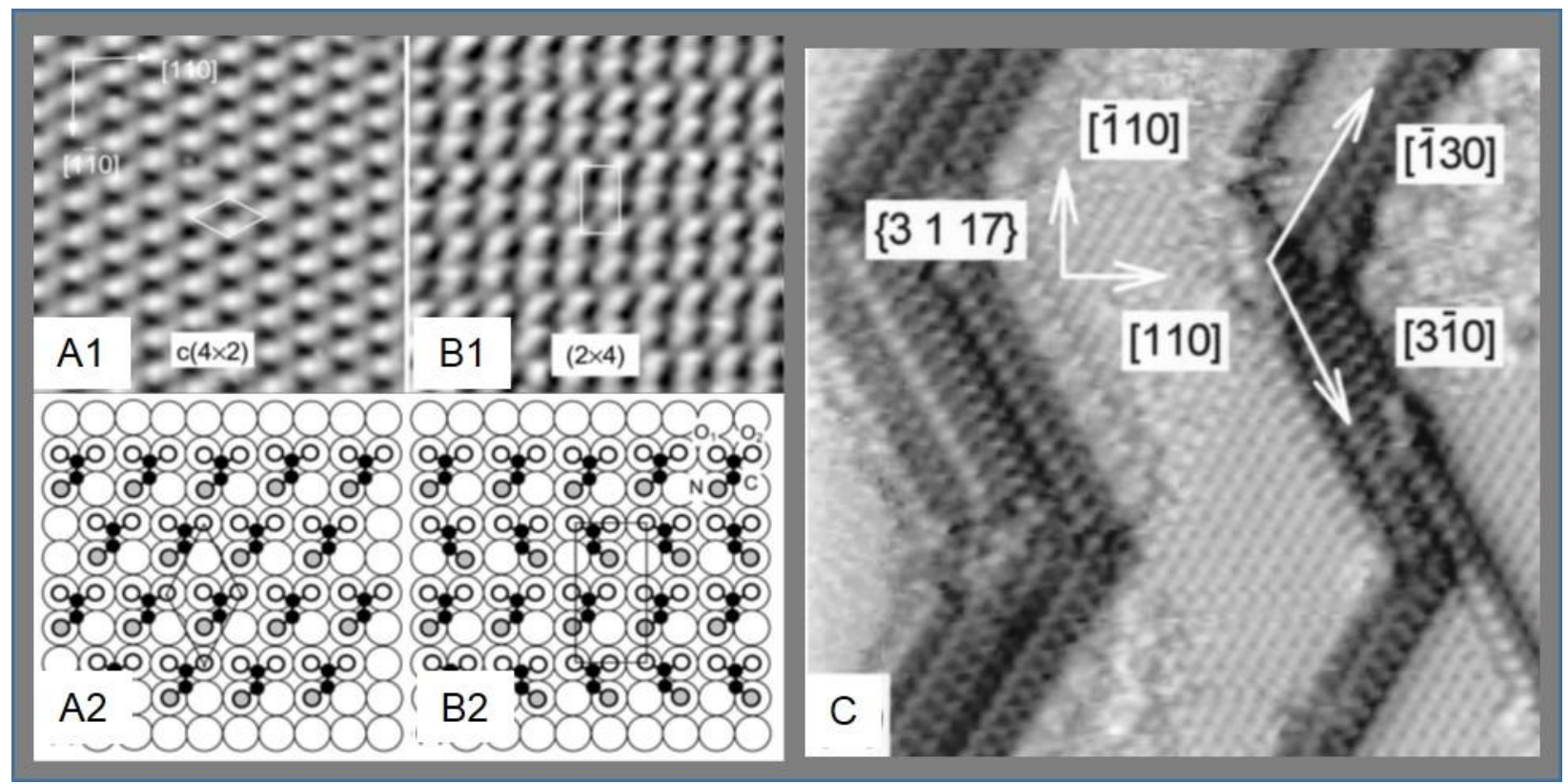

Figure 12. STM images $\left(49 \times 49 \AA^{2}\right)$ and schematic drawing of the proposed adsorbing geometries of: A) the $\mathrm{Cu}(001) \mathrm{c}(4 \times 2)$ - glycine superstructure $(\mathrm{V}=-0.5 \mathrm{~V}, \mathrm{I}=1.0 \mathrm{nA})$; B) the $\mathrm{Cu}(001)(4 \times 2)$ glycine superstructure (V=-1.2 V, I=0.5 nA). C) High-resolution STM image (175x $175 \AA^{2}, \mathrm{~V}=-1.2$ $\mathrm{V}, \mathrm{I}=0.5 \mathrm{nA}$ ) showing that, in areas with higher step density, steps have not only faceted to <310> directions but also bunched together forming (3 1117 ) facets. The $c(2 \times 4)$-glycine and the $1 \times 1-$ Gly superstructures are present on (100) and $\left(\begin{array}{lll}3 & 1 & 17\end{array}\right)$ facets, respectively. The angle between two adjacent $\langle 310\rangle$ steps can take all possible allowed values $\left(37^{\circ}, 53^{\circ}, 90^{\circ}, 127^{\circ}\right.$, and $\left.143^{\circ}\right)$ except $37^{\circ}$, although the dominant value is $127^{\circ}$. Taken with permission from refs. [95; 98].

The adsorption of glycine on $\mathrm{Cu}(111)$ was investigated in parallel to the $\mathrm{Gly} / \mathrm{Cu}(100)$ system, and was then reconsidered in the very recent years. STM experiments [110] showed that, upon Gly deposition at RT (and possibly further annealing to $400 \mathrm{~K}$ ) different structures form depending on coverage and deposition rate. At low $\Theta$ the adsorbate forms a 2D gas phase in which the molecules are oriented broadly perpendicular to the surface. At $\Theta_{\text {sat }}$ and if the deposition rate is not too high, the glycinate units adopt a $\mu 2$ conformation and are connected into chains of $5.05 \AA$ width by $\mathrm{H}$ bonds. These chains are aligned in the three equivalent $\langle 110\rangle$ directions and can further aggregate into domains separated by thin boundaries. Upon annealing to $400 \mathrm{~K}$, the molecules take the flatlying orientation with the $\mathrm{O}_{2}-\mathrm{C}-\mathrm{C}-\mathrm{N}$ backbone essentially parallel to the surface. They are connected by H-bonds and arrange in two different superstructures with $(4 \times 8)$ and $(2 \sqrt{ } 13 \times 2 \sqrt{ } 13)$ geometry, respectively. The former geometry is consistent with the previously observed $(8 \times 8)$ LEED pattern [179] due to the superposition of the diffraction patterns generated by equivalent domains of different orientation. High resolution STM images show round features, which have been interpreted as formed by three glycinate molecules in a circular and homochiral arrangement. There are two of these features in each $(4 \times 8)$ unit cell, which consists therefore of six molecules. The interpretation of the $(2 \sqrt{ } 13 \times 2 \sqrt{ } 13)$ superstructure is more complex. In the STM images of ref. [110] it appears to be formed by small rings surrounded by weaker features. The model proposed suggested that the rings are actually closed chains of molecules in the $\mu 2$ conformation connected by $\mathrm{H}$ bonds, while the external weaker features are individual molecules in the flat-lying, $\mu 3$, conformation. Both conformations co-exist in the unit cell, which is formed by nine glycinate units. 
This model was overcome a few years later by low temperature STM and STS investigation of the same system [6]. In the low coverage limit, upon Gly deposition and prolonged annealing to $350 \mathrm{~K}$, triangular, three-lobed structures are observed (see isolated features in Figure 13a), which are divided into two types with a mirror plane along the [11-2] direction. With increasing coverage, a higher-order structure based on the trimer units forms. The layer has $(2 \sqrt{ } 13 \times 2 \sqrt{ } 13) \mathrm{R} 13.9^{\circ}$ periodicity and shows the presence of two co-existing homochiral domains ( $\rho$ and $\lambda$ regions in Figure 13ab) corresponding to the different orientations of the constituent trimers. Hence the enantioselectivity which rules the formation of trimer units also plays an important role in the growth of the large-scale network. By combining high resolution STM images recorded at different bias voltage, $\mathrm{dI} / \mathrm{dV}$ images and STS spectra, the authors could work out the model proposed in Figure $13 \mathrm{c}$ for the $\rho$ orientation. Neighbouring trimers (circled in blue and red) exhibit $180^{\circ}$ rotational symmetry and each glycinate builds four hydrogen bonds between the amino and carboxyl groups, enabling intra and inter-trimer coupling (dotted green lines) and producing strong interactions. In ref. [6] the nanocavities formed within the $(2 \sqrt{13} \times 2 \sqrt{ } 13) \mathrm{R} 13.9^{\circ}$ layerhave been exploited as a template for the deposition of fullerene molecules. This example suggests a possible application of hierarchical self- assembly of amino acids also in the field of nanoelectronics.

The structural models of the $\mathrm{Gly} / \mathrm{Cu}(111)$ superstructures described so far do not provide a precise description of the relative positions of the atoms forming the Gly molecule with respect to the $\mathrm{Cu}$ atoms of the substrate. This was a goal of a more recent investigation on the $(4 \times 8)$ overlayer, combining energy-scanned photoelectron diffraction data and DFT calculations including dispersion forces [109]. The authors could identify the $\mathrm{N}$ adsorption site of the glycinate species on the $\mathrm{Cu}(111)$ to be close to atop. XPD confirms that glycinate binds to the surface through both the $\mathrm{N}$ and the $\mathrm{O}$ atoms.

Finally we mention that the $\mu 3$ adsorption geometry of Gly molecules was found to be the most stable also on the (311) and (110) nanofacets of the stepped $\mathrm{Cu}(531)$ surface [112]. Adsorption of various AA at chiral and stepped surfaces will be discussed in the dedicated section 3.1.5.

It is evident from above that glycine adsorbs on the $\mathrm{Cu}(100)$ and $\mathrm{Cu}(110)$ surfaces in its dehydrogenated form, $\mathrm{NH}_{2} \mathrm{CH}_{2} \mathrm{COO}$, at room temperature. In contrast, recent X-ray photoelectron spectroscopy experiments indicate zwitterionic adsorption of glycine to $\mathrm{Pd}(111)$. Using DFT, the adsorption of glycine on $\mathrm{Pd}(111), \mathrm{Cu}(100)$ and $\mathrm{Cu}(110)$ was studied to examine this apparent difference in chemical states on these surfaces[100]. It was concluded that at equilibrium, glycine is adsorbed preferentially to these surfaces in its dehydrogenated form, implying that observation of zwitterions is likely a kinetic effect due to the conditions of the experiment. However, for adsorbed glycine at relatively low temperatures, the kinetics of this reaction is sufficiently slow to prevent the formation of the energetically stable dehydrogenated adsorbates on the surface. 

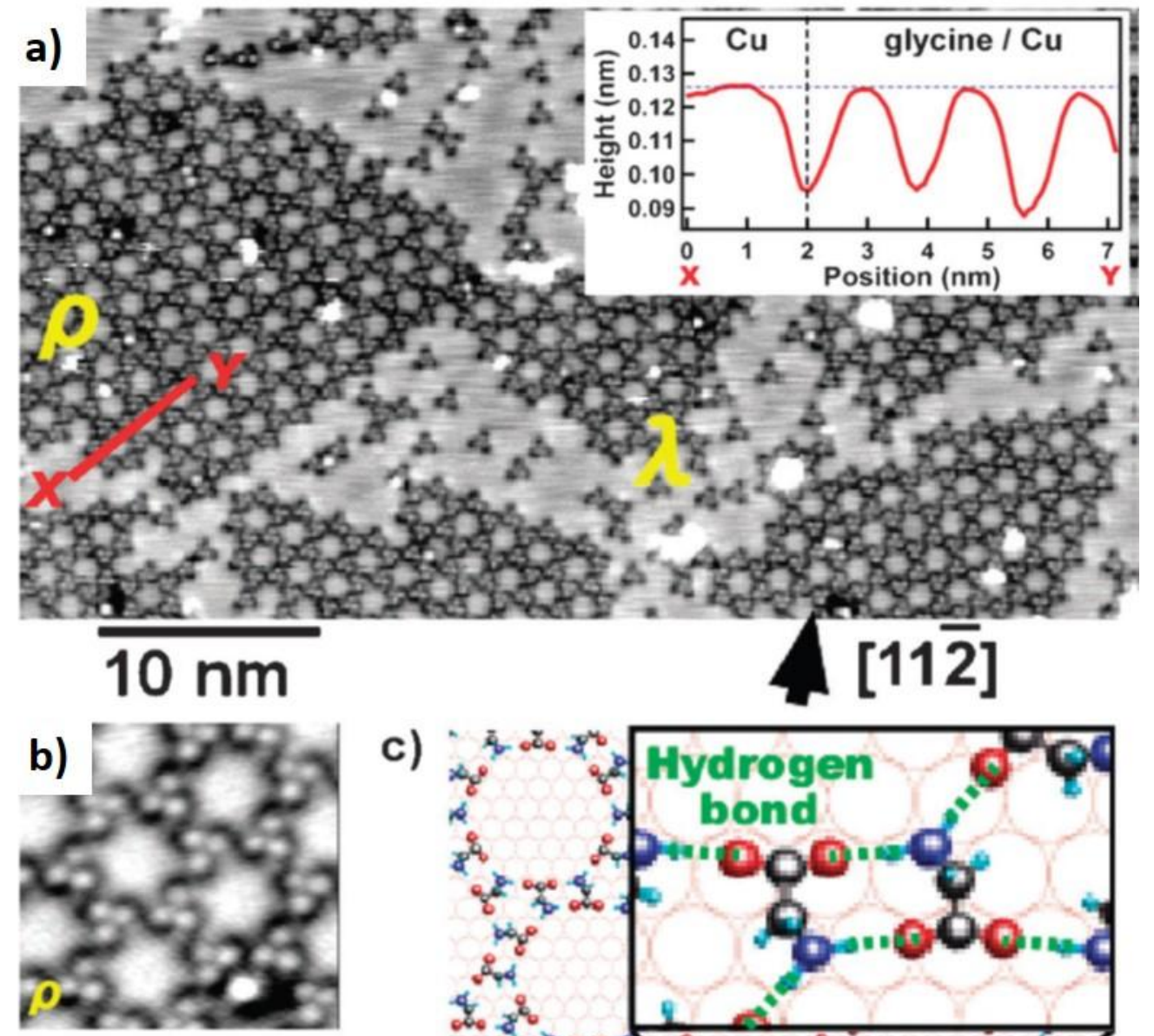

c)
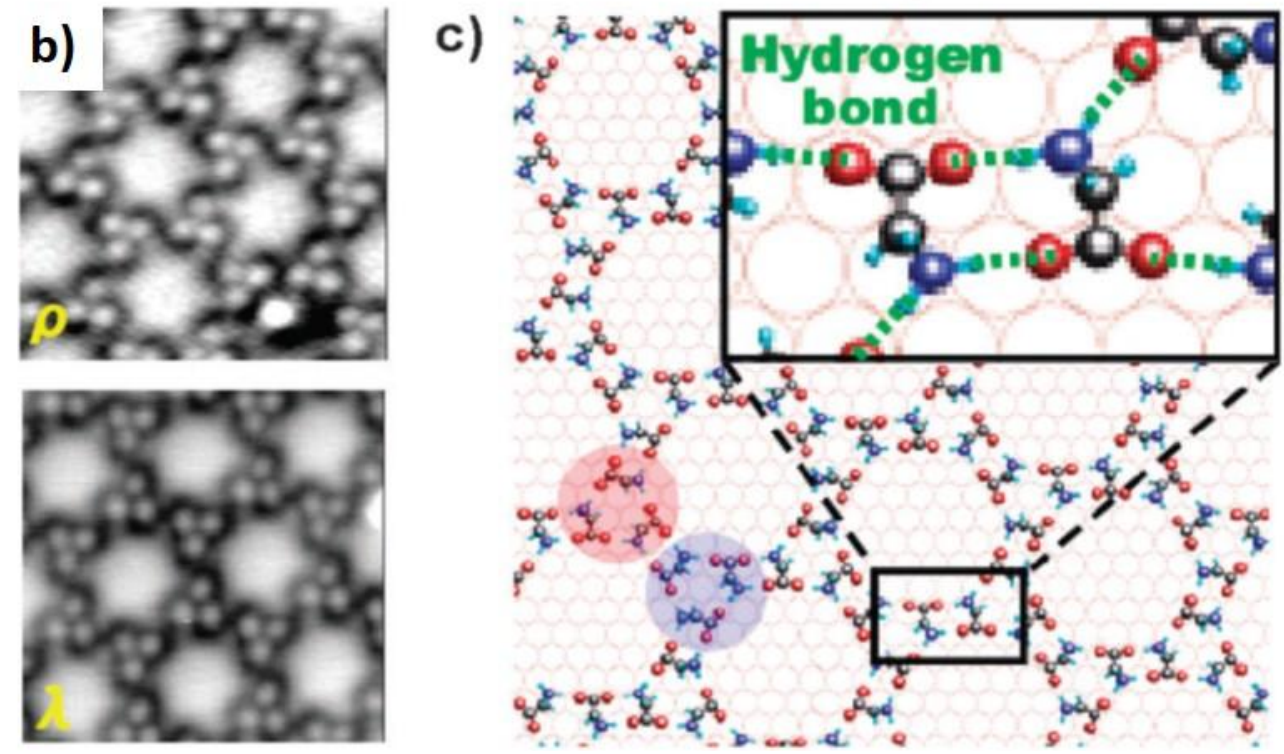

Figure 13. a) STM image recorded upon Gly exposure on $\mathrm{Cu}(111)$ and prolonged annealing to 350 $\mathrm{K}(\mathrm{V}=0.5 \mathrm{~V}, \mathrm{I}=0.5 \mathrm{nA})$. No isolated Gly molecules are observed. Gly-trimers are the elemental units and are present on the surface either isolated or arranged in a second order self-assembled network. The red line indicates the cross-section along $\mathrm{X}-\mathrm{Y}$. Regions with specular orientation with respect to the $\langle 11-2\rangle$ directions are marked as $\rho$ and $\lambda$. b) High-resolution STM images of $\rho$ and $\lambda$ regions in a). c) Schematic of the molecular arrangement of structure $\rho$ (H: light blue, O: red, $\mathrm{N}$ : blue, C: black). The green dotted lines in the box show the hydrogen bonds between molecules. Taken with permission from ref. [6]. 


\subsubsection{Alanine adsorption at $\mathrm{Cu}$ surfaces}

Being the simplest chiral amino acid, alanine is the natural candidate for the prosecution of the studies of AA interaction at $\mathrm{Cu}$ surfaces. Attention was focussed on the nature of the $\mathrm{AA} / \mathrm{metal}$ interaction, on the self-assembly mechanism but also on the transfer of the chiral properties to the surface. Since chirality is not the main topic of this review, we will mention this latter point only when it is useful for our discussion.

The Ala/Cu(110) system was deeply investigated by the group of Raval [50; 69; 105] by means of RAIRS, XPS, STM and LEED.

In the multilayer regime, stable below room temperature, the molecules are predominantly in a zwitterionic state, as expected for solid alanine and as revealed by the binding energy of the N1s doublet detected in the XPS spectra (see Table 3) [141]. The presence of a doublet in the C1s region and of two convoluted peaks in the $\mathrm{O} 1 \mathrm{~s}$ one indicates that adsorption occurs in two different moieties, either neutral and zwitterionic species (as in the case of Gly/Pt(111) [38]) or surface and bulk species.

According to RAIRS data, adsorption of S-alanine molecules on the $\mathrm{Cu}(110)$ surface in the 300 $470 \mathrm{~K}$ temperature range always yields to the formation of an alaninate species. We refer the reader to Table 2 for a detailed description of the observed vibrational frequencies and of their assignment. We mention here that both $\mu 2$ and $\mu 3$ conformations were identified by application of the strict RAIRS dipole selection rules (see Figure 14). In both cases the methyl group protrudes towards the vacuum. The similarity to the conformations determined for $\mathrm{Gly} / \mathrm{Cu}$ is evident, so that also for alaninate on $\mathrm{Cu}(110)$ very similar adsorption positions (near-on-top) were inferred [105] and then confirmed by DFT calculations [107] and photoelectron diffraction [108] for the $\mu 3$ geometry.
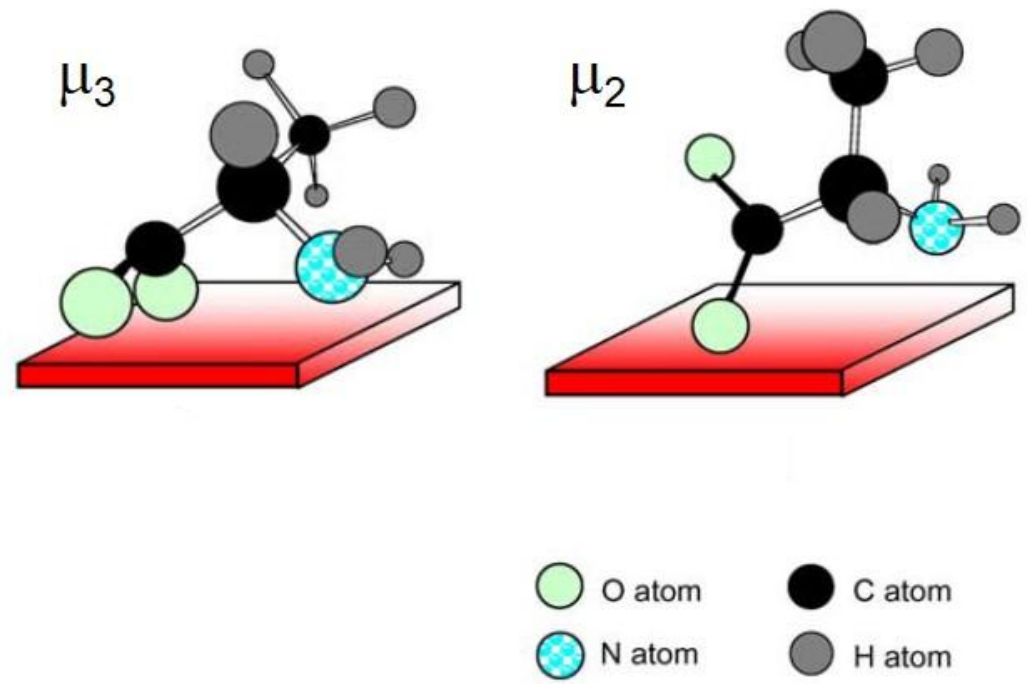

Figure 14. Scheme of the orientation and bonding of $\mu_{3}$ and $\mu_{2}$ species of S-alaninate on $\mathrm{Cu}(110)$, as deduced from RAIRS data. In the $\mu_{3}$ orientation the two carboxylate oxygens are equidistant from the surface. This is evidenced in RAIRS by the intensity of the symmetric carboxylate stretch vibration, $v_{\mathrm{s}}(\mathrm{COO})=1411 \mathrm{~cm}^{-1}$, and by the absence of the corresponding asymmetric stretch, $v_{\mathrm{as}}(\mathrm{COO})$, expected around $1625 \mathrm{~cm}^{-1}$. Since the $v_{\mathrm{s}}(\mathrm{COO})$ is active but quite weak, the OCO plane of the carboxylate group must be tilted towards the surface plane due to the additional interaction of the $\mathrm{N}$ atom with $\mathrm{Cu}$. Further analysis of the RAIR spectrum suggests that the plane of the $\mathrm{NH}_{2}$ group 
lies almost parallel to the surface while the $\mathrm{C}-\mathrm{H}$ and $\mathrm{C}-\mathrm{H}_{3}$ related bands are tilted from the surface normal. In the $\mu_{2}$ orientation, on the contrary, one $\mathrm{O}$ atom points away from the surface, as revealed by the strong intensity of the $v_{\text {as }}(\mathrm{COO})$ mode at $1626 \mathrm{~cm}^{-1}$, an the methyl group is held almost vertical along the surface normal. The $\mu_{3}$ species is present in all phases, the $\mu_{2}$ only in phases II and III. Taken with permission from ref. [105].

\begin{tabular}{|c|c|c|c|c|c|}
\hline $\begin{array}{l}\text { System } \\
\end{array}$ & $\mathrm{O}(1 \mathrm{~s})$ & $\mathrm{N}(1 \mathrm{~s})$ & $\mathrm{C}(1 \mathrm{~s})$ & $\mathrm{S}\left(2 \mathrm{p}_{3 / 2}\right)$ & Ref. \\
\hline $\begin{array}{l}\mathrm{Ala} / \mathrm{Cu}(110) \\
\text { monolayer }\end{array}$ & $\begin{array}{l}531.6 \\
531.3\end{array}$ & $\begin{array}{l}399.9 \\
399.5\end{array}$ & $\begin{array}{l}285.5 / 287.9 \\
285.2 / 285.9 / 288.0\end{array}$ & & $\begin{array}{l}{[105 ;} \\
141]\end{array}$ \\
\hline $\begin{array}{l}\mathrm{Ala} / \mathrm{Cu}(110) \\
\text { multilayer }\end{array}$ & $533.1 / 534.3$ & $401.2 / 403.0$ & $287.3 / 288.4 / 290.4$ & & [141] \\
\hline $\mathrm{Cys} / \mathrm{Cu}(110)$ & 531.6 & $399.9 / 401.0$ & $285.1 / 286.1 / 288.2$ & $162.0 / 163.9$ & [48] \\
\hline $\mathrm{Met} / \mathrm{Cu}(111)$ & 531.5 & $399.5 / 401.3$ & & & [62] \\
\hline $\mathrm{Ser} / \mathrm{Cu}(110)$ & $531.5 / 533.3$ & 399.8 & $285.6 / 286.6 / 288.2$ & & [43] \\
\hline $\begin{array}{l}\text { His/Cu(110) } \\
\text { multilayer }\end{array}$ & $\begin{array}{l}\sim 532.2 \\
\end{array}$ & $399.6 / 401.15$ & $\begin{array}{l}286 / 288.7 \\
\sim\end{array}$ & & [46] \\
\hline $\begin{array}{l}\text { His/Cu(110) } \\
1.0 \mathrm{ML}, 425 \mathrm{~K}\end{array}$ & $\sim 531.9$ & 398.6/399.6/ 400.4 & $\sim 285.8 / \sim 288$ & & {$[46]$} \\
\hline $\begin{array}{l}\text { His/Cu(110) } \\
0.4 \mathrm{ML}, 425 \mathrm{~K}\end{array}$ & 532 & $398.6 / 399.6 / 400.35$ & $285.1 / 288.1$ & & {$[46]$} \\
\hline $\begin{array}{l}\mathrm{His} / \mathrm{Cu}(110)-\mathrm{O} \\
\mathrm{p}(2 \times 2) ; 0.4 \mathrm{ML}\end{array}$ & & $398.6 / 399.4$ & $\sim 284.8 / \sim 287.8$ & & {$[46]$} \\
\hline $\begin{array}{l}\mathrm{Cu}\left(\begin{array}{lll}5 & 3 & 1\end{array}\right) \\
\mathrm{p}(6 \mathrm{x} 1) \text { overlayer }\end{array}$ & 531.3 & 399.5 & & & [112] \\
\hline $\begin{array}{l}(\mathrm{S})-\mathrm{Ala} / \mathrm{Cu}(531) \\
\mathrm{p}(4 \mathrm{x} 1) \text { overlayer }\end{array}$ & $\sim 531.2$ & $\sim 399.6$ & $285.3 / 285.9 / 288.0$ & & [143] \\
\hline $\begin{array}{l}(\mathrm{S})-\mathrm{Ala} / \mathrm{Cu}(421) \\
\mathrm{p}(1 \mathrm{x} 2) \text { overlayer }\end{array}$ & 531.2 & 399.5 & $285.4 / 285.9 / 288.0$ & & [145] \\
\hline $\begin{array}{l}\mathrm{Cys} / \mathrm{Cu}(531) \\
\text { monolayer }\end{array}$ & 531.5 & 399.9 & $285.0 / 286.2 / 288.1$ & 161.6 & {$[115]$} \\
\hline $\mathrm{Met} / \mathrm{Cu}(531)$ & 531.5 & 399.7 & $286.0 / 288.1$ & 163.1 & {$[115]$} \\
\hline $\begin{array}{l}\mathrm{Ser} / \mathrm{Cu}(531) \\
0.26 \mathrm{ML}\end{array}$ & $530.8 / 531.5$ & $398.0 / 400.0$ & $284.6 / 286.1 / 288.1$ & & {$[144]$} \\
\hline $\begin{array}{l}\mathrm{Ser} / \mathrm{Cu}(531) \\
0.5 \mathrm{ML}\end{array}$ & $531.4 / 532.8$ & $397.9 / 399.8$ & $284.7 / 286.0 / 288.1$ & & [144] \\
\hline Met/Ag(111) & 531.2 & 401.15 & & & [63] \\
\hline Tyr/Ag(111) & $532.0 / 533.5$ & 402.2 & & & [45] \\
\hline $\begin{array}{l}\text { Cys/Ag(111) - } \\
\text { multilayer }\end{array}$ & 531.6 & $\sim 401.4$ & $285.1 / 286.1 / 286.9 / 288.8$ & 164.4 & [44] \\
\hline Cys/Ag(111) - $\alpha$ & $531.4 / 532.3 / 533.5$ & 402.0 & $285.0 / 286.5 / 288.5 / 289.5$ & 161.8 & [44] \\
\hline Cys/Ag(111) - $\beta$ & $531.0 / 532.0 / 533.2$ & 399.2 & $285.0 / 286.0 / 288.0 / 289.0$ & 162.0 & [44] \\
\hline Glu/Ag(100) & $531.6 / 533.3$ & 400.1 & 285.6/286.8/288.3/289.2 & --- & [54] \\
\hline Glu/Ag(111) & $531.6 / 533.2$ & 400.1 & $285.3 / 286.6 / 288.3 / 289.3$ & --- & [53] \\
\hline $\begin{array}{l}\text { Gly/Pt(111) } \\
\text { monolayer }\end{array}$ & 531.7 & $399.9 / 401.3$ & $284.4 / 286.3 / 289.2$ & --- & [38] \\
\hline $\begin{array}{l}\text { Gly/Pt(111) } \\
\text { multilayer }\end{array}$ & 532.3 & $400.5 / 402.5$ & $285.7 / 287.3 / 289.4$ & --- & {$[38]$} \\
\hline $\begin{array}{l}\text { Gly/Pd(111) } \\
\text { monolayer }\end{array}$ & --- & $397.0 / 400.7$ & $284.6 / 287.3$ & --- & {$[40]$} \\
\hline $\begin{array}{l}\text { Gly/Pd(111) } \\
\text { multilayer }\end{array}$ & $-\overline{---}$ & $398.8 / 401.4$ & $285.9 / 288.5$ & --- & {$[40]$} \\
\hline $\begin{array}{l}\text { Ala/Pd(111) } \\
\text { monolayer }\end{array}$ & --- & $398.8 / 401.2$ & $285.2 / 288.0$ & --- & [39] \\
\hline $\begin{array}{l}\text { Ala/Pd(111) } \\
\text { multilayer }\end{array}$ & --- & $399.4 / 401.6$ & $285.8 / 288.5$ & --- & [39] \\
\hline
\end{tabular}




\begin{tabular}{|l|l|l|l|l|l|}
\hline $\begin{array}{l}\text { Pro/Pd(111) } \\
\text { monolayer }\end{array}$ & -- & 400.6 & $285.1 / 288.3$ & & {$[41]$} \\
\hline $\begin{array}{l}\text { Pro/Pd(111) } \\
\text { multilayer }\end{array}$ & --- & $399.8 / 401.5$ & $285.7 / 288.4$ & & {$[41]$} \\
\hline
\end{tabular}

Table 3. Comparison among the O1s, N1s, C1s and S2p binding energies of the different AA/metal systems.

In spite of the relatively simple picture presented by IR data, STM analysis reveals a polymorphic behaviour of the $\mathrm{Ala} / \mathrm{Cu}(110)$ system $[69 ; 105]$. Four different surface structural phases are identified (see Figure 15), depending on alanine coverage and surface temperature:

i) Phase I (low coverage and room temperature). Both the absence of LEED superstructures and inspection of STM images indicate that this is a disordered phase, in which the alaninate molecules (in $\mu 3$ orientation) are found concentrated in small step areas. Due to the high mobility of individual $\mu_{3}$ alaninate units, no STM images have been obtained for the molecular species at terraces (Figure 15a), even when RAIRS data show a significant growth of the $\mu_{3}$ species.

ii) Phase II (high coverage, $300 \mathrm{~K}$ ). Spectroscopic analysis indicates the coexistence of alaninate molecules both in $\mu_{3}$ and in $\mu_{2}$ conformation. The creation of the $\mu_{2}$ species at higher coverages can be directly attributed to kinetic factors since only the $\mu_{3}$ species is formed at all $\Theta$. In addition to the molecules already identified at the step edges in phase I, now molecular chains aligned in the $<-112>$ direction and developing for tens of $\mathrm{nm}$ across the surface cover large areas of the $\mathrm{Cu}(110)$ terraces (see Figure 15b). Since $<-112>$ is not a symmetry direction of the $\mathrm{Cu}(110)$ surface, the system is starting to organise in a chiral fashion. Interestingly, the presence of the $\mu_{2}$ species is suggested by the authors to trigger chain formation at the terraces, and thus to be essential for supramolecular chiral organization. The chain structure is not homogeneous; single chains (formed by individual alaninate units) and double chains (consisting of paired molecules) exist and turn into each other every few molecular units along the chain length. This behaviour was interpreted as an indication of some barriers to the propagation of the preferred chain structure, possibly due to strains from molecule-molecule versus molecule-metal interactions.

iii) Phase III (high coverage, annealing to $430 \mathrm{~K}$ ). Upon thermal treatment of phase II the RAIRS spectra remain unaltrered, indicating that molecules in both $\mu_{3}$ and $\mu_{2}$ configuration are still present. The short chains of alaninate molecules evolve to form a highly ordered chiral overlayer (Figure 15c). The two-dimensional chirality is expressed at the macroscopic length scale. The mirror image domain is achieved by repeating the experiment with $\mathrm{R}$ alanine. Alaninate molecules self-assemble into defined chiral clusters of 6 or 8 units lined up in pairs in the $\langle 110\rangle$ direction and with an overall growth direction broadly parallel to the non-symmetric <-112>. A clear LEED signature of the superstructure was found only for the hexamers, on which the authors concentrate to draw a model of the cluster assembly and of the long range order of the overlayer. Several models of individual hexamers are proposed in Refs. [69; 105], involving a different proportion of molecules in $\mu_{3}$ and in $\mu_{2}$ conformation on an unreconstructed or a reconstructed $\mathrm{Cu}(110)$ surface. The optimised model contains a larger fraction of $\mu_{2}$ alaninate molecules and has $\mathrm{Cu}$ atoms bound to only one functional unit. The determination of the long range order is complicated by the discrepancy between LEED and STM in the determination of the unit cell (see Figure 15c), 
which is possibly related to the presence of a surface reconstruction. However it is clear that size-defined chiral clusters self-assemble into a defined chiral array with chiral channels and spaces of bare metal left between them.

iv) Phase IV (high coverage, annealing to $470 \mathrm{~K}$ ). Substantial changes in the RAIR spectra (see Table 2) indicate irreversible conversion of $\mu 2$ alaninate units into the $\mu 3$ geometry. The latter appears indeed to be thermodynamically preferred, being adopted in all phases. LEED and STM data indicate the formation of a new, achiral (identical for both S- and R-alaninate) organisation of the molecules on the surface (Figure 15d): a $(3 \times 2)$ superstructure with two molecules per unit cell $(\Theta=1 / 3)$ and a pseudo-glide plane in the $<001\rangle$ direction. This overlayer is stable until $520 \mathrm{~K}$. Rankin and Sholl [107] calculated the preferred adsorption footprint for a monomer of $S$-alaninate and found that the $\mathrm{O}$ atoms are displaced differently in the <001> direction from atop $\mathrm{Cu}$ atom sites, to give a slightly tilted footprint. Furthermore, they confirmed that the $(3 \times 2)$ enantiopure overlayer structure contains two molecules per unit cell; the lowest energy configuration is the one in which the two molecules adopt opposite footprint chiralities. Photoelectron diffraction measurements [108] of $S$-alanine on $\mathrm{Cu}(110)$ confirmed the existence of two molecules with slightly different local adsorption sites within the $(3 \times 2)$ unit cell.

From the behaviour described above, it is clear that the chiral chains of phase II are precursors to the size selected clusters of phase III. Above a critical cluster size, the stress of maintaining optimum adsorption sites for the alaninate molecules and of maximising the intermolecular interactions leads to a fracture in the assembly of the chains, with two possible scenarios: either intermolecular interactions progressively force subsequent pairs of molecules further away from their optimum bonding positions or the strength of the chemisorption bond forces surface reconstruction in order to optimise adsorption geometry. The former mechanism may well dominate at room temperature (phase II), where the barrier to significant reconstruction cannot be overcome. On the other hand, the higher temperature required to create phase III suggests that metal reconstruction may accompany the formation of regular size selected clusters. We mention that this behaviour is indicative of a strong molecule-metal interaction and is indeed not observed on less reactive substrates as $\mathrm{Ag}[51 ; 54]$. 


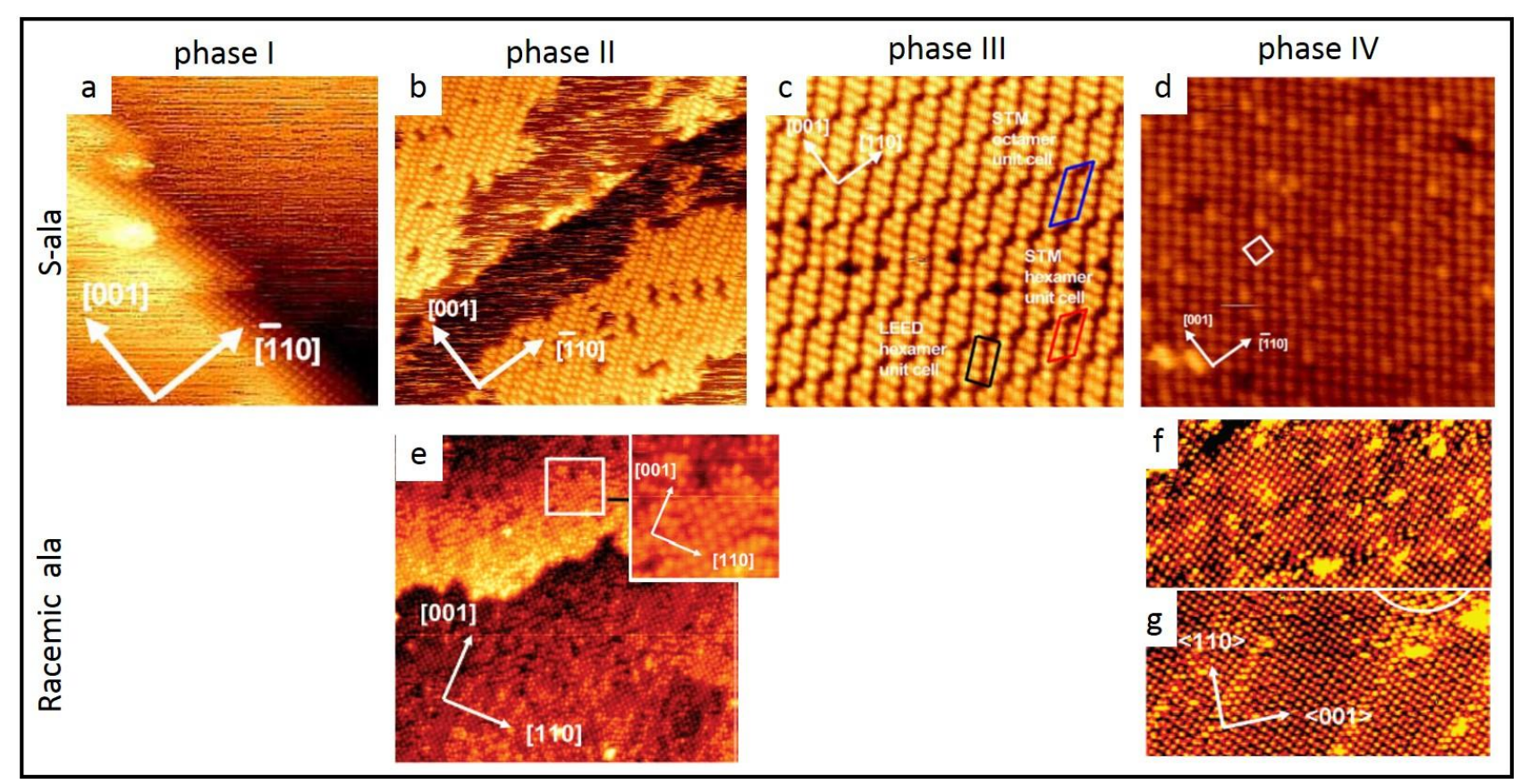

Figure 15. STM images of alaninate/Cu(110). Top row: S-alanine/Cu(110). (a) Phase I, showing alaninate molecules at step edges and streaky areas on terraces. (Image size $292 \AA \times 246 \AA, V=1.02$ $\mathrm{V}, \mathrm{I}=1.08 \mathrm{nA}$ ). (b) Phase II, showing the details of chiral chains covering large terraces. (Image size $254 \AA \times 246 \AA, V=2.08 \mathrm{~V}, \mathrm{I}=1.97$ nA.) (c) Phase III. The image shows size-selected clusters of 6 or 8 molecules. (Image size $127 \AA \times 123 \AA, \mathrm{V}=2.08 \mathrm{~V}, \mathrm{I}=1.97 \mathrm{nA}$ ). Hexamer clusters occupy a repeat unit mesh of $17.5 \AA \times 9 \AA$ and octamer clusters a repeat unit mesh of $21.5 \AA \times 9 \AA$. The corresponding unit cells as determined by STM and the hexamer unit cell determined by LEED are marked. The hexamer unit cell in matrix notation is $(-5,-3,3,1)$ according to STM and $(2,-2,5,3)$ according to LEED. (d) Phase IV. The STM image shows the achiral $(3 \times 2)$ reconstruction (image size $127 \AA \times 123 \AA, \mathrm{V}=2.83 \mathrm{~V}, \mathrm{I}=1.14 \mathrm{nA}$, annealing temperature $453 \mathrm{~K}$ ). The unit cell (marked in the figure) contains two molecules and measures $7.2 \AA \times 7.6 \AA$. Bottom row: racemic alanine/Cu(110). (e) Phase II showing patches of achirally ordered molecules (image size $400 \AA \times$ $400 \AA, \mathrm{V}=-0.36 \mathrm{~V}, \mathrm{I}=0.15 \mathrm{nA})$. The inset is an enlargement of the area marked by the square and shows the details of the local order of racemic phase II. (f) Racemic phase IVa associated with the distorted (3×2) LEED pattern (image size $224 \AA \times 112 \AA, \mathrm{V}=1.05 \mathrm{~V}, \mathrm{I}=0,17 \mathrm{nA}$ ). (g) Racemic phase IVb associated with the true $(3 \times 2)$ LEED pattern (image size $224 \AA \times 112 \AA, \mathrm{V}=1.49 \mathrm{~V}$, $\mathrm{I}=0,59 \mathrm{nA})$. Taken with permission from refs. [105; 140].

The strong similarity of the XPS and NEXAFS spectra recorded for the $\left(\begin{array}{cc}2 & -2 \\ 5 & 3\end{array}\right)$ and the achiral pseudo- $(3 \times 2)$ chemisorbed overlayers [141] suggests that the molecules in phases III and IV are in similar environments in terms of alignment of the two oxygen atoms with respect to the $\langle 1-10\rangle$ direction and the out-of-plane tilt of the carboxylate group. XPS results [141] find large chemical shifts in the $\mathrm{C} 1 \mathrm{~s}, \mathrm{~N} \mathrm{1s}$, and $\mathrm{O}$ 1s levels of the alanine multilayer and of the chemisorbed and pseudo- $(3 \times 2)$ alaninate species. Furthermore, from $\mathrm{C}, \mathrm{N}$, and $\mathrm{O}$ K-shell NEXAFS spectra the tilt angles of the carboxylate group (about $26^{\circ}$ in plane with respect to $\langle 1-10\rangle$ and about $45^{\circ}$ out of plane) and the $\mathrm{C}-\mathrm{N}$ bond angle with respect to $<1-10>$ could be determined for the pseudo- $(3 \times 2)$ overlayer. Based onthis information five $p(3 \times 2)$ structures (See Figure 16a-e) with almost identical heats of adsorption in the DFT calculations (between -1.40 and $-1.47 \mathrm{eV} /$ molecule, which can be 
considered as iso-energetic) were identified. Since the five configurations are all very close in energy, it is likely that they, or at least two most energetically favoured geometries (b and d),coexist on the surface at room temperature (See Figure 16).

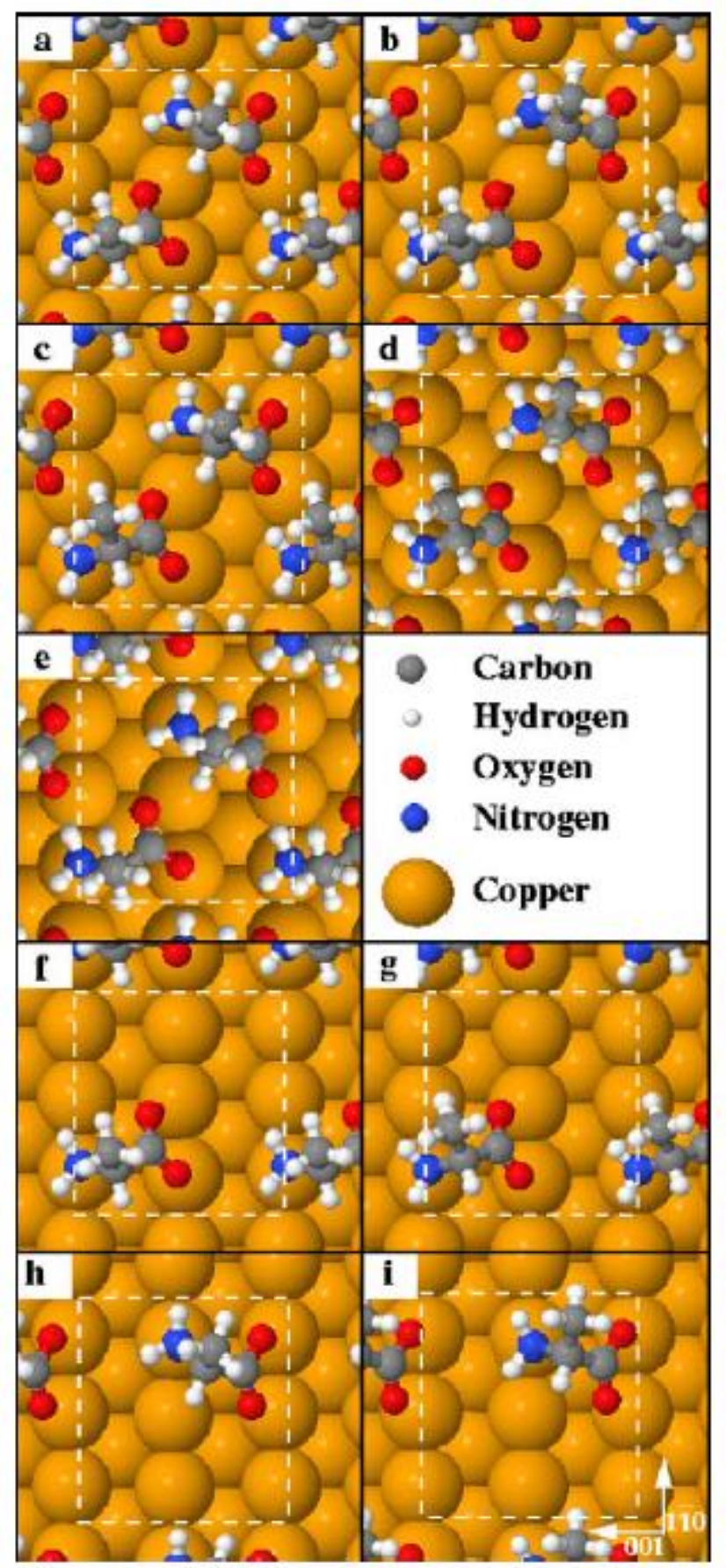

Figure 16 (a)-(e) Top view of the five enantiopure $R$-alanine $p(3 \times 2)$ overlayer geometries on $\mathrm{Cu}(110)$ 1/3 ML that led to similar calculated heats of adsorption. Each structure represents a local conformational change primarily noticeable in the backbone orientation of the molecule: (a), (e) kinked/kinked, (b) kinked/linear, (c) linear/kinked, (d) linear/ linear. (f)-(i) monomer geometries (1/6 ML with a $\mathrm{p}(3 \times 2)$ overlayer periodicity): (f), (h) kinked, different footprints; (g), (i) linear, different footprints. The unit cells are shown as dashed white lines. Taken with permission from ref. [141]. 
All the experiments reported so far dealt with enantio-pure amino acid species. Ref. [105] showed that the $\mathrm{S}$ - and R-alanine assemble on $\mathrm{Cu}(110)$ generating mirror image patterns both in LEED and in STM images. The natural question, which follows, is: what happens if a racemic mixture is employed? Separation of enantiomers within racemic crystals has been known for long, while chiral recognition at surfaces has been investigated more recently but is nowadays a well-established phenomenon [175]. In spite of that, surface chiral segregation - i.e. the formation of enantio-pure domains of opposite chirality - needs to be tracked separately in each single case and for each single phase of the AA/metal system. In general, if homochiral interactions dominate, chiral segregation of enantiomers should be favoured; conversely, if heterochiral interactions prevail, a racemic layer is expected.

In the case of adsorption of racemic alanine on $\mathrm{Cu}(110)$ [140], the surprising result is that, not only there is no chiral resolution and the consequent formation of achiral domains, but not even all the phases reported for the enantio-pure compound are present. In more details, a disordered phase I, with alaninate units in the $\mu_{3}$ conformation, is detected at low coverage and $300 \mathrm{~K}$ for both the enantio-pure and racemic compounds. Significant differences are present, on the contrary, already for phase II. In this case STM images show achirally ordered patches with poor long-range order (Figure 15e). The LEED spots are split and very faint, forming a pattern consistent with an achiral organization of the adsorbate at the surface. These organizational differences are the first indicators of a significant departure in how the enantio-pure and the racemic systems evolve with temperature. In fact, racemic phase II converts directly into the achirally organized phase IV at $\mathrm{T}=358 \mathrm{~K}$, thus completely bypassing phase III. The $(3 \times 2)$ overlayer of the racemic alanine mixture differs from the one described for pure $\mathrm{S}$-alaninate since: a) the energy barrier to produce this phase is smaller when both $S$ - and $R$-alaninate molecules are present, possibly because each molecule can now adopt its preferred adsorption footprint. b) The $(3 \times 2)$ structure presents initially a glide plane (phase IVa Figure 15f), as in the case of S-alaninate, but the coverage can be further increased to create a "true" $(3 \times 2)$ LEED pattern with no glide plane (phase IVb - Figure 15f). The profound difference between enantio-pure and racemic alaninate/ $\mathrm{Cu}(110)$ is that in the latter system both the molecular chirality and the footprint chirality can be heterochiral. Both the energetically preferred footprints of $S$ alaninate and $R$-alaninate can be accommodated at the surface, giving a $(3 \times 2)$ arrangement with one molecule of each enantiomer in the unit cell and a true glide plane[105]. This is indeed the lowestenergy structure according to DFT calculations [107]. Interestingly, the calculated energy difference between the favoured enantiopure and racemic structures is very small $(<0.2 \mathrm{eV} /$ unit cellwith 2 molecules/unit cell), so there is little driving force for the system to either form an ordered racemic compound or segregate to give a racemic conglomerate. Therefore, it is argued $[107 ; 140]$ that the most likely racemic structure is probably one where there is a disordered array of enantiomers but an overall 1:1 ratio of S- and R- species. Finally, STM images show a lack of long range periodicity either for the $(3 \times 2) \mathrm{S}$-alaninate layer and for the racemic $(3 \times 2)$ structures of phase IVa and phase $\mathrm{IVb}$. This effect may depend on a number of factors. The nearest and next-nearest adsorbateadsorbate interactions may drive the local structure, which creates an incommensurate overlayer;conversely, metal-adsorbate interactions might be so great that they cause the underlying copper atoms to be moved from their bulk-truncation positions and the surface to undergo a reconstruction. 
At variance with the case of DL-Ala/ $\mathrm{Cu}(110)$, deposition of the racemic mixture on $\mathrm{Cu}(100)$ at 310 $\mathrm{K}$ causes molecular segregation and the formation of separated $\mathrm{c}(2 \times 4)$ domains of $\mathrm{D}$ - and L-alanine units equivalent to those observed for the enantiopure molecule at the same $T$ [136-138]. Indeed the image in Figure 17A shows a number of row structures aligned in the equivalent $\langle 110\rangle$ directions and running across the terrace in two regions labelled as A and B. In each region, the row structures consist of two domains aligned along the $\langle 130\rangle$ and $\langle 310\rangle$ directions and the protrusions, identified with individual alanine molecules, show a $c(2 \times 4)$ periodicity, corresponding to $\Theta=0.25$ ML and coherent with the observed LEED pattern. RAIRS data (see Table 2) confirm that adsorption occurs in the alaninate form, in accord with the previously estimated values of binding energy $(1.7 \mathrm{eV})$ and diffusion activation energy $(0.83 \mathrm{eV})$ [139]. The proposed adsorption configuration [136], $\mu 3$ with the methyl group pointing away from the surface (see Figure 17B), is very similar to the one already reported for $\mathrm{Gly} / \mathrm{Cu}(100)$ [95; 98] and $\mathrm{Ala} / \mathrm{Cu}(110)$ [105]. The $\mathrm{c}(2 \times 4)$ structure is probably stabilized by $\mathrm{N}-\mathrm{H}^{1} \ldots \mathrm{O}^{2}$ hydrogen bonds between adjacent molecules at terraces and by $\mathrm{N}-\mathrm{H}^{2} \cdots \mathrm{O}^{1}$ hydrogen bonds at the domain boundary [95; 98]. Therefore, also in the present case, intermolecular hydrogen bonds are responsible for stabilization of D- and L-alanine molecules aligned along <130> and $<310\rangle$ directions, respectively. The presence of domains with two different orientations but with the same $\mathrm{c}(2 \times 4)$ periodicity is indicative that on the terraces $\mathrm{D}$ and L-alanine molecules segregate to form their own $\mathrm{c}(2 \times 4)$ domains separated by the boundaries aligned along <110> direction (see Figure 17). The commensurability and periodicity of the two domains strongly suggests that one functional group occupies the same local bonding site in all molecules.

At step sites on the surface, intrinsically chiral $\mathrm{Cu}(3,1,17)$ and $\mathrm{Cu}(3,1,17)$ facets form by adsorbateinduced reconstruction [139].
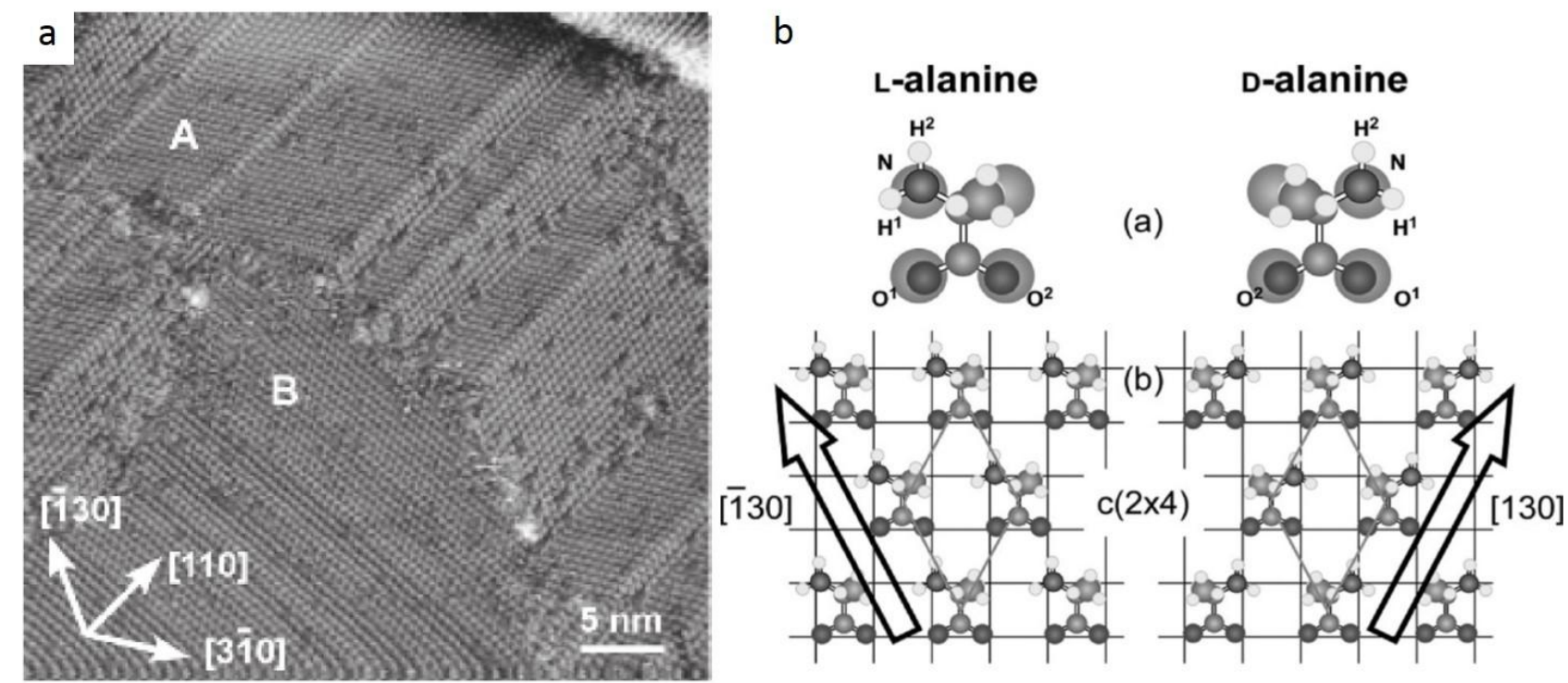

Figure 17. a) STM image of a saturated layer of DL-alanine on $\mathrm{Cu}(001)\left(40 \times 40 \mathrm{~nm}^{2}, \mathrm{~V}=0.3 \mathrm{~V}\right.$, $\mathrm{I}=0.1 \mathrm{nA})$. b) Schematic drawing of local geometries for D- and L-alanine adsorbed on $\mathrm{Cu}(001)$ surface (top) and of the $\mathrm{c}(2 \times 4)$ ordered structures of D- and L-alanine domains on $\mathrm{Cu}(001)$ (bottom). Notation of $\mathrm{H}$ and $\mathrm{O}$ atoms of amino and carboxylate group (H1, H2, O1, O2) is defined as shown in the figures; the intersection point of grids correspond to the position of $\mathrm{Cu}$ atoms on the terrace. Taken with permission from ref. [136]. 
Dense adlayers of glycine and alanine structures on the $\mathrm{Cu}(110)$ and $\mathrm{Cu}(100)$ surfaces have been determined using plane wave density functional theory [142]. These calculations resolve several experimental controversies regarding these structures. Glycine exists on $\mathrm{Cu}(110)$ as a single adlayer structure, while on $\mathrm{Cu}(100)$ two distinct glycine adlayers coexist. The glycine structures serve as useful starting points for constructing alanine adlayer structures. The adsorption of enantiopure alanine and racemic alanine has been considered separately on each surface. Adlayers of enantiopure alanine are found to be closely related to the adlayers observed for glycine. Racemic alanine adlayers on $\mathrm{Cu}(110)$ are structurally analogous to those observed for glycine on this surface and adopt a pseudo-racemate ordering. $\mathrm{On} \mathrm{Cu}(100)$, in contrast to glycine, racemic alanine is found to adopt a single adlayer structure, that is an ordered racemate.

\subsubsection{Amino acids with a linear side chain and one additional functional group.}

\subsubsection{S-containing amino acids.}

While we have shown in the previous sections that the simplest amino acids share very similar $\mu 2$ and $\mu 3$ binding configurations, there are situations in which the side chain of the amino acid plays an important role. One example is provided by amino acids containing sulphur, whose strong affinity to metals is determinant of the molecular behaviour[182].

Among the 20 amino acids, cysteine is the only one to bear an additional terminal thiol group, which is very likely to act as anchoring point to the metal surface. Most of the work on Cys adsorption was performed at $\mathrm{Au}$ surfaces, little on more reactive metals. Furthermore, Cys may undergo dimerization into cystine via the oxidation of two thiol groups. The SH group can also establish intramolecular hydrogen bonds, hence playing a role in the stabilization of the secondary structure of proteins [183]. Cysteine is often on the outer side of proteins, being a potential link to anchor them to inorganic or organic supports.

The interaction of Cys with $\mathrm{Cu}(110)$ was investigated by means of RAIR and photoemission spectroscopies by comparing the outcome of gas phase deposition and deposition from liquid solution, under different $\mathrm{pH}$ conditions [48]. According to RAIR spectra (see Table 4), (S)-cysteine adsorbs intact, mostly in the zwitterionic form, when evaporated under UHV conditions. Conversely, XPS spectra recorded after transferring the sample in air show a fraction of anionic adsorption (seeTable 3). After deposition from solution, even at very low $\mathrm{pH}$, a significant fraction of the molecules were not positively charged but kept an amine group. Careful analysis of XPS data recorded both at normal and at $45^{\circ}$ emission also indicates that the COO- group and $\mathrm{S}$ atoms are, on average, at the same distance from the surface. Combining FT-RAIRS and XPS results, the authors proposed the model reported in Figure 18. Since the calculated thickness leads to a value higher than the one expected for a Cys monolayer (although lower that the one estimated upon adsorption from solution), rather tightly bound cysteine molecules are likely to form multilayers already at room temperature. This is at variance with what reported for the simplest amino acids, e.g. glycine on $\mathrm{Cu}(110)$ [42] and $\mathrm{Pt}(111)$ [38] and alanine on $\mathrm{Cu}(110)$ [141], for which multilayer formation was observed only below RT. The different stability is related both to the stronger adsorption energy and to the stronger intermolecular interaction between Cys layers allowed by the thiol group. We mention that multilayers of cysteine were also observed on $\mathrm{Cu}(110)$ upon immersion at $\mathrm{RT}$ and 
explained by the formation of a complex ionic structure involving copper surface atoms or dissolved copper ions [182]. Upon deposition from solution, differently ionised Cys species form on the surface depending on $\mathrm{pH}$ conditions, as witnessed by the different vibrational modes detected by RAIRS (see Table 4).

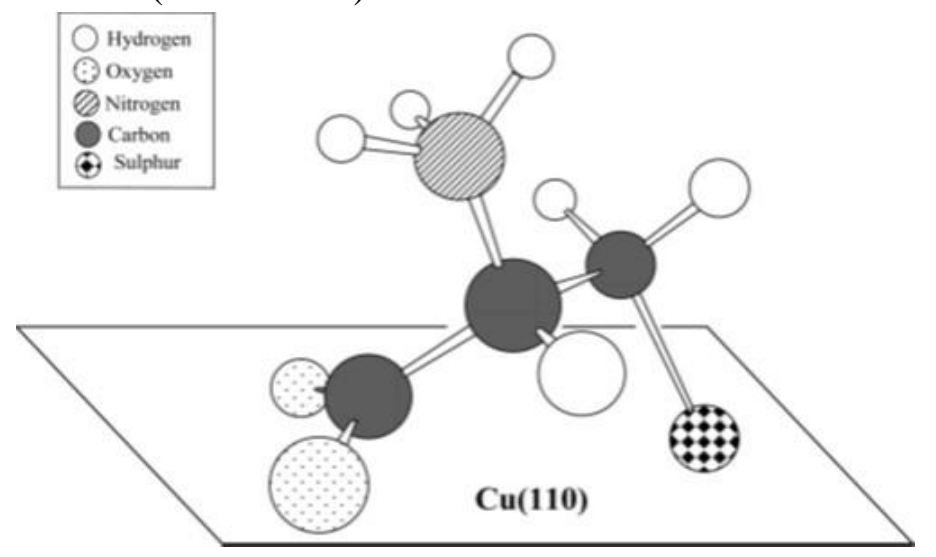

Figure 18. Probable orientation of $(\mathrm{S})$-cysteine on $\mathrm{Cu}(110)$ after adsorption from the gas phase. Taken with permission from ref. [48].

\begin{tabular}{|c|c|c|c|c|}
\hline \multirow{3}{*}{ Vibrational mode } & \multicolumn{4}{|c|}{ Cysteine/Cu(110) } \\
\hline & \multirow[t]{2}{*}{ Gas Phase } & \multicolumn{3}{|l|}{ Solution } \\
\hline & & pH 1.0 & pH 5.4 & pH 10.5 \\
\hline$v_{\text {asym }}\left(\mathrm{NH}_{3}{ }^{+}\right)$ & 3144 & & & \\
\hline$v_{\text {sym }}\left(\mathrm{NH}_{3}{ }^{+}\right)$ & 2992 & & & \\
\hline$v_{\text {assm }}\left(\mathrm{CH}_{2}\right)$ & 2957 & & & \\
\hline $\mathrm{v}(\mathrm{CH})$ & 2908 & & & \\
\hline$v_{\text {sym }}\left(\mathrm{CH}_{2}\right)$ & 2870 & & & \\
\hline$v(\mathrm{SH})$ & & & & \\
\hline$v(C=0)$ & & 1735 & & \\
\hline$\delta_{\text {asym }}\left(\mathrm{NH}_{3}{ }^{+}\right)$ & 1652 & 1625 & 1630 & \\
\hline$v_{\text {asym }}\left(\mathrm{COO}^{-}\right)$ & & 1590 & 1606 & 1606 \\
\hline$\delta_{\text {sciss }}\left(\mathrm{NH}_{2}\right)$ & & & & $\mid 1570$ \\
\hline$\delta_{\text {sym }}\left(\mathrm{NH}_{3}{ }^{+}\right)$ & 1510 & 1495 & 1508 & \\
\hline bend $\mathrm{NH}_{2}$ & & & & 1465 \\
\hline$\delta_{\text {sciss }}\left(\mathrm{CH}_{2}\right)$ & 1423 & 1426 & & 1420 \\
\hline$v_{\text {sym }}\left(\mathrm{COO}^{-}\right)$ & 1396 & 1404 & 1403 & 1407 \\
\hline$\omega\left(\mathrm{CH}_{2}\right)$ & 1340 & 1339 & & \\
\hline Bend $\mathrm{CH}$ & & 1315 & & \\
\hline$v(C-0)$ & & 1260 & & \\
\hline$v(C-0)$ (acid dimers?) & & 1211 & & \\
\hline $\mathrm{r}\left(\mathrm{NH}_{3}{ }^{+}\right)$ & 1130 & 1125 & 1104 & \\
\hline$\delta\left(\mathrm{NH}_{2}\right)$ & & 1080 & 1080 & 1080 \\
\hline$v(C-N)$ & 1061 & 1050 & 1050 & 1050 \\
\hline$v(C-C)$ & 900 & 882 & 882 & 877 \\
\hline
\end{tabular}

Table 4. Vibrational frequencies measured for $\mathrm{Cys} / \mathrm{Cu}(110)$ upon different adsorption conditions, expressed in $\mathrm{cm}^{-1}$. Data taken from ref. [48]. 
Methionine is also an S-containing AA, but with thesulphur atom inserted in a C-S-C bond (sulfide) instead of forming a thiol end-group like in Cys. Adsorption of L-methionine has recently been characterized on $\mathrm{Cu}(110)$, under low pressure conditions $\left(2 \times 10^{-9}\right.$ Torr), by combining in-situ PMIRRAS and XPS [154]. Like other amino acids, adsorbed methionine is anionic. At low coverage, the molecule interacts with the copper surface via weak interactions of sulphur and oxygen atoms, likely in a random geometry. At higher $\Theta$, the molecule stands up on the surface, interacting with it via the $\mathrm{N}$ and $\mathrm{O}$ atoms; such a modification in the binding mode was deduced from changes in the S2p and N1s XPSspectra (see Figure 19, panels a and b). Note that the adsorption continues until attaining one saturated monolayer, corresponding to $3.7 \times 10^{14}$ adsorbed molecules per $\mathrm{cm}^{2}$ (approximately 1 molecule per 3 surface copper atoms). Two adsorption geometries were proposed: randomly oriented molecules at low coverage and molecules standing up with the $\mathrm{S}-\mathrm{CH}_{2}$ terminal groups far from the surface at higher coverage (see Figure 19c).

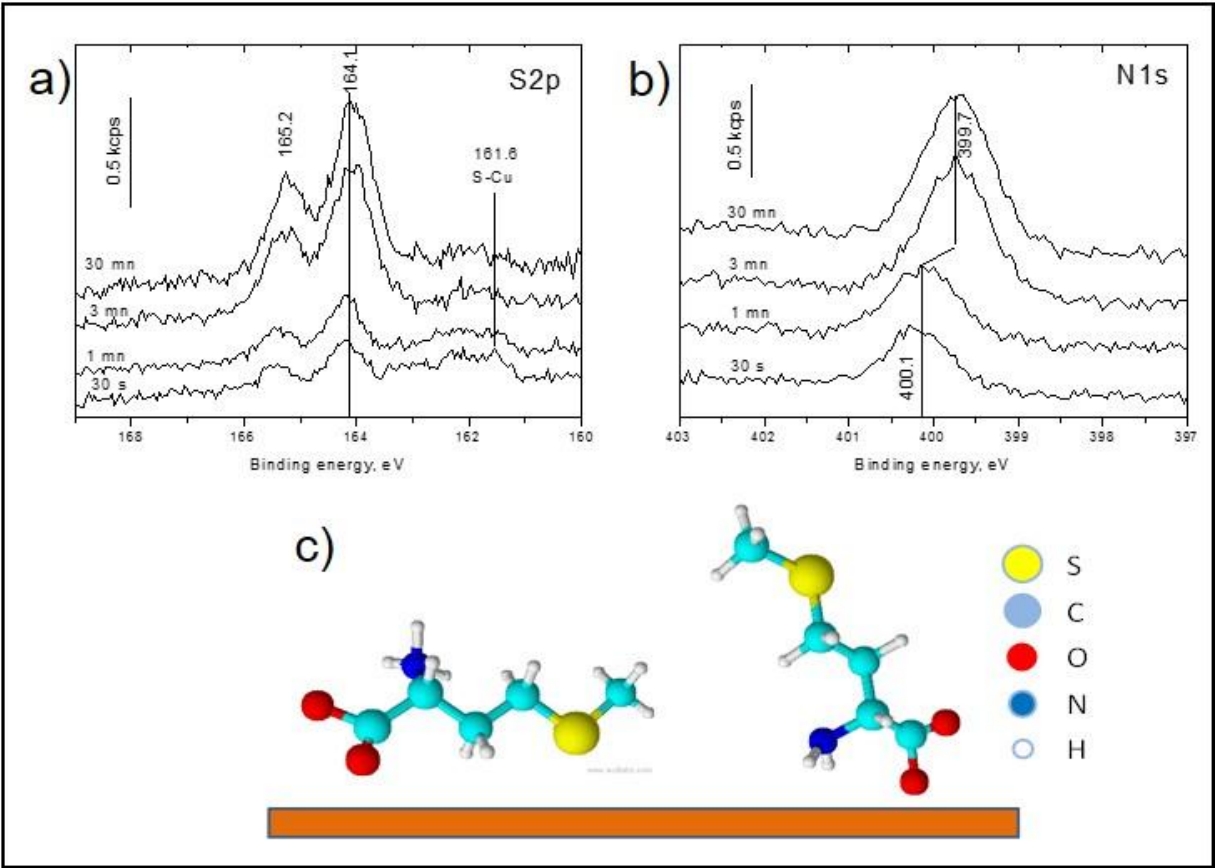

Figure 19. L-Met on $\mathrm{Cu}(110)$. a) S2p XPS peaks recorded after exposure from $30 \mathrm{~s}$ to $30 \mathrm{~min}$. At low exposure, the main doublet $\left(2 \mathrm{p}_{3 / 2}, 2 \mathrm{p}_{1 / 2}\right.$ at 164.1 and $165.3 \mathrm{eV}$ respectively) is characteristic of $\mathrm{S}$ in S-C bonds, while a smaller contribution, at lower $\mathrm{E}_{\mathrm{b}}$ indicates the presence of a small fraction of sulphur bound to copper. This latter feature becomes negligible at higher exposure. b) Same as a) for the N1s region. The N1s peak shifts from 400.1 to $399.7 \mathrm{eV}$ with increasing coverage, suggesting a stronger interaction of the molecule with the surface via the nitrogen atom. c) Proposed geometries for L-methionine adsorbed on $\mathrm{Cu}(110)$, at low (left) and high (right) coverage; taken with permission from ref.[154]

L-Methionine had been previously characterized on $\mathrm{Cu}(111)$, by combining STM, XPS and HAS [62]. This work is the follow up of the investigation on Met/Ag(111) by the same authors [63] (see section 3.2.2.1), and it offers therefore the possibility to correlate the adsorption behaviour to the different substrates. $\mathrm{On} \mathrm{Ag}(111)$ self-assembly in extended one-dimensional nanostructures running 
parallel to the close-packed crystallographic directions of the substrate and formation of regular biomolecular gratings occur. Vice versa, on $\mathrm{Cu}(111)$ the molecular ordering is strongly influenced by the higher reactivity and by the smaller lattice constant of the surface $\left(a_{\mathrm{Cu}(111)}=2.55 \AA ; a_{\mathrm{Ag}(111)}=\right.$ $2.89 \AA$ A). Methionine self-assembles into two distinct supramolecular structures, $\alpha$ and $\beta$, depending on the substrate temperature during deposition. At $\mathrm{T}_{\mathrm{ev}}=243 \mathrm{~K}$, the molecules form short strings $(\alpha$ structure - see Figure 20 a) oriented $\sim 10^{\circ}$ off the high-symmetry directions of the surface. Increasing $\mathrm{T}_{\mathrm{ev}}$, the system gradually evolves into the $\beta$ phase, dominant above RT and consisting in 1D arrays with long-range order and orientation $10^{\circ}$ counter-clockwise with respect to the high-symmetry axes of the substrate (Figure 20b).
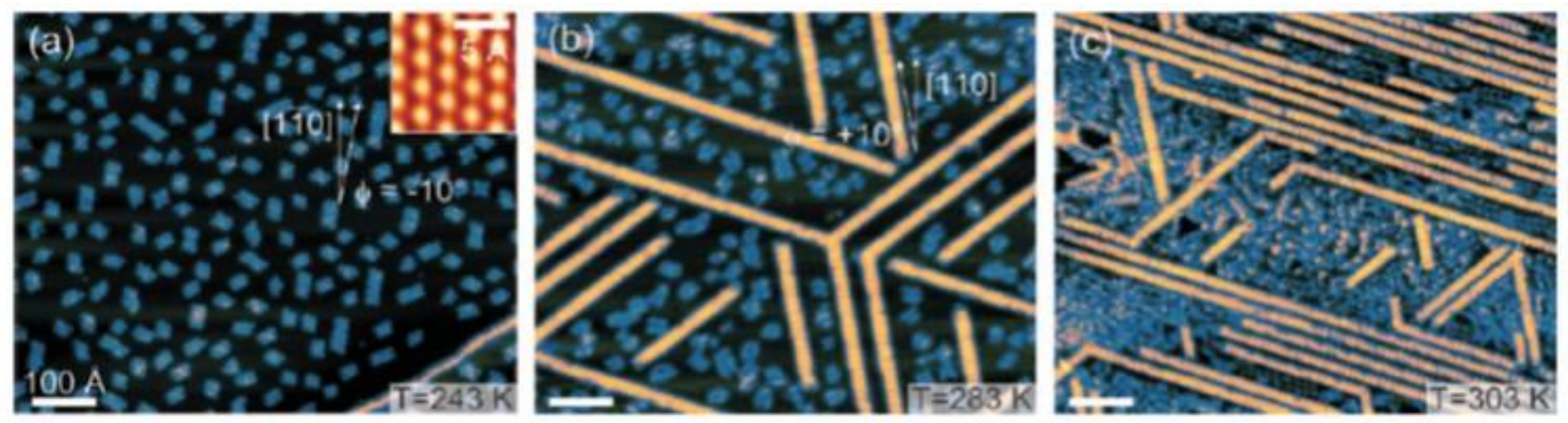

\section{$\mathrm{T}[\mathrm{K}]$}

Figure 20. Homochiral self-assemblies of L-Met/Cu(111). (a) L-Met strings ( $\alpha$ structure) following deposition on $\mathrm{Cu}(111)$ at $\mathrm{T}_{\mathrm{ev}}=243 \mathrm{~K}(\mathrm{I}=0.1 \mathrm{nA}, \mathrm{V}=-100 \mathrm{mV}, \Theta=(0.25 \pm 0.05 \mathrm{ML}))$. Inset: atomic resolution of the $\mathrm{Cu}(111)$ substrate. (b) At $\mathrm{T}_{\mathrm{ev}}=283 \mathrm{~K}$ extended $1 \mathrm{D}$ chains emerge $(\mathrm{I}=0.1 \mathrm{nA}, \mathrm{V}=-$ $100 \mathrm{mV}, \Theta=(0.42 \pm 0.05 \mathrm{ML}))$. (c) At $\mathrm{T}_{\mathrm{ev}}=303 \mathrm{~K}$ small domains of a nanograting ( $\beta$ structure) start to appear $(\mathrm{I}=0.06 \mathrm{nA}, \mathrm{V}=-100 \mathrm{mV}, \Theta=(0.90 \pm 0.05 \mathrm{ML}))$. Scale bars: $100 \AA$. Taken with permission fom ref. [62].

HAS experiments confirm the increasing degree of surface order with increasing temperature and allow estimating the biomolecular long-range correlation to exceed several tens of nm. Molecular resolution STM measurements of the ordered 1D arrangement, in addition, indicate dimerization and a second-order commensurability with the atomic lattice of the substrate along the chain direction. The investigation of the chemical nature of methionine adsorbed at $\mathrm{Cu}(111)$ results in a complex picture (see Table 3 for the $\mathrm{E}_{\mathrm{b}}$ values). In fact the $\alpha$ phase is characterised by the coexistence of anionic molecules at steps and of zwitterionic ones at terraces. The $\beta$ phase, on the contrary, is purely anionic. The anionic chemical state with a neutral amino group and a negatively charged carboxylate group is identical to the one of alanine and glycine adsorbed on low-index $\mathrm{Cu}$ surfaces at room temperature $[42 ; 66 ; 101 ; 108]$, so that a similar adsorption configuration can be suggested. For Met, indeed, the binding of the biomolecule through its $\mathrm{N}$ atom is enabled by the thermally activated deprotonation of the ammonium group (as observed by XPS) and reveals the directing role of the $\mathrm{Cu}$ substrate on the supramolecular chemistry and morphology. This influence of the $\mathrm{Cu}(111)$ surface is emphasized by comparison with the Met/Ag(111) system, for which a temperature independent zwitterionic chemical state of the adsorbate and no evidence of increased reactivity at the step-edges was reported [63]. Furthermore, the thermal excitation of the system also modifies the morphology of the substrate step-edges upon amino acid adsorption. At $283 \mathrm{~K}$ Met 
decorated step edges become indented, a phenomenon in agreement with the step faceting already reported for various systems and discussed in section 3.1.5.

At variance with the highly regular gratings found for $\operatorname{Met} / \operatorname{Ag}(111)$ [63], no systematic parallel adsorption of the molecular species could be determined for the zwitterionic $\alpha$-phase assembly of Met on $\mathrm{Cu}(111)$ and only restrained 1D extensibility is shown. The different behaviour is significant and reflects the different properties for the $\mathrm{Cu}$ and $\mathrm{Ag}$ substrates. The molecules have indeed reduced mobility and higher nucleation density on the more reactive $\mathrm{Cu}(111)$ substrate, which would kinetically limit the self-assembly process. Nevertheless, the zwitterionic nature of this phase and the tendency of the amino acid to dimerize along its long axis in a non-perfectly parallel fashion suggests a model of chain formation similar to the case of L-methionine on $\mathrm{Ag}(111)$ [63] (see also section 3.2.2.2).

Long range ordered structures are present, on the contrary, in the high temperature, anionic $\beta$-phase, for which a self-assembled structure with adjacent molecules in slightly non-equivalent binding sites is deduced from high resolution STM images. The molecular dimerization and the topographic characteristics of the self-assembly suggest a hydrogen bond driven head-to-head assemblage between two molecules along their main axis, where one of the negatively charged oxygen atoms of the carboxylate group faces an amino hydrogen atom. The linear growth is explained by adjacent $\mathrm{H}$ bonding involving again the carboxylate and amino groups, in agreement with what proposed for the L-methionine/Ag(111) system [63]. The regular mesoscopic ordering of the $\beta$-phase could be the effect of long-range indirect interactions mediated by the $\mathrm{Cu}(111)$ surface electronic structure. In fact, the self-assembled biomolecular chains scatter and confine the $\mathrm{Cu}(111)$ (Shockley) surfacestate electrons in a way similar to the previously studied L-methionine/Ag(111) system [2]. In comparison with the case of $\mathrm{Ag}(111)$, this substrate-mediated interaction could be stronger in the Lmethionine/ $\mathrm{Cu}(111)$ system due to a larger surface-state electronic density.

\subsubsection{Serine.}

Serine has an $\mathrm{OH}$ group in $\beta$-position in addition to the amino acid functional groups. Although magic number gas phase clusters of serine have been widely investigated [184; 185], there are not many examples of serine adsorption on metal surfaces.

Barlow and Raval [175] reported an ordered LEED pattern for the high coverage of L-serine on $\mathrm{Cu}(110)$ when annealed to $400 \mathrm{~K}$. At room temperature, on the contrary, RAIR spectra show an adsorption pattern with two coverage dependent phases similar to the one reported for alanine and glycine. The authors propose that intermolecular $\mathrm{H}$-bonding involving the $\beta$-OH groups affects the ordering of the molecules on the surface.

More recent studies suggest that both on $\mathrm{Cu}(110)$ [43] and on $\mathrm{Cu}(001)$ [160] adsorption occurs with the formation of Ser-dimers which are, however, stabilised by different H-bonds on the two surfaces.

Serine interaction with $\mathrm{Cu}(001)$ was investigated first and it was found that, at $310 \mathrm{~K}$, a domain of thick lines with a $\left(\begin{array}{cc}2 & -1 \\ 2 & 4\end{array}\right)$ periodicity initially appears along the <-130> direction regardless of serine exposure (see Figure 21a). With time, thick lines get gradually replaced by thin lines dominantly along the $<-310>$ directions, showing a $\left(\begin{array}{ll}2 & 1 \\ x & y\end{array}\right)$ pattern along the same directions. The 
thick serine lines show an internal "peanut-shaped" structure (see Figure 21b) originated by dimer formation. The authors assume that L-serine adsorbs through the carboxylate and amino groups, as other small amino acids on $\mathrm{Cu}(001)$, and consider additional interactions formed in the <-130> and $<310>$ directions by the $\mathrm{OH}$ groupspointing towards the surface (see Figure 21c), similarly to the case of $\mathrm{Lys} / \mathrm{Cu}(001)[181]$. Serine molecules in the gas phase form a stable octameric cluster [186] consisting of four dimers connected by a 3D-network of hydrogen bonds; therefore it is most likely that the dimer unit in the cluster of serine in the gas phase is maintained upon deposition, leading to the lines. The molecules of the Ser dimers have opposite orientation and are bound to each other by two hydrogen bonds between amino group and carboxylate group (N-H...O1) in the <-130> direction (see Figure 21).In addition, the dimers are connected by hydrogen bonds between hydroxymethyl groups $(\mathrm{O} 3-\mathrm{H}-\mathrm{O} 3)$ in the $<-130\rangle$ direction and between amino group and carboxylate group $(\mathrm{N}-\mathrm{H}-\mathrm{O} 1)$ in the $\langle 310\rangle$ direction.

On the contrary, the thermodynamically stable thin lines grow along the <-310> direction suggestan adsorption structure similar to that of Ala/Cu(001)[136].

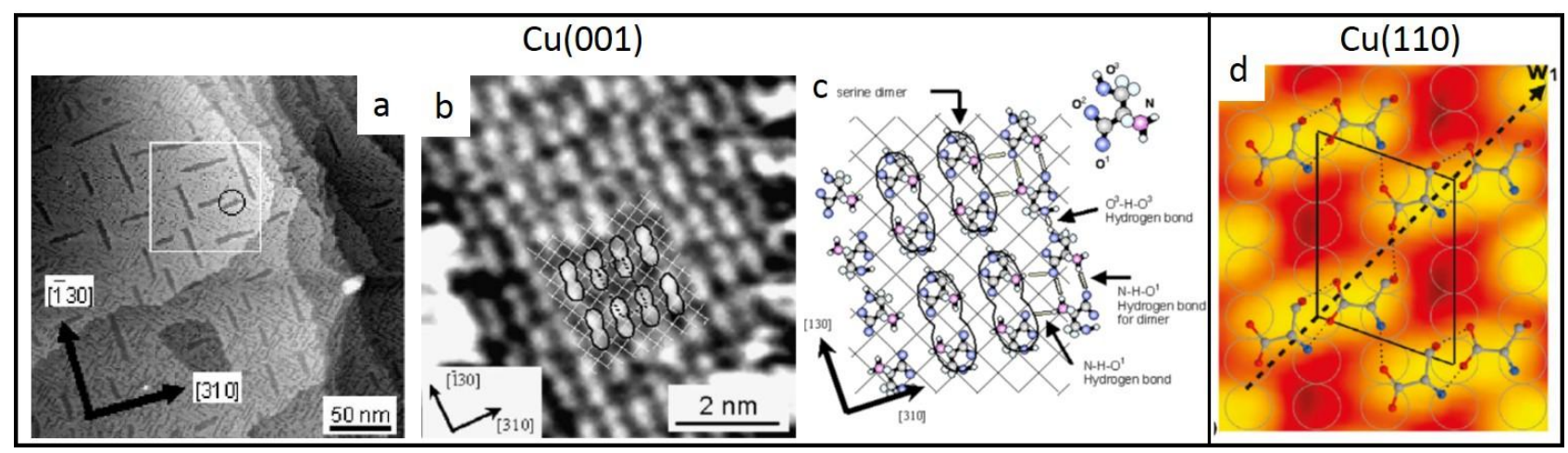

Figure 21. a) STM images after the deposition of $0.4 \mathrm{~L}$ of $\mathrm{L}$-serine on $\mathrm{Cu}(001)$ at $310 \mathrm{~K}(\mathrm{~V}=+0.1 \mathrm{~V}$, $\mathrm{I}=0.3 \mathrm{nA}$ ). Coexistence of thick and thin lines of serine is evident. b) Enlarged STM image of thick lines. Grid mesh indicates the lattice of the $\mathrm{Cu}(001)$ substrate. Single dimers (indicated by peanut shapes) and the periodic $\left(\begin{array}{cc}2 & -1 \\ 2 & 4\end{array}\right)$ structure (indicated by a dashed rectangle) are marked $(\mathrm{V}=+0.1$ $\mathrm{V}, \mathrm{I}=0.1 \mathrm{nA})$. c) Scheme of the L-serine overlayer on $\mathrm{Cu}(001)$. d) Model proposed for serine dimers on $\mathrm{Cu}(110)$ superimposed to the experimental STM image of the ordered L-serine layer. H-bonds and unit cells are indicated. The layer was produced by deposition of $0.25 \mathrm{ML}$ of serine (full coverage) and annealing to $420 \mathrm{~K}$. Taken with permission from refs. [43; 160].

On $\mathrm{Cu}(110)$ dimer formation is observed by STM, but a different organization is proposed [43]. Indeed, the combination of LEED and STM data demonstrate that, at full monolayer coverage and after annealing to $420 \mathrm{~K}, \mathrm{D} / \mathrm{L}$-serine overlayers on $\mathrm{Cu}(110)$ form well-ordered superstructures with $(-1 \pm 2 ; 40)$ periodicity after annealing. Each dimer occupies one unit cell of the superstructure and its axis is tilted by $\beta= \pm 73^{\circ}$ with respect to the $\langle 110\rangle$ direction of the $\mathrm{Cu}$ substrate. XPS indicates that adsorption occurs in the anionic form (see Table 3 ) and the $\mu 3$ adsorption model typical of other small amino acids is proposed also in this case. The $\beta-\mathrm{OH}$ side group is intact and is available for hydrogen bonds with adjacent molecules. The structural investigation is completed by NEXAFS data, which allow to determine the azimuthal angles of the carboxylate groups of the two inequivalent serine molecules in the dimer. On this basis, the model proposed for the dimer is the one reported in Figure 21d, in which two H-bonds form within each dimer, one of which involving 
the additional $\mathrm{OH}$ group. As evident from comparison of panels c) and d) of Figure 21, this model differs from the one proposed for serine dimers on $\mathrm{Cu}(001)$ since in that case the hydroxymethyl groups are not involved in intradimer bondings but only in the bondings between adjacent dimers. We mention that a significant number of monomers within hydrogen-bonded aggregates is detected upon deposition on $\mathrm{Cu}(110)$ at $\mathrm{RT}$, but they are all converted into dimers after annealing to $420 \mathrm{~K}$. Such behaviour indicates that dimer formation has a relatively high activation barrier once the molecule is involved in a hydrogen-bonded network. The network plays an important role in stabilizing the dimers, which must be considered as subunits of an extended hydrogen-bonded structure rather than as stand-alone units.

Dimer formation has not been observed for glycine, alanine, or proline on $\mathrm{Cu}(110)$ or $\mathrm{Cu}(100)$ [104; $105 ; 107 ; 108 ; 137 ; 142 ; 157 ; 175]$. The pairing observed for alanine and glycine on $\mathrm{Cu}(531)$ cannot be considered as true dimer formation because it involves only one hydrogen bond rather than two per pair $[43 ; 187]$. Cysteine on $\mathrm{Au}(110)$, on the contrary, forms homochiral dimers through two $\mathrm{OH} \cdots \mathrm{O}$ bonds involving the intact carboxylic acid groups [188], while Met arranges in dimer chains both on $\mathrm{Cu}(111)$ [62] and on $\mathrm{Ag}(111)$ [63]. This variety of behaviours suggests that it is not possible to point out a particular side group that is necessary for dimer formation, which is explained by the fact that they can indeed originate in several ways.

Finally, after investigating the adsorption of enantio-pure D- and L-serine, Eralp et al. [43] deposited also the racemic mixture. The differences between L- and D-serine in dimer orientation and dimer stacking with respect to the achiral $\mathrm{Cu}(110)$ substrate lead to spontaneous chiral resolution of the racemic mixture and into small enantio-pure domains, the chirality of which can be determined from their orientation. The structure of these domains is the same observed for the corresponding enantio-pure molecules, which strongly indicates that dimer formation occurs only between equal enantiomers. However, both STM images and TPD results indicate that enantiopure domains are smaller in the racemic layer than in the enantiopure layers, and that their separation is not perfect even after annealing to $370 \mathrm{~K}$. Heterochiral stacking faults within the domains disrupt then the formation of large islands.

\subsubsection{Lysine.}

Lysine is an essential amino acid characterized by the presence of a relatively large side group, which consists of a4-atom long alkane chain terminated by an additional $\mathrm{NH}_{2}$ group at the $\varepsilon$ position. 


\begin{tabular}{|c|c|c|c|c|c|}
\hline \multirow{3}{*}{ Vibrational mode } & \multicolumn{5}{|c|}{ L-Lysine/Cu(110) } \\
\hline & \multicolumn{2}{|c|}{$300 \mathrm{~K}$} & \multicolumn{2}{|c|}{$400 \mathrm{~K}$} & \multirow{2}{*}{$\begin{array}{l}\text { L-Lys- } \\
p(2 \times 1)-0- \\
\mathrm{Cu}(110)\end{array}$} \\
\hline & Low $\Theta$ & High $\Theta$ & Low $\Theta$ & High $\Theta$ & \\
\hline$v_{\text {sym }}\left(\mathrm{CH}_{2}\right)$ & \multirow{5}{*}{$\begin{array}{l}2930 \\
2860 \\
1624\end{array}$} & \multirow{4}{*}{$\begin{array}{l}2930 \\
2860 \\
1634 \\
1611\end{array}$} & \multirow{3}{*}{$\begin{array}{l}2934 \\
2864 \\
1618\end{array}$} & \multirow{2}{*}{$\begin{array}{l}2930 \\
2857\end{array}$} & \multirow{2}{*}{$\begin{array}{l}2934 \\
2857\end{array}$} \\
\hline$v_{\text {asym }}\left(\mathrm{CH}_{2}\right)$ & & & & & \\
\hline$\delta_{\text {asym }}\left(\mathrm{NH}_{3}{ }^{+}\right)$ & & & & 1628 & 1618 \\
\hline$\delta_{\text {sciss }}\left(\mathrm{NH}_{2}\right)$ & & & \multirow{4}{*}{1554} & 1614 & \\
\hline$v_{\text {asym }}\left(\mathrm{COO}^{-}\right)$ & & 1577 & & 1582 & \\
\hline$\delta_{\text {sym }}\left(\mathrm{NH}_{3}{ }^{+}\right)$ & \multirow[t]{2}{*}{1524} & 1530 & & 1549 & \multirow[b]{2}{*}{1447} \\
\hline$\delta_{\text {sciss }}\left(\mathrm{CH}_{2}\right)$ & & 1442 & & 1444 & \\
\hline$v_{\text {sym }}\left(\mathrm{COO}^{-}\right)$ & \multirow{4}{*}{$\begin{array}{l}1416 \\
1328\end{array}$} & 1411 & \multirow{4}{*}{$\begin{array}{l}1411 \\
1319\end{array}$} & 1407 & 1400 \\
\hline$\omega\left(\mathrm{CH}_{2}\right)$ & & 1336 & & 1322 & 1340 \\
\hline $\mathrm{r}\left(\mathrm{NH}_{3}{ }^{+}\right)$ & & & & & 1148 \\
\hline$v(C-N)$ & & & & & 1038 \\
\hline
\end{tabular}

Table 5. Characteristic vibrational bands (expressed in $\mathrm{cm}^{-1}$ ) and relative assignments for lysine adsorbed from the gas-phase on the $\mathrm{Cu}(110)$ and $\mathrm{p}(2 \times 1)-\mathrm{O}-\mathrm{Cu}(110)$ surfaces. Datataken from ref. [151].

a) $\operatorname{Lys} / \mathrm{Cu}(110)$

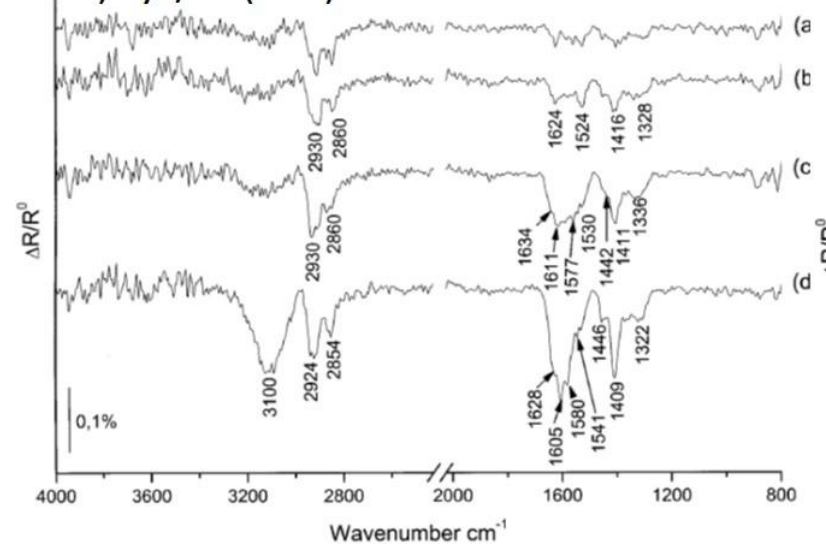

b) Lys/p(2x1)-O-Cu(110)

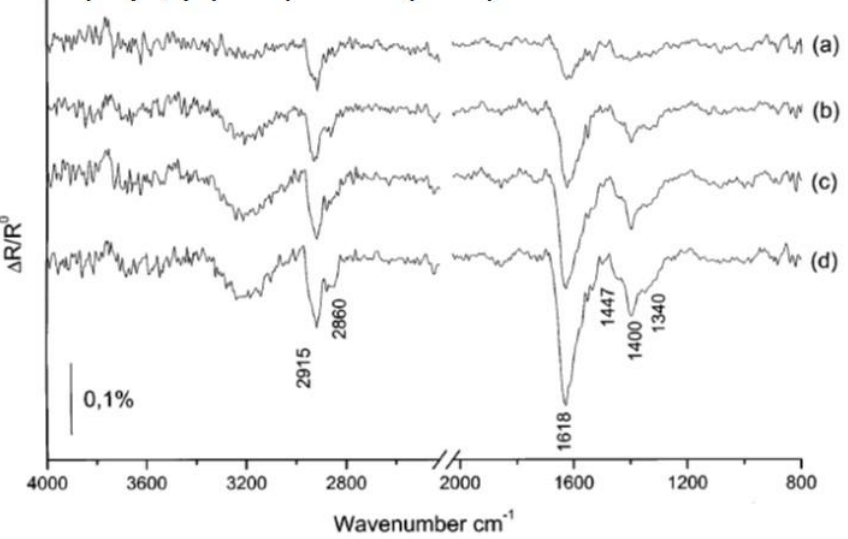

Figure 22. a) RAIR spectra following the adsorption of L-lysine on $\mathrm{Cu}(110)$ at $300 \mathrm{~K}$ as a function of coverage. Exposure times are (a) 5, (b) 15, (c) 22, and (d) $40 \mathrm{~min}$. b) RAIR spectra following the adsorption of L-lysine on $\mathrm{O}-\mathrm{p}(2 \times 1)-\mathrm{Cu}(110)$ at $300 \mathrm{~K}$ as a function of coverage. Exposure times are (a) 5, (b) 18, (c) 34 and (d) 43 min. Taken with permission from ref. [151].

The adsorption of L-Lys on $\mathrm{Cu}(110)$ was studied by Humblot et al. [61; 151; 189] by combining FT-RAIRS and STM with quantum chemical modelling. Upon deposition from solution, four different chemical states are found for lysine, depending on $\mathrm{pH}$ [151]. This behaviour is not surprising, as it has been observed by the same research group also for other amino acids [48]. When lysine is sublimated under UHV conditions, different types of molecular conformations and overlayer geometries are detected by STM [189] and RAIRS [151], depending on coverage, deposition/annealing temperature and surface pre-treatments: 
a) At low coverage and $\mathrm{T}_{\mathrm{ev}}=300 \mathrm{~K}$, mirror domains of a $(60 ; \pm 12)$ superstructure with two molecules per unit cell (local coverage $\Theta=0.17 \mathrm{ML}$ ) form. RAIRS analysis (see Table 5 and Figure 22a) and DFT calculations predict that the molecule, in its zwitterionic state, adopts a $\mu 3$ adsorption geometry with bonds through the two oxygen atoms of the deprotonated carboxylate group and the $\alpha-\mathrm{NH} 3^{+}$group [61].

b) For the saturated chemisorbed layer at $300 \mathrm{~K}$ a $(-2-2 ; 5-1)$ superstructure is determined by inspection of both STM images and LEED diffraction pattern, with a cluster-like arrangement of 4 molecules per unit cell $(\Theta=0.33 \mathrm{ML})$. A change in the molecular conformation is deduced from RAIRS spectra (see Table 5 and Figure 22a): as the coverage is increased, the carboxylate group tilts and the amino group is pushed away from the surface, lying almost parallel to the surface. Therefore the interaction between the surface and the lysine molecules proceeds mainly through one oxygen atom. Additional molecules may then adsorb in a disordered way on the top of the saturated "asymmetric" lysine monolayer.

c) On the reconstructed missing/added rows $\mathrm{p}(2 \times 1)-\mathrm{O}-\mathrm{Cu}(110)$ surface at $\mathrm{RT}$, lysine shows a drastic change of its adsorption properties: a poorly oriented adsorption mode with no changes when the exposure increases is determined from RAIRS (see Table 5 and Figure $22 \mathrm{~b}$ ) and Auger measurements. Adsorption does not proceed layer by layer but more likely in the form of aggregates from the beginning, with no clear saturation coverage.

d) If $\mathrm{T}_{\mathrm{ev}}=400 \mathrm{~K}$, the geometry of the Lys $/ \mathrm{Cu}(110)$ layer is very similar to that observed at room temperature, i.e. symmetric at low $\Theta$ and asymmetric at high $\Theta$. No multilayer forms.

e) Annealing the saturated $\mathrm{Lys} / \mathrm{Cu}(110)$ layer from $300 \mathrm{~K}$ to $425 \mathrm{~K}$ and $450 \mathrm{~K}$ causes partial desorption of the molecules and the formation of simpler and less dense structures with $\mathrm{p}(3 \times \mathrm{n})$ and $\mathrm{p}(3 \times 2)$ symmetry, respectively [189]. Since the $\mathrm{p}(3 \times 2)$ overlayer is the most stable assembly for several amino acids $[101 ; 105 ; 190]$, the authors claim that these results confirm the general behaviour adopted by the same kind of amino acids when adsorbed under similar conditions.

In a parallel DFT study of the same system [61], the adsorption geometry is discussed in terms of the molecular electrostatic interactions and electronic properties, such as the electronic density of states (DOS). From comparison of simulated and experimental STM images, the authors deduced the most stable adsorption configuration, depicted in Figure 23. Compelling evidence of the $\mu 3$ adsorption geometry and of the open row adsorption pattern (2 rows occupied 1 free, forming a pairing row - pattern shown in Figure 23) of the adlayer is provided. The stability of the adlayer is explained in terms of a subtle interplay between inter-molecular repulsive interactions, inter-molecular H-bonds, and surface tension of the copper surface. The presence of the "pairing" row has a destabilizing effect, compared to the single row assembly, but it is compensated by H-bond interactions between the molecules (See Figure 23). The adsorption energy of a L-Lys unit is $-1.31 \mathrm{eV}$ and $-0.77 \mathrm{eV}$ for molecules in single and in double rows, respectively, the latter configuration being $0.54 \mathrm{eV}$ less stable.The relative orientation of the L-lysine molecules is discussed using the electrostatic potential, explaining the formation of chiral adsorption assemblies. 


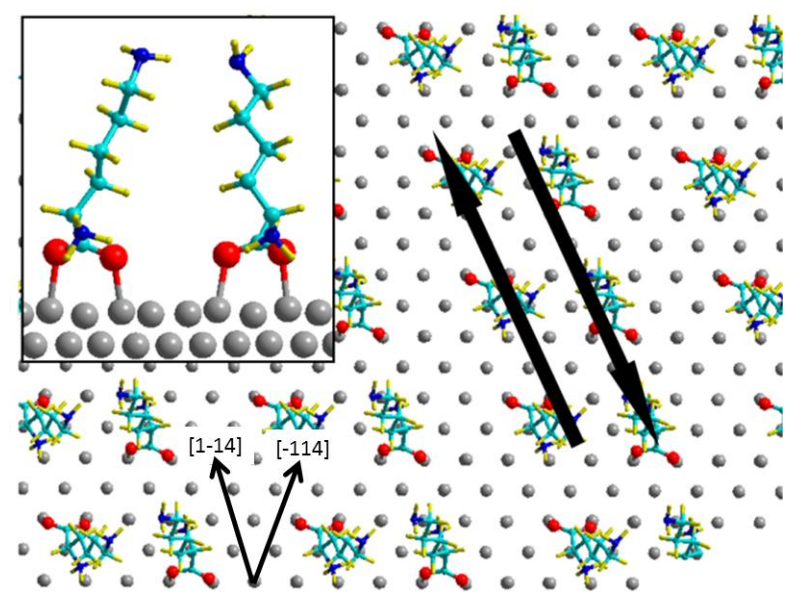

Figure 23. Top and side view of the most stable L-Lys/Cu(110) conformation according to combined STM and DFT results. The Lys coverage is $0.167 \mathrm{ML}$ and the molecules are arranged in a $\left(\begin{array}{ll}6 & 0 \\ 1 & 2\end{array}\right)$ assembly, i.e. growing along the $\langle 1-14\rangle$ and $\langle-114\rangle$ axes. The relative directions of the lysine molecules are indicated with arrows. Taken with permission from ref. [61].

The model proposed for the high temperature phases of $\mathrm{L}-\mathrm{Lys} / \mathrm{Cu}(110)$ has been recently revised by Eralp et al. [152] in light of new XPS and NEXAFS experiments. First of all, we mention that these authors reinterpreted the $\mathrm{Lys} / \mathrm{Cu}(110)$ overlayers forming at $\mathrm{T}=425 \mathrm{~K}$ and $450 \mathrm{~K}$ as best described by a $\mathrm{p}(6 \times 4)$ and a $\mathrm{p}(3 \times 4)$ geometry, respectively. Secondly, XPS data (see Table 3 for the details of the binding energies) indicate that at least $70 \%$ of the adsorbed molecules are in the zwitterionic state at room temperature, while they partially desorb or convert into the anionic form upon annealing above $420 \mathrm{~K}$. Moreover, the presence of a single $\mathrm{O} 1 \mathrm{~s}$ peak indicates that both oxygens of the carboxylate group interact with the substrate. Figure 24a shows the outcome of NEXAFS analysis performed on the $\mathrm{O} 1 \mathrm{~s} \rightarrow \pi^{*}$ transition. At $300 \mathrm{~K}$, no significant angular dependence is present in the $\mathrm{O} \mathrm{K}$-edge NEXAFS spectra while more modulation appears in the $\sigma$-resonance region; this observation is compatible with the cluster model proposed by Humblot [189], which envisages multiple orientations of the lysine molecules. On the contrary, the curve corresponding to the Lys/Cu(110) surface annealed to $420 \mathrm{~K}$ shows a maximum around $(-85 \pm 6)^{\circ}$, indicating that the O-C-O groups of all lysine molecule are aligned nearly parallel to the <-110>direction of the $\mathrm{Cu}(110)$ surface. On the basis of XPS and NEXAFS data, the authors propose a three-point adsorption model (Figure 24b) similar to the one already established for Gly and Ala (see refs. [104; $107 ; 108 ; 141 ; 142 ; 175]$ and Figure 6 ). The $\varepsilon$-amino group forms a fourth bond with a $\mathrm{Cu}$ atom, which, for steric reasons, must be 2 rows away from the carboxylate group, so that an overall $\mu 4$ bonding geometry is present, in which the additional anchoring point is provided by the side chain functionality. This result is, however, not compatible with the model proposed previously [189] on the basis of STM and RAIRS data, in which the side chain was lifted from the surface. The reason for this discrepancy is most probably a consequence of the different periodicity of the Lys overlayers detected in the two series of experiments. 

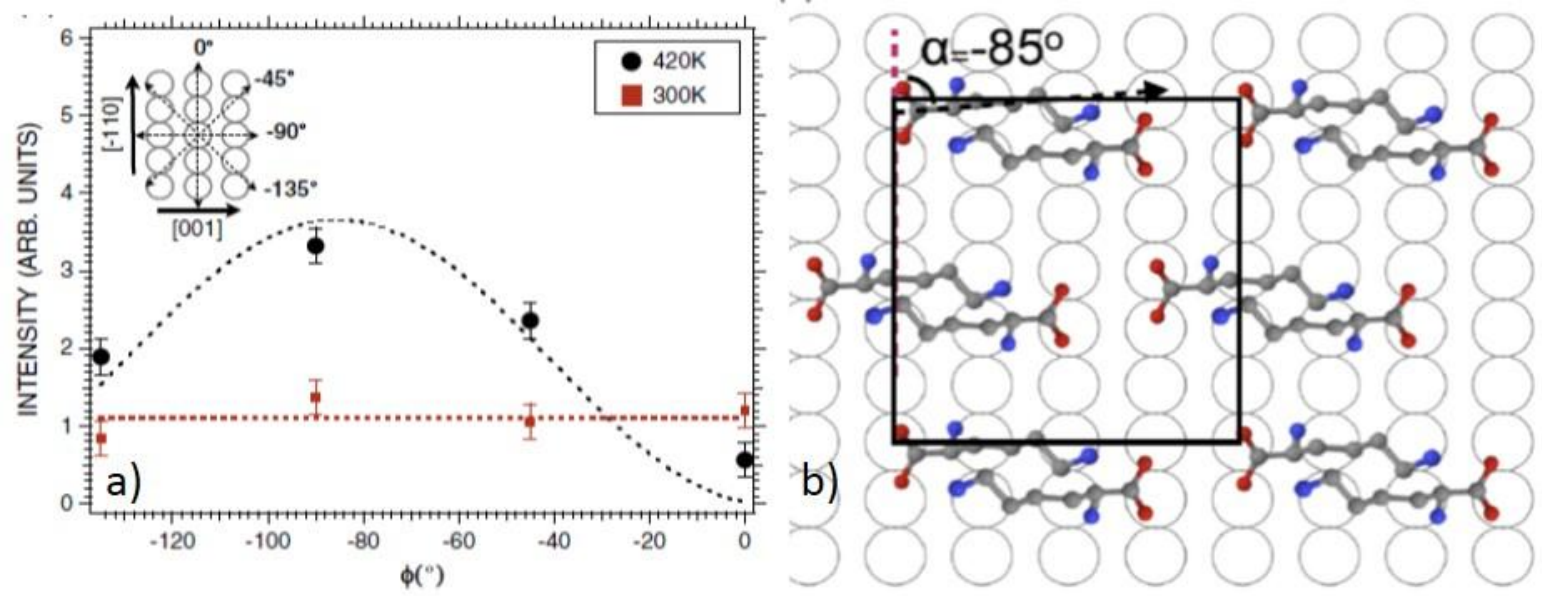

Figure 24. a) Intensities of the NEXAFS O $1 \mathrm{~s} \rightarrow \pi^{*}$ resonance vs azimuthal angle of the polarization vector for the $\mathrm{Lys} / \mathrm{Cu}(110)$ chemisorbed layer saturated at room temperature and after annealing to $420 \mathrm{~K}$. Inset: schematic diagram of $\mathrm{Cu}(110)$. b) Adsorption model of a $\mathrm{p}(6 \mathrm{x} 4)$ Lys overlayer on $\mathrm{Cu}(110)$ proposed on the basis of the NEXAFS and XPS data. Taken with permission from ref. [152].

Adsorption of Lysine on $\mathrm{Cu}(001)$ was performed by Zhao et al. [150; 181], and investigated by STM, using the same approach as for other amino acids[95; 139]. From the estimated chemisorption energy of $\sim-1.6 \mathrm{eV} /$ molecule, the authors deduce that Lys adsorbs in the anionic form, coherently with what reported for the $\mathrm{Ala} / \mathrm{Cu}(100)$ system [139].

At $300 \mathrm{~K}$ and at the lowest exposure, lysine forms a 2D-gas of molecules which may diffuse on terraces and/or undergo fast conformational changes. Once the coverage of the 2D gas phase reaches the saturated value, the excess adsorbed molecules segregate to form superstructure islands. In the few hours after Lys deposition, these structures change from small and narrow 2D solid islands, randomly distributed on $\mathrm{Cu}(001)$ terraces, to larger islands (reported in Figure 25a). This behaviour indicates that the homogeneous nucleation mechanism of superstructure islands is dominant and that subsequent "Ostwald ripening" occurs; this is markedly different from what observed for the other AA/Cu systems reported so far. To explain the coexistence of the two phases and the nucleation of 2D solid islands with increasing coverage, the authors draw the following picture: in the 2D gas-phase anionic lysine molecules stand upright on the surface at the atop sites [42] and experience a repulsive force between each other, due to their net negative charge and dipole momentum. These repulsive forces prevent the molecules from forming clusters or islands but generate a 2D gas homogeneously covering the entire surface. Above a critical coverage, it is energetically more favourable for the excess molecules to organize in the denser 2D solid phase or in superstructure islands, in which the molecules lie down on the surface. The major diving force for this process is, presumably, the formation of hydrogen bonds between adjacent Lys anions. Moreover, the repulsive forces between dipoles can also be reduced for molecules lying flat on the surface.

Further increasing the Lys coverage, all (001) terraces of the as-deposited surface can be covered only by the $2 \mathrm{D}$ solid phase, which organises in two different superstructures: $\mathrm{Cu}(100)-$ $\left(\begin{array}{cc}4 & 1 \\ -3 & 4\end{array}\right)-\mathrm{L}-$ Lys and $\mathrm{Cu}(100)-\left(\begin{array}{cc}4 & 1 \\ -2 & 4\end{array}\right)-\mathrm{L}$ - Lys (see Figure 25b-c for high resolution STM images). Annealing at $430 \mathrm{~K}$ leads to the conversion of the former assembly into the latter, so 
that the surface is uniformly covered by a single overlayer. STM images show only two protrusions in each unit cell of either superstructure. This suggests that: a) the unit cell consists of two Lys units, which is also reasonable considering the van der Waals dimensions of the L-lysine molecule; b) each lysine molecule is imaged as a single protrusion, as in the case of glycine and alanine on $\mathrm{Cu}(001)$ [95; 139]. Based on the anionic nature of the chemisorbed Lys molecules, on the adsorption sites already determined for simpler AA as Gly and Ala and on previous literature on Lys crystals [191], the unified model of Figure 25d was proposed for both the $\left(\begin{array}{cc}4 & 1 \\ -3 & 4\end{array}\right)$ and $\left(\begin{array}{cc}4 & 1 \\ -2 & 4\end{array}\right)$ superstructures. The $\mathrm{Cu}(100)$ substrate is assumed to be bulk-like, except for some relaxation; the two oxygen atoms of the carboxylate group and the two nitrogen atoms of the two amine groups locate approximately at the atop site while the side chain stays in the favourable trans conformation. The two superstructures are qualitatively similar, consisting of molecular chain pairs formed through a H-bond network. They differ, mainly, in the arrangement of the molecule-chain pairs and in the relevant coverage of lysine. Since the $\left(\begin{array}{cc}4 & 1 \\ -2 & 4\end{array}\right)$ arrangement allows the formation of more H-bonds, it is reasonably more stable, and in fact it is the structure surviving above room temperature. Bonding of the $\mathrm{N}$ atoms of both the amine group and side chain to the $\mathrm{Cu}$ substrate may also contribute to the stabilization of the superstructures.

Finally, we mention that step faceting and bunching into facets occurs upon Lys adsorption at $\mathrm{Cu}(100)$ and subsequent annealing at $430 \mathrm{~K}$ (Figure 25e). Details of this phenomenon are discussed in the dedicated paragraph of Section 3.1.5. 


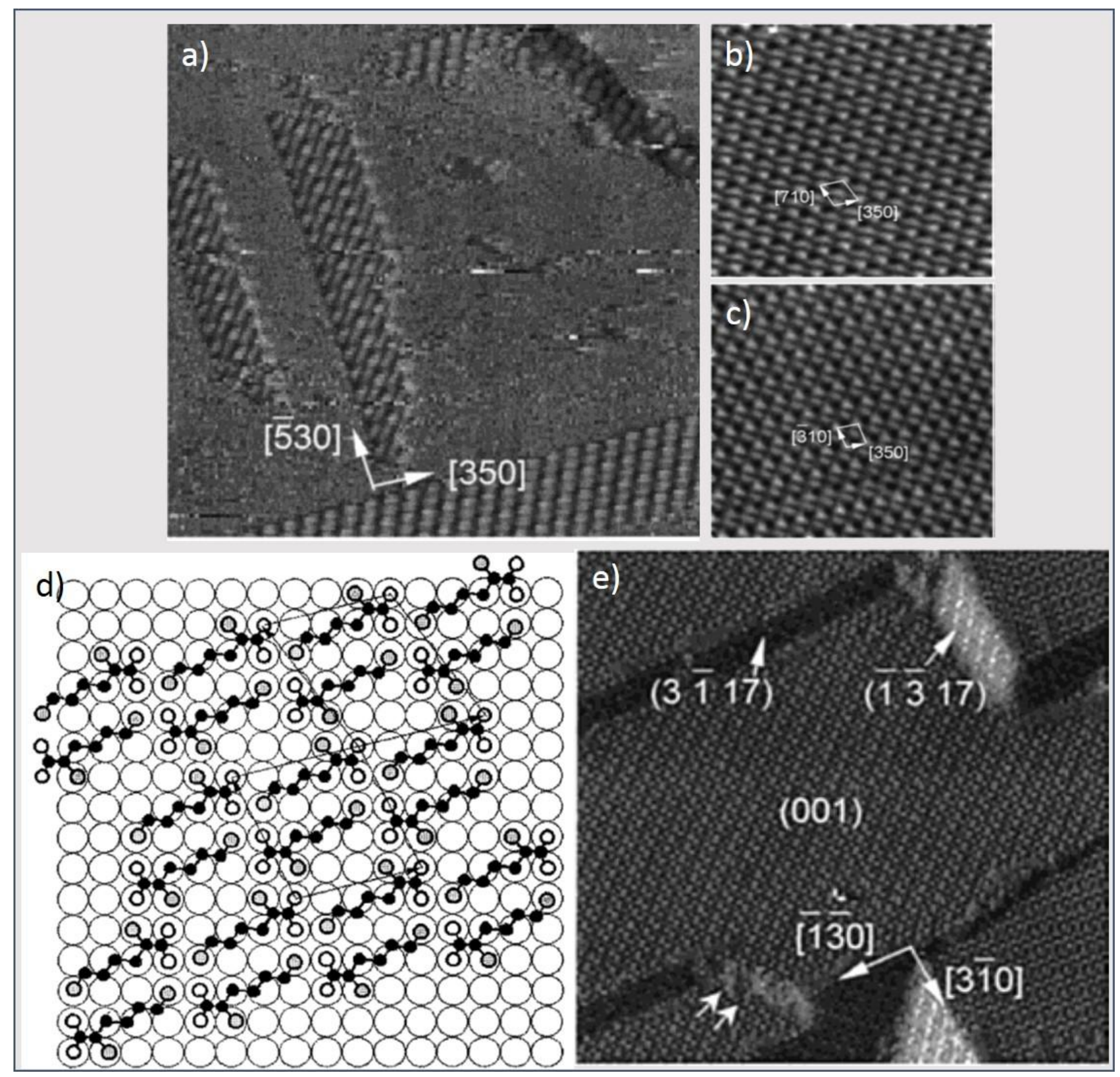

Figure 25. a) STM image of a Lys/Cu(001) surface $14 \mathrm{~h}$ after deposition at room temperature, showing the growth of ordered domains (Image size: $270 \AA$ x $270 \AA$, V=1.0 V, I=1.0 nA). The $<350>$ and

$<-350>$ directions are marked. b) High-resolution STM image of the Cu(001)- $\left(\begin{array}{cc}4 & 1 \\ -3 & 4\end{array}\right)$-L-lysine structure on the as-deposited surface, with a unit cell outlined. c) Same as b) for the $\mathrm{Cu}(001)$ $\left(\begin{array}{cc}4 & 1 \\ -2 & 4\end{array}\right)$-L-lysine structure produced upon annealing the surface to $430 \mathrm{~K}$. (In both cases, image size: $133 \AA \times 133 \AA, \mathrm{V}=1.0 \mathrm{~V}, \mathrm{I}=0.6 \mathrm{nA}$ ). d) Schematic drawing of the model proposed for the superstructures of panels b) and c) (top and bottom unit cells, respectively), corresponding to Lys coverage of 2/19 and 2/18 ML, respectively. e) STM image (image size: $550 \AA \times 550 \AA, \mathrm{V}=1.0 \mathrm{~V}$, $\mathrm{I}=0.6 \mathrm{nA})$ acquired from the L-lysine $/ \mathrm{Cu}(001)$ surface annealed at $430 \mathrm{~K}$ for $20 \mathrm{~min}$, showing coexistence of (001) terraces and $(31$ 17)facets formed through bunching of $\langle 310\rangle$-faceted steps. Due to the differential acquisition mode, the grey scale has nothing to do with the actual surface height but the same grey scale represents the same crystal plane. Taken with permission from refs. $[150 ; 181]$. 


\subsubsection{Amino acids with an aromatic or pyrrolic ring.}

\subsubsection{Proline}

The peculiarity of the amino acid proline is that the nitrogen atom is rigidly inserted in the pyrrolidine ring. This limits the conformational mobility of the $\mathrm{N}-\mathrm{H}$ bond with respect to the carboxyl group, which is itself restricted in mobility by the pyrrolidine cycle [192]. This rigidity is one of the factors that determines the important role played by proline in the folding of proteins and peptides, where steric effects arising from the proline side chains help to determine the stabilities and positions of the protein folds [193]. It can also be expected to influence the way the molecule adsorbs and self-assembles at a metal surface. In addition, Pro and its derivatives are commonly used as chiral modifiers in heterogeneous diastereoselective catalysis [194; 195], e.g. in the hydrogenation of the $\alpha, \beta$-unsaturated ketones or in the hydrogenation of substituted aromatic compounds, such as o-toluic acid.

Proline adsorption at $\mathrm{Cu}(110)$ was deeply investigated by Raval's group [156-158; 196; 197]. RAIRS analysis (see Table 6) evidences that proline adsorbs on $\mathrm{Cu}(110)$ at $300 \mathrm{~K}$ in the anionic form, as observed for a number of other amino acids $[42 ; 105 ; 140 ; 151]$. LEED studies revealed the formation of a $(4 \times 2)$ overlayer [158] already at the smaller coverage. This information, later confirmed by STM analysis [157], is indicative of an island growth mechanism. The picture is coherent with the very small changes observed in the RAIR spectra with increasing coverage, which suggests a very minor readjustment of the layer in contrast to the case of other amino acids, such as Gly and Ala [42; 50]. This difference arises from the rigidity of the S-proline structure, where reorientation of the carboxylate group would also induce a reorientation of the imino group and pyrrolidine ring, leading to either unfavourable steric repulsion with the surface or loss of bonding interactions.

Furthermore, combining the geometrical information provided by LEED, the one derived from the qualitative application of the metal surface selection rules to the RAIR spectra and the established results for other AA/Cu systems, the authors proposed the schematic models reported in Figure 26a and $\mathrm{b}$ for the molecular conformation and for the Pro overlayer, respectively. The pyrrolidine ring is oriented largely parallel to the surface and the plane of the carboxylate tilted significantly toward the surface plane. The self-assembled layer consists then of a $(4 \times 2)$ unit mesh in which - by analogy with previously investigated systems $[104 ; 107 ; 108 ; 141 ; 142 ; 175]$ - the molecules are disposed with their carboxylate group across short bridge sites and they straddle across the long bridge site to enable the $\mathrm{N}$ atom of the imino group to adsorb on the adjacent close packed row. Thermal desorption studies further demonstrated that this phase is stable until around $500 \mathrm{~K}$, highlighting the robust nature of this molecular assembly and its ability to survive at elevated temperatures. 


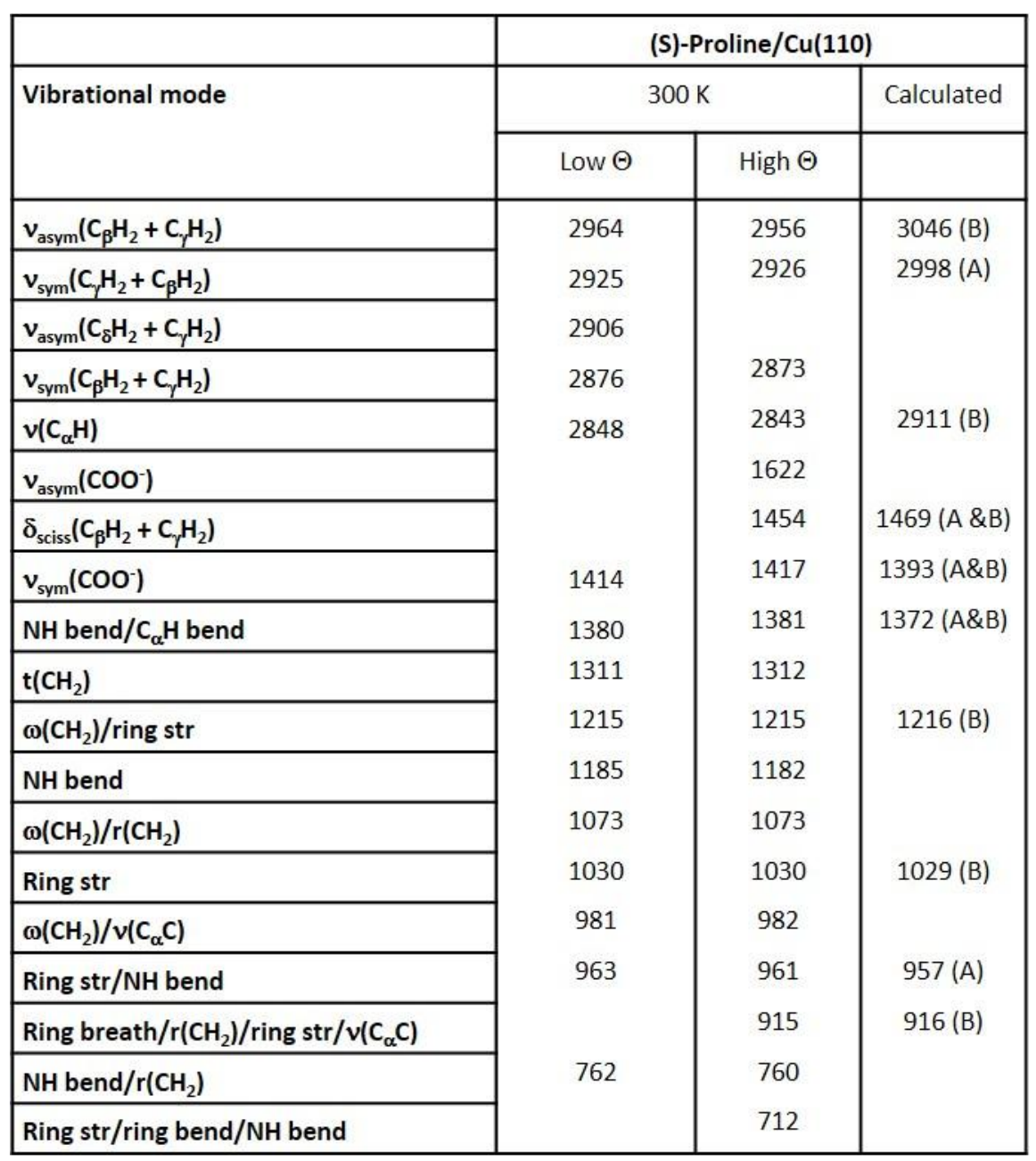

Table 6. Observed absorption bands (in $\mathrm{cm}^{-1}$ ) for $(\mathrm{S})$-proline adsorbed on $\mathrm{Cu}(110)$ at $300 \mathrm{~K}$ and corresponding assignments as reported in ref. [158]. The peaks at lower frequency show intermixing and have contributions from a number of vibrational motions making firm assignments difficult in this region. The right column reports the values calculated for the adsorption model for the $(4 \times 2)$ overlayer described in ref. [157] and in Figure 28b. The conformer involved in each vibration is indicated in brackets. 

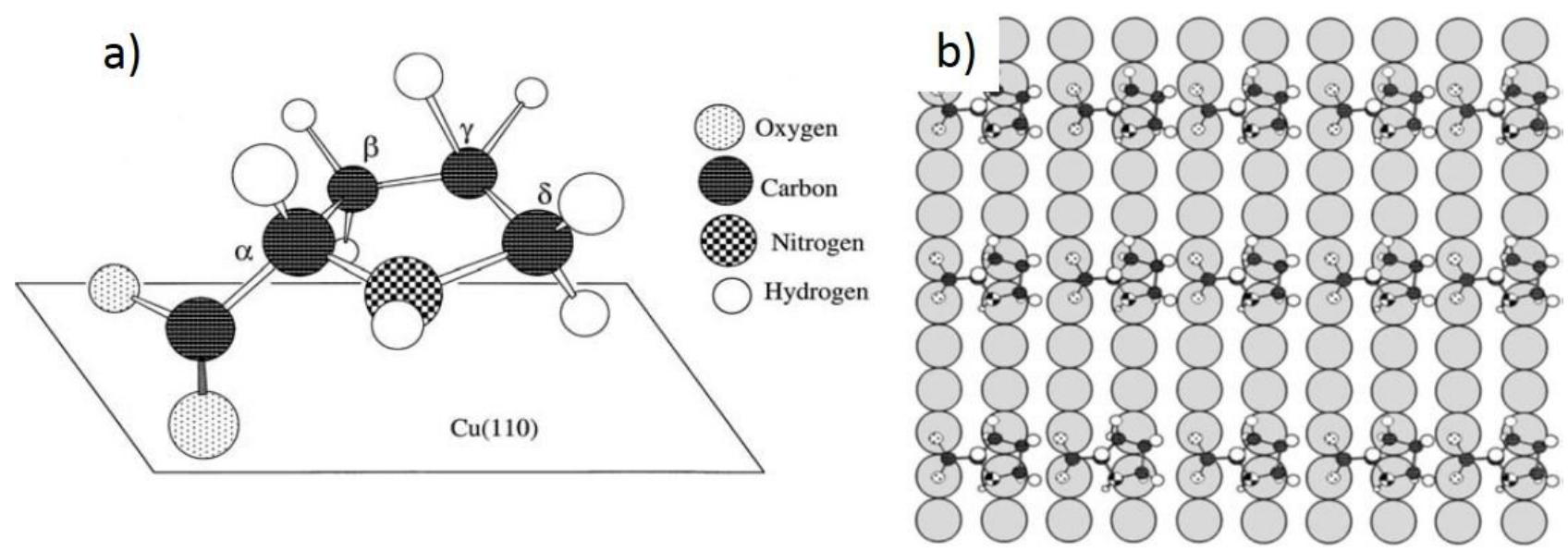

Figure 26. a) Schematic model showing the orientation adopted by (S)-Pro on $\mathrm{Cu}(110)$, as deduced by RAIR spectra. b) Schematic model of a simple $(4 \times 2)(\mathrm{S})-\mathrm{Pro} / \mathrm{Cu}(110)$ adlayer in the hypothesis of one molecule per unit cell. Taken with permission from ref. [158].

Although the model of Figure $26 \mathrm{~b}$ presents only one Pro unit per $(4 \times 2)$ unit mesh, the author admit that it is not possible to determine whether a second molecule is present. This is indeed the case, as revealed by a later STM and DFT investigation of the same system [157].

The STM data presented in Figure 27 contribute significantly to a more accurate description of the Pro/Cu(110) biomolecular assembly. First, they clearly confirm the island growth mechanism, showing that small islands with $(4 \times 2)$ periodicity are created at the earliest stages of adsorption (Figure 27a) and evolve with increasing exposure towards larger islands, elongated in the <1-10> direction, that coalesce together (Figure $27 b$ and c). Further exposure leads to the formation of multilayers (not shown). In addition, high resolution images reveal the presence of two Pro molecules per $(4 \times 2)$ unit cell, with the second molecule accommodated in the centre (Figure $27 \mathrm{~d}$ ) and showing in general a weaker contrast at all the voltage conditions (see high profile in Figure 27g). Finally, the STM images show that locally defected unit meshes occur in which the two molecules appear identical, i.e. either all weak or all bright (Figure 27e and f). The overall observation suggests therefore that the primitive $(4 \times 2)$ unit cell with two non-equivalent molecules is energetically favoured, but that the energetic advantage compared to the centred $(4 \times 2)$ unit mesh where all molecules are equivalent is small. As a general consideration, we notice that the $(4 \times 2)$ prolate assembly is very similar to the $(3 \times 2)$ overlayers of alaninate and glycinate on $\mathrm{Cu}(110)$, with the larger unit mesh of the $(4 \times 2)$ phase arising from the increased molecular dimensions of prolate. 

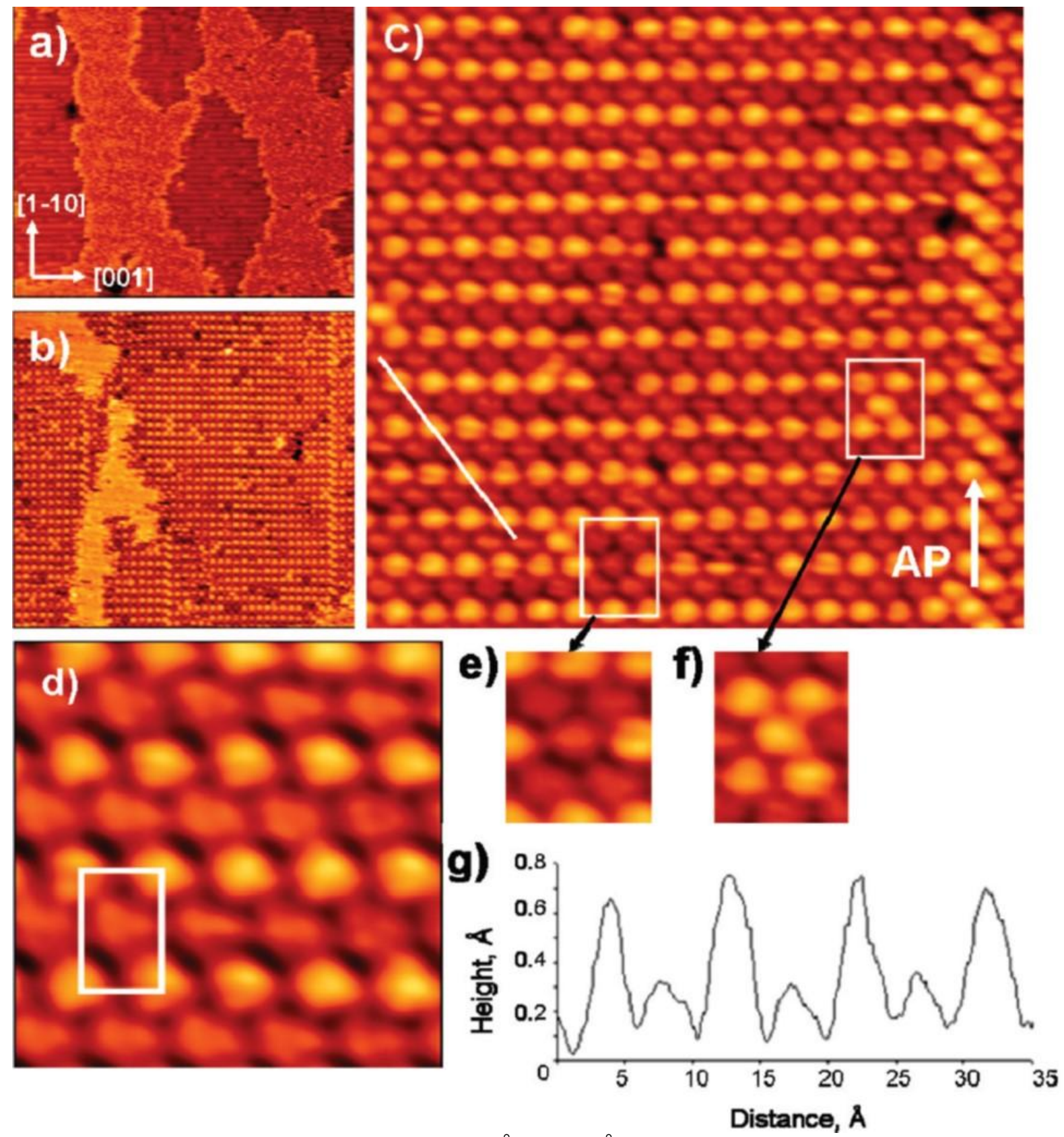

Figure 27.(a) STM image (image size $451 \AA \times 420 \AA$, I=-0.34 nA, V=-1.25 V) showing the growth of $(4 \times 2) \mathrm{Pro} / \mathrm{Cu}(110)$ islands at low coverage. $\mathrm{b}$ and c) STM images showing $(4 \times 2)$ islands joining together at increasing coverage, with the creation of antiphase boundaries, indicated in $\mathrm{c}$ as AP (Image size: $132 \AA \times 128 \AA$, I=-0.52 nA, V= -1.58 V and image size: $140 \AA \times 128 \AA, \mathrm{I}=-0.40 \mathrm{nA}, \mathrm{V}=-$ $0.81 \mathrm{~V}$, respectively). (d) High resolution STM image with the $(4 \times 2)$ unit indicated (Image size: $37 \AA \times 39 \AA, \mathrm{I}=-0.47 \mathrm{nA}, \mathrm{V}=-0.42 \mathrm{~V}$ ). (e and f) Expansion STM images from c) showing defect unit meshes containing all 'weak' or all 'bright' molecules, respectively. (g) Line scan cut along the white line marked in panel c) and showing the height contrast within the overlayer. Taken with permission from ref. [157]. 


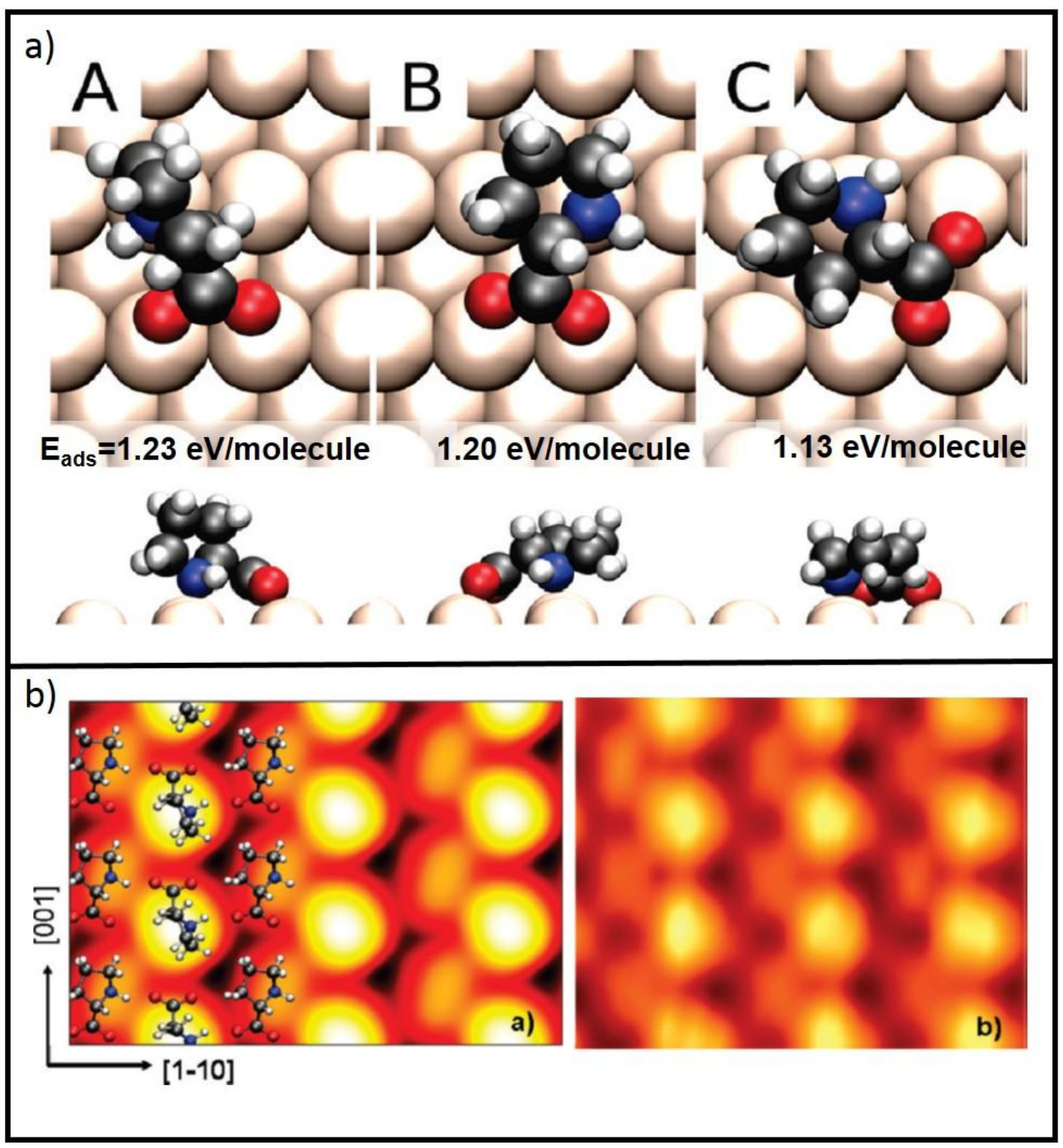

Figure 28. a)Relaxed adsorption geometries for the three most stable isolated prolate anions in a $(4 \times 4)$ unit cell on $\mathrm{Cu}(110)$. First Row: shown from above. Second Row: along the close-packed $<1$ $-10>$ rows. The absolute value of the adsorption energy is reportedfor each geometry. b) Left panel: simulated STM image of the most stable molecular arrangement; the positions of the atoms in the prolate anions are included. The calculated image is shown in a $(12 \times 6)$ unit cell and simulated at a bias voltage of $-1.25 \mathrm{~V}$ with an average tip-surface height of $7.5 \AA$. Right panel: High resolution STM image of $(4 \times 2)$ phase, reported for comparison. Image size $31.1 \times 21.7 \AA^{2}, \mathrm{I}=-0.43 \mathrm{nA}, \mathrm{V}=-$ $1.25 \mathrm{~V}$. Taken with permission from ref. [157].

The experimental investigation of the $\mathrm{Pro} / \mathrm{Cu}(110)(4 \times 2)$ overlayer was combinedwith DFTcalculations, providing a good example of how the combination of the two methods can lead to unravel details not allowed by the use of a single technique. Forster et al. [157] started to simulate the adsorption of an isolated prolate molecule, for which the three low energy structures reported in 
Figure 28a were found. Structures A and B, nearly isoenergetic (see values inFigure 28), possess the highest adsorption energies and are preferred over structure C. Both structures A and B are adsorbed with the two oxygen atoms on neighbouring copper atoms along a close-packed row and the nitrogen atom on a copper atom in the next row, in agreement with the intuitive picture of Figure 26b. We note that, having prolate a three-point bonding interaction with the substrate, the adsorption geometry can be defined in terms of its adsorption footprint. Specifically, the triangular footprints projected by structures $\mathrm{A}$ and $\mathrm{B}$ are chiral and represent non-superimposable mirror images as they are confined in two dimensions and thus unable to flip over. Importantly, the chirality of the adsorption footprint leads to a concomitant reorientation of the pyrrolidine ring. Thus, the more stable structure A, with left-handed triangular footprint, has its ring tilted away from the surface whereas structure $\mathrm{B}$, which describes a right-handed triangular footprint, possesses a flat-lying ring and is less stable by just $30 \mathrm{meV}$.

Conformers A and B can generate eight possible $(4 \times 2)$ overlayers, with a variety of adsorption footprint arrangements. Besides energetic considerations, simulation of STM images for each structural model provideda direct comparison with the experiments and led to one specific structural model. The result, reported in Figure 28b, indicates that the arrangement comprises two homochiral molecules in different conformation, thus having heterochiral adsorption footprints. The stability of the assembly is strengthened by the formation of hydrogen bonds along the $\langle 110\rangle$ direction, which justifies the preferential growth direction of the prolate islands. This behaviour is common to amino acids with tail groups that form strong H-bonds between them (See e.g. Lysine discussed in the previous section). The calculated RAIR spectrum derived from this model is in good agreement with the experimental data (see Table 6).

The identical investigation was repeated using (S)-3-pyrroline-2-carboxylic acid (PCA), a (S)proline derivative obtained by the addition of a double bond within the pyrrolidine ring between the $\beta$ and $\gamma$ carbon positions [197]. This subtle structural alteration is sufficient to promote a $(4 \times 2)$ organization containing a single conformer species, in particular the one with the ring lying flat on the surface (B conformer). This leads to the projection of a truly homochiral footprint assembly, in direct contrast to the heterochiral footprint assembly observed for the $(4 \times 2)$ organization of $(\mathrm{S})$ proline on $\mathrm{Cu}(110)$ [157]. The authors underline the importance of this work in showing how the chirality of a molecule-surface system can be engineered to achieve a complete control of the chirality of the interface by careful consideration of the molecular structure. Considering the focus of the present review, we are more interested in how simple changes of the molecular structure can deeply influence both the anchoring of the molecule and the geometry of the self-assembled layer. The addition of a double bond within the pyrrolidine ring of PCA provided indeed a fourth bonding point for the molecule. Combination of STM results and DFT calculations show that this is sufficient to make the B conformer slightly more stable than the A conformer. As a consequence, the most stable assembly becomes the one in which all molecules have their rings flat on the surface and occupy two distinct orientations rotated by $180^{\circ}$ with respect to each other; this configuration reduces the strain between adjacent carboxylate groups and maximises the H-bonding network.

After clarifying the interaction of enantiopure Pro with $\mathrm{Cu}(110)$, Forster et al. deposited the racemic mixture [196]. Although their focus is mainly on the chiral properties of the compound and on how they are transferred to the surface, the work is of interest since it unravels the details of the (R,S)$\mathrm{Pro} / \mathrm{Cu}(110)$ system at the molecular level. Enantiopure (S)- and (R)-proline can be distinguished in 
STM images since the corresponding A conformers are imaged as bright ovals slanted in mirror directions with respect to the $\langle 1-10\rangle$ axis, and the B conformers appear as fainter but distinct triangles, also slanted in mirror directions. Thus, STM images can provide information both on the molecular chirality and on the adsorption-footprint chirality. Upon (R,S)-proline adsorption on $\mathrm{Cu}(110)$ at $300 \mathrm{~K}$, a pseudo $(4 \times 2)$ adlayer appears. Both conformers $\mathrm{A}$ and $\mathrm{B}$ are present on the surface but, at variance with the very ordered pattern observed for the enantionpure layer, their distribution is random. The novelty of this work was the observation that molecules forming a row in the $<001>$ direction present a random mixture of conformers but all with the protrusions oriented in the same direction. This situation corresponds to a random distribution of enantiomers but all with the same adsorption footprint. For adjacent rows above and below, the protrusions are oriented in the opposite direction, adopting the mirror adsorption footprint. So it was concluded that the adsorption footprints adhere strictly to a specific heterochiral pattern and the arrangement is dictated by the chirality of the adsorption footprint, and not by that of the molecule. This conclusion was corroborated by DFT calculations modelling the racemic $(4 \times 2)$ overlayer. When using only conformers A and B separately (with equal population of R- and S- species), adsorption energies of -1.40 and $-1.36 \mathrm{eV} /$ molecule were found for the $\mathrm{A}$ and $\mathrm{B}$ conformers, respectively. The corresponding value for the enantiopure system in the same footprint arrangement was -1.38 $\mathrm{eV} / \mathrm{molecule}$ [157], showing that there is no energy penalty for accommodating molecules of opposing chirality into the heterochiral footprint template. For the mixed-conformer, the most energetically favourable configuration, also producing a simulated STM image in excellent agreement with the experimental ones, is the one in which the enantiomer is changed but the same orientation of the three bonding points (i.e. the same footprint arrangement) is maintained.

The strict heterochiral adsorption-footprint template governs the organization of the adsorbed racemic mixture, minimizing repulsive interactions and maximizing hydrogen bonding. Each adsorption position in the template can be occupied by either enantiomer, resulting in a $2 \mathrm{D}$ random solid solution at the surface. Given that amino acids crystallize in 3D as racemic compounds, this result highlights that, compared to $3 \mathrm{D}$, there are greater opportunities at surfaces for creating random solid solutions, induced by adsorption effects. This work also suggests that targeted modifications that influence adsorption footprints at surfaces are a vital step towards controlling chiral organization in molecular monolayers.

\subsubsection{Histidine}

The amino acid histidine is characterised by an imidazole (IM) side chain containing two $\mathrm{N}$ atoms with different properties (see Figure 29a): the ring amino nitrogen (N3) is bound to hydrogen and donates its lone pair to the aromatic ring and as such is slightly acidic; the imino nitrogen (N2) donates only one electron pair to the ring, so it has a free lone pair and it is basic. The IM ring can readily switch between various protonated or unprotonated states [147; 148]. Interaction of imidazole with copper at zero oxidation state yields metal imidazolates that passivate the surface [198], which makes imidazole-containing compounds of interest for applications as corrosion inhibitors and as adhesion promoters.

The structures of metal-histidine complexes depending on the pDwere deduced from IR data and revealed the presence of $\mu 1, \mu 2$, and $\mu 3$ HIS complexes depending on the solution acidity [199]. Xray studies in the solid state [200] showed that histidine can use each of the three potential 
coordination sites to bind metal ions. However, the copper-histidine system remains controversial, especially for what concerns the binding mode of histidine to copper(II), and requires a deep analysis of the $\mathrm{Cu}$-HIS interface.

Adsorption of histidine on $\mathrm{Cu}(110)$ at $300 \mathrm{~K}$ was investigated by Marti et al. by means of AES, LEED and RAIRS (see Table 7 for vibrational frequencies and their assignment) [147; 148]. Histidine preferentially binds to the $\mathrm{Cu}(110)$ surface with the carboxylic acid group deprotonated, in the HHis ${ }^{-}$form [147], according to the adsorption model reported in Figure 29a. Adsorption proceeds via the carboxylate group $\left(\mathrm{COO}^{-}\right)$, holding the two oxygen atoms nearly equidistant from the surface, and via the dehydrogenated nitrogen of the imidazole group, which adopts an upright position with respect to the copper surface. The amino group $\left(\mathrm{NH}_{2}\right)$ is maintained close to the surface, facilitating the interaction with the metallic surface. In a second model the ring stays close to the surface but without bonding[148]. Pre-adsorption of oxygen on the copper surface and the consequent formation of a $\mathrm{p}(2 \times 1)$ (See Figure 29b) or of a $\mathrm{c}(6 \times 2)$ superstructure forces a readjustment of the molecular orientation. More interesting, the $\mathrm{NH}$ group of the heterocycle interacts with the $\mathrm{Cu}-\mathrm{O}$ row of the $\mathrm{p}(2 \times 1)-\mathrm{O}-\mathrm{Cu}-(110)$ reconstructed substrate. To help in the interpretation of the infrared spectra and find some information on the adsorbed molecular geometry, the authors used a molecular force field calculation to estimate the different vibrational frequencies and the orientation of HHis ${ }^{-}$adsorbedon the solid surface with preadsorbed oxygen. An embedded cluster approach was chosen andthe MMFF calculation led to an equilibrium geometry in which the attractive force between the negatively charged carboxylate group and the copper surface governed the molecular adsorption process. On the $\mathrm{Cu}-(110)-\mathrm{O}-\mathrm{p}(2 \times 1)$ surface, the carboxylate group of the HHisentity likely interacts with Lewis cationic sites located between $\mathrm{Cu}-\mathrm{O}$ rows in the $<001>$ direction (bidentate form), the COOplane being nearly parallel to this direction in agreement with the experimental results (b). The distance between the carboxylate oxygen atoms and the nearest positively charged copper ion is about $0.26 \mathrm{~nm}$. The computed vibration frequencies nicely reproduce the experimental IRAS spectrum (c).

A few years later, Feyer et al. [46] revisited the same system by HR-XPS and NEXAFS, investigating a wider range of temperatures. They concluded that, at high coverage ( $\Theta \sim 1.5 \mathrm{ML})$, the IM side chains of the histidine molecules are randomly oriented. The molecules bind to the copper surface via the carboxylate group, and the IM ring lies close to the surface. This deduction is supported by the observation in XPS spectra of a single $\mathrm{O}$ 1s peak and of features in the $\mathrm{N}$ 1s region due to two amino and imino nitrogen atoms (seeTable 3). Upon annealing to $375 \mathrm{~K}$, desorption of molecules exceeding $1 \mathrm{ML}$ occurs. The conformation of the amino acid units remaining on the surface changes a little; in particular the IM ring gets tilted close to the surface and the N3 nitrogen becomes deprotonated. Further annealing of the sample to $425 \mathrm{~K}$ causes the appearance of a new feature in the low $E_{b}$ side of the $N$ 1s spectrum, indicating the strong interaction of the IM ring with the copper surface via the imino nitrogen atom (N2).

At low coverage and room temperature the model is essentially coherent with the one of Marti et al. [147]. HIS interacts with the $\mathrm{Cu}(110)$ surface only via the carboxylate group and ring imino (N2) nitrogen atoms with a small tilt angle of the IM ring with respect to the surface. After annealing to $425 \mathrm{~K}$ the $\mathrm{N} 3$ nitrogen loses an $\mathrm{H}$ atom, and both nitrogen atoms of the IM ring (N2 and N3) and the nitrogen atom of the amino group (N1) bind to the surface covalently. The carboxylate group $\left(\mathrm{COO}^{-}\right)$forms a strong ionic bond to the $\mathrm{Cu}(110)$ surface, thus confirming that histidine binds to the 
surface mainly in its HHis ${ }^{-}$ionic form. This study also completes the picture of His adsorption at $p(2 \times 1)-\mathrm{O}-\mathrm{Cu}(110)$ provided by RAIRS studies [147; 148]. XPS and NEXAFS analysis suggests indeed that, on thissurface, the N2 nitrogen and carboxylate group are involved in the interaction; in addition pre-adsorbed oxygen reacts with $\mathrm{H}$ from histidine, which then also binds to the surface via the dehydrogenated N3 nitrogen atom, with the IM ring tilted to a more upright orientation.

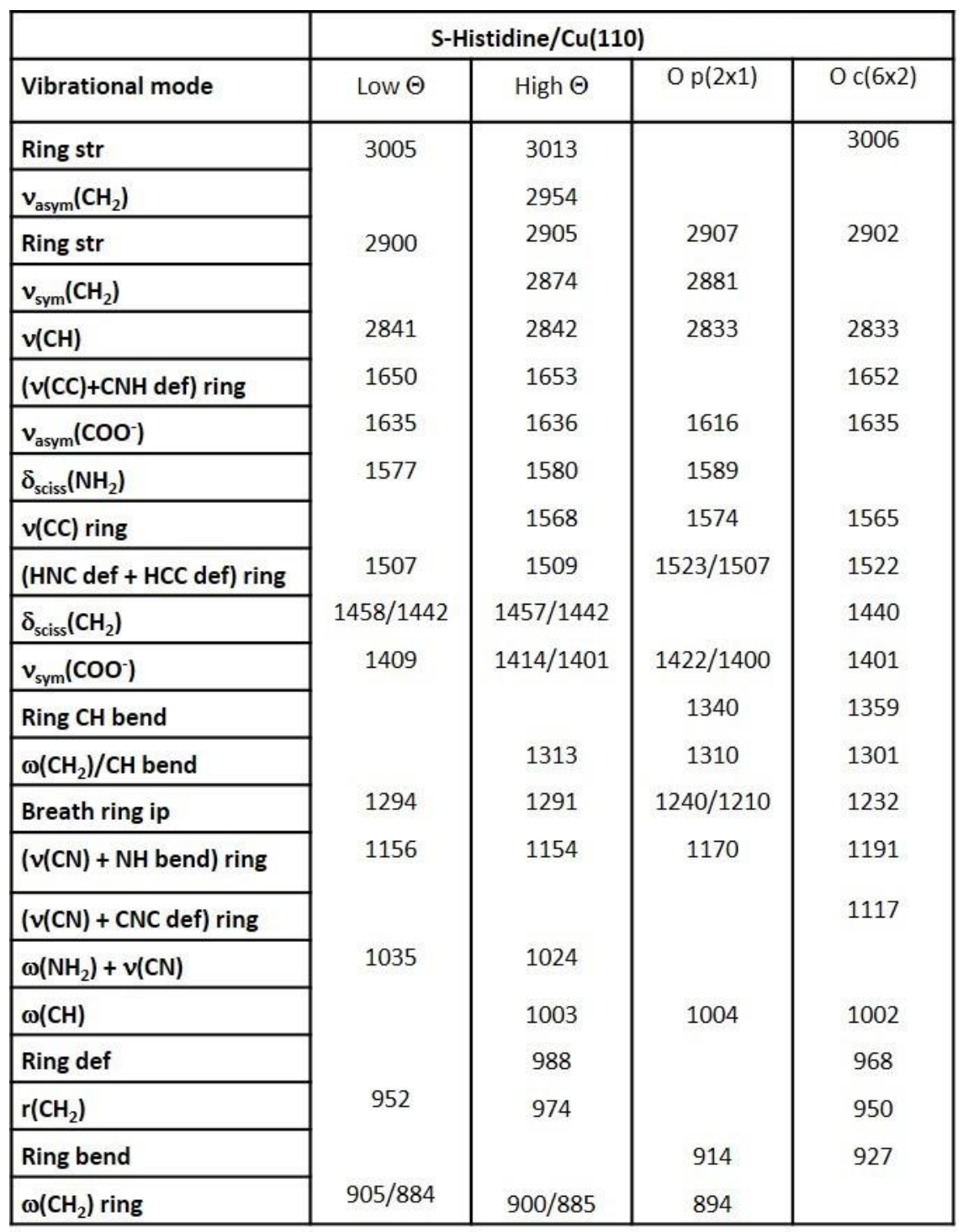

Table 7. Characteristic Vibrational Bands and relative assignments for HIS Adsorbed on $\mathrm{Cu}(110)$, $\mathrm{Cu}(110)-\mathrm{O} \mathrm{p}(2 \times 1)$ and $\mathrm{Cu}(110)-\mathrm{O} \mathrm{c}(6 \times 2)$. Taken with permission from ref. [147] 
a)

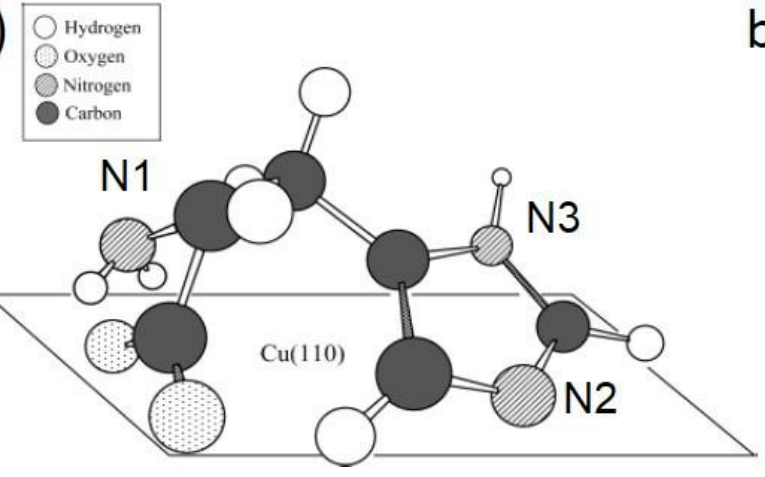

b)

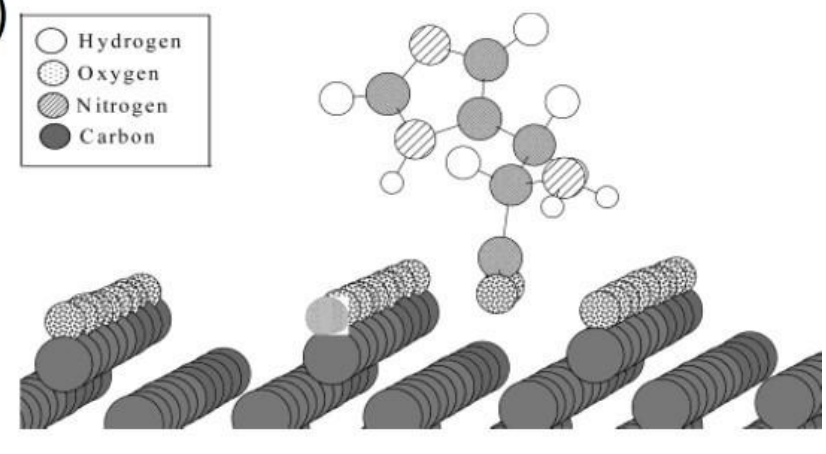

c)

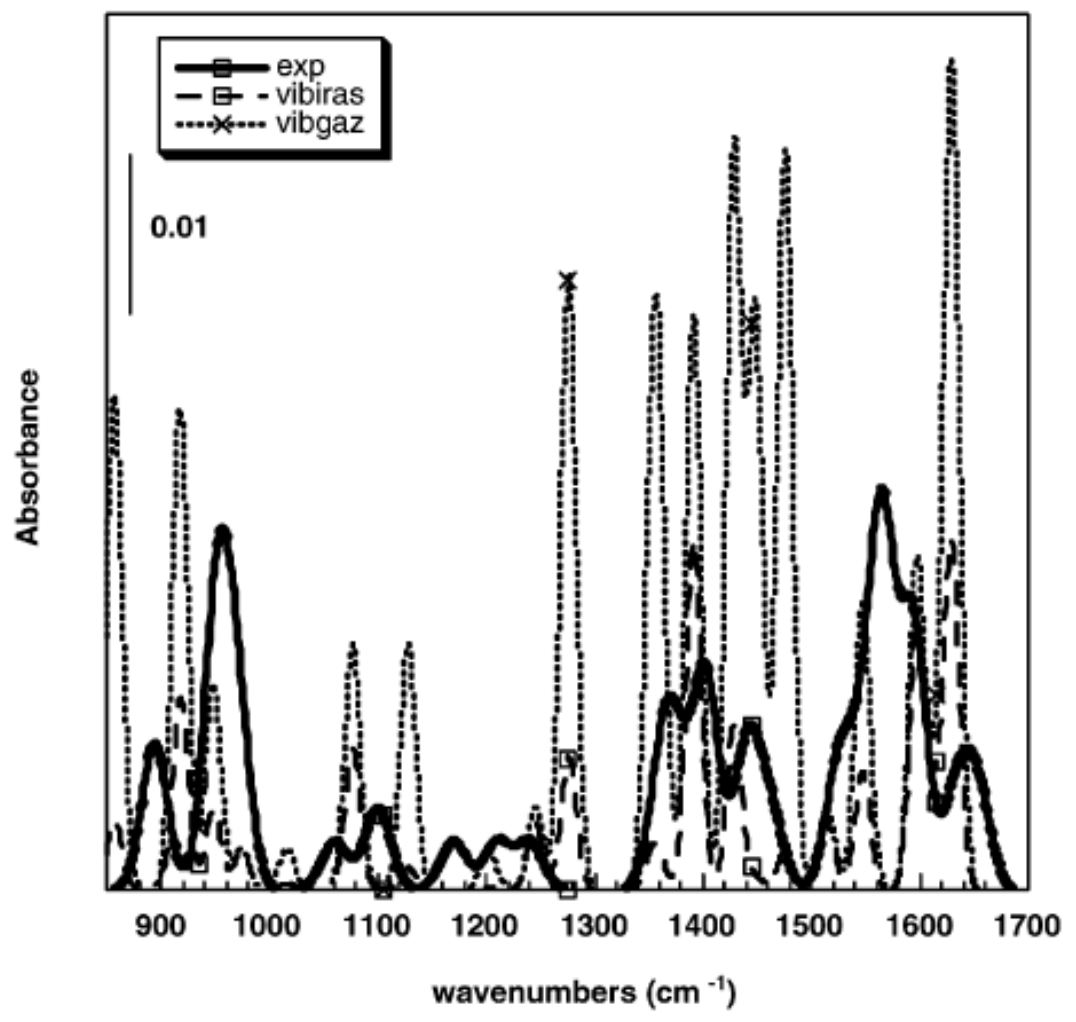

Figure 29 Orientation adopted by (S)-histidine adsorbed on: a) $\mathrm{Cu}(110)$; b) $\mathrm{Cu}(110)-\mathrm{O} p(2 \times 1)$ c) comparison of experimental and computed spectra with (vibiras) and without (vibgaz) surface selection rules.Taken with permission from ref. [147].

\subsubsection{Tryptophan.}

Tryptophan is the largest amino acid (204 amu), since its side group consists of a methylene group $\left(-\mathrm{CH}_{2}-\right)$ and a large active indole group $\left(-\mathrm{C}_{8} \mathrm{H}_{6} \mathrm{~N}\right)$. Probably for this reason, specific studies on this molecule are very few, especially under controlled conditions. Its adsorption on $\mathrm{Cu}(001)$ was described by Zhao et al. [161] in the frame of the extensive STM investigation on the interaction of seven AA species on this surface $[95 ; 98 ; 139 ; 150 ; 201]$.

Figure 30 shows STM images obtained after L-Tryptophan deposition on $\mathrm{Cu}(001)$ at RT and after subsequent annealing to $450 \mathrm{~K}$. At a first glance, it is evident that this molecule organizes in a much 
richer zoology of superstuctures with respect to smaller amino acids. After $1 \mathrm{~L}$ of exposure little diffusion occurs and individual adsorbed molecules randomly aggregate into small clusters (Figure 30a). At medium coverage almost the entire surface is covered by the adsorbate and some small domains of ordered structures appear (see Figure 30b). This behaviour is at variance with the one previously reported for smaller AA as Gly, Ala, Phe and Lys, which initially forms a 2D gas phase and then organizes into short rows through $\mathrm{H}$-bonds at intermediate coverage [98; 181]. Annealing the surface to $450 \mathrm{~K}$ greatly improves the order of the overlayer. As evident in Figure 30c, at this stage domains with $(77,-77)$ and $(64,-54)$ superstructure coexist. If the initial dose increases slightly, the latter geometry prevails. At saturation coverage, the denser $(4 \times 4)$ and $(8-1,-12)$ superstructures form (see Figure $30 \mathrm{~d}$ and e). In addition, the smallest terraces, especially those neighbouring to faceted steps, are often occupied by molecules arranged in a $(40,12)$ geometry. Indeed annealing may cause non-chiral faceting in the <310> directions, as in the case of Gly[98] and Ala.

The high coverage superstructures persist even after annealing to $620 \mathrm{~K}$ for $10 \mathrm{~min}$, which allowed the authors to deduce a minimum adsorption energy of $-1.9 \mathrm{eV} /$ molecule, well suited for anionic adsorption. Furthermore, the desorption temperature of adsorbed L-tryptophan,at least $65 \mathrm{~K}$ higher than the decomposition point of the molecule in a solid crystal,suggests that the thermal stability of tryptophan is enhanced by adsorption on $\mathrm{Cu}(001)$.

As pointed out by the authors, it is not easy to determine the adsorption geometry of a rather complex molecule only on the basis of STM images. The (7 7, -7 7), (6 4, -5 4), (4×4), (4 0, 12 ), and $(8-1,-12)$ unit cells are found to consist of eight, four, two, one, and two molecules, respectively, equivalent to a surface density of one Try unit every 12.25, 11.0, 8.0, 8.0, and 7.5 Cu atoms, respectively. Empirical models are proposed for each structure, based on the consideration that: i) the $\mu 3$ adsorption geometry of the amino-acid groupholds, but also the indole group is attached to the surface; ii) the superstructures are stabilised by intermolecular hydrogen bonds and, at high coverage, by $\pi$-stacking between the indole groups of neighbouring molecules [161]. 

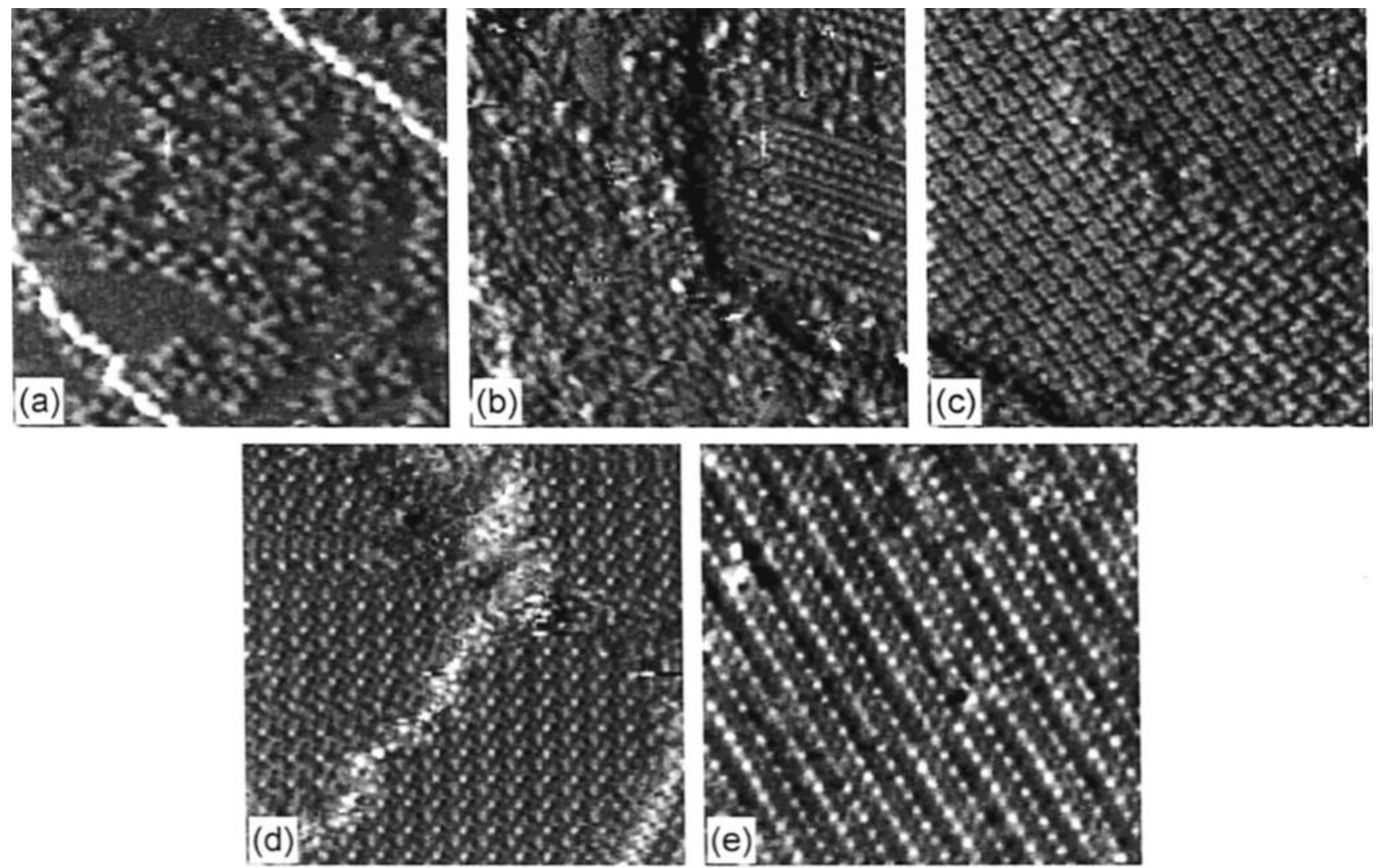

Figure 30. STM images of the L-tryptophan/Cu(001) surface: (a) after exposure to $1 \mathrm{~L}$ of tryptophan at RT and annealing at $450 \mathrm{~K}$ (image size: $200 \AA \times 200 \AA, \mathrm{V}=1.0 \mathrm{~V}, \mathrm{I}=0.6 \mathrm{nA}$ ). (b) Obtained from the as-deposited surface with $5 \mathrm{~L}$ of tryptophan exposure (image size: $270 \AA 270 \AA$, $\mathrm{V}=-2.5 \mathrm{~V}, \mathrm{I}=0.6 \mathrm{nA})$. (c) Same surface as in (b), but after annealing at $450 \mathrm{~K}$. The $(77,-7$ 7) superstructure (the lower-right portion) and the $(64,-54)$ one (rest of the image) coexist (image size: $270 \AA \times 270 \AA, \mathrm{V}=1.0 \mathrm{~V}, \mathrm{I}=0.6 \mathrm{nA})$. (d) $(4 \times 4)$ superstructure imaged for the surface with high Trycoverage and after annealing at $450 \mathrm{~K}$ (image size: $140 \AA \times 140 \AA, \mathrm{V}=1.5 \mathrm{~V}, \mathrm{I}=0.4 \mathrm{nA})$. (e) (8 -1, -1 2) superstructure imaged from the same surface as in (d) (image size: $155 \AA \times 155 \AA, \mathrm{V}=1.0 \mathrm{~V}$, $\mathrm{I}=0.4 \mathrm{nA})$. Taken with permission from ref. [161].

\subsubsection{Amino acid adsorption on stepped and chiral $\mathrm{Cu}$ surfaces.}

Chirality at surfaces has become a strong focus within the surface science community. A particular motivation is the prospect of using heterogeneous catalysis over chiral solid surfaces for asymmetric synthesis, which is of clear relevance to the pharmaceutical industry. Small amino acids adsorbed on metal surfaces have emerged as important model systems for investigations at the fundamental level. In this respect, chirality can be introduced into the system at different levels:

i) By the adsorption of a chiral modifier on an achiral surface.

ii) By step faceting and bunching of LMI surfaces upon amino acid adsorption.

iii) By using intrinsically chiral metal surfaces.

A few words on point i) have already been spent discussing the $\mathrm{Pro} / \mathrm{Cu}(110)$ experiments and we will deal with it in more details in section3.3.2[168-170; 202]. Points ii) and iii), on the contrary, have been investigated mainly on $\mathrm{Cu}$ surfaces. 


\subsubsection{Step faceting and bunching of LMI surfaces upon amino acid adsorption.}

Adsorbate-induced step faceting and bunching was observed for $\mathrm{Gly} / \mathrm{Cu}(001)$ (see Figure 12c), $\mathrm{Ala} / \mathrm{Cu}(001)$ and other $\mathrm{AA} / \mathrm{Cu}(001)$ at room temperature by Zhao et al. [95; 98; 139; 201]. A similar mechanism is proposed for Gly and Ala: the formation of $\mathrm{H}$-bond chains parallel to the steps is suggested to be the driving mechanism for the step faceting in the $<310>$ directions and for the bunching of highly stepped regions to form $\left(\begin{array}{lll}3 & 1 & 17\end{array}\right)$ nanofacets. Both amino acids form a $(1 \mathrm{x} 1)$ overlayer on the (3 1117$)$ nanofacets.

The behaviour of lysine is, on the contrary, markedly different. First, L-lysine molecules do not induce the step reconstruction at RT but annealing to $430 \mathrm{~K}$ is required. At this temperature faceting and bunching of all steps occurs, to form very regular (3 1117 ) facets covered by a $(4 \times 1)$-L-lysine overlayer (see Figure 25e). A more interesting peculiarity is related to the geometry of the reconstructed facets. Indeed the symmetry of the (001) surface of face-centred-cubic copper is $C 4 \mathrm{v}$, so that eight equivalent $\langle 310\rangle$ directions and eight equivalent (3 11 17) vicinal surfaces around the (001) surface exist. They can be divided into two groups of opposite chirality, each one including four (3 117 ) vicinal surfaces rotationally equivalent relative to the (001) normal direction. Upon LLys adsorption, only the $R$-( $\left.\begin{array}{lll}3 & 1 & 17\end{array}\right)$ facets emerge, which clearly indicates that adsorption of Llysine induces chiral restructuring of steps on $\mathrm{Cu}(001)$. From this phenomenon, one may reasonably assume that there exists a difference in adsorption energies of L-lysine on bare (3 117 )- $R$ and $-S$ facets, which has important implications for the use of these substrates in enantio-selective processes.

While it may be obvious that the chiral restructuring of the steps does not occur with the achiral amino acid glycine, it is less evident why the same phenomenon is not observed with Ala, Phe or Try. This can be well understood on the basis of the "three-point interaction model" [203], which states that at least three side groups of the chiral centre must interact with the receptor for successful chiral recognition. Only two anchoring points are present for alanine (amino and carboxylate groups), while a third one is available for lysine, which has an additional functional group in the side chain. The ring-structure of the radical group of Phe and Try, evidently, does not provide a third anchoring point either.

Since no or very little experimental information about the geometry of the molecules on these chiral facets is available, DFT calculations of the structure of glycine and alanine adlayers on $\mathrm{Cu}(3 \quad 117)$ were performed. At moderate coverage $(\Theta \sim 1 / 2$ ML)[146] - as it might be expected - molecules prefer to bind at the step edges rather than on (100)-oriented terraces (see Figure 31). The adsorption of enantiopure alanine on $\mathrm{Cu}(3 \mathrm{l} 17)$ is predicted to be weakly enantiospecific, with $\mathrm{S}$ Ala being more stableby $0.03 \mathrm{eV}$ than R-Ala on $\mathrm{Cu}(3 \quad 1 \quad 17)-S$. The authors compared surface energies of the unreconstructed and reconstructed surfaces before and after adsorption. The bare

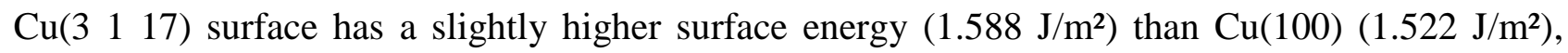
being thus less stable.This order is reversed by adsorption : the surface energy difference between $\mathrm{Cu}\left(\begin{array}{lll}3 & 1 & 17\end{array}\right)$ and $\mathrm{Cu}(100)$ after Gly adsorption is calculated between -0.017 and $-0.047 \mathrm{~J} / \mathrm{m}^{2}$. This means thatthe reconstruction of the $\mathrm{Cu}(100)$ surface to form $\mathrm{Cu}(31$ 17) facets in the presence of Gly decreases the surface energy, but only by a very small amount. This result suggests that, at equilibrium, an annealed $\mathrm{Cu}(100)$ surface likely contains a mixture of (3 11 17) facets and (100) terraces.Using the same method, surface energy differencesassociated to reconstruction, were 
estimatedbetween -0.015 and $-0.045 \mathrm{~J} / \mathrm{m}^{2}$ for $\mathrm{S}-\mathrm{Ala} / \mathrm{Cu}(3 \quad 1 \quad 17)-S$ or $\mathrm{R}-\mathrm{Ala} / \mathrm{Cu}(3 \quad 117)-R$, and between-0.004 and $-0.034 \mathrm{~J} / \mathrm{m}^{2}$ for $\mathrm{R}-\mathrm{Ala} / \mathrm{Cu}(3 \quad 117)-S$ or $S$-Ala/Cu(3 117 )- $R$. These values predict that if enantiopure $S$-alanine is adsorbed on $\mathrm{Cu}(100)$, the annealed surface will exhibit facets of both $\mathrm{Cu}(3$ 1 17)-Rand $\mathrm{Cu}(3 \quad 117)-S$, the latterin slightly greater abundance than the former ones.

For dense glycine and alanine adlayers( $\Theta=1 \mathrm{ML})$ on $\mathrm{Cu}(3 \quad 117)-S[204]$, an exhaustive comparison of different adlayers was performed. For glycine, one adlayer was found $0.1 \mathrm{eV} /$ cell more stable than all others.These calculations show that the appearance of $\mathrm{Cu}(3,1,17)-\mathrm{S}$ facets on $\mathrm{Cu}(100)$ is quite energetically favourable when a dense adlayer of Gly is present on the surface (with a gain of surface energy of $0.1 \mathrm{~J} / \mathrm{m}^{2}$ )[204].For enantiopure alanine, faceting is also confirmed with a gain of surface energy of $0.06 \mathrm{~J} / \mathrm{m}^{2}$ as compared to $\mathrm{Cu}(100)$. The authors have also considered the adsorption of racemic alanine on the same surface. Although the most stable structure identified relevant to this situation involves enantiopure regions of alanine on the $\mathrm{Cu}(3,1,17)-S$ and $\mathrm{Cu}(3,1,17)-R$ facets, other structures with only slightly less favourable energies $\left(0.03 \mathrm{~J} / \mathrm{m}^{2}\right)$ exist on the same facets with racemic distributions of the adsorbates. Thus, there does not appear to be a strong driving force for the spontaneous segregation of adsorbed enantiomers bysurface faceting when racemic alanine is adsorbed on $\mathrm{Cu}(100)$. The overall stability of glycine and alanine adlayers on $\mathrm{Cu}(3 \mathrm{l} 17)-S$ is confirmed to arise from an interplay between the formation of chemical bonds with the $\mathrm{Cu}$ surface, deformations in the adsorbed molecules during adsorption, and intermolecular hydrogen bonds within the adlayer; none of these factors were found to individually dominate.

The adsorbed Gly molecules have a strong energetic preference $(0.4 \mathrm{eV} / \mathrm{molecule})$ to adsorb at the step edge. For Ala, all local minima were found to lie within $0.2 \mathrm{eV} / \mathrm{molecule}$ with respect to the most stable state. For the most energetically favoured configuration of S-Ala (R-Ala) on $\mathrm{Cu}$ (3 1 17)-S, a N-Cu bond length of 2.07 (2.06) $\AA$ and $\mathrm{O}-\mathrm{Cu}$ bond lengths of 2.06 (2.07) and 2.02 (2.01) $\AA$ were found. For S-Ala, the calculated NCC bond angle is $106.8^{\circ}$, whereas for R-Ala it is $105.6^{\circ}$. These values support that the footprint of the two enantiomers of alanine on this surface are extremely similar. It should be noted that the S-Ala and R-Ala have almost similar energies, which are too close to each other to discriminate between the two enantiomers. 


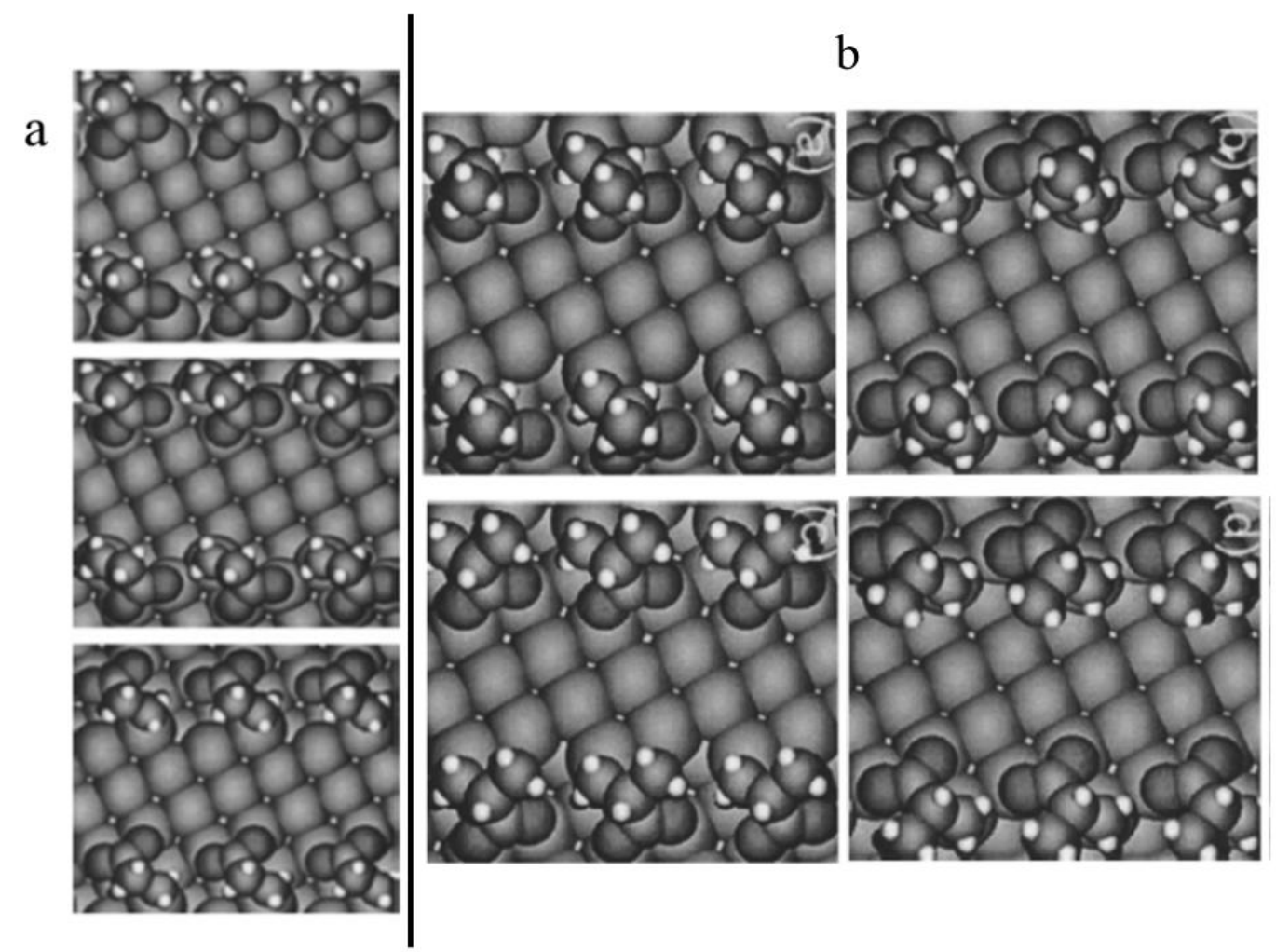

Figure 31. a) The three most stable bonding configurations of Gly on $\mathrm{Cu}(3 \quad 117)-S$. b) The two most stable configurations for S-Ala (top) and R-Ala (bottom) on $\mathrm{Cu}(3117)-S$. Taken with permission from refs. [146].

\subsubsection{Amino acid adsorption at intrinsically chiral metal surfaces.}

It is well reported in literature that chiral molecules adsorbed at intrinsically chiral single-metal surfaces may show significant enantiomeric differences in the adsorption geometry and in the desorption behaviour $[187 ; 205 ; 206]$.

Surfaces of face-centred cubic metals, such as $\mathrm{Pt}, \mathrm{Ni}$, or $\mathrm{Cu}$, are chiral when the Miller indices are different from each other and from zero. Being HMI surface, for their very nature they consist in a sequence of nanofacets of different geometry, which may deeply influence the adsorption configuration of the molecules. Among all possibilities, (531) is the fcc surface with the smallest surface unit cell that fulfils this condition, and it is thus among the most used for this kind of fundamental studies. All chiral fcc single-crystal surfaces have 6-fold coordinated kink atoms, which are surrounded by (111), (100), (110)nanofacets. In analogy to the Cahn-Ingold-Prelog rules, the surfaces are termed R/D or S/L if the $(111) \rightarrow(100) \rightarrow(110)$ series describes a clockwise or counter clockwise rotation, respectively [207].

The adsorption of glycine and of both enantiomers of alanine on the chiral $\mathrm{Cu}(531)-R$ surface was investigated by the group of Held by means of LEED, XPS, and NEXAFS [112; 143]. The comparison of the only non-chiral amino acid with the simplest chiral one allows clarifying the adsorption behaviour on a chiral substrate. In both cases, after deposition of a full monolayer at 300 $\mathrm{K}$ and further annealing, a regular $\mathrm{p}(\mathrm{n} \times 1)$ LEED pattern is observed ( $\mathrm{n}=4$ for Ala, $\mathrm{n}=6$ for Gly), which is dissolved within tens of seconds by the electron beam. 
XPS analysis reveals that both species adsorb in the deprotonated form. The chemical shifts of the $\mathrm{C} 1 \mathrm{~s}, \mathrm{~N}$ 1s and $\mathrm{O}$ 1s core level signals are essentially the same as for glycinate and alaninate on $\mathrm{Cu}(110)$ at low coverage (see Table 3), which suggests that the molecules assume the same $\mu 3$ adsorption geometry. Furthermore, for alanine, a difference in the O1s intensities for the (S)- and (R)- enantiomers (ascribed to photoelectron diffraction effects) suggests a difference in the adsorption conformation, which is confirmed by the NEXAFS results summarised in Figure 32a. In contrast to $\mathrm{Cu}(110)$, NEXAFS spectra of Gly and Ala on $\mathrm{Cu}(531)-R$ show only limited intensity variations of the $\pi$-resonance, with minima well above the zero level. This behaviour is indicative that the signal arises from molecules with at least two different azimuthal orientations. Although for different reasons, it is likely that both the Gly- $p(1 \times 6)$ and the Ala-p $(1 \times 4)$ overlayers contain two non-equivalent molecules per unit cell. DFT model calculations for glycinate strongly favour the $\mu 3$ adsorption model common to many AA at LMI surfaces; in this case, for both the (110) and the (311) nanofacets of the $\mathrm{Cu}$ (531) surface, two atoms of the first $\mathrm{Cu}$ layer (kink atoms) and one of the second $\mathrm{Cu}$ layer are involved in the adsorbate bonds. The adsorption geometry deduced on these theoretical premises (schematically shown for alanine in Figure 32b) allows all first layer $\mathrm{Cu}$ atoms of the surface to participate in the adsorbate bond and the molecules on (110) nanofacets to form strong hydrogen bonds between their amine groups and the oxygen atoms of neighbouring molecules.

For Ala the picture is complicated by the chiral nature of the molecule. NEXAFS spectra indicate distinct changes in the angular intensity between the $R$ and the $S$ enantiomer (see Figure 32a). This is interpreted as an indication that both triangular adsorption sites on the (311) ( $\alpha 1)$ and (110) $(\alpha 2)$ nanofacets of the $\mathrm{Cu}(531)$ surface are occupied independently of their chirality but that a change in the adsorption configuration occurs, induced by steric interaction of the methyl group with the metal atoms. According to the authors, the more plausible scenario is that the adsorption configuration remains unchanged at (311) nanofacets but (R)-Ala molecules are significantly less distorted than (S)-Ala ones at (111) nanofacets. As a consequence, (R)-Ala shows preferential adsorption at (111) sites, which causes a reduction of the long range order.

We mention that the $\mathrm{Gly} / \mathrm{Cu}(531)$ system does not show a coverage dependent behaviour, since also at $\Theta=0.44 \Theta_{\text {sat }}$ adsorption occurs at (111) and (311) nanofacets in equal amount [112]. At variance, NEXAFS spectra recorded for a dilute Ala layers $\left(\Theta=0.55 \Theta_{\text {sat }}\right)$ show a significant change in the angular dependence [187], which is now common to the (S)- and (R)- enantiomers. Careful analysis allows to conclude that, on the (311) facets, molecules, rotated by $\sim 20^{\circ}$ with respect to the adsorption geometry at saturation coverage, are preferred. This behaviour, consequence of the missing intermolecular interactions, allows drawing the following important conclusions: a) The isolated molecules have higher adsorption energy on the (311) facets, where alanine can adsorb without major lateral distortions. b) The adsorption energy difference between (311) and (110) must be higher than possible energy gains from the formation of hydrogen bonds with molecules on the (110) adsorption site. c) The enantiospecific adsorption behaviour at higher coverage is induced by (repulsive or attractive) intermolecular interactions between molecules on (311) and (110) sites or by repulsive molecule-substrate interactions on the (110) adsorption sites. The most important type of inter-molecular interactions are hydrogen bonds, while repulsive interactions are most likely related to the methyl group. 
Since the same basic triangular adsorption complex is adopted for $\mathrm{Gly} / \mathrm{Cu}(531)$ and for $\mathrm{Ala} / \mathrm{Cu}(531)$, the different behaviour at low coverage must be due to the methyl side group of alaninate. This effect must be similar in magnitude to the hydrogen bond energy.
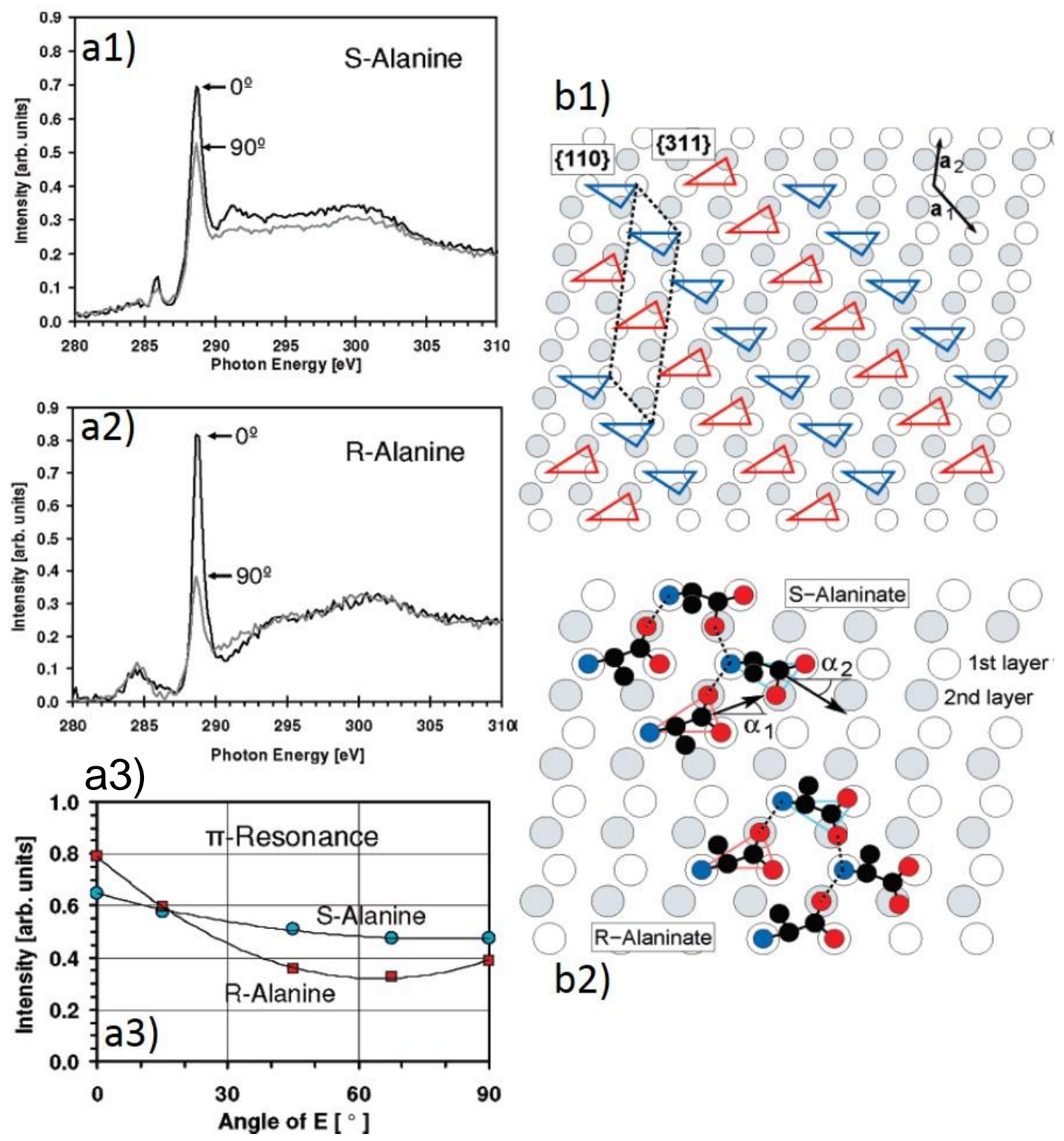

Figure 32. a1-a2) Normalized carbon K-edge NEXAFS spectra recorded with horizontal $\left(0^{\circ}\right)$ and vertical polarization $\left(90^{\circ}\right)$ of $\vec{E}$ for $(\mathrm{S})$ - and (R)-alanine on $\mathrm{Cu}(531)-R$. (a3) Angular variation of the $\pi$-resonance for (R)- and (S)-alanine.b1) Long-range arrangement of molecules on (311) and (110) nanofacets in the $\mathrm{p}(1 \times 4)$ superstructure of alaninate on $\mathrm{Cu}(531)-R$. For clarity the molecules are represented by their triangular footprints and only the first and second layer atoms of the substrate are shown as open and shaded circles, respectively; the dotted parallelogram indicates the unit cell. (b2) Geometry model for the adsorption of (R)- and (S)-alaninate on $\mathrm{Cu}(531)-R$. The dotted lines indicate hydrogen bonds; hydrogen atoms are omitted for clarity. The $\alpha 1$ and $\alpha 2$ adsorption sites are marked by the red and blue triangles, respectively. Taken with permission from ref. [143]. 
The natural prosecution of the above research is the investigation of the Ala/ $\mathrm{Cu}(421)$ system [145]. $\mathrm{Cu}(421)$ has (311) and (110)nanofacets identical to those of $\mathrm{Cu}(531)$, but the unit cell is one atom longer in the $\langle-110\rangle$ direction. Hence the surface kink-site density is lower and the chiral adsorption sites are further apart. This generates a slightly larger distance between adsorbed molecules and substantially decreases the intermolecular interactions on the surface. In this way, it is possible to target the contribution of intermolecular bonding and adsorption site interaction to enantioselectivity by comparison of the two chiral surfaces.

Thomsen et al. [145] demonstrated that the adsorption of alanine on $\mathrm{Cu}(421)$ shows a considerable decrease in enantioselectivity compared to $\mathrm{Cu}(531)$. This is deduced from the $\mathrm{C}=\mathrm{O} \pi^{*}$ transition in carbon K-edge NEXAFS spectra, which are very similar for both enantiomers and indicate that (311) and (110) nanofacets are populated in similar amount. In spite of that, XPS reveals differences in the thermal stability and decomposition products of the (R)- and (S)-alanine on the $\mathrm{Cu}(421)-R$ surface and LEED shows a different long range order, with (R)- and (S)- enantiomers self-assembling in a $\mathrm{p}(2 \times 3)$ and in a $\mathrm{p}(1 \times 2)$ overlayer, respectively.

The fact that the angular dependence of the $\pi^{*}$ resonance between the two enantiomeric species is similar even though they generate such dramatically different LEED images on the surface is not totally surprising. Indeed, NEXAFS allows to determine the local adsorption geometry, which must therefore be very similar for both enantiomers. It is reasonably proposed that it consists in the three binding points geometry by analogy with the $\mathrm{Ala} / \mathrm{Cu}(531)$ system and with several AA on $\mathrm{LMI} \mathrm{Cu}$ faces. LEED on the contrary, provides information on the long range order, which is related to the way in which adsorption sites are distributed on the surface. By comparison of results on $\mathrm{Cu}(421)$ and $\mathrm{Cu}(531)$, it is concluded that the intermolecular hydrogen bonding in conjunction with steric effects play a significant role in determining the stereochemistry on these surfaces.

Cysteine and methionine differ from alanine in having the methyl termination replaced with a Scontaining group. Thomsen et al. [115] repeated the XPS and NEXAFS analysis performed on Ala for $\mathrm{Cys} / \mathrm{Cu}(531)$ and $\mathrm{Met} / \mathrm{Cu}(531)$. Careful analysis of the photoemission spectra (see Table 3) suggests that in both cases the local chemical environment of $\mathrm{C}, \mathrm{O}$ and $\mathrm{N}$ atoms is very similar to the one of Ala. Furthermore, the presence of a single O1s peak indicates adsorption though a deprotonated carboxylic group, while the core level shifts of the S2p lines of L-Met and L-Cys indicate that the S-atom interacts directly with the surface only in the latter case. Additional analysis of the angular dependence of the $\mathrm{C} 1 \mathrm{~s} \pi^{*}$ resonance leads to the following proposed picture: both Met and Cys adsorb on both (110) and (311) nanofacets of the $\mathrm{Cu}(531)$ surface with a triangular footprint similar to the one of alanine. In addition, Cys has a fourth binding point at the $\mathrm{S}$ atom, while in the case of methionine the radical is protruding towards the vacuum.

Synchrotron-based structural techniques where applied also to the study of serine adsorbed on the pure and Au modified $\mathrm{Cu}(531)$ surface [144]. Combination of LEED, XPS, NEXAFS and DFT results shows that: i) Enantiomeric differences between (D)-Ser and (L)-Ser exist in both cases. The observed LEED pattern depends indeed on the chirality of both the surface and the molecule, although at saturation coverage of $0.5 \mathrm{ML}$ of serine is common to the D- and L- species. ii) Serine adsorbs on the pure surface with the dehydrogenated carboxyl group, in a $\mu 4$ conformation (with de-protonated $\beta$-OH group) at low coverage and in a $\mu 3$ conformation (with the $\mathrm{OH}$ group not interacting with the surface) at saturation coverage. The change of adsorption geometry above a critical coverage of $\sim 0.3 \mathrm{ML}$ is evident from the O1s spectra reported in Figure 33. The main peak 
is always observed around $531.4 \mathrm{eV}$, characteristic of oxygen atoms of the carboxylate group[43; $141 ; 143]$. However, the O1s component related to the hydroxyl termination is found at $\mathrm{E}_{\mathrm{b}}=530.8$ $\mathrm{eV}$ at low coverage and at $\mathrm{E}_{\mathrm{b}}=532.8 \mathrm{eV}$ close to saturation coverage. Such a shift is related to a change in the hydroxyl chemical environment: for $\Theta<0.3 \mathrm{ML}$, this oxygen atom is bound to the substrate, resulting in an overall $\mu 4$ configuration. When $\Theta$ approaches saturation, the $\beta-\mathrm{OH}$ group is detached from the surface, which leads to a $\mu 3$ binding mode. The transition occurs for both enantiomers at similar coverage and is driven by the fact that the $\mu 3$ substrate bond has a smaller footprint than the $\mu 4$ bond, and hence the surface can accommodate more molecules [208]. iii) Similarly to the case of $\mathrm{Ala} / \mathrm{Cu}(531)$ [143], both (110) and (311) nanofacets are populated, but in different relative amount and with different orientation, depending on the chirality of the molecule. DFTmodel calculations for the different possible configurations confirm that the $\mu 4$ geometry (reported in Figure 34) has de-protonated $\beta-\mathrm{OH}$ and carboxylate groups, while the $\beta$-OH group is intact in the $\mu 3$ geometry. The simulation shows thus perfect agreement with the NEXAFS results.
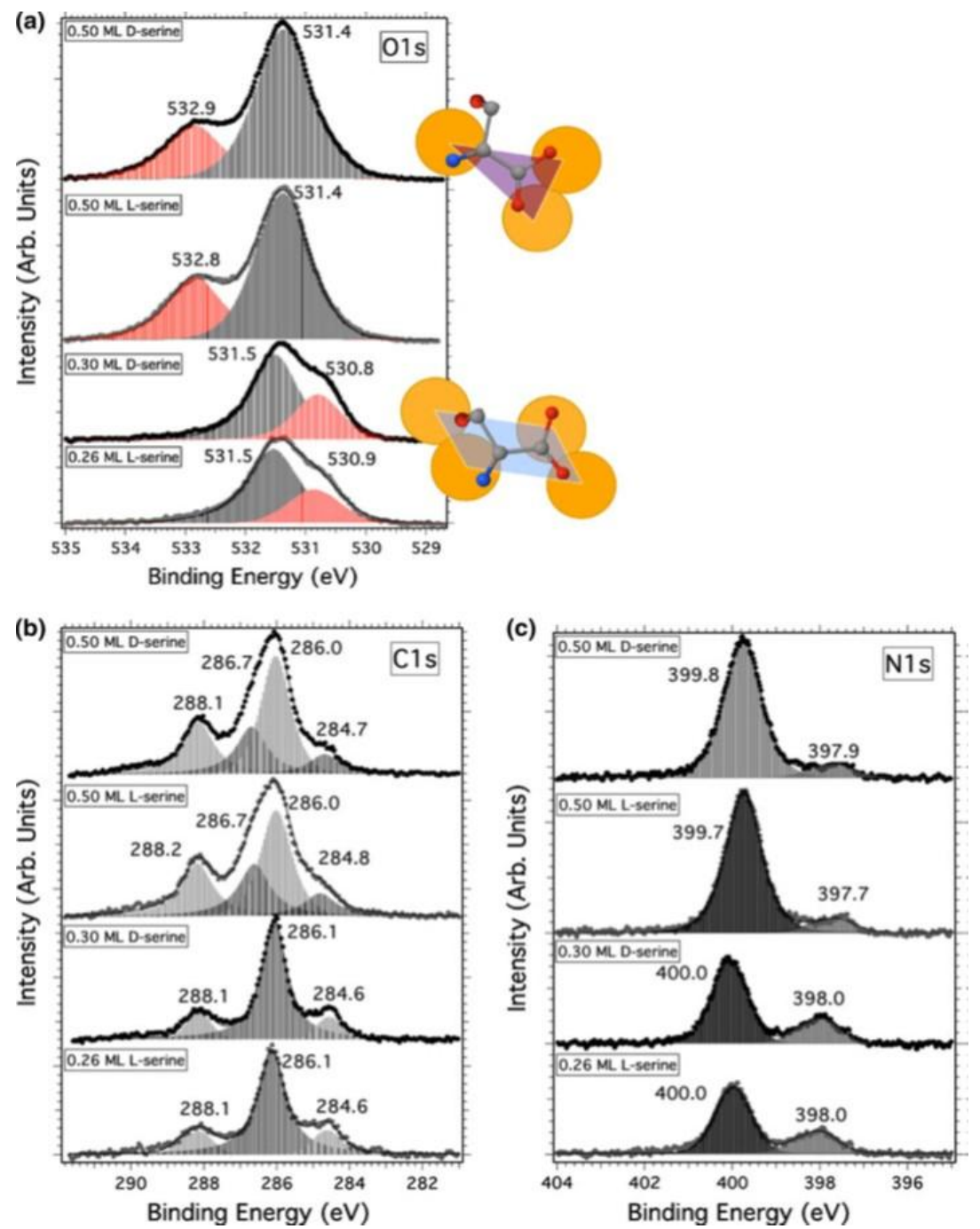

Figure 33. O1s (a), C1s (b) and N1s (c) photoemission spectra of different coverages of L- and Dserine adsorbed on the clean $\mathrm{Cu}(531)-S$ (photon energy $=630 \mathrm{eV}$ ). Panel (a) also reports the proposed adsorption geometries for the molecule at low and at saturation coverage, respectively. Taken with permission from ref. [144]. 


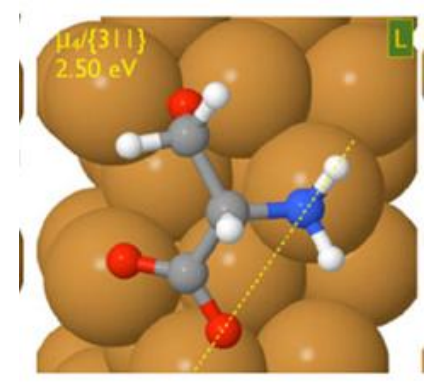

Figure 34. DFT-optimized $\mu 4$ adsorption geometry for L-Ala on the (311) nanofacet of $\mathrm{Cu}(531)-S$. The absolute value of corresponding adsorption energy $\left(\mathrm{E}_{\mathrm{ads}}=-2.50 \mathrm{eV}\right)$ is indicated in the figure; the dotted line $\left(0^{\circ}\right)$ marks the $<1-1-2>$ crystallographic direction (red: oxygen, blue: nitrogen, grey: carbon, white: hydrogen). Taken with permission from ref. [144].

When $\mathrm{Cu}(531)$ is covered by $1 \mathrm{ML}$ or $2 \mathrm{ML}$ of $\mathrm{Au}$, the orientation of Ser molecules at saturation coverage is significantly different from that on the clean surface. For $1 \mathrm{ML}$ of $\mathrm{Au}$, it is impossible to quantify enantiomeric differences in the molecular orientation by NEXAFS, but the thermal decomposition behaviour indicates that they exist. Since XPS spectra indicate a $\mu 3$ bond configuration for saturation coverage and a similar involvement of all gold atoms, the authors suggest that the arrangements should be a typical $\mu 3$ geometry with the gold atoms replacing the top-layer copper atoms. The second layer of gold is not pseudomorphic, which is reflected by the very different molecular orientations on the $2.2 \mathrm{ML} \mathrm{Au} / \mathrm{Cu}(531)$ surface compared to $1 \mathrm{ML} \mathrm{Au}$. Again, the XPS spectra indicate that all surface Au atoms interact with the molecules in a $\mu 3$ bond configuration, however substantial deviations from a bulk-terminated pseudomorphic substrate geometry have to be considered. 


\subsection{Amino acid interaction with poorly reactive surfaces}

\subsubsection{Amino acid adsorption at Au surfaces}

For its weak reactivity and its biocompatibility properties, $\mathrm{Au}$ is a natural choice as a substrate for the deposition of molecules of biological interest. Although several studies have been performed also on $\mathrm{Au}(110)-(1 \times 2), \mathrm{Au}(111)$ is a surface of election for amino acid adsorption experiments and derived applications. This is justified at least by the following reasons: a) it is stable also in contact with air and water (although it can be easily contaminated by hydrophobic molecules); b) its typical $22 \times \sqrt{3}$ reconstruction is well-characterized (also in water) and does not profoundly affect the $\mathrm{Au}(111)$ reactivity; c) due to its low reactivity, the adsorbate is able to keep its molecular and electronic properties, although weakly interacting with the gold substrate and with its neighbouring molecules.

The success of the use of $\mathrm{Au}$ as a substrate is shown by the following examples: a) it has been the target surface for one of the best characterized surface-specific peptide, i.e. the gold-binding peptide [209-211]; b) it has been employed in several experimental studies on the adsorption of small molecules under controlled conditions [212-214]; c) it is important for nano-bioelectronics applications, since it represents the most used substrate for creating contacts of nano-objects with the macroscopic world; d) it is the metal of choice in optical detection systems, such as the widely employed surface plasmon resonance (SPR) technique.

One of the first families of molecules adsorbed on $\mathrm{Au}$ surfaces is that of thiols; the earlier investigations date back to the 1980s, and interesting results have been appearing in the literature from that date to very recent years $[4 ; 61 ; 74 ; 215-218]$. Theoretical and experimental studies of $\mathrm{AA} / \mathrm{Au}$ systems started with Cys $[118 ; 126 ; 182 ; 219-222]$ in the early 1990s [182; 222]. The choice of this S-containing amino acid took into account the strong affinity of $\mathrm{S}$ to Au and thus the possibility to obtain stable adsorption at the poorly reactive metal. Cysteine adsorption was indeed exploited already in the early 2000s in studies of enantioselectivity processes at surfaces [65; 188]. Later on, the interaction of other amino acids with Au has been investigated using surface science methods (Phe, Ala, Tyr, Try, His), but the literature on Cys remains the most abundant.

\subsubsection{Cysteine adsorption at Au surfaces.}

When deposited from the liquid phase, the structural properties of cysteine self-assembled monolayers (SAMs) subtly depend on the $\mathrm{pH}$ and on the electrochemical potential conditions, which affect the charge state of the molecule [223-227]. Upon sublimation in UHV conditions, a rich variety of interesting nanostructures forms [116; 120; 127], so that Cys SAMs have been proposed as case studies to investigate hydrogen bonds in 2D networks [52].

The first theoretical studies on Cys at $\mathrm{Au}(111)$, performed by R. Di Felice [128; 129] in 2003and 2004, offered a deeper insight into the role played by sulphur head groups in the attachment of molecules to metallic substrates. Besides providing the energetics of different adsorption reactions, they showed the modified energy levels of the molecules, thus allowing to determine the lineup between the molecular HOMO with respect to the metal Fermi level: this information is crucial for 
the interpretation of transport phenomena in molecular electronic devices[228]. It emerges that the Fermi level cannot be simply placed in the middle of the HOMO-LUMO gap but depends on the interface bonding features. In the cited works, R. Di Felice et al. that the $\mathrm{S}$ headgroup preferentially sits on a bridge site, similarly to the short chain thiols. The energetics are comparable with thiol SAMs $\left(E_{a d s}=-0.74 \mathrm{eV}\right)$, which suggests that the amino acid group of Cys does not influence the local structural and electronic details of the $\mathrm{Au}-\mathrm{S}$ bonding. The chain length or/and the nature of the thiol tailgroup is only relevant in the quantitative determination of the adsorption energy. This first analysiswas later confirmed in the case of thiols by Santos and Tielens [215] and reviewed by Hakkinen et al. [229].

The self-assembly of S-cysteine on $\mathrm{Au}(111)$ was investigated thoroughly both by DFT calculations and experimental means. From the adsorption of individual cysteine molecules on $\mathrm{Au}(111)$ surface at room temperature, it was shown that the AA diffuse freely on the surface due to low-energy barriers (lower than $60 \mathrm{meV}$; estimated from single point calculations), and can be regarded as a 2D molecular gas. The balance between molecule-molecule and molecules-substrate interactions induces molecular condensation and evaporation from steps, reconstruction edges, etc. as revealed by STM images. These processes progressively lead to the formation of a number of stable arrangements such as single-molecular rows, trimers and 2D islands.[130; 131]
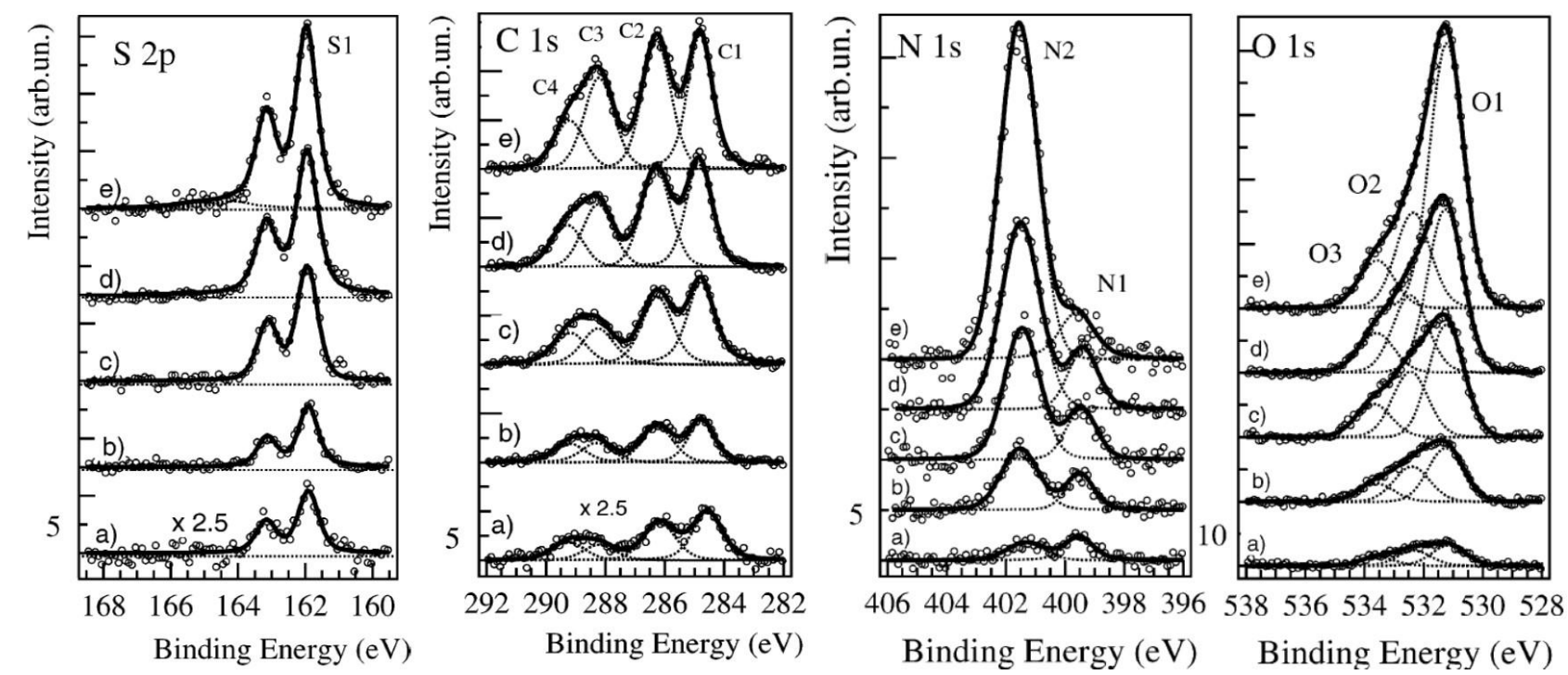

Figure 35. Cys/Au(110)-(2×1). HR-XPS spectra as a function of coverage in the submonolayer adsorption regime: a) 0.14 ML; b) $0.33 \mathrm{ML}$; c) $0.62 \mathrm{ML}$; d) $0.8 \mathrm{ML}$; e) 1 ML. Photon energy: 710 eV. Circles: experimental data (after normalization to the photon beam flux and background subtraction). Continuous lines: best fit. Dotted lines: deconvolution with doublets of pseudo-Voigt peaks. S2p core level region: the S1 doublet corresponds to the thiolate species. The very small component at $\mathrm{E}_{\mathrm{b}} \sim 164 \mathrm{eV}$ in spectrum e) is related to initial formation of the second layer. C1s region: the four components are assigned to carbon bonded to sulphur $(\mathrm{C} 1)$, to the $\alpha-\mathrm{C}(\mathrm{C} 2)$, to carboxylate carbon (C3) and to carboxyl carbon (C4), respectively. N 1s region: the N1 and N2 components are related to $\mathrm{N}$ atoms in $\mathrm{NH}_{2}$ and in $\mathrm{NH}_{3}{ }^{+}$groups, respectively. $\mathrm{O}$ 1s region: $\mathrm{O} 1$ is assigned to the two equivalent oxygen atoms belonging to the so-called resonant state of the deprotonated carboxyl group; the $\mathrm{O} 2$ and $\mathrm{O} 3$ states are put in relation to the presence of $\mathrm{COOH}$ groups. Taken with permission from ref. [121]. 
Several XPS investigations were performed on $\mathrm{Cys} / \mathrm{Au}(111)$ and $\mathrm{Cys} / \mathrm{Au}(110)-(1 \times 2)$ after deposition of the amino acid either by vapour [52; 121; 222] or solution [224; 230] (seeTable 3). As an example, we present inFigure 35 high resolution spectra obtained with synchrotron radiation upon Cys evaporation onto the $\mathrm{Au}(110)-(2 \times 1)$ surface [121]. The general picture is that Cys adsorbs on the reconstructed $\mathrm{Au}(110)$ surface at room temperature and in the monolayer regime as a thiolate $\left(\mathrm{E}_{\mathrm{b}}\left(\mathrm{S} 2 \mathrm{p}_{3 / 2}\right) \sim 162 \mathrm{eV}\right)$, while molecules in the second layer show a dangling $\mathrm{SH}$ termination $\left(\mathrm{E}_{\mathrm{b}}(\mathrm{S}\right.$ $\left.\left.2 \mathrm{p}_{3 / 2}\right) 164 \mathrm{eV}\right)$.

$\mathrm{On} \mathrm{Au}(111)$ a thiolate species forms when Cys is deposited from solution or evaporated in UHV at $\mathrm{T}_{\mathrm{ev}} \geq 330 \mathrm{~K}$; on the contrary, a large contribution of weakly interacting molecules is present for $\mathrm{T}_{\mathrm{ev}}=\mathrm{RT}$ and monolayer coverage. These results were later confirmed by analysis of the valence band region performed by metastable deexcitation spectroscopy [118]. As already mentioned, the optimum sulphur adsorption site is the Au bridge site [128]. From these calculations, it was also demonstrated that the binding of cysteine becomes stronger if also the $\mathrm{N}$ atom of the amino group interacts with the surface. The result is coherent with early infrared spectroscopy measurements [182] but it was not confirmed by the subsequent XPS studies [224; 230]. All XPS data indicate, indeed, that Cys adsorbs on Au surfaces mainly in the zwitterionic form. A small fraction of neutral molecules was observed on $\mathrm{Au}(111)$ after deposition from solution [230] and on $\mathrm{Au}(110)$ in the early stages of the adsorption process (possibly related to adsorption of isolated molecules or dimers at defects) [121]. TDS experiments on vapour deposited films [231] showed that Cys molecules on $\mathrm{Au}(111)$ desorb from multilayers at 353-373 K, while the first, covalently bound, monolayer decomposes above $458 \mathrm{~K}$. The vibrational spectrum of Cys SAMs on $\mathrm{Au}(111)$ was investigated by De Renzi et al. [52] when passing from the quite heterogeneous layer deposited from the gas-phase at RT [127] to the more homogeneous and ordered interface obtained for the compact layer at 330 $\mathrm{K}$. In the latter case, a sharp feature localized at $3335 \mathrm{~cm}^{-1}$ (attributed to a stretching mode of the N$\mathrm{H}$... O bond) and an extremely low-frequency feature at $74 \mathrm{~cm}^{-1}$ ( $\mathrm{H}$ mode - assigned to a collective mode) are proposed as fingerprints of the formation of a highly organized H-bonding network.

The lateral order in Cys/Au(111) adlayers was initially addressed by STM in a series of studies under non-comparable conditions [223; 224][225; 226], finding - as a consequence - a variety of results. The system was firstly studied under controlled, UHV conditions by Kuhnle et al. [127], who identified and compared several structures forming upon RT deposition and those obtained after thermal annealing of the substrate to approximately $380 \mathrm{~K}$. At RT and submonolayer coverage, islands with no apparent molecular order coexist with a highly ordered molecular layer with square symmetry (see Figure 36a and b). Increasing $\Theta$, a multitude of slightly differing structures with short-range hexagonal order covers the surface (Figure 36c). No epitaxial or rotational relationship to the underlying $\mathrm{Au}(111)$ substrate was found neither for the quadratic nor for the hexagonal assemblies, suggesting that no specific molecular adsorption positions on the gold surface are involved in the formation of these structures.

Annealing the Cys/Au(111) layer produced at RT to $380 \mathrm{~K}$ leads to significant changes in the adsorption structure and surface morphology (Figure 36d and e). In particular, beyond the first monolayer, the well-known $(\sqrt{3} \times \sqrt{ } 3) \mathrm{R} 30^{\circ}$ superstructure forms, accompaniedbythe development of monatomically deep etch pits, both characteristic of alkanethiol SAMs on Au(111). Although no definite conclusion about the binding properties of cysteine to the Au(111) surface can be drawn 
from STM data alone, the authors suggest that covalent S-Au bonding occurs only upon thermal activation of the vacuum deposited cysteine molecules.

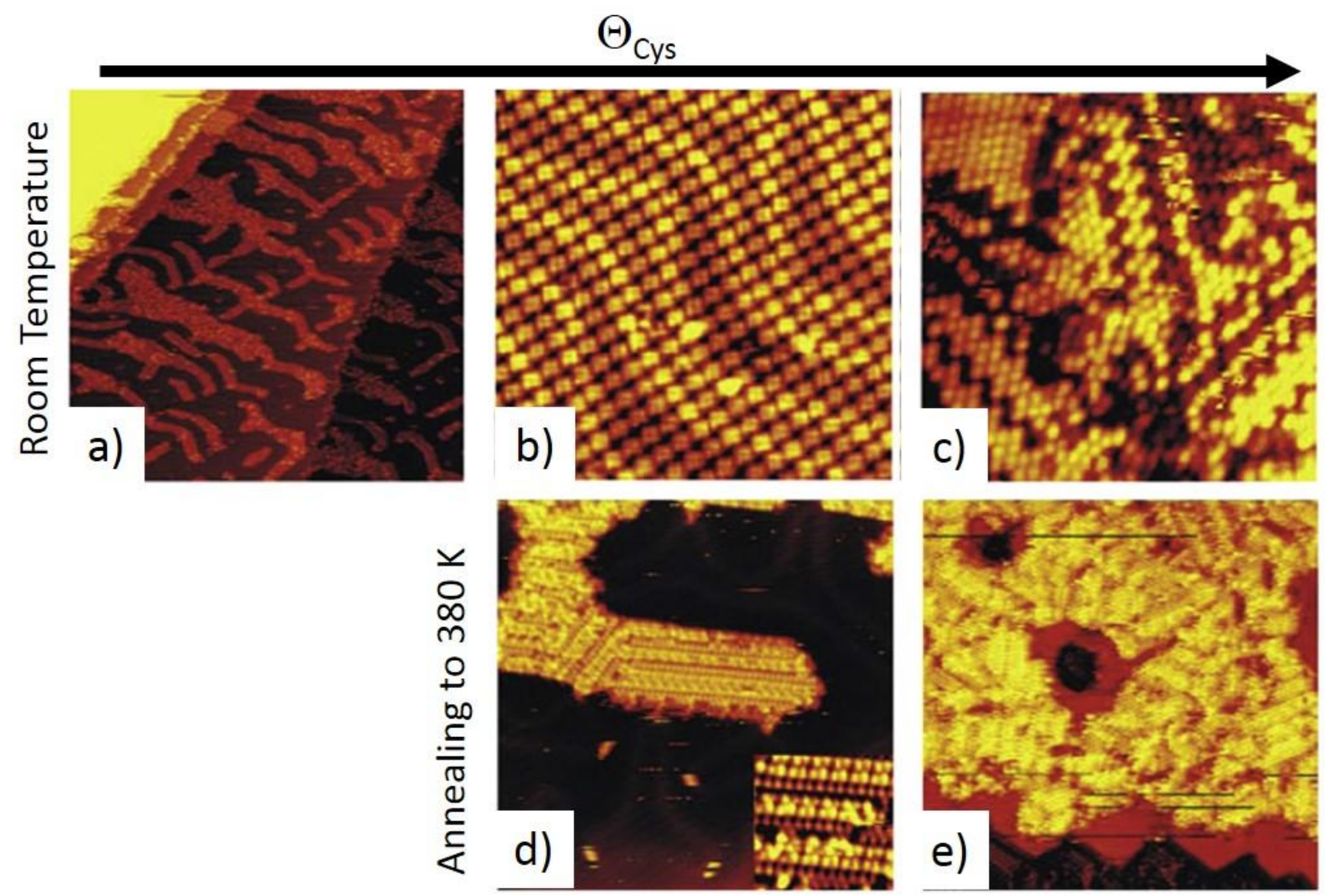

Figure 36. Overview over the multitude of different molecular structures of L cysteine on $\mathrm{Au}(111)$ in dependence of substrate temperature and surface coverage. a) Upon low-coverage deposition at room temperature, unordered cysteine islands are formed and grow from the step edges and the elbows of the herringbone reconstruction (Image size: $111 \times 116 \mathrm{~nm}^{2}$ ). b) An ordered, square assembly coexists with the unordered islands. Its presence, however, does not affect the herringbone reconstruction of the $\mathrm{Au}(111)$ surface, which is evident in the STM image (Image size: $22 \times 23 \mathrm{~nm}^{2}$ ). c) Molecular structures observed after RT deposition at higher cysteine coverages. Variations of a hexagonal pattern are found (Image size $15.2 \times 17.4 \mathrm{~nm}^{2}$ ). d) Low cysteine coverage after annealing to $380 \mathrm{~K}$ revealing an internal structure within the cysteine islands (Image size: $38 \times 44 \mathrm{~nm}^{2}$. Inset: $\left.7.6 \times 8.7 \mathrm{~nm}^{2}\right)$. e) High cysteine coverage after annealing to $380 \mathrm{~K}$. Locally the $(\sqrt{3} \times \sqrt{3}) \mathrm{R} 30^{\circ}$ superstructure is formed (Image size $38 \times 44 \mathrm{~nm}^{2}$ ). Reprinted with permission from ref. [132].

With respect to the case of Cys/Au(111), the self-organization of Cys molecules on the $\mathrm{Au}(110)$ $(1 \times 2)$ surface presents an even richer variety of structures depending on coverage and temperature; in addition, it shows interesting features related to the chirality of the molecules. Evaporation of Cys on the substrate at $120 \mathrm{~K}$ leads to the formation of unordered islands consisting of several molecular species (see Figure 37a) [133]. Upon annealing to $270 \mathrm{~K}$ the molecules arrange in identical, monodisperse clusters that are composed of eight subunits (two at the centre and three at each side) of different apparent height (see Figure 37b and c) [133]. The internal structure breaks the mirror symmetry of the system, indicating the chiral nature of the adsorbed molecules. If the 
experiment is performed with D-Cys, identical but mirror-imaged clusters are observed. Upon deposition of a racemic mixture, no new structures suggestive of heterochiral assemblies were observed. The racemate thus appears to segregate into homochiral clusters, indicating intermolecular chiral recognition during the self-assembly process. The brighter corner units of the clusters are less strongly bound than fainter ones; moreover, the clusters can be manipulated along the $\langle 1-10\rangle$ direction at $260 \mathrm{~K}$ leaving behind an unperturbed gold surface. The overall picture suggests then that the clusters are physisorbed to the substrate rather than chemisorbed. A tentative model for the assembly is proposed in Figure 37d [132]. According to it, all Cys molecules are physisorbed to the gold surfaces and form an H-bond network. The mercapto group is likely involved in the physisorption. Although the detailed S adsorption site cannot be deduced from the STM data alone, a bridge position is proposed on the basis of previous results [128; 129]. Furthermore, the upper left and lower right corner entities are imaged brighter and are less strongly bound to the cluster since they are connected to it with only one hydrogen bond.
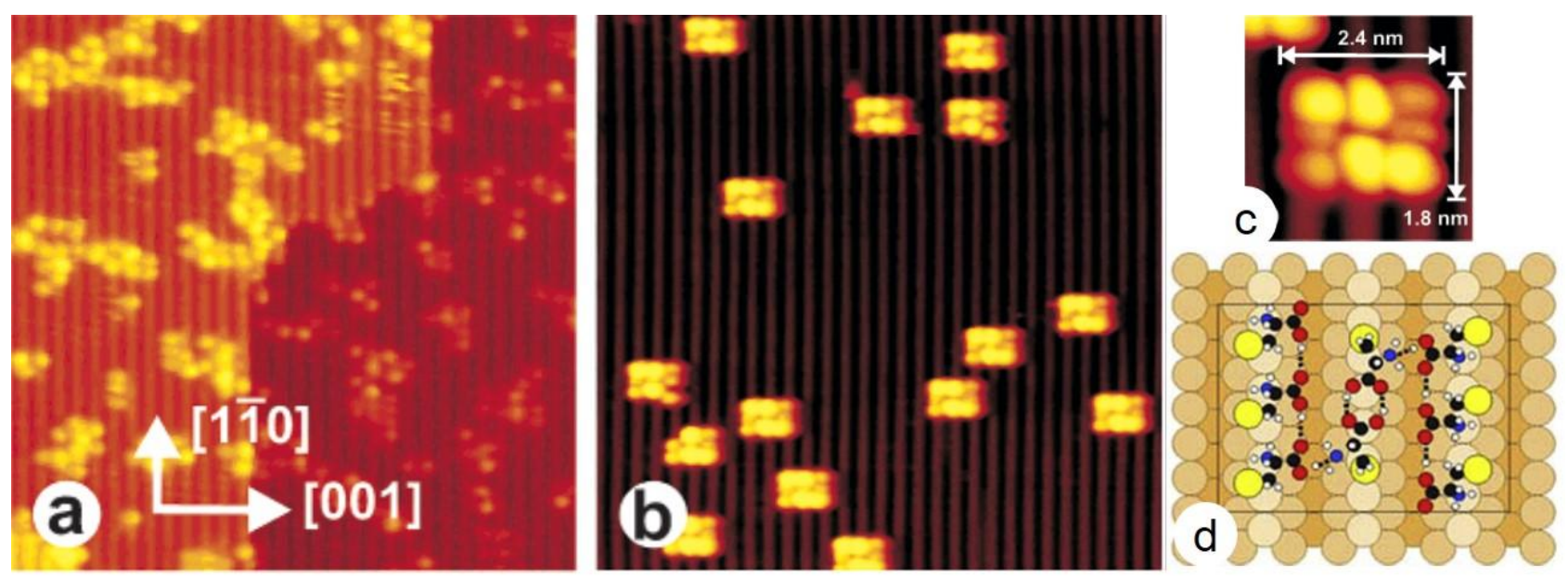

Figure 37. STM images of cysteine deposited on $\mathrm{Au}(110)-(1 \times 2)$ below RT. The missing-row reconstruction results in the close-packed gold rows along the $\langle 1-10\rangle$ direction seen running vertically in all the images. (a) Irregular cysteine agglomerates formed after deposition at $120 \mathrm{~K}$ $\left(24.5 \times 24.5 \mathrm{~nm}^{2}\right)$. b) L-Cysteine nanoclusters formed after annealing to $270 \mathrm{~K}\left(24.5 \times 24.5 \mathrm{~nm}^{2}\right)$. (c) Close-up of an L-cysteine nanocluster $\left(3.2 \times 3.2 \mathrm{~nm}^{2}\right)$. d) Tentative model for the L-Cys cluster of panel c). Taken with permission from refs. [132; 133]. 

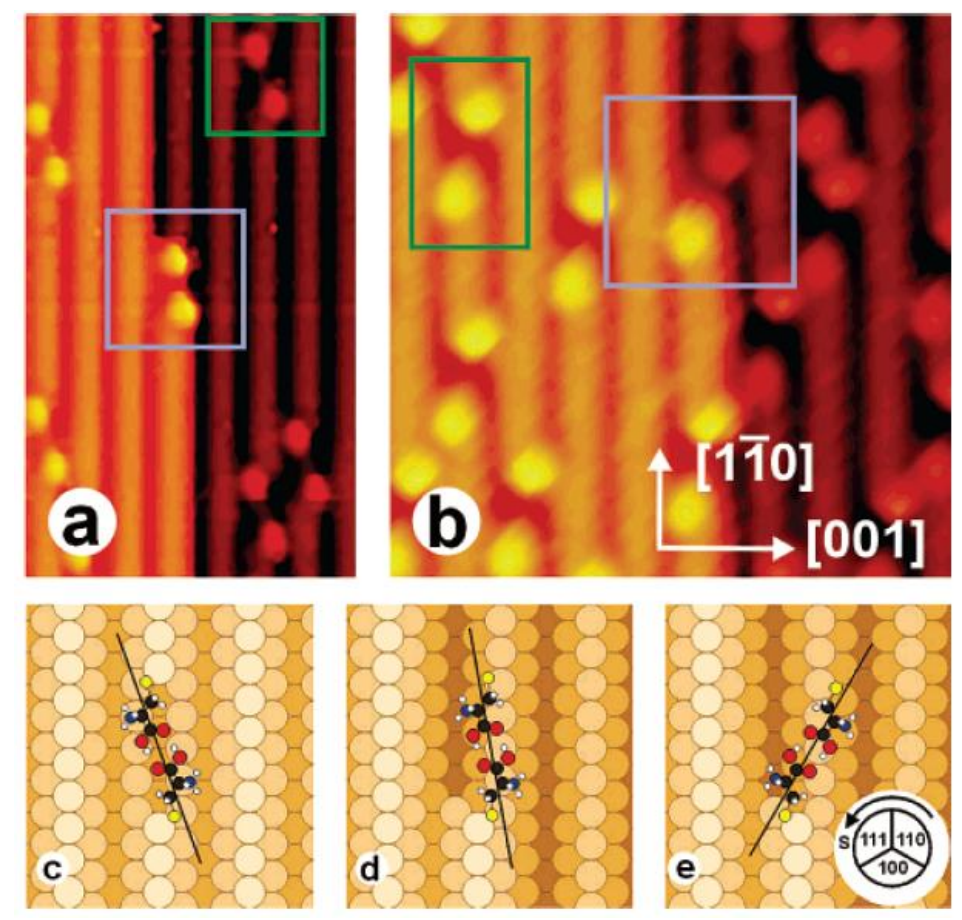

Figure 38.STM images and models for cysteine dimers at S kinks (blue boxes) and at terraces (green boxes). a) D-Cysteine dimers. b) L_Cysteine dimers. c) Adsorption geometry of a DCysteine dimer on a four-atom-long vacancy structure [188] showing a $20^{\circ}$ rotation compared to the $<1-10>$ direction. d) Adsorption geometry of a D-cysteine dimer at an S kink site, showing a $10^{\circ}$ rotation. e) Adsorption geometry of an L-cysteine dimer at and S-kink site, showing a $30^{\circ}$ rotation. Taken with permission from ref. [127].

Upon deposition/annealing at RT, Cys forms a poorly ordered, anisotropic structure, which rearranges into particularly interesting features once the system is annealed to $340-380 \mathrm{~K}$ :

i) A double-lobe structure is formed with a characteristic $20^{\circ}$ rotation with respect to the closepacked gold rows (see Figure 38a and b, green boxes). The size of each protrusion fits with the size of an individual cysteine molecule, indicating the formation of cysteine dimers. The clockwise/counter-clockwise rotation of the cysteine dimer depends on the chirality of the adsorbate. Interestingly, upon evaporation of the racemic mixture exclusively homochiral dimers form, thus electing this system to a molecular-scale example for chiral recognition [188]. Under special (and accidental) tip conditions, the dimers appear transparent, revealing the presence of holes in the underlying gold surface and of gold rows collecting part of the ejected metal atoms. Therefore, the molecular dimers formation induces a restructuring of the gold substrate through an activated process (and indeed annealing of the surface is required). Attempts to manipulate the cysteine dimers failed, suggesting a much stronger binding of the dimers to the gold surface compared to the clusters. This is in line with a transition from physisorbed cysteine below RT to chemisorbed cysteinate species above RT and with the presence of thiolate species observed by XPS [121]. Theoretical DFTmodelling showed that the adsorption geometry can be understood from a simultaneous optimization of three bonds for each molecule of the dimer, as illustrated in Figure 38c: the sulphur atom is covalently bound at a bridge site next to a low-coordinated gold atom in the topmost row; the nitrogen atom coordinates via a lone pair to the gold surface; the carboxylic group forms $\mathrm{H}$-bonds with the carboxylic group of the other molecule in the dimer. 
ii) While the appearance of DD-Cys and LL-Cys dimers is identical (but mirror imaged) for adsorption at $\mathrm{Au}(110)-(1 \times 2)$ terraces, this is no longer true for adsorption at kinked steps [124]. As evident from inspection of Figure 38a and b (blue boxes), DD-Cys dimers adsorbed at an $S$ kink are only rotated by $10^{\circ}$ counter-clockwise with respect to the $\langle 1-10\rangle$ direction, LL-Cys dimers are rotated by $30^{\circ}$ clockwise. This difference can be understood within the framework established for cysteine dimers at terrace vacancy sites. The kink site presents an atomic structure identical to that found at the termination of the vacancies, so it is assumed that one Cys unit adsorbs with the sulphur atom at this site (Figure 38d and e). The sulphur atom of the second molecule, however, must find a bridge adsorption site at the lower terrace, which causes a different rotation of the molecule for the two enantiomers. Dimers at kinked steps are demonstrated to act as nucleation centres for homochiral islands of Cys molecules packed in a denser structure [124; 132].

iii) When increasing the coverage and annealing to $340 \mathrm{~K}$, a second structure is observed coexisting with the molecular dimers: molecular double rows running along the $\langle 1-10\rangle$ direction and formed by bean-shaped units of the size of individual cysteine molecules (Figure 39a) [116]. The STM image suggests that the molecules in the double-row structure interact both along the $<001\rangle$ and the $\langle 1-10\rangle$ directions. DFT calculations do, however, indicate that the molecules in the double-row structures only interact along the $<001\rangle$ direction, forming individual cysteine dimers, with an adsorption energy of $-1.45 \mathrm{eV}[116]$. The solution to this discrepancy is again found in a massive substrate restructuring underneath the double-row structure. The most stable conformation found by DFT corresponds indeed to Cys-dimers on the unreconstructed $\mathrm{Au}(110)$ surface $(-2.10 \mathrm{eV})$ (two added gold rows underneath the row structure are removed- marked by the black arrows in Figure 39b). The two Cys units are anchored to the surface through the $\mathrm{S}$ atoms, adsorbed in the preferred bridge position, and are bound to each other by two OH-O hydrogen bonds (see scheme in Figure 39b). The removal of an atom from a perfect surface is energetically demanding $(0.90$ $\mathrm{eV} /$ dimer) as this involves the reduction of the coordination number of highly coordinated atoms. However, if an atom is removed from an already existing vacancy structure, the energy needed is much smaller $(0.40 \mathrm{eV} /$ dimer $)$. This explains the formation of dimer rows and their nucleation at descending steps. The molecule-induced surface rearrangement provides an efficient attraction driving the unidirectional growth of the molecular structure. Subsequent NEXAFS and XRD results [120] proved that the paired rows consist indeed of Cys zwitterions with both the C-S bond and the carboxylic group almost parallel to the surface. However, a different orientation of the molecules within the row was proposed, in which molecules in the same row are rotated by $180^{\circ}$ with respect to adjacent ones and $\mathrm{H}$-bonds occur between the carboxyl group of one molecule and the $\mathrm{NH}_{3}{ }^{+}$ groups of both one consecutive molecule in the same row and of the twin molecule of the adjacent row.

iv) Dissociative Cys desorption is observed after annealing to $440 \mathrm{~K}$, leaving the surface covered by a $\mathrm{c}(4 \times 2) \mathrm{S}$-overlayer. At this stage, the surface is re-facetted and exhibits rectangular terraces, again indicating a substantial mass transport upon formation of this $\mathrm{c}(4 \times 2)$ sulphur structure.

We further mention that a significant enantiomeric excess of LL-Cys dimers is observed upon partial desorption/decomposition of racemic cysteine [117]. The possible origin of this interesting effect was deeply discussed by the authors, who were however unable to reach a conclusion. 

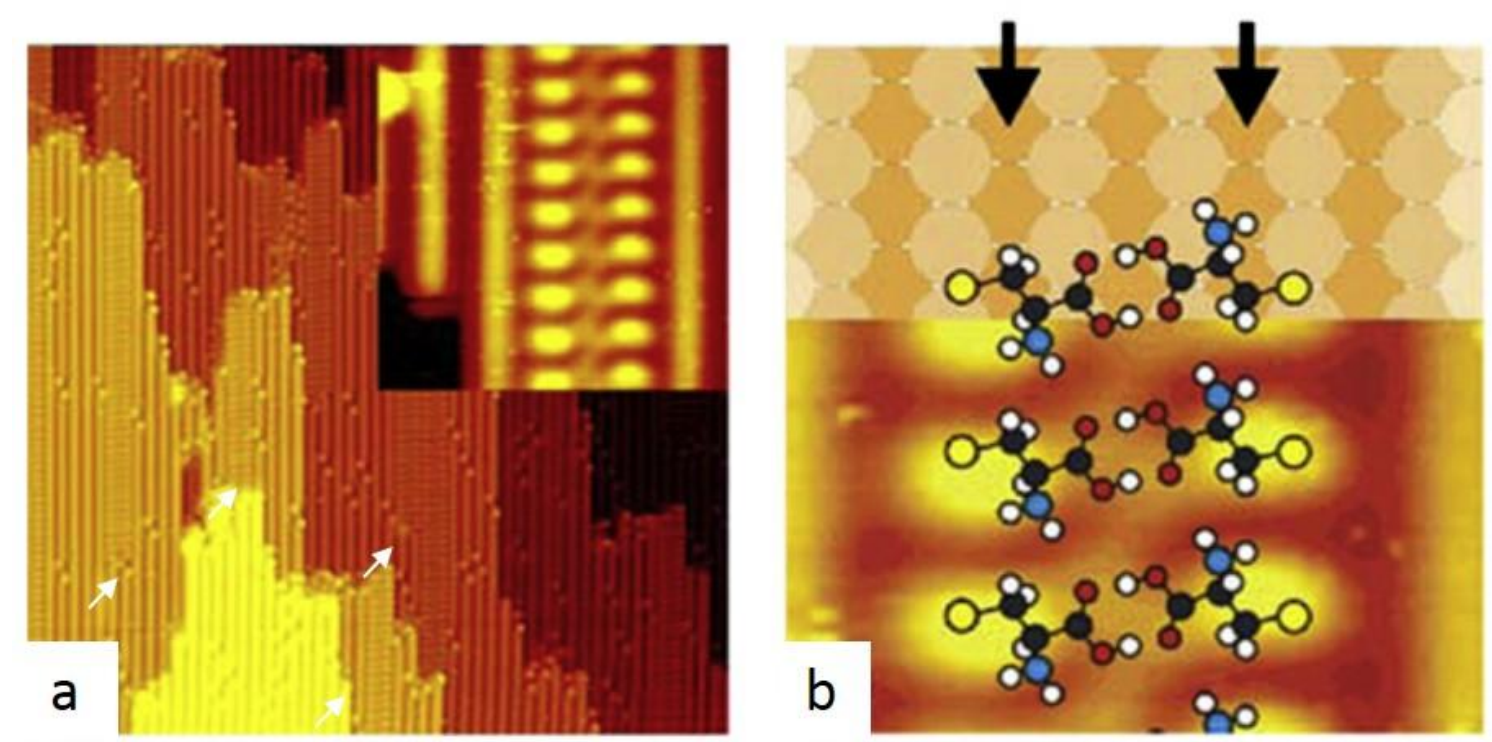

Figure 39. a) STM image of the D-Cys/Au(110)-(1×2) surface showing the coexistence of dimers and of molecular double rows (some of them evidenced by white arrows). Image size $42.5 \mathrm{~nm} \times$ $42.5 \mathrm{~nm}$. In the insert, a zoom into such a double-row structure is shown (Image size $4 \mathrm{~nm} \times 4 \mathrm{~nm}$ ). b) Most stable cysteine double-row structure as obtained from the DFT calculations superimposed onto a ball model showing the gold surface and onto an STM image of the double-row structure. The arrows indicate the position of the removed Au atoms. Taken with permission from ref. [132].

The interplay of molecule-surface and molecule-molecule interactions in the self-assembly process of monomers, dimers, and trimers of L-cysteine on a $(1 \times 2)$-reconstructed $\mathrm{Au}(110)$ surface, and the implications for chiral recognition were re-examined in details recently [125]. Multiple adsorbed configurations (over sixteen geometries are presented) of L-cysteine and L-cysteinate in neutral and zwitterionic form were generated using molecular dynamics simulations, serving as starting points for further density functional theory (DFT)-based optimizations. It was found that binding for both monomers and dimers was stronger at kink sites formed on the surface during the chemisorption process and was unlikely to occur along the highly coordinated through sites. In this, DFT calculations disagreed with MD simulations using centrosymmetric potentials, which tended to maximize the coordination of the adsorbate groups and ignore differences in reactivity of the various $\mathrm{Au}$ sites, unless specifically included in the force field. Homochiral L-cysteine dimers bound at kink sites were particularly stable with respect to both heterochiral and trimer structures, while molecules more weakly bound at more stable surface locations did not exhibit chiral recognition. Nevertheless, the anchoring of cysteinate monomers occurs, via the thiol and amine groups. If barriers to the diffusion of $\mathrm{Au}$ atoms along the surface can be overcome, the four-atom vacancy structures proposed by Kühnle et al. [188] (See Figure 38) provide reactive kink sites, ideally spaced for binding homochiral cysteinate dimers, with highly stable $\mathrm{COOH}$-based hydrogen bonding (See Figure 40).[125] 

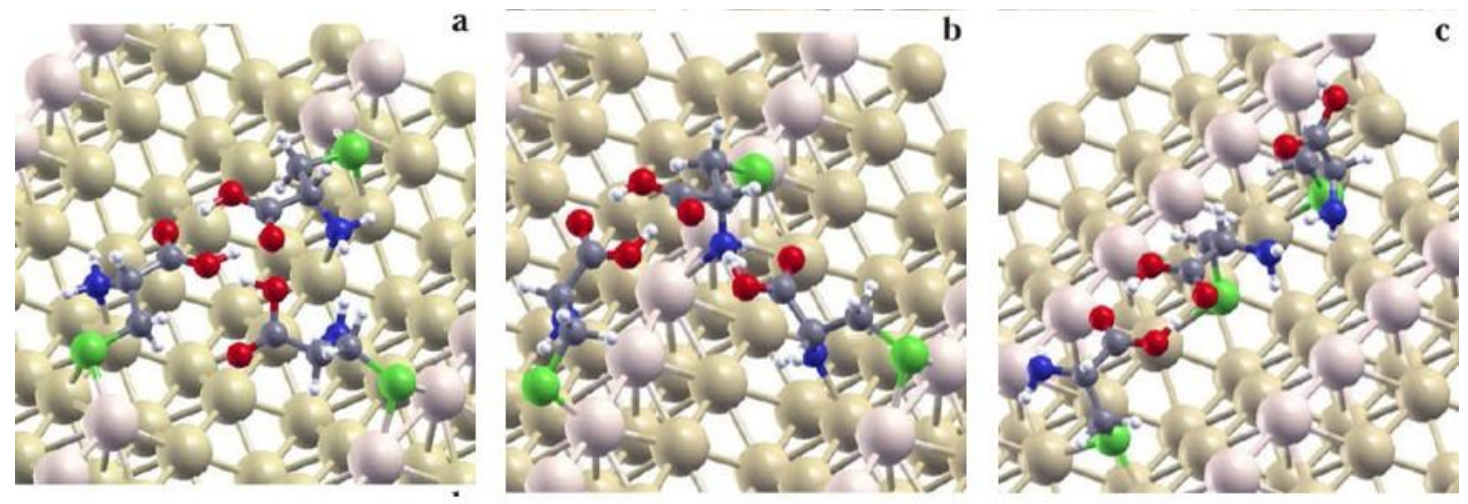

Figure 40. Stable equilibrium structures of LLL cysteinate trimers on (1×2)-Au(110): (a) at a 4atom vacancy site; (b) along the ridge, with binding to the third molecule; (c) along trough, $\mathrm{NH}_{2}$ based H-bonds to third molecule. Taken with permission from ref. [125].

Also Cys dimers on Au(110)-(1×1) were studied by periodic DFT [126]. Three types of rotational conformers (rotamers) of a cysteine dimer (See Figure 41 and Figure 42) were investigated in the protonated and unprotonated forms. With this model, Morari et al.[126] were able to describe the formation of new nucleation centres formed by Cys pairs on the surface as well as the properties of long chains of cysteine molecules adsorbed on the $\mathrm{Au}(110)-(1 \times 1)$ surface.
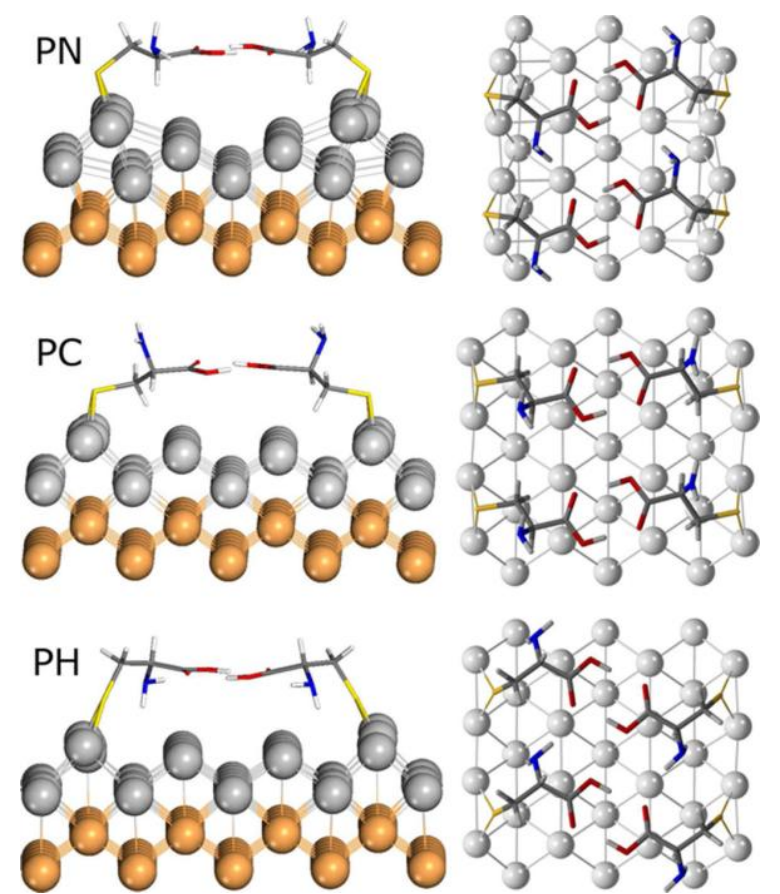

Figure 41Graphical representation (side view, left; top view, right) of the Cys rotamers PN, PC and PHs (unprotonated periodical model; for the Newman projection see under) adsorbed on $\mathrm{Au}(111)$ (1x1). Yellow is used to represent deep Au layers and light gray for the top layers. Taken with permission from ref. [126]. 


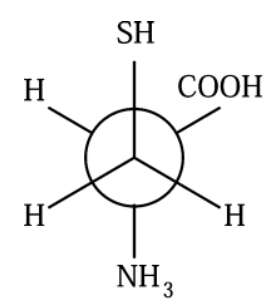

$\mathrm{PN}$

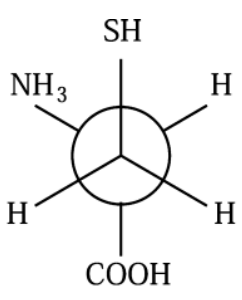

PC

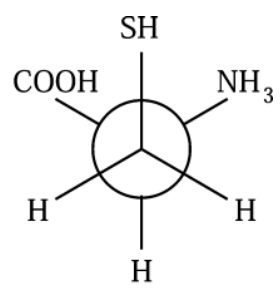

$\mathrm{PH}$

Figure 42 Newman projection for the PN, PC, and PH rotamers of cysteine. Taken with permission from ref. [126].

The PC conformer is found to be the most stable structure as well as the one having the most convenient shape of the potential energy surface. Interestingly, in this conformers the $\mathrm{NH}_{2}$ group is located at a high position relative to the surface (i.e., about $5 \AA$ ), which reduces significantly its interaction with it. The formation of nucleation centres(the dimers) as well as the formation of rows along the $\langle 1-10\rangle$ direction are favoured for the PC conformer of cysteine. The PH conformer is the least favourable since the $\mathrm{NH}_{2}$ group lies about $3 \AA$ above the Au surface and thus interacts directly with it, which makes the formation of rows unfavourable.

Some work has been performed also on the adsorption of cysteine onto the intrinsically chiral gold surfaces, namely $\mathrm{Au}(321)$ [134] and $\mathrm{Au}(1711$ 9) [65; 135].

By means of a periodic DFT-D approach [134], Fajin et al. investigated and compared their results on Cys/Au(321)-Rand $\mathrm{Au}(321)-S$ with those obtained at the same level of theory for a nonchiral surface with the same terrace orientation, namely $\mathrm{Au}(111)$ (See Figure 43). Neutral and zwitterionic forms of L- and D-Cys are systematically considered on all the surfaces and surface coverage effects are taken into account. At full monolayer coverage, the zwitterionic form is found to be more stable for both enantiomers and both on $\mathrm{Au}(321)^{-} R$ and $\mathrm{Au}(321)-\mathrm{S}$. The zwitterionic L- and D-Cys forms on $\mathrm{Au}(321)-R, \mathrm{Au}(321)-S$ are all isoenergetics (with adsorption energies of $-1.32 \pm$ $0.02 \mathrm{eV}$, dispersion correction $-1.58 \pm 0.01 \mathrm{eV})$. The same trend was foundon $\mathrm{Au}(111)$ surfaces ( $-0.97 \mathrm{eV}$, dispersion corrected: $-1.38 \mathrm{eV}$; see Figure 43). This leads to highly organized cysteine monolayers. However, at low coverage (about $1 / 2 \mathrm{ML}$ ) the adsorption of cysteine dimers, with the pairs interacting through their carbonyl groups, is more or equally favourable than adsorption of single cysteine molecules on both surfaces. Moreover, adsorption on the stepped surface is clearly more favourable than on the flat surface, revealing the importance of the low coordinated gold atoms in the adsorption of these species. Non-negligible differences between the adsorption energy of the enantiomers of neutral cysteine were found on $\mathrm{Au}(321)$ both at high (1 ML) and low coverage $(1 / 2 \mathrm{ML})$, thus showing the enantiospecificity of this intrinsically chiral surface regarding cysteine adsorption. In summary, the adsorption energy difference between neutral L-Cys and DCys on $\mathrm{Au}(321)-S$ is always in favour of L-Cys (by $0.46 \mathrm{eV}$, dispersion corrected: $0.32 \mathrm{eV}$ at low coverage and by $0.29 \mathrm{eV}$, dispersion corrected: $0.21 \mathrm{eV}$ at high coverage). On the contrary, on $\mathrm{Au}(321)$-Rsuch quantity is in favour of L-Cys at low coverage $(0.14 \mathrm{eV}$, dispersion corrected 0.23 $\mathrm{eV})$ and in favour of D-Cys at high coverage $(0.01 \mathrm{eV}$, dispersion corrected $0.17 \mathrm{eV})$. Adsorption occurs with the cysteine binding the surface through only one contact point (its sulphur atom on a bridge site of a step), in contrast to a previous work [232] where the enantiospecific adsorption of 
cysteine has been related to two non-equivalent binding sites of the cysteine enantiomers with the surface.
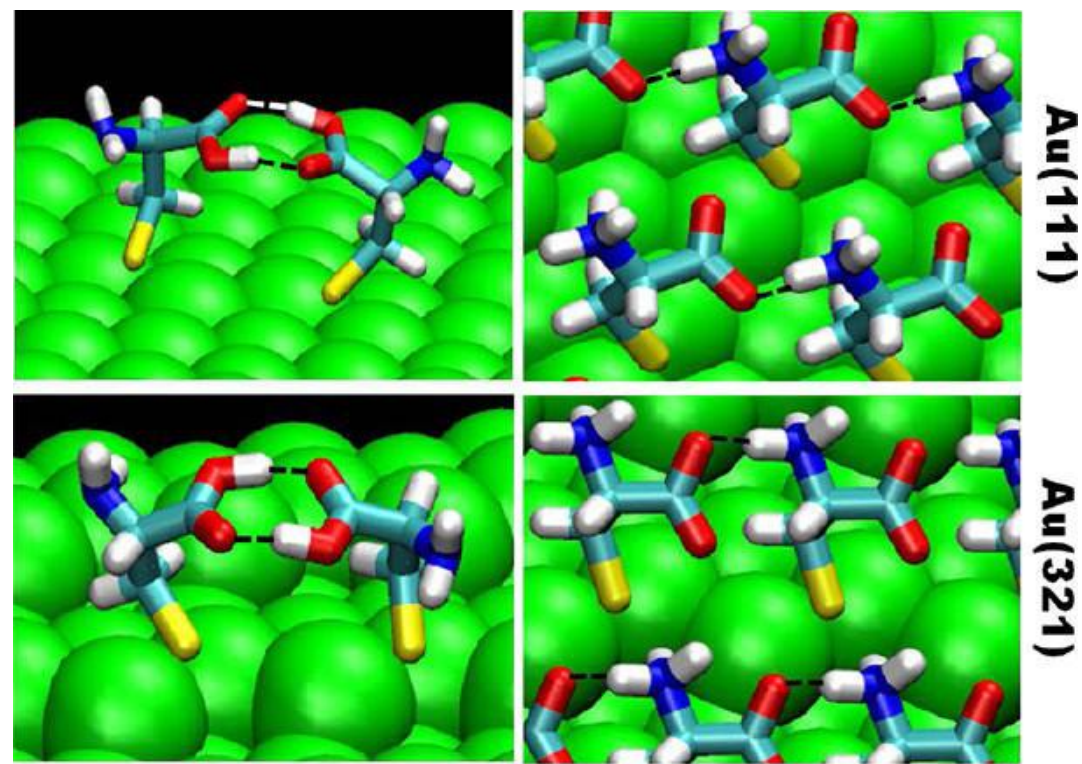

\section{Low coverage vs. high coverage}

Figure 43. Most stable conformation for Cys on $\mathrm{Au}(111)$ and $\mathrm{Au}(321)$ at high and low coverage, showing the pair and the row formation of Cys on the surface. Taken with permission from ref.[134].

Two distinct, mirror symmetric conformations were found also for L- and D-Cys adsorption at $\mathrm{Au}(1711$ 9) [65; 135]. This system was studied by combining XPS experiment (including a detailed analysis of the N1s forward scattering peaks) and DFT calculations. Figure 44A displays the hard sphere model of the theoretically determined adsorption structures of $D$ - and $L$-cysteine on $\mathrm{Au}(17119)$. The calculated adsorption energies and the experimental and theoretical values of the $\mathrm{N}-\mathrm{C}_{\alpha}$ bonds are reported inFigure 44B. The overall information indicates that cysteine forms a thiolate and an amino bond with the gold surface, while the carboxyl group "flies" above the gold substrate. The trend that molecules bind to the lowest coordinated metal atoms [233] is nicely reproduced. However, $D$-cysteine binds with the amino group at the kink atom and the thiol group in a bridge site at the step. Vice versa, L-Cys adsorbs with the $\mathrm{S}$ atom at the kink bridge site and with the amino group at the upper step edge. The third bond, i.e. the carboxyl group, is not significant or even weakly repulsive. Therefore, enantiospecific steric repulsion and deformation of receptor and target molecule leads to the situation that $L$-cysteine does not bind in the same way to $S$ kinksas does $D$-cysteine. The latter binds more strongly to the surface $(-2.03 \mathrm{eV}$ and $-2.17 \mathrm{eV}$ for L- and D-Cys, respectively), as witnessed also by the adsorption energy of all functional groups. In particular, the amino group is $0.19 \mathrm{eV}$ more strongly bound for the $\mathrm{D}$ - than for the L- enantiomer. As in the case of $\mathrm{Au}(321)$ [134], also for $\mathrm{Cys} / \mathrm{Au}(17$ 11 9) the three point contact model for chiral recognition [234; 235] fails.

Further spectroscopic evidence for chiral hetero- recognition is given by the XPS spectra ofFigure $44 \mathrm{C}$, which clearly shows different core levels for $D$ - and $L$-cysteine [135]. This is an independent proof that the two Cys enantiomers bind differently on a Au (17 11 9)-S surface. Furthermore, there are significant enantiospecific differences in the hydrogen abstraction from the amino group of 
cysteine. This indicates the presence of enantiospecific reaction pathways. DFT calculations confirm, indeed, a higher hydrogen affinity to the amino group of L-Cys/Au(17 11 9) than to the one of D-Cys.

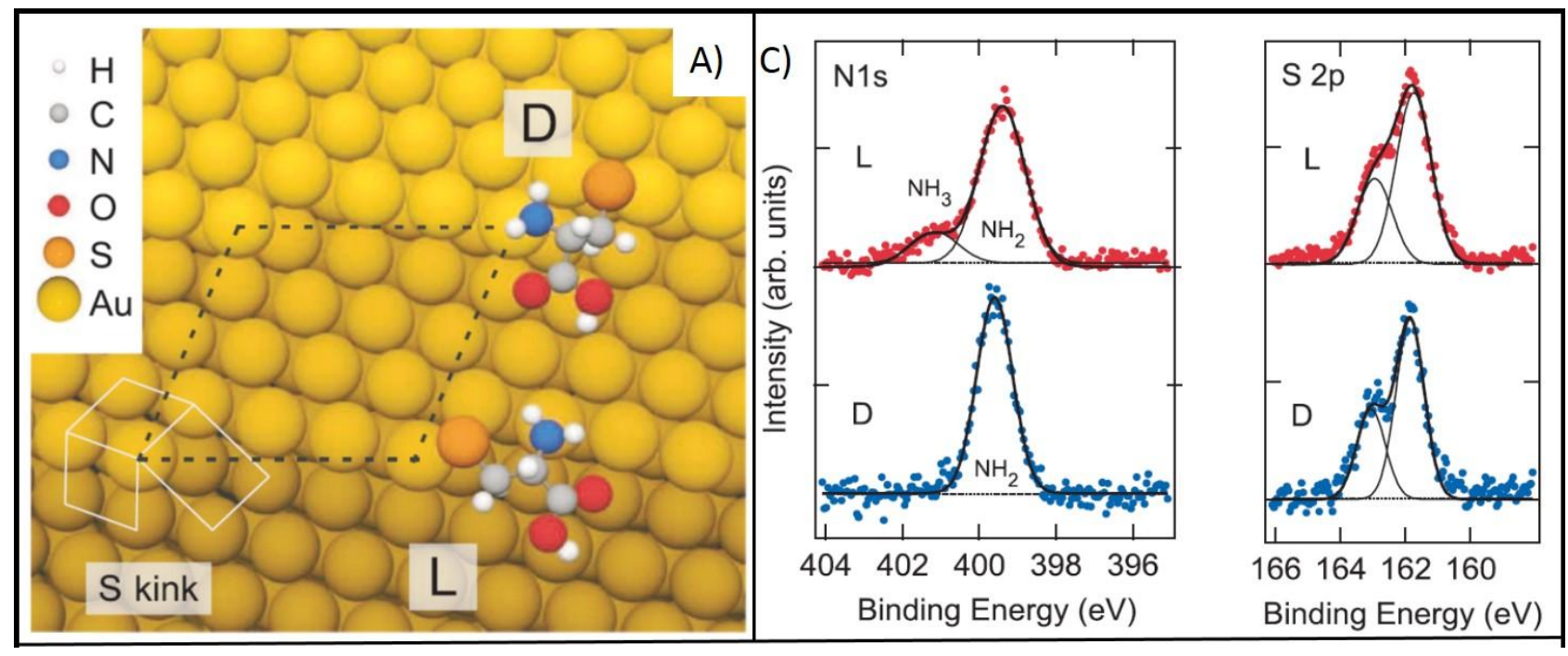

\begin{tabular}{|c|c|c|c|c|c|}
\hline \multirow[t]{2}{*}{ B) } & & \multicolumn{2}{|l|}{$D$-cysteine } & \multicolumn{2}{|l|}{$L$-cysteine } \\
\hline & & Experiment & Theory & Experiment & Theory \\
\hline \multirow[t]{2}{*}{$\mathrm{N}-\mathrm{C}_{\alpha}$} & $\theta$ (deg.) & 74 & 74 & 76 & 69 \\
\hline & $\phi$ (deg.) & 35 & 37 & 116 & 126 \\
\hline Energy (eV) & $E_{\text {model }}$ & & -2.17 & & -2.03 \\
\hline
\end{tabular}

Figure 44. A) Structural model of Cys/Au(17 11 9)-S. The unit cell of the (17 11 9) surface is marked with dashed lines. In the case of L-cysteine the amino group binds to a step atom, while for D-cysteine it binds to the kink atom. The color code of the atoms is given on the left. B) Orientation of the $\mathrm{N}-\mathrm{C}$ bond and calculated adsorption energies of $\mathrm{D}$ - and L-cysteine on $\mathrm{Au}(1711$ 9). The polar angle is measured from the surface normal, the azimuthal angle is measured clockwise from the <14-3> direction. C) XPS spectra for D- (blue) and L-cysteine (red) on $\mathrm{Au}(17$ 11 9)-S. In the N 1s region (left), two or one Gaussians were necessary to fit the L- or D-cysteine spectra, respectively. The $\mathrm{NH}_{2}$ component dominates but, in the case of L-cysteine, also a $\mathrm{NH}_{3}{ }^{+}$component is visible as a high binding-energy shoulder. Spectra in the S2p region (right) are fitted with a doublet of Gaussians with a fixed spin-orbit splitting of $1.2 \mathrm{eV}$ and an intensity ratio of 1:2. Taken with permission from refs. $[65 ; 135]$.

\subsubsection{Other amino acids at Au surfaces.}

Much less work has been performed on the interaction of amino acids others than Cys, at least under controlled UHV conditions. Glycine was found to physisorb on $\mathrm{Au}(110)$ at room temperature [113]. An STM investigation was performed as a function of coverage and deposition rate. At low deposition rate, imaging of the $(1 \times 2)$ reconstruction becomes difficult, indicating that the adsorbates 
exist in the 2D gas phase as in the case of adsorption of amino acids on $\mathrm{Cu}(001)$ [98]. In the present case individual molecules are not visible by means of STM because the diffusion barrier is low, smaller than in the caseof chemisorption, and thus the molecules diffuse too fast to be imaged. The relevance of this work lies in the fact that a physisorbed system can induce a significant restructuring of the substrate. In fact, already at low deposition rate, the $(1 \times 2)$ reconstruction converts into a well-developed $(1 \times 3)$ geometry (see Figure $45 \mathrm{a})$. Chemisorption-induced changes in surface reconstruction are well documented[236; 237]. A physisorption-induced change, however, is a more surprising phenomenon; it is driven by the fact that the atomic corrugation of the $(1 \times 3)$ reconstructed surface is significantly larger than that of the $(1 \times 2)$ one, with $(111)$ nanofacets long enough to accommodate one Gly molecule. Deposition of glycine at medium rate resulted in many different visible features as well as in significant surface morphology changes. A chain phase with molecules aligned into chains of $\sim 45 \AA$ length, further aggregated into domains separated by domain walls, coexists with at least three other different superstructures: $\mathrm{Au}(110) \mathrm{c}(4 \times 4)-\mathrm{Gly}$, $\mathrm{Au}(110) \mathrm{X}$-Gly (not identified in the paper) and $\mathrm{Au}(110) \mathrm{c}(2 \times 4)$-Gly (see Figure 45b). Deposition with the high rate resulted in a dramatic change in the surface morphology, where larger and smaller pits as well as facets exist everywhere (Figure 45c). High resolution images show that the molecules are actually well ordered, especially in the $\langle 1-10\rangle$ direction (as evidenced in the high resolution image of Figure 45d), similarly to the images of the 2D solid phase of amino acid adsorbates on the $\mathrm{Cu}(100)$ surface $[95 ; 98 ; 139 ; 181]$. Therefore the authors propose that, as in the case of $\mathrm{Cu}(100)$, molecules are flat-lying on the surface and stabilized by $\mathrm{H}$ bonds. At variance with $\mathrm{Cu}(100)$, in the present case the $2 \mathrm{D}$ molecular net is incommensurate with the surface, at least in the direction perpendicular to $\langle 1-10\rangle$, as a consequence of the physisorption character of the present system.

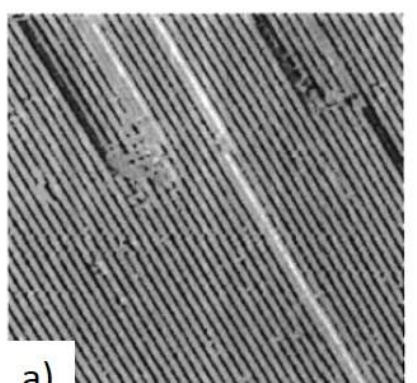

a)

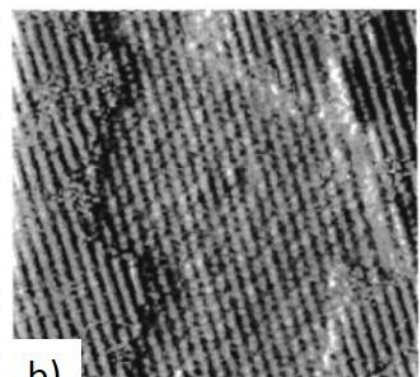

b)

Figure 45. a) STM image (500 $₫ \times 500 \AA, 1.0 \mathrm{~V}, 0,6 \mathrm{nA})$ showing the (1 x 3)-Au(110) surface obtained at the end of the low-rate Gly deposition process. B) Coexistence of different structures upon Gly deposition at intermediate rate: the $\mathrm{Au}(110) \mathrm{c}(4 \times 4)$-Gly superstructure is surrounded by the chain phase (image size: $250 \AA \times 250 \AA, \mathrm{V}=1.0 \mathrm{~V}, \mathrm{I}=0.6 \mathrm{nA}$ ). c) Large-scale image ( $820 \AA \mathrm{x} 820$ $\AA, V=1.2 \mathrm{~V}, \mathrm{I}=0.6 \mathrm{nA}$ ), showing the surface faceting during high rate deposition of Gly at room temperature. D) Enlarged image ( $55 \AA \times 55 \AA, \mathrm{V}=1.0 \mathrm{~V}, \mathrm{I}=0.6 \mathrm{nA})$ of the ordered adsorbates on facets. Taken with permission from ref. [113].

(S)-Lysine adsorption at $\mathrm{Au}(111)$ induces the formation of $\mathrm{Au}$ nanofingers growing from faceted step-edges [153]. As evident in Figure 46a, these nanostructures are already present at $300 \mathrm{~K}$, become more dense and longer upon annealing (Figure 46b) and disappear at $400 \mathrm{~K}$. The hearing bone reconstruction is not lifted by Lys adsorption. Au nanofingers formation had already been 
produced by nanomanipulation with the STM tip [238], but a clear conclusion of ref. [153] is that the displacement of $\mathrm{Au}$ atoms from step edges and their reorganisation into nanofingers is induced by Lys adsorption. A similar molecular mechanism was reported by Arima et al. [239], who found that thiol immobilisation enhanced the mobility of $\mathrm{Au}$ adatoms on $\mathrm{Au}(111)$. Domains of ordered lysine molecules could be observed at RT only after several hours (see Figure 46c), suggesting high diffusion rates. They are rotated by $120^{\circ}$ with respect to each other, indicating that the underlying substrate influences the packing of the amino acid molecules. Each lysine unit is typically observed as a bright "head" with a fainter "tail", so that the authors propose a structure consisting of chains of lysine molecules aligned head-to-tail and running parallel to the $\langle 4-51\rangle$ direction, with adjacent chains running in opposite directions. As a consequence, "head-to-head" alignment occurs in the $<1-54>$ direction (the short diagonal of the unit cell -Figure 46c), suggesting an H-bonding interaction between $\mathrm{COOH}$ groups on adjacent lysine molecules. The empirical model was followed by DFT calculations proving that the most stable conformation is a dimer of two neutral lysine molecules bound by two $\mathrm{O}-\mathrm{H}$... O hydrogen bonds and attached to the surface by the nitrogen of the $\varepsilon-\mathrm{NH}_{2}$ group sitting directly atop a gold atom (see Figure 46d-f). The $\alpha-\mathrm{NH}_{2}$ does not bind to the substrate since it is unable to preferentially bind at an on top site, which is the energetically favoured one. The dimer units are connected by an $\mathrm{H}$-bonded $\mathrm{COOH}-\mathrm{COOH}$ bridge and the ordered structure is stabilised by these intermolecular hydrogen bonds and, possibly, via $\mathrm{O}$... $\mathrm{H}-\mathrm{N}$ interactions with the $\varepsilon-\mathrm{NH}_{2}$ groups. The direction of growth of the gold nanofingers appears to correlate very closely with the direction of growth of the chains of Lys dimers, but unfortunately no images of Lys molecules close to nanofingers are available. Therefore the authors speculate that the atomic structure at the edges of the nanofingers is controlled by interactions with lysine molecules. Furthermore, no nanofingers growing along the $\langle-1-45\rangle$ direction, i.e. the mirror equivalent surface azimuthal direction to the <1-54> direction, were ever observed in these experiments, providing evidence that (S)-lysine causes chiral faceting of the Au surface. 


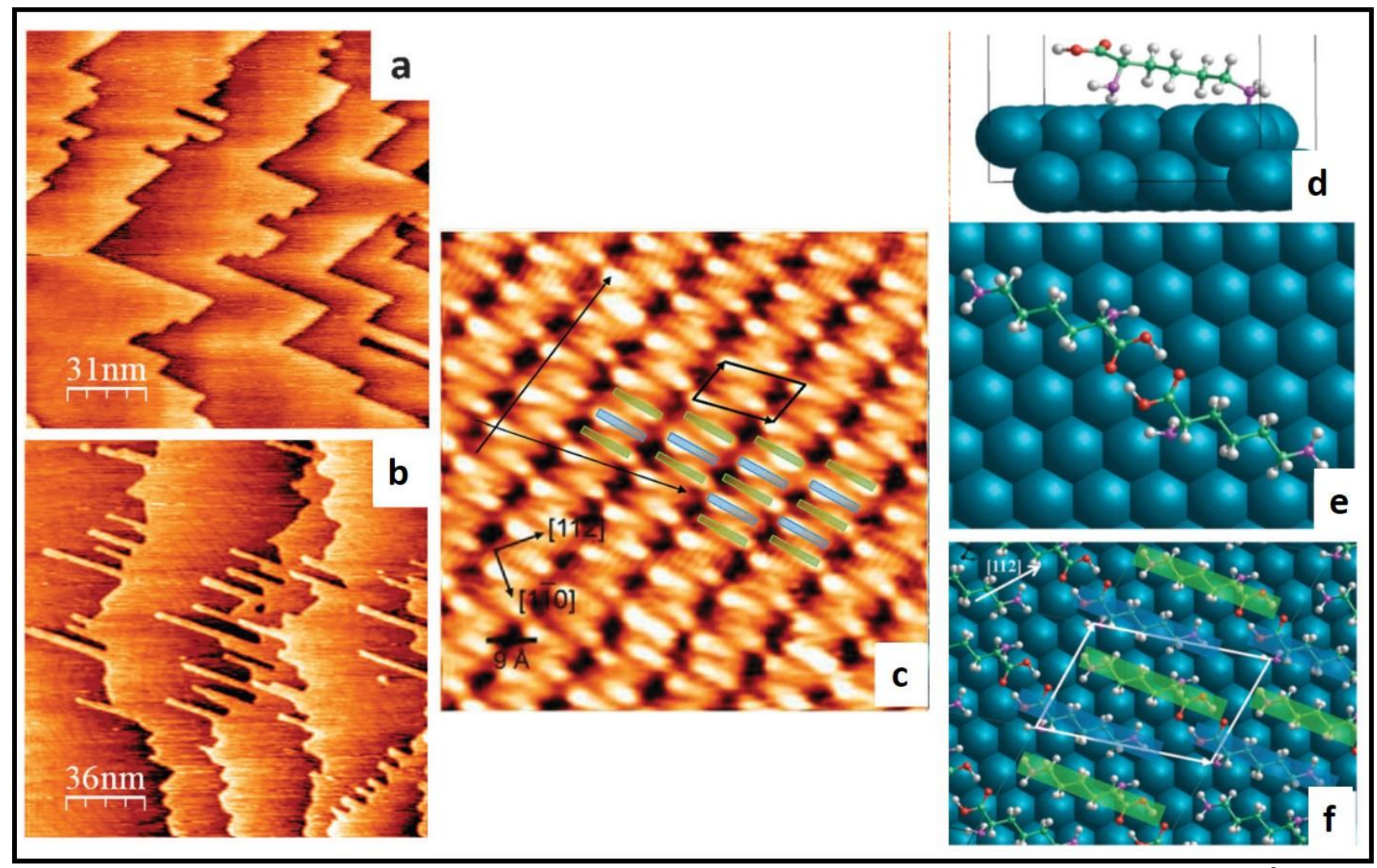

Figure 46. a) STM images of (S)-lysine on $\mathrm{Au}(111)$ at $\mathrm{T}=300 \mathrm{~K}$ (Image size $155 \times 155 \mathrm{~nm}^{2}, \mathrm{~V}=0.4$ $\mathrm{V}, \mathrm{I}=0.2 \mathrm{nA}$ ). Same as a), after flashing the Lys-covered sample to $373 \mathrm{~K}$ (image size $180 \times 180$ $\left.\mathrm{nm}^{2}, \mathrm{~V}=0.8 \mathrm{~V}, \mathrm{I}=1 \mathrm{nA}\right)$. The apparent height of the nanofingers is $(2.30 \pm 0.06) \AA$, consistent with the $\mathrm{Au}(111)$ step height $(2.35 \AA)$, while their length is variable. The longest finger found was 85 $\mathrm{nm}$. C) STM image of an ordered (S)-Lys domain at $300 \mathrm{~K}$ (Image size: $8 \times 8 \mathrm{~nm}^{2}, \mathrm{~V}=0.9 \mathrm{~V}, \mathrm{I}=0.6$ $\mathrm{nA})$. The high symmetry directions are marked. In addition, a unit cell with lattice constants of (8.7 $\pm 0.3) \AA \times(13.3 \pm 0.4) \AA$ and an angle between the unit cell vectors of $109^{\circ}$ is shown. d-f $)$ Optimized adsorption geometry of a single lysine molecule (d- side view), of a lysine dimer (e top view) and of the extended overalyer ( $\mathrm{f}$ - top view). Taken with permission from ref. [153].

The interaction of proline with the $\mathrm{Au}(111)$ surface has also been recently investigated by the same group by means of STM, XPS and HREEL spectroscopy [159]. It was shown that Pro is able to adsorb onto $\mathrm{Au}(111)$ at $300 \mathrm{~K}$ forming a 2D gas which condenses into self-assembled structures upon cooling to $77 \mathrm{~K}$. Nucleation occurs at surface defects such as step edges and soliton boundaries (e.g. protrusion) of the herringbone reconstruction, coherently with previous observations, e.g., for cysteine [127]. Two kinds of assemblies are observed by STM: 1D ordered islands of few tens of $\mathrm{nm}$ in length and a few $\mathrm{nm}$ in width and other islands with a more random internal molecular arrangement. The adsorbed proline interacts only weakly with the Au surface; at submonolayer coverage almost complete desorption occurs around $370 \mathrm{~K}$. XPS indicates that proline exists as a mixture of the neutral, zwitterionic, and anionic forms (see Table 3), while HREELS experiments were only able to detect the last two forms. It is proposed that this inconsistency may be due to the much longer time scale of HREELS experiments with respect to XPS measurements: with time the neutral form may be able to deprotonate with the driving force being the facile liberation of $\mathrm{H}_{2}$ into the gas phase. The dimensions of the ordered structures are consistent with the formation of a 2-D analogue of the 3-D crystal structure of proline (which exists 
in the zwitterionic form in the solid state). It is speculated that the disordered regions consist of a random mixture of the neutral, zwitterionic, and anionic proline units.

Methionine adsorption on $\mathrm{Au}(111)$ is interesting since this amino acid self-assembles into structures which are quite similar to those observed on $\mathrm{Cu}(111)$ [62] and $\mathrm{Ag}(111)$ [63]. It is thus possible to evidence the influence of the substrate in the overlayer formation. XPS analysis of Met/Au(111) samples (see Table 3 for the $E_{b}$ values) indicates that, in the monolayer regime, the molecules adsorb in the zwitterionic form and do not undergo any chemical change with increasing coverage. STM data show that the self-assembly of D-, L-methionine layers on Au(111) occurs already at RT through the formation of parallel molecular rows into three well-ordered domains $(\alpha, \beta$ and $\delta)$ rotated by $120^{\circ}$ from each other [155]. Among them, the $\alpha$ orientation is predominant and corresponds to the most favourableconformation. Due to the lack of a functional group with a strong affinity to gold, such as for cysteine, the effect of the substrate geometry contributes to the orientation of molecules only at coverage largely below surface saturation. Therefore, at the early stage of growth, the nucleation occurs only on the fcc regions of the Au(111) surface, owing to its low coordinated Au atoms. Following the behaviour of the three domains with coverage, the authors conclude that the 2D ordering of Meton $\mathrm{Au}(111)$ is a result of an intermolecular equilibrium within the film rather than a substrate induced arrangement. In addition, the molecule-surface interactions are rather weak, allowing significant mobility and rearrangement of molecules. This is further proved by the low desorption temperature of the methionine layer, which does not survive annealing to $365 \mathrm{~K}$, and by the recovery of the original herringbone structure of the $\mathrm{Au}(111)$ surface. These data point towards a non-covalent bonding character of the interactions between molecules and $\mathrm{Au}(111)$. 


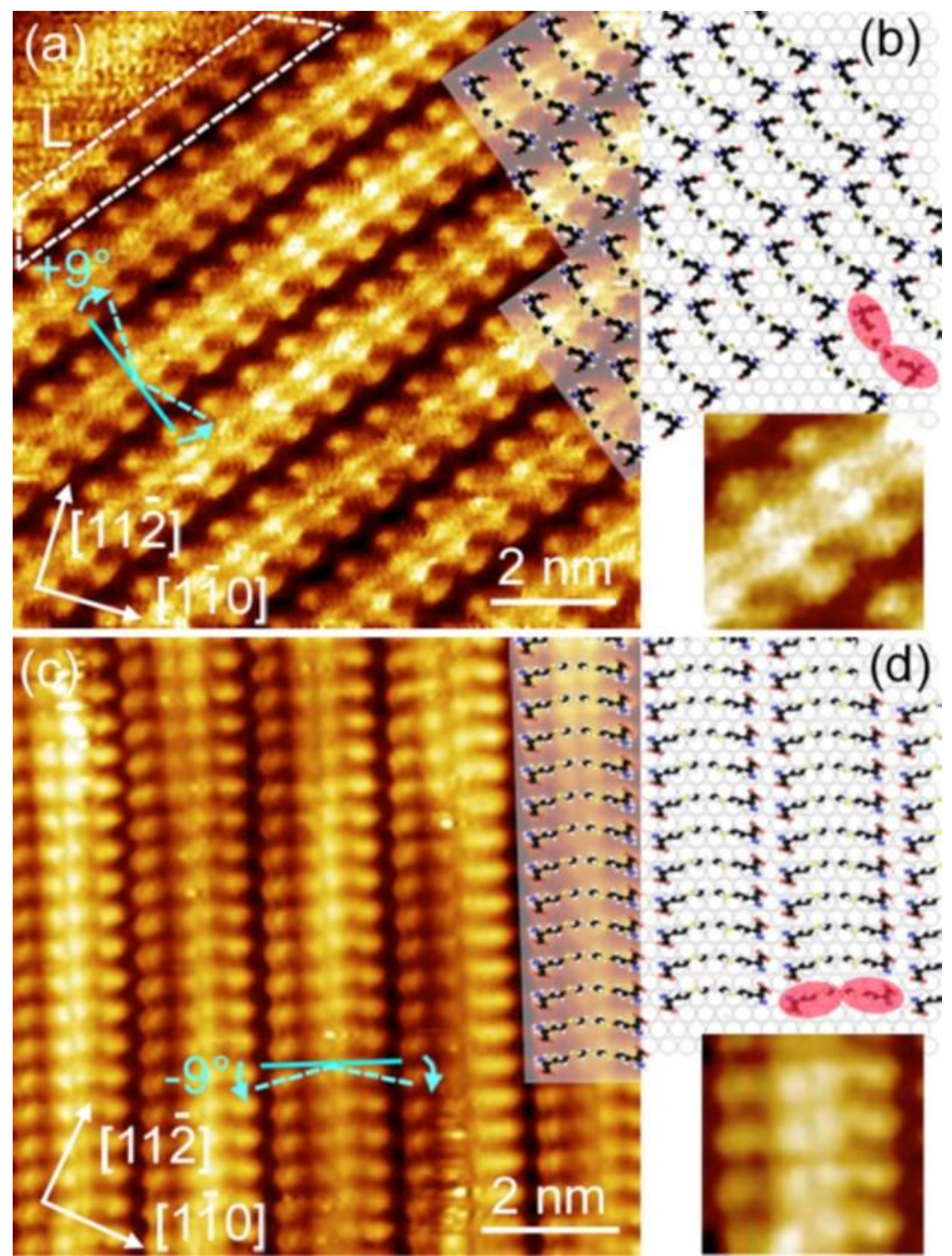

Figure 47. a) and c) Molecular resolution STM images ( $\mathrm{V}=-1 \mathrm{~V}, \mathrm{I}=200 \mathrm{pA}$ ) acquired on a $\alpha$ domain area from $\mathrm{D}$ - and L-Met on $\mathrm{Au}(111)$, respectively. B) and d) illustrate the corresponding model structures. The insets in $b$ and $d$ are STM images magnifications to highlight molecular dimers. Taken with permission from ref. [155].

The arrangement of D- and L-Met units within the self-assembled pattern in deduced on the basis of high resolution STM images as those reported in Figure $47 \mathrm{a}$ and c. The structure consists of molecular dimers organised into stable and parallel rows separated by a distance of $2.6 \AA$. The row width of $13.1 \AA$, too large to be the length of one single Met molecule $(8 \AA)$ and too narrow for a dimer, indicates that the pair of molecules in one chain is not lying flat. It is rather standing with an angle of $32^{\circ}$ with respect to the surface plane (also confirmed by IR data). D-met (L-Met) chains in the $\alpha$ domains are always running $40^{\circ}$ clockwise (counter clockwise) with respect to the closepacked $\langle 11-2\rangle$ direction, which breaks the mirror symmetry of the gold surface. This result is related to the chirality of methionine molecules themselves.

On the basis of the images of Figure 47 the authors drew the following adsorption model, which was recently confirmed by DFT calculations and simulation of the STM images [68]: a single pair of Met units consists of two molecules that are tilted by $9^{\circ}$ towards the chain axis, and thus form an acute angle with each other (as marked in Figure 47a and c). This tilt is related to the tendency of molecules to arrange in order to minimize the short-range steric repulsion from two adjacent 
$\mathrm{CH}_{3}$ groups of their tails and to accommodate the attractive interactions between the positively charged $\mathrm{NH}_{3}{ }^{+}$head with the negatively charged $\mathrm{COO}^{-}$group of the nearest moleculeof the neighbouring chain. The separation of $2.6 \AA$ between two parallel chains is consistent with a zwitterionic hydrogen-bonding distance. On the contrary, the periodicity along a chain is $6.9 \AA$, which indicates that the interactions between two neighbouring pairs in this direction are likely weak. This periodicity is incidental and the pair formation is driven by hydrogen bonding that contributes strongly to the stabilization of the chains and consequently to the $2 \mathrm{D}$ arrangement. In fact, no single chain of pairs was observed in the middle of $\alpha$ domains, but at least two adjacent chains are required for a stable domain to settle. Therefore, one can exclude the mechanism, active on $\mathrm{Cu}$, by which the molecules build up as pairs first, followed by a single chain formation. The self-assembly consists rather in an equilibrium within the film, in which at least two adjacent chains are required for stability, whereby the interchain interactions play a crucial role.

Finally, we mention that the same Met/Au(111) film was produced by deposition from solution. In this case, of course, the characteristics of the layer were also dependent on the solution $\mathrm{pH}$. Such substrate was successfully employed for chiral recognition of a relatively long, chiral polypeptide, i.e. L-gramicidine [240].

Histidine interaction with $\mathrm{Au}$ surfaces attracted some attention due to the good affinity of the imidazole ring with Au. This system was studied by means of XPS and NEXAFS spectroscopies first on polycrystalline $\mathrm{Au}(111)$ [149] surfaces and eventually on a $\mathrm{Au}(111)$ single crystal [241; 242]. Adsorption in the monolayer and multilayer regimes was investigated on the former surface, while coverages of $0.5 \mathrm{ML}$ and 1.0 ML of His were explored on the latter. On the single Au(111) crystal adsorption from the gas phase and from aqueous solution yield identical spectroscopic signatures [242]. Typical XPS spectra are reported in Figure 48, while the $\mathrm{E}_{\mathrm{b}}$ values are summarised in Table 3. The $\mathrm{C} 1 \mathrm{~s}, \mathrm{~N} 1 \mathrm{~s}$ and $\mathrm{O} 1 \mathrm{~s}$ peaks, corresponding to the $0.5 \mathrm{ML}$ film, are all slightly downshifted in binding energy with respect to thoserecorded for the full monolayer film. This effect is ascribed to final state screening, which is more efficient in thinner films [149]. The A and B peaks in the $\mathrm{C} 1 \mathrm{~s}$ region are assigned to the core level of the carboxylate $\mathrm{C}$ and to the unresolved contributions of the other $\mathrm{C}$ atoms, respectively. The N1s spectra show two main peaks, whose ratio changes with coverage. Peak B is identified with the imino nitrogen atom, while the two amino nitrogen atoms (of the amino group and the IM ring) contribute to the A peak. The peak separation of $2.05 \mathrm{eV}$ is significantly larger than the one observed between the two nitrogen atoms of imidazole in the solid and gas phases [243] or in multilayers. Moreover, the amino peak position shifts by only $0.1 \mathrm{eV}$ between the multilayer and monolayer spectra (due to more efficient screening by the metal), while the corresponding shift of the imino peak is $0.6 \mathrm{eV}$. From comparison of the spectra recorded for different His coverage values, and also considering the lack of an effect on the $\mathrm{N}$ 1s core level spectra when to annealing to $400 \mathrm{~K}$, the authors suggest that histidine binds to the surface via the imino nitrogen atom, so that the imidazole ring is oriented to allow its interaction with the surface. This is in agreement with results on polycrystalline Au [149] but in contradiction with previous RAIRS studies [244]. Furthermore, we note that Zubavichus et al. proposed that also the amino group contributes to the histidine-substrate bonding [149]but no signature of this interaction is present in the spectra of Figure 48b. The authors of this last work concluded that probably the nitrogen atom of the amino group is not strongly bound to the Au(111) surface. The discrepancy may be related to the different crystallinity of the samples employed for the two 
experiments. Only one feature is present in the $\mathrm{O} 1 \mathrm{~s}$ region, which indicates the presence of a deprotonated carboxylic group with the two oxygen atoms in very similar chemical states. This is in accord with RAIRS data of His/Cu(110) [147] but inconsistent with the results of Liedberg et al. [244], who proposed coordination of the carboxylate group of His on gold by only one oxygen atom, and olny in partial agreement with the data of Zubavichus et al. [149], who showed one or two peaks in the O1s spectra depending on sample preparation. The overall picture proposed by Feyer et al. [242], also on the basis of complementary NEXAFS analysis, is that His molecules interact with the gold surface in the anionic form via the two oxygen atoms of the carboxylate group and the imino nitrogen atom of the imidazole ring, as schematically represented in Figure 48d. The bond to the carboxylate group is stronger than to the imino group, so that at high coverage not all His molecules bond through the IM group. From the $\mathrm{N}$ and $\mathrm{O}$ K-edge NEXAFS spectra measured at different incidence angles with respect to the surface, tilt angles of the IM ring and COO- triangle of $\sim 35^{\circ}$ and $\sim 27^{\circ}$ with respect to the $\mathrm{Au}(111)$ surface, respectively, were deduced.

We further mention that His/Au(111) and His/Au(110) were investigated by the same experimental means also to unravel the role of $\mathrm{pH}$ on the final adsorption form of His, when deposited from solution [241]. Finally, the work on polycrystalline films also evaluated the damage induced on the monolayer or multilayer His films by intense or prolonged X-ray exposure and the effect of water coadsorption [149]. They found that soft X-ray irradiation induces a discharge of the zwitterions present in the multilayer films to form neutral molecules and a mass loss via decarboxylation. Water is dissolved in the multilayer histidine film and partly dissociated, producing hydroxyl anions and increasing the degree of protonation of the histidine molecules. 


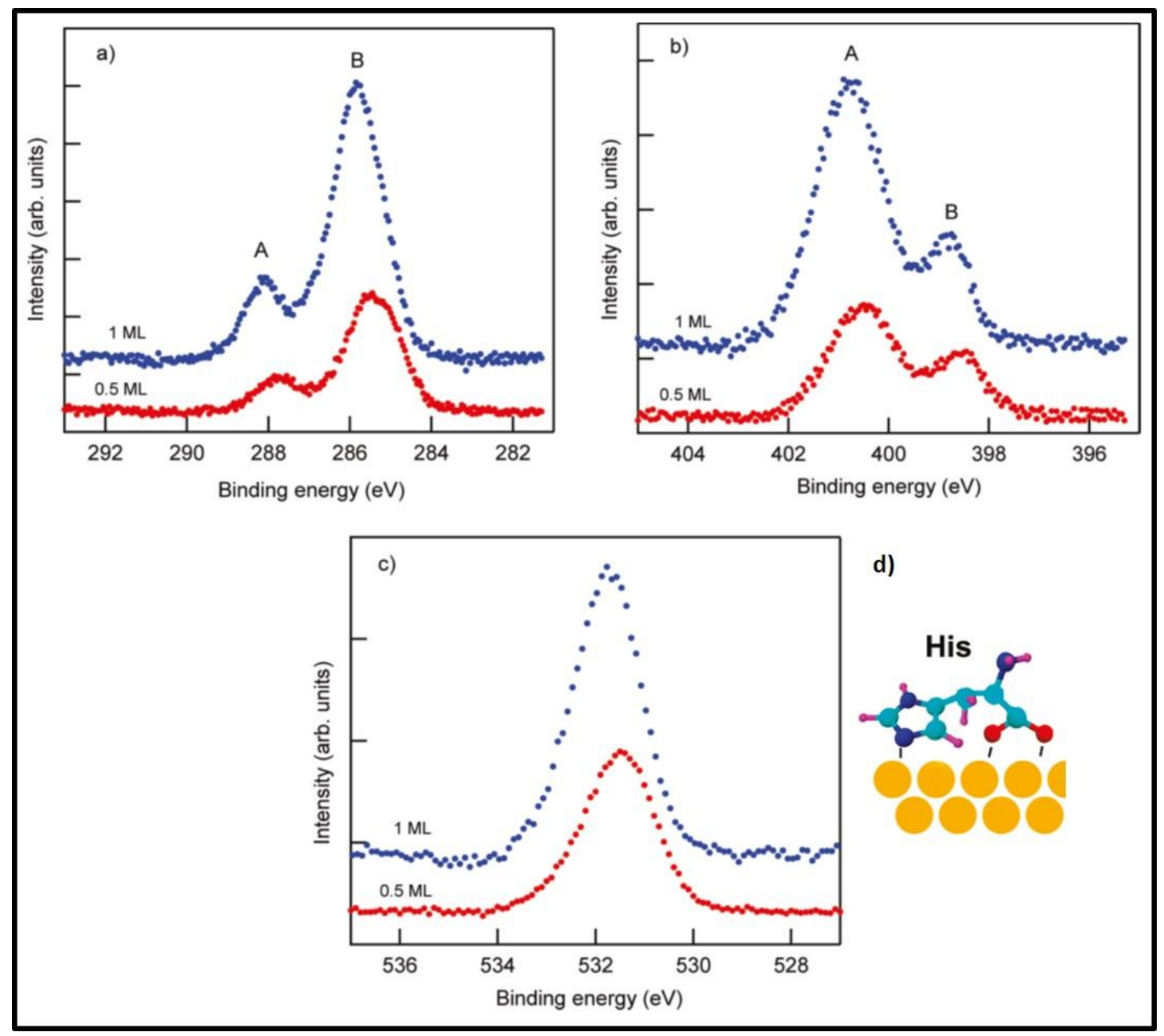

Figure 48. C 1s (a), N 1s (b), and O 1s (c) core level spectra of histidine adsorbed by evaporation in UHV onto Au(111). (d) Adsorption model of His on the same surface. Taken with permission from ref. [242].

A complete study of L-3,4-dihydroxyphenylalanine (L-DOPA, a catechol containing AA) (see Figure 49d for the molecular structure) on $\mathrm{Au}(110)$ [219] was performed to describe the selforganization pattern at the surface. The study includes many experimental techniques such as LEED, FTIR, XPS, HREELS, and STM, combined with DFT. It was found that zwitterionic LDOPA interacts with the gold surface through two of its functional groups, the phenyl ring and the carboxylate group. The other groups are involved in the intermolecular interactions of the assembly. The (2×1)-Au(110) reconstruction is not lifted. The L-DOPA molecules form dimers, within a super structure matrix $\left(\begin{array}{cc}1 & 2 \\ -1 & 3\end{array}\right)$, as determined by LEED images. Since L-DOPA interacts with the surface through its catechol group, it is expected that L-DOPA containing peptides and proteins play a key role in the anchoring of these molecules to a gold surface. 


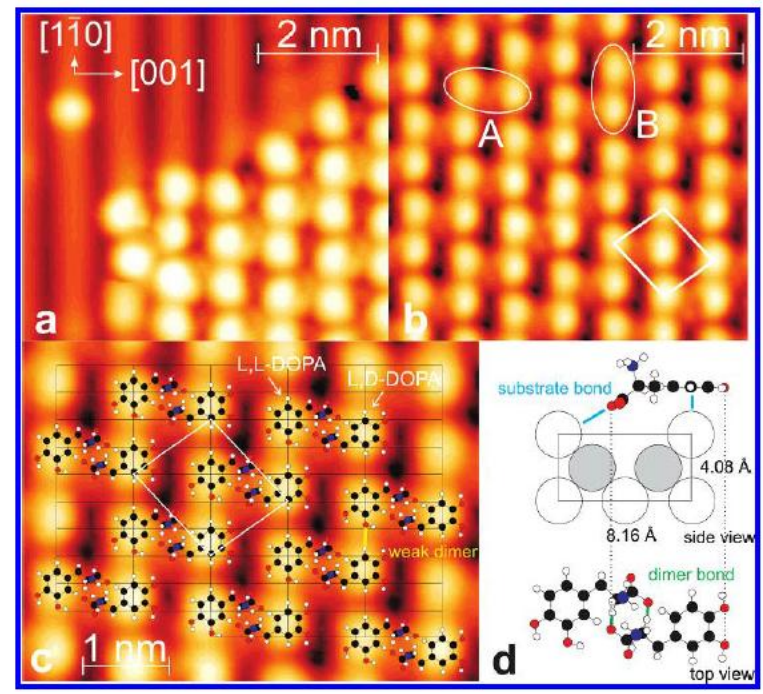

Figure 49. STM images recorded from a submonolayer of L-DOPA on $\mathrm{Au}(110)-(1 \times 2)(\mathrm{V}=313 \mathrm{mV}$, $\mathrm{I}=75 \mathrm{pA}$ ). a) Image size $6.1 \times 5.3 \mathrm{~nm}^{2}$, taken close to the boundary of a L-DOPA island. In the upper left-hand part of the image ridges and grooves of the reconstructed Au surface are visible. B) Image size $6.5 \times 5.7 \mathrm{~nm}^{2}$, recorded inside a large L-DOPA island. The superstructure unit cell $\left(b_{1}=9.8 \AA\right.$, $\mathrm{b}_{2}=11.5 \AA, \theta=100^{\circ}$ ) is shown in white. Two types of dimerizations (A, B) are indicated. C) Enlarged section of image $b$, with $(2 \times 1)$ reconstructed unit cells (black lines) and superstructure unit cell (white lines) marked. The structure model proposed is superimposed to the experimental image. D) Side view of L-DOPA on the $(2 \times 1)$ reconstructed $\mathrm{Au}(110)$ surface (top) and top view of a dimer from part c. In parts c and d, various intermolecular and molecule substrate bonds are indicated in yellow, blue, and green. Taken with permission from ref. [219].

Finally, we wish to mention a few studies of amino acid adsorption at gold surfaces which were not performed under UHV conditions but which are however significant with respect to our dissertation, since the combination of experimental data and theoretical simulations provides a nice description of the adsorption configuration at the molecular level.

Aromatic amino acids (phenylalanine, tyrosine, and tryptophan) immobilized on single gold nanoplates were investigated by Tip Enhanced Raman Scattering (TERS) and DFT [245]. In contrast to observations in Raman or SERS, in all TERS spectra the characteristic ring breathing modes of the aromatic unit of the molecules were hardly detected. This fact can be readily explained by the unique geometric rigidity of the TERS setup that is comparable to single-crystal Raman experiments and is consistent with aromatic rings lying flat on the gold surface. The main interaction of the molecules within the gold-silver sandwich (Au nanoplate - amino acid - Ag covered tip) occurs via the carboxyl and amino functionalities and can be perceived in the enhanced signal intensities in the TERS spectra. The experimental results were supported by theoretical calculations of the molecules taking into account the presence of the metal tip. It is interesting to note that the stable geometries flat and parallel to the surface are not significantly perturbed by the presence of the tip, whereas the carboxylic groups can suffer a rotation. Such a comparison, never performed before, provides insight into the behaviour of biomolecules between two metal layers.

The adsorption and oxidation of glycine [246], L-alanine [247] and L-serine [247] at Au single crystals and at evaporated thin-film electrodes with preferential (111) orientation was studied in acidic solution. Cyclic voltammetry experiments were combined with external reflection IR 
spectroscopy (for single crystals) or surface-enhanced infrared reflection-absorption spectroscopy under attenuated total reflection conditions (ATR-SEIRAS, for thin films). Theoretical harmonic vibrational frequencies obtained from DFT calculations of the AA species adsorbed on Au clusters with (111) orientation were used to interpret the experimental spectra. For all the molecules, the optimized geometry obtained from DFT calculations for a zwitterion plus a water molecule corresponds to a $\mu 2$ asymmetrical bridge configuration under the application of an external electric field of 0.01 atomic units. The absence of the asymmetric OCO stretching mode in the experimental infrared spectrum, confirms the $\mu 2$ bonding of the amino acidthrough the oxygen atoms of the carboxylate group, irrespective of the crystallographic orientation of the electrode surface, the adsorbate coverage, and/or the electrode potential.

In addition, we wish to mention an overview of theoretical studies on AA adsorption at Au surfaces [248]. DFT calculations of amino acid fragments in vacuum and molecular dynamics simulations of the interaction of all amino acids with a $\mathrm{Au}(111)$ surface in explicit solvent were reported, using the polarizable force field GolP. The preferred orientations of the amino acids on the metal surface were described. It is found that all amino acids preferably interact with the gold surface at least partially with their backbone, underlining an unfolding propensity of gold surfaces. In this nice overview[248],combining DFT and MD simulations the adsorption properties of all amino acids were described (see Figures 1 and 2 in ref. [248]). An intriguing interplay between steric and electrostatic factors was found. In passing from molecules adsorbed on gold in vacuum to molecule adsorbed in water, microscopic effects tend to favour orientations, where the charges are exposed to the solvent.

\subsubsection{Amino acid adsorption at Ag surfaces}

The choice of $\mathrm{Ag}$ as a substrate for AA adsorption came only after that several studies on $\mathrm{Cu}$ and some on Au. The interest in this system lies in the search for a substrate which is reactive enough to allow AA to stick at RT also in absence of a S-group, but less reactive that $\mathrm{Cu}$ and other transition metals. Controlling the self-assembly of molecules at surfaces and thus the interplay between binding to the surface and cohesion between the molecules is a challenge for surface chemistry. Indeed, the binding energy should be low enough to avoid chemisorption, which might be followed by reaction (activation of the molecule on a heterogeneous catalyst), and to maintain the original properties of the adsorbing molecules. Therefore one should prefer weak adsorption compensated by strong cohesive interactions, which is the reason at the basis of the richness of structures observed on the $\mathrm{Ag}(100)$ surface. The intermediate reactivity of $\mathrm{Ag}$ allows indeed to let intermolecular bonds prevail over substrate-molecule attraction and to minimize substrate-induced molecular deformation. In this respect it is worth mentioning as an example that the calculated adsorption energy of phenylalanine in its most stable configuration is $-0.3 \mathrm{eV}$ on $\mathrm{Ag}(111)$ and -0.5 $\mathrm{eV}$ and $-1.3 \mathrm{eV}$ on the corresponding $\mathrm{Cu}$ and $\mathrm{Pt}$ surfaces, respectively [249]. From the experimental point of view, glycine does not chemisorb on clean Ag at $300 \mathrm{~K}$ due to the inability of this substrate to deprotonate it into glycinate [162].

Since much less systems have been investigated so far with respect to the case of AA adsorption on $\mathrm{Cu}$ surfaces, a systematic report is not possible for Ag. However all the studies aim at unravelling two major points, i.e. the chemical state of the adsorbed molecules and the formation of self- 
assembled layers of well-defined geometry. Although for sake of clarity we discuss them separately, of course the two topics are strictly correlated and treated together in most of the papers.

\subsubsection{Adsorption state of AA at Ag surfaces}

A systematic study of the chemical state of AA adsorbed at electrochemically roughened $\mathrm{Ag}$ surfaces was performed by Stewart and Fredericks by Surface Enhanced Raman Scattering (SERS) [172]. Their analysis over 19 AA species allowed them to conclude that in all cases adsorption occurred via the ionized carboxylate group and that the side chain of most of the molecules was also in close proximity to the surface. In contrast, the amino group was protonated and quite far away from the substrate. For S-containing species an additional molecule-substrate interaction was provided by the presence of the sulphur atom. For our purposes, the importance of this experiment is in the fact that it is the first methodical study of AA adsorption on a silver surface. However, SERS measurements were performed in air and after deposition from a liquid solution. Therefore it is not surprising that contrasting results are found in UHV experiments after AA deposition from the gas-phase.

As already mentioned, Barth et al. studied the interaction of some amino acids with noble metal surfaces using a multi-technique approach (XPS, NEXAFS, STM and molecular mechanics calculations). In particular, they investigated the adsorption of L-Cysteine, L-Tyrosine and LMethionine on $\operatorname{Ag}(111)[2 ; 44 ; 45 ; 63]$. In these papers, the chemical state of the molecule is strictly correlated to the formation of well-defined molecular architectures based on strong intermolecular interactions. In the last two cases the XPS signature clearly reveals a zwitterionic nature of the adsorbates for all investigated conditions (see Table 3; $\mathrm{E}_{\mathrm{b}}(\mathrm{N} 1 \mathrm{~s})=401.15 \mathrm{eV}$ and $402.2 \mathrm{eV}$ for Met and Tyr, respectively). The difference in $\mathrm{E}_{\mathrm{b}}(\mathrm{N} 1 \mathrm{~s})$ observed between the two AA is ascribed to an increased distance of the Tyr ammonium group from the surface, resulting in a reduced screening process of the $\mathrm{N}$ core-hole by the substrate conduction electrons. The $\mathrm{E}_{\mathrm{b}}(\mathrm{O} 1 \mathrm{~s})$ values are coherent with this picture, showing a single peak at $531.2 \mathrm{eV}$ for Met and a doublet at $532.0 \mathrm{eV}$ (carboxylic oxygen) and $533.5 \mathrm{eV}$ (oxygen from the additional hydroxyl group) for Tyr.

On the contrary, three different adsorption regimes are identified for Cys/Ag(111) [44]: 1) the multilayer, in which Cys zwitterions physisorb on top of an already present monolayer of molecules and maintain their SH-group intact; 2) the $\alpha$ phase, consisting of a single layer of zwitterionic molecules chemisorbed through their deprotonated S-group; 3) the $\beta$ phase, produced upon annealing the $\alpha$ phase to $\mathrm{T}>393 \mathrm{~K}$ and consisting in a mixture of anionic and neutral molecules. While many amino acids adsorb in an anionic form upon deposition [175], thermally activated deprotonation of the ammonium group was previously reported only for Met on $\mathrm{Cu}(111)$ [62]. However, in that case deprotonation was caused by the significant reactivity of the underlying crystal, a condition which does not hold with the more inert silver surface.

L-Cys was also investigated in detail by DFT methods in the group of Santos [163-166]. The AA was adsorbed on $\mathrm{Ag}(111)$ as a radical $\left(\cdot \mathrm{S}-\mathrm{CH}_{2}-\mathrm{CH}-\mathrm{NH}_{2}-\mathrm{COOH}\right)$ and as a zwitterionic radical (.S$\mathrm{CH}_{2}-\mathrm{CH}-\mathrm{NH}_{3}{ }^{+}-\mathrm{COO}^{-}$). At low coverage, it was found to adsorb almost horizontally on the surface, showing the role of the carboxyl group in the interaction with the substrate. At higher coverage, using $4 \times 4$ and $(\sqrt{3} \times \sqrt{3}) \mathrm{R} 30^{\circ}$ unit cells (seeFigure 50), Cys was found to adsorb as a zwitterion and almost on a bridge site. In this case special attention was paid to the electronic structure of the 
system in order to understand the chemical bonds formed during chemisorption. A complex interplay between sp and d states of silver in the formation of covalent bonds between the AA and the surface was found.

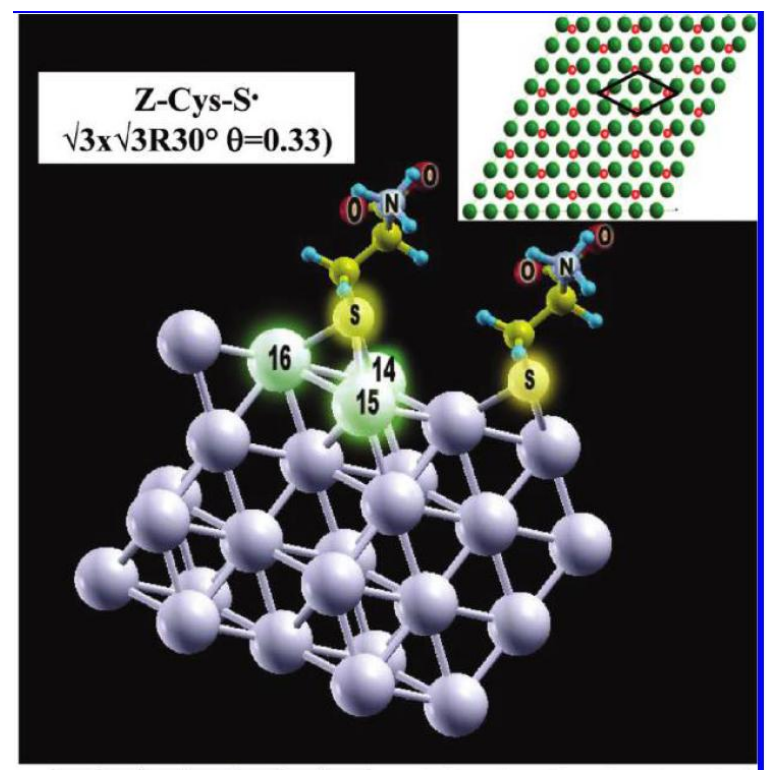

Figure 50Optimised Z-cys adsorbed at a bridge shifted toward hep site $(\theta=0.33 \mathrm{ML})$. The unit cell $(\sqrt{ } 3 \times \sqrt{ } 3) \mathrm{R} 30^{\circ}$ is also shown. Taken with permission from ref.[163].

The similarity of the chemical behaviour of Met, Tyr and Cys adsorbed on $\operatorname{Ag}(111)$ at RT and the observation of similar self-assembled the patters for the first two systems (see next section) lead the authors to suggest that the zwitterionic non-covalent intermolecular bonding scheme may represent a general construction motif on weakly reactive close-packed metal surfaces [45]. This behaviour may indeed be common also to other, not yet investigated, AA systems, but the idea of a universal trend was proved to be wrong by the case of Glu/Ag(111)[53].

The chemical form of Glutamic acid adsorbed at low Miller index Ag faces was thoroughly studied both by photoemission and by vibrational spectroscopy [51; 53; 54] (see Figure 51 and Table 8). From the analysis of RAIR spectra of the Glu/Ag(110) system at $\mathrm{T}=300 \mathrm{~K}$ (Figure 51a) the following adsorption model is drawn by the authors: Glu adsorbs in its anionic form (carbonyl bands frequency never higher than $1723 \mathrm{~cm}^{-1}$ ) with carbonyl groups involved either in intermolecular interactions or in direct interactions with the Ag substrate. Only one carbonyl group is deprotonated and, since the $\mathrm{pKA}$ of the amino acid $\mathrm{COOH}$ group is considerably lower than that of the aliphatic acid group, deprotonation is likely to involve the amino acid functionality. The $\mathrm{N}$ atom of the $\mathrm{NH}_{2}$ group interacts directly with the surface. 


\begin{tabular}{|c|c|c|c|c|c|}
\hline Vibrational mode & (a) Glu/Ag(111) & (b) $\mathrm{Glu} / \mathrm{Ag}(100)$ & (c) Glu/Ag(110) & $\begin{array}{l}\text { (d) Glu } \\
\text { (Zwitterionic) }\end{array}$ & $\begin{array}{l}\text { (e) Glu (Non- } \\
\text { Zwitterionic) }\end{array}$ \\
\hline$\delta(\mathrm{COOH})$ & 540,612 & 516,620 & & 571 & $430,525,599-618$ \\
\hline$\gamma(\mathrm{HOCC})$ & 790 & \multirow[t]{2}{*}{$725-757$} & & 731,757 & 792 \\
\hline$\delta_{s}\left(\mathrm{COO}^{-}\right)$ & & & & & \\
\hline $\mathrm{r}\left(\mathrm{CH}_{2}\right)$ & \multirow[t]{2}{*}{$895-918$} & \multirow[t]{2}{*}{951} & & 809,965 & 846,1043 \\
\hline$v(C C)$ & & & 891 & 899,937 & 897,1043 \\
\hline \multicolumn{6}{|l|}{$w\left(\mathrm{NH}_{2}\right)$} \\
\hline$v(\mathrm{CN})$ & & $1046-1080$ & & 965,1029 & 1110 \\
\hline $\mathrm{r}\left(\mathrm{NH}_{3}{ }^{+}\right)$ & & & & 1320,1353 & \\
\hline $\mathrm{t}\left(\mathrm{CH}_{2}\right)$ & \multirow[t]{2}{*}{1265} & \multirow[t]{2}{*}{1265} & & 1151,1204 & $1246,1304,1376$ \\
\hline$w\left(\mathrm{CH}_{2}\right)$ & & & 1330 & $1307-1320$ & 1322,1418 \\
\hline$v_{s}\left(\mathrm{COO}^{-}\right)$ & $1378-1427$ & 1394 & 1387 & 1422 & \\
\hline$\delta_{s}\left(\mathrm{NH}_{3}{ }^{+}\right)$ & & & & 1521 & \\
\hline$v(\mathrm{CO})$ & \multirow[t]{2}{*}{$1378-1427$} & & & 1390 & 1367 \\
\hline$\delta\left(\mathrm{CH}_{2}\right)$ & & 1394 & 1462 & 1441,1506 & $1492-1515$ \\
\hline$v_{a}\left(\mathrm{COO}^{-}\right)$ & \multirow[t]{2}{*}{1652} & \multirow[t]{2}{*}{$1628-1693$} & & 1618 & \\
\hline$\delta\left(\mathrm{NH}_{2}\right)$ & & & 1574 & & 1634 \\
\hline$\delta_{a}\left(N_{3}{ }^{+}\right)$ & & & & $1618-1646$ & \\
\hline$v(\mathrm{COOH})$ & 1725 & $1628-1693$ & & & \\
\hline$v(C=0)$ & 1725 & (1628-1693) & $1651,1692,1723$ & 1654 & $1765-1778$ \\
\hline$v_{s}\left(\mathrm{CH}_{2}\right)$ & \multirow[b]{2}{*}{ 2950-2974 } & \multirow[t]{2}{*}{2958} & 2946 & $2942-2952$ & 2972 \\
\hline$v(\mathrm{CH})$ & & & & 2987-3006, 3055 & 3022 \\
\hline$v_{a}\left(\mathrm{CH}_{2}\right)$ & & 3087 & & 2987-2999 & 3089 \\
\hline$v_{s}\left(\mathrm{NH}_{3}{ }^{+}\right)$ & & & & 3089 & \\
\hline$v_{a}\left(N_{3}{ }^{+}\right)$ & & & & 3147-3167 & \\
\hline$v_{s}\left(N_{2}\right)$ & & & & & 3402 \\
\hline$v_{a}\left(\mathrm{NH}_{2}\right)$ & & & & & 3507 \\
\hline
\end{tabular}

Table 8. Summary of the vibrational frequencies observed for Glu adsorption on the different HMI Ag faces and of their assignment. The calculated vibrational frequencies for isolated molecules $[250 ; 251]$ are reported for comparison. We remark that, for $\mathrm{Glu} / \mathrm{Ag}(110)$, the band at $1651 \mathrm{~cm}^{-1}$ is very close in frequency to the $v(\mathrm{C}=\mathrm{O})$ band of the zwitterionic form [250]. However, it may be explained as a carbonyl band strongly downshifted by intramolecular interactions. The alternative hypothesis that the zwitterionic species is present at low coverage [170] is not true for the other LMI Ag surfaces and it must therefore be considered less probable. Data taken from refs. [53] (column a); [54] (column b); [51] (column c); [250] (column d) and [251]. 


\section{a) $\mathrm{Glu} / \mathrm{Ag}(110)$}

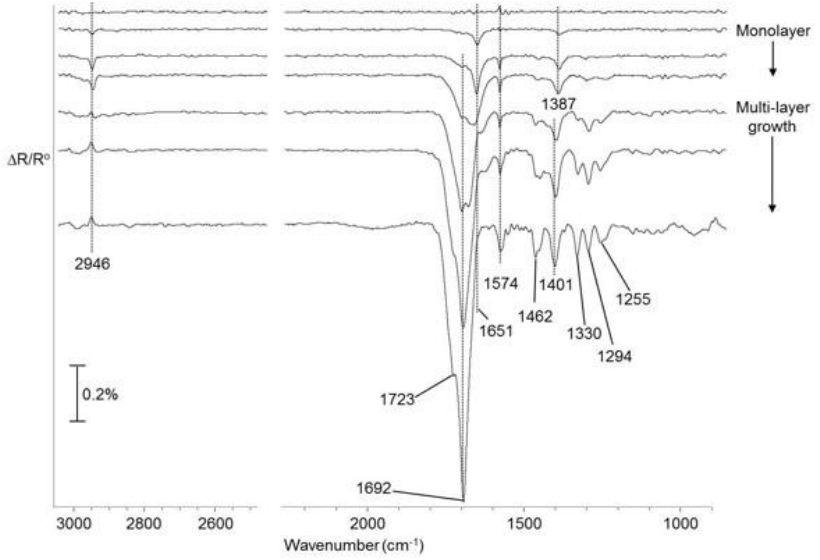

b) $\mathrm{Glu} / \mathrm{Ag}(100)$

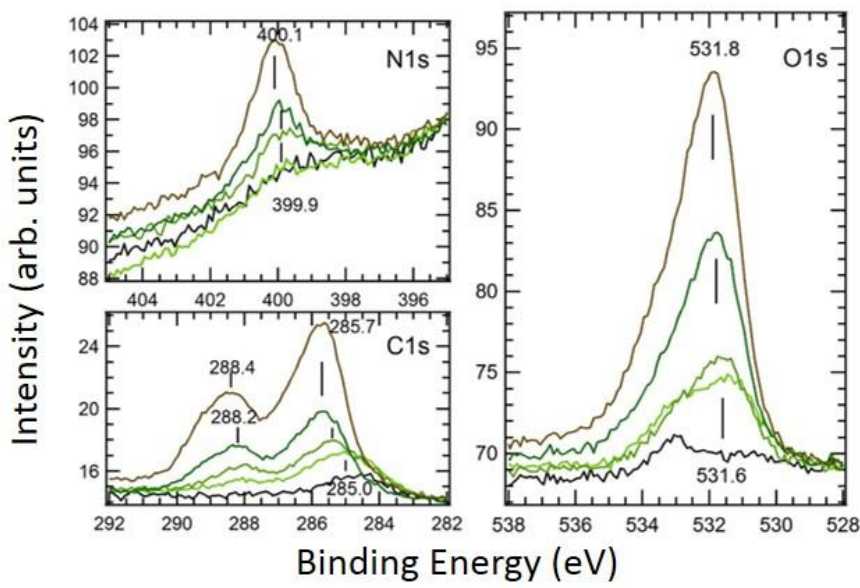

c) $\mathrm{Glu} / \mathrm{Ag}(111)$

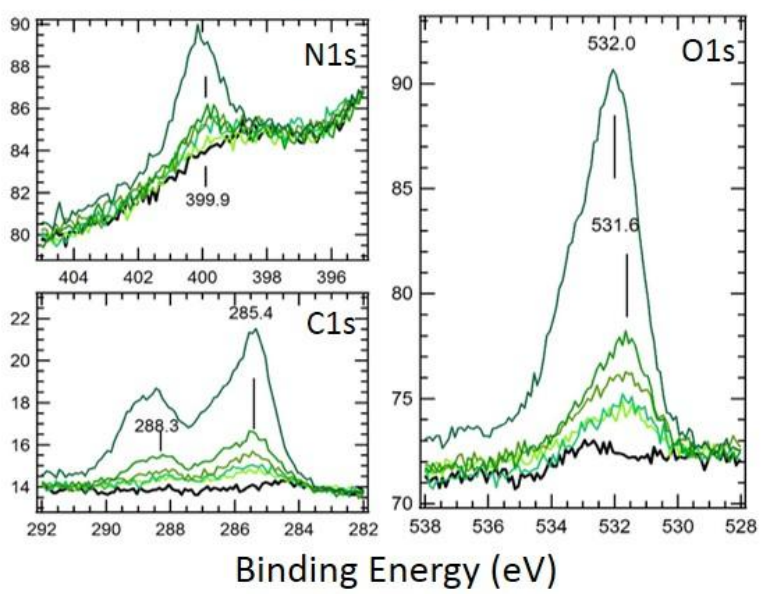

Figure 51. Glu adsoption at LMI Ag faces at $\mathrm{T}=300 \mathrm{~K}$. a) Glu/Ag(110). RAIRS spectra. B) Glu/Ag(100). XPS spectra of the N1s, C1s and O1s regions after Glu exposures of $0 \mathrm{~s}, 30 \mathrm{~s}, 60 \mathrm{~s}$, $120 \mathrm{~s}, 720 \mathrm{~s}$, respectively. C) Same as b) for Glu/Ag(111). Exposure times are $0 \mathrm{~s}, 30 \mathrm{~s}, 60 \mathrm{~s}, 120 \mathrm{~s}$, $240 \mathrm{~s}$ and $480 \mathrm{~s}$. Partly taken with permission from ref.[51]

Figure 51b and c reports the parallel experiments performed on $\operatorname{Ag}(100)$ and $\operatorname{Ag}(111)$ and monitored by XPS, which allows for a much more straightforward interpretation of the results. At $300 \mathrm{~K}$ there is evidence from STM that adsorption occurs in the monolayer regime. Under these conditions, the $\mathrm{E}_{\mathrm{b}}(\mathrm{N} 1 \mathrm{~s})$ line is stably around $400.0 \mathrm{eV}$, revealing a clear non-zwitterionic adsorption on both surfaces. Varying the adsorption temperature in the range $250 \mathrm{~K} \leq \mathrm{T} \leq 400 \mathrm{~K}$ does not change the outcome except for a small shift to $400.5 \mathrm{eV}$ at $\mathrm{T}=250 \mathrm{~K}$ and high coverage, when the second layer starts to form. To distinguish between adsorption in the neutral or in the anionic form from the XPS spectra of Figure 51 requires a quantitative analysis of the $\mathrm{O} 1 \mathrm{~s}$ region [48; 252]. Both for Glu/Ag(100) and Glu/Ag(111), a single peak is present at $531.3 \mathrm{eV} \leq \mathrm{E}_{\mathrm{b}} \leq 532.6 \mathrm{eV}$, the lower values corresponding to higher temperature and smaller coverage. It can be separated into two components (O533 and $\mathrm{O} 531$ in the following), corresponding to the $\mathrm{O}$ atoms involved in $\mathrm{OH}$ groups (at higher $\mathrm{E}_{\mathrm{b}}$ ) and to the $\mathrm{O}$ atoms double bonded to $\mathrm{C}(\mathrm{C}=\mathrm{O})$ or in carboxylate groups $\left(\mathrm{COO}^{-}\right.$ ). The neutral or anionic nature of the amino acid layers is then deduced from the O533/0531 ratio. Considering the structure of Glu (see Figure 2) and applying this strict model, one should expect a 
1:1 ratio for adsorption in the neutral form, and a 1:3 ratio for adsorption in the anionic form. Experimentally, the O533/O531 ratio is always lower than 1, ranging from $\sim 0.85$ to $\sim 0.33$ depending on dosing conditions on both surfaces [53; 54]. A mixture of neutral and anionic adsorption must therefore be present. Since the O533/O531 ratio decreases with coverage, the latter species must become dominant as soon as a critical Glu density is reached on the surface. Note that the model applied is quite rigid and does not keep into account that the O1s binding energy may be affected also by other effects, e.g. by hydrogen bonds [51]. Theoretical DFT-D modelling of the Glu layers forming on $\mathrm{Ag}(100)$ at $\mathrm{T}=350 \mathrm{~K}$ confirms however this empirical analysis, by suggesting the existence of two stable structures consisting of neutral molecules [59] and of a mixture neutral and anionic molecules in equal amounts[253], respectively.

The adsorption probability of Glu on $\operatorname{Ag}(100)$ and $\operatorname{Ag}(111)$ is strongly temperature dependent, as demonstrated by the experiments summarised in Figure 52[53; 54]. No multilayer formation was detected on $\operatorname{Ag}(100)$ for $\mathrm{T} \geq 300 \mathrm{~K}$ and on $\mathrm{Ag}(111)$ for $\mathrm{T} \geq 250 \mathrm{~K}$ within the investigated exposure range. The sticking probability, which is proportional to the slope of these curves, decreases with increasing $\mathrm{T}$ (see inset of Figure 52A), indicating a precursor mediated adsorption mechanism. Both the final coverage and the adsorption probability are systematically lower for $\mathrm{Ag}(111)$, confirming the weaker reactivity of the closed packed surface.

The $\mathrm{C} 1 \mathrm{~s}$ region shows peaks in the $\mathrm{E}_{\mathrm{b}}$ ranges [285.0, 286.2] $\mathrm{eV}$ and [288.0, 288.9] eV, respectively. As for the O1s and N1s peaks, Ebslightly upshifts with coverage. Each peak consists of two components, which are related to the four non-equivalent $\mathrm{C}$ atoms of the deprotonated molecule.

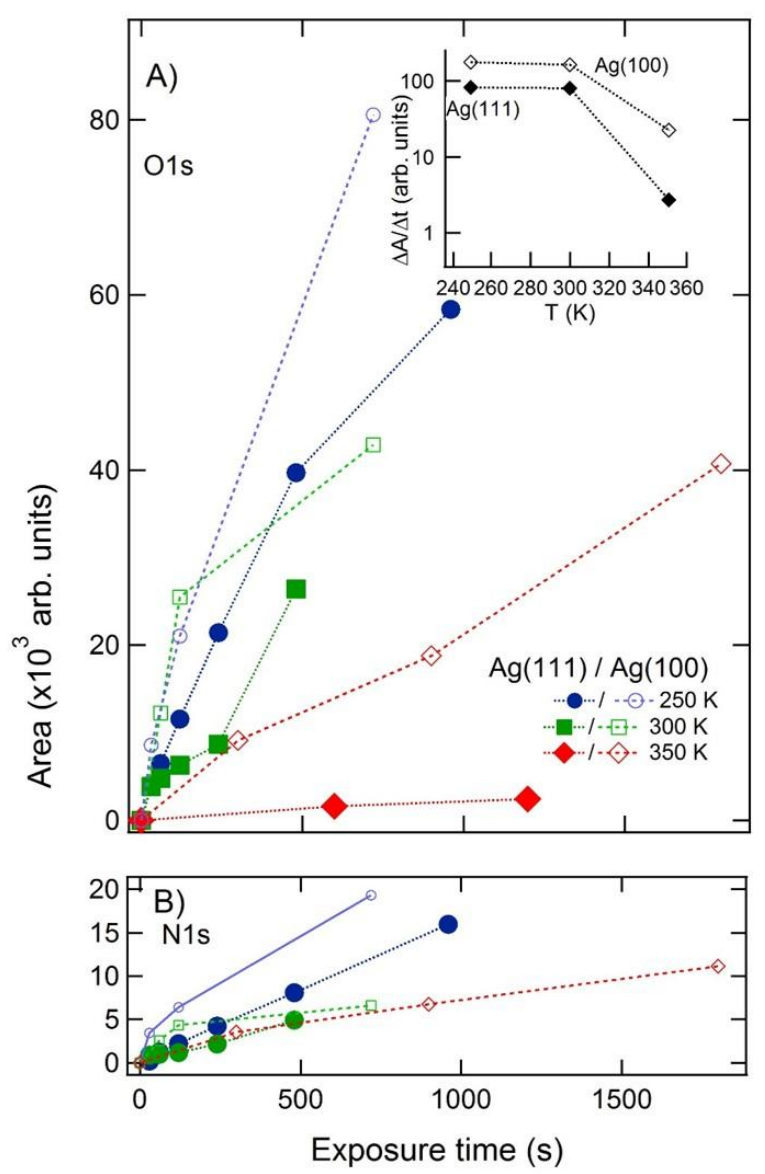

Figure 52. Total area of the O1s and N1s photoemission signals vs exposure time and parametric in deposition temperature for Glu adsorption on $\mathrm{Ag}(111)$ (full symbols) and $\mathrm{Ag}(100)$ (empty 
symbols). The inset in panel A reports the ratio between the area of the O1s peak and the exposure time, which is proportional to the sticking coefficient, vs T. Figure made from data of refs. [53; 54].

Finally, we report the case of Gly adsorption on $\mathrm{Ag}$ films deposited on $\mathrm{Cu}(001)$ and on mica [162]. On the one hand, Zhao and co-workers based their investigation on the premises that Ag surfaces should be able to chemisorb glycinate, since it has a bonding orbital very similar to the one of formate, which chemisorbs on Ag. On the other hand, SERS data [172] indicate that only zwitterionic adsorption (indicating physisorption) occurs for Gly/Ag. For $\mathrm{Ag} / \mathrm{Cu}(001)$ there is no intermixing of $\mathrm{Ag}$ and $\mathrm{Cu}$ atoms, and the former species stays at the surface. At submonolayer coverage, $\mathrm{Ag}$ atoms arrange into islands with the $\mathrm{c}(2 \times 10)$ superstructure, separated by $\mathrm{Cu}$ regions with a small amount of alloyed Ag atoms (see Figure 53a). For $\Theta_{\mathrm{Ag}}>1 \mathrm{ML}$ the areas covered by two Ag layers show a (111)-like geometry, whereas single layers maintain the $c(2 \times 10)$ structure. The structure of the Ag film on mica always shows hexagonal close packing. Upon exposure of Gly at $\mathrm{T}_{\mathrm{ev}}=300 \mathrm{~K}$, no adsorption is detected on mica and on $\mathrm{Ag} / \mathrm{Cu}(001)$ for $\Theta_{\mathrm{Ag}}>1 \mathrm{ML}$. On the contrary, if $\Theta_{\mathrm{Ag}}<1 \mathrm{ML}$, features of glycine adsorbates can cover the entire surface. STM inspection (see next section) revealed the formation of Gly stripes very similar to those already observed on $\mathrm{Cu}$ [110] and persisting on the surface after 5 minutes of annealing to $450 \mathrm{~K}$. Such behaviour allows to estimate a binding energy of the adsorbed molecules not lower than $1.4 \mathrm{eV} /$ molecule and, in turn, that the adsorption of the glycine molecules on the $\mathrm{Ag} / \mathrm{Cu}(001)$ surface must be chemisorption. The explanation provided by the authors is that pure Ag surfaces have the ability to chemisorb glycinate molecules, but not to deprotonate Gly molecules into glycinate. The latter must originate from the spillover source, i.e., the nearby $\mathrm{Cu}$ nanopatches.

\subsubsection{Self-assembly of AA at Ag surfaces.}

The tendency of AA to self-assemble in ordered patterns is confirmed also in the case of poorly interactive substrates as the Ag ones, whenever the molecule-substrate attraction is strong enough to favour stable adsorption either in the physisorbed or in the chemisorbed state.

As a first example we provide in Figure 53b an STM image of a Gly layer formed on the reconstructed $\mathrm{Ag} / \mathrm{Cu}(001)$ film described in the previous section [162]. Specifically, in presence of the $\mathrm{c}(2 \times 10)$ superstructure and at low Gly coverage, the surface is uniformly covered by chains lying exclusively in the $<110>$ directions of the substrate and having a width of about $5 \AA$ (see Figure 53b), just like the molecular chains observed from the Gly/Cu systems [110]. At saturation coverage, on the contrary, the narrow $\mathrm{Cu}$ areas evident on the surface prior to Gly deposition coalesce into larger patches covered by the typical $\mathrm{Cu}(001)-\mathrm{c}(2 \times 4)$-glycine superstructure [98], and the Gly chains on Ag are now aligned in the four equivalent <320> directions (see Figure 53c). The similarity of these molecular self-assembly with the one reported for $\mathrm{Gly} / \mathrm{Cu}$ surfaces [95; 110] suggests that also in this case glycine molecules adsorb in the two-points $(\mu 2)$ adsorption conformation and are connected by hydrogen bonds. The formation of linear chains of molecules is observed also upon Gly deposition on $\mathrm{Ag} / \mathrm{Cu}(111)$ only if the surface is not fully covered by $\mathrm{Ag}$ atoms. 

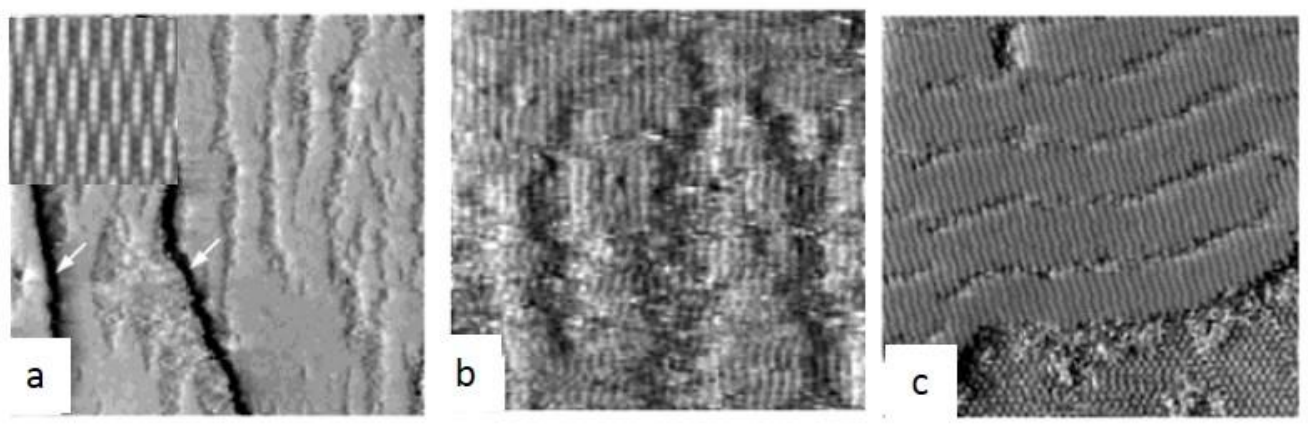

Figure 53. a) Low-magnification STM image (500 $₫ \times 500 \AA, 1.0 \mathrm{~V}, 0.6 \mathrm{nA})$ of the $\mathrm{Cu}(001)$ surface covered with $\sim 0.7 \mathrm{ML}$ of Ag atoms. The two dark lines on the left (marked with white arrows) are surface steps. Ag atoms substitute only the first layer of $\mathrm{Cu}$ atoms and form the $\mathrm{c}(2 \times 10)$-Ag islands (the brighter areas) separated by slightly lower and thus darker regions, which consist of $\mathrm{Cu}$ atoms and a small amount of Ag atoms. Inset: Atom-resolved STM image (image size: $40 \AA \times 40 \AA, \mathrm{V}=20$ $\mathrm{mV}, \mathrm{I}=3 \mathrm{nA})$ of the $\mathrm{c}(2 \times 10)-\mathrm{Ag}$ structure. B) STM image (image size: $180 \AA \times 180 \AA, \mathrm{V}=1.0 \mathrm{~V}$, $\mathrm{I}=0.6 \mathrm{nA})$ of the same $\mathrm{Ag} / \mathrm{Cu}(001)$ surface as in (a) but after adsorption of glycine. The adsorbed glycine molecules form chains lying in the <110> direction. C) STM image ( $230 \AA \times 230 \AA, V=-1.0$ $\mathrm{V}, \mathrm{I}=0.6 \mathrm{nA}$ ) obtained after further deposition of glycine onto the surface imaged in $\mathrm{b}$ ). In this case the molecular chains on the $\mathrm{c}(2 \times 10)-\mathrm{Ag}$ structure align in the $\langle 320\rangle$ directions, whereas the $\mathrm{Cu}$ regions now coalesce into larger islands covered by the $\mathrm{c}(2 \times 4)$-glycine superstructure (see the lower portion). Taken with permission from ref. [162].

a)

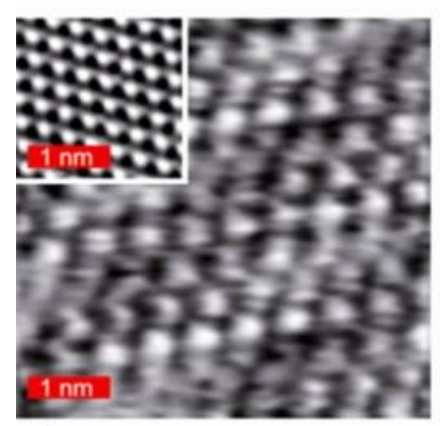

c) b)

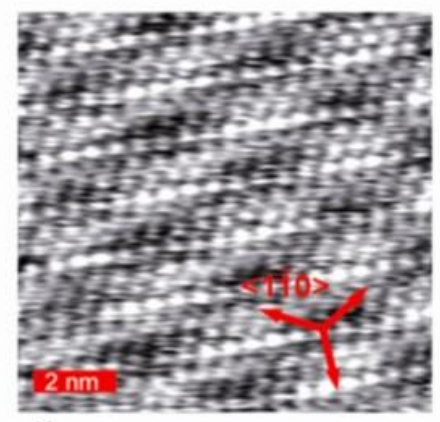

d)

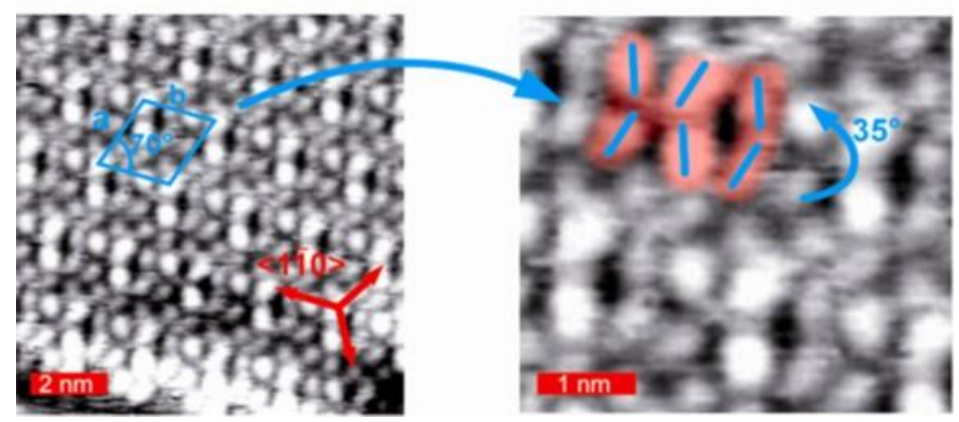

Figure 54. STM images of L-cysteine adsorbed on the $\operatorname{Ag}(111)$ : (a) Hexagonal structure $\alpha$ after room-temperature deposition $(\mathrm{I}=100 \mathrm{pA}, \mathrm{V}=1.25 \mathrm{~V})$. Inset: atomically resolved image of the $\operatorname{Ag}(111)$ substrate $(\mathrm{I}=-170 \mathrm{pA}, \mathrm{V}=-0.88 \mathrm{~V}) . \mathrm{b})$ Moiré pattern of the $\alpha$ structure $(\mathrm{I}=100 \mathrm{pA}, \mathrm{V}=$ $1.25 \mathrm{~V})$. c) $\beta$-structure formed after annealing the system to $393 \mathrm{~K}(\mathrm{I}=320 \mathrm{pA}, \mathrm{V}=1.25 \mathrm{~V})$; the rombic unit cell (marked in blue) consists of nine molecules and is described by the lattice vectors $a=(1.80 \pm 0.04) \mathrm{nm}$ and $b=(1.86 \pm 0.09) \mathrm{nm}$, forming an angle of $70^{\circ}$ with each other. In $\left.b\right)$ and $\left.c\right)$, 
the high symmetry axes of the substrate are marked in red.D) Enlargement of c) with the molecular pairs lining up along the $\langle 1-10\rangle$ direction, highlighted in red and their molecular orientation indicated in blue. Taken with permission from ref. [44].

The Cys/Ag(111) system was investigated thoroughly by STM both in the $\alpha$ and $\beta$ phases (see section3.2.2.1)[44]. In the $\alpha$ phase (zwitterionic), the molecules build up a close-packed hexagonal structure with a nearest neighbour distance of $(5.8 \pm 0.2) \AA$ and a density of 3.66 molecules $/ \mathrm{nm}^{2}$ (Figure 54a). The Moirépattern evident in Figure 54b indicates that the dense-packed layer is incommensurate with the underlying $\operatorname{Ag}(111)$ substrate. This suggests the presence of multiple adsorption sites for cysteine units on $\operatorname{Ag}(111)$, similarly to what observed for layers of organic thiols on $\mathrm{Ag}(111)$ [254] and $\mathrm{Au}(111)$ [255]. The unit cell axes of the $\alpha$ phase are rotated by $30^{\circ}$ with respect to the silver $\langle 1-10\rangle$ directions. In the submonolayer regime, molecules assemble into islands extending over $50 \mathrm{~nm}$ and occasionally covering the entire atomically flat terrace. Annealing to $393 \mathrm{~K}$ causes the transition to $\beta$ phase, which is accompanied by a complete reorganization of the organic layer (Figure 54c). Such conformational change, confirmed also by NEXAFS experiments, is driven by the different charge distribution within the molecule when passing from the zwitterionic $\alpha$ phase to the non-zwitterionic $\beta$ phase. The lower molecular density of the latter layer (2.78 molecules $/ \mathrm{nm}^{2}$ ) and the disappearance of the Moiré pattern indicate a change of the binding site selectivity of the molecules during heat treatment. The chirality of L-Cys is reflected by the appearance of three clockwise symmetrically equivalent domains. The $\beta$ phase is characterized by molecular rows of L-Cys pairs (marked in red in Figure 54d) running along the $\mathrm{Ag}(111)<1-10>$ axis and sandwiched between molecular rows with lower corrugation. The molecules within a pair form an angle $<180^{\circ}$ and are mirror images of each other with respect to the $\operatorname{Ag}(111)<1-10>$ direction. We note that, since the AA remains attached to the surface through the S-group in both the $\alpha$ and $\beta$ configurations, the utility of Cys molecules as a fundamental unit in metal-tethered AA sequences is confirmed.
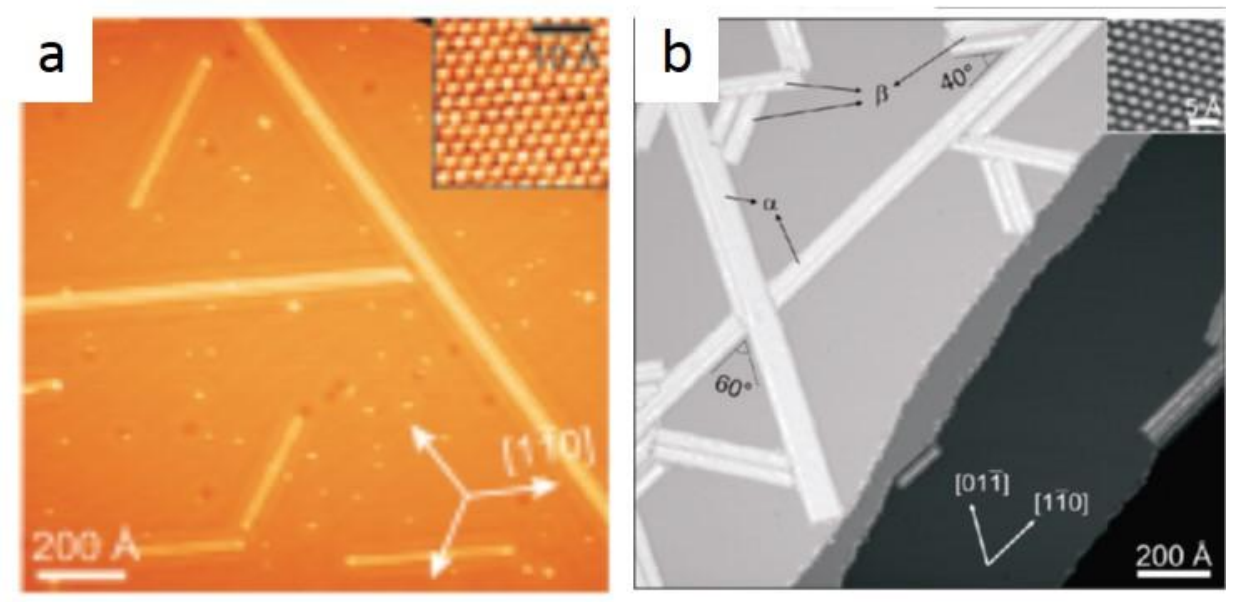

Figure 55. Constant-current STM topographs of L-methionine (a) and L-tyrosine (b) on $\mathrm{Ag}(111)$. In both cases the molecules self-assemble in extended 1D arrangements following the close-packed <110> orientation of the substrate. A): $\mathrm{I}=0.7 \mathrm{nA}, \mathrm{V}=-120 \mathrm{mV}$; L-Met was deposited on the substrate held at $\mathrm{T}_{\mathrm{ev}} \sim 320 \mathrm{~K} . \mathrm{b}$ ): $\mathrm{I}=0.1 \mathrm{nA}, \mathrm{V}=500 \mathrm{mV}$; the L-Tyr layer was produced by deposition at $170 \mathrm{~K}$ and annealing of the crystal to $320 \mathrm{~K}$. Insets: atomic resolution image of the $\operatorname{Ag}(111)$ substrate. Taken with permission from refs.[45; 63]. 
As mentioned in the previous section, the same research group performed interesting studies on the self-assembly of Met and Tyr on $\mathrm{Ag}(111)$. In both cases at $\mathrm{T}=320 \mathrm{~K}$ the molecules organize in stripes oriented along the three equivalent $\langle 1-10\rangle$ directions and with very long linear extension (see Figure 55), to indicate that site-specific bonding at the surface is decisive in both the Lmethionine and in the L-tyrosine self-assembly 110ormats110. The mesoscopic ordering and the supramolecular interactions in the high coverage limit are however different. A comparative analysis is therefore useful to correlate the behaviour of the molecules with the role of the different functional groups in the self-assembly process. The Met coverage plays an important role in the mesoscopic ordering and domain formation of the molecular stripes. In fact, while at low coverage any orientation along the close-packed substrate is equi-probable, beyond a critical coverage $\Theta_{\text {Met }} \sim 0.10$ ML domains with mutual alignment appear. The inter-stripe distance, initially in the 85$190 \AA$ A range, becomes smaller and more regular with increasing coverage (see Figure 56), creating regular linear patterns extending up to the micrometer range. Thus, tuneable and regular nanogratings can be fabricated. The correlated orientation of the stripes witnesses the presence of long-range interactions. Related cases in which surface-state electrons drive adatom array formation [256; 257] and influence molecular ordering [258] on metal substrates have been reported, and it is likely that for Met/Ag(111) the surface states interfere in the molecular self-assembly. The same mesoscopic ordering is not observed for $\mathrm{Tyr} / \mathrm{Ag}(111)$ in spite of the almost identical stripes forming at low coverage. Instead, with increasing coverage the isolated stripes coalesce in 2D nanoribbons possibly crossing each other. This is explained by the authors as a consequence of the increased reactivity of the tyrosine phenol side chain and of the conformational surface adaptation.

Nanogratings with tuneable spacing may be exploited as templates for the design of functional linear arrangements such as nanowires. In this context, the thermal stability of the Met/ $\operatorname{Ag}(111)$ structures at RT, with molecular desorption occurring only above T 370 K, is encouraging, while the wide variety of AA allows to think of the possibility to engineer nanogratings with a high degree of tuneability. On the other hand, the outcome on the $\mathrm{Tyr} / \mathrm{Ag}(111)$ experiment, although negative in this respect, represents a further contribution to the understanding of supermolecular assemblies and remembers about the specificity of each AA species.
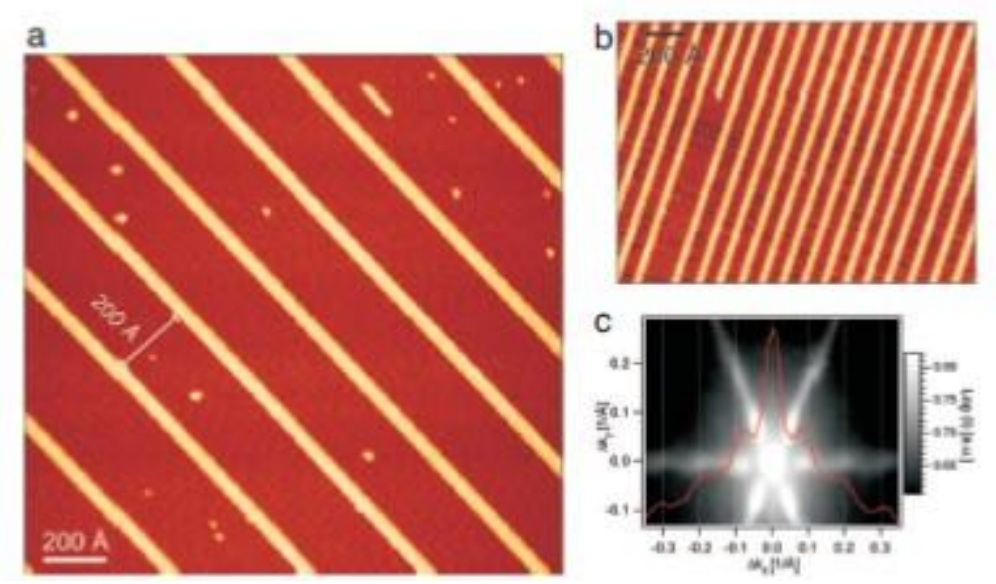

Figure 56. Tuning the self-assembly of $1 \mathrm{D}$ L-methionine nanogratings at intermediate coverages. A) $275 \AA$ periodicity, corresponding to $\Theta_{\mathrm{Met}} \cong 0.15 \mathrm{ML}(\mathrm{I}=0.8 \mathrm{nA}, \mathrm{V}=-800 \mathrm{mV})$; b) $94 \AA$ periodicity, corresponding to $\Theta_{\mathrm{Met}} \cong 0.38 \mathrm{ML}(\mathrm{I}=0.1 \mathrm{nA}, \mathrm{V}=-500 \mathrm{mV})$. C) The 2D HAS 
diffraction pattern of L-Met on $\operatorname{Ag}(111)\left(\mathrm{T}_{\mathrm{ev}} \approx 300 \mathrm{~K}, \Theta_{\mathrm{Met}} \cong 0.6 \mathrm{ML}\right)$ shows the amino acid selfassembling after the six-fold symmetry of the underlying substrate. The red curve corresponds to a single scan at $\Delta \mathrm{k}_{\mathrm{y}}=0$. Symmetrically placed satellite peaks aside the specular peak demonstrate the periodicity of the nanogratings. Taken with permission from ref. [63].

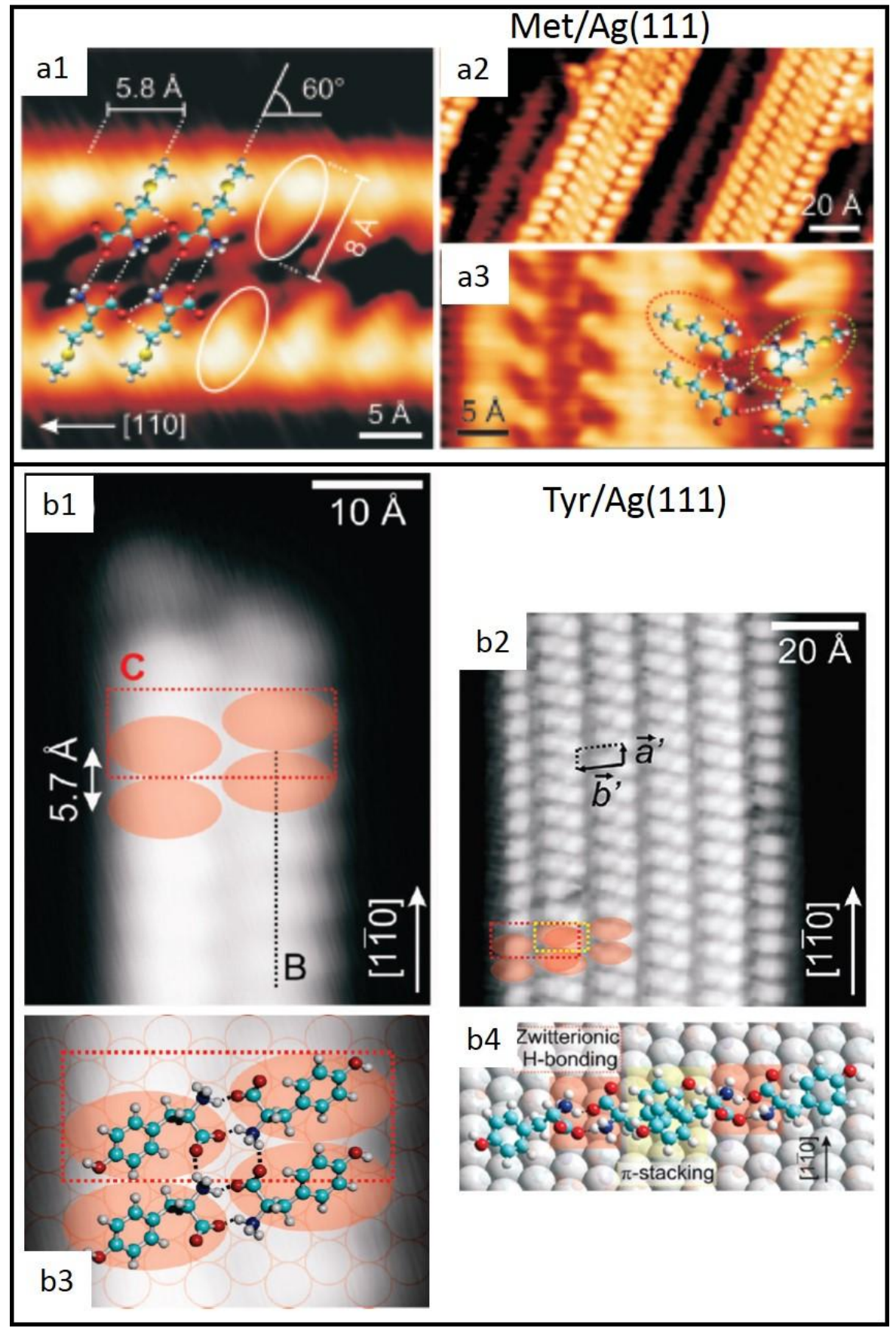

Figure 57. a) Molecular resolution imaging of L-methionine stripes self-assembled on $\mathrm{Ag}(111)$ and tentative models of molecular arrangements. A1) Individual molecules appear as elliptical features with a long axis of $\approx 8 \AA$ arranged in pairs, with a lateral separation of $5.8 \AA$ corresponding to twice the nearest-neighbour distance between silver surface atoms. This separation and the $60^{\circ}$ angle of the molecules with respect to the stripe orientation reflect the influence of the substrate on the molecular self-assembly $(\mathrm{I}=0.9 \mathrm{nA}, \mathrm{V}=-80 \mathrm{mV})$. A2) Quadruple methionine rows with $30 \AA$ 
spacing, observed for $\Theta_{\mathrm{Met}} \cong 0.50 \mathrm{ML}$. Chevron and parallel mutual row orientations coexist $(\mathrm{I}=$ $0.65 \mathrm{nA}, \mathrm{V}=-1,1 \mathrm{~V})$. a3) Quadruple molecular row with parallel and chevron configurations. The chevron dimers correspond to a down molecule (green oval) bonded to an up molecule (red oval). B) High resolution STM data and molecular model of the submonolayer biomolecular self-assembly of L-tyrosine on $\mathrm{Ag}(111)$. After annealing the Tyr covered surface to $320 \mathrm{~K}$, either ordered biomolecular chains extending along the $\langle 1-10>$ direction ( $\mathrm{b} 1-\mathrm{I}=0.2 \mathrm{nA}, \mathrm{V}=500 \mathrm{mV}$ ) or wider nanoribbons ( $\mathrm{b} 2-\mathrm{I}=0.1 \mathrm{nA}, \mathrm{V}=500 \mathrm{mV}$ ) appear, depending on coverage. Molecules dimerize along their long axis and bind adjacently and commensurably with the atomic lattice along the chain direction. The two ellipses framed in red represent two zwitterions forming a hydrogen-bonded dimer. B3) Molecular model of the hydrogen-bonded dimer on the $\mathrm{Ag}(111)$ atomic lattice. H-bonds involving the carboxylate and ammonium groups of the zwitterions are shown as dashed lines. B4) Schematic of the intermolecular bonding mechanism within the wider amino acid nanostructure: the zwitterion dimerizes via head-to-head hydrogen bonds between carboxylate and ammonium groups (red rectangle), and wider commensurate supramolecular sheets result from merging of simple bimolecular chains via parallel-displaced interactions between the phenol side chains (yellow rectangle). Taken with permission from refs.[45; 63].

The configuration and the intermolecular interactions of Met and Tyr within the 1D stripes or the 2D nanoribbons are deduced from the combination of high resolution STM images, NEXAFS data and molecular mechanics calculations and are schematised in Figure 57. For both AA, the stripes comprise elliptical features, each of them corresponding to a single molecule in the zwitterionic form. The AA units appear always in pairs within the 1D chains, which consist therefore of a sequence of dimers laterally coupled with each other and forming an angle with the stripe direction. Both amino-amino dimerization and lateral coupling are accomplished through hydrogen bonds involving the ammonium and carboxylate groups. The common behaviour observed for different systems and for different conformations of the same system is indicative that dimerization is mediated by the reactive amino-acid group. In the low coverage limit, the bonding scheme for Met and Tyr is the same. However the latter molecule shows only a linear configuration (i.e. the two molecules of the dimer are co-axial), while methionine can organize in double or quadruple rows and with molecular dimers either parallel or at an angle of $120^{\circ} \pm 10^{\circ}$ (chevron rows) with respect to each other. NEXAFS data and molecular mechanics calculations agree that the carboxylate group resides parallel to the surface. The sulphur atom also may play a secondary role in the lateral or surface bonding through its lone electron pair. A related zwitterionic bonding scheme was identified in the formation of layered amino acid crystals, where it is associated with appreciable bonding energies [259; 260]. The presence of the chevron rows and their co-existence with parallel rows is explained in terms of coupling of molecules in the "up" configuration (i.e. with the $\mathrm{H}$ atom of the $\alpha$-carbon pointing upward with respect to the substrate) and in the "down" configuration (obtained by flipping the "up"-molecule about its axis). The mirror symmetry of the chevron rows with respect to the line defined by the molecular dimerization could naturally be explained by the pairing of a row of methionine molecules in the energetically preferred "up"-configuration (circled in red in Figure 57, panel a3) with another one, in a "down"-configuration (circled in green), being energetically close. Lateral coupling would imply the changed orientation.

The formation of the quadruple rows from the merging of two-molecule chains is not due to lateral hydrogen bonding because the terminal $\mathrm{CH}_{3}$ group at the tail of the molecules is unreactive and 
because wider stripes should also form with increasing coverage. The deposition rate is suspected to determine whether the double or quadruple row is more favourable. For $\Theta_{\text {Met }}$ exceeding $0.65-0.70$ ML, methionine stripes evolve into highly anisotropic 2D molecular islands, but the interatomic interaction scheme remains substantially unchanged. The same does not hold for Tyr, for which there is evidence of a different supramolecular arrangement with more protruding rows within the self-assembled nanoribbon. From the geometrical point of view, this implies the partial merging and overlapping of narrower supramolecular rows, with intercalation of the phenol-moieties. This is consistent with the oblique orientation of the aromatic groups determined by NEXAFS.

The role of the deposition temperature in the self-assembly process was investigated in detail for the case of Glu adsorption at $\mathrm{Ag}(110)$ [51] and $\mathrm{Ag}(100)$ [54]. Glu molecules organize on $\operatorname{Ag}(110)$ in different ordered structures depending on coverage and adsorption temperature $300 \mathrm{~K} \leq \mathrm{T}_{\mathrm{ev}} \leq$ to 475 K. Such geometries are determined by the combined analysis of LEED superstructures and STM images and are often associated to adsorbate-induced faceting of the surface. Since this is the first systematic work on AA adsorption at a single crystal Ag surface performed under controlled UHV conditions, the results are naturally compared with those achieved on the more reactive $\mathrm{Cu}$ samples. So the authors underline the subtle difference in the nature of the adlayers produced on $\operatorname{Ag}(110)$ and $\mathrm{Cu}(110)$, starting from the formation of extended layers of AA instead of observing fragmentation into small clusters $[69 ; 261]$. On Ag, in fact, the adsorbate-molecule and molecule-molecule interactions are of a similar strength, making the adsorption site of (S)-glutamic acid more flexible. This work was followed by a theoretical investigation by Blankenburg and Schmidt [167], who simulated the experimentally observed Glu/Ag(110) (4×8) overlayer with DFT-D (Figure 58). The interaction energy is discussed by its contributions and it is shown that H-bonds and dispersion forces are important in the self-assembly. Simulated STM images and calculated vibrational frequencies contribute to an extensive description of the Glu/Ag(110) system.

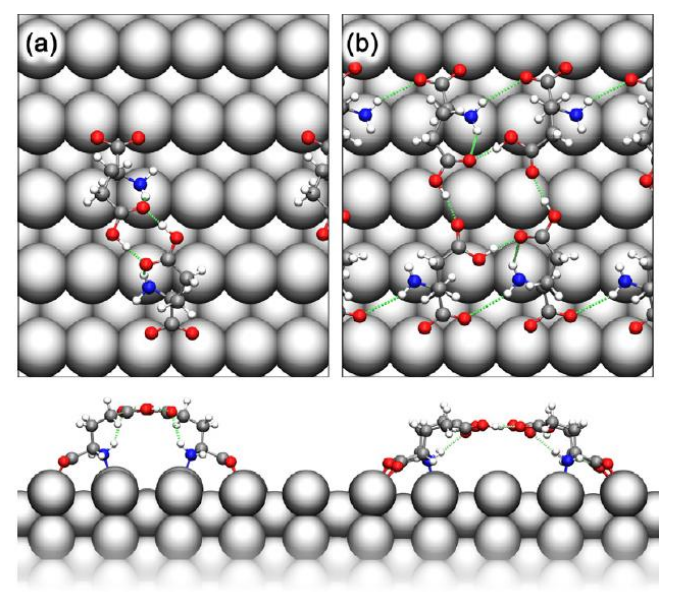

Figure 58. Most stable adsorption structure ofGlu/Ag(110)-(4x8) overlayer calculated by (a) DFTGGA and (b) DFT-GGA + VDW. Taken with permission from Ref. [167].

A similar study was performed on two different assemblies of Glu on $\operatorname{Ag}(100)$ [59; 253]. (S)Glutamic acid organizes on $\mathrm{Ag}(100)$ in different self-assembled structures depending on surface temperature [54]. Four different assemblies (see Figure 59) were detected in the $\mathrm{T}$ range between $250 \mathrm{~K}$ and $350 \mathrm{~K}$ : a row-like structure at $\mathrm{T}_{\mathrm{ev}}=250 \mathrm{~K}(\mathrm{~A})$, a comb-like structure at $\mathrm{T}_{\mathrm{ev}}=300 \mathrm{~K}(\mathrm{~B})$, a 
flower-like (C) and a square-like (D) structure coexisting at $T_{e v}=350 \mathrm{~K}$. In all cases, the molecules form single layer islands, the extension of which is larger at lower $\mathrm{T}_{\mathrm{ev}}$. The co-presence of comblike and flower-like domains at the transition temperature $T_{e v}=325 \mathrm{~K}$, combined with the thermal evolution measured by XPS, suggests the existence of several local minima in the energy diagram of the Glu/Ag(100) system. Upon deposition at $390 \mathrm{~K}$, little or no adsorption takes place, in accord with the vanishing sticking probability measured under such conditions. Contrary to the case of $\mathrm{Ag}(110)$, no superstructures are observed in the LEED pattern for any of these preparations, also at full monolayer coverage.

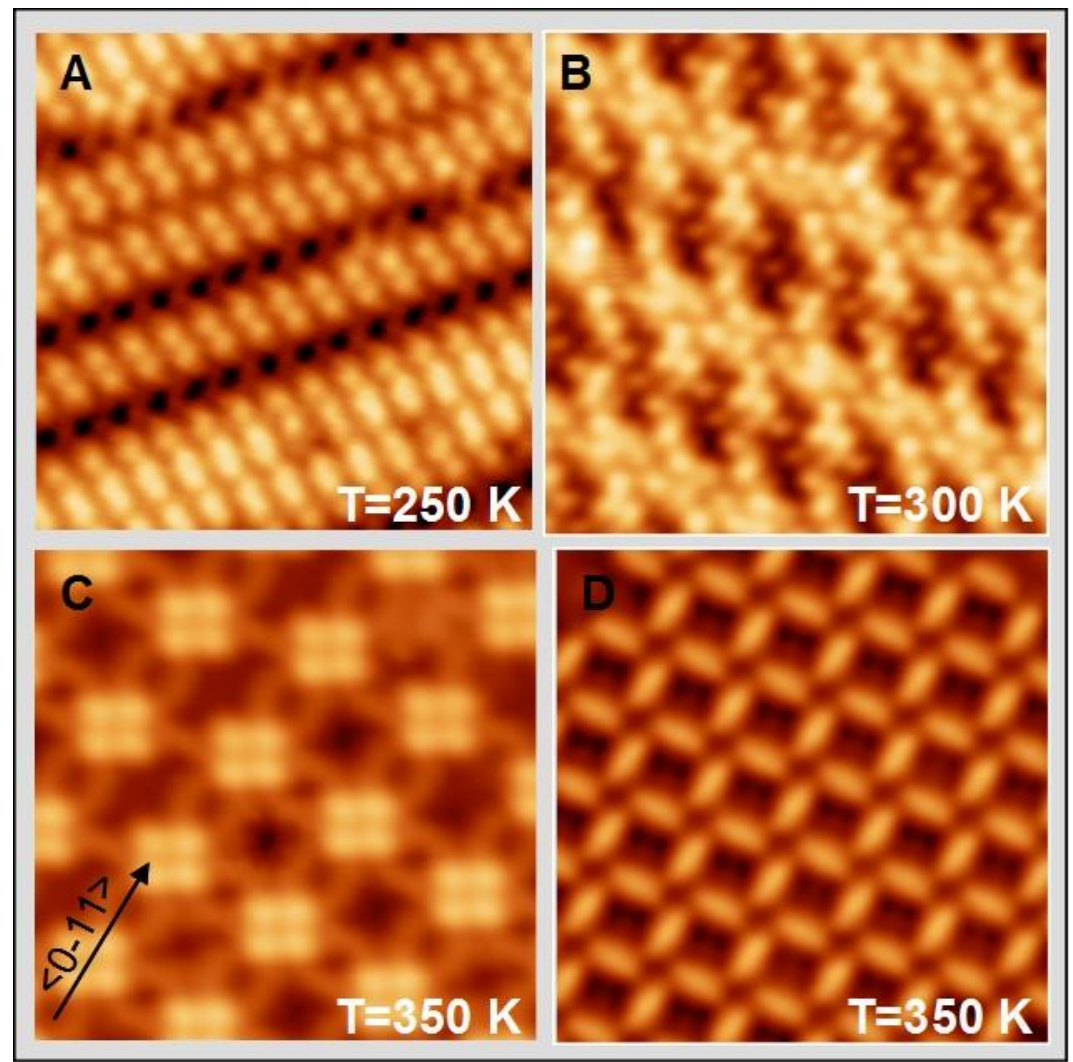

Figure 59. Overview of the different self-assembled structures produced by evaporating Glu on $\operatorname{Ag}(100)$ at different $\mathrm{T}_{\mathrm{ev}}$ (image size: $80 \AA \mathrm{X} 80 \AA$ ). A) "row" structure forming at $250 \mathrm{~K}$. The amino acids organize in long, one-molecule-wide rows oriented approximately $40^{\circ}$ off the $<0-11>$ direction. Within each row, single molecules lie flat on the surface, separated by $(6.5 \pm 0.2) \AA$ and with the molecular axis forming an angle of $\sim 65^{\circ}$ with respect to $<0-11>$. The estimated local coverage is $\Theta_{\mathrm{Glu}} \sim 0.09 \mathrm{ML}$. B) "Comb" structure forming at $300 \mathrm{~K}$. The amino acids arrange in large monolayer islands consisting of elongated features oriented along the $<0-11>$ directions and repeating with a periodicity of $26 \AA$. Each feature consists of a linear backbone formed by two lines of molecules separated by $(5.6 \pm 0.2) \AA$, lying flat on the surface with their axis aligned with the high symmetry <0 -1 $1>$ direction. Additional Glu units are inserted as "teeth" with an inclination of $\sim 45^{\circ}$ and a periodicity of $(14.0 \pm 0.2) \AA$. Also in this case the estimated $\Theta_{\mathrm{Glu}}$ is $\sim 0.09$ ML. C) "Flower" structure forming at $350 \mathrm{~K}$. Groups of four molecules (the "flowers") are linked by "sticks"showing a lower contrast. These tetramers organize in a square pattern with the main axes aligned within $5^{\circ}$ with the $<0-11>$ directions and with a periodicity of $\sim 21 \AA$. The sides of the single tetramers are rotated by $\sim 30^{\circ}$ with respect to the same direction, while adjacent maxima in the same tetramer are separated by $5.8 \AA$ and $5.4 \AA$, respectively. The sticks are also oriented in 
the high symmetry direction of the substrate. According to the proposed model, the Glu local coverage is $\Theta_{\mathrm{Glu}}=0.14 \mathrm{ML}$ but, considering that only a fraction of the surface is covered by Glu islands, the overall coverage is likely to be significantly smaller. D) "Square" structure forming at $350 \mathrm{~K}$. Amino acids arrange in a square geometry oriented $\sim 20^{\circ}$ off $\langle 1-10\rangle$. Each side of the square consists of a single Glu molecule lying almost flat on the surface. The axis of the Glu units is oriented $\sim 10^{\circ}$ off the $\langle 1-10\rangle$ direction, so that a chiral pattern forms in self-assembled islands. The average dimensions of the single squares are $\mathrm{a}=(13.2 \pm 0.8) \AA$ and $\mathrm{b}=(12.2 \pm 0.7) \AA$ for the sides and $\mathrm{c}=(19.7 \pm 0.8) \AA$ for the diagonal, from which a local coverage $\Theta_{\mathrm{Glu}} \sim 0.10 \mathrm{ML}$ is deduced. Taken with permission from ref.[54].

The observed self-assembled structures were described with empirical models deduced from high resolution STM images [54]. Starting from these models, the two assemblies observed at $350 \mathrm{~K}$ were also reproduced by DFT-D calculations[59; 253].

At variance with the majority of cases reported in literature, the "square" structure consists of neutral molecules physisorbed on the surface, $4.6 \AA$ above the surface plane (see Figure 60). The interaction with the poorly reactive Ag substrate is only due to weak van der Waals forces, while $\mathrm{H}$ bonds between carboxyl groups and the formation of a OCOH-OCOH-OCOH-OCOH cycle at the vertex of the squares are the main responsible for the self-assembly.

The flower structure is formed by $50 \%$ of deprotonated Glu, which assume an upstanding conformation and arrange in the bright tetrameric units, and by $50 \%$ of neutral molecules (Figure 61). The latter ones lie flat on the surface and appear in STM images as the sticks interconnecting the flowers. This configuration is characterised by strong chemical bonds between glutamic acid deprotonated Glumolecules and the surface, in contrast to the former "square"-structure in which physisorption dominates. Whereas the chemisorbed central Glu tetramer interacts strongly with the surface, the physisorbed surrounding Glu molecules conserve some degree of freedom in the layer, which counterbalances the weak adsorption energy.

The "flower" and the "square" assemblies have similar dispersion energy and H-bond interaction energy; as a consequence of the different chemical state of the GLU molecules, however, they differ by the formation of covalent bonds in the flower structure and by a different surface coverage. Moreover, it is important to understand the thermodynamics behind the coexistence of the two molecular assemblies[262]. Their thermodynamical iso-stability at $350 \mathrm{~K}$ is explained by considering entropic factors which have a strong stabilizing impact on the poorly adsorbed "square" structure. Interestingly, the calculated desorption temperatures fairly agree with experimental values. It is expected that the transition from one structure to the other is kinetically possible thanks to the low reactivity of the $\mathrm{Ag}(100)$ surface. Indeed on this surface, experiments and calculations suggest that intermediary species such as isolated Glu and atomic H, are poorly adsorbed [59], and can diffuse without kinetically controlled barriers. Finally, for both assemblies, simulated STM images in very good agreement with the experimental ones confirm the validity of the proposed models. 


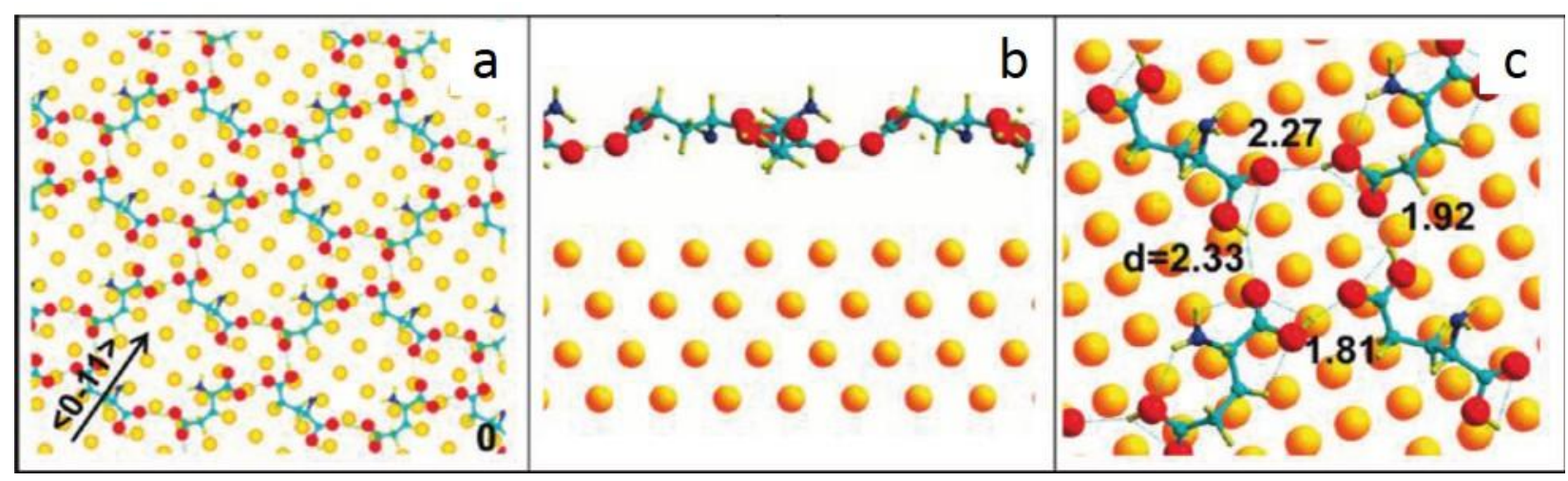

Figure 60. a) Optimized geometry of the most stable, square structure found for $\mathrm{Glu} / \mathrm{Ag}(100)$. B) Side view of the square structure, showing that no chemical bonds form between Glu and Ag. C) Detail of the H-bond ring forming between four adjacent carboxyl groups at the vertex of the squares. (figure partly taken from ref. [59]).
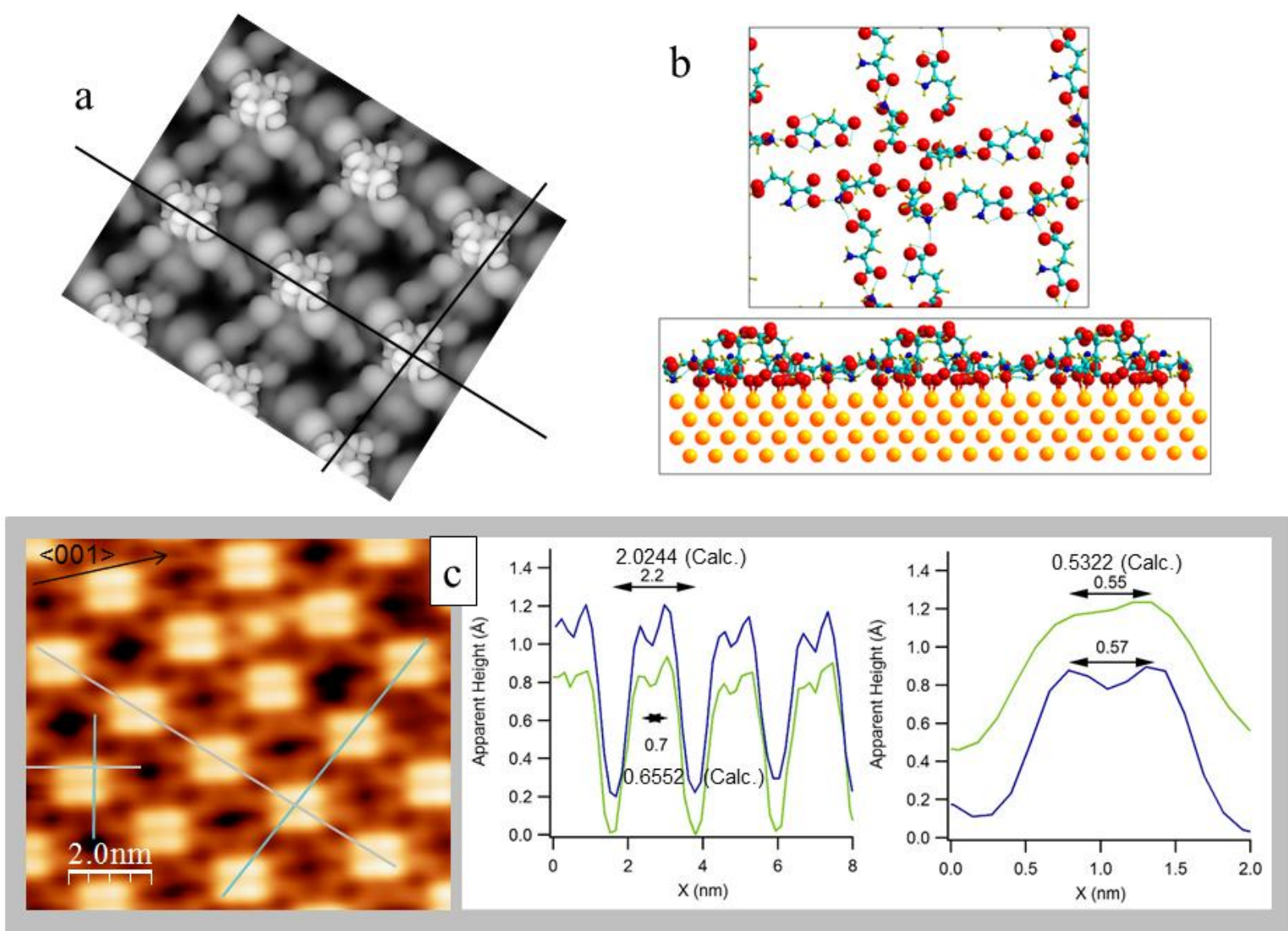

Figure 61. A) Simulated STM image of the most stable "flower" assembly obtained for Glu/Ag(100). B) Molecular top and side view of this structure. C) Height profiles of the Glu/Ag(100) structure cut along the lines drawn on the picture. The calculated periodicity of amino acid units along the rows is compared with the experimentally measured one. Distances (experimental and calculated) along the rows are in nanometers. Taken with permission from ref.[253]. 


\subsection{Amino acids adsorption at transition metals}

Amino acid adsorption at reactive metals has received, so far, less attention compared to the coinage metals (See Table 1). The research has concentrated in particular on $\mathrm{Ni}, \mathrm{Pd}$, and Pt surfaces and on a limited selection of molecules. These transition metals, which occupy the column immediately on the left of noble metals in the periodic table of elements, are characterised by the same electronic configuration of the outer shell and share therefore similar electronic properties. The characterisation of the organic adsorbed layer was performed initially by spectroscopic means, while more recently a few STM studies and ab-initio calculations have appeared.

The increasing interest in the adsorption of amino acids at transition metal surfaces is linked to the role of these materials as base catalysts for enantioselective reactions. The importance of enantioselective catalysis, stigmatised by the award of the 2001 Nobel Prize for Chemistry to three pioneering researchers in the area of homogeneous chiral catalysis (Knowles, Noyori and Sharpless), is continuously increasing due to the demand of pharmaceutical and chemical industries for larger quantities of optically pure chemicals. The development of heterogeneous catalysts capable of selectively producing one optical isomer of a chiral molecule would meet this demand, since heterogeneous processes have usually high product yields and relatively low cost. However, few - if any - industrial enantioselective processes are heterogeneously catalysed at present.

Academic research focussed so far on few classes of enantioselective heterogeneous catalytic processes: the enantioselective hydrogenation of $\alpha$-keto esters over Pt based systems and the asymmetric hydrogenation of $\beta$-keto esters over Ni based catalysts [263-265], while Pd is used as base material for the hydrogenation of ketones [266-270]. In all cases enantioselectivity is conferred to the metal nanoparticles by the adsorption of chiral molecules (modifiers). In Ni systems, successful modifiers include $\alpha$-hydroxy acids (e.g. R,R-tartaric acid) or $\alpha$-amino acids (e.g. Salanine) [263-265]. Pd is successfully modified by proline adsorption [268-270].

Since fundamental understanding of the reason why surface chemistry induces preferential formation of one enantiomeric product is lacking, attention of surface scientists has focussed on attempts to elucidate the nature of the chirally modified metal nanoparticles as well as to relate the nature of the surface species and the resulting structures to their enantioselectiviy. We underline that, besides the work on transition metal surfaces partly reported in this section, several studies have been performed on $\mathrm{Cu}$, which is the most investigated substrate also in this respect [175].

The investigation of other transition metals is seldom. The study on Cys adsorption at a magnetic metal surface, namely Fe(100), by Lavagnino et al. [271] showed that the growth dynamics is apparently similar to the one already reported on the $\mathrm{Cu}(100)$ and $\mathrm{Ag}(100)$ surfaces, with the formation of a strongly-bound thiolate species ( $\mathrm{S}-\mathrm{Fe}$ bond) and weakly-bound second layer molecules. Spin polarized metastable deexitation spectroscopy neatly revealed the spin-polarized character of an Auger deexcitation peak related to Cys. Spin-polarization effects are ascribed to the interaction with the magnetized substrate and vanish before ML completion, suggesting a possible correlation with the layer assembly properties. A complementary structural investigation is solicited by the authors but, to the best of our knowledge, this research had no further developments so far.

\subsubsection{Adsorption at $\mathrm{Pt}(111)$ and $\mathrm{Pd}(111)$}


The choice of Gly for pioneering studies on AA adsorption on Pt and Pd surface is a quite natural one since it is an ideal starting point for the understanding of the more structurally complicate, chiral amino acids. The Pt surface [38; 272] was initially chosen as a possible weakly reactive substrate before Ag, but proved to be reactive enough to cause significant interaction with the molecules. $\mathrm{Pt}(111)$ was taken as model since it is very well-characterized, it is stable and it can be modified in a controlled manner by pre-adsorption of small inorganic molecules. In principle, this last point opens up a possibility of studying several different well-defined surface modifications with one single substrate. However, to the best of our knowledge, it was not developed further by the authors. As already mentioned, the interest in the $\operatorname{Pd}(111)$ surface is focussed on the investigation of enantioselective chemisorption. Indeed, after glycine [40], the authors adsorbed on the same substrate also alanine [39; 273] and proline [41].

For all the systems Gly/Pt(111), Gly/Pd(111), Ala/Pd(111) and Pro/Pd(111), non-dissociative adsorption and multilayer formation at $\mathrm{T} \leq 300 \mathrm{~K}$ is reported. The final adsorption state was characterised by XPS while, to gain a more detailed picture of the reaction pathways for chemisorption, also TPD (TPS) analysis was performed. Very few systems have been investigated by microscopic means and DFT methods (such as, e.g., Ala/Pd(111)).

XPS spectra present common features for all systems, which are summarisedin Table 3 and discussed in the following:

i) At any coverage, spectra present a doublet in the $\mathrm{C} 1 \mathrm{~s}$ region, which is ascribed to the $\alpha$ carbon or to the pyrrolidine ring (low $\mathrm{E}_{\mathrm{b}}$ feature) and to the deprotonated carboxyl group $\left(\mathrm{E}_{\mathrm{b}} \sim 288 \mathrm{eV}\right)$, in agreement with what is observed for $\mathrm{Cu}$ and noble metals (see sections 3.1 and 3.2). Only one, asymmetric feature at $531.7 \mathrm{eV} \leq \mathrm{E}_{\mathrm{b}} \leq 532.3 \mathrm{eV}$, suitable for deprotonated carboxyl, appears in the $\mathrm{O} 1 \mathrm{~s}$ region (reported only for the Gly/Pt(111) system [38]). The high energy tailing indicates that multiple binding configurations may be present.

ii) For adsorption at $\mathrm{T}<300 \mathrm{~K}$, the $\mathrm{N}$ ls spectral region shows a wide, asymmetric feature, clearly consisting of two components in the range typical of the protonated $\mathrm{NH}_{3}{ }^{+}(\sim 401 \mathrm{eV})$ and of the neutral $\mathrm{NH}_{2}$ groups $(\sim 399.5 \mathrm{eV})$, respectively. Therefore coexistence of molecules in the zwitterionic and in the non-zwitterionic form occurs for all systems, although the zwitterionic form seems to prevail at low temperature. Detailed analysis of the N1s peak areas suggests indeed that $65-70 \%$ of the $\mathrm{Ala} / \mathrm{Pd}(111)$ layer[39], 70\% of the $\operatorname{Pro} / \mathrm{Pd}(111)$ layer [41], $70-80 \%$ of the Gly/Pd(111) layer [40] and up to $90 \%$ of the Gly/Pt(111) layer [38] consists of zwitterions. Adsorption in the purely zwitterionic form was observed for Proline on $\operatorname{Pd}(111)$ at $300 \mathrm{~K}$, although with a significantly reduced sticking probability. For $\mathrm{Ala} / \mathrm{Pd}(111)$ the situation is more controversial. The same research group initially indicated full zwitterionic adsorption at $300 \mathrm{~K}$ [39] but, in a very recent paper [273], shows the formation of mixed zwitterionic-anionic structures with no long range order on the surface at $\mathrm{T}=290 \mathrm{~K}$. The authors do not discuss explicitly the difference between the two series of experiments.

iii) The C1s, N1s and Ols binding energies upshift with increasing coverage. This was observed also for adsorption at noble metals, as in the case of Glu/Ag [53; 54] and His/Au(111) [149; 242]. The increase is rapid below a critical coverage and sets then to a reduced, constant slope. It can be due either to relaxation shifts or chemical shifts. The more efficient relaxation of electronic excitations coupled to metal electronic states, compared to the corresponding relaxation in the insulating solid amino acid, is indeed identified as the cause of the shift for 
Gly/Pd(111) and Ala/Pd(111) between 0 and $3 \mathrm{ML}$. The binding energy in the monolayer results thus lower that the one of multilayers. The much slower shift observed at multilayer coverage is due to charging of the relatively thick AA layer.

iv) Both alanine and proline show a different ratio between the $\mathrm{C} 1 \mathrm{~s}$ component due to the carboxylic group $(\sim 288 \mathrm{eV})$ and the one due to the remaining $\mathrm{C}$ atoms $(\sim 285 \mathrm{eV})$ when adsorbed at low $\mathrm{T}$ or at RT. This indicates a conformational change of the adsorbed layer with $\mathrm{T}$, in particular when passing from the multilayer to the monolayer regime and, possibly, screening of some $\mathrm{C}$ atoms in presence of multilayers.

Desorption experiments present also a common behaviour, since molecules in multilayers always desorb intact, while those in contact with the metal substrate undergo partial dissociation before desorption. There are however subtle differences which are worth to be mentioned.
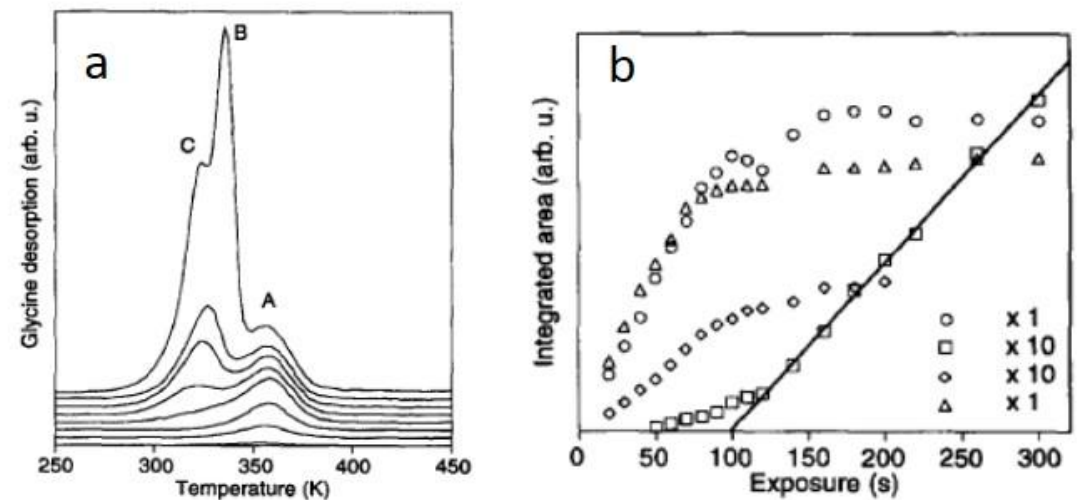

Figure 62. a) Thermal desorption spectra for intact glycine $(\mathrm{m} / \mathrm{z}=30)$ following adsorption of glycine on Pt(111) at $200 \mathrm{~K}$. Exposure times (from bottom up): 60 s, 100 s, 140 s, 180 s, 220 s, 260 s, $300 \mathrm{~s}$ and $600 \mathrm{~s}$. A saturated monolayer is formed after an exposure of $200 \mathrm{~s}$. b) Desorption yields (integrated desorption peak areas) of the intact glycine molecule and of the dissociation products as a function of glycine exposure on clean $\mathrm{Pt}(111)$ : ( $\square$ ) glycine (both from the first ML and multilayers), $(\mathrm{O}) \mathrm{H}_{2},(\diamond) \mathrm{CO}^{2}$ and $(\triangle) \mathrm{H}_{2} \mathrm{CN} / \mathrm{CO}(>400 \mathrm{~K})$. The solid line is a linear curve fit for intact glycine at the highest (> $120 \mathrm{~s}$ ) glycine exposures. Taken with permission from ref. [38].

Figure 62 shows typical desorption spectra of Gly adsorbed on $\operatorname{Pt}(111)$ at $\mathrm{T}<250 \mathrm{~K}$ (panel a) and the integrated desorption yields of the molecule and of the dissociation products (panel b). The desorption information indicates that Gly adsorbs molecularly on $\operatorname{Pt}(111)$ at $\mathrm{T}<250 \mathrm{~K}$, forming either monolayers or multilayers depending on coverage. For $\Theta_{\mathrm{Gly}} \leq 0.25 \mathrm{ML}$ all glycine molecules dissociate prior to desorption, in the range $350 \mathrm{~K}<\mathrm{T}<400 \mathrm{~K}$. Part of the so-produced fragments $\left(\mathrm{H}_{2}, \mathrm{CO}_{2}\right.$ and $\left.\mathrm{H}_{2} \mathrm{O}\right)$ desorb immediately, while others are detected at higher temperature $(\mathrm{CO}$ at $\mathrm{T}=$ $450 \mathrm{~K}, \mathrm{H}_{2} \mathrm{CN}, \mathrm{N}_{2}$ and $\mathrm{C}_{2} \mathrm{~N}_{2}$ at $500 \mathrm{~K}<\mathrm{T}<900 \mathrm{~K}$ ). In the coverage range 0.25-1 ML some molecules dissociate and some desorb intact (peak A in Figure 62a), allowing estimating a binding energy of $0.95 \mathrm{eV}$ molecule ${ }^{-1}$. The branching ratio dissociative/non-dissociative desorption at $1 \mathrm{ML}$ is nearly unity. For $\Theta_{\mathrm{Gly}}>1 \mathrm{ML}$, two new non-dissociative (intact glycine) binding states emerge, one associated with the second (and probably also the third) layer (peak B) and the other associated with multilayers (peak $\mathrm{C}$ ). For both states the estimated binding energy is $(0.87 \pm 0.05) \mathrm{eV}$ molecule $^{-1}$. The overall picture shows therefore that Gly adsorbs on $\operatorname{Pt}(111)$ at $\mathrm{T}<250 \mathrm{~K}$ 
exclusively in the non-dissociative form, mainly as a zwitterion and populating up to three different states depending on the coverage regime.
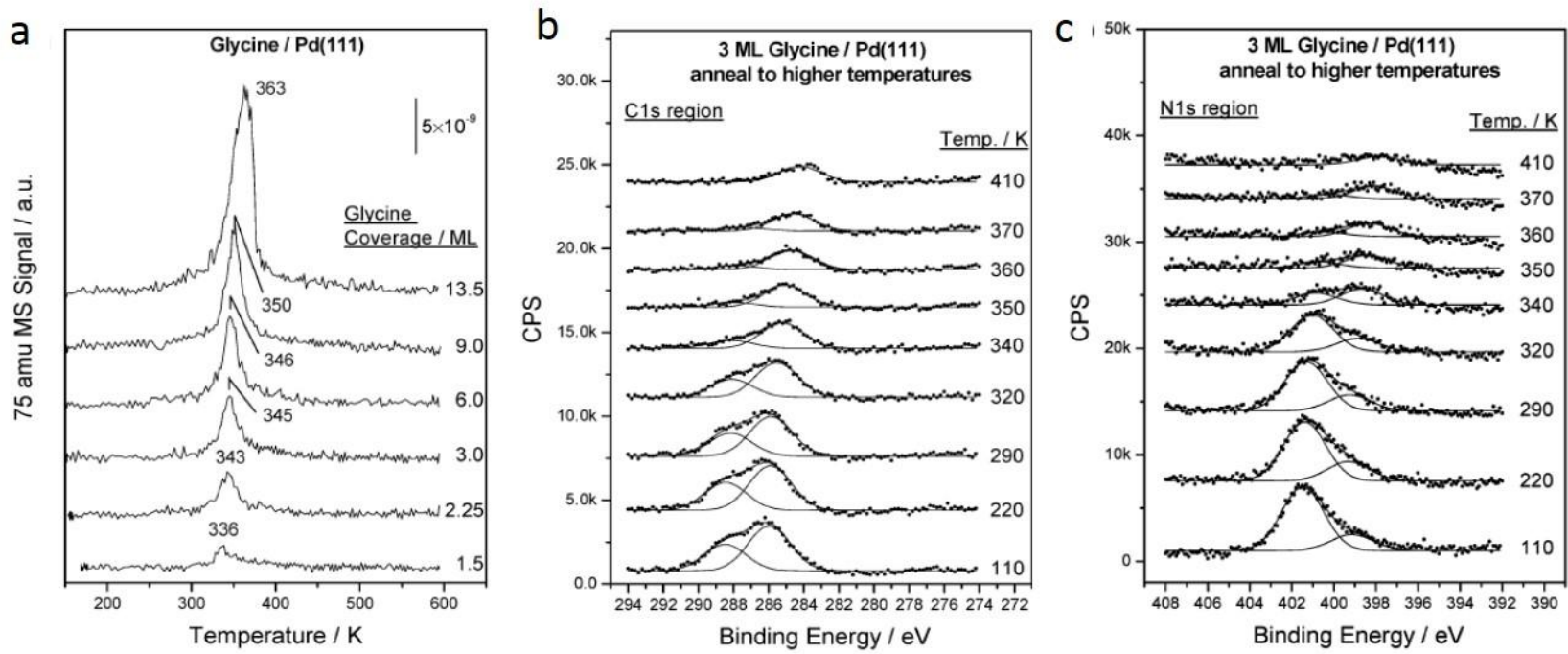

Figure 63. a) Glycine (75 amu) desorption profiles as a function of glycine exposure collected at a heating rate of $6.5 \mathrm{~K} / \mathrm{s}$. b) and c): XPS spectra of the $\mathrm{C} 1 \mathrm{~s}$ and $\mathrm{N} 1 \mathrm{~s}$ regions recorded after deposition of $3 \mathrm{ML}$ of glycine on $\mathrm{Pd}(111)$ and annealing to different temperature. The surface was cooled back to $\mathrm{T}=100 \mathrm{~K}$ to record the spectra. Taken with permission from ref. [40].

Only one desorption peak is observed for the different AA adsorbed on $\operatorname{Pd}(111)$ [39-41]. Figure 63 refers to the Gly/Pd(111) system, but a very similar analysis was performed for $\mathrm{Ala} / \mathrm{Pd}(111)$ and for $\mathrm{Pro} / \mathrm{Pd}(111)$ as well. For all the systems desorption occurs at $315 \mathrm{~K} \leq \mathrm{T} \leq 382 \mathrm{~K}$, the exact temperature depending on the aminoacid species and coverage. The corresponding XPS spectra after annealing (recorded for a 3 ML Gly film in panels b and c) present in the $\mathrm{C} 1 \mathrm{~s}$ region both the features corresponding to the $\mathrm{COO}^{-}(\sim 288 \mathrm{eV})$ and to the $\alpha-\mathrm{C}$ and aliphatic or pyrrolidine terminations $(\sim 285 \mathrm{eV})$. The low $\mathrm{E}_{\mathrm{b}}$ component may also include carbon from dissociation products, especially at higher temperatures. The N1s spectrum shows both the $\mathrm{NH}_{3}{ }^{+}$and $\mathrm{NH}_{2}$ related components. For Gly/Pd(111) no significant changes in the spectra are observed between $110 \mathrm{~K}$ and $290 \mathrm{~K}$, indicating that the majority of adsorbed glycine remains intact over this temperature range. Heating above $\mathrm{T}=320 \mathrm{~K}$ leads, on the contrary, to a reduction of the peaks intensity, consistent with the TPD information, and to a change in the relative weight of the peak components. In particular the $\mathrm{NH}_{3}{ }^{+}$signal almost disappears, suggesting that zwitterionic glycine decomposes and/or converts to glycinate. A similar behaviour was observed for the other systems. To determine the nature of the dissociation products, additional TPD experiments were performed, monitoring a wider range of masses and varying the heating rate. As in the case of Gly/Pt(111), decomposition prior to desorption is dominant at submonolayer coverage. In principle, two straightforward pathways are possible for dissociation: C-COO bond and/or C-N bond scission. The charge distribution of the molecule in the zwitterionic form weakens the $\mathrm{C}-\mathrm{C}$ bond thus facilitating cleavage [272] and indeed TPD and XPS results demonstrate that $\mathrm{C}-\mathrm{C}$ bond scission dominates for all investigated systems. The carboxylate moiety desorbs as $\mathrm{CO}$ and $\mathrm{CO}_{2}$. For Gly and Ala, the nitrogen-containing moiety desorbs as an amine and $\mathrm{HCN}$, while the behaviour of proline is more complex. A number of desorption products are detected in addition to $\mathrm{HCN}$, including pyrrole $(\mathrm{T}=$ $390 \mathrm{~K})$, pyrrolidine $(\mathrm{T}=410 \mathrm{~K})$ and not fully identified species that desorbs at $\mathrm{T}=450 \mathrm{~K}$. 
As already mentioned, the adsorption of alanine on $\mathrm{Pd}(111)$ close to room temperature was investigated recently in deeper detail by combining XPS, IRAS and STM with DFT results[273]. After adsorption of $0.09 \mathrm{ML}$ of alanine at $\sim 270 \mathrm{~K}$ the surface consists primarily of dimers with their axis oriented $\left(30^{\circ} \pm 5^{\circ}\right)$ relative to the $\langle 1-10\rangle$ directions. Adsorption and imaging at $290 \mathrm{~K}$ reveal the formation of more complex surface structures (see Figure 64) which, however, do not present the long range order already observed for adsorption at $\mathrm{Cu}$ surfaces. Two distinct structural motifs can be identified. The first consists of dimer rows with their long axes aligned along the $\langle 1$ $10\rangle$ directions. Many of these comprise hexamers, although a number of longer dimer chains also form. The second structure consists of tetramers with one edge oriented along the close-packed $\langle 1$ 10) directions and the other edge oriented at $\sim 30^{\circ}$ to this direction. Similar structures form at different coverage values but, as the $\Theta_{\text {Ala }}$ approaches saturation, the dimer-row structures evolve into a disordered surface. Both XPS (see Table 3) and RAIRS data (seeTable 9) suggest the coexistence of alanine molecules in the zwitterionic and anionic form.

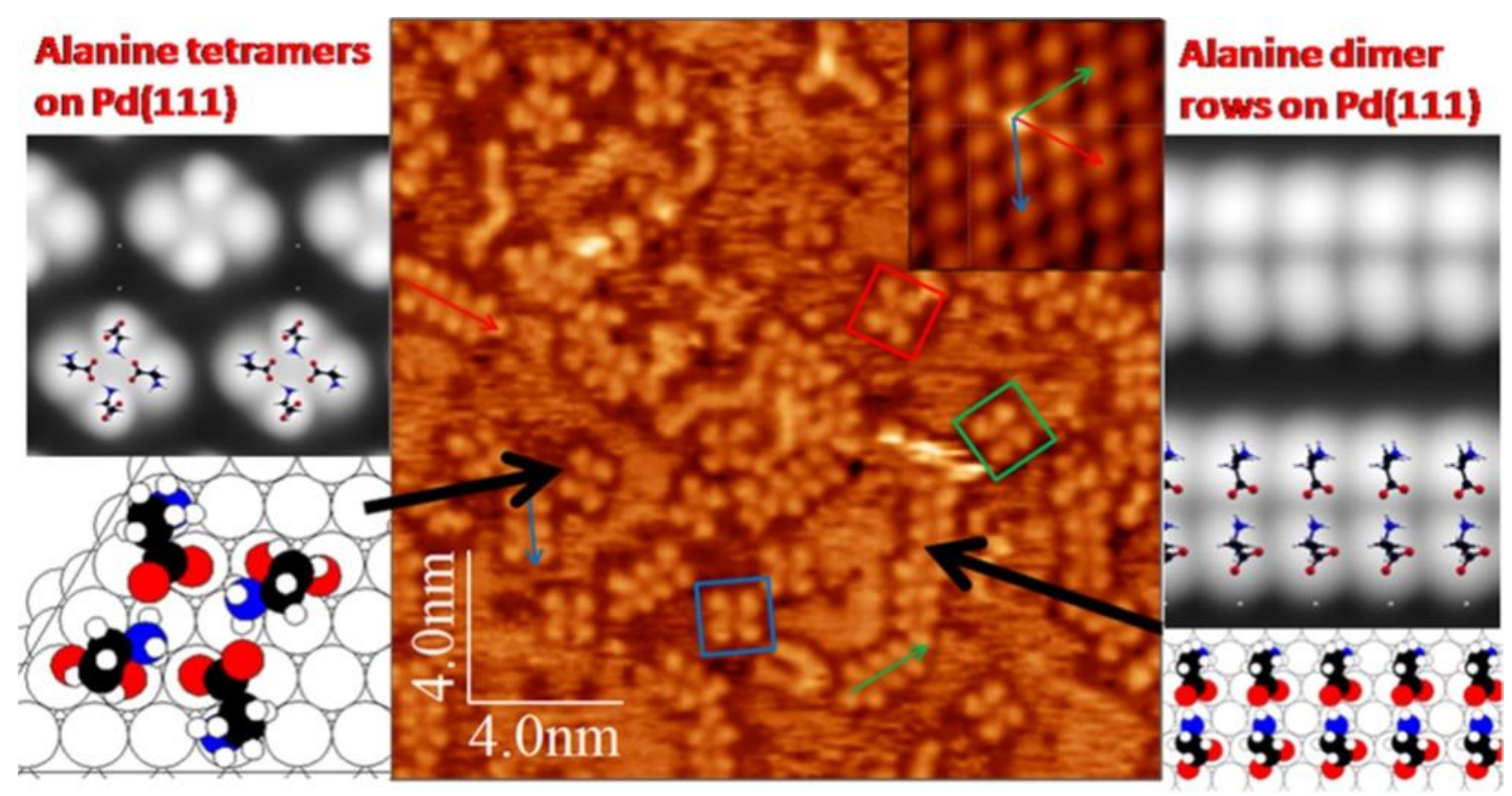

Figure 64. Central panel: STM image of an absolute coverage of $0.052 \mathrm{ML}$ of alanine on $\operatorname{Pd}(111)$ dosed and imaged at $290 \mathrm{~K}(\mathrm{~V}=-161 \mathrm{mV}, \mathrm{I}=20.0 \mathrm{pA})$. The inset shows an atomic-resolution image of the clean $\operatorname{Pd}(111)$ surface, in which the three equivalent $\langle 1 \overline{10}\rangle$ directions are indicated by arrows of different colours. Three dimer rows are indicated in the image by color-coded arrows related to the corresponding $\langle 1-10\rangle$ direction. The intermolecular spacing (gauged from the brightest part of the spots in the image) along the chains is $(0.59 \pm 0.02) \mathrm{nm}$, and perpendicular to the chain is $(0.58$ $\pm 0.01) \mathrm{nm}$. Tetramers are marked by squares. The intermolecular spacing in the tetramers $(0.65 \pm$ $0.02 \mathrm{~nm}$ ) is larger than in the dimer rows. Left panel: Calculated structure (bottom) and simulated STM image (top) of the tetrameric features. Right panel: same as left panel, for the dimer rows. Taken with permission from ref. [273]. 


\begin{tabular}{|c|c|}
\hline \multicolumn{2}{|c|}{ Alanine/Pd(111) } \\
\hline Vibrational mode & $\left(\mathrm{cm}^{-1}\right)$ \\
\hline $\mathrm{v}_{\text {asym }}\left(\mathrm{NH}_{3}{ }^{+}\right)$ & 3226 \\
\hline$v_{\text {sym }}\left(\mathrm{NH}_{3}{ }^{+}\right)$ & 3183 \\
\hline$v_{\text {sym }}\left(\mathrm{CH}_{3}\right), v(\mathrm{CH})$ & 2938 \\
\hline $2 \times \delta_{a}\left(\mathrm{CH}_{3}\right), v_{\text {sym }}\left(\mathrm{CH}_{3}\right)$ & 2889 \\
\hline$v_{\text {asym }}\left(\mathrm{COO}^{-}\right)$ & 1606 \\
\hline$\delta\left(\mathrm{NH}_{2}\right), \delta\left(\mathrm{NH}_{3}{ }^{+}\right)$ & 1565 \\
\hline$v_{\mathrm{sym}}\left(\mathrm{COO}^{-}\right)+\delta_{\mathrm{s}}\left(\mathrm{CH}_{3}\right)$ & 1402 \\
\hline$\delta_{\text {sym }}\left(\mathrm{CH}_{3}\right)+v_{\text {sym }}\left(\mathrm{COO}^{-}\right)$ & 1374 \\
\hline$v_{\text {asym }}(\mathrm{CCN}), \rho\left(\mathrm{CH}_{3}\right)$ & 1101 \\
\hline$v(\mathrm{CN}), \rho\left(\mathrm{NH}_{3}{ }^{+}\right)$ & 922 \\
\hline$v(\mathrm{CN})+\delta_{\text {sym }}(\mathrm{OCO})$ & \\
\hline$\delta_{\text {sym }}(\mathrm{OCO})+v(\mathrm{CN})$ & 770 \\
\hline
\end{tabular}

Table 9. Vibrational frequencies of alanine adsorbed on $\operatorname{Pd}(111)$ at $300 \mathrm{~K}$. Data taken from ref. [273].

In order to gauge the relative stability of anionic and zwitterionic alanine on $\mathrm{Pd}(111)$, DFT calculations were performed for isolated alanine species ( $0.02 \mathrm{ML}$ coverage). The calculations indicate that the molecule in the anionic form is more stable by $0.21 \mathrm{eV}$ with respect to the zwitterion (adsorption energies of $-0.68 \mathrm{eV}$ vs $-0.47 \mathrm{eV}$ for the two species, respectively). This confirms previous DFT calculations by Sholl and James, who found the anionic species to be more stable on $\mathrm{Pd}(111)$ by $\sim 0.44 \mathrm{eV}$ at a higher coverage of $0.111 \mathrm{ML}$ [274]. In both studies and for both anionic and zwitterionic species, the individual alanine units adsorb with the oxygen atoms of the carboxylate groups located near atop sites and the amine group bound to the $\mathrm{Pd}$ surface, i.e. in a three-points fashion similar to the one reported for many amino acids on $\mathrm{Cu}$ surfaces. From the electronic point of view, the amine group has a lone pair of electrons available on the nitrogen atom, which allows it to form a strong bond with the surface. Such bond will be weaker for an $\mathrm{NH}_{3}{ }^{+}$ group, accounting for the energy difference between the two forms. However, alanine units in the dimeric structures must have a different conformation with respect to the isolated molecules. Different combinations were studied, finding that the dimer must consist of one alaninate unit and one zwitterion. The Z-A dimer has one oxygen atom in the carboxylate group moved away from the palladium atop site, in agreementwith the detection of an asymmetric carboxylate mode at $\sim 1606$ $\mathrm{cm}^{-1}$ (see Table 9), and it is stabilized by an interaction between the carboxylate and the $\mathrm{NH}_{3}{ }^{+}$ groups, causing the carboxylate group to reorient. Therefore the dimer rows are assigned to the assembly of Z-A dimers and the tetramer to the assembly of two dimers in which three of the alanine molecules undergo a concerted rotation by $30^{\circ}$ (see Figure 64). Finally, we note that the similarity between the tetrameric structures observed here and those reported for $\mathrm{Glu} / \mathrm{Ag}(100)$ is only apparent since in the present case the strong interaction with the surface keeps the amino group bound to the surface, while on the poorly reactive $\operatorname{Ag}(100)$ it was lifted and involved in H-bonds with the nearby glutamate molecules [253]. 
Experimental studies have reported that glycine adsorbsin its deprotonated form on the $\mathrm{Cu}(110)$ [42] and $\mathrm{Cu}(100)$ [97] surfaces at room temperature, but in its zwitterionic form on $\operatorname{Pd}(111)$ [40] and $\mathrm{Pt}(111)$ [38]. Density functional theory (DFT) calculations indicated that the deprotonated molecules are thermodynamically favoured on $\mathrm{Cu}(110), \mathrm{Cu}(100)$ but also on $\mathrm{Pd}(111)$. To explore the source of this controversy for the case of $\operatorname{Pd}(111)$, DFT calculations were performed [275]. Calculations are performed at low coverage, i.e. in the limit of isolated molecules. It was shown that the kinetic barrier on $\mathrm{Pd}(111)$ is larger than on $\mathrm{Cu}(110)$ or $\mathrm{Cu}(100)$ (see also section 3.1.1). Indeed, the calculated activation energies for the deprotonation reaction of glycine on $\operatorname{Pd}(111), \mathrm{Cu}(110)$ and $\mathrm{Cu}(100)$ are $0.73,0.27$, and $0.02 \mathrm{eV}$, respectively. Then it was also found that the presence of excess hydrogen has little influence on the observed experimental results, especially for $\mathrm{Pd}(111)$.

In the same study [275], Monte Carlo simulations using the calculated adsorption energiesdemonstrated that isolated molecules adsorb in the neutral form, whereas the aggregates of zwitterionic species on $\mathrm{Pt}(111)$ are energetically preferred to those of neutral species. Indeed, the interaction energy $\left(E_{\text {int }}\right)$ between the aggregated neutral molecules is $>0$, reflecting that individual neutral adsorbates are energetically favoured relative to aggregated ones. Once the number of interacting molecules becomes sufficiently large, the sign of the $\mathrm{E}_{\text {int }}$ difference reverses and the zwitterionic molecules become energetically favoured. This situation occurs when more than 12 molecules are available to interact with one another on the surface. These results strongly suggest that the formation of aggregates with relatively large numbers of adsorbed molecules (up to 15 Gly molecules) is favoured at $300 \mathrm{~K}$ and that the adsorbate-adsorbate interactions in these aggregates stabilize the zwitterionic species.
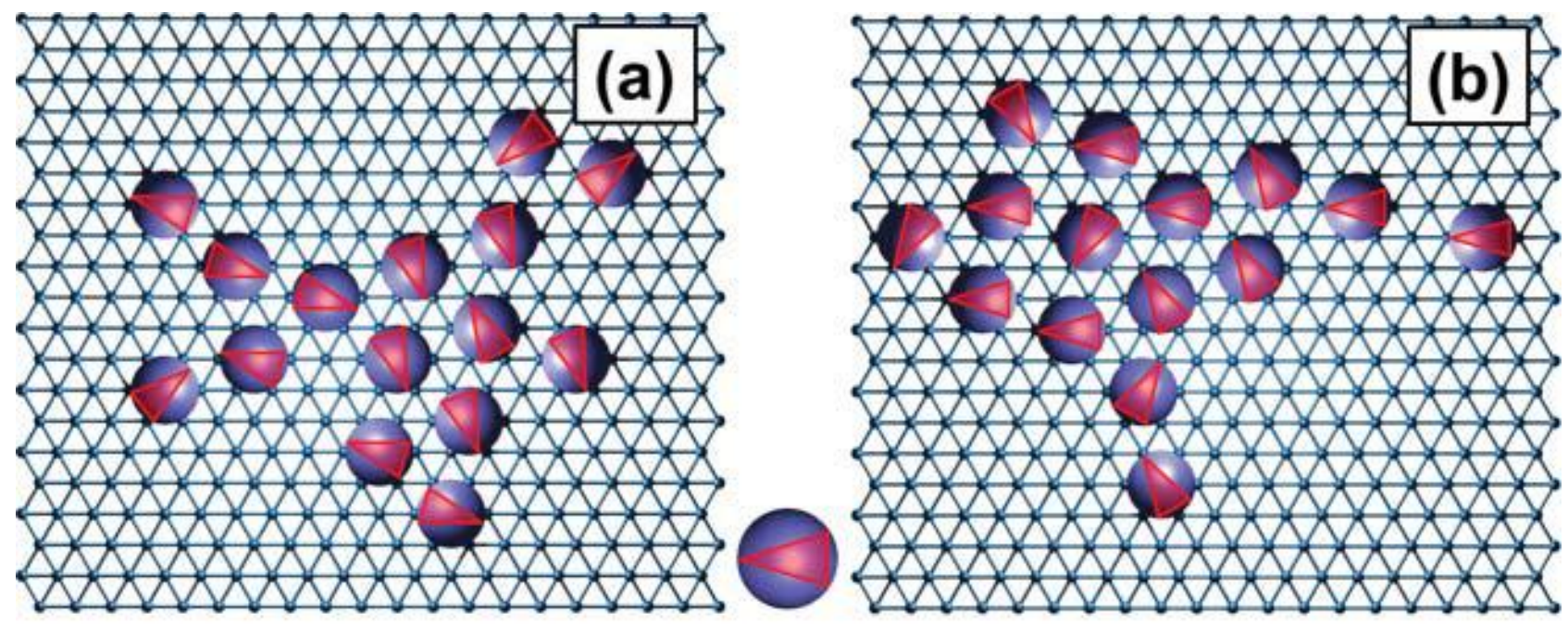

(a) $\mathrm{H}_{2} \mathrm{~N} \rightarrow \mathrm{COOH}$

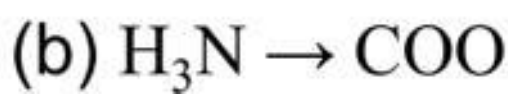

Figure 65. Snapshots of aggregations of 15 (a) neutral and (b) zwitterionic glycine molecules on a $20 \times 20$ hexagonal lattices at $300 \mathrm{~K}$. The hexagons for modelling each species are shown as spheres for illustrative purposes. The orientation of each molecule is marked as a triangle inside the sphere, 
with the thin end of the triangle denoting $\mathrm{H}_{2} \mathrm{~N}\left(\mathrm{H}_{3} \mathrm{~N}\right)$ in the neutral (zwitterionic) molecules. Taken with permission from ref. [275]

Finally we mention a recent DFT study of the adsorption of isolated and deprotonated glycine, alanine, nor-valine, valine, proline, cysteine, and serine on $\operatorname{Pd}(111)$ at low coverage [276] (see Figure 66). Most amino acids were found to adsorb onto the surface in the common $\mu 3$ conformation. For cysteine, an additional bond is formed with the surface due to the strong affinity of the sulphur atom, resulting in a significantly larger adsorption energy (i.e.> $0.4 \mathrm{eV}$, See Figure 66). The proposed adsorption patterns of amino acids are supported by the shifts in vibrational frequencies associated with $\mathrm{NH}_{2}$ and $\mathrm{COO}$ groups (See Table 2 in ref. [276]). The overall adsorption trend for the investigated Aas is the following: Pro $<$ Nor $<$ Ser $<$ Val $<$ Gly $<$ Ala $<$ Cys, can be explained in terms of electrostatic considerations beside the chemical interactions of specific functional groups such thiol groups e.g. with the surface. The absolute adsorption energy is a balance between molecular dipole moments, and the flexibility of the functional chain that can more or less adapt to accommodate at the surface. The Sulphur containing AA (Cyst) shows the highest affinity for Pd, whereas the AA having the highest geometrical constraint (i.e. having a pyrrole ring, Pro)shows the lowest affinity.The adsorption strength of amino acids depends on how much the molecules deform or relax during the adsorption process, with the maximum deformation energy found for Ala.
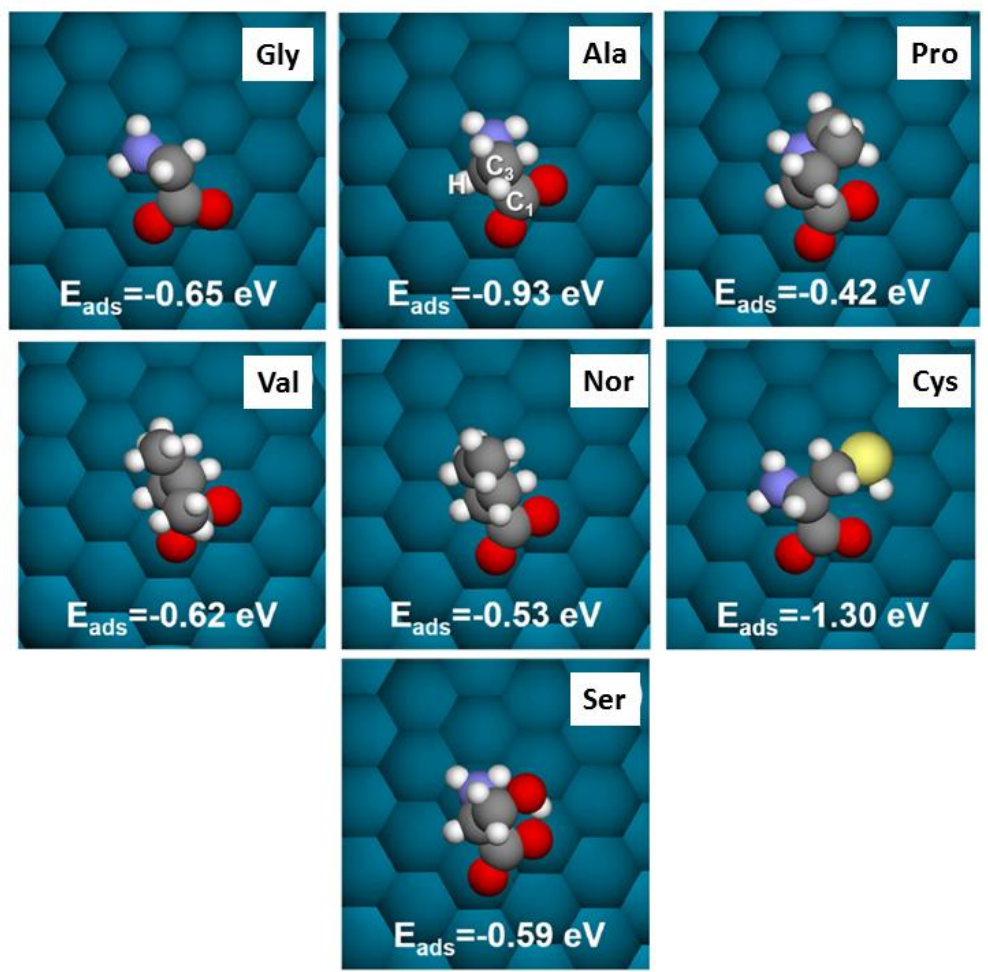

Figure 66.Most Stable optimized DFT-geometries for a series of AA on Pd(111) surface. Composed of figures of ref. [276].

\subsubsection{Amino acids as chiral modifiers: the case of Glutamic acid at Ni and Ni/Au surfaces.}




\subsubsection{Ni crystals.}

Being relevant for enantioselective catalysis, Ni-based materials and their interaction with chiral modifiers have been deeply investigated. The most studied molecules are (R,R)-tartaric acid [277][49] and (S)-Glutamic acid [170]. In the present review, we will focus our attention on the latter species, which shows a peculiar behaviour as a modifier since the dominant enantiomer produced in the catalytic reaction depends on $\mathrm{T}_{\mathrm{ev}}$. Glu adsorption below $350 \mathrm{~K}$ results in a preference for the $\mathrm{R}$ enantiomeric product in the hydrogenation of $\beta$-keto-esters, while adsorption above $350 \mathrm{~K}$ favours the S product (Figure 67) [263].

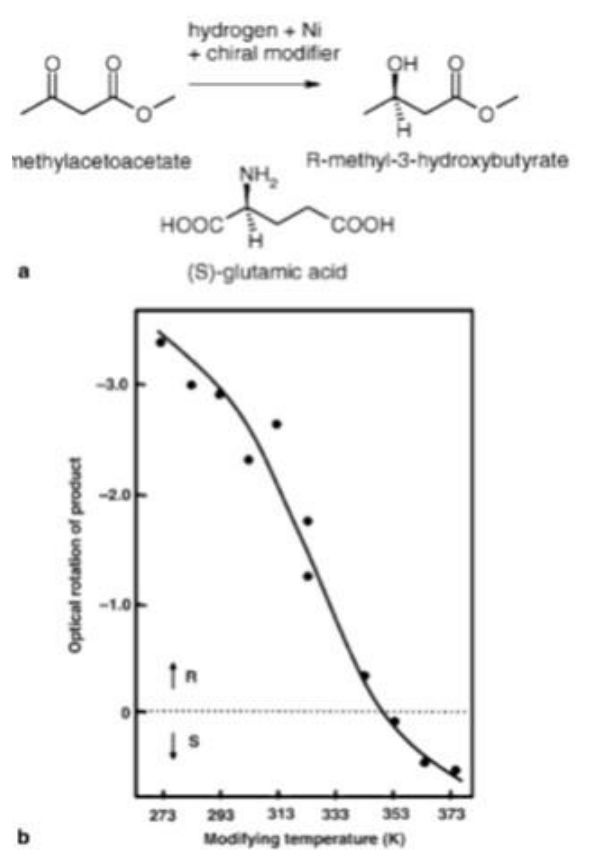

Figure 67. a) Reaction scheme showing the enantioselective hydrogenation of $\beta$-keto esters over Ni catalysts; b) Net optical rotation of the methyl-3-hydroxybutyrate product formed over (S)-Glu modified Ni catalysts as a function of modification temperature.Taken with permission from ref. [170].

The (S)-Glu/Ni(111) system was thoroughly studied by the group of Baddeley, who started from the characterisation of the Glu layer [170] and moved than to the investigation of the chemical activity of the chirally-modified ( $\mathrm{S}$ )-Glu/Ni catalyst with respect to the simplest $\beta$-keto ester, i.e. methylacetoacetate (MAA) [168; 169]. Figure 68 reports STM images recorded after Glu deposition on $\mathrm{Ni}(111)$ under different conditions. At low Glu coverage and $300 \mathrm{~K}$ (Figure 68a) molecular features are found predominantly close to the upper step edges, with no obvious short-range order. With increasing coverage the terraces are populated (Figure 68b) and ordered arrangements with a $(\sqrt{7} \times \sqrt{7}) \mathrm{R} 19.1^{\circ}$ geometry (local coverage, $\left.0.14 \mathrm{ML}\right)$ are observed in small areas of the surface. Only one of the two mirror equivalent domains of this type is present, in agreement with the chiral nature of the amino acid molecules. In addition, ordered structures consistent with a $(3 \sqrt{3} \times 3 \sqrt{3}) \mathrm{R} 30^{\circ}$ overlayer (local coverage, $0.11 \mathrm{ML}$ ) are detected [168]. Both structures are stabilized by intermolecular hydrogen bonding. It should be noted that the extent of ordering is relatively limited (the authors estimate that $10-20 \%$ of the surface consists of ordered domains, 
whose typical diameter is $\sim 10 \mathrm{~nm}$ ). This lack of long-range order has been reported for (R,R)tartaric acid on $\mathrm{Ni}(111)$ [49] and $\mathrm{Ni}(110)$ [277] and contrasts to the behaviour observed for (S)-Glu on $\operatorname{Ag}(110)$ [51] and for (R,R)-tartaric acid [261], succinic acid [278] and other amino acids on $\mathrm{Cu}$ surfaces $[69 ; 101 ; 158 ; 181]$.

The degree of surface ordering increases by annealing the surface to $350 \mathrm{~K}$ (Figure 68c), while further heating to $400 \mathrm{~K}$ (Figure 68d) changes the nature of the adlayer: molecules tend to order in 1-D structures extending along the close packed Ni direction, but no large 2-D ordered domains are observed. Adsorption of Glu directly at $350 \mathrm{~K}$ (Figure 68e) reveals an ordering similar to the one characteristic of the annealed layer. In addition, evidence is found for the growth of second layer islands, which possess a local ordering similar to the one of monolayer molecular features. The step edges take a "saw-tooth" appearance. The restructuring of the step edges by a chiral molecule has been shown to cause chiral faceting of metal single crystalse.g. in the case of lysine on $\mathrm{Cu}(001)$ [150] and of glutamic acid on $\operatorname{Ag}(110)$ [51]. This observation may be important in explaining the behaviour of (S)-Glu modified Ni catalysts as a function of modification temperature. In fact at room temperature, where little step-edge corrosion is observed, the enantioselective properties of the catalyst are different from the one observed following modification at $350 \mathrm{~K}$. 

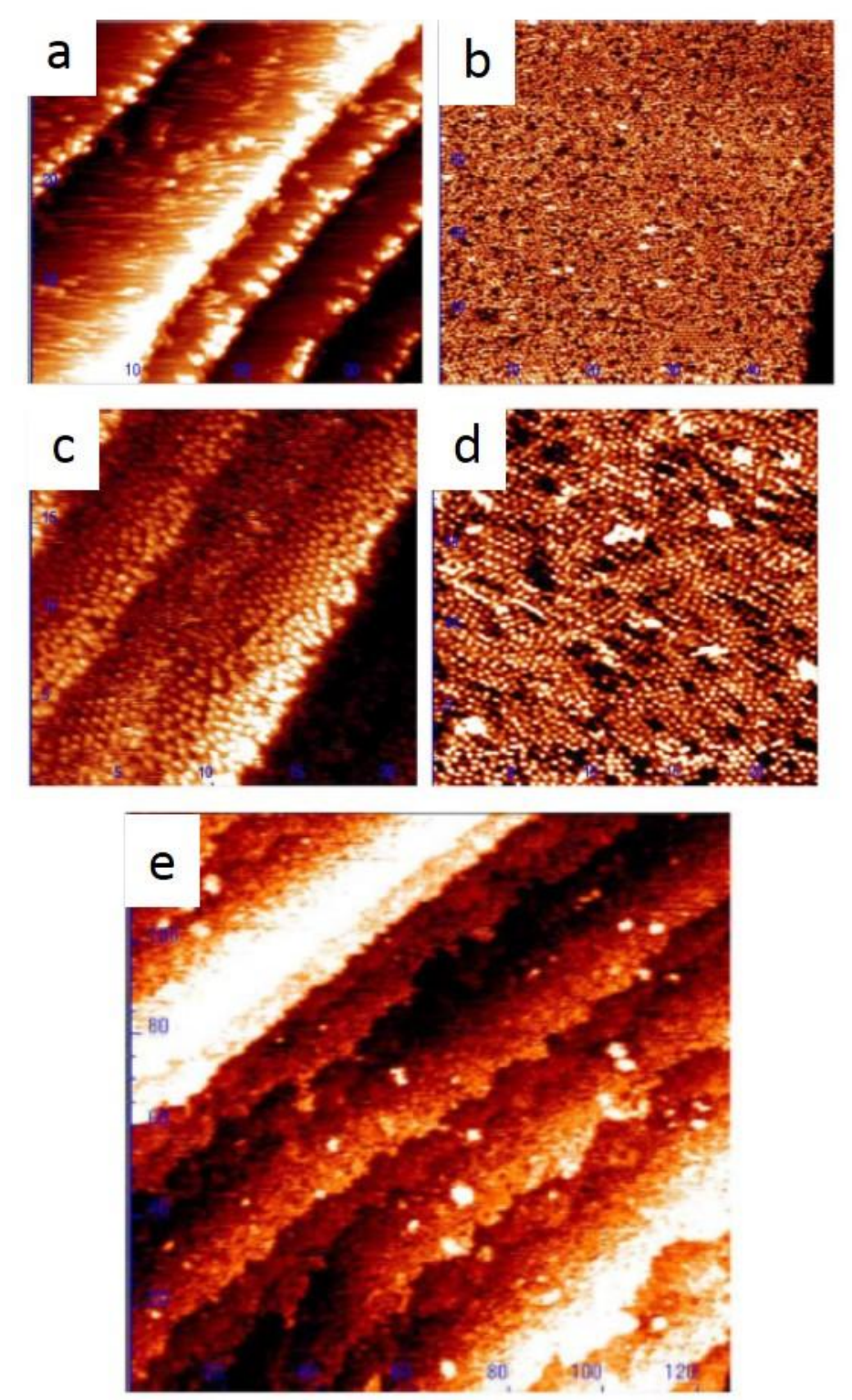

Figure 68. STM images of the Glu/Ni(111) layers under different experimental conditions. A) low coverage at $300 \mathrm{~K}$ (image size: $36.1 \times 36.1 \mathrm{~nm}^{2} ; \mathrm{I}=0.36 \mathrm{nA} ; \mathrm{V}=0.48 \mathrm{~V}$ ); b) high coverage at 300 $\mathrm{K}$ (image size: $49.7 \times 49.7 \mathrm{~nm}^{2} ; \mathrm{I}=0.34 \mathrm{nA} ; \mathrm{V}=0.55 \mathrm{~V}$ ); c) high coverage annealed to $350 \mathrm{~K}$ (image size: $21.4 \times 21.4 \mathrm{~nm}^{2}, \mathrm{I}=0.32 \mathrm{nA} ; \mathrm{V}=0.36 \mathrm{~V}$ ); d) high coverage annealed to $400 \mathrm{~K}$ (image size: $23.5 \times 23.5 \mathrm{~nm}^{2} ; \mathrm{I}=0.30 \mathrm{nA} ; \mathrm{V}=0.54 \mathrm{~V}$ ); e) adsorption at $350 \mathrm{~K}$, showing corrosion of step edges (image size: $128.4 \times 128.4 \mathrm{~nm}^{2}, \mathrm{I}=0.32 \mathrm{nA} ; \mathrm{V}=0.54 \mathrm{~V}$ ). Taken with permission from ref. [170].

The chemical state of the adsorbed Glu molecules was investigated by means of RAIRS. Sample spectra are reported in Figure 69, while vibrational frequencies and their assignment are summarized in Table 10. The overall picture emerging is that:

1) At low coverage and $\mathrm{T}=300 \mathrm{~K}$, glutamate exists in the zwitterionic form. By applying the metal surface selection rule, one deduces that the $\mathrm{C}-\mathrm{N}$ bond has a significant component perpendicular to the surface and that the two oxygen atoms of the carboxylate functionality are equidistant from the surface. A similar carboxylate geometry has been observed for glycine [42] and alanine [50] on $\mathrm{Cu}(110)$. The STM data suggest that the zwitterionic species adsorb in 
the vicinity of step edges on the upper terrace. This adsorption site may be stabilised by the interaction of the ionic adsorbates with the high electric field at the step edges.

2) At higher coverage, a new phase coexists with the zwitterionic one. On the basis of the additional bands appearing at $1716 \mathrm{~cm}^{-1}(\mathrm{C}=\mathrm{O}$ stretching frequency of a carboxylic acid $)$ and at $1418 \mathrm{~cm}^{-1}\left(v_{\mathrm{s}}(\mathrm{OCO})\right)$, it is identified with anionic mono-glutamate with the carboxylate and amino groups of the amino acid functionality interacting directly with the Ni surface.

3) Adsorption of (S)-Glu at $350 \mathrm{~K}$, leads to the formation of an adsorbed anionic species. The formation of the 1-D ordered structure observed in STM images is probably driven by Hbonding interactions between neighbouring molecules.
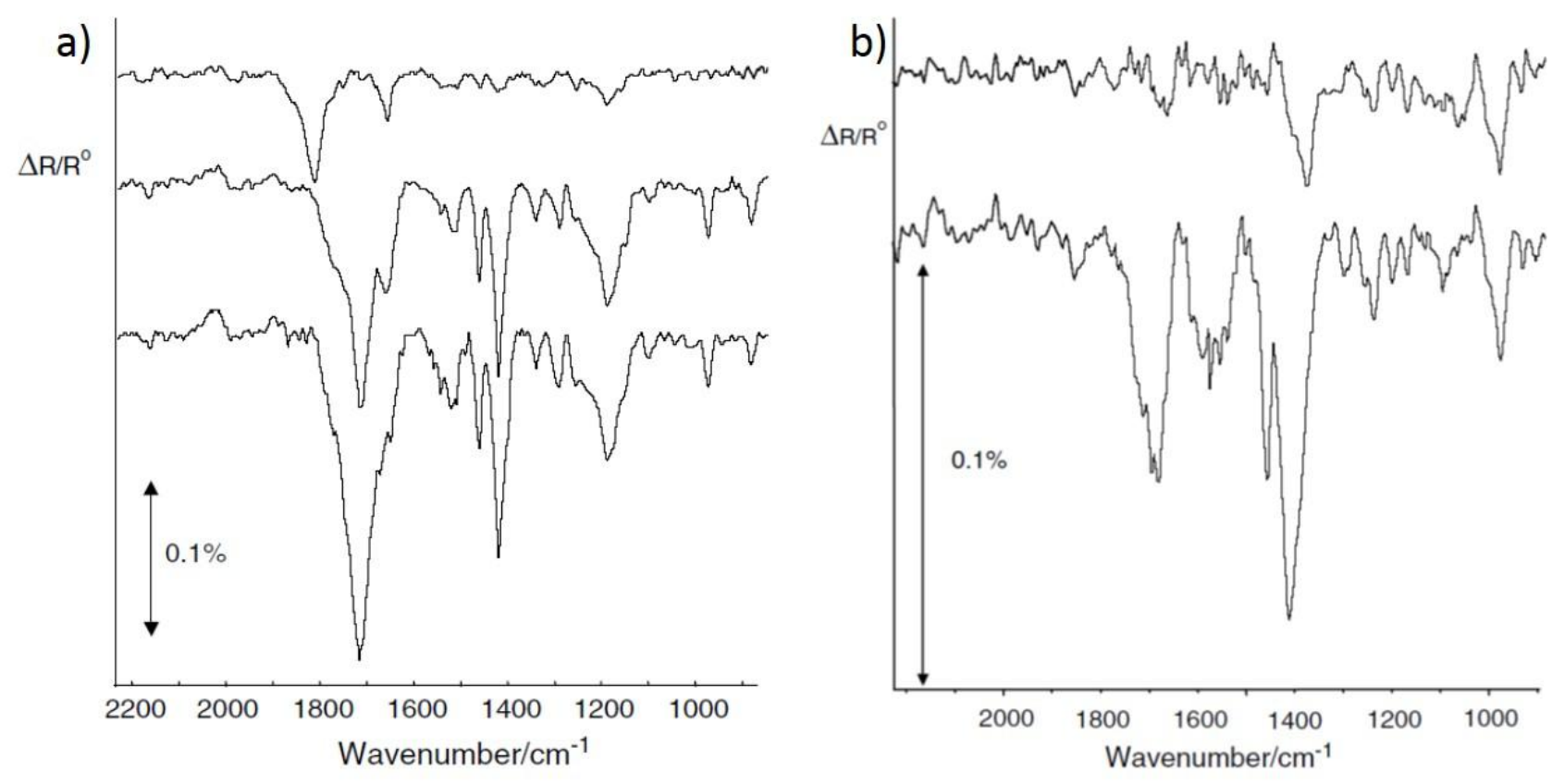

Figure 69. a) RAIR spectra following (S)-Glu adsorption on $\mathrm{Ni}(111)$ at $300 \mathrm{~K}$. From top down: low; intermediate and saturation exposure. B) RAIR spectra following (S)-Glu adsorption on Ni(111) at 350 K. Top: low coverage; bottom: high coverage. Taken with permission from ref. [170]. 


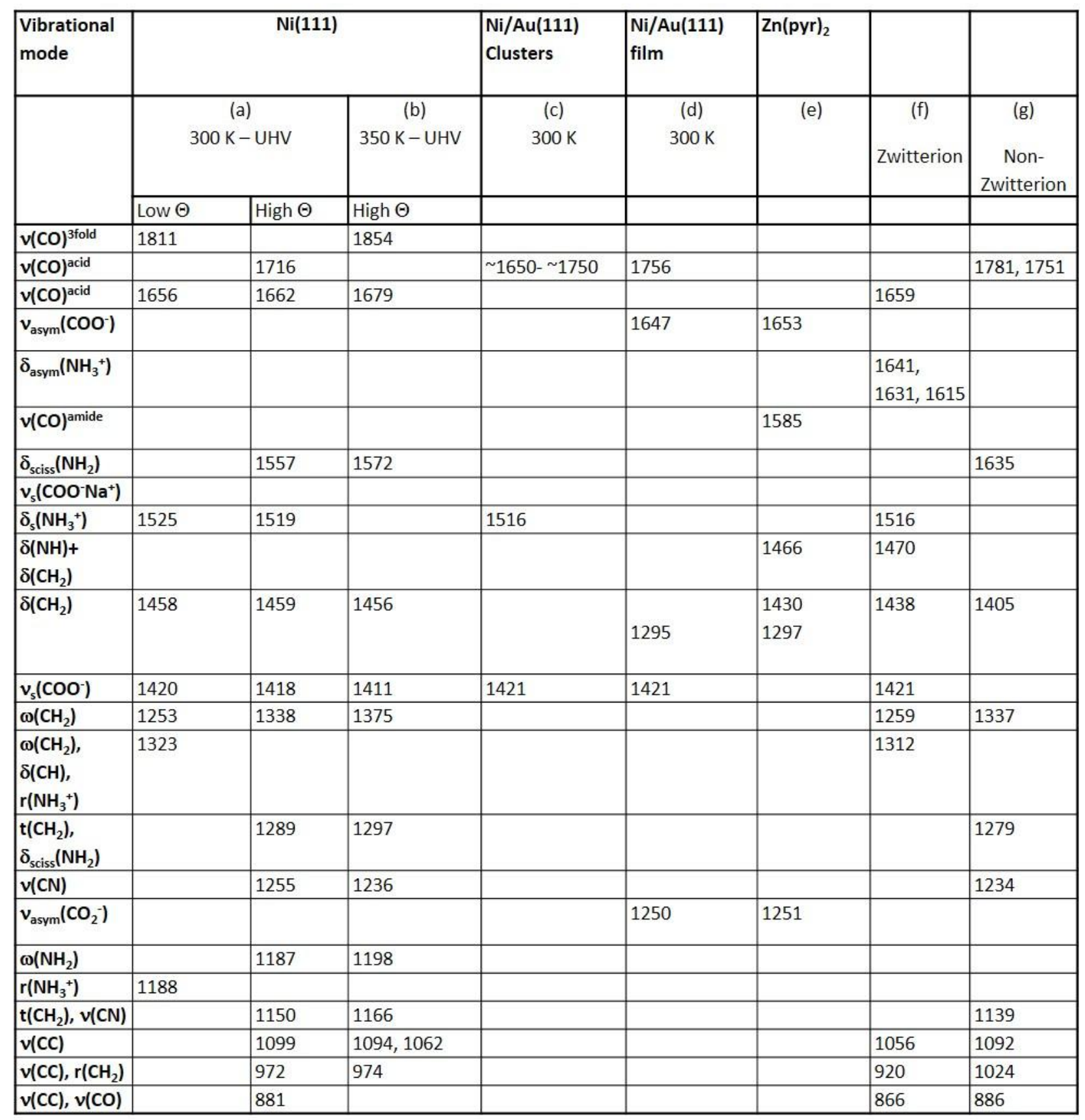

Table 10. Summary of the assignments of the different vibrational frequencies observed for Glu adsorption at $\mathrm{Ni}(111)$ and $\mathrm{Ni} / \mathrm{Au}(111)$ surfaces under the different conditions reported in the papers discussed in this section. Data for zinc pyroglutammate and theoretical predictions for isolated molecules in the zwitterionic and non-zwitterionic form are reported for comparison. Frequencies are expressed in $\mathrm{cm}^{-1}$. Data taken from refs. [170] (col. A and b), [279] (column c), [171] (columnd), [280] (column e), [250] (column f) and [251] (column g).

After complete characterisation of the chirally modified ( $\mathrm{S})-\mathrm{Glu} / \mathrm{Ni}(111)$ surface, Jones and Baddeley proceeded in their investigation with the adsorption of methylacetoacetate $\left(\mathrm{C}_{5} \mathrm{H}_{8} \mathrm{O}_{3}\right)$ and tested two preparation protocols. Initially both the chiral modifier and the reactive molecule were dosed under UHV conditions, thusproviding a complete characterisation of the MAA hydrogenation reaction as a function of the modifier coverage and modification temperature [168]. Afterwards, the sample was prepared by immersion in solutions of suitable concentration [169], thus allowing 
control over the influence of $\mathrm{pH}$ over the (S)-Glu layer and the enantioselective process. A detailed description of the results goes beyond the scope of the present review. Here we just mention that:

1) When the (S)-Glu/Ni(111) layer is prepared from solution, RAIR spectra show a significant $\mathrm{pH}$ dependence of the adsorbed Glu layer which, at RT, is in the cationic/zwitterionic form at low $\mathrm{pH}$ and gets progressively deprotonated with increasing $\mathrm{pH}$. After modification at $350 \mathrm{~K}$, nickel glutamate or a mixture of nickel and sodium glutamate is detected depending on the acidity/basicity of the solution [169].

2) At $300 \mathrm{~K}$ and under UHV conditions, the sticking probability of MAA on $(S)$-Glu/Ni(111) with different Glu coverage suggests that clean metal patches are required for adsorption [168]. Since the chiral modifier arranges in ordered structures only at high coverage, ordered modifier layers do not seem to play a significant role in the catalytic reaction: the local coverage in the arrays is too high to accommodate extra molecules.

3) After adsorption of $(S)$-Glu at $300 \mathrm{~K}$, subsequent adsorption of MAA at $300 \mathrm{~K}$ occurs mainly in the diketo tautomeric form, with a direct interaction between the $\mathrm{NH}_{3}{ }^{+}$group of zwitterionic Glu and the ketone group of the MAA molecules. After modification at $350 \mathrm{~K}$, on the contrary, MAA adsorbs at $300 \mathrm{~K}$ exclusively in the enol form [168]. The nature of the final product depends also on $\mathrm{pH}$; essentially, the more protonated the Glu modifier is, more diketones tend to form.

This is an example of the, already mentioned, dependence of the enantioselectivity of (S)-glutamic acid-modified Ni catalysts with temperature. Modification at $\mathrm{T}<350 \mathrm{~K}$ results in predominantly the I-enantiomeric product, whileT> $350 \mathrm{~K}$ favours the $(S)$-enantiomeric product (Figure 67). The optimum enantioselective catalytic performance occurs following modification at $\mathrm{pH} 5$, at which the keto:enol ratio continues to drop with increasing modification temperature. From these pieces of information, it is evident that the diketone form of MAA is the precursor $\mathrm{I}(R)$-methyl-3hydroxybutyrate when interacting with $(S)$-glutamic acid-modified Ni(111). In contrast, the enol form appears to be the precursor to the $(S)$-enantiomeric product. Therefore, the control over the tautomeric form present on the surface appears as the key to optimizing the enantioselectivity of the catalytic process. Since (S)-Glu adsorbs in the zwitterionic form at $300 \mathrm{~K}$ and low coverage and in the anionic form at $350 \mathrm{~K}$, the switch in the preferred enantiomeric product with modification temperature may be related to the ability of Glu to behave either as an amino acid (analogous to alanine) or as a chiral diacid (analogous to tartaric acid).

\subsubsection{Ni/Au systems.}

Amino acid adsorption at bimetallic surfaces may show peculiar properties with respect to the interaction of the same molecules with a single metal, as already observed for Gly/Ag/Cu [162] (see section 3.2.2). The case of Glu adsorption at Ni clusters [279] and thin films [171] deposited on $\mathrm{Au}(111)$ is another interesting case, although characterised by a completely different chemistry.

Upon $\mathrm{Ni}$ deposition on $\mathrm{Au}(111)$ at $300 \mathrm{~K}, 2-\mathrm{D}$ islands nucleate at the elbows of the herringbone reconstruction [281] (Figure 70a) and extend then to larger patches of the surface (Figure 70c). Subsequent exposure to (S)-Glu leads to the formation of two distinct adsorbate species (see Table 10): at low coverage, $(S)$-Glu adsorbs in the zwitterionic form at the centre of the Ni islands. As the latter ones become filled, glutamate species are forced to populate sites at the edge of islands, with the $\mathrm{COOH}$ group pointing away from the islands and thus unable to interact with other incoming molecules (not sticking on Au sites under the same conditions). 
At the highest Glu doses, Ni clusters with initial diameters $<3 \mathrm{~nm}$ disappear, while larger clusters are modified in shape and, presumably, passivated by the adsorbed glutamate layer. Annealing these surfaces to $\mathrm{T} \sim 360 \mathrm{~K}$ results in the corrosion of clusters and the formation of 1 -D chains of molecular features aligned along preferential directions (see Figure 70, panels $b$ and $d$ ). The authors conclude that the periodicity of the molecular features is too short to be consistent with the formation of nickel(II) glutamate. Instead, they propose that glutamate undergoes an internal cyclization process, forming pyroglutamate, with the liberation of a water molecule. Then, two types of chains are identified. The former, growing in the <101> direction, is formed by intermolecular $\mathrm{H}$-bonding interactions between neighbouring pyroglutamate units. The latter, aligned with the $\langle 112\rangle$ direction, is more likely stabilized by ionic interactions between $\mathrm{Ni}^{2+}$ and the carboxylate functionalities of pyroglutamate.
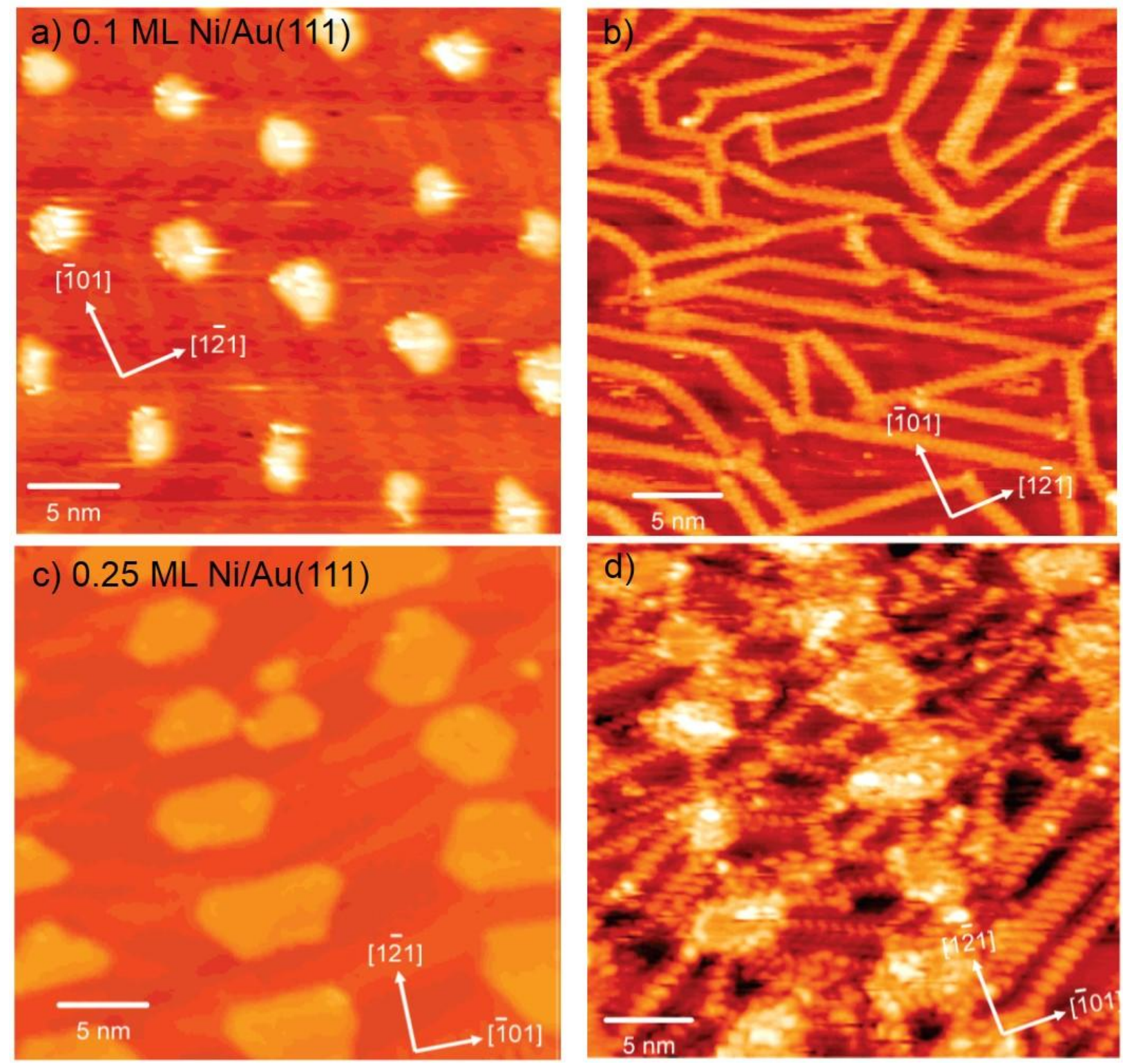

Figure 70. a) STM image recorded after deposition of $0.1 \mathrm{ML} \mathrm{Ni}$ on $\mathrm{Au}(111)$ at $300 \mathrm{~K}(\mathrm{~V}=-0.80$ $\mathrm{V}, \mathrm{I}=0.19 \mathrm{nA})$. b) Same as a) after a exposure to (S)-Glu at $300 \mathrm{~K}$ up to saturation and subsequent flash to $360 \mathrm{~K}(\mathrm{~V}=-0.92 \mathrm{~V}, \mathrm{I}=0.14 \mathrm{nA})$. Chains are aligned approximately along both $\langle 101\rangle$ and $<112>$ azimuthal directions on the surface in comparable numbers. Typically, parallel chains are 
separated by 3-4 nm. Neighbouring repeating features within a given chain are separated by 7-8 A. This periodicity appears to be approximately independent of the growth direction of the chain. c) STM image recorded after deposition of $0.25 \mathrm{ML} \mathrm{Ni}$ on $\mathrm{Au}(111)$ at $300 \mathrm{~K}$ (V =-0.90 V, I =2.0 nA). Now the 2-D Ni clusters have well defined straight edges that are aligned parallel to the closepacked <-101> directions of the underlying Au surface. d) Same as c) after exposure to saturation coverage of (S)-Glu with the substrate at $300 \mathrm{~K}$ and subsequent annealing to $360 \mathrm{~K}(\mathrm{~V}=-0.90 \mathrm{~V}$, $\mathrm{I}=0.16 \mathrm{nA}$ ). Ni clusters are still visible but their edges are now much less defined. Clusters are connected via similar 1-D chains of molecular features extending in two preferred growth directions. One type of chain has a periodicity of $\sim 7.3 \AA$ with one of the three rotationally equivalent growth directions aligned at an angle of $\sim 80^{\circ}$ to the $\langle 1-21\rangle$ direction; the other has a periodicity of $\sim 6.3 \AA$ with one of the three rotationally equivalent growth directions at an angle of $\sim 57^{\circ}$ with respect to the $\langle 1-21>$ direction. For all panels, image size: $30 \mathrm{~nm}$ x $30 \mathrm{~nm}$. Taken with permission from ref. [279].

The strong affinity between glutamic acid and nickel is demonstrated in the case of Glu adsorption at $\mathrm{Ni}$ films on $\mathrm{Au}(111)$ [171]. Since $\mathrm{Au}$ diffuses easily through $\mathrm{Ni}$, for a $8 \mathrm{ML} \mathrm{Ni}$ film it was possible to move gradually from a Ni-rich to an Au-rich surface by thermal treatment. By carefully combining RAIRS (see Table 10), TPD and MEIS results, the authors demonstrate that, at RT, Glu adsorbs as pyroglutamate on the Ni-rich bimetallic surface but in the non-zwitterionic form on the Au-rich one (it converts into pyroglutamate only upon annealing). Pyroglutamate formation, not observed on $\mathrm{Ni}(111)$ at the same temperature, is likely catalysed by steps or other defect sites on the relatively rough Ni films[279]. Moreover, AA-induced segregation of Ni occurs, as expected for bimetallic surfaces exposed to a reactive adsorbate. Since desorption experiments strongly suggest the formation of pyroglutamate on Au patches, this compound is likely the thermodynamic driving force underpinning the segregation of $\mathrm{Ni}$ to the surface. At $300 \mathrm{~K}$, glutamic acid is able to dehydrate to form pyroglutamate more efficiently on a Ni-rich surface than on a Au-rich surface, and may cause the segregation of $\mathrm{Ni}$ to the surface. Interestingly, segregation is reversed by annealing to 400 $\mathrm{K}$, a temperature sufficient to initiate decomposition and desorption of $\mathrm{Ni}(\mathrm{pyr})_{2}$, resulting in a return to the Au-rich surface favoured under UHV conditions.

\subsubsection{Adsorption of other amino acids at Ni surfaces.}

The adsorption of alanine and phenylalanine was studied theoretically on Ni(111) using DFT and classical atomistic simulations[282; 283], taking into account both quantum and statistical aspects of the molecular conformations of the AA at the surface. A model was presented for the adsorption of these molecules both in isolated and aqueous conditions. These models were subsequently used in classical atomistic simulations to study the physical-chemical aspects of amino acids at a $\mathrm{Ni}(111) /$ water interface. Alanine adsorption in isolated conditions was studied at the DFT level by Ghiringhelli et al.[282; 283]. Alanine was investigated in its neutral and zwitterionic form (see Figure 71 and Figure 72), yielding maximum adsorption energies of -0.57 and $-0.74 \mathrm{eV}$, respectively. The adsorption site for the neutral form is on top. Although two competing geometries are observed (See Figure 71), adsorption through the $\mathrm{N}$ of the amino group, and with the methyl group oriented upwards due to the high repulsion with the surface, is proposed to be favoured. The interaction with Nickel is visualized by a very small displacement of the Ni adsorption siteout of the surface. 

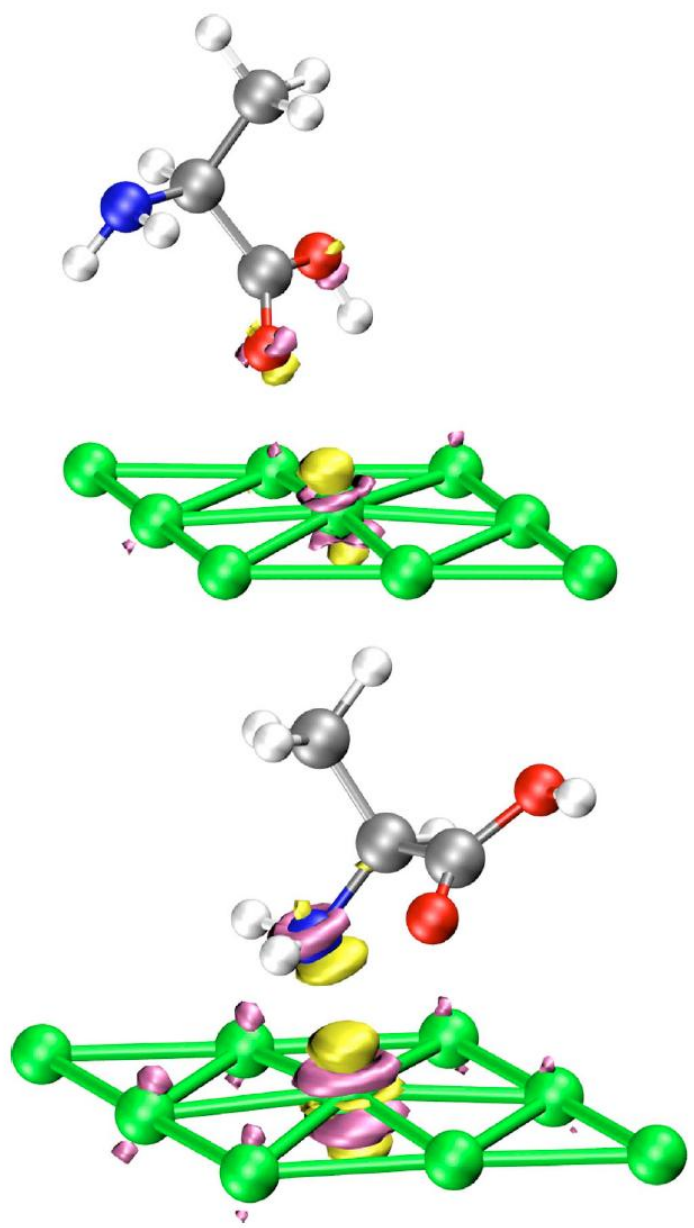

Figure 71. Two adsorption geometries for the neutral alanine adsorbed in $\mathrm{Ni}(111)$, with electron density difference plots (not discussed in the text). Top panel: the configuration with one oxygen bonded to the surface, with $\mathrm{E}_{\mathrm{ads}}=-0.37 \mathrm{eV}$. Bottom panel the configuration with the nitrogen bonded to the surface, with $\mathrm{E}_{\mathrm{ads}}=-0.57 \mathrm{eV}$.Taken with permission from reference [283].

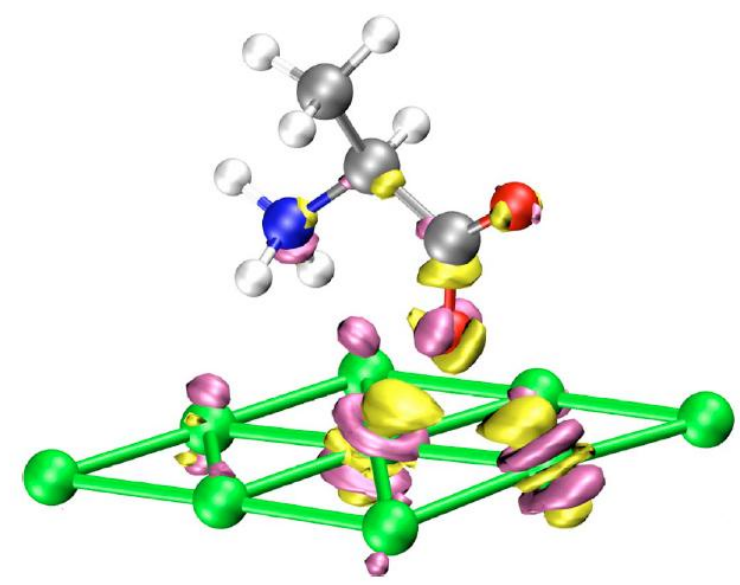


Figure 72. Perspective view of the final configuration of the adsorbed zwitterionic alanine, with electron density difference map (not discussed in the text) $\left(\mathrm{E}_{\mathrm{ads}}=-0.74 \mathrm{eV}\right)$. Taken with permission from reference [283].

Lysine adsorption on submonolayer $\mathrm{Ni}$ films deposited on $\mathrm{Au}(111)$ was investigated by experimental methods, namely by STM, RAIRS and TPD [284]. This study is the natural prosecution of the one performed on $\mathrm{Glu} / \mathrm{Ni} / \mathrm{Au}(111)$ [279] and described in the previous section. Some common behaviour but also significant differences, related to the specific molecular structure of the adsorbates, were observed. (L)-Lys was deposited at room temperature on the $\mathrm{Au}(111)$ substrate covered by $0.1 \mathrm{ML}$ or $0.5 \mathrm{ML}$ of $\mathrm{Ni}$ clusters produced in the same way as those reported in Figure 70a and c. Two different lysine overlayers were found, being dominant at $300 \mathrm{~K}$ and upon annealing at $350 \mathrm{~K}$, respectively. As in the case of $\mathrm{Glu} / \mathrm{Ni} / \mathrm{Au}(111)$, adsorption of lysine is associated with the corrosion of the Ni clusters, which completely disappear below a critical size. The data are interpreted with the formation of $\mathrm{Ni}$ (lysinate) ${ }_{2}$, which organizes into self-assembled arrays according to the following proposed mechanism. Initial adsorption occurs on the Ni clusters. Atoms at the edges of $\mathrm{Ni}$ clusters are easily oxidised and removed from the clusters by lysine. However, adsorption of lysine on the centre of Ni clusters stabilises the clusters such that there exists a threshold cluster size above which the cluster survives the etching process albeit reduced in size. Nickel-lysinate complexes are able to self-assemble into two ordered arrangements. The $\left(\begin{array}{cc}7 & -2 \\ 1 & 2\end{array}\right)$ one, dominant at RT, is stabilised by intermolecular H-bonding between the terminal amine groups in an essentially flat lying geometry. The $\left(\begin{array}{cc}6 & -2 \\ 2 & 4\end{array}\right)$ assembly has a higher molecular density and it is believed to form at higher temperature via the reorientation of the aliphatic backbone of lysine to reduce the surface footprint. If $\mathrm{Ni}$ clusters are totally corroded by Lys adsorption, no ordered structures are observed, probably due to the high diffusion rate of nickellysinate complexes at low coverage. Under these conditions, however, rearrangement of step atoms into chiral nanofingers is observed. This effect is typical of the molecular species, being observed already for Lys adsorption at $\mathrm{Au}(111)$ (see ref. [153] and Figure 46). It is likely that the direction of nanofinger growth is closely associated with the preferred surface orientation of nickel-lysinate complexes. The ability to 'spill over' nickel lysinate species onto Au surfaces with a high degree of control over the supramolecular assembly has potential to be adapted in bimetallic catalytic systems. 


\subsection{Introduction: From metals to oxides.}

At the exception of gold, all metals are oxidized in ambient, non reducing conditions. The metal can be fully oxidized in a bulk oxide or be covered by an oxide layer, as e.g. in the case of $\mathrm{TiO}_{2}$ on $\mathrm{Ti}$. Mostly, adsorption of biomolecules on oxide surfaces happens in wet conditions. Works performed in UHV are very little and the hydrated conditions are the natural prosecution of the research.

The domains of interest ofinteraction of AA with natural oxides is wide, ranging from the origin of life (on silica, alumina, clays), to geochemistry or biomineralization (adsorption on minerals and impacts on dissolution), including the issue of adsorption of amino acids on minerals (e.g. iron and aluminium oxides). For industrial and medical applications, amino acids adsorption at oxides is also a key question, e.g., in chromatography (silica) or on medical devices (stainless steel)[7].They are used to monitor nanoparticles growth and shape (e.g. boehmite, $\mathrm{CuO}, \mathrm{TiO}_{2}$ ) and are also candidates for green corrosion inhibition (steel, $\mathrm{Al}$ alloys)[285]. AA adsorption on metals and oxides (in this case, often an ultrathin oxide film formed on the metal) has been studied in the search for green inhibitors as an alternative for chromate inhibition. Because the information brought by those studies (especially on aluminium alloys[286; 287] and mild steel) is at the macroscopic but not at the molecular level, it will not be detailed here. It is however worth to mention that amino acids have an impact on corrosion inhibition, a direct consequence of their adsorption at the metal/oxidized surface at various potential/pH. In other words, the change of macroscopic properties observed is explained by the formation of a full layer of amino acids at the surface.

Adsorption on oxides generally relies on different concepts compared to metals; the first difference is that a perfect metal surface bears equivalent sites and acts (unless an anodic electrochemical field is applied) as an electron reservoir. In contrast, oxides exhibit cations and anions and are most of the time insulators, sometimes are semi-conductors of $\mathrm{n}$ or $\mathrm{p}$ type. Thus, unless non specific, van der Waals interactions explain adsorption, metals and oxides behave very differently.

Adsorption of amino acids on oxide surfaces has been studied from the gas phase. It generally implies a neutral molecule $\mathrm{RH}(\mathrm{R}=\mathrm{COO}, \mathrm{NH}, \mathrm{S}$, etc $)$ and a neutral oxide surface. When the oxide surface is non hydroxylated, $\mathrm{M}$ Lewis cations and surface $\mathrm{O}$ bases $(=\mathrm{M}-\mathrm{O})$ are present on the surface, then the AA-surface interaction occurs through the formation of a covalent M-R bond, associated with the formation of a surface $\mathrm{OH}$ group. Surface science studies on well-defined surfaces are helpful to characterize these types of bonds, as it will be described hereafter.

An oxide surface that has reacted with water is hydroxylated. Adsorption of amino acid vapour on a hydroxylated surface is - to a certain e-tent - a way to study the role of water in the system. The reactions taking place can be creation of hydrogen bonds by adsorption above the hydroxylated surface (or outer sphere adsorption), and/or bond breaking and making with formation of a covalent bond and desorption of a water molecule in the gas phase (or inner sphere adsorption). The adsorption type is distinguishable through vibrational frequency and photoemission analysis. There have been very few experiments of that type, but samples produced by immersion in aqueous solution and analysed then in UHV conditions are - to a certain extent - similar to those produced by gas phase adsorption.

In this chapter we review experimental and theoretical works performed to characterize the adsorption of amino acids on oxide surfaces at the molecular level and at the vacuum interface.As evidenced in Erreur ! Source du renvoi introuvable., much less surface science studies have been 
devoted to oxides as compared to metals. Furthermore, we note that for all the AA/oxide systems the atomic information (nature of the bond with the surface) is brought mainly by spectroscopic studies. The reason is the insulating character of oxides, that makes surface science tools difficult to be used. One exception is $\mathrm{TiO}_{2}$, which has a two-fold interest: i) $\mathrm{TiO}_{2}$ has semi-conducting properties that allow surface science experiments to be performed; ii) it is a highly relevant system for biomolecule adsorption as $\mathrm{Ti}$ used as a biomaterial is covered with oxide.In addition, on $\mathrm{TiO}_{2}$ the gap between gas phase and UHV to liquid phase has been bridged; indeed $\mathrm{TiO}_{2}$ is of interest for the modelling of prebiotic systems (in gas phase and high temperature conditions)[288] as well as for biomedical applications (in biological solution), thus from the relative simplicity to the extreme complexity.A bottom up approach exists for $\mathrm{TiO}_{2}$,including many surface science studies and different atomistic modelling methods, and studies on $\mathrm{TiO}_{2}$ are illustrative of the wide range of atomistic modelling tools.

Apart from $\mathrm{TiO}_{2}$, to the best of our knowledge, adsorption from the gas phase has been studied for four oxides, namely $\mathrm{SiO}_{2}, \mathrm{Al}_{2} \mathrm{O}_{3}$ and $\mathrm{Cr}_{2} \mathrm{O}_{3}$ and $\mathrm{ZnO}$. Adsorption on silica[7] was treated recently and is not considered here. In the following, carboxylates and amines are first mentioned as models of amino acids. Then the adsorption of glycine/ $/ \mathrm{TiO}_{2}$, other $\mathrm{AA} / \mathrm{TiO}_{2}, \mathrm{Gly} /$ other oxides and other AA/other oxides is described.

\begin{tabular}{|c|c|c|c|c|c|}
\hline & 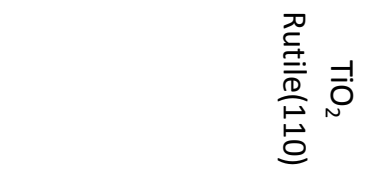 & 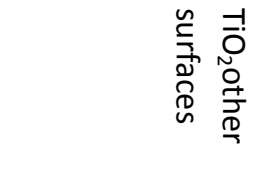 & 今̃ & 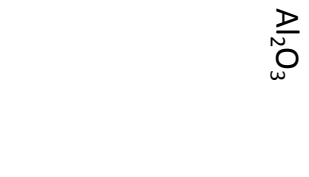 & 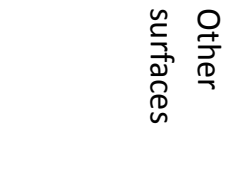 \\
\hline Gly & $\begin{array}{l}\text { DFT [289] } \\
\text { XPD [290] } \\
\text { STM [64] } \\
\text { SR-XPS }[291 ; 292] \\
\text { DFT [19; 293] } \\
\text { Classical DM FF[294]91 }\end{array}$ & $\begin{array}{l}\text { Rutile (011): } \\
\text { XPS, TPD [295] } \\
\text { Anatase (101): } \\
\text { DFT [296] } \\
\text { TDS [297] } \\
\text { IR[288] }\end{array}$ & $\begin{array}{l}\mathrm{ZnO}(0001): \\
\text { DFT[298] } \\
\text { ZnO(10-10): } \\
\text { DFT } \\
{[299]}\end{array}$ & $\begin{array}{l}\text { Thin film } \mathrm{Al}_{2} \mathrm{O}_{3} / \mathrm{NiAl} \text { : } \\
\text { XPS, UPS, NEXAFS } \\
{[300]} \\
\alpha-\mathrm{Al}_{2} \mathrm{O}_{3}(0001) \text { : } \\
\text { DFT [301] } \\
\text { NMR on } \\
\text { powder[302] }\end{array}$ & $\begin{array}{l}\text { DFT } \\
\mathrm{Cr}_{2} \mathrm{O}_{3}(0001) \\
{[303-305]}\end{array}$ \\
\hline Pro & $\begin{array}{l}\text { XPS [306-308] } \\
\text { DFT [293] }\end{array}$ & & & & \\
\hline Cys & $\begin{array}{l}\text { XPS [309] } \\
\text { DFT [310] }\end{array}$ & & & & \\
\hline Asp & DFT [311] & & & & \\
\hline Arg & $\begin{array}{l}\text { DFT on O deficient } \\
\text { surface[312] }\end{array}$ & $\begin{array}{l}\text { Anatase } \\
(100)[313]\end{array}$ & & & \\
\hline Ala & $\begin{array}{l}\text { XPS and NEXAFS on } \\
\text { Phenyl-alanine } \\
\text { [314] } \\
\text { DFT[315] }\end{array}$ & & $\begin{array}{l}\mathrm{ZnO}(10-10) \\
\mathrm{XPS}[316] \\
\mathrm{ZnO}(10-10) \\
\text { DFT [317] }\end{array}$ & & \\
\hline many & & $\begin{array}{l}\text { Car Parinello Cys, } \\
\text { Glu, Lys, His } \\
\text { rutile (100), } \\
\text { [318] } \\
\text { Anatase (101) } \\
\text { and Anatase }\end{array}$ & $\begin{array}{l}\text { DM on } \\
\text { amino acids } \\
\text { on } \mathrm{ZnO}(10- \\
\text { 10) surface } \\
\text { [320] }\end{array}$ & & \\
\hline
\end{tabular}


\begin{tabular}{|l|l|l|l|l|l|}
\hline & (001) [318][319] & & & \\
\hline
\end{tabular}

Table 11 Adsorption of amino acids on oxides from the gas phase (experimental and theoretical works).

\subsection{Adsorption of amino acids on $\mathrm{TiO}_{2}$}

$\mathrm{TiO}_{2}$ surfaces are the substrates on which much work has been performed, and perhaps the most studied oxide surfaces in surface science. Detailed information is available at the molecular level, including structural level, from surface science tools[321]. Here we benefit of a battery of experimental techniques, from STM, LEED, HREELS, XPS (applied to surfaces), to ATR-FTIR and other techniques more convenient for dispersed samples, thus providing a possible bridge from surface science on model surfaces to "real life" samples containing defects, polycrystalline or amorphous surfaces, powders or (nano)particles.

The most studied monocrystalline $\mathrm{TiO}_{2}$ surface is rutile (110). The (1x1) surface consists of rows of bridging oxygen ions $\left(\mathrm{O}_{\mathrm{br}}\right)$ with 5 fold $\mathrm{Ti}^{4+}$ ions $(\mathrm{Ti} 5 \mathrm{c})$ and in plane oxygen ions (see Figure 73

Figure 73. Schematic view of the rutile (110) surface: a) unreconstructed surface; b) reconstructed). The Ti surface atoms density is $5.10^{14} / \mathrm{cm}^{2}$. With reference to the surface chemistry and electronic structure, some of the most interesting information obtained concerns surface oxygen vacancies in the bridging oxygen rows. The reviews by Diebold [321] and Pang et al. [322] describe the structures of clean $\mathrm{TiO}_{2}$ surfaces in more details.

As detailed in the next section, acidic lateral chains (e.g. aspartic or glutamic acid residues in a peptide) and basic ones (e.g. lysine) have been shown to have affinities with the $\mathrm{TiO}_{2}$ surfaces. Therefore, before entering into details of surface science studies on amino-acid adsorption, we will briefly mention some key results on the characterization of the simplest carboxylate, formic acid, and model amines on $\mathrm{TiO}_{2}$ single crystals and particles surfaces. Here we benefit of a number of experimental data that allow us to report a bottom up approach from the single crystal to the nanoparticules, and from UHV conditions to solid-liquid interface.

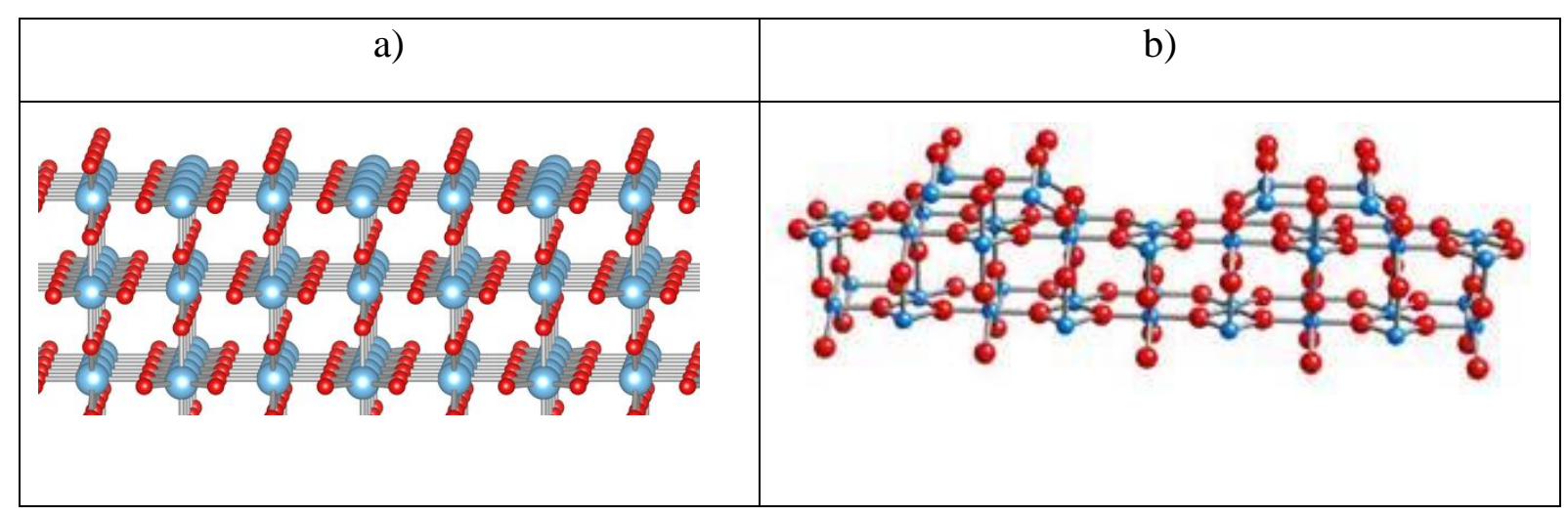

Figure 73. Schematic view of the rutile (110) surface: a) unreconstructed surface; b) reconstructed $1 \mathrm{x} 2$ surface. Oxygen is red, Ti is blue. Taken with permission from ref. [321]. 


\subsubsection{Carboxylic acids as models of amino acids: adsorption on $\mathrm{TiO}_{2}$}

The simplest carboxylic acid, formic acid, was adsorbed on $\mathrm{TiO}_{2}$ rutile (110)[323-325] and rutile (111)[326]. It is interesting to examine the vibration frequencies recorded. In fact, frequency analysis allows to scrutinize the adsorption mode of carboxylates on surfaces, especially on oxides where XPS data are less numerous than on metals. Three adsorption modes are envisaged (Figure $74)$ and $v(\mathrm{COO})$ frequency analysis $\left(v_{\text {sym }}\right.$ and $\left.v_{\text {asym }}\right)$ can help discriminating bridge or bidentate $(\mu 2)$

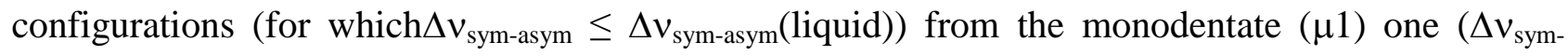
asym $>\Delta v_{\text {sym-asym }}($ liquid) $)[327]$. The $\Delta v_{\text {sym-asym }}$ of free formate in solution is $(1580-1351)=229 \mathrm{~cm}^{-}$ ${ }^{1}[328]$. Bridge and $\mu 2$ configurations are not distinguishable from frequency analysis.
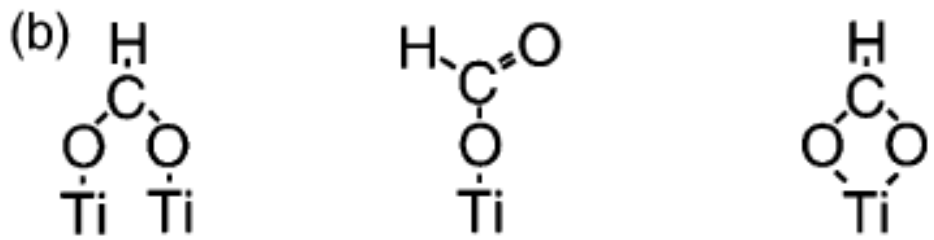

\section{bridge monodentate bidentate}

Figure 74. Different possible adsorption configurations of carboxylic acid (dissociated) on the $\mathrm{TiO}_{2}$ surface.

Formic acid adsorbed on $\mathrm{TiO}_{2}$ single crystals was analysed by HREELS[323; 324; 326; 329] and IRAS[330]. Adsorption on thin films and nanoparticles was also performed, and the frequencies were analysed by HREELS[324] or ATR-FTIR[331; 332].

There is a general agreement that, in 138ormatsrmates and hydroxyl species are produced on dry $\mathrm{TiO}_{2}(110)$ by dissociative adsorption of formic acid at room temperature, $(2 \times 1)$ ordered overlayers of formate being formed at saturation exposure. The corresponding coverage is 0.5 monolayer (ML), referenced to the number of exposed Ti sites. STM images of the $(2 \times 1)$ overlayer indicate that formate adsorbs on the rows of fivefold coordinated $\mathrm{Ti}^{4+}$ cations along the $\langle 001\rangle$ direction, with some evidence for a bond geometry in which the carboxylates are $\mu 2$ and bonded to adjacent Ti atoms. Indeed one single peak was observed at $1365 \mathrm{~cm}^{-1}$ in HREEL spectra for formic acid on rutile (110) and it was assigned to bridge adsorption[330]. Larger carboxylates follow the same trends, as evidenced by the comparison of formate, acetate and propanate adsorption on rutile (110) using K-edge NEXAFS[325]. Theoretical calculations confirm that the bridge coordination is more stable than the $\mu 1$ one[289; 333-335].

Formate species were also characterized on $\mathrm{TiO}_{2}(110)$ by IRAS[330].Adsorption at $300 \mathrm{~K}$ produces a formate coverage of $0.59 \mathrm{ML}$, higher than expected for a perfect $(2 \times 1)$ overlayer observed by lowenergy electron diffraction (LEED). This is attributed to adsorption at defective sites. A $v_{\text {as }}(C O O)$ band at $1536 \mathrm{~cm}^{-1}$ was attributed to bridging $\mu 2$ species adsorbed on 5-fold-coordinated Ti(IV) sites along the $<001>$ direction, whereas a second $v_{\text {as }}(\mathrm{COO})$ band at $1566 \mathrm{~cm}^{-1}$ was assigned to bridging $\mu 2$ species adsorbed at oxygen vacancies.

On the (111) surface, STM observation revealed nanometer-scale domains of $(1 \times 1)$ and $(1 \times 2)$ phases on the surface[326]. Formic acid was dissociatively adsorbed on the surface at room 
temperature. Three species were envisaged and HREELS could help discriminating the 2 from the $\mu 1$ one.

Formate species were produced also by dissociative adsorption of formic acid, either from gas phase or from water, on thin films exhibiting mostly the $\mathrm{TiO}_{2}(110)$ surface[331]. The FT-IRAS spectra recorded after adsorption from the gas phase and those at the liquid interface were nearly identical (with peak differences of 6-7 $\mathrm{cm}^{-1}$ ) and indicated that only one kind of adsorption occurs. On the basis of the measured frequency splitting $\Delta v_{\text {sym-asym }}(\mathrm{COO})=191 \mathrm{~cm}^{-1}$, the $\mu 1$ binding mode could be excluded[331]. Chang and Thorthon[324] also showed that native $\mathrm{TiO}_{2}(110)$ surface and $\mathrm{TiO}_{2}(110)$ thin films behave very similarly, both interacting with formic acid, and leading to its complete dissociation. $\Delta v_{\text {sym-asym }}(C O O)$ is $172 \mathrm{~cm}^{-1}$ for the film and $220 \mathrm{~cm}^{-1}$ for the native surface, both being consistent with $\mu 2$ bonding.

Moving to nanostructured systems, two types of nanoporous rutile layers (nanorod and nanowire thin films) were studied[332]. The most significant morphological differences of the two films consisted in the size of the nanocrystals as well as in their aspect ratio (nanorods, $7 \mathrm{~nm} \times 40 \mathrm{~nm}$; nanowires, $2 \mathrm{~nm} \times>50 \mathrm{~nm}$ ). The adsorpti139ormatsrmates from gas phase onto these films was investigated by ATR-IR spectroscopy,leading to $\Delta v_{\text {sym-asym }}(\mathrm{COO})=191 \mathrm{~cm}^{-1}$, characteristic of a bridge adsorption. The same results was obtained after adsorption from an aqueous solution.

Finally, vibrational frequencies calculated by ab initio methods could be successfully compared with the experimental ones and the attributions for $\mu 1$ and $\mu 2$ species were confirmed. The general scheme is that $\Delta v_{\text {sym-asym }}(\mathrm{COO})=305-530 \mathrm{~cm}^{-1}$ corresponds to a $\mu 1$ conformation and $\Delta v_{\text {sym- }}$ $\operatorname{asym}(C O O)=204-303 \mathrm{~cm}^{-1}$ corresponds to a bridge configuration[289].

Theoretical calculations yield an energy of adsorption of $-1.35 \mathrm{eV}$ for the bridge adsorption of formic and acetic acids on rutile (110), only $0.10 \mathrm{eV}$ more exothermic than the $\mu 1$ adsorption[289]. B3LYP calculations conclude in adsorptions of -0.36 (respectively-0.26) eV for bi-and $\mu 1$ adsorption[336]. DFT $+\mathrm{U}$ calculations conclude to energies of $-1.84 \mathrm{eV}$ and $-1.31 \mathrm{eV}$ for the $\mu 2$ and $\mu 1$ adsorption, respectively[337]. All in all, calculated energies of adsorption on the rutile (110) surface are between 1.3 and $1.8 \mathrm{eV}$. On anatase, the HCOOH DFT-calculated adsorption energies are -0.9 and $-1.7 \mathrm{eV}$ on the (101) and (001) surfaces, respectively[338].

To conclude this part, formate adsorption from the gas phase occurs with a direct Ti-OCO bond. The adsorption mode is site specific, and depends on the Ti coordination number. The adsorption of larger carboxylic acids follows the trends evidenced for formic acid[325]. Frequency analysis nicely helps to understand the adsorption mode. This information is useful when considering carboxylic acids as models of acidic lateral chains/residues of amino acid/peptides.

\subsubsection{Amines as models of amino acids adsorbed on $\mathrm{TiO}_{2}$}

Adsorption of several organic molecules, including amines, on $\mathrm{TiO}_{2}$ was briefly reviewed by Thomas and Syres[339]. Farfan-Arribas and Madix[340] used amine adsorption to determine the Lewis acidity of surface $\mathrm{Ti}^{4+}$ ions in rutile $\mathrm{TiO}_{2}(110)$ surfaces. Ethylamine and diethylamine were both found to adsorb on the stoichiometric surface through formation of an N-Ti bond. On defected surfaces bonding also occurred at oxygen-vacancy sites. In both cases, the amines remained intact,with less amines adsorbedon the defected surface. This may be due to adsorption at the defect 
sites blocking adsorption at more than one neighbouring Ti5c site. The authors also found that the desorption activation energy decreased in the following order: diethylamine > ethylamine > ammonia, which is also the order of decreasing Lewis basicity. The authors suggested that this was evidence of adsorption driven by a Lewis acid-base interaction with the $\mathrm{Ti}^{4+}$ ions behaving as Lewis acids. A DFT study of adsorption of diaminoethane on anatase $\mathrm{TiO}_{2}(101)$ predicted an interaction around $-0.5 \mathrm{eV}$ (-0.7 eV includingdispersion forces), a value indicating a less exergonic processthan for carboxylates on the same anatase surface $(-0.9 \mathrm{eV}$, calculated without dispersion).

\subsubsection{Glycine on $\mathrm{TiO}_{2}$}

Glycine differs from acetic acid by the presence of the $\mathrm{NH}_{2}$ functionality. The first guess for glycine adsorption was derived from carboxylate adsorption. There are thus several possible adsorption schemes for glycine on $\mathrm{TiO}_{2}$, as schematized in Figure 75.

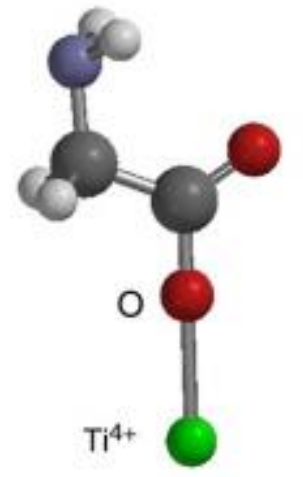

I $\mu 1$

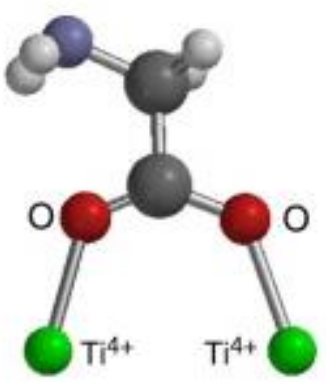

II

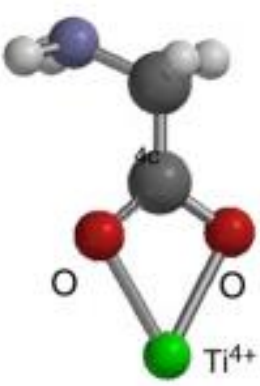

III

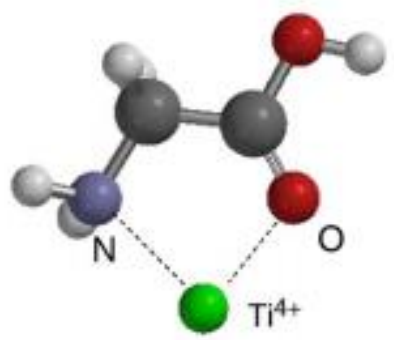

IV

intercalating

Figure 75. Possible modes (non exhaustive) of interaction of glycine with Ti cations. Taken with permission from ref. [295].

Several works using surface science techniques have been devoted to the elucidation of adsorption mode and deprotonation state of amino acids on rutile (110)[64; 291; 292]. The first surface science study on glycine adsorption at $\mathrm{TiO}_{2}(110)$ (and more generally on adsorption of an amino acid on an oxide surface) was performed by Soria et al.[291; 292] on both unreconstructed $(1 \times 1)$ and $(1 \times 2)$ reconstructed rutile (110). Using synchrotron light with hv=55 eV, Gly layers of increasing thickness, from sub-ML to $7 \mathrm{ML}$, were investigated at room temperature. In the submonolayer range, the molecules adsorb dissociatively with the carboxylate radical adopting a bridging configuration over $\mathrm{Ti}^{4+}$ cation sites. Glycine in multilayers adsorbs in the zwitterionic form, a structure reminiscent of the bulk phase.

A STM investigation of glycine adsorbed on the $\mathrm{TiO}_{2}(110)-(1 \times 1)$ at $\mathrm{RT}$ [64] showed that, at saturation coverage, the Gly overlayer exhibits a periodicity of $0.68 \mathrm{~nm}$ in the $\langle 110\rangle$ direction and of $0.59 \mathrm{~nm}$ in the $<001>$ one (Figure $76 \mathrm{a}$ and $\mathrm{b}$ ). This demonstrates that glycine forms an ordered $(2 \times 1)$ structure on $\mathrm{TiO}_{2}(110)-(1 \times 1)$. The similarity of the structures obtained for aliphatic 
carboxylic acids, including formate, suggeststhat the local bonding configuration is similar to that of formate, i.e. glycinate $\left(\mathrm{NH}_{2} \mathrm{CH}_{2} \mathrm{COO}^{-}\right)$bridging adjacent $\mathrm{Ti}$ cations along the $\langle 001\rangle$ direction (configuration II in Figure 75, see also Figure 77). The glycinate features in the STM image do not show any preferred orientation, shape or displacement; they appear as uniform bright spots well aligned along the <001> rows. The same uniformity was observed for the aliphatic carboxylates, suggesting in all cases that there is free rotation about the $\mathrm{C}-\mathrm{C}$ bond at room temperature.Results on $\mathrm{TiO}_{2}(110)$ - $(1 \times 2)$ reconstructed surface are very different. No structure was observed with STM but only disordered glycine patches.

a)

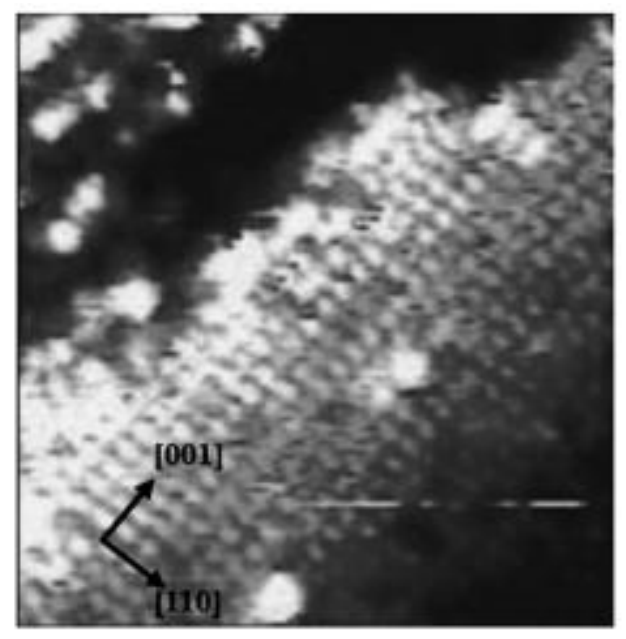

b)

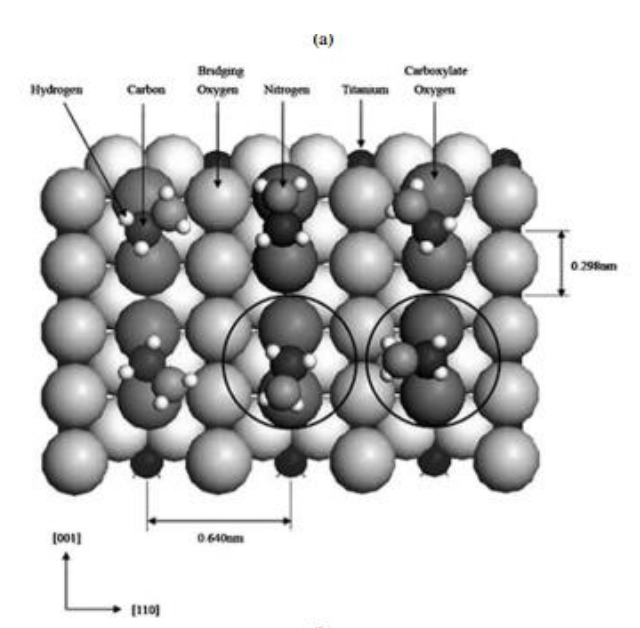

Figure 76. a) STM image (image size: $\left.15 \times 15 \mathrm{~nm}^{2}, \mathrm{~V}=+1.5 \mathrm{~V}, \mathrm{I}=0.1 \mathrm{nA}\right)$ of theTiO $2(110)-(1 \times 1)$ surface exposed to $4.2 \mathrm{~L}$ of Gly at $\mathrm{T}=300 \mathrm{~K}$. b) Scheme of the corresponding (2x1) structure. The $\mathrm{NH}_{2}$ moiety is free to rotate around the $\mathrm{C}-\mathrm{C}$ backbone. Reprinted with permission from reference [64].

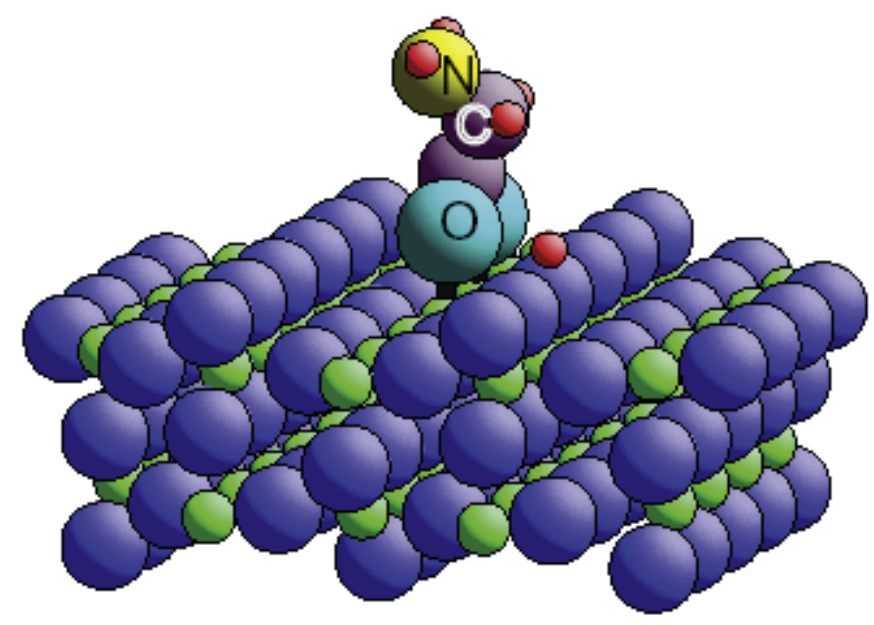

Figure 77 Ball model of the optimised structure for glycinate on $\mathrm{TiO}_{2}(110)$ based on the determined positions of the carboxylate $\mathrm{O}$ atoms and the implication from the results that the molecule is 'standing up'; the positions of the $\mathrm{C}, \mathrm{N}$ and $\mathrm{H}$ atoms are indicative only and were not determined explicitly in the cited investigation. Reprinted with permission from ref.[290]. 
Ojamäe et al. modelled with DFT glycinates adsorbed on the $\mathrm{TiO}_{2}(110)-(1 \times 1)$ surface[289] . Several configurations were considered: two bridging configurations (configuration II in Figure 75), one anionic and one zwitterionic, and two unidentate configurations, one with a Ti-OCO bond (scheme I in Figure 75), and the second with a $\mathrm{Ti}^{-} \mathrm{NH}_{2}$ bond. The bridging configuration (with the $\mathrm{C}-\mathrm{C}$ bond normal to the surface and no $\mathrm{NH}_{2}$-surface interactions) was found more stable than the $\mu 1$ configuration by ca. $11 \mathrm{~kJ} / \mathrm{mol}(0.1 \mathrm{eV})$, a difference similar to those calculated for formic and acetic acids. The most stable among the calculated configurations was the bridging zwitterion (Figure 78a), in which the $\mathrm{NH}_{2}$ group is protonated in $\mathrm{NH}_{3}{ }^{+}$, the $\mathrm{COO}$ group is bound in a $\mu 2$ coordination and the $\mathrm{C}-\mathrm{C}$ bond is tilted in the $\langle 110\rangle$ direction to permit hydrogen bonding between the $\mathrm{NH}^{+3}$ group and the adjacent row of oxygen atoms. This configuration was calculated to be more stable by $36 \mathrm{~kJ} / \mathrm{mol}(0.3 \mathrm{eV})$ than the simple anionic bridging configuration shown in Figure 77. However, the anionic standing up configuration fitted better withwith the previous STM data obtained by Qiu and Barteau.

a)

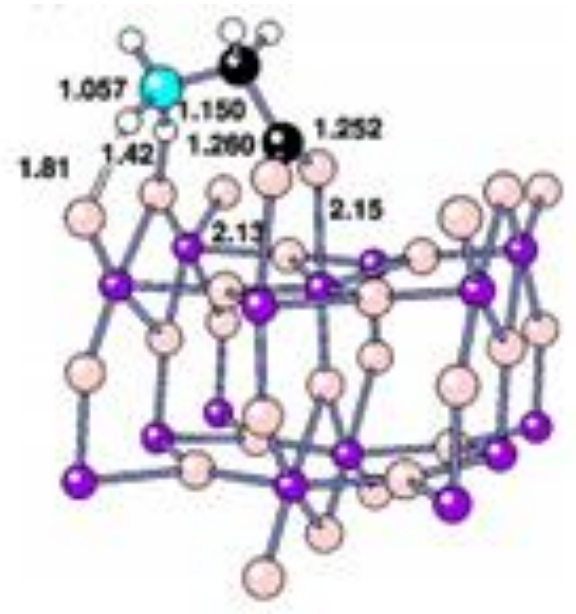

b)

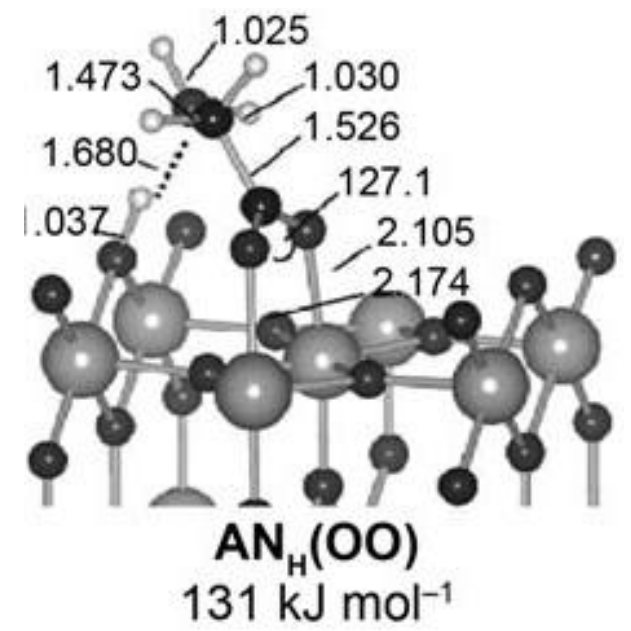

Figure 78. DFT- Optimized adsorption geometries of glycine on $\mathrm{TiO}_{2}(110)$, most stable configurations found by a) Ojamae et al.[289](by $9 \mathrm{~kJ} / \mathrm{mol}$ ) b) Tonner[293].

Carravetta and Monti performed DFT calculations on the same system and found this difference to be much larger, with the $\mu 2$ adsorption configuration again more stable by $0.8 \mathrm{eV}$ [315].

Tonner[293]confirmed the trend found by Ojamae[289], but a new anionic adsorption mode (Figure $78 \mathrm{~b})$ was identified as being even more stable by $9 \mathrm{kJmol}^{-1}(0.1 \mathrm{eV} /$ molecule $)$ compared to the previous minimum configuration, this time in agreement with the STM study of Qiu and Barteau which suggested adsorption in the anionic form. Among the other considered configurations, the anionic bifunctional adsorption with $\mathrm{COO}$ and $\mathrm{NH}_{2}$ binding to Ti atoms in a bridging mode was found $23 \mathrm{~kJ} / \mathrm{mol}(0.2 \mathrm{eV} /$ molecule $)$ less stable than the most favoured configuration. The work of Tonner also provides the calculated vibrational frequencies for anionic glycine adsorbed on $\mathrm{TiO}_{2}$ (110) in the binding $\mu 2$ configuration: $1528 \mathrm{~cm}^{-1}$ (C=O stretch) and $1566 \mathrm{~cm}^{-1}$ (HNH bending mode). The final confirmation was obtained later from high-resolution core-level photoemission and scanned-energy mode photoelectron diffraction of the O 1s and N 1s states of glycine[290]. While there was clear evidence for the presence of the $\mathrm{NH}^{3+} \mathrm{CH}_{2} \mathrm{COO}^{-}$zwitterion with multilayer deposition, at low coverage only the deprotonated glycinate species, $\mathrm{NH}_{2} \mathrm{CH}_{2} \mathrm{COO}^{-}$was identified. 
Multiple-scattering simulations of the $\mathrm{O} 1 \mathrm{~s} \mathrm{PhD}$ data show the glycinate is bonded to the surface through the two carboxylate $\mathrm{O}$ atoms which occupy near-atop sites above the five-fold coordinated surface Ti atoms, with no $\mathrm{NH}_{2}$-surface interaction. The Ti-O bond length is $2.12 \pm 0.06 \AA$. Atomic hydrogen, arising from the deprotonation, is coadsorbed to form hydroxyl species at the bridging oxygen sites with an associated Ti-O bond length of $2.01 \pm 0.03 \AA$. The bridging mode (model II in

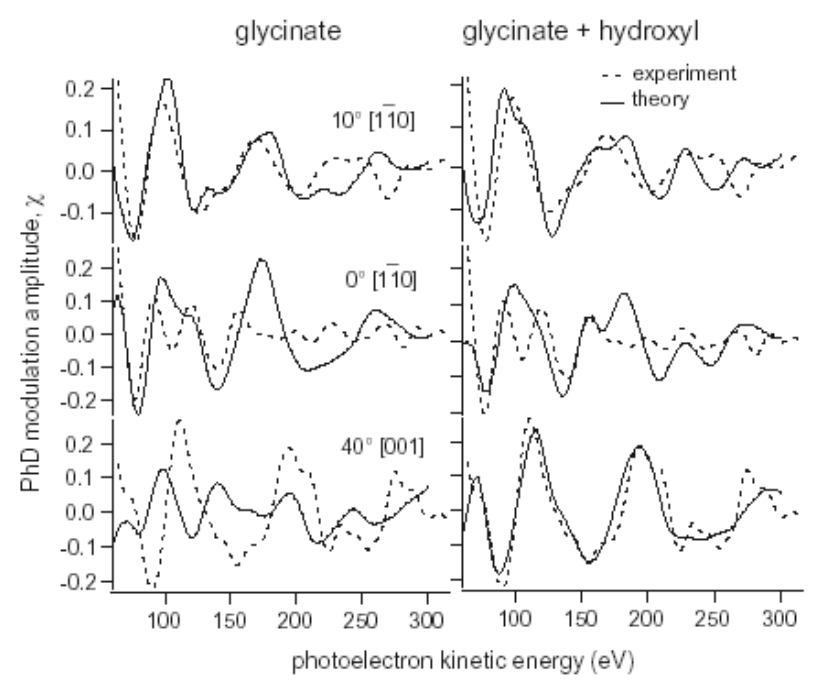

Figure 75) fits best the experimental data (

Figure 79) than the $\mu 1$ one (fit not shown). The experimental data unambiguously show that below and at the monolayer coverage, glycine is deprotonated and $\mu 2$.

This exemplifies one main difficulty of atomistic simulation, and especially ab initio simulations, in exploring the potential energy surface. In fact DFT calculations cannot be exhaustive and it is important, when possible, to check the obtained theoretical results in the light of experimental ones.

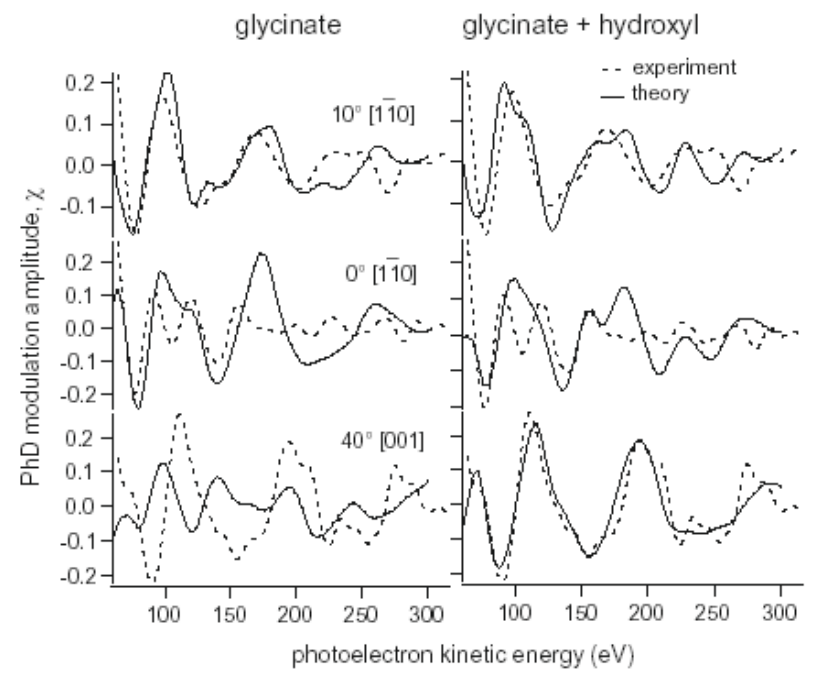

Figure 79. Comparison of the experimental $\mathrm{O} 1 \mathrm{~s} \mathrm{PhD}$ spectra of adsorbed Gly on $\mathrm{TiO}_{2}(110)-(1 \mathrm{x} 1)$ with the results of theoretical simulations for the best-fit structural models of adsorbed glycinate alone (left panel) and of coadsorbed glycinate and hydroxyl species (right panel).Figure taken with permission from ref.[290].

Adsorption of glycine was performed on the rutile (011) surface and followed by XPS and TPD techniques[295]. This surface consistsof zigzag-rows of two-fold coordinated oxygen atoms 
asymmetrically bonded to fivefold titanium atoms (see ref.[295] and refs. therein). Both the twofold $\mathrm{O}$ atoms and five-fold $\mathrm{Ti}$ atoms accommodate the dissociative adsorption of the amino acids and naturally explain the results of the many carboxylic acids studied previously. The surface coverage is very close to $0.5 \mathrm{ML}$ using either the XPS Ti2p or O1s attenuation lines upon adsorption. Glycine forms two stable species on the surface upon dosing at $300 \mathrm{~K}$, the zwitterion and the anionic ones.

\subsubsection{Glycine and water on $\mathrm{TiO}_{2}$ from the gas phase}

Lausmaa and co-workers studied the coadsorption of water and glycine on a $\mathrm{TiO}_{2}$ thin film by TDS[297]. Glycine was coadsorbed with $\mathrm{H}_{2} \mathrm{O}$ by sequential adsorption of first $\mathrm{H}_{2} \mathrm{O}$ and then glycine, or by the reverse sequence.

Intact glycine molecules desorb from the saturated monolayer at around 400K (Figure 80). The estimated coverage is $(1.0 \pm 0.5) \times 10^{14}$ Gly molecules $\mathrm{cm}^{-2}$ i.e. 1 glycine per five Ti atoms or 0.20 ML. This number is comparable to the molecule density in low-index planes of solid crystalline glycine, which is in the range of $1 \times 10^{14}-4 \times 10^{14} \mathrm{~cm}^{-2}$ [341]. This suggests that glycine-glycine interactions determine the maximum surface coverage by steric hindrance.

No changes were observed with either sequence in the TD spectra of glycine after coadsorption with $\mathrm{H}_{2} \mathrm{O}$ coverages up to a few monolayers. This applies to all studied glycine coverages, from submonolayer to several multilayers. In contrast, significant differences with and without coadsorption of glycine are observed in the desorption spectra of $\mathrm{H}_{2} \mathrm{O}$ : the total amount of desorbed water remained identical, but a peak recorded without glycine (peak $\alpha$ inFigure 81 ) was shifted to a lower temperature in the presence of glycine (peak $\beta$, Figure 81). This means that some sites able to react with pure water (and giving rise to the apeak upon desorption) were "poisoned" by glycine in the case of co-adsorption. Peaks $\alpha$ and $\beta$ were assigned to water molecularly adsorbed at regular terrace sites on the $\mathrm{TiO}_{2}$ surface, $\mathrm{Ti}^{4+}$ and $\mathrm{O}$ anions respectively. This result implies that glycine, when adsorbing on the water-covered surface, has the ability to displace water from its original sites to weaker ones. There is a thermodynamic driving force, where displacement of water from stronger to weaker binding sites is energetically overcompensated by binding of glycine to the strong sites. 

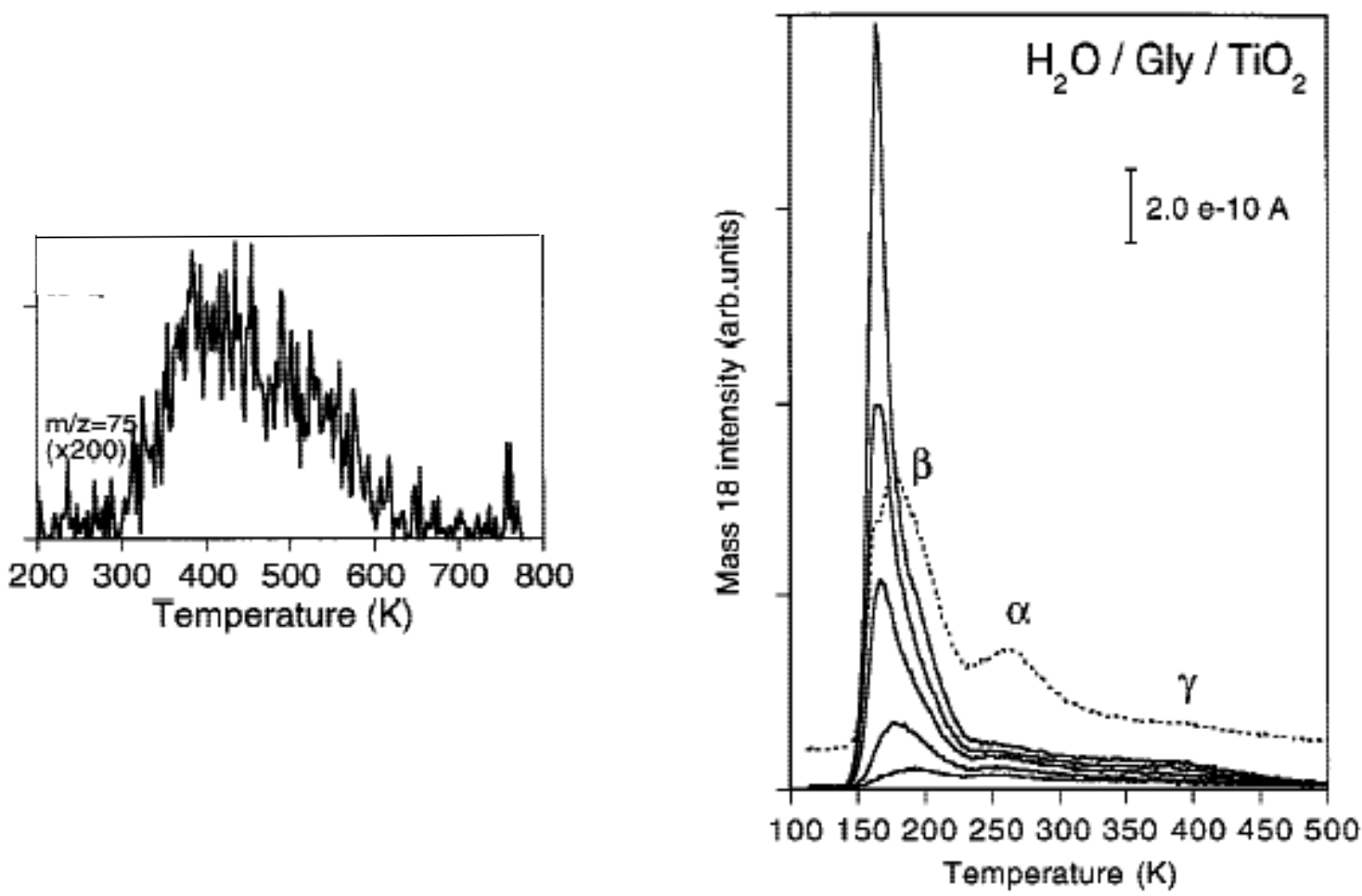

Figure 80. TDS spectrum for intact Figure 81. Water desorption from a $\mathrm{TiO}_{2}$ glycine $(\mathrm{m} / \mathrm{z}=75)$ desorbing from the $\mathrm{TiO}_{2}$ surface that first was precovered with $\sim 2 \mathrm{ML}$ of surface after $25 \mathrm{~s}$ of exposure at low $\mathrm{T}_{\mathrm{ev}}$. glycine and then was exposed to different water Coadsorption of water and glycine does not doses (solid lines). Water exposures change the glycine desorptionspectrum. (coverages) are, from bottom up: $40 \mathrm{~s}(0.1$ ML), $100 \mathrm{~s}$ (0.2 ML), $200 \mathrm{~s}$ (0.4 ML), $300 \mathrm{~s}$ (0.6 ML), and $400 \mathrm{~s}$ (0.8 ML). For comparison, the TD spectrum of clean $\mathrm{TiO}_{2}$ covered with $0.8 \mathrm{ML}$ of water is also shown (dashed line).The latter spectrum has been upshifted for clarity.

Recently, Martra et al. adsorbed glycine on $\mathrm{TiO}_{2}$ from the gas phase and subsequently exposed it to water vapour. They observed glycine polymerization (poly-Gly) in a $\beta$-sheet form[288]. Water vapour adsorption confirmed the expected hydrophobic character of the poly-gly layer.

Glycine adsorbed on dry rutile (110) from the gas phase was modelled with a reactive Force Field (see section 2.2.3) at monolayer and bilayer coverage[294]. Molecules reacted with the rutile(110) surface forming a glycinate and atomic hydrogen. $\mathrm{OH}$ dissociation seemed to proceed through a multistep mechanism, where first glycine molecules formed a prebonded state orienting the lone pair of the hydroxyl oxygen toward a titanium site and then released the proton to the closer oxygens (out of plane oxygens of the slab). Reactions took place when favourable environmental conditions were met, i.e. when the species were close to each other, adopted specific orientations and were not restrained in their conformational readjustments. As a consequence, not all of the system components exchanged. The final result was a chemisorption of both the anion 
$\left(\mathrm{NH}_{2} \mathrm{CH}_{2} \mathrm{COO}^{-}\right)$and cation $\left(\mathrm{H}^{+}\right)$at the interface for about $60 \%$ of the Gly molecules. The adsorbates were quite stable, underwent relatively limited motions when in direct contact with the interface, and had the tendency to react with one another and with the inorganic layer in order to improve their binding. The outer glycine layer, even though hydrogen-bonded to the first layer, was more mobile, and unable to penetrate the quite densely packed network of adsorbed molecules. Instead, they diffused above the first amino acid layer guided by the evolution of their hydrogen bonds. The settled molecules had a reasonable propensity to preserve their orientations with the carboxyl group providing the main anchoring point to the substrate. This interaction was sometimes reinforced by other strong coordination bonds (involving for example the nitrogen atom) or weaker hydrogen bonds. Although not all possible configurations of the glycine bilayer have been fully sampled, the main features of the adsorption process have been identified.

\subsubsection{Other amino acids adsorbed on $\mathrm{TiO}_{2}$ single crystals from the gas phase}

An experimental investigation of DL-proline, $\left(\mathrm{C}_{4} \mathrm{NH}_{8} \mathrm{COOH}\right.$, a major constituent of collagen $)$ on the stoichiometric and reduced (oxygen defective) $\mathrm{TiO}_{2}(110)$ single crystal surfaces was performed using HR-XPS[307; 308]. It indicates that both zwitterionic and dissociated (deprotonated) forms are present on the surface. The zwitterionic structure $\left(\mathrm{C}_{4} \mathrm{H}_{7}-\left(\mathrm{NH}_{2}{ }^{+}\right) \mathrm{COO}^{-}\right)$is less stable and desorbs at a lower temperature. The reduced surface appears to increase the concentration of this structure, most likely because it provides less oxygen for the proton originating from the dissociated proline adsorption.

The reaction of proline on the surface of the stoichiometric and reduced $\mathrm{TiO}_{2}(001)$ single-crystal surfaces was also conducted and characterized with TPD in order to highlight the effect of surface defects on the reaction pathways[306]. On a stoichiometric (001) surface, proline does not seem to decompose, and the only probable reaction is the deprotonation of the carboxylic group, as on $\mathrm{TiO}_{2}(110)$. In contrast, proline appears to be strongly adsorbed on the defected (001) surface, as evidenced by a shift of the desorption temperature from $350 \mathrm{~K}$ on stoichiometric $\mathrm{TiO}_{2}(001)$ to 530 $550 \mathrm{~K}$ on the oxygen-defected surface.

Theoretical calculations reveal that proline shows similar adsorption behaviour than glycine but zwitterionic forms and coordination via the nitrogen atom are much less viable[293]. The most stable mode for both molecules is theone schematized in Figure 77, with an anionic amino acid moiety bridging two surface titanium atoms via the carboxyl oxygen atoms and a proton transferred to an oxygen atom at the surface. This is similar to carboxylic acids, but the amino acids are able to form hydrogen bonds with the surface, thus stabilizing the adsorbed molecules by $15-20 \mathrm{kJmol}^{-1}$ $(0.15 \mathrm{eV})[293]$.

Ataman et al.[309] used XPS to study the adsorption of L-cysteine on a rutile $\mathrm{TiO}_{2}(110)$ surface at room temperature and at $\mathrm{T}=-65{ }^{\circ} \mathrm{C}$. For the molecules in direct contact with the surface, the results suggest that they bind dissociatively to the fivefold-coordinated $\mathrm{Ti}$ atoms through their deprotonated carboxylic groups, in agreement with previous results on glycine. Interestingly, a second, dissociative interaction occurs between the molecular thiol groups and the surface. Most likely, the thiol groups are deprotonated and a bond is formed between the thiolates and defects (the bridging oxygen vacancies).

Later this system was investigated with DFT by Muir and Idriss[310]. Thirteen configurations were scrutinized having various adsorption modes and protonation states. The two most stable 
configurations are shown in Figure 82. Cysteine adsorption occurs at low (25\%) and high coverage (50\% with respect to the surface $\mathrm{Ti}$ atoms density) through the carboxyl group in a preferred bridging mode (Figure $82 \mathrm{a}-\Delta \mathrm{E}_{\mathrm{ads}}=-1.37 \mathrm{eV}$ and $-1.47 \mathrm{eV}$ at low and high coverage respectively). The bridging adsorption through the $\mathrm{COO}$ and $\mathrm{NH}_{2}$ moieties is slightly less stable (Figure 82b $\Delta \mathrm{E}_{\mathrm{ads}}=-1.31$ and $-1.39 \mathrm{eV}$ ). The most stable configuration is thus geometrically (bridge adsorption) and energetically similar to that of formic acid. The configurations involving the formation of a direct Ti-S bond are far less stable, by $0.5-0.7 \mathrm{eV}$. This confirms the view of Ataman et al. [309] that the thiol does not adsorb on the perfect surface but, rather, on defects (O vacancies).

a)

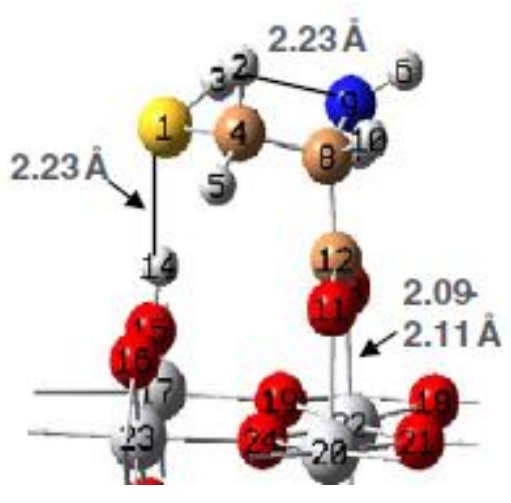

b)

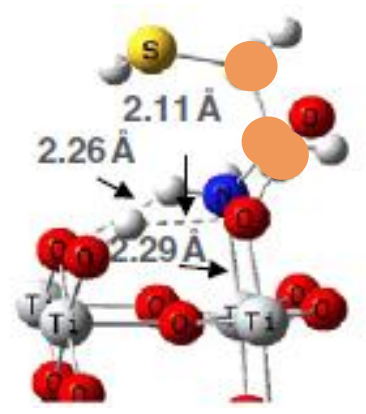

Figure 82. Images of the two most stable adsorption configurations found for cysteine on rutile (110). $\mathrm{S}$ is yellow, $\mathrm{C}$ is brown, Nitrogen is blue, Hydrogen is white, $\mathrm{O}$ is red and Ti is grey. Reprinted with permission from ref.[310].

Alanine on rutile $\mathrm{TiO}_{2}(110)$ was also calculated to adsorb at the monolayer coverage in a bridging configuration forming a Ti-O-C-O-Ti cycle, that was more stable by $0.8 \mathrm{eV}$ than a $\mu 1$ conformation [315].

The adsorption of the aromatic amino acid phenylalanine on a rutile $\mathrm{TiO}_{2}(110)$ single crystal has been investigated by XPS and NEXAFS spectroscopy. The results indicate initial adsorption via the carboxylate group in a $\mu 2$ configuration with the phenyl ring oriented at approximately $25^{\circ}$ to the surface normal (Figure 83). The amino group remains as $\mathrm{NH}_{2}$. Subsequent layers of phenylalanine appear to adsorb as neutral molecules with $\mathrm{H}$-bonding between $\mathrm{NH}_{2}$ and $\mathrm{COOH}$ groups, in contrast with glycine which forms zwitterionic multilayers. 


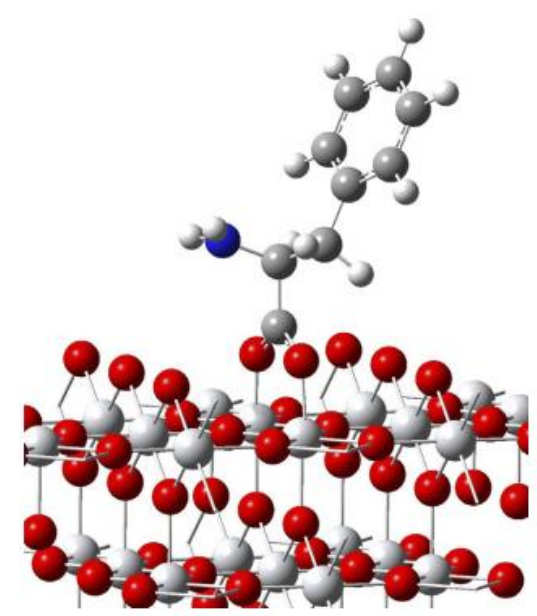

Figure 83. Ball and stick model of the adsorption of a phenylalanine molecule adsorbed on the rutile $\mathrm{TiO}_{2}(110)$ surface as deduced from XPS and NEXAFS experiments. Reprinted with permission from ref. [314].

The case of an amino acid with two carboxylate functions (aspartic acid or glutamic acid) is of interest. Here, the question is whether the molecule binds through the alpha- or lateral $\mathrm{COO}$, or both.Figure 84 exemplifies some possible adsorption modes ofGlu, through the carboxylate moieties. It is interesting to notice that adding one carboxylate group (as compared to glycine) considerably increases the numbers of possible anchoring points to the surface. These molecules belong to the family of dicarboxylic acids, which have been shown to adsorb more easily than the monocarboxylic acids on several oxides like $\mathrm{TiO}_{2}, \mathrm{Al}_{2} \mathrm{O}_{3}, \mathrm{ZrO}_{2}, \mathrm{Ta}_{2} \mathrm{O}_{5}[342]$.
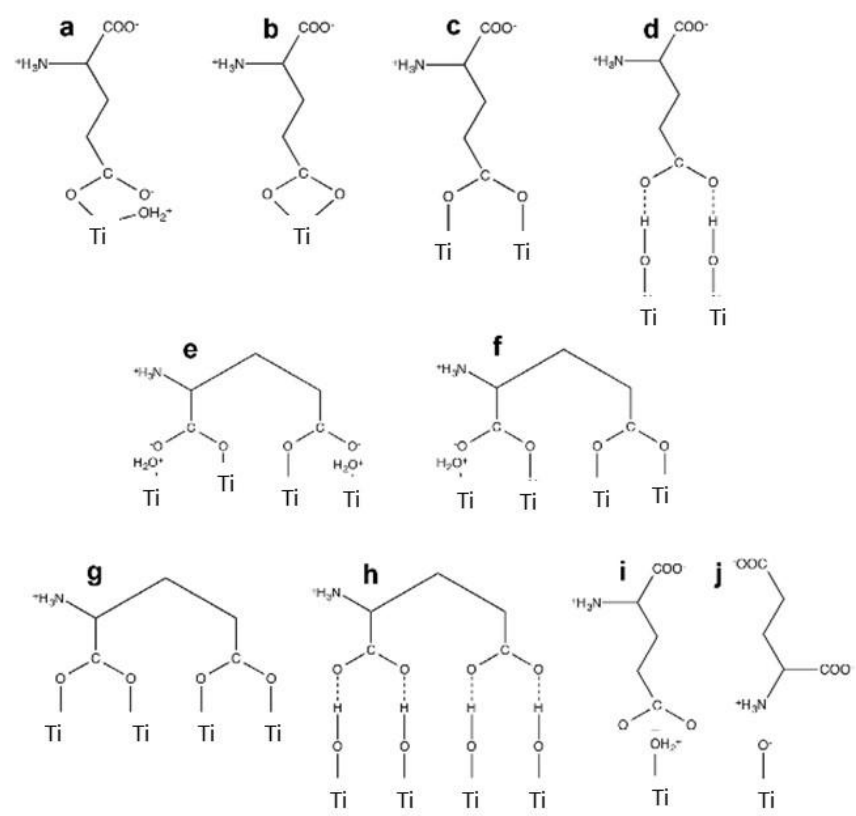

Figure 84.Scheme of possible binding modes through the carboxylate moieties of Glu on $\mathrm{TiO}_{2}$, inspired from ref.[343]. a-c) covalent bonds with the lateral COO moiety, d) H-bonding between the lateral $\mathrm{COO}$ and hydroxyls, e-h), bridging adsorption involving the two COO moieties,(with covalent and/or H-bonds) $\mathrm{i}-\mathrm{j}$ ) electrostatic interactions. The same possible configurations hold for Asp. Adsorption configurations involving the amine species are not considered in these schemes. 
Guo et al.[311] studied by DFT the aspartic acid adsorption on rutile $\mathrm{TiO}_{2}(110)$ both on the dry and hydrated (water adsorbed on surface $\mathrm{Ti}$ atoms) surface, and in a microsolvated form. It was found that, in vacuum on the dry surface, the best adsorption configuration is a bridging mode forming a Ti-COOH--- $\mathrm{NH}_{2}$-Ti cycle. However the authors did not consider the dissociation of aspartic acid and adsorption of an anionic species with the formation of an $\mathrm{OH}$ surface group. The anionic form of Asp (which is the stable form in biological medium) was considered when studying the microsolvated form. Two types of water environment were built: i) The water environment model (WE), in which eight water molecules surrounded the anionic Asp molecule and several of them were near the functional groups of the anionic Asp. ii) The surface water layer model (WL), in which four water molecules were on the top of $\mathrm{Ti}_{5 \mathrm{c}}$ with a $\mathrm{O}_{\text {-ater }}$ - $\mathrm{Ti}$ distance of about $2.300 \AA$ to form a water layer. The anionic Asp molecule was on top of the water layer and four additional water molecules were added near the functional groups of Asp. For both model the presence of water was found to weaken the Asp adsorption on the rutile $\mathrm{TiO}_{2}(110)$ surface. Comparison between the results of the WE and WL models shows that there is competition between Asp and water molecules during the adsorption process. If Asp occupies the adsorption site first, the interaction between Asp and the rutile $\mathrm{TiO}_{2}(110)$ surface is still relatively strong, as in the WE model. However, Asp almost cannot interact with $\mathrm{Ti}$ surfaces if water molecules block the interaction sites, as in the WL model. Thus, the adsorption energy of the WL model $(-0.3 \mathrm{eV})$ is much smaller than that of the WE model $(-1.0 \mathrm{eV})$.

The adsorption of arginine was studied with DFT (dispersion forces corrected) in vacuum on ideal rutile $\mathrm{TiO}_{2}(110)$, on rutile $\mathrm{TiO}_{2}(110)$ with $\mathrm{O}$ vacancies and on rutile $\mathrm{TiO}_{2}(110)$ with an adsorbed water layer[312]. The strongest adsorption on the ideal surface occurred when the aliphatic chain of Arg was parallel to the surface $\left(\mathrm{E}_{\mathrm{ads}}=-4.6 \mathrm{eV}\right.$, to be compared to $-2.0 \mathrm{eV}$ and $-1.4 \mathrm{eV}$ for configurations in which Arg is perpendicular to the surface). For those configurations, it was found that the interaction between Argandrutile $\mathrm{TiO}_{2}(110)$ involving the $-\mathrm{COOH}$ and $-\mathrm{NH}_{2}$ functional groups $(-2.0 \mathrm{eV})$, was more stablethan that between the surface and the guanidinium group (-1.4 $\mathrm{eV})$. The Vander Waals interactions increased the adsorption energy between Argandrutile $\mathrm{TiO}_{2}(110)$, but the trends did not change. The adsorption was due to bond formation between surface Ti atoms and $\mathrm{O}$ and $\mathrm{N}$ atoms from Arg, together with Hbonds from $\mathrm{COOH}$ and $\mathrm{NH}_{2}$ of $\mathrm{Arg}$ to surface $\mathrm{O}$ atoms. Deficiency of either in-plane oxygen or bridging oxygen weakened the Arg adsorption on the rutile surface. The influence of the former is more significant than that of the latter. A water layer adsorbed on rutile surface hindered the Arg adsorption. However, again, substitution of preadorbed water by Arg and direct Arg-Ti bond formation moieties was not considered.

Arginine adsorption on $\mathrm{TiO}_{2}$ anatase (100) was modelled using a cluster approach[313]. The $\mathrm{TiO}_{2}$ (100) surface is one of the two major cleavage planes of anatase, together with the (101) surface. It possesses small grooves formed by oxygen atoms on either side of the groove along with both $\mathrm{Ti}$ and $\mathrm{O}$ atoms at the bottom. Only the interaction of the arginine lateral chain inside the groove was considered. The most stable conformation obtained (Figure 85) (-272 kJ/mol, i.e. $-2.7 \mathrm{eV} / \mathrm{molecule}$ ) involves $\mathrm{H}$-bonding between the $\mathrm{NH}_{2}$ moieties and the surface $\mathrm{O}$ atoms. This very high energy of adsorption despite only H-bonds formation is not commented by the authors. It might be an artefact 
of the method of calculations, the number of $\mathrm{TiO}_{2}$ layers being too small, and the BSSE effects not corrected.

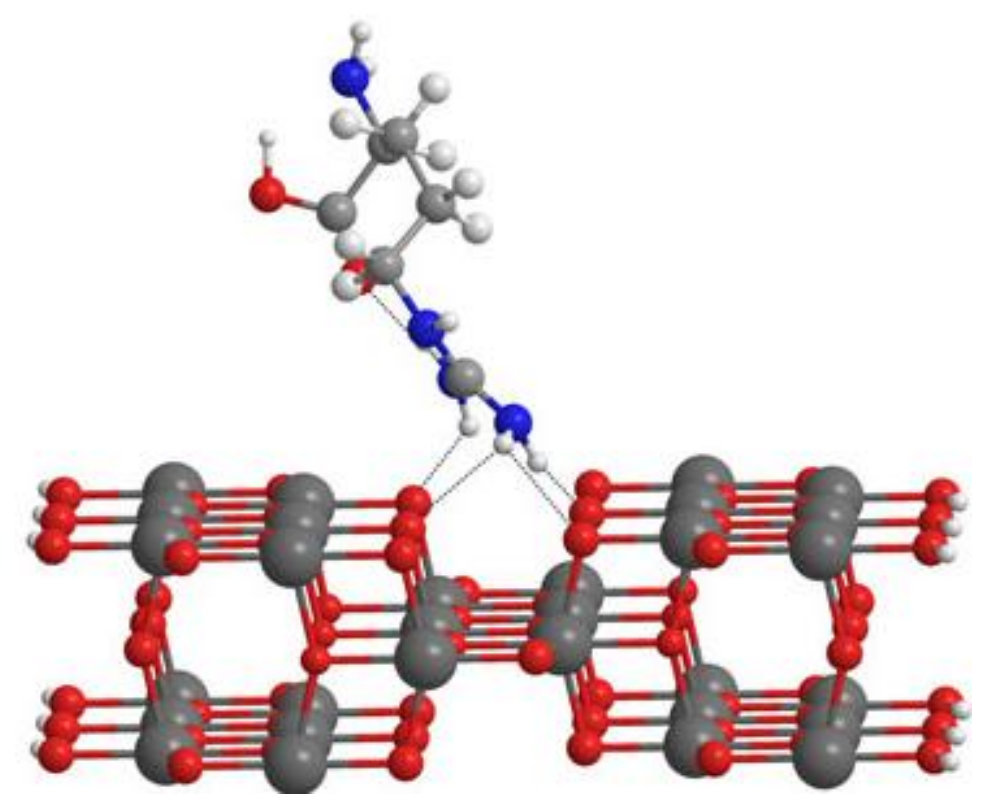

Figure 85. Adsorption of arginine on $\mathrm{TiO}_{2}$ anatase (100) grooves. Taken with permission from ref. [313].

\subsection{Adsorption of amino acids on other oxides}

\subsubsection{Adsorption of glycine}

\subsubsection{Adsorption of glycine on alumina}

As already mentioned, the number of papers on single crystal oxides other than $\mathrm{TiO}_{2}$ is low.

Alumina and aluminium polymorphs can be inhaled and incorporated in the body as food additives or vaccine adjuvants[344]. The fate of alumina in the body fluid is thus of interest. Alumina may also have played a role in prebiotic chemistry. The interaction between glycine layers and an ultrathin $\mathrm{Al}_{2} \mathrm{O}_{3}$ film (5 $\AA$ ) grown epitaxially onto $\mathrm{NiAl}(110)$ was studied by temperatureprogrammed desorption, X-ray and ultra-violet photoelectron spectroscopy, work function measurements and NEXAFS[300]. The authors claim that this oxide film is well ordered, with ay$\mathrm{Al}_{2} \mathrm{O}_{3}(111)$ surface structure, and purely oxygen-terminated, the sequence of layers from bulk to surface being NiAl-Al-OAl-O.However, several works on ultrathin polar oxide films show that the polarity (and thus the surface charge) is lost by reconstruction in an amorphous layer, where $\mathrm{Al}$ ions and $\mathrm{O}$ anions coexist in the same surface plane (see ref.[345] and references therein).

At monolayer coverage and at $110 \mathrm{~K}$, there are two coexisting molecular forms of Gly on $\mathrm{Al}_{2} \mathrm{O}_{3}$, the anionic and the zwitterionic, as shown by the $\mathrm{N}$ 1s spectral region in XPS data (peaks at 400.8 and $402.6 \mathrm{eV}$ for the $\mathrm{NH}_{2}$ and $\mathrm{NH}_{3}{ }^{+}$species respectively, $\mathrm{Z} / \mathrm{A}$ ratio $=\mathrm{NH}_{3}{ }^{+} / \mathrm{NH}_{2}=0.8$ ). The TPD data suggest that zwitterionic species form Gly clusters in the monolayer regime. At higher exposures, multilayers of condensed Gly develop andthe zwitterionic configuration of the adsorbed species prevails $\left(\mathrm{NH}_{3}{ }^{+} / \mathrm{NH}_{2}=3.8\right)$. 
In contrast, the monolayer formed at $300 \mathrm{~K}$ consists primarily of glycine molecules in the anionic state, as suggested by XPS (ratio $\mathrm{NH}_{3}{ }^{+} / \mathrm{NH}_{2}=0.2$ ), UPS and NEXAFS results. The characteristics of the monolayer at 300Kare independent of the preparation pathway, that is, adsorption of Gly on the $\mathrm{Al}_{2} \mathrm{O}_{3} / \mathrm{NiAl}(110)$ surface at $300 \mathrm{~K}$ or annealing of the Gly multilayers at the same temperature result in the presence of essentially identical species on the surface. This suggests that the anionic state is the stable state achieved once a kinetic barrier is overcome starting from the neutral/zwitterionic adsorption. Glycinate is adsorbed with the oxygen atoms of the carboxylic group pointing toward the substrate (Figure 86). The polarization-dependent $\mathrm{C} \mathrm{K}$ - and $\mathrm{O} \mathrm{K}$ edgeNEXAFS spectra indicate that the glycinate species is oriented nearly perpendicular to the surface, with an electrostatic interaction between the carboxylic oxygen atoms and the surface $\mathrm{Al}^{3+}$ cations andwith the amino group pointing away from the surface.

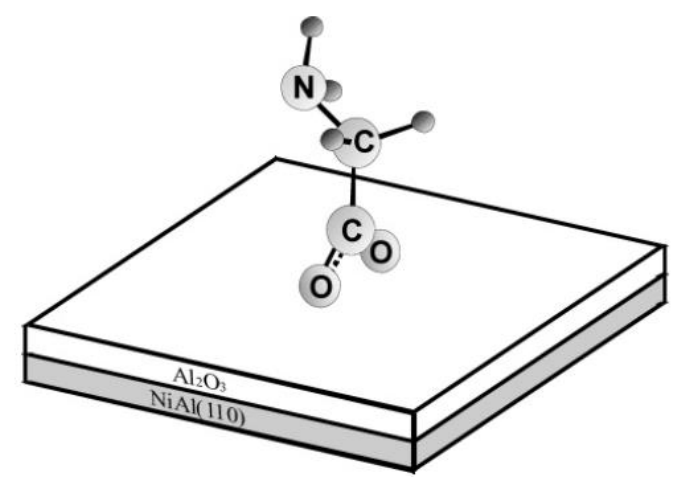

Figure 86.Scheme of glycinate adsorbed on a thin film of $\mathrm{Al}_{2} \mathrm{O}_{3}$ on NiAl. Taken with permission from reference [300].

Glycine was adsorbed from the gas phase on alumina powders and the C NMR signal was recorded. The powder was hydroxylated by exposure to air. The broad signal has a component at 170ppm and one at $177 \mathrm{ppm}$ (Figure 87), attributed to coordinative bonds to $\mathrm{Al}^{3+}$ and $\mathrm{H}$-bonds formation respectively[302]. This example shows that both coordinative bonds and H-bonds driven adsorption may occur on a hydroxylated surface.

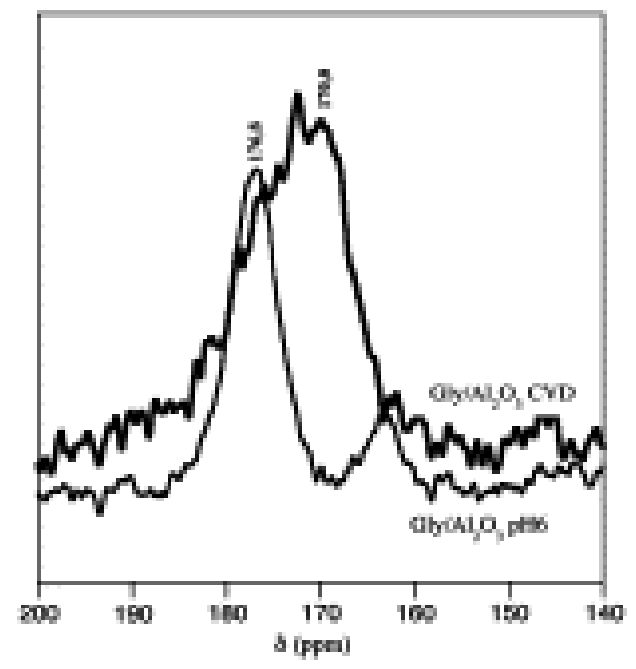

Figure 87.CP-MAS NMR in the carboxylate region of glycine/ $\mathrm{Al}_{2} \mathrm{O}_{3}$ deposited from a water solution at $\mathrm{pH} 6$ (thin line: the signal at 160ppm is due to surface carbonates) and deposited from the gas phase (bold line: CVD procedure). Taken with permission from ref. [302]. 
Adsorption of glycine from the gas phase and in the low coverage limit was modelled on alpha$\mathrm{Al}_{2} \mathrm{O}_{3}$ with terminations modelling increasing water activity, $\mathrm{O}_{3} \mathrm{Al}$ (anhydrous) and $\left(\mathrm{AlO}_{3}-\mathrm{H}, \mathrm{O}_{3} \mathrm{Al}-\right.$ $\mathrm{OH}$ ) (oxy-hydroxide-like)[301]. Different possible glycine conformers have been considered (neutral (NG), zwitterion (ZG), anion (AG)), as well as different binding modes and orientations toward the surface. In all cases, glycine interacts preferentially with the surface through the carboxylic group. The binding with the $\mathrm{O}_{3} \mathrm{Al}$ termination of the anhydrous oxide surface may be unidentatIl-O(C) or bridging Al-OCO-Al. The anion perpendicular unidentate form is the most stable one with an energy of adsorption $\mathrm{E}_{\mathrm{ads}}=-2.21 \mathrm{eV}$. The nature of the $\mathrm{Al}-\mathrm{O}(\mathrm{C})$ bond is found to be iono-covalent. On the hydroxylated surface with $\left(\mathrm{AlO}_{3}-\mathrm{H}, \mathrm{O}_{3} \mathrm{Al}-\mathrm{OH}\right)$ terminations, two types of adsorption may arise: either only $\mathrm{H}$ bonds $\left(\mathrm{E}_{\mathrm{ads}}=-0.73 \mathrm{eV}\right)$ or both $\mathrm{Al}-\mathrm{OC}$ and $\mathrm{H}$ bonds $\left(\mathrm{E}_{\mathrm{ads}}=-\right.$ $1.64 \mathrm{eV}$ )form with the surface. An additional scenario was considered, where glycine substitutes a surface $\mathrm{OH}$ group and forms an $\mathrm{Al}-\mathrm{O}-\mathrm{C}$ bond, whereas the $\mathrm{OH}$ group combines with a proton from the surface to form a water molecule that coadsorbs with gycine. This reaction is thermodynamically favoured $\left(\mathrm{E}_{\mathrm{ads}}=-2.21 \mathrm{eV}\right)$. The $\mathrm{ZG}$ and $\mathrm{AG}$ forms are isoenergetic in a unidentate parallel mode of adsorption. It is shown that coadsorbed water has a stabilizing effect, thus influencing the nature of the most stable Gly conformer. These calculations confirm the occurrence of covalent bonding as well as $\mathrm{H}$-bonding adducts at hydroxylated $\mathrm{Al}_{2} \mathrm{O}_{3}$ surfaces, even without bulk water explicitly added.

\subsubsection{Adsorption of glycine on $\mathrm{ZnO}$}

$\mathrm{ZnO}$ has potential applications in biosensing, or as peptide-linker, though its possible toxicity is still unknown. It is therefore of interest to understand how the surface evolves in contact with biomolecules. Theoretical studies of adsorption of glycine on zinc oxide from the gas phase have been performed with ab initio tools and are described in the following in order of increasing water activity. On the dry surface and with adsorption from the gas phase, glycine adsorbs and forms only covalent bonds with a surface cation, either through the $\mathrm{COOH}$ moiety, the $\mathrm{NH}_{2}$ one or both. On zinc-terminated zincite, (0001)-( $\mathrm{ZnO})$, adsorption of neutral, zwitterionic and anionic glycine as well as several coverages were considered (from the limit of the isolated molecule to monolayer and bilayer coverage) [298]. The $\mathrm{Zn}$ density at the surface of $\mathrm{ZnO}$ is $1.06 \times 10^{16} \mathrm{Zn}$ atom per $\mathrm{cm}^{2}$, or 10.6 Zn/ $\mathrm{nm}^{2}$. The maximum coverage defining the monolayer was unknown and several Gly/Zn ratios were explored (1:4, 1:2 and 1:1 Gly/Zn), corresponding to $1.3,2.6$ and $5.3 \mathrm{Gly} / \mathrm{nm}^{2}$, respectively. The interaction of each glycine functionality (carboxylate and amine) with the $\mathrm{Zn}-$ $\mathrm{ZnO}$ surface was investigated separately, and then the adsorption through both functional groups was studied. Glycine adsorbs on the $\mathrm{Zn}-\mathrm{ZnO}$ surface at low coverage preferentially in the anionic form, in a parallel orientation to the surface. The carboxylate moiety forms a $\mathrm{Zn}-\mathrm{OCO}-\mathrm{ZnO}$ ring with two surface $\mathrm{Zn}$ atoms, and an additional $\mathrm{Zn}-\mathrm{N}$ bond is formed between the amine function and a surface $\mathrm{Zn}$ atom; the proton, product of dissociation, forms a hydride like $\mathrm{Zn}-\mathrm{H}$ at the surface. The formation of $\mathrm{OH}$ groups with oxygens of the underlying layer was also considered, but it is less stable. At higher coverage, (2.6 Gly/nm², corresponding to $1: 2 \mathrm{Gly}: \mathrm{Zn})$ the dissociation of glycine into a glycinate ion is again the most stable configuration. An organized glycinate layer is formed, in which the glycine molecules adopt the $\mathrm{ZnO}$ honeycomb structure. Still increasing the coverage to $5.3 \mathrm{Gly} / \mathrm{nm}^{2}$ leads to the formation of a $\mathrm{Zn}$-glycinate bilayer as shown by ab initio molecular 
dynamics simulations. In the first layer glycine molecules adopt the honeycomb $\mathrm{ZnO}$ lattice. The second layer consists of a $\mathrm{Zn}$-glycinate complex, with a $\mathrm{Zn}$ atom extracted from the surface.

\subsubsection{Adsorption of glycine on $\mathrm{Cr}_{2} \mathrm{O}_{3}$}

Stainless steel composes many medical devices. The surface of stainless steel is covered with a passive film of 1-2 $\mathrm{nm}$ thickness, enriched in $\mathrm{Cr}_{2} \mathrm{O}_{3}$. Increasing water activity by considering hydroxylated surfaces and glycine plus water co-adsorption at various Gly coverages was applied to the study of glycine adsorption on dry and hydroxylated $\mathrm{Cr}_{2} \mathrm{O}_{3}[304 ; 305]$. The GGA + U method was employed and the role of coverage on the adsorption mode and the self-assembly properties were explored. The dispersion contribution to the adsorption energies was also calculated. Glycine adsorbs at low and high coverage in the anionic form. The adsorption is driven by the formation of iono-covalent bonds with the $\mathrm{Cr}$ surface atoms. The most stable configurations correspond to maximization of the bonds $(\mathrm{Cr}-\mathrm{O}$ and $\mathrm{Cr}-\mathrm{N})$, where the $\mathrm{O}$ or $\mathrm{N}$ atoms replace $\mathrm{O}$ atoms from the missing anionic plane above the terminal $\mathrm{Cr}$ plane. To maximize bond formation and minimize lateral repulsion, glycine lies parallel to the surface at low coverage and has a bent orientation at high coverage. The inclusion of dispersion forces strengthens this trend. At saturation, a glycinate monolayer with a 1:1 Gly:Cr ratio is formed. The roles of glycine coverage and surface termination (hydroxylated $\mathrm{Cr}$ - and $\mathrm{O}$-terminated surfaces) on the adsorption mode and self-assembly properties were explored. The hydroxylated Cr-terminated $\mathrm{Cr}_{2} \mathrm{O}_{3}$ surface, which presents two types of $(\mathrm{H}) \mathrm{OH}$ groups exhibiting different acidic characters, is more reactive than the hydroxylated O-terminated surface, where one single type of $\mathrm{OH}$ group is present, for all adsorption modes and coverages considered. Outer sphere adsorption occurs in the zwitterion form, stabilized at low coverage through H-bond formation with coadsorbed water molecules, and at the monolayer coverage by glycine self-assembling. The $\mathrm{OH}$ substitution by glycinate is favoured on the hydroxylated $\mathrm{Cr}-$ terminated surface and not on the O-terminated one. The inclusion of dispersion forces does not change the observed tendencies.

\subsubsection{Adsorption of other amino acids}

Alanine adsorption on $\mathrm{ZnO}(10-10)$ was studied combining XPS and DFT calculations[316]. Deposition at $200 \mathrm{~K}$ leads to the formation of multilayers of Ala zwitterions, as indicated by the peak at $\mathrm{E}_{\mathrm{b}}(\mathrm{N} 1 \mathrm{~s})=401.8 \mathrm{eV}$ in the XPS spectrum. Heating to $300 \mathrm{~K}$ resulted in the removal of most of the multilayer, with the remaining surface coverage estimated to be $0.4 \mathrm{ML}$ with respect to the density of $\mathrm{Zn}$ cations. At this temperature most of the alanine molecules are anionic $\left(\mathrm{E}_{\mathrm{b}}(\mathrm{N} 1 \mathrm{~s})=\right.$ $\left.400.3 \mathrm{eV}, \mathrm{E}_{\mathrm{b}}(\mathrm{Cl})=288.7 \mathrm{eV}\right)$. The anion $\mathrm{NH}_{2}-\mathrm{CH}\left(\mathrm{CH}_{3}\right)-\mathrm{COO}$ is adsorbed with the protonation of a surface oxygen to an $\mathrm{OH}$ species. To resolve the atomic structures and compare the adsorption energy of alanine on the nonpolar surface of $\mathrm{ZnO}(10-10)$, ab initio DFT calculations were carried out for a periodic slab setup. A total of 13 different alanine binding geometries at monolayer coverage were investigated. The most stable structure was found when alanine was dissociatively adsorbed on the surface with the carboxylate in a 2 mode (bridging two $\mathrm{Zn}$ ions configuration) and the dissociated $\mathrm{NH}_{2}$ moiety forming a $\mathrm{H}$ bond with the formed $\mathrm{OH}$ group, a configuration very similar to that found for glycine on $\mathrm{TiO}_{2}$ (Figure 78b).With a calculated adsorption energy of -1.75 
$\mathrm{eV}$, this conformationwas found to be more stable than the $\mu 1$ species by about $0.7 \mathrm{eV}$. Further heating of the surface resulted in a gradual decrease of the surface coverage and by $500 \mathrm{~K}$ a large fraction of adsorbed alanine molecules have desorbed from the surface.

All amino acids interaction with the stable $\mathrm{ZnO}(10-10)$ surfacewas studied with force fields[320]. The adsorption followed the range: HID GLU HIE $\sim$ ASP $(-1.69--2.07 \mathrm{eV})>\operatorname{HIP}(-1.87 \mathrm{eV})>$ ASN $(-1.76 \mathrm{eV}) \sim \mathrm{LYS} \sim \mathrm{CYS}(-1.76 \mathrm{eV})>\mathrm{TRP} \sim \mathrm{GLN}(-1.66 \mathrm{eV})>\mathrm{LEU} \sim \mathrm{SER} \sim \mathrm{THR}(-1.45$ $\mathrm{eV})>\mathrm{VAL} \sim \mathrm{TYR} \sim \mathrm{ILE} \sim \mathrm{ARG}(-1.35)>\mathrm{MET} \sim \mathrm{PRO} \sim \mathrm{GLY}(-1.24)>$ PHE $\sim$ ALA (-1.14eV). We note the high binding energy of histidine, including its unprotonated form (HID), and of acidic amino acids. The corresponding energy is the highest obtained among theuncharged AAs. Unfortunately, details of bond formations between individual AA and the surface are not given.

\section{Adsorption of peptides on metals and oxides surfaces}

\subsection{Why studying peptides at metals and oxides surfaces?}

Peptides, like proteins, are assemblies of amino acid residues, ordered in a controlled sequence and forming 3D structures thanks to intramolecular interactions. While proteins are macromolecules whose functionality is guided by the sequence of their constitutive amino acids, peptides may be considered as small protein fragments, which can be specifically designed in order to have similar chemical groups and, even better, similar activity as the proteins from which they are derived. Designing such peptides is not only a dream. Some authors have indeed shown that site selectivity in biomolecules can be reproduced in relatively short peptides, not requiring the entire and complex scaffolds of natural proteins[346]; even tripeptides were shown to bear topological characteristics of much larger proteins[347]. The ultimate goal is, of course, to elucidate the relationship between chemistry, structure and functionality of these peptides/proteins, in particular to explain the mystery of specific molecular recognition or metal binding.

Unravelling the nature of peptide-metal interactions, together with the mechanism of peptide transformation in the vicinity of such materials, brings invaluable clues to the understanding and control of many physiological processes.

Peptides are currently used to increase cell adhesion[348] or, on the contrary, to prevent biocontamination, just because, once immobilized on a metal, they are able to interact with macromolecules in a biological environment in a specific way. In all cases, peptide activity is very dependent on the way they are immobilized, or grafted, on the surface. This further proves the importance of elucidating the nature of the interaction between peptides and metal or oxide surfaces.

Protein-metal (in neutral of charged forms) interactions also play a crucial role in the metabolism. Proteins usually possess a binding site, very specific to given metals depending on the resulting function[94], thus proving that fragments of the macromolecules are able to specifically bind to metal atoms or ions. Already in 1997, Brown constructed a large population of repeating polypeptides and tested their interaction with gold. Two categories of peptides have thus been identified, namely gold binding or non-gold- binding peptides (GBP or NGBP). Some of them 
contain amino acids which, individually, all have a strong or low affinity to gold, yet leading macromolecules of opposite affinities[349].

In this review, we will limit the discussion to the interaction of peptides with metals and oxides, where information exists at the molecular level. This implies the study of the interaction of very short peptides with well-defined surfaces, in an approach very similar to that of AA with metallic single crystals; it also comprises the investigation of the interaction of longer peptides with any type of inorganic surface, using techniques which help to elucidate the factors governing these interactions, as well as to locate and possibly identify the anchoring sites.

We will limit our contribution in this section to the most significant and recent examples reported in the literature, rather than pretending to report an exhaustive review of all works dealing with peptides on surfaces. Examples will be ordered from the simplest to more complex cases, where a large pallet of techniques is often applied to bring complementary information.

The following table reports all peptide-substrate systems which are described in this section, together with the techniques used and the references of the corresponding papers.

\begin{tabular}{|c|c|c|c|c|c|c|c|}
\hline Surface & $\mathrm{Cu}(110)$ & $\mathrm{Cu}(100)$ & $\mathrm{Au}(110)$ & $\mathrm{Au}(111)$ & $\begin{array}{l}\mathrm{Au} \\
\text { polycry } \\
\text { st. }\end{array}$ & $\mathrm{TiO}_{2}$ & Other oxides \\
\hline di-L-Ala & $\begin{array}{l}\text { STM, } \\
{[350]} \\
\end{array}$ & & & & & & \\
\hline Gly-Gly & $\begin{array}{l}\text { XPS, } \\
\text { Nexafs } \\
{[351]} \\
{[352]}\end{array}$ & & & & & & \\
\hline Tri-Ala & $\begin{array}{l}\text { RAIRS } \\
\text { [353] } \\
\text { MD [354] }\end{array}$ & & & & & & \\
\hline Tri-Leu & $\begin{array}{l}\text { RAIRS } \\
\text { [353] }\end{array}$ & & & & & & \\
\hline Gly-Pro & $\begin{array}{l}\text { PM-RAIRS } \\
\text { XPS [355] }\end{array}$ & & $\begin{array}{l}\text { PM-RAIRS } \\
\text { XPS LEED } \\
{[57]}\end{array}$ & & & & \\
\hline $\begin{array}{l}\text { Gly-Pro- } \\
\text { Glu (IGF) }\end{array}$ & $\begin{array}{l}\text { PM-RAIRS } \\
\text { XPS } \\
{[355]}\end{array}$ & & $\begin{array}{l}\text { PM-RAIRS } \\
\text { XPS, LEED } \\
{[57]} \\
\text { DFT[356] }\end{array}$ & $\begin{array}{l}\text { STM } \\
\text { [357] }\end{array}$ & & & \\
\hline Di-Phe & $\begin{array}{l}\text { STM } \\
{[358]}\end{array}$ & $\begin{array}{l}\text { STM } \\
\text { [359] }\end{array}$ & & & & & \\
\hline $\begin{array}{l}\text { Gly-Cys- } \\
\text { Glu (GSH) }\end{array}$ & & & $\begin{array}{l}\text { PM-RAIRS } \\
\text { STM } \\
\text { LEED } \\
{[57]}\end{array}$ & $\begin{array}{l}\text { PM-RAIRS } \\
\text { XPS [360] }\end{array}$ & $\begin{array}{l}\text { DFT on } \\
\text { clusters } \\
{[361]}\end{array}$ & $\begin{array}{l}\text { PM- } \\
\text { RAIRS } \\
\text { XPS } \\
{[360]}\end{array}$ & \\
\hline Gly-His & & & & $\begin{array}{l}\text { XPS, } \\
\text { Nexafs }\end{array}$ & & & \\
\hline
\end{tabular}




\begin{tabular}{|c|c|c|c|c|c|}
\hline & & $\begin{array}{l}{[362]} \\
M D[363]\end{array}$ & & & \\
\hline $\begin{array}{l}\text { Gly-His-Gly } \\
\text { And } \\
\text { Gly-Gly-His }\end{array}$ & & $\begin{array}{l}\text { XPS } \\
\text { Nexafs } \\
{[362]} \\
\text { MD [363] }\end{array}$ & & & \\
\hline $\begin{array}{l}\text { Gly-Cys- } \\
\text { Glu (GSH) }\end{array}$ & & $\begin{array}{l}\text { PM- } \\
\text { IRRAS, } \\
\text { XPS [364] }\end{array}$ & $\begin{array}{l}\text { ATR-IR } \\
\text { [365] }\end{array}$ & & \\
\hline $\begin{array}{l}\text { Arg-Gly- } \\
\text { Asp (RGD) }\end{array}$ & & & & $\begin{array}{l}\text { DFT in } \\
\text { vacuu } \\
m \\
{[366-} \\
369]\end{array}$ & \\
\hline Cys-Try & $\begin{array}{l}\text { RAS } \\
{[370]}\end{array}$ & & & & \\
\hline TAT & & $\begin{array}{l}\text { FT-IRRAS } \\
\text { XPS [371; } \\
372]\end{array}$ & & & \\
\hline RKLPDA & & & & $\begin{array}{l}{[348] \mathrm{Q}} \\
\mathrm{CM}\end{array}$ & \\
\hline EAK-RGD & & & $\begin{array}{l}\text { [373] } \\
\text { NEXAFS } \\
\text { ' XPS, } \\
\text { IR }\end{array}$ & $\begin{array}{l}{[373]} \\
\text { NEXAFS } \\
\text { IR XPS, }\end{array}$ & \\
\hline $\begin{array}{l}\text { hexapepti } \\
\text { des- } \mathrm{N} \text { - } \\
\text { terminal } \\
\text { tryptopha } \\
\mathrm{n}\end{array}$ & & & & & $\begin{array}{l}\text { [374]TM } \\
\text { oxides, } \\
\text { SERS }\end{array}$ \\
\hline Collagen & & & & $\begin{array}{l}\mathrm{MD} \\
{[375]}\end{array}$ & $\mathrm{Al}_{2} \mathrm{O}_{3}, \mathrm{MD}[375]$ \\
\hline $\begin{array}{l}\text { Hexa- } \\
\text { homo- } \\
\text { peptides }\end{array}$ & & & & & $\begin{array}{l}\text { TM oxides, } \\
\text { Fluorescence [376] }\end{array}$ \\
\hline $\begin{array}{l}\text { 3GBP, } \\
6 \mathrm{GBP} \text { and } \\
6 \mathrm{NGBP}\end{array}$ & & $\mathrm{MD}$ & & & \\
\hline $\begin{array}{l}\text { MRKDV, } \\
\text { ADEDRDA, } \\
\text { and } \\
\text { LGRGISL }\end{array}$ & & $\begin{array}{l}\text { SERS and } \\
\text { Hückel } \\
\text { theory } \\
\text { [377] }\end{array}$ & & & \\
\hline
\end{tabular}




\begin{tabular}{|l|l|l|l|l|l|l|l|}
\hline CLPFFD & & & $\begin{array}{l}\text { SERS and } \\
\text { Hückel } \\
\text { theory } \\
{[378]}\end{array}$ & & & \\
\hline $\begin{array}{l}\text { Hexa- } \\
\text { homo- } \\
\text { peptides }\end{array}$ & & & & & & & $\begin{array}{l}\text { TM Fluorescence [376] } \\
\text { Foides, }\end{array}$ \\
\hline
\end{tabular}

Table 12. Peptides on metal and oxide surfaces.

\subsection{Systems of very low complexity (short peptides on single crystal surfaces, adsorbed under UHV conditions)}

This last decade has shown an increasing number of experimental investigations aiming at describing the interaction of short peptides with coinage metals, like gold, silver or copper, as well as with some "functional" oxides, like titanium or silicon oxides. In particular, gold and copper have been the metals of choice in many "model" studies, experimental and theoretical, leading to unravel some clear tendencies regarding the affinity and likely anchoring points of small peptides with these coinage metals.

\subsubsection{Di- and Tri-peptides on $\mathrm{Cu}(110)$ and $\mathrm{Cu}(100)$}

Let's start with the simple case of a di-peptide on metals. Compared to AA, the presence of a peptidic link may fully change the adsorption geometry, and thus the reactivity, of molecules yet very similar from a chemical point of view[351].

After having widely described the adsorption of AA[175], Raval et al. investigated the organization of some homo-di and tri-peptides on copper, and in particular on $\mathrm{Cu}(110)$. They were precursors in this field of research in the sense that they extended the techniques and conditions they had developed for AA to form and characterize layers of rather complex molecules at a molecular level. In these pioneering experiments, peptides were adsorbed under UHV conditions, thus demonstrating that peptides can be evaporated in a UHV chamber and adsorb intact on the surface.

They mainly used STM and FT-IRRAS. Let's recall that the first technique gives access to the 2D structure of adsorbed layers, while the second one enables to unravel the chemical form of the peptide, orientation of its main groups but, thanks to the sensitivity to the amide I position, also its conformation.

The first experiments were performed on tri-L-alanine and tri-L-Leucine, differing by the side chain (see Figure 88). Both peptides adsorb on $\mathrm{Cu}(110)$ in an anionic form resulting from the deprotonation of the terminal carboxyl group, which is the binding point (or one of the binding points) of the molecule with the surface[353]. Both molecules rearrange on the surface when increasing the coverage or the temperature, but in different ways. Tri-L-alanine adsorbs and binds to the surface via its terminal $\mathrm{COO}^{-}$group and probably the $\mathrm{NH}_{2}$ one, in a disordered manner. The rather intense amide I band (1674-1678 $\mathrm{cm}^{-1}$, see top spectra in Figure 89) suggests an orientation of the $\mathrm{C}=\mathrm{O}$ group perpendicular to the surface. This band broadens when the coverage increases; this evidences the existence of H-bond interactions between molecules and a probable growth and 
arrangement of alanines in bilayers resembling the antiparallel $\beta$-sheets of the crystalline peptide[354].

The behaviour of the layer when stopping the exposure and waiting in vacuum iseven more interesting (Figure 89, bottom spectra). The amide I band intensity decreases, not due to a loss of molecules but rather because of a rearrangement of the layer into pairs of molecules letting the $\mathrm{C}=\mathrm{O}$ groups parallel to the surface. The story is quite different with tri-L leucine, which also adsorbs via a depr-onated $\mathrm{COO}^{-}$-group but which does not form bilayers. This is likely due to the alkyl side groups of the molecule that hinder any possible interaction between $\mathrm{C}=\mathrm{O}$ and $\mathrm{NH}$ groups of a second molecule.

(a)<smiles>C[C@@H](CN)NC(=O)[C@H](C)N(C)C(=O)[C@@H](C)N</smiles>

(b)<smiles>CC(C)CC(CC(C)C)NC(=O)[C@H](CC(C)C)NC(=O)[C@H](C)CN</smiles>

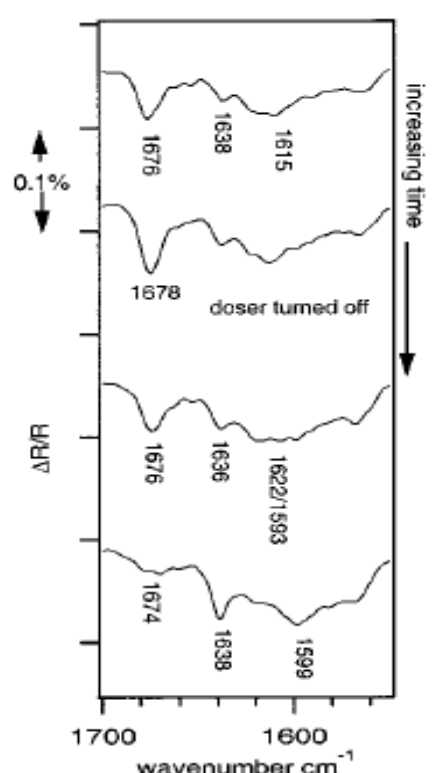

Figure 89. Tri-alanine layer growth on $\mathrm{Cu}(110)$ at $300 \mathrm{~K}$, during exposure, and after stopping it. Taken with permission from ref. [353].

It is interesting to compare the behaviours of tri- with that of di-alanine on the same surface. Little after Raval's work, Stensgaard characterized the adsorption of di-L-alanine on $\mathrm{Cu}(110)$ by variable temperature STM; they evidenced the formation of elongated molecular islands along the <-110> direction, indicating strong directional interactions between molecules, likely between $\mathrm{COO}^{-}$and $\mathrm{NH}_{2}$ or $\mathrm{NH}_{3}{ }^{+}$groups. At high coverage islands coalesce and two types of domains of opposite direction appear (Figure 90 and Figure 91)[350]. For the first time, quasi chiral domains were observed on a $\mathrm{Cu}(110)$ surface and, conversely to tri-alanine, di-alanine tends to form a dense and organized monolayer before growing into multilayers. These dense molecular rows are stabilised by $\mathrm{H}$-bonds between $\mathrm{NH}$ and $\mathrm{CO}$ groups in neighbouring strands. 


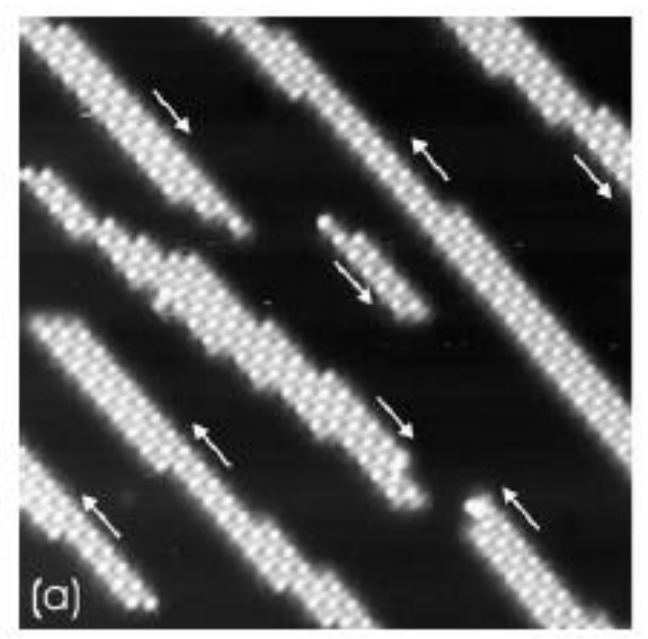

Figure 90. STM images of di-alanine islands on $\mathrm{Cu}(110)$. Each molecule is imaged as a pair of one bright and one grey bumps; they are aligned in two possible directions, either parallel or antiparallel to the <-332> direction as indicated by the arrows (image size: $250 \AA \times 250 \AA$ ). Takenwith permission from ref. [350].

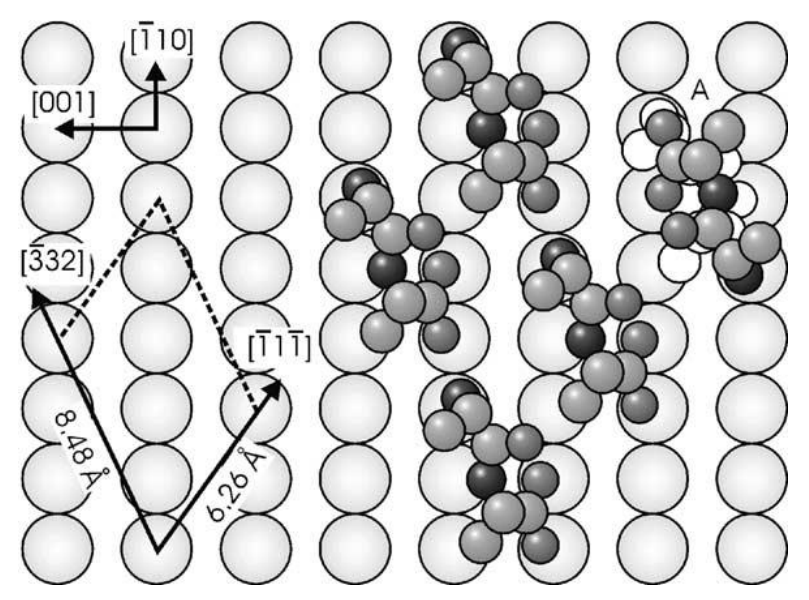

Figure 91. Schematic model of the di-L-alanine layer on $\mathrm{Cu}(110)$, deduced from STM observations. The molecule was adsorbed under UHV conditions. N: black, O: grey, C: light grey. Note the molecule marked $\mathrm{A}$ in the upper right part of the figure is rotated $180^{\circ}$ with respect to the other ones; this represents alanine molecules in either the two domains identified by STM. Taken with permission from ref. [350].

An important question was thus raised by the two above described examples: do peptides form multilayers? The just cited example shows the key role of H-bondings, which are driving forces for 2D arrangement as well as for further growth of peptide multilayers. Another example is that of Glycine-Proline (Gly-Pro) and Glycine-Proline-Glutamic acid (Gly-Pro-Glu) both of which were adsorbed on $\mathrm{Cu}(110)$ under UHV conditions[355]. For both molecules, two binding points were identified by combining PM-IRRAS and XPS, namely the $\mathrm{COO}^{-}$groups and the $\mathrm{N}$ atom of $\mathrm{NH}_{2}$ groups (contribution at low $\mathrm{E}_{\mathrm{b}}$ in the XPS spectra of the $\mathrm{N} 1 \mathrm{~s}$ region, see Figure 92). No 2D organization could even be seen by LEED. Striking differences were observed at long exposure (more than 60 min under $\mathrm{P}=10^{-9}$ Torr): the Gly-Pro-Glu layer keeps growing, while a saturated monolayer of Gly-Pro is attained, with no further adsorption of the peptide. Such a difference was 
attributed to the presence of an additional glutamate residue on Gly-Pro-Glu which enables Hbonding between molecules adsorbed in the first layer, and incoming new ones (Figure 93). Note also that, when forming multilayers, Gly-Pro-Glu adsorbs under a zwitterionic form as indicated by the high binding energy of the appearing $\mathrm{N} 1$ s contribution (Figure 92, right).
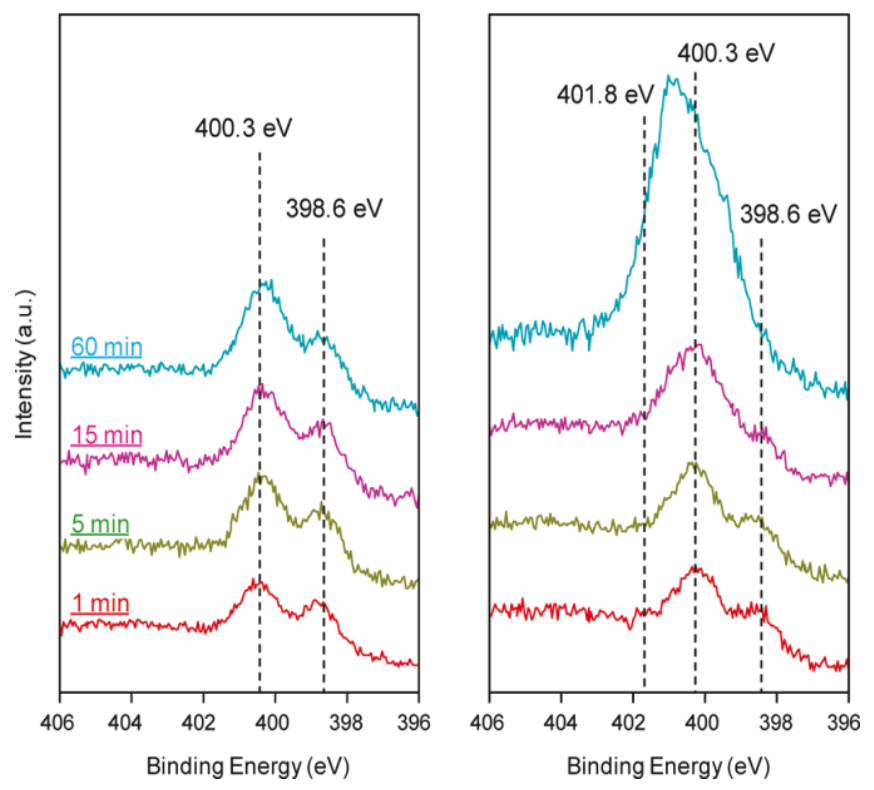

Figure 92. XPS N1s peaks after Gly-Pro (left) and Gly-Pro-Glu (right) adsorption on $\mathrm{Cu}(110)$. Both peptides were adsorbed under UHV conditions. The left series of spectra shows a low $\mathrm{E}_{\mathrm{b}}$ contribution at $398.6 \mathrm{eV}$ and a rapid saturation of the intensities, while in the right series the peaks grow continuously with exposure time. After 60 min., a new contribution at $401.8 \mathrm{eV}$ witnesses the appearance of $\mathrm{NH}_{3}{ }^{+}$groups, likely associated to the formation of multilayers. Taken with permission from ref. [355].

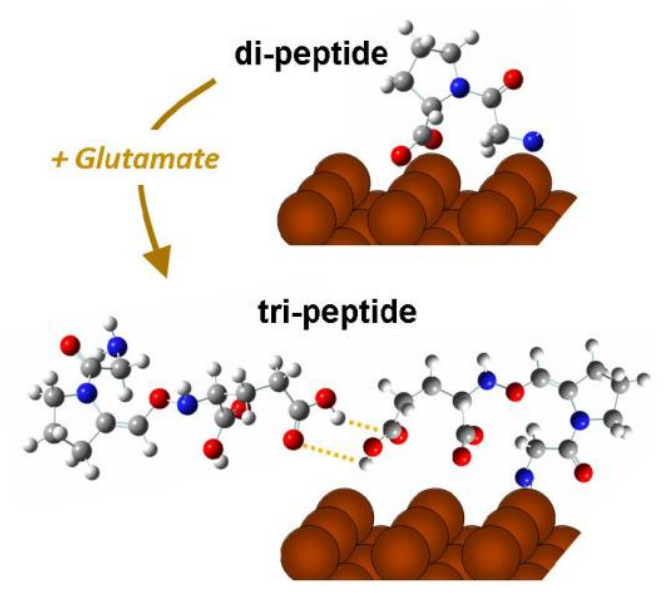

Figure 93. Schematic representation of the Gly-Pro and Gly-Pro-Glu molecules adsorbed on $\mathrm{Cu}(110)$. The figure evidences how the presence of an additional glutamate residue permits the formation of multilayers. Fig. taken from ref. [355].

Feyer's group was also amongst the first ones to investigate the exciting field of di- or tri-peptide adsorption on well-defined surfaces. They combined X-ray absorption, X-ray photoelectron spectroscopy and DFT(using a cluster approach) to describe the adsorption of the simplest dipeptide, Gly-Gly on $\mathrm{Cu}(110)[351]$. At variance with many other theoretical studies described here, the approach is non-periodic, so only non-interacting adsorbed molecules can be modelled. The purpose of this work was, rather than perform exhaustive search of adlayers organization, to compare experimental XPS data obtained with a synchrotron beam with ionisation potentials calculated with DFT. To this purpose, several adsorption modes of GlyGly on $\mathrm{Cu}(100)$ were considered, including anions and zwitterions in different configurations. 


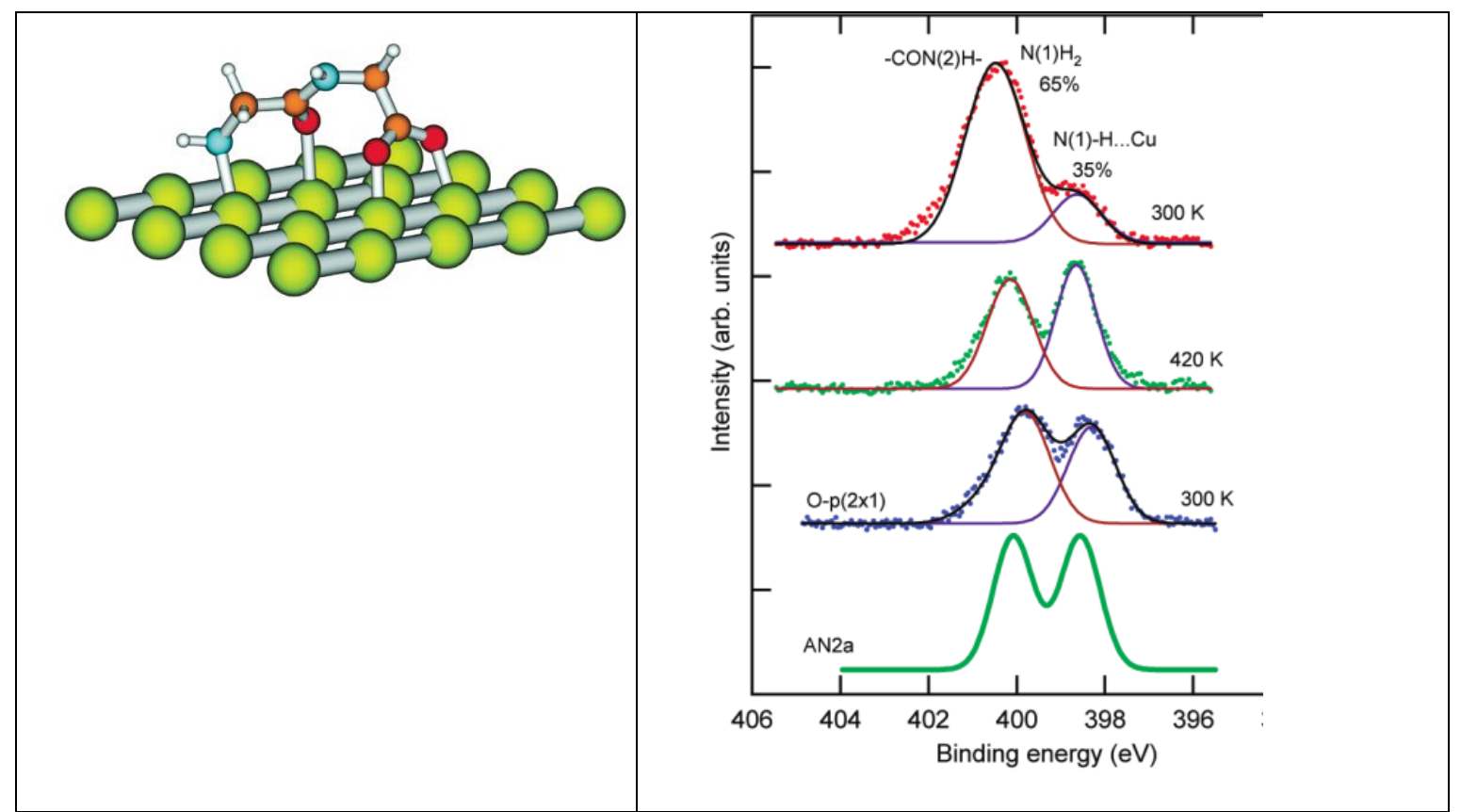

Figure 94. Proposed adsorbed Figure 95. Core level spectra of N 1s, measured geometry of Gly-Gly on $\mathrm{Cu}(110)$, at photon energy hv= $490 \mathrm{eV}$ on the clean and optimized by DFT calculation. Such oxygen-modified $\mathrm{Cu}(110)$ surface. Solid lines a configuration corresponds to the are fitted curves. The lowest spectrum is a calculation performed for a $1 \mathrm{ML}$ calculated spectrum corresponding to the coverage. Fig. taken from ref. [351]. geometry shown in Figure 94. Fig. taken from ref. [351].

According to DFT calculations, the most stable conformation of Gly-Gly molecules on $\mathrm{Cu}(110)$, in the anionic form and in the submonolayer regime, is schematised in Figure 94. The molecules are preferentially bound through the two oxygen atoms of the carboxylate and the amide group.Note that the combination of DFT calculation and XPS data led the authors to exclude a direct $\mathrm{N}-\mathrm{Cu}$ interaction.Indeed, this would lead to shift of the $\mathrm{E}_{\mathrm{b}}(\mathrm{N}$ 1s) with respect to the value expected for $\mathrm{N}$ in $\mathrm{NH}_{2}$ groups much larger than the $\sim 2 \mathrm{eV}$ observed experimentally. A further interaction of the peptidic $\mathrm{C}=\mathrm{O}$ bond was also considered. Moreover, after adsorption at $300 \mathrm{~K}$ and heating to $420 \mathrm{~K}$, all Gly-Gly species interact with copper via the hydrogen of the amino group (see the increase of the low $E_{b}$ contribution inFigure 95 . Core level spectra of $N$ s, measured at photon energy hv= 490 $\mathrm{eV}$ on the clean and oxygen-modified $\mathrm{Cu}(110)$ surface. Solid lines are fitted curves. The lowest spectrum is a calculated spectrum corresponding to the geometry shown in Figure 94. Fig. taken from ref. [351]. When forming monolayer and multilayers, upon further exposure at $300 \mathrm{~K}$, anionic and zwitterionic forms of Gly-Gly coexist (upper spectrum ofFigure 95) and both electrostatic interactions and hydrogen bonding ensure intermolecular interactions. From the analysis of the $\mathrm{C} 1 \mathrm{~s}$ spectra and comparison with computed values, it was concluded that the $2 \mathrm{eV}$ shift corresponds to the anionic state bonded through $\mathrm{COO}$ and $\mathrm{Cu}-\mathrm{NH}$ (Figure 95). Examination of N1s and O1s peaks confirmed this trend. These authors also demonstrated that pre-adsorption of oxygen on copper forces the formation of mostly anionic species $\left(\mathrm{COO}^{-}\right)$, bound to copper via their $\mathrm{NH}$ groups. By the way, they explained why the binding of carboxylates to the copper atoms of a (110) surface is so strong and commonly observed; this may be simply due to the $\mathrm{Cu}-\mathrm{Cu}$ distance along the close- 
packed $\left\langle 1-10>\right.$ direction, very close to the $\mathrm{O}-\mathrm{O}$ one in $\mathrm{COO}^{-}(2.556 \AA)$, allowing a $\mu 2$ adsorption of the carboxylate.

Reactive dynamics simulationsby means of ReaxFF in the gas phase were carried out to study diglycine adsorption on a perfect $\mathrm{Cu}(110)$ surface slab. In agreement with theoretical data and experimental observation, the slab model relaxed its configuration and reconstructed part of the interface, forming a terrace-like structure. A few copper atoms diffused on the topmost layer and the adsorbed molecules, which were already in contact with the interface, migrated to better locations close to the step edge where the interaction with the copper atoms was stronger. The peptides were interconnected by a network of hydrogen bonds which was denser than in the frozenslab simulation. Heterogeneous tridentate bonding was the preferred connection and the anionic form the best way of binding (See Figure 96). An evaluation of the adsorptionenergies (up to -4.77 $\mathrm{eV}$ for the conformation shown inFigure 97) suggests that antiparallel $\beta$-sheet configurations are highly preferred, which actually turns out to be in line with experimental observations.

Although the $\mathrm{Cu}-\mathrm{N}$ interaction was predicted, until the contrary is proven we tend to privilege the $\mathrm{Cu}-\mathrm{HN}$ geometry since it was obtained with a more reliable calculation method (DFT) compared with the FF based (ReaxFF) 


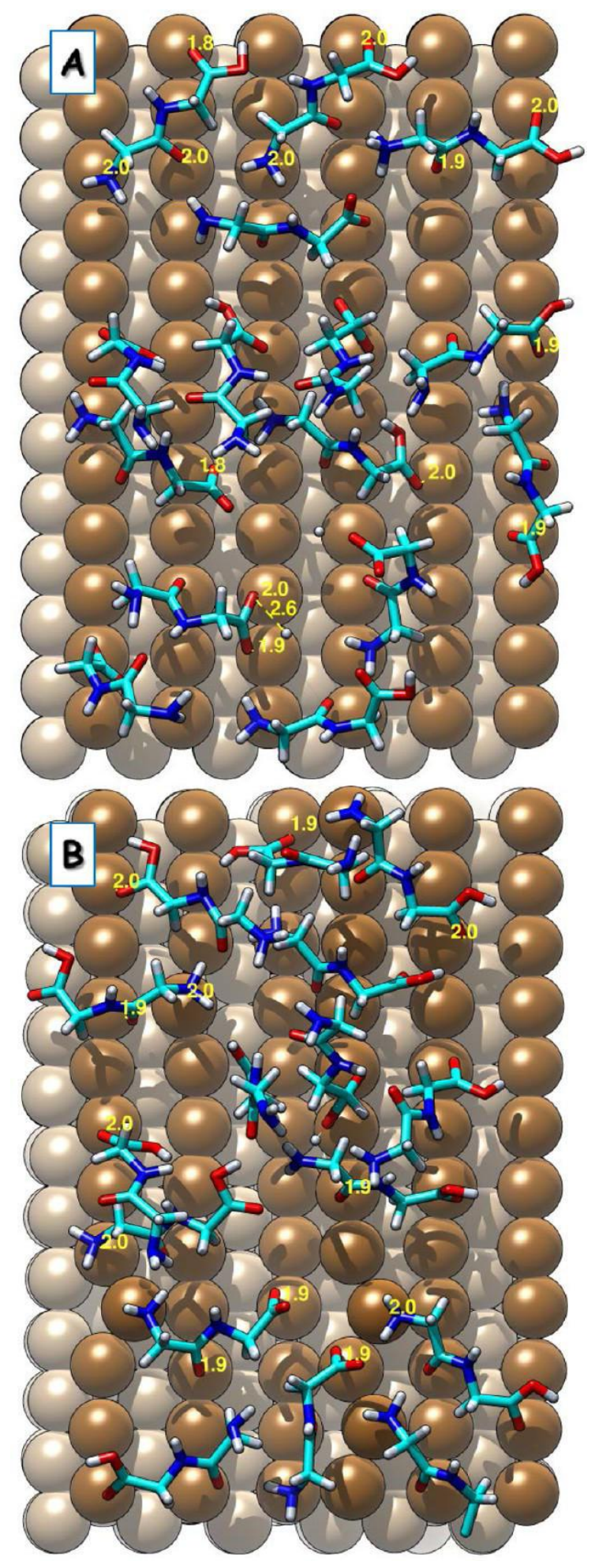

Figure 96. Final configurations of the Gly_Gly monolayers obtained after 2 ns of MD in the NVT ensemble (A: frozen-slab; B: free-slab). Peptides on top of the adsorbed molecules have been removed for clarity. Different adsorption modes are displayed, and the characteristic adsorption distances of the coordinated atoms are reported (yellow labels). A chiral domain is visible in the frozen-slab model (top-left region of the surface). Taken with permission from ref. [352]. 


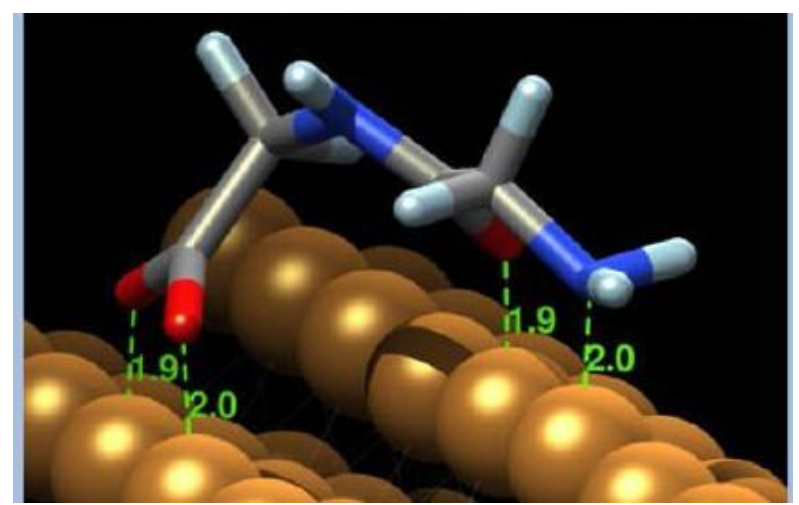

Figure 97. Most stable adsorption conformation for Gly-Gly on $\mathrm{Cu}(110)$. Distances in $\AA$. Taken with permission from ref. [352].

In addition to peptide-substrate binding, inter- and intramolecular interactions play a major role in peptide organisation at surfaces. Let's take the example of diphenylalanine (L-Phe-L-Phe) molecules deposited under ultrahigh vacuum on $\mathrm{Cu}(110)$ surfaces and then annealed to $420 \mathrm{~K}$ for five minutes.Molecules arrange in linear supramolecular chains, probably by interaction between the carboxylic group of one molecule with theamino group of the neighbouring one (

Figure 98, left). These chains are isolated, and no extended ordered structures could be observed; this is obviously related to some repulsive interactions between them.

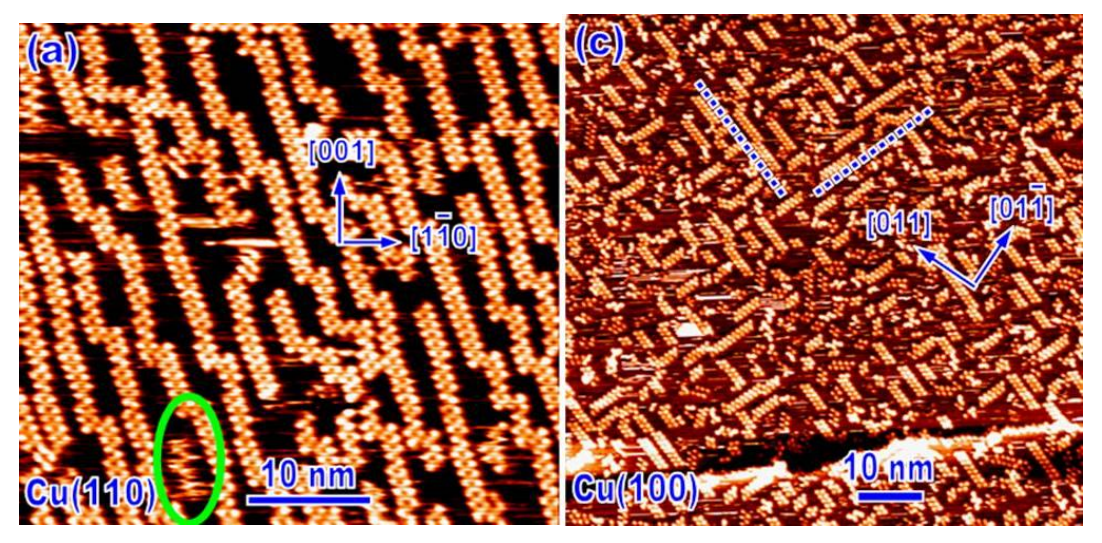

Figure 98.STM images of the $\mathrm{Cu}(110)$ (left), and $\mathrm{Cu}(100)$ surface (right), after L-Phe-L-Phe adsorption. On $\mathrm{Cu}(110)$ the chains, extending mainly along the<-114> direction, are isolated and characterized by a high density of kinks. On the isotropic $\mathrm{Cu}(100)$, the L-Phe-L-Phe chains grow along four possible directions. Two main chain orientations can be distinguished along the substrate $<021>$ and <012> directions, as illustrated by the dashed lines. Fig. taken with permission from ref. [359]. 

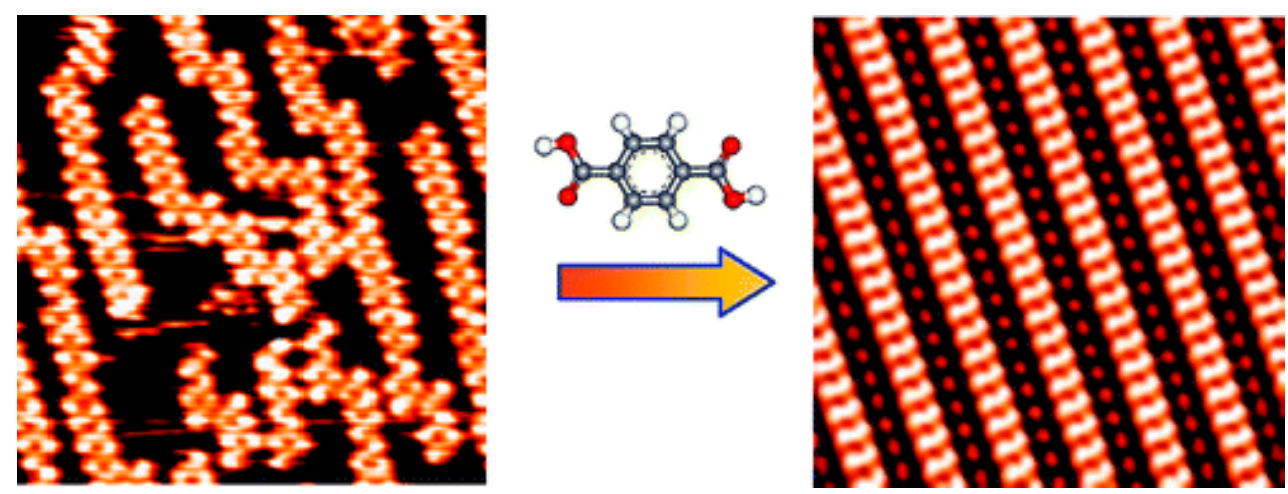

Figure 99. Ordering of the dipeptide chains by co-crystallisation with terephtalic acid (TPA) on $\mathrm{Cu}(110)$. Taken with permission from ref. [359].

On the $\mathrm{Cu}(100)$ surface, the same dipeptide arrange along four possible directions. This $90^{\circ}$ rotational symmetry of the supramolecular arrangements reflects the four-fold symmetry of the $\mathrm{Cu}(100)$ surface (see

Figure 98, right).

On both surfaces, $\mathrm{Cu}(110)$ and $\mathrm{Cu}(100)$, terephtalic acid (TPA), having two carboxyl end groups, was then co-deposited with L-Phe-L-Phe molecules, with the objective of making connections between isolated peptide chains. Indeed, 2D organized structures, namely large domains of highly ordered molecular chains, could be formed, as evidenced on the right STM image of Figure 99, TPA playing a role of interchain molecular glue[359]. This happened on either $\mathrm{Cu}(100)$ or $\mathrm{Cu}(110)$ surfaces, showing that the ability of TPA to link, and order, peptide chains is independent of the substrate. Note eventually that, on $\mathrm{Cu}(100)$, only two differently chiral oriented domains were formed, upon TPA co-adsorption, opening new routes to fabricate extended peptide architectures on surfaces.

The L-L and D-D enantiomers of di-phenyl-alanine, which contain two chiral carbon centres linked through a central amide bond, were adsorbed on $\mathrm{Cu}(110)$ [358]. After co-deposition of these two enantiomers, STM imaging revealed the creation of two homochiral domains, where the molecules form linear chains rotated $34^{\circ}$ clockwise, or anticlockwise, from the $\langle 1-10\rangle$ substrate direction (see Figure 100). Classical MD simulations at room temperature in the gas phase indicated that the LPhe-L-Phe molecular conformation with the carboxylic and the amino groups located on the same side with respect to the main molecular axis (See Figure 102) is more stable than the other possible conformation, in which the two functional groups point in opposite directions (See Figure 102). DFT Calculations show the minimum energy structure of adsorbed isolated molecules (Figure 101); in such a three-point configuration, molecules bind to the metal via the nitrogen atom of the amino group, one oxygen of the carboxyl and the oxygen of the central carbonyl $\left(\mathrm{E}_{\mathrm{ads}}=-1.6 \mathrm{eV}\right)$. They also suggest the existence of strong hydrogen bonds between the carboxyl group of one molecule and the amino group of the adjacent one (Figure 102). In addition, spontaneous proton transfer between the two groups occurs during the simulations, further strengthening the bond and yielding a chain of zwitterionic molecules. This finding is in perfect agreement with the XPS analysis of the same dipeptide molecule on $\mathrm{Cu}(110)$, reported by Barlow and Raval [175]. 

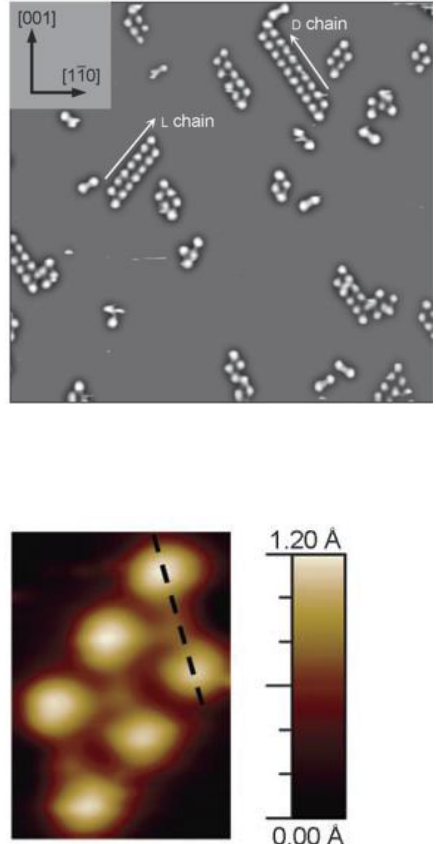

Figure 100.STM image $\left(36 \times 34 \mathrm{~nm}^{2}\right)$ of coadsorbed L-Phe-L-Phe and D-Phe-D$\mathrm{Phe}$ on $\mathrm{Cu}(110)$ at room temperature. The arrows indicate the growth direction of the homochiral chains. Taken with permission from ref. [358].
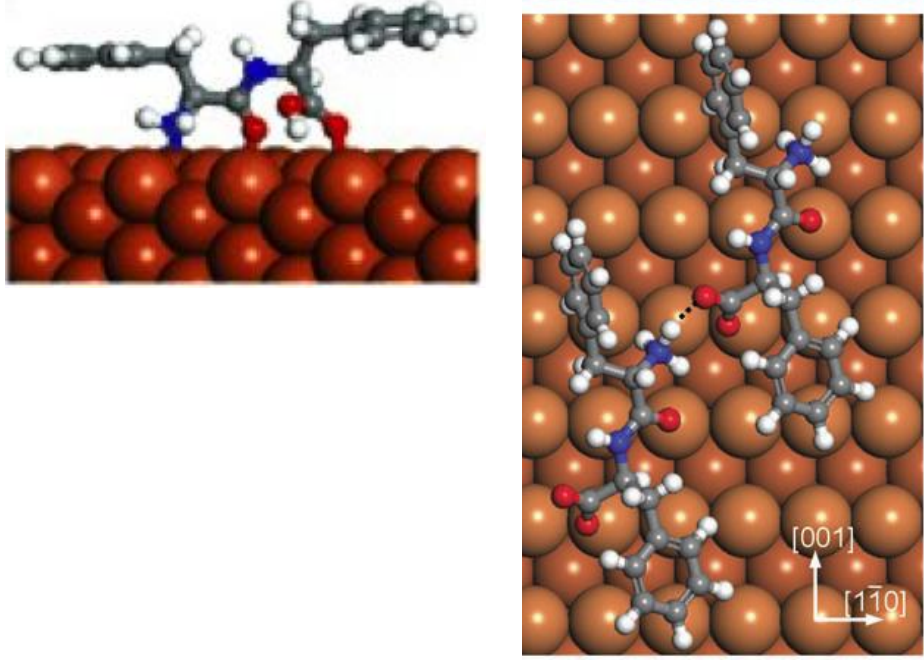

Figure 101.Minimum- Figure geometry 102.Adsorption energy structure of an isolated

L-Phe-L-Phe dipeptide

(DFT simulations). The carboxylic and amino groups are located on the same side dipeptides of L-Phe-L-Phe homochiral chains. The carboxylic and amino groups are located on opposite sides with respect to the molecular axis. with respect to the Taken with permission from ref. molecular main axis. Taken [358]. with permission from ref. [358].

Investigating surface chirality is indeed another intriguing issue related to peptides on surfaces. In this context, the possible stereoselective recognition between two molecules with opposite chirality (L-Phe-L-Phe and D-Phe-D-Phe) was investigated by co-depositing dipeptide enantiomers of either identical, or of opposite, chirality on $\mathrm{Cu}(110)$. STM video revealed first a longer distance between the two peptide when they are of opposite chirality than when similar; then, the enantioselective recognition only occurs when the arrangement of peptides of identical chirality permits electrostatic or polar hydrogen bond type interactions between amino and carboxylic groups of the two molecules. The authors have rationalized the process using first-principles and classical moleculardynamics simulations and came to the very important conclusion that chiral recognition is a dynamic process that involves conformational adjustments when molecules come close to each other and interact[358].

\subsubsection{Di- and Tri-peptides on $\mathrm{Au}(110)$ and $\mathrm{Au}(111)$}

A question one may ask is the influence of the nature of the metal on peptide binding and organization. When passing from $\mathrm{Cu}$ to $\mathrm{Au}(110)$, the adsorption of Gly-Pro and Gly-Pro-Glu (IGF) 
shows slight differences [57]. Both the neutral and zwitterionic forms of these two peptides were observed on $\mathrm{Au}(110)$, whereas the anionic forms were favoured on copper. More important is the difference in the peptide binding mode; the identified nitrogen binding point, revealed by an XPS contribution at low $E_{b}$, was not (or hardly) observed on $\mathrm{Au}(110)$, which suggests that the gold interaction (and electron transfer) with amino or amide nitrogen atoms is not favoured. This difference changes the strength of the interaction and the geometry of the molecules. As a consequence, also the growth mode is different, in particular for Gly-Pro which does continuously adsorb without attaining saturation. This can be directly related to the fact that the binding configuration of the molecule leaves $\mathrm{NH}_{2}$ terminal groups available for $\mathrm{H}$-bond interactions with other molecules.

Note that the adsorption behaviour of IGF on $\mathrm{Au}(111)$ and on $\mathrm{Au}(110)$ is very similar; the only difference is the ability of IGF to form some ordered 2D arrangement on $\mathrm{Au}(110)$ whereas no LEED patterns or STM images revealed any superposed surface structure on Au(111). An STM study was performed to elucidate the reason why IGF did not form any stable 2D layer on that surface at room temperature. This investigation strongly suggests that IGF is capable of chelating gold atoms, at kinks and steps of the (111) surface, in a multipoint binding mode, and induce some surface reconstruction but no ordered adlayers[357]. Due to its chelating properties IGF can displace individual gold atoms from the edges and kinks of the $\mathrm{Au}(111)$ surface. This phenomenon was investigated using theoretical calculations at the DFT level (B3LYP for the $\mathrm{Au}_{2}$ cluster model (Figure 103) and PBE for the periodic model (Figure 104)). It was postulated that the adsorption energy of IGF overpasses the cohesion energy of gold surface atoms.Surface reconstruction is thus favoured, explaining the strong reconstruction phenomenon of what was called the "finger" formation.

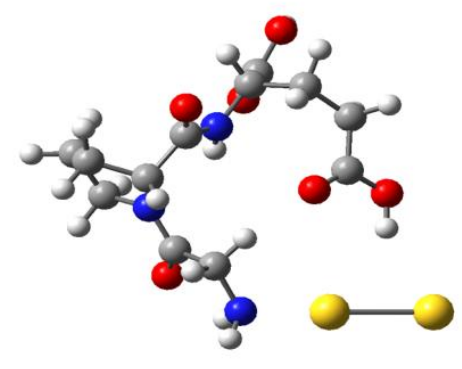

Figure 103. Most stable IGF-Au 2 geometry showing the chelating effect of the $\mathrm{NH}_{2}$ and $\mathrm{COOH}$ groups. Taken with permission from ref. [356]. 


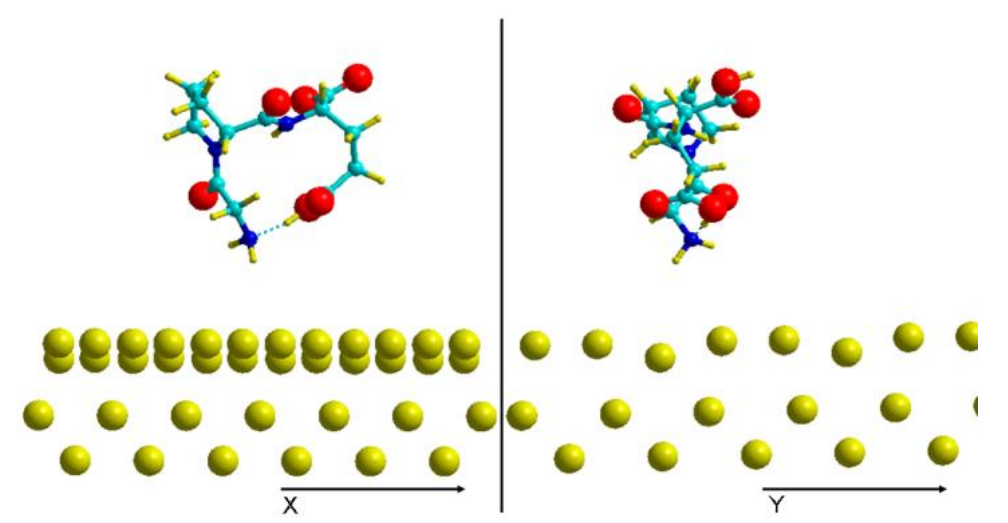

Figure 104. Optimized IGF molecule adsorbed on $\mathrm{Au}(110)$ surface ( $\mathrm{XZ}$ and $\mathrm{YZ}$ view), showing the intra-molecular $\mathrm{H}$-bond between the $\mathrm{NH}_{2}$ and $\mathrm{COOH}$ zwitterionic precursor groups and the adsorption via those groups. Taken with permission from ref. [356].

On the $\mathrm{Au}(110)$ surface the IGF molecule adsorbs in a geometry similar to that of the $\mathrm{Au}_{2}$ cluster but stabilizing both end groups implicated in the chelation with an intra-molecular hydrogen bond (See Figure 104), thus forming $\mathrm{COO}^{-}$and $\mathrm{NH}_{3}{ }^{+}$zwitterionic groups, in line with the experimental observations. Displacement of gold atoms was also observed upon adsorption of IGF. The high adsorption energy $(-3.79 \mathrm{eV}$, periodic DFT) calculated on the $\mathrm{Au}(110)$ surface was explained partly by the higher dipole moment and higher conformational flexibility of IGF compared to related amino acids.

On the basis of reaction energetics, obtained on model calculations simulating step sites on $\mathrm{Au}(111)$ using a reconstructed $\mathrm{Au}(110)$ surface, it is expected that the strong adsorption is responsible for the unusual surface reconstruction observed on $\mathrm{Au}(111)$ (Figure 105). The reconstruction is expected to originate from the displacement of terrace $\mathrm{Au}$ atoms which are replaced by underlying bulk $\mathrm{Au}$ atoms, giving rise to this original "candle melting" phenomenon.
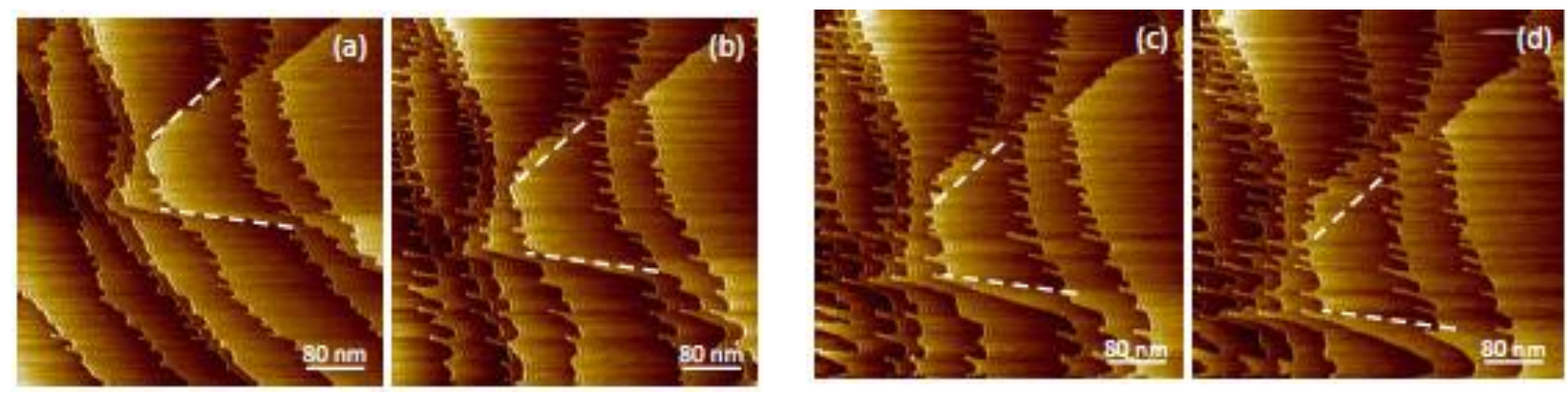

Figure 105 Consecutive STM images $(500 \mathrm{~nm} \times 500 \mathrm{~nm})$, showing the surface reconstruction during IGF adsorption on $\mathrm{Au}(111)$, one image every 4 min and 25 s.; the dashed line shows the evolution of one flat terrace. Taken with permission from ref. [356].

Considering Gluthatione (Gly-Cys-Glu, or GSH), which differs from IGF only by its central fragment (cysteine instead of proline), results are again different. GSH adsorbs on $\mathrm{Au}(111)$ and $\mathrm{Au}(110)$ under a mainly neutral form $\left(\mathrm{NH}_{2} / \mathrm{COOH}\right)$, and it first interacts with the surface via the $\mathrm{S}$ atom of its central fragment (low $\mathrm{E}_{\mathrm{b}}$ doublet in Figure 106). When increasing the exposure, and thus the coverage, unbound sulphurcould be identified ( $\mathrm{SH}$, high $\mathrm{E}_{\mathrm{b}}$ doublet in Figure 106),suggesting 
that the coverage increases by interaction of additional molecules with those which are already bound. This was confirmed by the simultaneous appearance of a $\mathrm{C}=\mathrm{O}$ stretching band of carboxylic groups at low wavenumber $\left(1697 \mathrm{~cm}^{-1}\right.$, to be compared to the unique band at $1730 \mathrm{~cm}^{-1}$ observed at low coverage), indicative of intermolecular H-bonds (formation of GSH dimers, see Figure 107). Note that,neither on $\mathrm{Au}(111)$ nor on $\mathrm{Au}(110)$, any 2D organization of GSH layers could be observed, likely due to the preferential formation of dimers[57; 360].

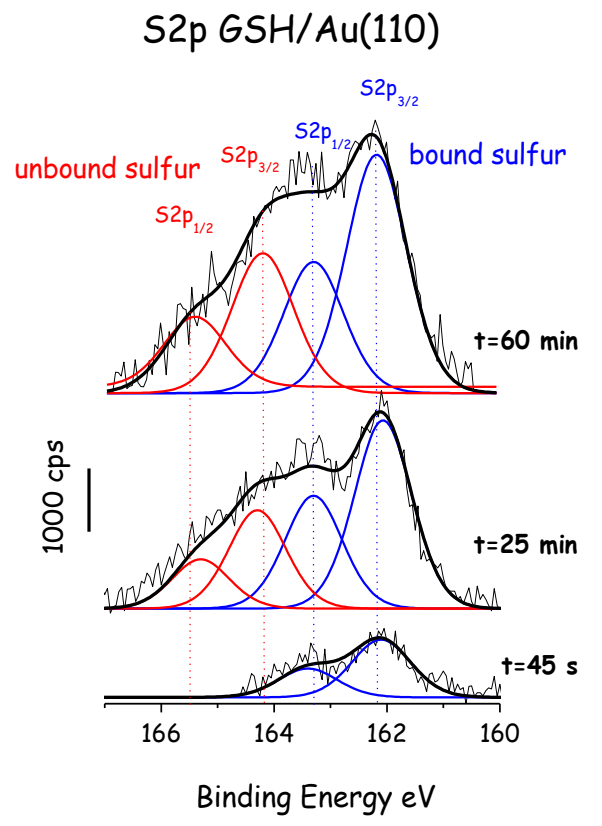

Figure 106.GSH exposure $\left(\mathrm{P}=10^{-9}\right.$ Torr $)$ on $\mathrm{Au}(110)$ at RT. Evolution of the S2p signal vs exposure time. Taken with permission from ref. [361].

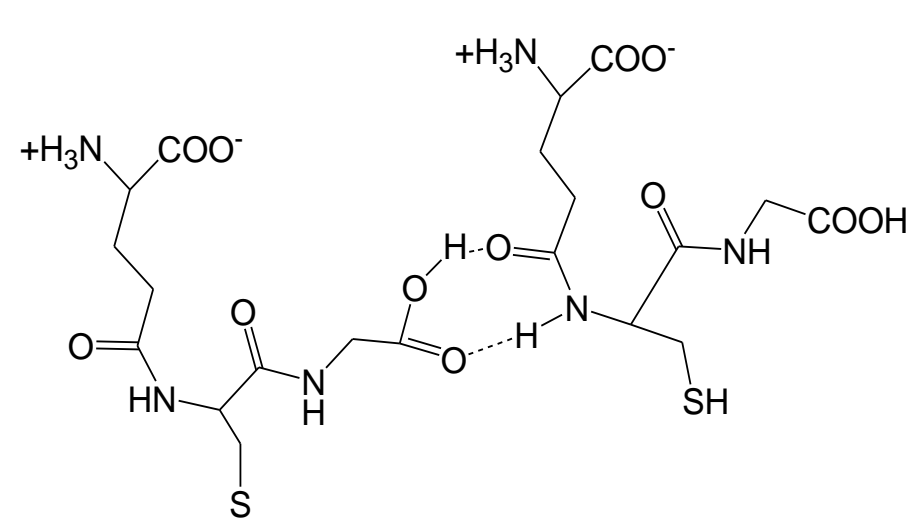

Figure 107.GSH/Au(110). Schematic representation of the peptide dimers formed at intermediate coverage (still below $1 \mathrm{ML}$ ). Taken with permission from ref. [361].

The adsorption of GSH on $\mathrm{Au}(111)$ has been widely described after adsorption from aqueous solutions, revealing another interesting result: after a relatively soft rinsing in pure water, the peptides retain the ionic form they have in solution at various pHs. It was also shown that charges born by the adsorbed peptides influence the further adsorption of proteins [379].

Histidine, and three different histidine-containing di- or tri-peptides were adsorbed on $\mathrm{Au}(111)$. Histidine is another intriguing molecule, having three reactive groups, namely amine, imidazole and carboxyl groups; histidine is also known to easily complex metal ions. Gly-His, Gly-His-Gly, GlyGly-His were selected with the objective of investigating the role of additional $\mathrm{NH}_{2}$ or $\mathrm{COOH}$ groups in a side or end position. While His was adsorbed under UHV conditions, other peptide layers were deposited from the liquid phase. Core level XPS results suggest that His binds to $\mathrm{Au}(111)$ via two functional groups: the imino nitrogen atom of the imidazole,so that the electronrich imidazole ring comes in interaction with the surface, and the two oxygen atoms of deprotonated carboxylic groups (see Section 3.2.1.2 for more details). When adsorbed from solution, both His and the investigated His-containing peptides also bind to gold via the carboxylate and the imino groups (see Figure 108). XPS and NEXAFS data suggest that the peptide chain is oriented rather 
perpendicular to the surface. Note that the geometry of the adsorbed molecule is also influenced by the sequence within the peptide (see differences in Gly-His-Gly and Gly-Gly-His, Figure 108)[362].

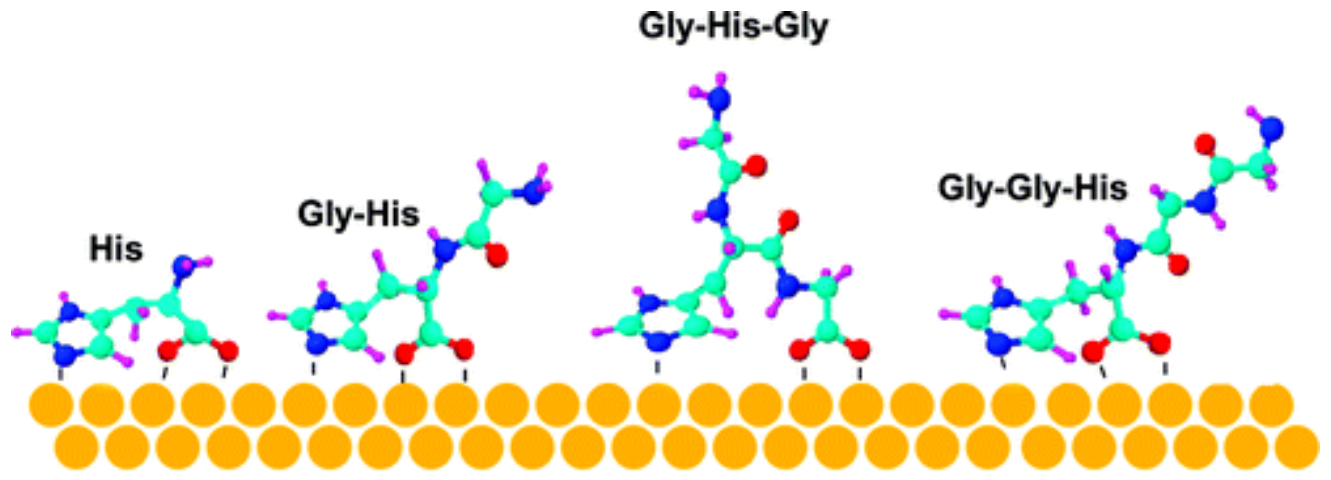

\section{$\mathrm{Au}(111)$}

Figure 108. Model of His amino acid or His-containing peptides adsorbed on Au(111). Taken with permission from ref. [362].

Interestingly, the adsorption behaviour of His, Gly-His, Gly-His-Gly and Gly-Gly-His on Au(111) was also the focus of a theoretical studybased on classical molecular dynamics simulations[363], which essentially agrees with the above mentioned experimental work. As expected, all the four kinds of molecules adsorb from the liquid phase (including up to 2000 water molecules in the model) onto the $\mathrm{Au}(111)$ surface after a $3 \mathrm{~ns}$ MD run. Many statistical properties, like the interaction energy of adsorption, were analysed after the systems reached the equilibrium. It was confirmedat the molecular level that His and His-derived peptides adsorb on Au(111) via the imino nitrogen in the imidazole (IM) ring and the carboxylic acid group (See Figure 109). Among the four molecules, the dipeptide Gly-His adsorbs more rapidly on $\mathrm{Au}(111)$; this information is not trivial since the sequence of residues in a peptide can significantly influence theadsorption rate and the adsorption geometry of the individual amino acid constituents.

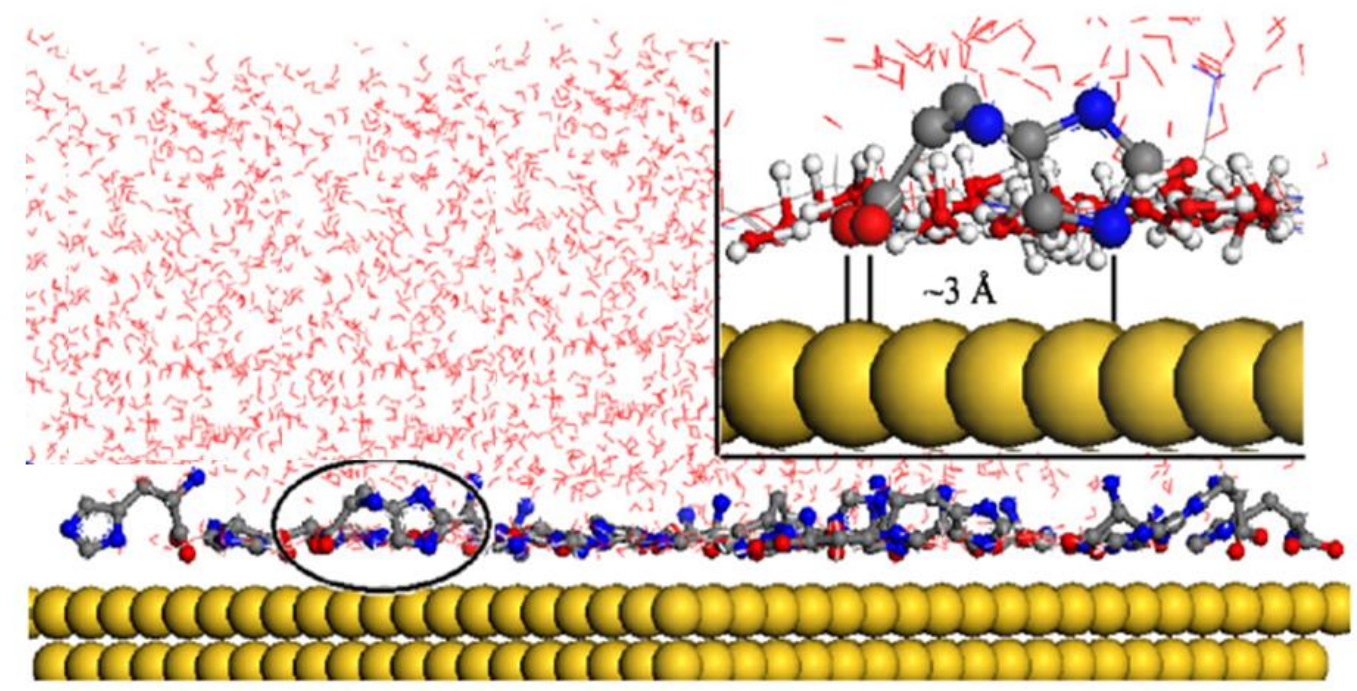


Figure 109. The final snapshot of adsorption structures of histidine molecules on $\mathrm{Au}(111)$. The upper-right panel shows the area highlighted by the dark circled area on an enlarged scale. Taken with permission from ref.[363].

Although highly valuable, such studies are seldom. Indeed, focussing on studies concerning the adsorption of small peptides on metal surfaces using modelling techniques and, in particular, quantum chemical calculations such as DFT, one can say that this topic is still in its child shoes. A simple literature search with key words such as: "metal", "peptide" and "modelling" results in only one hundredth of papers. If one types "DFT"in the search, one obtains less than ten. Nevertheless, we have to mention that some works exist on classical MD, in which the parameterization of the metal polarization of the substrate is the main challenge but also scanning of the enormous number of possible configurations of peptides is a hard goal. In section 5.4.1, we will summarize the results of a few representative examples of studies on peptides. When possible, we will discuss of dry/UHV conditions, according to the main scope of this review; however, an example of a solvated system is also included for the sake of completeness.

More than just giving a catalogue of results, implying various peptides on several types of surfaces, this short survey shows at least two important tendencies:

- Like most amino acids, peptides may bind to metallic surfaces (copper or gold) via the oxygen atoms of deprotonated carboxyl groups.

- Behaviours are different on gold or on copper in the sense that deprotonation of $\mathrm{COOH}$ groups is not total on gold thus favouring the interaction of the peptides via another functional groups ( $\mathrm{SH}$ or, in some cases, nitrogen).

- Adsorption from aqueous solution, vs in UHV conditions, changes the ionic form of the peptide drastically; it is for instance possible to reduce the fraction of deprotonated carboxyl groups. We underline the important possibility to dictate the charge of the adsorbed peptide by adsorbing it from solutions at selected $\mathrm{pH}$ values.

\subsection{Peptides on more complex surfaces}

\subsubsection{Adsorption of peptides on oxides from the gas phase}

Modelling adsorption of peptides from the gas phase is extremely rare, especially on oxides. Zhang et al. modelled RGD (see Figure 110a for structure) adsorption on $\mathrm{TiO}_{2}$ anatase and rutile surfaces in vacuum[367]. The two topmost layers were annealed to generate atomistic disorder on $\mathrm{TiO}_{2}$ surfaces, thus to simulate real surfaces. RGD adsorbed best on (100) anatase, with formation of a Ti-OCO bond involving the Asp branch (Figure $110 \mathrm{~b}$ and c). Comparison of surfaces is uneasy because the surfaces reconstruction and amorphisation is not described.

a)

b) c) 

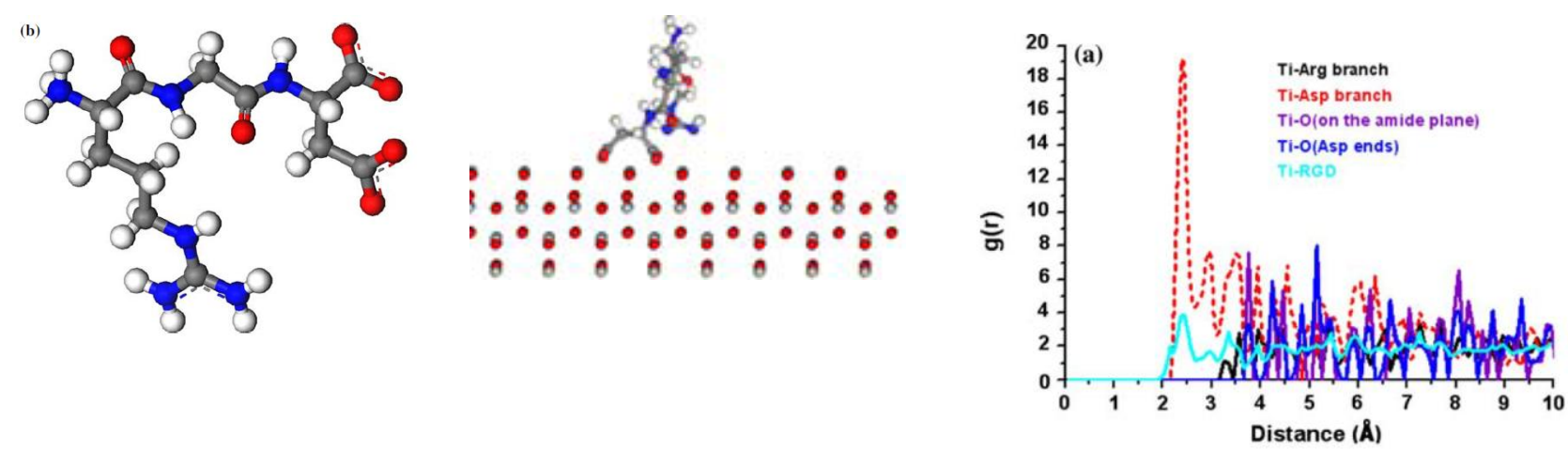

Figure 110. (a) Balland- Stick model of RGD. Colour code: carbon, grey; hydrogen, white; nitrogen, blue; and oxygen, red. (b) Snapshot of the RGD peptide configuration: RGD peptide on the anatase (001) surface after MD simulation. (c) The pair correlation functions: RGD-anatase (001). The blue peaks indicate the surface Ti atoms-RGD distance; the red peak corresponds to the Ti-Asp distance; the Asp residue is clearly responsible of the interaction with the surface. Taken with permissionfrom ref. [367].

Muir et al. [366] performed ab initio MD at 300K of RGD adsorption on rutile $\mathrm{TiO}_{2}(110)$ surface in vacuum; RGD adsorbs best through the aspartic acid carboxy groups and not through the arginine side groups, in agreement with previous results[367]. Dissociative bridging and molecular adsorption are similar in energy, with molecular adsorption being slightly preferred. The carboxy group on the end of the aspartic acid side chain is the preferred bonding site due to the additional carbonyl-surface bond it enables.

\subsection{Adsorption of longer peptides}

\subsubsection{Adsorption of longer peptides on metal surfaces}

A hint into the behaviour of a rather long peptide (TAT= CGISYGRKKRRQRRR) on Au (111) brings valuable information. The gold surface was characterized by XPS and PM-IRRAS after adsorption of TAT following three different procedures, namely from solution, by microcontact printing, or by dip pen nanolithography [372]. In the frame of this report,we underline the following interesting result: whatever the method, TAT is adsorbed in similar amounts on the gold surface. Moreover, a careful analysis of the PM-IRRAS data shows: i) shifts of the $v_{\mathrm{C}-\mathrm{H}}$ stretch and changes in the ratio of the $v_{\mathrm{CH}}$ intensities corresponding to $\mathrm{CH}_{3}$ and $\mathrm{CH}_{2}$ groups, indicating that all TAT layers form disordered chains in a liquid-like structure; ii) various positions of the amide I band in the $1620-1680 \mathrm{~cm}^{-1}$ region. These changes were ascribed to different $\mathrm{H}$-bond interactions with the C-O carbonyl, related to the flexibility of the peptide and to the way it accommodates on the surface depending on deposition conditions; this may explain why the recognition properties of the peptide may change with the conditions of immobilization on a surface [371;372].

In the work by Verde et al. [380] all-atom classical MD simulations following adsorption of goldbinding (3GBP and 6GBP)and non-gold-binding (6NGBP) peptides on gold surfaces modelled with dispersive interactions are presented (See Figure 111Erreur ! Source du renvoi introuvable.). The 
adsorption properties were examined considering both the type of the amino acids and the mobility of the peptides. Within the limitations of the approach, results indicate that when the peptides are solvated, adsorption requires both configurational changes and local flexibility of the individual amino acids. This is achieved when peptides consist mostly of random coils or when their secondary structural motifs (helices, sheets) are short and connected by flexible hinges. In the absence of solvent, only affinity for the surface is required: mobility is not important. In combination, these results suggest that the barrier to adsorption present by the displacement of water molecules requires conformational sampling enabled through mobility. The limitations of this approach include the lack of structural data with which to obtain solution structures of the peptides, the period of time over which behaviour is monitored and the nature of interactions with the gold surface.

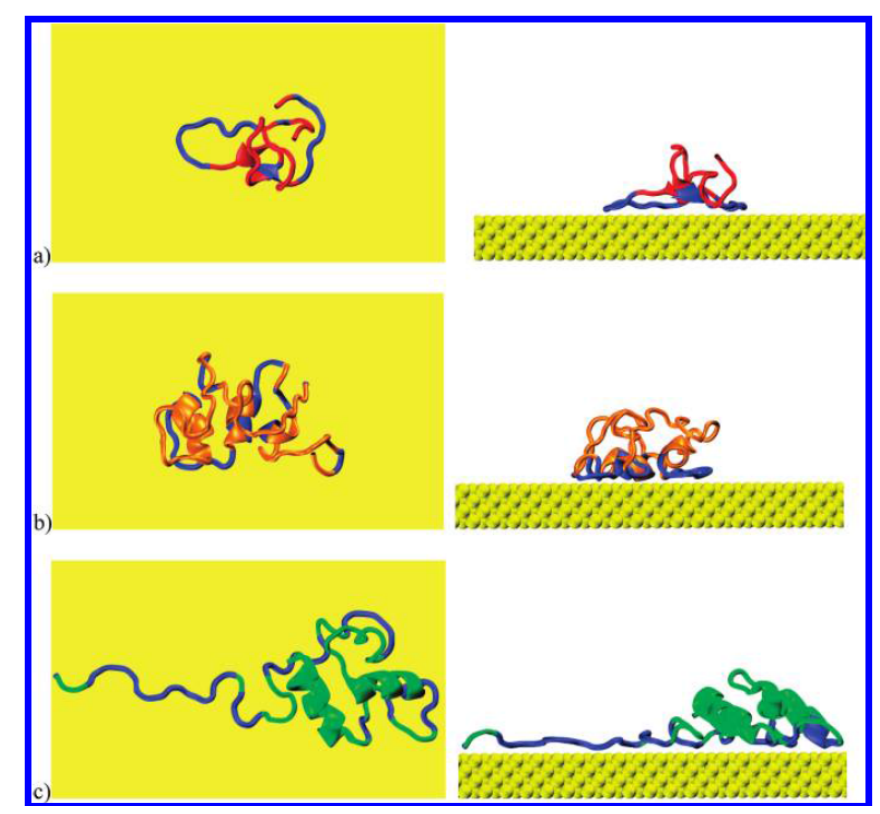

Figure 111 Final adsorbed conformation of a) 3GBP, b) 6GBP, and c) 6NGBP on Au(001) from MD simulation in vacuum at $310 \mathrm{~K}$. The tube represents the backbone of the protein. Adsorbed sections are shown in blue. The texture of the gold surface (in yellow) is omitted from the top views for clarity. Taken with permission from ref. [380].

The MRKDV peptide (Amino Acid letter code can be found in the acronym list), structurally associated with an immune-modulatory protein, as well as model peptides ADEDRDA and LGRGISL with common amino acid residues were studied using surface enhanced Raman scattering (SERS) supported by quantum chemical computations[377]. The MRKDV SERS spectrum is dominated by signals coming from the guanidinium moiety of the argininelino acid (R); guanidinium is the intrinsic probe which drives the orientation of the peptide on the metal surface. LGRGISL interacts with the metal surface through the guanidinium group and other amino acid residues; a single structural conformation of the peptide on the surface was proposed. ADEDRDA interacts with the metal surface through various amino acid residues, also including the guanidinium moiety; at least two different structural conformations seem to coexist on the surface. Theoretical calculations performed by using extended Hückel theory/6-31G* methods for a model of arginine interacting with a silver cluster support the structural conformation observed experimentally. 


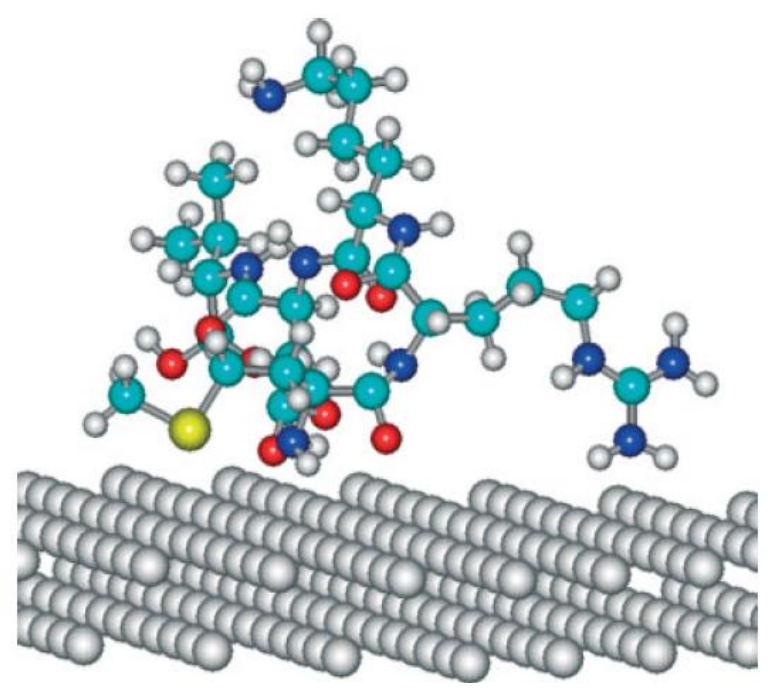

Figure 112. Predicted molecular model for the MRKDV-Ag surface interaction. Taken with permission from ref. [377].

As final (and most recent) example, we cite a work [378] which is mainly based on SERS, but which is supported by theoretical data obtained from molecular mechanics and Extended Hückel Theory (EHT) calculations. MM was employed to optimize the peptide-Au system keeping constant the metallic layer geometry. EHT was used to calculate the wave function of CLPFFD as a fully ionized isolated system (isoelectric point structure) and interacting with the metal surface. The authors investigate the interactions of CLPFFD with Au nano structures of $10 \mathrm{~nm}$ average diameter. The SERS data suggest that phenylalanine displays its aromatic ring coplanar to the surface, which was confirmed by theoretical calculations. The vibrational frequency of the breathing mode band at $1007 \mathrm{~cm}^{-1}$ and the $\mu \mathrm{CS}$ band at $623 \mathrm{~cm}^{-1}$ of the thiol group were calculated since they were expected to be close to the surface and thus be enhanced in agreement with the SERS spectra. This study is also an interesting example of the use of quantum chemical based calculations other than DFT.

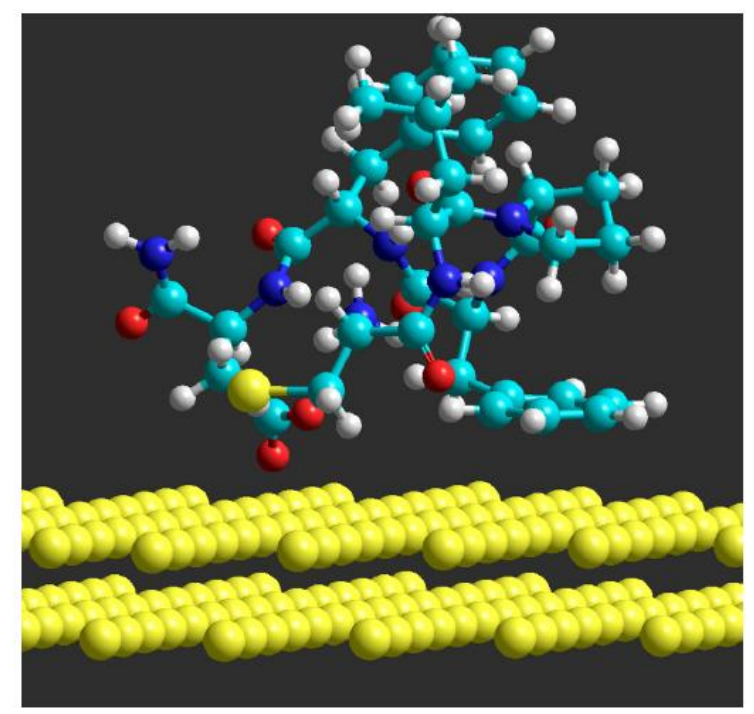


Figure 113 Molecular model for the CLPFFD-Au layer interaction. Figure taken with permission of ref.[378].

\subsubsection{Adsorption of longer peptides on $\mathrm{TiO}_{2}$ and other oxides}

Since titanium holds a particular place for its use for medical implants, several studies, either experimental or theoretical, aimed at unravelling the behaviour of peptides on such a surface. In particular, knowing the nature of amino acid residues that are involved in the binding of peptides to $\mathrm{Ti}$ is of crucial importance. In this context, by testing more than 40 phages, Sano and Shiba identified a hexapeptide motif, RKLPDA, as playing a role in phage adsorption on $\mathrm{TiO}_{2}$ [348]. They then constructed a group of 11 peptides, just by substituting alanine to one of the amino acid fragments, and tested the affinity of these new peptides to titanium oxide. The conclusion is that only N-terminal hexapeptide RKLPDA is sufficient to bind to Ti. They propose a model (see Figure 114 ) in which the terminal arginine $\mathrm{NH}_{2}$ groups interact with $\mathrm{Ti}^{-} \mathrm{O}^{-}$(Lewis base site), and the $\mathrm{COO}^{-}$ of aspartic acid interacts with $\mathrm{Ti}-\mathrm{OH}_{2}{ }^{+}$(lewis acid site). In fact, this model relies on an amphoteric behaviour of a titanium oxide surface. They also demonstrated that increasing the surface $\mathrm{OH}$ concentration increases the peptide binding [348].

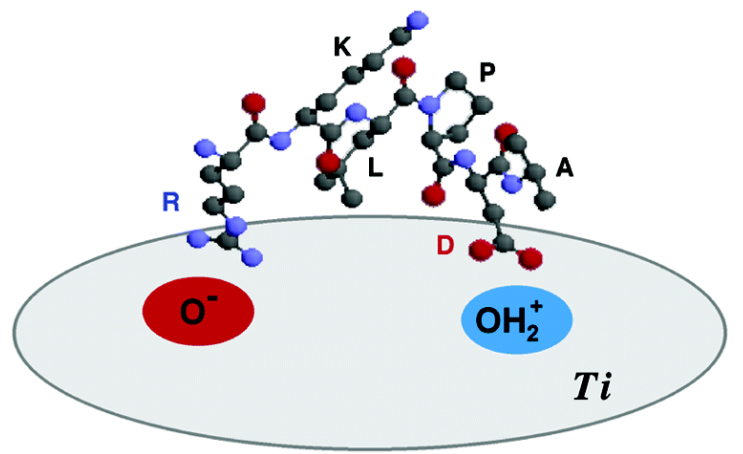

Figure 114. Model of the RKLPDA peptide interaction with a titanium oxide surface. Taken with permission from ref.[348].

Type $\mathrm{I}$ collagen adhesion on $\mathrm{TiO}_{2}$ and on $\alpha-\mathrm{Al}_{2} \mathrm{O}_{3}$ perfect and rough surfaces was investigated[375]. The initial configuration of collagen was a triple-helix collagen molecule, composed of 1299 atoms. Collagen adsorbed more on $\mathrm{TiO}_{2}$ than $\mathrm{Al}_{2} \mathrm{O}_{3}$, and more on rough surfaces. Details on residues responsible of adsorption are not given, neither details on the perfect and rough surfaces. 


\section{Conclusions}

In this manuscript we have presented an extended overview of the interaction of amino acids and small peptides with metal and oxide surfaces. The systems of interest are characterised at the molecular level and with experimental and theoretical methods, which are state-of-the-art in surface science. We have tackled this topic starting from the simplest cases, i.e. one amino acid at a flat metal surface, and moved then to the description of systems with increasing degree of complexity, up to some hints on peptides on an oxide substrate.Both for the most fundamental and for the more complex systems,we reported studies of adsorption from the gas phase as well as analyses performed in UHV after adsorption at the interface with water.

Each kind of system presented its peculiarities and preferred analysis techniques. In spite of that, some common points which characterise the bio-interface can be retrieved. E.g., the adsorption configuration of the amino acids is always quite similar, presenting - with a -ew exceptions - 2 or 3 anchoring points in correspondence of the amino and carboxylic/carboxylate functional groups. This is indicative that the adsorption mechanism is mainly ruled by the AA functionalities, while the radical tail is less involved. On the contrary, the neutral, anionic or zwitterionic nature of the adsorbed species depends on the nature of the substrate, since it is influenced by the charge transfer between the latter and the molecules. Indeed, anionic adsorption is quite common on reactive metals and dry oxides, while zwitterions are mainly observed on inert metals, hydroxylated oxides and in the presence of co-adsorbed water. Amino acids can also compete with water for adsorption on the oxide surfaces. Therefore biomolecules present a high degree of versatility. When the moleculesurface interaction is weak, complex and exotic self-assembly structures ruled by intermolecular interactions form, as clearly shown in the case of Ag surfaces. On the contrary, if the formation of covalent bonds prevails, as for reactive metals and oxides, the hard matter dictates the formation of a pseudo-epitaxial (bio)-organic layer on the inorganic substrate. In some cases, especially on metals, self-assembled monolayers are still observed, but their structure and geometry are mainly determined by the molecule-substrate interaction.

In spite of the well-defined limits we have imposed to the present work, i.e. the choice of describing only metal and oxide systems interacting with molecules generally adsorbed from the gas phase, analysed in vacuum and at the molecular level, we believe we have given quite a wide overview of the fundamental interactions of small protein constituents with surfaces. We also hope that we have provided a significant example of the validity of the bottom-up approach, i.e of the way in which the investigation of simplified systems can provide insight into the nature of more complex phenomena. In this respect, it has been shown in the manuscript that the anchoring mode of AA and peptides at surfaces does not change much if the adlayer is sublimated in UHV or if it is deposited from the liquid phase and then dried (with all other parameters unchanged, in particular the chemical form of the biomolecule). Thus, gas phase experiments (and calculations in vacuum) help to understand the binding sites under the condition that long range electrostatic on charged surfaces/biomolecules does not prevail in the adsorption.

Another important message we wanted to convey concerns complementarity of the experimental and theoretical mtehods and the added value that the integrated use of these tools gives to the 
characterisation of the adlayers. Among all possible systems investigated in surface science, this benefitis particularly evident for the bio-interfaces, because of their intrinsic complexity and of the typical size of the unit cells (up to a few hundreds of atoms). The match between experimental and theoretical methods has shown to be impressive in a number of cases, demonstrating that DFT associated to experimental characterization tools can be used to retrieve most of the stable states in a potential energy landscape, a capacity that was not straightforward. As already stated, this agreement between $0 \mathrm{~K}$ calculations and experiments was achieved for small size molecules in gas phase, thus systems for which entropic and kinetics contributions could be neglected.

Concerning modelling, most of the works cited here used first principles methods (usually DFT).We underline the significant improvement given by the recent inclusion of different descriptions for the dispersion forces in the periodic DFT packages. This upgrade allows describing all the forces of a system at a reliable level of approximation and, for simple systems (some hundreds of atoms in the unit cell) it has already become the standard level of calculation (including the case of BOMD for time scales of a few some ps). Some works have been performed with classical force fields, that may be reactive, and thus allow bonds formation. Note that the use of classical force fields allowed the authors to perform exhaustive studies of all AAs adsorption on a given surface. Some works using a multi-scale approach combining energetics and kinetics data were also presented.

The great challenge to face in a bottom up approach is to characterise the AA or peptides adlayer at the solid/liquid interface. In fact, in situ experimental methods that give local molecular information have been developed, as SFG/SHG, operando XPS, ATR, IRAS, as well as in liquid AFM, but the interpretation of the results is complicated by the higher complexity of the system and by the presence of coadsorbates from the environment (e.g. of water molecules from the solution). Therefore the degree of accuracy in the characterisation of the system is still not comparable with the one achieved at the solid/vacuum interface. On the theoretical side, we predict that molecular dynamics (both abinitio including dispersion forces and reactive force fields including bond breaking and making) will be more and more applied.For DFT the challenge will be the need of optimised and adapted algorithms for the computing power available and still increasing and, on the methodological side, the development of more accurate exchange correlation functionals.Lighter atomistic methods as semi empirical electronic approaches (DFTB) and classical the reactive force field will certainly gain an increasing role and come nearer to real size systems (including solvent) and real time scales.

The perspectives of the amino acids, peptides and proteins interface with surfaces are immense and we have given here only a partial view limited to the information which can be retrieved with surface science methods. We are however convinced that, in this particular case, as always in material science, the understanding of basic, simplified cases, opens the way to the interpretation of more complex systems, for which other approaches will be required. We also believe that the effort in the route towards complexity must be pursued and it is well justified by the many applications of these systems, which have been discussed in the Introduction. 


\section{References}

[1] A.E. Nel, L. Maedler, D. Velegol, T. Xia, E.M.V. Hoek, P. Somasundaran, F. Klaessig, V. Castranova, M. Thompson, Nature Materials 8 (2009) 543-557.

[2] Y. Pennec, W. Auwaerter, A. Schiffrin, A. Weber-Bargioni, A. Riemann, J.V. Barth, Nature Nanotechnology 2 (2007) 99-103.

[3] S. Mann, Nature Materials 8 (2009) 781-792.

[4] J.C. Love, L.A. Estroff, J.K. Kriebel, R.G. Nuzzo, G.M. Whitesides, Chemical Reviews 105 (2005) 11031169.

[5] C. Compere, M.N. Bellon-Fontaine, P. Bertrand, D. Costa, P. Marcus, C. Poleunis, C.M. Pradier, B. Rondot, M.G. Walls, Biofouling 17 (2001) 129-145.

[6] K. Kanazawa, A. Taninaka, H. Huang, M. Nishimura, S. Yoshida, O. Takeuchi, H. Shigekawa, Chemical Communications 47 (2011) 11312-11314.

[7] A. Rimola, D. Costa, M. Sodupe, J.-F. Lambert, P. Ugliengo, Chemical Reviews 113 (2013) 4216.

[8] P. Ardalan, G. Dupont, C.B. Musgrave, Journal of Physical Chemistry C 115 (2011) 7477-7486.

[9] L.E. Bain, S.A. Jewett, A.H. Mukund, S.M. Bedair, T.M. Paskova, A. Ivanisevic, Acs Applied Materials \& Interfaces 5 (2013) 7236-7243.

[10] S.J. Jung, D.H. Kim, S.M. Jeon, S. Hong, S. Kim, Journal of Physical Chemistry C 115 (2011) 46364641.

[11] J.S. Kachian, S.J. Jung, S. Kim, S.F. Bent, Surface Science 605 (2011) 760-769.

[12] Y. Kumada, T. Ootsuka, M. Asada, S. Yoshizuka, M. Chiyama, M. Sakane, H.M.D. Fida, K. Sawada, K. Okumura, M. Kishimoto, Journal of Biotechnology 184 (2014) 103-110.

[13] F.R. Rahsepar, L. Zhang, H. Farkhondeh, K.T. Leung, Journal of the American Chemical Society 136 (2014) 16909-16918.

[14] J. Katoch, S.N. Kim, Z.F. Kuang, B.L. Farmer, R.R. Nalk, S.A. Tatulian, M. Ishigami, Nano Letters 12 (2012) 2342-2346.

[15] R.B. Pandey, Z. Kuang, B.L. Farmer, S.S. Kim, R.R. Naik, Soft Matter 8 (2012) 9101-9109.

[16] H. Vovusha, S. Sanyal, B. Sanyal, Journal of Physical Chemistry Letters 4 (2013) 3710-3718.

[17] T. Roman, W.A. Dino, H. Nakanishi, H. Kasai, Thin Solid Films 509 (2006) 218-222.

[18] B. Akdim, R. Pachter, S.S. Kim, R.R. Naik, T.R. Walsh, S. Trohalaki, G. Hong, Z. Kuang, B.L. Farmer, Acs Applied Materials \& Interfaces 5 (2013) 7470-7477.

[19] M.-h. Wang, Y.-n. Guo, Q. Wang, X.-s.-y. Zhang, J.-j. Huang, X. Lu, K.-f. Wang, H.-p. Zhang, Y. Leng, Chemical Physics Letters 599 (2014) 86-91.

[20] R. de la Rica, H. Matsui, Chemical Society Reviews 39 (2010) 3499-3509.

[21] M. Sarikaya, C. Tamerler, A.K.Y. Jen, K. Schulten, F. Baneyx, Nature Materials 2 (2003) 577-585.

[22] B.J. Pepe-Mooney, R. Fairman, Current Opinion in Structural Biology 19 (2009) 483-494.

[23] M. Schuler, D. Trentin, M. Textor, S.G.P. Tosatti, Nanomedicine 1 (2006) 449-463.

[24] J.F. Lambert, Origins of Life and Evolution of Biospheres 38 (2008) 211-242.

[25] M. Mrksich, G.M. Whitesides, Annual Review of Biophysics and Biomolecular Structure 25 (1996)

55-78.

[26] I.A. Shkrob, S.D. Chemerisov, Journal of Physical Chemistry C 113 (2009) 17138-17150.

[27] C.J. vanOss, Current Opinion in Colloid \& Interface Science 2 (1997) 503-512.

[28] V. Mailander, K. Landfester, Biomacromolecules 10 (2009) 2379-2400.

[29] J.E. Raynor, J.R. Capadona, D.M. Collard, T.A. Petrie, A.J. Garcia, Biointerphases 4 (2009) FA3-FA16.

[30] S.M. Barlow, R. Raval, Current Opinion in Colloid \& Interface Science 13 (2008) 65-73.

[31] C. Gervais, J. Hulliger, Crystal Growth \& Design 7 (2007) 1925-1935.

[32] A. Kuhnle, Current Opinion in Colloid \& Interface Science 14 (2009) 157-168.

[33] K.C. Timberlake, Chemistry, Haper-Collins, New York, 1992.

[34] J.K.a.K.-H. Röhm, Color atlas of biochemistry, Thieme, Stuttgard, 1996.

[35] S. Blanco, A. Lesarri, J.C. Lopez, J.L. Alonso, Journal of the American Chemical Society 126 (2004) 11675-11683.

[36] A. Lesarri, S. Mata, J.C. Lopez, J.L. Alonso, Review of Scientific Instruments 74 (2003) 4799-4804.

[37] S. Iannotta, T. Toccoli, Journal of Polymer Science Part B-Polymer Physics 41 (2003) 2501-2521. 
[38] P. Lofgren, A. Krozer, J. Lausmaa, B. Kasemo, Surface Science 370 (1997) 277-292.

[39] F. Gao, Z. Li, Y. Wang, L. Burkholder, W.T. Tysoe, Surface Science 601 (2007) 3276-3288.

[40] F. Gao, Z. Li, Y. Wang, L. Burkholder, W.T. Tysoe, Journal of Physical Chemistry C 111 (2007) 9981-

9991.

[41] F. Gao, Y. Wang, L. Burkholder, W.T. Tysoe, Surface Science 601 (2007) 3579-3588.

[42] S.M. Barlow, K.J. Kitching, S. Haq, N.V. Richardson, Surface Science 401 (1998) 322-335.

[43] T. Eralp, A. Shavorskiy, Z.V. Zheleva, G. Held, N. Kalashnyk, Y. Ning, T.R. Linderoth, Langmuir 26 (2010) 18841-18851.

[44] S. Fischer, A.C. Papageorgiou, M. Marschall, J. Reichert, K. Diller, F. Klappenberger, F. Allegretti, A. Nefedov, C. Woell, J.V. Barth, Journal of Physical Chemistry C 116 (2012) 20356-20362.

[45] J. Reichert, et al., Acs Nano 4 (2010) 1218-1226.

[46] V. Feyer, O. Plekan, T. Skala, V. Chab, V. Matolin, K.C. Prince, Journal of Physical Chemistry B 112 (2008) 13655-13660.

[47] A. Vallee, V. Humblot, C. Methivier, C.-M. Pradier, Surface Science 602 (2008) 2256-2263.

[48] E.M. Marti, C. Methivier, C.M. Pradier, Langmuir 20 (2004) 10223-10230.

[49] T.E. Jones, C.J. Baddeley, Surface Science 513 (2002) 453-467.

[50] J. Williams, S. Haq, R. Raval, Surface Science 368 (1996) 303-309.

[51] T.E. Jones, C.J. Baddeley, A. Gerbi, L. Savio, M. Rocca, L. Vattuone, Langmuir 21 (2005) 9468-9475.

[52] V. De Renzi, L. Lavagnino, V. Corradini, R. Biagi, M. Canepa, U. del Pennino, Journal of Physical Chemistry C 112 (2008) 14439-14445.

[53] M. Smerieri, L. Vattuone, M. Rocca, L. Savio, Langmuir 29 (2013) 6867-6875.

[54] M. Smerieri, L. Vattuone, T. Kravchuk, D. Costa, L. Savio, Langmuir 27 (2011) 2393-2404.

[55] E. Ozensoy, D.C. Meier, D.W. Goodman, Journal of Physical Chemistry B 106 (2002) 9367-9371.

[56] T. Buffeteau, B. Desbat, J.M. Turlet, Applied Spectroscopy 45 (1991) 380-389.

[57] A. Vallée, V. Humblot, C. Méthivier, C.M. Pradier, J. Phys. Chem. C

113(21) (2009) 9336-9344.

[58] R. Wiesendanger, Scanning Probe Microscopy and Spectroscopy: Methods and Applications, Cambridge University Press, 1994.

[59] M. Smerieri, L. Vattuone, D. Costa, F. Tielens, L. Savio, Langmuir 26 (2010) 7208-7215.

[60] V. Humblot, F. Tielens, N.B. Luque, H. Hampartsoumian, C. Methivier, C.M. Pradier, Langmuir 30 (2014) 203-212.

[61] F. Tielens, V. Humblot, C.-M. Pradier, Surface Science 602 (2008) 1032-1039.

[62] A. Schiffrin, et al., Journal of Physical Chemistry C 113 (2009) 12101-12108.

[63] A. Schiffrin, A. Riemann, W. Auwarter, Y. Pennec, A. Weber-Bargioni, D. Cvetko, A. Cossaro, A. Morgante, J.V. Barth, Proceedings of the National Academy of Sciences of the United States of America 104 (2007) 5279-5284.

[64] T. Qiu, M.A. Barteau, Journal of Colloid and Interface Science 303 (2006) 229-235.

[65] T. Greber, Z. Sljivancanin, R. Schillinger, J. Wider, B. Hammer, Physical Review Letters 96 (2006).

[66] J.H. Kang, R.L. Toomes, M. Polcik, M. Kittel, J.T. Hoeft, V. Efstathiou, D.P. Woodruff, A.M. Bradshaw, Journal of Chemical Physics 118 (2003) 6059-6071.

[67] J. Hasselstrom, O. Karis, M. Weinelt, N. Wassdahl, A. Nilsson, M. Nyberg, L.G.M. Pettersson, M.G. Samant, J. Stohr, Surface Science 407 (1998) 221-236.

[68] V. Humblot, F. Tielens, N.B. Luque, H. Hampartsoumian, C. Methivier, C.-M. Pradier, Langmuir 30 (2014) 203-212.

[69] S.M. Barlow, S. Louafi, D. Le Roux, J. Williams, C. Muryn, S. Haq, R. Raval, Langmuir 20 (2004) 71717176.

[70] L. Barbosa, P. Sautet, Journal of the American Chemical Society 123 (2001) 6639-6648.

[71] A. Ulman, Chemical Reviews 96 (1996) 1533-1554.

[72] Y.N. Xia, J.A. Rogers, K.E. Paul, G.M. Whitesides, Chemical Reviews 99 (1999) 1823-1848.

[73] C. Joachim, J.K. Gimzewski, A. Aviram, Nature 408 (2000) 541-548.

[74] C. Vericat, M.E. Vela, G. Benitez, P. Carro, R.C. Salvarezza, Chemical Society Reviews 39 (2010) 18051834. 
[75] Q.J. Chi, J.D. Zhang, J.U. Nielsen, E.P. Friis, I. Chorkendorff, G.W. Canters, J.E.T. Andersen, J. Ulstrup, Journal of the American Chemical Society 122 (2000) 4047-4055.

[76] R. Rinaldi, A. Biasco, G. Maruccio, R. Cingolani, D. Alliata, L. Andolfi, P. Facci, F. De Rienzo, R. Di Felice, E. Molinari, Advanced Materials 14 (2002) 1453-+.

[77] J.H. Harding, D.M. Duffy, M.L. Sushko, P.M. Rodger, D. Quigley, J.A. Elliott, Chemical Reviews 108 (2008) 4823-4854.

[78] J. Antony, B. Brueske, S. Grimme, Physical Chemistry Chemical Physics 11 (2009) 8440-8447.

[79] J. Antony, S. Grimme, Physical Chemistry Chemical Physics 8 (2006) 5287-5293.

[80] J. Antony, S. Grimme, Journal of Computational Chemistry 33 (2012) 1730-1739.

[81] J.G. Brandenburg, S. Grimme, Topics in current chemistry 345 (2014) 1-23.

[82] S. Ehrlich, J. Moellmann, S. Grimme, Accounts of Chemical Research 46 (2013) 916-926.

[83] S. Grimme, Abstracts of Papers of the American Chemical Society 245 (2013).

[84] S. Grimme, M. Steinmetz, Physical Chemistry Chemical Physics 15 (2013) 16031-16042.

[85] J. Moellmann, S. Grimme, Journal of Physical Chemistry C 118 (2014) 7615-7621.

[86] T. Risthaus, S. Grimme, Journal of Chemical Theory and Computation 9 (2013) 1580-1591.

[87] J. Klimes, D.R. Bowler, A. Michaelides, Physical Review B 83 (2011).

[88] M. Dion, H. Rydberg, E. Schroder, D.C. Langreth, B.I. Lundqvist, Physical Review Letters 92 (2004).

[89] H.M. Aktulga, S.A. Pandit, A.C.T. van Duin, A.Y. Grama, Siam Journal on Scientific Computing 34

(2012) C1-C23.

[90] C. Li, S. Monti, V. Carravetta, Journal of Physical Chemistry C 116 (2012) 18318-18326.

[91] S. Monti, C. Li, V. Carravetta, Journal of Physical Chemistry C 117 (2013) 5221-5228.

[92] S. Monti, A.C.T. van Duin, S.-Y. Kim, V. Barone, Journal of Physical Chemistry C 116 (2012) 5141-

5150.

[93] P.J. Bond, J. Holyoake, A. Ivetac, S. Khalid, M.S.P. Sansom, Journal of Structural Biology 157 (2007) 593-605.

[94] R.H. Holm, P. Kennopohl, E.I. Solomon, Chem. Rev. 96 (1996) 2239-2314.

[95] X.Y. Zhao, Z. Gai, R.G. Zhao, W.S. Yang, T. Sakurai, Surface Science 424 (1999) L347-L351.

[96] V. Efstathiou, D.P. Woodruff, Surface Science 531 (2003) 304-318.

[97] S.P. Ge, X.Y. Zhao, Z. Gai, R.G. Zhao, W.S. Yang, Chinese Physics 11 (2002) 839-845.

[98] X.Y. Zhao, H. Wang, R.G. Zhao, W.S. Yang, Materials Science \& Engineering C-Biomimetic and Supramolecular Systems 16 (2001) 41-50.

[99] K. Kanazawa, A. Taninaka, O. Takeuchi, H. Shigekawa, Physical Review Letters 99 (2007).

[100] J.N. James, D.S. Sholl, Journal of Molecular Catalysis a-Chemical 281 (2008) 44-48.

[101] Q. Chen, D.J. Frankel, N.V. Richardson, Surface Science 497 (2002) 37-46.

[102] J. Hasselstrom, O. Karis, M. Nyberg, L.G.M. Pettersson, M. Weinelt, N. Wassdahl, A. Nilsson, Journal of Physical Chemistry B 104 (2000) 11480-11483.

[103] M. Nyberg, J. Hasselstrom, O. Karis, N. Wassdahl, M. Weinelt, A. Nilsson, L.G.M. Pettersson, Journal of Chemical Physics 112 (2000) 5420-5427.

[104] R.B. Rankin, D.S. Sholl, Surface Science 548 (2004) 301-308.

[105] S.M. Barlow, S. Louafi, D. Le Roux, J. Williams, C. Muryn, S. Haq, R. Raval, Surface Science 590

(2005) 243-263.

[106] M. Sacchi, S.J. Jenkins, Physical Chemistry Chemical Physics 16 (2014) 6101-6107.

[107] R.B. Rankin, D.S. Sholl, Surface Science 574 (2005) L1-L8.

[108] D.I. Sayago, M. Polcik, G. Nisbet, C.L.A. Lamont, D.P. Woodruff, Surface Science 590 (2005) 76-87.

[109] D.A. Duncan, M.K. Bradley, W. Unterberger, D. Kreikemeyer-Lorenzo, T.J. Lerotholi, J. Robinson,

D.P. Woodruff, Journal of Physical Chemistry C 116 (2012) 9985-9995.

[110] X.Y. Zhao, H. Yan, R.G. Zhao, W.S. Yang, Langmuir 19 (2003) 809-813.

[111] R.L. Toomes, J.H. Kang, D.P. Woodruff, M. Polcik, M. Kittel, J.T. Hoeft, Surface Science 522 (2003)

L9-L14.

[112] T. Eralp, A. Shavorskiy, Z.V. Zheleva, V.R. Dhanak, G. Held, Langmuir 26 (2010) 10918-10923.

[113] X.Y. Zhao, H. Yan, R.G. Zhao, W.S. Yang, Langmuir 18 (2002) 3910-3915.

[114] X.Y. Zhao, J. Rodriguez, Surface Science 600 (2006) 2113-2121. 
[115] L. Thomsen, M.T. Wharmby, D.P. Riley, G. Held, M.J. Gladys, Surface Science 603 (2009) 1253-1261.

[116] A. Kuhnle, L.M. Molina, T.R. Linderoth, B. Hammer, F. Besenbacher, Physical Review Letters 93 (2004).

[117] A. Kuhnle, T.R. Linderoth, F. Besenbacher, Topics in Catalysis 54 (2011) 1384-1391.

[118] M. Canepa, L. Lavagnino, L. Pasquali, R. Moroni, F. Bisio, V. De Renzi, S. Terreni, L. Mattera, Journal of Physics-Condensed Matter 21 (2009).

[119] M. Canepa, P. Pelori, L. Lavagnino, F. Bisio, R. Moroni, S. Terreni, L. Mattera, Nuclear Instruments \& Methods in Physics Research Section B-Beam Interactions with Materials and Atoms 256 (2007) 324-327.

[120] A. Cossaro, S. Terreni, O. Cavalleri, M. Prato, D. Cvetko, A. Morgante, L. Floreano, M. Canepa, Langmuir 22 (2006) 11193-11198.

[121] G. Gonella, S. Terreni, D. Cvetko, A. Cossaro, L. Mattera, O. Cavalleri, R. Rolandi, A. Morgante, L. Floreano, M. Canepa, Journal of Physical Chemistry B 109 (2005) 18003-18009.

[122] B. Hoeffling, F. Ortmann, K. Hannewald, F. Bechstedt, Physica Status Solidi C: Current Topics in Solid State Physics, Vol 7, No 27 (2010) 149-152.

[123] B. Hoeffling, F. Ortmann, K. Hannewald, F. Bechstedt, Physical Review B 81 (2010).

[124] A. Kuhnle, T.R. Linderoth, F. Besenbacher, Journal of the American Chemical Society 128 (2006) 1076-1077.

[125] C.R.L. Chapman, E.C.M. Ting, A. Kereszti, I. Paci, Journal of Physical Chemistry C 117 (2013) 1942619435.

[126] L. Buimaga-larinca, C. Morari, Journal of Physical Chemistry C 117 (2013) 20351-20360.

[127] A. Kuhnle, T.R. Linderoth, M. Schunack, F. Besenbacher, Langmuir 22 (2006) 2156-2160.

[128] R. Di Felice, A. Selloni, E. Molinari, Journal of Physical Chemistry B 107 (2003) 1151-1156.

[129] R. Di Felice, A. Selloni, Journal of Chemical Physics 120 (2004) 4906-4914.

[130] E. Mateo-Marti, C. Rogero, C. Gonzalez, J.M. Sobrado, P.L. de Andres, J.A. Martin-Gago, Langmuir 26 (2010) 4113-4118.

[131] E. Mateo-Marti, C. Briones, C. Rogero, C. Gomez-Navarro, C. Methivier, C.M. Pradier, J.A. MartinGago, Chemical Physics 352 (2008) 11-18.

[132] A. Kuehnle, Current Opinion in Colloid \& Interface Science 14 (2009) 157-168.

[133] A. Kuhnle, T.R. Linderoth, F. Besenbacher, Journal of the American Chemical Society 125 (2003)

14680-14681.

[134] J.L.C. Fajin, J.R.B. Gomes, M. Cordeiro, Langmuir 29 (2013) 8856-8864.

[135] R. Schillinger, Z. Sljivancanin, B. Hammer, T. Greber, Physical Review Letters 98 (2007).

[136] H. Iwai, C. Egawa, Langmuir 26 (2010) 2294-2300.

[137] H. Iwai, M. Tobisawa, A. Emori, C. Egawa, Surface Science 574 (2005) 214-218.

[138] C. Egawa, H. Iwai, M. Kabutoya, S. Oki, Surface Science 532 (2003) 233-236.

[139] X.Y. Zhao, R.G. Zhao, W.S. Yang, Surface Science 442 (1999) L995-L1000.

[140] S. Haq, A. Massey, N. Moslemzadeh, A. Robin, S.M. Barlow, R. Raval, Langmuir 23 (2007) 10694-

10700.

[141] G. Jones, L.B. Jones, F. Thibault-Starzyk, E.A. Seddon, R. Raval, S.J. Jenkins, G. Held, Surface Science 600 (2006) 1924-1935.

[142] R.B. Rankin, D.S. Sholl, Journal of Physical Chemistry B 109 (2005) 16764-16773.

[143] M.J. Gladys, A.V. Stevens, N.R. Scott, G. Jones, D. Batchelor, G. Held, Journal of Physical Chemistry C 111 (2007) 8331-8336.

[144] T. Eralp, A. Cornish, A. Shavorskiy, G. Held, Topics in Catalysis 54 (2011) 1414-1428.

[145] L. Thomsen, A. Tadich, D.P. Riley, B.C.C. Cowie, M.J. Gladys, Journal of Physical Chemistry C 116

(2012) 9472-9480.

[146] R.B. Rankin, D.S. Sholl, Journal of Chemical Physics 124 (2006).

[147] E.M. Marti, C. Methivier, P. Dubot, C.M. Pradier, Journal of Physical Chemistry B 107 (2003) 1078510792.

[148] E.M. Marti, A. Quash, C. Methivier, P. Dubot, C.M. Pradier, Colloids and Surfaces a-Physicochemical and Engineering Aspects 249 (2004) 85-89. 
[149] Y. Zubavichus, M. Zharnikov, Y.J. Yang, O. Fuchs, C. Heske, E. Umbach, G. Tzvetkov, F.P. Netzer, M. Grunze, Journal of Physical Chemistry B 109 (2005) 884-891.

[150] X.Y. Zhao, Journal of the American Chemical Society 122 (2000) 12584-12585.

[151] V. Humblot, C. Methivier, C.M. Pradier, Langmuir 22 (2006) 3089-3096.

[152] T. Eralp, A. Shavorskiy, G. Held, Surface Science 605 (2011) 468-472.

[153] K.E. Wilson, H.A. Fruechtl, F. Grillo, C.J. Baddeley, Chemical Communications 47 (2011) 10365-

10367.

[154] C. Méthivier, V. Humblot, C.-M. Pradier, Surface Science 632 (2015) 88-92.

[155] A. Naitabdi, V. Humblot, Applied Physics Letters 97 (2010).

[156] M. Forster, M.S. Dyer, M. Persson, R. Raval, Topics in Catalysis 54 (2011) 13-19.

[157] M. Forster, M.S. Dyer, M. Persson, R. Raval, Journal of the American Chemical Society 131 (2009) 10173-10181.

[158] E.M. Marti, S.M. Barlow, S. Haq, R. Raval, Surface Science 501 (2002) 191-202.

[159] R.T. Seljamaee-Green, G.J. Simpson, F. Grillo, J. Greenwood, S.M. Francis, R. Schaub, P. Lacovig, C.J. Baddeley, Langmuir 30 (2014) 3495-3501.

[160] H. Iwai, A. Emori, C. Egawa, Surface Science 600 (2006) 1670-1673.

[161] X.Y. Zhao, R.G. Zhao, W.S. Yang, Langmuir 18 (2002) 433-438.

[162] X.Y. Zhao, H. Yan, X.W. Tu, R.G. Zhao, W.S. Yang, Langmuir 19 (2003) 5542-5545.

[163] N.B. Luque, P. Velez, K. Potting, E. Santos, Langmuir 28 (2012) 8084-8099.

[164] E. Santos, L.B. Avalle, R. Scurtu, H. Jones, Chemical Physics 342 (2007) 236-244.

[165] E. Santos, L. Avalle, K. Potting, P. Velez, H. Jones, Electrochimica Acta 53 (2008) 6807-6817.

[166] N.B. Luque, E. Santos, Langmuir 28 (2012) 11472-11480.

[167] S. Blankenburg, W.G. Schmidt, Journal of Physics-Condensed Matter 21 (2009).

[168] T.E. Jones, C.J. Baddeley, Langmuir 22 (2006) 148-152.

[169] T.E. Jones, A.E. Rekatas, C.J. Baddeley, Journal of Physical Chemistry C 111 (2007) 5500-5505.

[170] T.E. Jones, M.E. Urquhart, C.J. Baddeley, Surface Science 587 (2005) 69-77.

[171] A.G. Trant, T.E. Jones, T.C.Q. Noakes, P. Bailey, C.J. Baddeley, Surface Science 604 (2010) 300-307.

[172] S. Stewart, P.M. Fredericks, Spectrochimica Acta Part a-Molecular and Biomolecular Spectroscopy 55 (1999) 1641-1660.

[173] Z.V. Zheleva, T. Eralp, G. Held, Journal of Physical Chemistry C 116 (2012) 618-625.

[174] V. Humblot, C. Méthivier, R. Raval, C.-M. Pradier, Surface Science 601 (2007) 4189-4194.

[175] S.M. Barlow, R. Raval, Surface Science Reports 50 (2003) 201-341.

[176] N.A. Booth, D.P. Woodruff, O. Schaff, T. Giessel, R. Lindsay, P. Baumgartel, A.M. Bradshaw, Surface Science 397 (1998) 258-269.

[177] K.-H. Ernst, in: M. CregoCalama, D.N. Reinboudt (Eds.), Supramolecular Chirality, 2006, pp. 209-252.

[178] R. Raval, Chemical Society Reviews 38 (2009) 707-721.

[179] L.L.B. Atanasoska, J.C.; Somorjai, G.A., Surface Science 72 (1978).

[180] K. Mae, Y. Morikawa, Surface Science 553 (2004) L63-L67.

[181] X.Y. Zhao, R.G. Zhao, W.S. Yang, Langmuir 16 (2000) 9812-9818.

[182] A. Ihs, B. Liedberg, Journal of Colloid and Interface Science 144 (1991) 282-292.

[183] W. Qian, S. Krimm, Biopolymers 32 (1992) 1503-1518.

[184] R.G. Cooks, D.X. Zhang, K.J. Koch, F.C. Gozzo, M.N. Eberlin, Analytical Chemistry 73 (2001) 3646-

3655.

[185] S.C. Nanita, R.G. Cooks, Angewandte Chemie-International Edition 45 (2006) 554-569.

[186] C.A. Schalley, P. Weis, International Journal of Mass Spectrometry 221 (2002) 9-19.

[187] G. Held, M.J. Gladys, Topics in Catalysis 48 (2008) 128-136.

[188] A. Kuhnle, T.R. Linderoth, B. Hammer, F. Besenbacher, Nature 415 (2002) 891-893.

[189] V. Humblot, C. Methivier, R. Raval, C.-M. Pradier, Surface Science 601 (2007) 4189-4194.

[190] Q. Chen, C.W. Lee, D.J. Frankel, N.V. Richardson, Physchemcomm 2 (1999).

[191] G.S. Prasad, M. Vijayan, Acta Crystallographica Section B-Structural Science 47 (1991) 927-935.

[192] I.D. Reva, S.G. Stepanian, A.M. Plokhotnichenko, E.D. Radchenko, G.G. Sheina, Y.P. Blagoi, Journal of Molecular Structure 318 (1994) 1-13. 
[193] A.M. Sapse, L. Mallahlevy, S.B. Daniels, B.W. Erickson, Journal of the American Chemical Society 109 (1987) 3526-3529.

[194] M. Besson, B. Blanc, M. Champelet, P. Gallezot, K. Nasar, C. Pinel, Journal of Catalysis 170 (1997) 254-264.

[195] A. Tungler, G. Fogassy, Journal of Molecular Catalysis a-Chemical 173 (2001) 231-247.

[196] M. Forster, M.S. Dyer, M. Persson, R. Raval, Angewandte Chemie-International Edition 49 (2010) 2344-2348.

[197] M. Forster, M.S. Dyer, M. Persson, R. Raval, Journal of the American Chemical Society 133 (2011) 15992-16000.

[198] G. Xue, J. Dong, Y. Sun, Langmuir 10 (1994) 1477-1481.

[199] R.H. Carlson, T.L. Brown, Inorganic Chemistry 5 (1966) 268-277.

[200] R.J. Sundberg, R.B. Martin, Chem. Rev. 74 (1974) 471-517.

[201] H. Wang, X.Y. Zhao, R.G. Zhao, W.S. Yang, Chinese Physics Letters 18 (2001) 445-448.

[202] T.E. Jones, C.J. Baddeley, Surface Science 519 (2002) 237-249.

[203] W.H. Pirkle;, J. Finn;, in: M. J.D. (Ed.), Asymmetric Synthesis, Academic Press, New York, 1993, p. 87.

[204] R.B. Rankin, D.S. Sholl, Langmuir 22 (2006) 8096-8103.

[205] C.F. McFadden, P.S. Cremer, A.J. Gellman, Langmuir 12 (1996) 2483-2487.

[206] J.D. Horvath, A. Koritnik, P. Kamakoti, D.S. Sholl, A.J. Gellman, Journal of the American Chemical

Society 126 (2004) 14988-14994.

[207] S.J. Jenkins, S.J. Pratt, Surface Science Reports 62 (2007) 373-429.

[208] T. Eralp, A. levins, A. Shavorskiy, S.J. Jenkins, G. Held, Journal of the American Chemical Society 134

(2012) 9615-9621.

[209] S. Brown, Nature Biotechnology 15 (1997) 269-272.

[210] S. Brown, M. Sarikaya, E. Johnson, Journal of Molecular Biology 299 (2000) 725-735.

[211] M. Sarikaya, C. Tamerler, D.T. Schwartz, F.O. Baneyx, Annual Review of Materials Research 34 (2004) 373-408.

[212] S.M. Wetterer, D.J. Lavrich, T. Cummings, S.L. Bernasek, G. Scoles, Journal of Physical Chemistry B 102 (1998) 9266-9275.

[213] D. Syomin, B.E. Koel, Surface Science 498 (2002) 61-73.

[214] D. Syomin, B.E. Koel, Surface Science 498 (2002) 53-60.

[215] F. Tielens, E. Santos, Journal of Physical Chemistry C 114 (2010) 9444-9452.

[216] F. Tielens, V. Humblot, C.M. Pradier, M. Calatayud, F. Illas, Langmuir 25 (2009) 9980-9985.

[217] F. Tielens, D. Costa, V. Humblot, C.M. Pradier, Journal of Physical Chemistry C 112 (2008) 182-190.

[218] N.B. Luque, E. Santos, J. Andres, F. Tielens, Langmuir 27 (2011) 14514-14521.

[219] M. Weinhold, S. Soubatch, R. Temirov, M. Rohlfing, B. Jastorff, F.S. Tautz, C. Doose, Journal of Physical Chemistry B 110 (2006) 23756-23769.

[220] L. Buimaga-larinca, N. Tosa, Processes in Isotopes and Molecules (Pim 2011) 1425 (2012) 22-25.

[221] L. Buimaga-larinca, C. Morari, N. Tosa, European Biophysics Journal with Biophysics Letters 40

(2011) 103-103.

[222] K. Uvdal, P. Bodo, B. Liedberg, Journal of Colloid and Interface Science 149 (1992) 162-173.

[223] A.S. Dakkouri, D.M. Kolb, R. EdelsteinShima, D. Mandler, Langmuir 12 (1996) 2849-2852.

[224] G. Dodero, L. De Michieli, O. Cavalleri, R. Rolandi, L. Oliveri, A. Dacca, R. Parodi, Colloids and

Surfaces a-Physicochemical and Engineering Aspects 175 (2000) 121-128.

[225] J.D. Zhang, Q.J. Chi, J.U. Nielsen, E.P. Friis, J.E.T. Andersen, J. Ulstrup, Langmuir 16 (2000) 7229-

7237.

[226] Q.M. Xu, L.J. Wan, C. Wang, C.L. Bai, Z.Y. Wang, T. Nozawa, Langmuir 17 (2001) 6203-6206.

[227] J.D. Zhang, Q.J. Chi, R.R. Nazmutdinov, T.T. Zinkicheva, M.D. Bronshtein, Langmuir 25 (2009) 2232-

2240.

[228] Y.Q. Xue, S. Datta, M.A. Ratner, Journal of Chemical Physics 115 (2001) 4292-4299.

[229] H. Hakkinen, Nature Chemistry 4 (2012) 443-455.

[230] O. Cavalleri, G. Gonella, S. Terreni, M. Vignolo, L. Floreano, A. Morgante, M. Canepa, R. Rolandi, Physical Chemistry Chemical Physics 6 (2004) 4042-4046. 
[231] T. Shin, K.N. Kim, C.W. Lee, S.K. Shin, H. Kang, Journal of Physical Chemistry B 107 (2003) 1167411681.

[232] J.L.C.C. Fajín, M. N. D. S.; Gomes, J. R. B. , J. Phys. Chem. C 111 (2007) 17311-17321.

[233] M. Mavrikakis, P. Stoltze, J.K. Norskov, Catalysis Letters 64 (2000) 101-106.

[234] L.H. Easson, E. Stedman, The Biochemical journal 27 (1933) 1257-66.

[235] D.V. A., Chirality 9 (1997) 99-102.

[236] T. Gritsch, D. Coulman, R.J. Behm, G. Ertl, Physical Review Letters 63 (1989) 1086-1089.

[237] P. Fery, W. Moritz, D. Wolf, Physical Review B 38 (1988) 7275-7286.

[238] Q.M. Guo, F. Yin, R.E. Palmer, Small 1 (2005) 76-79.

[239] V. Arima, et al., Small 4 (2008) 497-506.

[240] V. Humblot, C.-M. Pradier, Journal of Physical Chemistry Letters 4 (2013) 1816-1820.

[241] V. Feyer, O. Plekan, S. Ptasinska, M. lakhnenko, N. Tsud, K.C. Prince, Journal of Physical Chemistry C 116 (2012) 22960-22966.

[242] V. Feyer, O. Plekan, N. Tsud, V. Chab, V. Matolin, K.C. Prince, Langmuir 26 (2010) 8606-8613.

[243] E. Apen, A.P. Hitchcock, J.L. Gland, Journal of Physical Chemistry 97 (1993) 6859-6866.

[244] B. Liedberg, C. Carlsson, I. Lundstrom, Journal of Colloid and Interface Science 120 (1987) 64-75.

[245] T. Deckert-Gaudig, E. Rauls, V. Deckert, Journal of Physical Chemistry C 114 (2010) 7412-7420.

[246] A.P. Sandoval, J.M. Orts, A. Rodes, J.M. Feliu, Journal of Physical Chemistry C 115 (2011) 16439-

16450 .

[247] A.P. Sandoval, J.M. Orts, A. Rodes, J.M. Feliu, Electrochimica Acta 89 (2013) 72-83.

[248] M. Hoefling, F. Iori, S. Corni, K.E. Gottschalk, Chemphyschem 11 (2010) 1763-1767.

[249] L.M. Ghiringhelli, L. Delle Site, Journal of the American Chemical Society 130 (2008) 2634-2638.

[250] F.J. Ramirez, J.T.L. Navarrete, Spectrochimica Acta Part a-Molecular and Biomolecular Spectroscopy 51 (1995) 293-302.

[251] J.T.L. Navarrete, L. Bencivenni, F. Ramondo, V. Hernandez, F.J. Ramirez, Theochem-Journal of Molecular Structure 330 (1995) 261-266.

[252] A. Vallee, V. Humblot, C. Methivier, C.-M. Pradier, Journal of Physical Chemistry C 113 (2009) 9336-

9344.

[253] I. Tranca, M. Smerieri, L. Savio, L. Vattuone, D. Costa, F. Tielens, Langmuir 29 (2013) 7876-7884.

[254] P. Fenter, P. Eisenberger, J. Li, N. Camillone, S. Bernasek, G. Scoles, T.A. Ramanarayanan, K.S. Liang, Langmuir 7 (1991) 2013-2016.

[255] Q. Xu, H. Ma, H. Yip, A.K.Y. Jen, Nanotechnology 19 (2008).

[256] M.L. Merrick, W.W. Luo, K.A. Fichthorn, Progress in Surface Science 72 (2003) 117-134.

[257] F. Silly, M. Pivetta, M. Ternes, F. Patthey, J.P. Pelz, W.D. Schneider, Physical Review Letters 92 (2004).

[258] E.C.H. Sykes, P. Han, S.A. Kandel, K.F. Kelly, G.S. McCarty, P.S. Weiss, Accounts of Chemical Research 36 (2003) 945-953.

[259] B. Dalhus, C.H. Gorbitz, Journal of Molecular Structure-Theochem 675 (2004) 47-52.

[260] B. Dalhus, C.H. Gorbitz, Acta Crystallographica Section C-Crystal Structure Communications 55

(1999) 1105-1112.

[261] M.O. Lorenzo, S. Haq, T. Bertrams, P. Murray, R. Raval, C.J. Baddeley, Journal of Physical Chemistry B 103 (1999) 10661-10669.

[262] D.S. Costa, M.; I. Tranca, I.; Savio, L. ; Vattuone, L ; Tielens, F., Journal of Physical Chemistry C 118 (2014) 29874-29879.

[263] Y. Izumi, Advances in Catalysis 32 (1983) 215-271.

[264] G. Webb, P.B. Wells, Catalysis Today 12 (1992) 319-337.

[265] A. Tai, T. Harada, Tailored Metal Catalysts, D. Reidel Publishing Company, 1986.

[266] A.K. Tungler, M.; Mathe, T.; Toth, G.; Fogassy, E.; Petro,, P.W.H.F. J., Catal. Today 5 (1989) 159-171.

[267] A.T. Tungler, T.; Máthé, T.; Petró, J., J. Mol. Catal. 70 (1991) L5-L8.

[268] A.T. Tungler, T.; Máthé, T.; Petró, J. , J. Mol. Catal. 67 (1991) 277-282.

[269] G.K.c. Tóth, A.; Tarnai, T.; Tungler, A., Tetrahedron: Asymmetry 4 (1993) 331-338.

[270] A.I.W. McIntosh, D. J.; Burton, J. W.; Lambert, R. M., J. Am. Chem. Soc. 128 (2006) 7329-7334. 
[271] L. Lavagnino, R. Moroni, F. Bisio, S. Terreni, L. Mattera, M. Canepa, Nuclear Instruments \& Methods in Physics Research Section B-Beam Interactions with Materials and Atoms 269 (2011) 932-935.

[272] K.H. Ernst, K. Christmann, Surface Science 224 (1989) 277-310.

[273] M.B. Mahapatra, L.; Bai, Y; Garvey, M.; Boscoboinik,J.A.; Hirschmugl, C.; Tysoe, W.T., J. Phys. Chem. C 118 (2014) 6856-6865.

[274] J.N.S. James, D. S., J. Mol. Catal. A: Chem. 281 (2008) 44-48.

[275] J.W. Han, J.N. James, D.S. Sholl, Journal of Chemical Physics 135 (2011).

[276] J.N. James, J.W. Han, D.S. Sholl, Applied Surface Science 301 (2014) 199-207.

[277] V. Humblot, S. Haq, C. Muryn, W.A. Hofer, R. Raval, Journal of the American Chemical Society 124

(2002) 503-510.

[278] V. Humblot, M.O. Lorenzo, C.J. Baddeley, S. Haq, R. Raval, Journal of the American Chemical Society 126 (2004) 6460-6469.

[279] A.G. Trant, T.E. Jones, C.J. Baddeley, Journal of Physical Chemistry C 111 (2007) 10534-10540.

[280] C.C. Wagner, E.J. Baran, Journal of Raman Spectroscopy 35 (2004) 395-400.

[281] D.D. Chambliss, R.J. Wilson, S. Chiang, Physical Review Letters 66 (1991) 1721-1724.

[282] P. Schravendijk, L.M. Ghiringhelli, L. Delle Site, N.F.A. van der Vegt, Journal of Physical Chemistry C 111 (2007) 2631-2642.

[283] L.M. Ghiringhelli, P. Schravendijk, L. Delle Site, Physical Review B 74 (2006).

[284] K.E. Wilson, C.J. Baddeley, Surface Science 629 (2014) 102-107.

[285] P.M. D. Costa, in: P.M.a.J.C. Taylor (Ed.), Modeling Corrosion, Wiley and sons, New York, 2014.

[286] G. Bereket, A. Yurt, Corrosion Science 43 (2001) 1179-1195.

[287] A. Yurt, G. Bereket, C. Ogretir, Journal of Molecular Structure-Theochem 725 (2005) 215-221.

[288] G. Martra, C. Deiana, Y. Sakhno, I. Barberis, M. Fabbiani, M. Pazzi, M. Vincenti, Angewandte Chemie-International Edition 53 (2014) 4671-4674.

[289] L. Ojamae, C. Aulin, H. Pedersen, P.O. Kall, Journal of Colloid and Interface Science 296 (2006) 71-

78.

[290] T.J. Lerotholi, E.A. Kroeger, M.J. Knight, W. Unterberger, K. Hogan, D.C. Jackson, C.L.A. Lamont, D.P. Woodruff, Surface Science 603 (2009) 2305-2311.

[291] E. Soria, I. Colera, E. Roman, E.M. Williams, J.L. de Segovia, Surface Science 451 (2000) 188-196.

[292] E. Soria, E. Roman, E.M. Williams, J.L. de Segovia, Surface Science 433 (1999) 543-548.

[293] R. Tonner, Chemphyschem 11 (2010) 1053-1061.

[294] S. Monti, A.C.T. van Duin, S.-Y. Kim, V. Barone, The Journal of Physical Chemistry C 116 (2012) 5141-

5150.

[295] J.N. Wilson, R.M. Dowler, H. Idriss, Surface Science 605 (2011) 206-213.

[296] D. Szieberth, A.M. Ferrari, X. Dong, Physical Chemistry Chemical Physics 12 (2010) 11033-11040.

[297] J. Lausmaa, P. Löfgren, B. Kasemo, Journal of Biomedical Materials Research 44 (1999) 227-242.

[298] S. Irrera, D. Costa, P. Mucus, Journal of Molecular Structure-Theochem 903 (2009) 49-58.

[299] A. Domínguez, N.H. Moreira, G. Dolgonos, T. Frauenheim, A.L. da Rosa, The Journal of Physical Chemistry C 115 (2011) 6491-6495.

[300] G. Tzvetkov, G. Koller, Y. Zubavichus, O. Fuchs, M.B. Casu, C. Heske, E. Umbach, M. Grunze, M.G. Ramsey, F.P. Netzer, Langmuir 20 (2004) 10551-10559.

[301] C. Arrouvel, B. Diawara, D. Costa, P. Marcus, Journal of Physical Chemistry C 111 (2007) 1816418173.

[302] J.F. Lambert, L. Stievano, I. Lopes, M. Gharsallah, L.Y. Piao, Planetary and Space Science 57 (2009) 460-467.

[303] D. Costa, P.-A. Garrain, M. Baaden, JOURNAL OF BIOMEDICAL MATERIALS RESEARCH Part A 101A (2013) 1210-1222.

[304] D. Costa, P.A. Garrain, B. Diawara, P. Marcus, Langmuir 27 (2011) 2747-2760.

[305] P.A. Garrain, D. Costa, P. Marcus, Journal of Physical Chemistry C 115 (2011) 719-727.

[306] G.J. Fleming, H. Idriss, Langmuir 20 (2004) 7540-7546.

[307] G.J. Fleming, K. Adib, J.A. Rodriguez, M.A. Barteau, H. Idriss, Surface Science 601 (2007) 5726-5731. 
[308] G.J. Fleming, K. Adib, J.A. Rodriguez, M.A. Barteau, J.M. White, H. Idriss, Surface Science 602 (2008) 2029-2038.

[309] E. Ataman, C. Isvoranu, J. Knudsen, K. Schulte, J.N. Andersen, J. Schnadt, Surface Science 605 (2011) 179-186.

[310] J.M.R. Muir, H. Idriss, Surface Science 617 (2013) 60-67.

[311] Y.N. Guo, X. Lu, H.P. Zhang, J. Weng, F. Watari, Y. Leng, Journal of Physical Chemistry C 115 (2011) 18572-18581.

[312] H.-p. Zhang, X. Lu, X.-g. Luo, X.-y. Lin, Y.-f. Zhou, Physica E: Low-dimensional Systems and Nanostructures 61 (2014) 83-89.

[313] R. Koch, A. Lipton, S. Filipek, V. Renugopalakrishnan, Journal of Molecular Modeling 17 (2011) 1467-

1472.

[314] A.G. Thomas, W.R. Flavell, C.P. Chatwin, A.R. Kumarasinghe, S.M. Rayner, P.F. Kirkham, D. Tsoutsou, T.K. Johal, S. Patel, Surface Science 601 (2007) 3828-3832.

[315] V. Carravetta, S. Monti, Journal of Physical Chemistry B 110 (2006) 6160-6169.

[316] Y.K. Gao, F. Traeger, O. Shekhah, H. Idriss, C. Woll, Journal of Colloid and Interface Science 338

(2009) 16-21.

[317] Y.K. Gao, F. Traeger, K. Kotsis, V. Staemmler, Physical Chemistry Chemical Physics 13 (2011) 1070910718.

[318] S. Koeppen, O. Bronkalla, W. Langel, Journal of Physical Chemistry C 112 (2008) 13600-13606.

[319] E.G. Brandt, A. Lyubartsev, Biophysical Journal 106 (2014) 208a.

[320] G. Nawrocki, M. Cieplak, Physical Chemistry Chemical Physics 15 (2013) 13628-13636.

[321] U. Diebold, Surf. Sci. Rep. 48 (2003) 53-229.

[322] R.L.a.G.T. C. L. Pang, Chem. Soc. Rev. 37 (2008) 2328-2353.

[323] M.A. Henderson, The Journal of Physical Chemistry B 101 (1997) 221-229.

[324] Z. Chang, G. Thornton, Surface Science 462 (2000) 68-76.

[325] A. Gutiérrez-Sosa, P. Martínez-Escolano, H. Raza, R. Lindsay, P.L. Wincott, G. Thornton, Surface Science 471 (2001) 163-169.

[326] H. Uetsuka, M.A. Henderson, A. Sasahara, H. Onishi, The Journal of Physical Chemistry B 108 (2004) 13706-13710.

[327] S. Crook, H. Dhariwal, G. Thornton, Surface Science 382 (1997) 19-25.

[328] S.E. Cabaniss, I.F. McVey, Spectrochimica Acta Part a-Molecular and Biomolecular Spectroscopy 51 (1995) 2385-2395.

[329] S.A. Chambers, M.A. Henderson, Y.J. Kim, S. Thevuthasan, Surface Review and Letters 5 (1998) 381385.

[330] B.E. Hayden, A. King, M.A. Newton, The Journal of Physical Chemistry B 103 (1998) 203-208.

[331] F.P. Rotzinger, J.M. Kesselman-Truttmann, S.J. Hug, V. Shklover, M. Grätzel, The Journal of Physical Chemistry B 108 (2004) 5004-5017.

[332] T. Berger, J.M. Delgado, T. Lana-Villarreal, A. Rodes, R. Gomez, Langmuir 24 (2008) 14035-14041.

[333] J. Ahdjoudj, C. Minot, Catalysis Letters 46 (1997) 83-91.

[334] P. Kackell, K. Terakura, Applied Surface Science 166 (2000) 370-375.

[335] P. Kackell, K. Terakura, Surface Science 461 (2000) 191-198.

[336] M. Nilsing, P. Persson, S. Lunell, L. Ojamae, Journal of Physical Chemistry C 111 (2007) 12116-

12123.

[337] A. Mattsson, S. Hu, K. Hermansson, L. Österlund, The Journal of Chemical Physics 140 (2014) -.

[338] A. Vittadini, M. Casarin, A. Selloni, Theoretical Chemistry Accounts 117 (2007) 663-671.

[339] A.G. Thomas, K.L. Syres, Chemical Society Reviews 41 (2012) 4207-4217.

[340] E. Farfan-Arribas, R.J. Madix, Journal of Physical Chemistry B 107 (2003) 3225-3233.

[341] R.W.G. Wyckoff, in: N.Y.W. Interscience (Ed.), Crystal structure, 1966.

[342] K.D. Dobson, A.J. McQuillan, Spectrochimica Acta Part a-Molecular and Biomolecular Spectroscopy 56 (2000) 557-565.

[343] E. Greiner, K. Kumar, M. Sumit, A. Giuffre, W. Zhao, J. Pedersen, N. Sahai, Geochimica Et Cosmochimica Acta 133 (2014) 142-155. 
[344] E.R. Jon I. Mujika, Jose M. Mercero, Fernando Ruipérez, Dominique Costa, Jesus M. Ugalde and Xabier Lopez, Computational and Structural Biotechnology Journal 09 (2014) e201403002. .

[345] D.R. Costa, T.; Mercuri, F.; Pacchioni, G.; Marcus, P., Advanced Materials Interfaces 1 (2014) 1300072.

[346] M. Matzapetakis, V. Pecoraro, L., J. Am. Chem. Soc. 127 (2005) 18229-18233

[347] K. Go, R. Parthasarathy, Biopolymers 36 (1995) 607.

[348] K. Sano, K. Shiba, J. Am. Chem. Soc. 125 (2003) 14234-14235.

[349] S. Brown, Nature Biotechnol. 15 (1997) 269-272.

[350] I. Stensgaard, Surf. Sci. 545 (2003) L747-L752.

[351] V. Feyer, O. Plekan, N. Tsud, V. Lyamayev, V.r. Cháb, V.r. Matolín, K.C. Prince, V. Carravetta, J. Phys.

Chem. C 114 (2010) 10922-10931.

[352] S. Monti, V. Carravetta, C. Li, H. Agren, Journal of Physical Chemistry C 118 (2014) 3610-3619.

[353] S.M. Barlow, S. Haq, R. Raval, Langmuir 17 (2001) 3292-3300.

[354] W. Qian, J. Bandekar, S. Krimm, Biopolymers 31 (1991) 193.

[355] C. Methivier, V. Lebec, J. Landoulsi, C.M. Pradier, J. Phys. Chem. C 115 (2011) 4041-4046.

[356] V. Humblot, A. Vallee, A. Naitabdi, F. Tielens, C.M. Pradier, Journal of the American Chemical Society 134 (2012) 6579-6583.

[357] V. Humblot, A. Naitabdi, A. Vallée, C.M. Pradier, J. Am. Chem. Soc. 134 (2012) 6579-6583.

[358] M. Lingenfelder, G. Tomba, G. Costantini, L. Colombi Ciacchi, A. De Vita, K. Kern, Angew. Chem., Int. Ed. 46 (2007) 4492-4495.

[359] Y. Wang, T. Classen, G. Costantini, K. Kern, J. Am. Chem. Soc. 129 (2007) 15742-15743.

[360] A. Vallée, V. Humblot, C. Méthivier, C.M. Pradier, Surf. Sci.

602 (2008) 2256-2263.

[361] Z.A. Tehrani, Z. Jamshidi, M.J. Javan, A. Fattahi, Journal of Physical Chemistry A 116 (2012) 4338-

4347.

[362] V. Feyer, O. Plekan, N. Tsud, V.r. Cháb, V.r. Matolín, K.C. Prince, Langmuir 26 (2010) 8606-8613.

[363] Z. Xu, S.-L. Yuan, H. Yn, C.-B. Liu, Coll. and Surf. A: Physicochem. Eng. Aspects 380 (2011) 135-142.

[364] A. Vallée, V. Humblot, C. Méthivier, C.M. Pradier Surf. Interf. Anal., 40 (2008) 395-399.

[365] M. Bieri, T. Bürgi, Langmuir 21 (2005) 1354-1363.

[366] D.C. J.M.R. Muir, H. Idriss, Surface Science

624 (2014) 8-14.

[367] H.P. Zhang, X. Lu, L.M. Fang, J. Weng, N. Huang, Y. Leng, Journal of Materials Science-Materials in Medicine 19 (2008) 3437-3441.

[368] D.-P. Song, M.-J. Chen, Y.-C. Liang, Q.-S. Bai, J.-X. Chen, X.-F. Zheng, Acta Biomaterialia 6 (2010) 684694.

[369] H.P. Zhang, X. Lu, Y. Leng, F. Watari, J. Weng, B. Feng, S.X. Qu, Journal of Biomedical Materials Research Part A 96A (2011) 466-476.

[370] B. Morozzo della Rocca, C.T.a. C.I. Smith b, A. Desideri a, P. Weightman, Surface Science 604 (2010) 2170-2176.

[371] Y. Cho, A. Ivanisevic, J. Phys. Chem. B 108 (2004) 15223-15228.

[372] Y. Cho, A. Ivanisevic, J. Phys. Chem. B 109 (2005) 6225-6232.

[373] G. Iucci, C. Battocchio, D. M., R. Gambaretto, C. Di Bello, F. Borgatti, V. Carravetta, S. Monti, G. Polzonetti, Surface Science 601 (2007) 3843-3849.

[374] E. Podstawka-Proniewicz, Y. Ozaki, Y. Kim, Y. Xu, L.M. Proniewicz, Appl. Surf. Sci. 257 (2011) 8246-

8252.

[375] S. Ebrahimi, K. Ghafoori-Tabrizi, H. Rafii-Tabar, Computational Materials Science 71 (2013) 172-178.

[376] S. Joshi, I. Ghosh, S. Pokhrel, L. Mädler, W.N. Nau, ACS Nano 6 (2012) 5668-5679.

[377] C. Garrido, A.E. Aliaga, J.S. Gomez-Jeria, R.E. Clavijo, M.M. Campos-Vallette, S. Sanchez-Cortes, Journal of Raman Spectroscopy 41 (2010) 1149-1155.

[378] A.M. Vera, J.J. Carcamo, A.E. Aliaga, J.S. Gomez-Jeria, M.J. Kogan, M.M. Campos-Vallette, Spectrochimica Acta Part a-Molecular and Biomolecular Spectroscopy 134 (2015) 251-256. 
[379] A. Vallée, V. Humblot, C. Méthivier, C.M. Pradier J. Phyics ;. Condensed. Mater., .

[380] A.V. Verde, J.M. Acres, J.K. Maranas, Biomacromolecules 10 (2009) 2118-2128. 


\section{Appendix}

9.1 Amino Acid letter code:

G - Glycine (Gly)

P - Proline (Pro)

A - Alanine (Ala)

V - Valine (Val)

L - Leucine (Leu)

I - Isoleucine (Ile)

M - Methionine (Met)

C - Cysteine (Cys)

F - Phenylalanine (Phe)

Y - Tyrosine (Tyr)

W - Tryptophan (Trp)

$\mathrm{H}$ - Histidine (His)

K - Lysine (Lys)

$\mathrm{R}$ - Arginine (Arg)

Q - Glutamine (Gln)

$\mathrm{N}$ - Asparagine (Asn)

E - Glutamic Acid (Glu)

D - Aspartic Acid (Asp)

$\mathrm{S}$ - Serine (Ser)

$\mathrm{T}$ - Threonine (Thr)

9.2 List of acronyms (in alphabetical order).

$\begin{array}{ll}\text { AA } & \text { Amino acid(s) } \\ \text { AES } & \text { Auger Electron Spectroscopy } \\ \text { AFM } & \text { Atomic Force Microscopy }\end{array}$


ATR-IR Attenuated Total Reflection Infra-Red Spectroscopy

BMP Bone Morphogenetic Protein

BOMD Born Oppenheimer Molecular Dynamics

BSSE Basis Set Superposition Error

CP-MAS NMRSolid-state Cross-Polarization Magic Angle Spinning Nuclear Magnetic Resonance

CVD Chemical Vapor Deposition

DOS Density Of States

$\mathrm{E}_{\mathrm{ads}} \quad$ Adsorption energy

$\mathrm{E}_{\mathrm{b}} \quad$ Binding Energy (in photoemission experiments)

$\mathrm{E}_{\mathrm{e}} \quad$ Primary electron energy (in HREELS)

$\mathrm{E}_{\mathrm{i}} \quad$ Electrical field in the plane of incidence (in IRAS)

$\mathrm{E}_{\text {int }} \quad$ Interaction energy

$\mathrm{E}_{\text {loss }} \quad$ Energy loss (in HREELS)

$\mathrm{E}_{\mathrm{is}} \quad$ Electrical field normal to the plane of incidence (in IRAS)

EHT Extended Hückel Theory

FF $\quad$ Force Field

FTIR Fourier Transform Infra Red

FT-RAIRS Fourier Transform RAIRS

GBP Gold Binding Peptide

GGA Generalized Gradient Approximation

$\mathrm{GGA}+\mathrm{U}$

HAS Helium Atom Scattering

HMI High Miller Index

HREELS High Resolution Electron Energy Loss Spectroscopy

IRAS Infra-Red Absorption Spectroscopy

L Langmuir

LDA Local Density Approximation

L-DOPA L-3,4-dihydroxyphenylalanine

LEED Low Energy Electron Diffraction 
LMI Low Miller Index

MD Molecular Dynamics

MEIS Medium Energy Ion Scattering

ML Monolayer

MM Molecular Mechanics

MMFF Molecular Mechanics Force Field

MPS Molecular Precursor State

NEXAFS Near Edge X-ray Absorption Fine Structure

NGBP Non Gold Binding Peptide

NMR Nuclear Magnetic Resonance

PES Photoelectron Spectroscopy

PhD Photoelectron Diffraction

PM-IRRAS Polarization Modulation IRAS

QCM Quartz Crystal Microbalance

QM Quantum Mechanics

QM/MM Quantum Mechanics/Molecular Mechanics

QMS Quadrupole Mass Spectrometer

RAIRS Reflection Absorption Infra-Red Spectroscopy

RT Room Temperature

S Sticking coefficient

SAM Self Assembled Monolayer

SERS Surface Enhanced Raman Scattering

SFG Sum Frequency Generation spectroscopy

SPM Scanning Probe Microscopy

SPR Surface plasmon resonance

SR-XPS X-ray Photoemission Spectroscopy performed with Synchrotron Radiation

STM Scanning Tunnelling Microscopy

t Time

T Sample temperature

$\mathrm{T}_{\mathrm{ev}} \quad$ Sample temperature during AA evaporation 
$\mathrm{T}_{\text {sub }} \quad$ Sublimation temperature

TDS Temperature Desorption Spectroscopy

TPD Temperature Programmed Desorption

TS Transition State

UHV Ultra High Vacuum

UPS Ultraviolet Photoemission Spectroscopy

vdW-DF van der Waals - Density Functional

WE Water Environment model

WL Water Layer model

XES X-ray Emission Spectroscopy

XPS X-ray Photoemission Spectroscopy

$\chi \quad$ Total exposure

$\Delta \mathrm{E}_{\mathrm{ads}} \quad$ Variation in the adsorption energy

$\Delta \mathrm{t} \quad$ Time increment

$\phi \quad$ Angle of incidence (with respect to the surface normal)

$\Theta, \theta \quad$ Total coverage

$\Theta_{\text {sat }} \quad$ Saturation coverage 